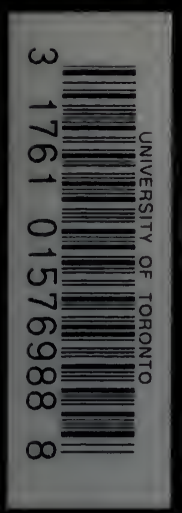





$$
\text { . }
$$


Digitized by the Internet Archive in 2008 with funding from Microsoft Corporation 



$$
\begin{array}{r}
\text { "Science, which biings man nearer } L \text { God" } \\
\text { Pastene }
\end{array}
$$

\title{
VERTEBRATE EMBRYOLOGY
}

\author{
MARSHALI
}




\section{BY THE SAME AUTHOR.}

Fourth Edition, Revised and Illustrated. Crown 8vo. $4 \mathrm{~s}$.

THE FROG: an Introduction to Anatomy, Histology, and Embryology.

\section{DR. MILNES MARSHALL AND C. HERBERT HURST.}

\section{A JUNIOR COURSE OF PRACTICAL ZOOLOGY.}

By A. Milnes Marshaul, M.D., D.Sc., M.A., F.R.S., Professor in the Victoria University; Beyer Professor of Zoology in Owens College ; late Fellow of St. John's College, Cambridge. Assisted by C. Herbert Hurst, Ph.D., Lecturer in the Victoria University; Demonstrator and Assistant-Lecturer in Zoology, Owens College, Manchester. Third Edition, Revised. With additional Illustrations. Crown 8vo. 10s. $6 \mathrm{~d}$.

'This book cannot fail to be of great value to those who are studying zoology in their laboratory work; and to such ue have great pleasure in strongly recommending it.'-LONDOX MEDICAL RECORD.

'We have, in all, a most successful

and important book. ... The illustrations are excellent, reflecting the greatest credit upon all concerned. ... The book is highly welcome and most admirable. It is provided with an exceedingly good index. and presented in a form demanding our sincere thanks.'-NATURE.

London: SMITH, ELDER, \& CO., 15 Waterloo Place. 


\section{VERTEBRATE EMBRYOLOGI}

A TEXT-BOOK

FOR STUDENTS AND PRACTITIONERS

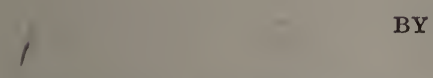

A. MILNES MaRSHaLL, M.D., D.Sc., M.A., F.R.S.

PROFESSOR IN THE VICTORIA UNIVERSITY; BEIER PROFESSOI

OF ZOOLOGY IN OWENS COLLEGE; LITE FELLOW OF

ST JOHN'S COLLEGE, CAMBRIDGE

\section{LONDON}

SMITH, ELDER, \& CO., 15 WATERLOO PLACE 1893 


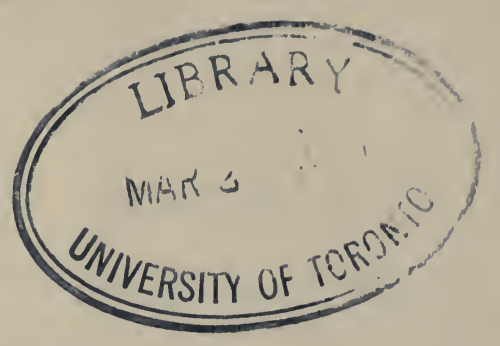

QL
959

M37 


\section{PREFACE.}

GREAT attention has of recent years been given to the study of Embryology, and yet it is curiously difficult to find straightforward accounts of the development even of the commonest animals. The special memoirs and monographs are usually limited to particular phases in the life-history of the forms with which they are concerned; while the text-books of embryology aim rather at explaining the general progress of development within the several groups than at supplying complete descriptions of individual examples.

Up to the present time there has been no reasonably complete account of the development of the common frog, or of the rabbit, in our own or in any other language; while in works professing to deal with human embryology it is more common than not to find that the descriptions, and the figures given in illustration of them, are really taken, not from human embryos at all, but from rabbits, pigs, chicken, or even dogfish.

This latter practice is a most unfortunate one, and has been the cause of much confusion. The student is led to suppose that our knowledge is more complete than is really the case, while at the same time he finds the greatest difficulty in obtaining definite information on any particular point in which he is interested. Moreover, the implication that the details of development are identical in members of the same or of allied groups is directly opposed to the results of recent investigations, which are showing more and more clearly that marked differences, 
both in the earlier and later stages of development, may occur between allied genera and species, or even amongst individual members of the same species.

The present book is an attempt to fill the gap, thus indicated, so far as the elements of Vertebrate Embryology are concerned. In it a few selected types are alone dealt with, and to each of these a separate chapter is devoted.

In the choice of types I have been mainly guided by the following considerations. Amphioxus is taken first, partly on account of its great morphological importance, and partly because of the extreme simplicity of its earlier developmental history, and of the clue which this affords to the more complicated conditions obtaining in the higher vertebrates. The next three chapters deal with the frog, the chick, and the rabbit respectively; these have been selected as good representatives of the classes to which they belong, and as being the most easily obtained and the most suitable forms for laboratory purposes. The final chapter, and the longest in the book, is devoted to the development of the Human Embryo; this has been included on account of its great intrinsic interest, and of the difficulty the student experiences, owing to the scattered and comparatively inaccessible nature of the original memoirs, in obtaining a reliable account of the present state of our knowledge. I have taken much pains to make this chapter as complete as our knowledge will allow, and venture to hope that it will be found useful not only by students of science and of medicine, but also by those engaged in medical practice.

I have not attempted to write a series of complete monographs; my purpose has been to give consecutive and straightforward accounts which shall contain, in a form convenient for reference, the main facts known to us concerning the development of the animals I have selected as types. Many points of detail have been purposely omitted, as have also some of the more recent statements which appear to me to require confirmation. Science is better served by clearly stating in what points our knowledge is defective than by ignoring or evading difficulties; 
and I have purposely emphasised the more important of these gaps in the hope of drawing to them the attention of those who may have opportunity of filling up the deficiencies. The bibliographical lists at the ends of the several chapters have been deliberately curtailed, and include only those books and papers which appear to me of real importance: my object in this, as indeed in all respects, has been to produce a book which shall be useful rather than encyclopædic.

I have, in the text, made no attempt to assign the several statements to their original authors: to do so would have burdened the book unduly. It will be well, however, to give here the main sources from which the facts are gathered, in order that I should not receive credit which is really due to others.

In the chapter on Amphioxus I have had to rely entirely on the work of other observers. The descriptions of the earlier stages are from the well-known accounts by Kowalevsky and by Hatschek: for the later stages I have depended mainly on the recent researches of Professor Lankester and Mr. Willey.

Except as regards the processes of maturation and fertilisation of the egg, which are described from Oskar Schultze's papers, the chapter on the Frog is based almost entirely on my own observations, supplemented by the work of some of my pupils.

'The development of the Chick has been described more often than that of any other animal. I have, however, worked over the greater part of the ground again, with special reference to this book. I have derived much assistance from the researches of Duval, especially in regard to the earlier stages of derelopment.

I have not myself studied the processes of segmentation, and formation of the blastodermic vesicle in the Rabbit; but I have had the advantage of examining a very excellent series of preparations by my friend Mr. Assheton. In my descriptions of these earlier stages I have relied mainly on the accounts of E. van Beneden, and of Kölliker. The later stages, from the first appearance of the embryo onwards, I have studied in con- 
siderable detail, and the descriptions are mainly from my own observations. In the study of the placenta, especially in its earlier stages, I have been greatly helped by Duval's careful investigations.

In the cliapter dealing with the Human Embryo I have been compelled to obtain my material almost entirely from the observations of others; and notably from the splendid and longcontinued work of Professor His, to whom it is due that our knowledge of human enibryology is in so many respects more precise than that of any other mammal. It is a source of great regret to me that my friend Professor Minot's important treatise on Human Embryology only came into my hands while the last sheets of my own book were passing through the press, and that I have been unable to avail myself of the rich store of facts, and of the numerous suggestive explanations which his work contains.

A large proportion of the figures are new, and have been made expressly for this book from my own drawings. In the new figures, as well as in a large number of those which $I$ have copied from the works of others, I have adopted, so far as practicable, a uniform mode of treatment and of lettering, which will, I hope, facilitate comparison of the figures of the several types with one another. I am under great obligations to the publishers of the works from which figures have been borrowed, for permission to reproduce these; and more especially to Messrs. Vogel, of Leipzig, for their ready consent to supply electrotypes, and to allow a large number of figures to be copied from Professor His' great monograph on the development of the Human Embryo; a courteous liberality that is not always to be met with in this country.

I wish also to record my indebtedness to my friends Dr. Robinson and Mr. Assheton for many valuable suggestions and criticisms in the course of the work, and for much kind assistance in the correction of the proofs. To Mr. P. Hundley and Mr. G. Pearson, by whom the drawings on the wood were made, and the blocks engraved, my thanks are due for the great care 
they have bestowed on what, in my judgment, is one of the most important parts of the book.

I shall be very grateful for corrections or suggestions from those who use the book. I would further venture to make an earnest appeal for assistance to those who have opportunity of obtaining human embryos, and who do not require them for their own purposes. Our knowledge of the early stages of development of the human embryo is still very imperfect, and it is of the utmost importance that any opportunities that may occur of extending it should not be lost. Embryos of any age, but more particularly those of the first month or six weeks, would be of the greatest service to myself: they should be put into strong spirit as quickly as possible, a little cotton-wool being placed in the bottle to support the embryos, and to prevent them from shaking about during transit; and any acts, such as the date of the last occurring menstruation, which would aid in determining the age, should be carefully recorded.

A. M. M.

()wexs College: March 1893. 



\section{CONTENTS.}

\section{CHAPTER I}

INTRODUCTION

General account of the development of animals-Structure of the egg-

Maturation or ripening of the egg-Fertilisation of the egg-The early stages of development of the embryo-Theory of fertilisationSegmentation of the egg-The germinal layers-The general history of development-The recapitulation theory-The origin of sexBibliography

\section{CHAPTER II}

\section{AMPIIIOXUS}

Structure of the adult Amphioxus-Morphological importance of Amphioxus-General account of the development of Amphioxus-The early embryonic development-The condition at the time of hatching -The later embryonic development-The condition at the close of the embryonic period-The larval period-The adolescent periodBibliography

\section{CHAPTER III}

\section{THE FROG}

General account of the development of the frog-The egg-The early stages of development-The nervous system - The sense organs-The alimentary canal-The gill-clefts and the gills-The heart and bloodvessels-The urinary and reproductive organs-The skeleton and teeth-Bibliography . . . . . . . . .

\section{CHAPTER IV}

\section{THE CHICK}

General account of the development of the chick-The egg-The early stages of development-The nervous system-The sense organs-The alimentary canal-The heart and blood-vessels-The urinary organs - The body cavity and the muscular system-The skeleton-The feathers-Bibliography 


\section{CHAPTER V}

\section{THE RABBIT}

Preliminary account of the development of the rabbit-The egg-The early stages of development-General history of the embryo-The nervous system-The sense organs-The digestive system-The heart and blood-vessels-The urinary organs-The colom-The muscular system-The skeleton-The skin-The placenta-Bibliography .

\section{CHAPTEIR VI \\ THE IIUMAN EMBRYO}

Preliminary account of the development of the human embryo--The human ovum-General history of the human embryo-The nervous system-The sense organs-The digestive system-The heart and blood-vessels-The urinary organs-The reproductive organs-The fœtal membranes and the placenta-Bibliography . . . .

INDEX 


\section{LIST OF ILLUSTRATIONS.}

\section{INTRODUC'TORY CHAPTER}

1. Stages in the maturation of the egg of the frog. (After O. Schultze) 9

2. Segmentation of the egg of Amphioxus. (After Hatschek) . 18

3. Segmentation of the frog's egg . . . . . . . . 20

4. The hen's egg, freshly laid . . . . . . . . . 21

5. Segmentation of the germinal disc of the hen's egg. (After Coste and Duval) . . . . . . . . . . . 21

6. Later stage in the segmentation of the germinal disc of the hen's egg. (After Coste and Duval) . . . . . . . 21

7. Vertical section of the germinal disc of the hen's egg at the close of segmentation. (After Duval) . . . . . . . 21

8. Vertical section of early larval stage of Amphioxus. (After Hatschek) • . . . . . . . . .

9. Horizontal section of early larval stage of Amphioxus. (After Hatschek) . . . . . . . . . . .

10. Transverse section through the head of a chick embryo at the end of the first clay of incubation . . . . . . . 24

\section{AMPHIOXUS}

11. Young specimen of Amphioxus, viewed as a transparent object. (From Marshall and Hurst) . . . . . . .

12. Transverse section through the anterior part of the pharynx of an adult Amphioxus. (From Marshall and Hurst). . . .

13. Transverse section through the posterior part of the pharynx of an adult female Amphioxus. (From Marshall and Hurst) . . 42

14. Segmentation of the egg of Amphioxus. (After Hatschek) . $\quad 50$

15. Formation of the gastrula of Amphioxus. (After Hatschek) . - 53

16. Later stage in the formation of the gastrula of Amphioxus. (After

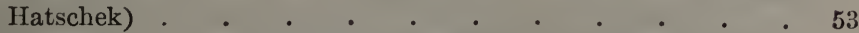

17. Further stage in the formation of the gastrula of Amphioxus. (After Hatschek) • • • • . • . . . . . 54

18. Completion of the gastrula of Amphioxus. (After Hatschek) . . $\quad 54$

19. The gastrula of Amphioxus, bisected vertically. (After Hatschek) . $\quad 56$

20. The gastrula of Amplioxus, bisected horizontally. (After Hatschek) 56

21-24. Transverse sections across the bodies of Amphioxus embryos, showing the mode of formation of the nervous system and of the mesoblastic somites. (After Hatschek) . . . . 58 
Fu.

25. Amphioxus embryo at the time of hatching; bisected vertically.

(After Hatschek) Amphiuxus embryo at the time of hatching; bisected horizontally.
26. (After Hatschek)

27. Amphioxus embryo shortly after hatching; seen in optical section from the right side. (After Hatschek)

28 and 29. Transverse sections of Amphioxus embryos shortly after hatching; showing stages in the formation of the notochord and mesoblastic somites. (After Hatschek) . . . . .

30. Amphioxus embryo with nine pairs of mesoblastic somites, seen in optical section from the right side. (After Hatschek) . .

31. Amphioxus embryo with nine pairs of mesoblastic somites, scen in horizontal section. (After Hatschek). . . . . .

32. Transverse section through the midclle of an Amphioxus embryo with nine pairs of mesoblastic somites. (After Hatschek)

33. Amphioxus embryo with fourteen pairs of somites; seen in optical section from the right side. (After Hatschek) .

34. Amphioxus larva at the commencement of the second or 'larval' period of development. (After Hatschek).

35. Young Amphioxus during the 'adolescent' period. (After Kowalevsky)

36. The anterior end of an Amphioxus larra with four primary gill-slits, seen from the left side. (After Lankester and Willey) . .

37. The anterior end of an Amphioxus larva with fourteen primary gillslits, seen from the right side. (After Willey).

38. The anterior end of an Amphioxus larva with thirteen primary and eight secondary gill-slits, seen from the right side. (After Willey)

39. The anterior end of an Amphioxus larva with twelve primary gillslits, of which the first and twelfth are disappearing, and eight secondary gill-slits; seen from the ventral surface. (After Willey)

40. Diagrammatic transverse section across an Amphioxus larva with eleven or twelve primary gill-slits, but no secondary ones. (Slightly modified from Lankester and Willey) .

41. Diagrammatic transverse section through an advanced Amphioxus larva with fully formed atrial cavity. (Slightly modified from Lankester and Willey, and from Boveri)

42. Diagrammatic transverse section across the intestinal region of an Amphioxus larva with five primary gill-slits. (After Hatschek)

43. Diagrammatic transverse section across a young Amphioxus immediately after the completion of the larval period. (After Hatschek)

\section{THE FROG}

44. Various stages in the development of the frog. (From Brehm's 'Thierleben')

45. Stages in the maturation of the egg of the frog. (After O. Schultze)

46-48. Segmentation of the frog's egg 
Fir.

49. 'The blastula stage in the development of the frog's egg . . . 103

50. The frog's egg at the close of segmentation . . . . . 103

51. Meclian sagittal section of a frog embryo, showing the spreading of the epiblast, and the commencing formation of the mesenteron.

52. Sagittal section of a frog embryo during the formation of the mesenteron . $\cdot$. $\cdot$. $\cdot$. .

53. Horizontal section of a frog embryo during the formation of the mesenteron . . . . . . . .

54. Sayittal section of a frog embryo just before the disappearance of the segmentation cavity . . . . . .

55. Sagittal section of a frog embryo after the disappearance of the segmentation cavity and completion of the mesenteron . .

5li. A transverse section through the middle of a frog embryo at about the stage represented in Fig. 55 . . . . . .

5i. $\Lambda$ frog embryo at the time of appearance of the neural folds: seen from the dorsal surface

5. Stages in the early development of the frog embryo, seen obliquely from the hinder end. (From a series of wax models by Dr. F. Ziegler of Freiburg i/B)

59. Transverse section through a frog embryo, showing the neural folds shortly before they meet each other to complete the neural tube

60. Sagittal section of a frog embryo, shortly before closure of the blastopore . . . . . . . . . . .

61. Sagittal section of a frog embryo, shortly after closure of the blastopore and formation of the anus . . . . . . 117

62. The brain of the adult frog: dorsal surface . . . . . 119

(3i). The brain of the adult frog: ventral surface . . . . . . 119

64. Sagittal section of the head end of a tadpole, just before the opening of the mouth $\quad$. . . . . . . . .

(55. Sagittal section through the head and body of a tadpole of $12 \mathrm{~mm}$. length, at the time of appearance of the hind limbs . . .

66. Diagrammatic horizontal section of a $12 \mathrm{~mm}$. tadpole, at the time of appearance of the hind limbs . . . . . . .

6i. Transverse section through the head of a tadpole of $6 \frac{1}{2} \mathrm{~mm}$. length, about the time of hatching. . . . . . .

68. Transverse section across the posterior part of the head of an adult frog, showing the position and relations of the auditory organs, Eustachian tube, and hyoid apparatus . . . . .

69. Sagittal section through a tadpole at the time of hatching . . 146

70. Transverse section across the middle of the length of a frog embryo $3 \frac{i}{2} \mathrm{~mm}$. in length . • . . . . . . . 147

71. Horizontal section of the head and body of a $12 \mathrm{~mm}$. tadpole . . 15\%

72. Side view of a tadpole at the time of hatching . . . . . 157

73. Ventral view of a tadpole at the time of hatcling . . . . $15 \pi$

it. Horizontal section of a tadpole at the time of hatching . . . 158

75. Transverse section through the head of a $12 \mathrm{~mm}$. tadpole . . . 162

76. Diagrammatic figure of a $12 \mathrm{~mm}$. tadpole, about the time of appearance of the hind limbs $. \quad . \quad . \quad . \quad . \quad . \quad . \quad .166$

77. Diagrammatic figure of the head and anterior part of the body of a 
FIG.

$7 \mathrm{~mm}$. tadpole shortly after hatching; showing the branchial blood-vessels from the ventral surface

PAGL

78. Diagrammatic figure of the same embryo as in fig. 77 , seen from the
right side

78. Diagrammatic figure of the same embryo as in fig. 77 , seen from the
right side

79. Diagrammatic transverse section across the head of a $7 \mathrm{~mm}$. tadpole

80. Diagrammatic figure of the head of a $12 \mathrm{~mm}$. tadpole from the right side, showing the heart and branchial blood-vessels . . .

81. Diagrammatic figure of the arterial system of an adult frog . hatehing, showing the nephrostomes of the head-kidney . 170

82. Transverse section through the body of a tadpole at the time of

83. Diagrammatic figure of a $12 \mathrm{~mm}$. tadpole dissected from the ventral surface, to show the heart and branchial vessels, and the headkidneys and commencing Wolffian bodies . . . . .

84. Transverse section across a $12 \mathrm{~mm}$. tadpole, passing through the middle of the head-kidney . . . . . . .

85. A $40 \mathrm{~mm}$. tadpole dissected from the ventral surface, to show the heart, the branchial vessels, and the urinary and reproductive organs . . . . . . . . . . .

86. A tailed frog, during the metamorphosis, dissected from the ventral surface to show the urinary and reproductive organs .

87. Transverse section through the hinder part of the body of a tailed frog during the metamorphosis . . . . . . .

88. Transverse section through the anterior part of the body of a tailed frog during the metamorphosis . . . . . . . 195

89. Sagittal section of a tailed frog during the metamorphosis . . 200

90. The skull of a $12 \mathrm{~mm}$. tadpole, seen from the right side . . 202

91. The skull of a $12 \mathrm{~mm}$. tadpole, from the dorsal surface . . 202

92. The skull of a $12 \mathrm{~mm}$. tadpole, from the ventral surface . . . 202

93. The skull of a tailed frog, towards the close of the metamorphosis, seen from the right side . . . . . . . 208

94. The skull of an adult frog, seen from the right side . . . . 209

95. The skull of an adult frog, seen from the ventral surface . . . 210

96. The skeleton of the frog, seen from the dorsal surface . . . 214

\section{THE CHICK}

97. The hen's egg at the time of laying - 98 . The yolk of a hen's egg at the thirty-sixth hour from the commencement of incubation

99. The yolk of a hen's egg at the end of the third day of incubation . 100. The hen's egg at the end of the fifth day of incubation . . 101. The hen's egg at the end of the ninth day of incubation . .

102. An early stage in the segmentation of the germinal disc of the hen's egg. (After Coste and Duval) . . . . . . .

103. A later stage in the segmentation of the germinal disc of the hen's egg. (After Coste and Duval) .

104. Section through the germinal disc and adjacent parts of the yolk of a hen's egg about the middle of its stay in the uterus. (After Duval).

105. Vertical section of the blastoderm and adjacent part of a hen's egg towards the close of segmentation. (After Duval) . . 
106. Vertical section of the blastoderm and adjacent part of the yolk of a hen's egg at the time of laying, but before the commencement of incubation. (After Duval) . . . . . . .

107. A diagrammatic figure of the blastoderm of a hen's egg about the twentieth hour of incubation. (In part after Duval) . .

108. Transverse section across the blastoderm of a hen's egg about the twentieth hour of incubation . . . . . .

109. A diagrammatic figure of the blastoderm of a hen's egg about the twenty-fourth hour of incubation. (In part after Duval) . .

110. A chick embryo at the twenty-fourth hour of incubation; seen from the dorsal surface . . . . . . . . .

111. A chick embryo at the thirty-sixth hour of incubation; seen from the dorsal surface . . . . . . . . .

112. A median longitudinal, or sagittal, section of a chick embryo at the thirty-sixth hour of incubation . . . . . . 251

113. $A$ chick embryo at the end of the third day of incubation . . 253

11t. A median longitudinal, or sagittal, section through a chick embryo at the end of the third day of incubation . . . . 255

115. A chick embryo at the end of the fifth day of incubation . .

116. A median longitudinal, or sagittal, section of the head and anterior part of the neck of a chick embryo at the end of the eighth day of incubation . . . . . . . . .

117. Transverse section across the body of a chick embryo at the twentyfourth hour of incubation. . . . . . .

118. Transverse section across the head of a chick embryo at the twentyfourth hour of incubation. . . . . . .

119. Transverse section across the head of a chick embryo at the fortythird hour of incubation . . . . . . . .

120. Transverse section across the head of a chick embryo at the fortythird hour of incubation, the section passing through the commencing auditory pits and the heart . . . . . .

121. Transverse section across the head of a chick embryo at the fortyeighth hour of incubation . . . . . . .

122. Transverse section across the fore-brain and eye of a chick embryo at the sixtieth hour of incubation . . . . . .

123. A median longitudinal, or sagittal, section through a chick embryo at the end of the fifth day of incubation . . . . .

124. A section through the head of a chick embryo at the end of the third day of incubation . . . . . . . . .

125. The head of an embryo chick at the end of the fifth day of incubation . . . . . . . . . .

126. The head of an embryo chick at the end of the seventh day of incubation . . . . . . . . .

127. The anterior end of a chick embryo at the thirty-sixth hour of incubation. . . . . . . . . .

128. A diagrammatic figure showing the arrangement of the bloodvessels in a chick embryo at the end of the fifth day of incubation.

129. A transverse section across the body of a chick embryo at the fortyeighth hour of incubation 
FIG.

130. The left lialf of the skeleton of the common fowl. (From Marshall and Hurst) . . . . . . . . .

131. The skull of a chick embryo at the end of the eighth day of incubation .

132. The skull of the fowl, from the right side. (From Marshall and Hurst) .

\section{THE RABBI'T}

133. Section through part of the ovary of an adult rabbit

134. A fully formed ovum of a rabbit, shortly before its discharge from the ovary. (After Bischoff)

135. A rabbit's ovum, from the upper end of the oviduct, after extrusion of the two polar bodies. (After Bischoff).

136. A rabbit's ovum, about twenty-two hours after copulation, showing division of the ovum into two cells. (After Bischoff)

137. A rabbit's ovum about the middle of the third day, showing the morula stage, shortly before the completion of segmentation. (After Bischoff) *

138. A rabbit's ovum seventy hours after copulation, showing the condition at the close of segmentation. (After Van Beneden). .

139. A rabbit's ovum seventy-five hours after copulation, showing the first stage in the formation of the blastodermic vesicle. (After Van Beneden)

140. Section of the blastodermic vesicle of a rabbit at the end of the fourth day. (After Van Beneden) . . . . . .

141. A vertical section across the embryonal area of the blastodermic vesicle of a rabbit at the end of the fifth day. (After Kölliker)

142. A transverse section across the hinder part of the embryonal area of a rabbit embryo at the end of the seventh day. (After Kölliker)

143. The blastodermic vesicle of a rabbit at the end of the seventh day. (Modified from Kölliker) . . . . . . . .

144. 'The embryonal area of a rabbit at the middle of the eighth day. (Modified from Kölliker)

145. A rabbit embryo and blastodermic vesicle at the end of the nintl day

146. A median longitudinal, or sagittal, section through a rabbit embryo and blastodermic vesicle at the end of the ninth day. (In part after Van Beneden and Julin)

117. A rabbit embryo and blastodermic vesicle at the end of the tenth day. (In part after Van Beneden and Julin) . . . .

148. A rabbit embryo and fotal appendages at the end of the twelfth day. (In part after Van Beneden and Julin) . . . . 366

149. A rabbit embryo of the twentieth day . . . . . . 367

150. A median longitudinal, or sagittal, section through a rabbit embryo at the end of the twelfth day

151. A median longitudinal, or sagittal, section through the head of a rabbit embryo of the eighteenth day .

152. The brain of an adult rabbit, dissected from above. (From Marshall and Hurst) .

153. A median longitudinal, or sagittal, section of the brain of an adult rabbit. (From Marshall and Hurst). 
154. The brain of an adult rabbit from the ventral surface. (From Marshall and Hurst) . . . . . . . . .

155. A transverse section across the head of a rabbit embryo of the fourteenth day . . . . . . . . . . 388

156. A transverse section across the head of a rabbit embryo of the twenty-first day . . . . . . . . . .

157. A diagrammatic section across the head of an adult rabbit, to show the relations of the internal ear, tympanic cavity and membrane, and the auditory ossicles. (From Marshall and Hurst) . .

158. A transverse section across the head of a rabbit embryo at the end of the eleventh day, the section passing through the medulla oblongata, the ears, and the pharynx . . . . .

159. A transverse section across the head of a rabbit embryo of the fifteenth day, passing through the medulla oblongata, the ears, and the pharynx .

160. A diagrammatic view of an adult male rabbit from the left side. (From Marshall and Hurst). . . . . . . .

161. A rabbit embryo at the end of the twelfth day, seen from the right side . . . . . . . . . .

162. The skull of the rabbit from the right side. (From Marshall and Hurst). . . . . . . . . . .

163. A transverse section across the thorax of a rabbit embryo of the sixteenth day . . . . . . . . . . . 409

164. A transverse section across the body of a rabbit embryo of the early part of the tenth day, showing the supposed epiblastic origin of the Wolffian duct. (After Hensen) . . . .

165. A transverse section across the body of a rabbit embryo at the end of the eleventh day . . . . . . . .

166. A transverse section across the hinder part of the body of a rabbit embryo of the fourteenth day . . . . . . . 424

167. Selected vertebræ from the rabbit. (From Marshall and Hurst) . 431

168. A transverse section across the uterus, with the contained blastodermic vesicle, of a rabbit at the end of the seventh day. (In part after Duval). . . . . . . . . .

169. A transverse section across the uterus and the contained blastodermic vesicle of a rabbit at the end of the ninth day. (In part after Duval) . . . . . . . . . .

170. A transverse section across the uterus and the contained embryo of a rabbit at the end of the nineteenth day . . . .

\section{THE HUMAN EMBRYO}

171. Part of a vertical section of the ovary of a new-born infant. (From Stricker's 'Histology') . • . . . . . . . 451

172. Front view of Reichert's ovum. (From Kölliker, after Reichert) . 472

173. Side view of Reichert's ovum. (From Kölliker, after Reichert) . 472

174. Diagrammatic section of Reichert's ovum. (From His) . . . 473

175. A longitudinal section of the uterus, with an ovum in situ, estimated as about the thirteenth day. (After Kollmann) . . 474

176. Outline figure of a human embryo lettered by Professor His, E, and estimated as about the thirteenth day. (From His) . • . 477 
FIC.

177. Outline figure of a human embryo deseribed by Allen 'Thomson, and estimated as about the thirteenth day. (From His) . . .

178. Outline figure of a human embryo, lettered by Professor His, S R, and estimated as of the thirteenth day. (From His) . .

179. Human embryo lettered by Professor His, S R, and estimated as of the thirteenth day. (After His). . . . . . .

180. Human embryo of about the thirteenth day, from the left side.

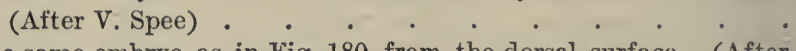

181. The same embryo as in Fig. 180, from the dorsal surface. (After V. Spee)

182. Transverse section across the head end of the human embryo shown in Figs. 180 and 181. (After V. Spee)

183. Transverse seetion across the middle of the body of the human embryo shown in Figs. 180 and 181. (After V. Spee)

184. Transverse section aeross the hinder end of the human embryo shown in Figs. 180 and 181. (After V. Spee) . . . .

185. Human embryo of about the fourteenth day, from the right side. (After Kollmann)

186-188. Diagrammatic longitudinal sections through human embryos, representing hypothetical stages intermediate between Reichert's ovum and His' embryos, E or S R. (From His).

PAGE

189. Outline figure of a human embryolettered by Professor His, Lg, and estimated as fifteen days old. (From His). . . . .

190. Outline figure of a human embryo lettered by Professor His, Sch, and estimated as fifteen days old. (From His).

191. Outline figure of a human embryo lettered by Professor His, M, and estimated as eighteen days old. (From His) . . . . 487

192. Outline figure of a human embryo figured by Allen Thomson, and probably about eighteen days old. (From His) . . . 487

193. Outline figure of a human embryo lettered by Professor His, B B, and estimated as about eighteen days old. (From His) . .

194. Outline figure of a human embryo lettered by Professor His, Kln, and estimated as about twenty days old. (From His) .

195. Outline figure of a human embryo lettered by Professor His, Lr, and estimated as twenty or twenty-one days old. (From His).

196. Human embryo at the commencement of the third week. (From His, after Coste) . . . . . . . . .

197. Human embryo lettered by Professor His, Lg, and estimated as - fifteen days old. (After His) . . . . . .

198. Human embryo lettered by Professor His, Lr, and estimated as twenty or twenty-one days old. (After His) . . . .

199. Outline figure of a human embryo figured and described by Coste, and estimated as about twenty-three days old. (From His) .

200. Outline figure of a human embryo lettered by Professor His, a, and estimated as about twenty-three days old. (From His) • .

201. Outline figure of a human embryo figured and described by Allen Thomson, and estimated as about twenty-three days old. (From His) . . . . . . . . . .

202. Outline figure of a human embryo lettered by Professor His, B, and estimated as twenty-seven days old. (From His) 
FIG.

203. Outline figure of a human embryo lettered by Professor His, A, and estimated as twenty-seven days old. (From.His) . . . 493

204. Human embryo lettered by Professor His, A, and estimated as twenty-seven days old. (After His) . . . . . . 494

205. Outline figure of a human embryo lettered by Professor His, Rg, and estimated as thirty-two or thirty-three days old. (From His) .

206. The under surface of the head of a human embryo lettered by Professor His, Hn, and estimated as about twenty-nine days old. (After His). . . . . . . . .

207. The under surface of the head of a human embryo lettered by Professor His, C.II., and estimated as about thirty-four days old. (After His). . . . . . . . • .

208. The left ear of a human embryo lettered by Professor His, Br. 2, and estimated as thirty-five days old. (From His) . . . .

209. The left ear of a human embryo lettered by Professor His, Dr, and estimated as thirty-eight days old. (From His). . . .

210. A pregnant uterus of about the fortieth day. (From Kölliker, after Coste). . . . . . • . • •

211. Outline figure of a human embryo about the middle of the sixth week. (From His) . . . . . . . . .

212. Outline figure of a human embryo at the end of the second month. (From His) . . . . . . . . . . .

213. Head of a human embryo at the end of the seventh week. (After His) . . . . . . . . . .

214. Head of a human embryo at the end of the second month. (After His) .. . . . . . . . . . .

215. The head and fore part of the body of a human embryo lettered by Professor His, Lr, and estimated as twenty or twenty-one days

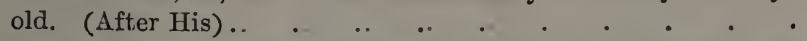

216. Human embryo lettered by Professor His, Pr, and estimated as twenty-eight days old. The brain is exposed from the left side, and the body of the embryo has been dissected to show the heart and aortic arches.and the alimentary canal. (After His)

217. The brain of a human embryo lettered by Professor His, ZW, and estimated as about the middle of the eighth week. (After His)

218. A human foetus three months old, dissected from the dorsal surface to expose the brain and spinal cord. (From Kölliker) . .

219. The brain of a human fœetus three months old, from the right side. (From Kölliker) . . . . . . . . . .

220. The brain of a human foetus three months old, dissected from the dorsal surface. (From Kölliker) . . . . . .

221. The brain of a human fœetus three months old, from the ventral surface. (From Kölliker) . . . . . . . . 515

222. The brain and spinal cord of a human fœitus four months old, from the dorsal surface. (From Kölliker) . . . • . .

223. The brain of a human fotus six months old, from the right side. (From Kölliker) . . . . . . . .

224. The brain of a human fotus of the fifth month, bisected, and seen from the inner surface. (From Kölliker) . . . • .

225. A transverse section through a portion of the wall of the spinal 
FIG.

cord of a human embryo at the beginning of the fourth week. (After His) . . . . . . . . . . 522

226. A diagrammatic transverse section across the spinal cord of a human embryo of the fourth week. ( $\Lambda$ fter His)

227. A diagrammatic figure of a human embryo lettered by Professor $\mathrm{His,} \mathrm{Ko}$, and estimated as thirty-one days old. The figure shows the brain and spinal cord, and the cranial and spinal nerves. (After His) .

228. Transverse section across the medulla oblongata of a human embryo lettered by Professor His, Ko, and estimated as thirty-one days old. The section passes through one of the roots of the hypoglossal nerve, and through both the motor and sensory roots of the pneumogastric nerve. (After His)

229. The left auditory vesicle of a human embryo four weeks old. (After W. His, jun.)

230. The left auditory vesicle of a human embryo five weeks old. (After W. His, jun.)

231. The left auditory vesicle, or internal ear, of a human embryo of the eighth week. (After W. His, jun.) • . . . . .

232. Human embryo lettered by Professor His, Lg, and estimated as fifteen days old. The brain and heart are exposed from the right side; the alimentary canal and the yolk-stalk are represented in median sagittal section. (After His)

233. Outline figure of the alimentary canal of a human embryo lettered by Professor His, Pr, and estimated as twenty-eight days old. (From His).

234. Outline figure of the alimentary canal of a human embryo lettered by Professor His, Sch, and estimated as thirty-five days old.

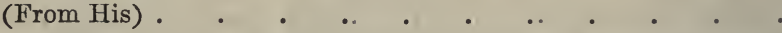

235. Outline figure of the alimentary canal of a human embryo estimated as thirty-two days old. (From His) . . . . . .

236. Outline figure of the alimentary canal of a human embryo estimated as thirty-five days old. (From His) . . . . . .

237. The floor of the pharynx of a human embryo fifteen days old, seen from above. (After His) . . . . . . .

238. The floor of the pharynx of a human embryo twenty-three days old, seen from above. (After His) . . . . . .

239. The floor of the pharynx of a human embryo twenty-eight days . old, seen from above. (After His) . . . . . .

240. The head and neck of a human embryo thirty-two days old, seen from the ventral surface. (After His) . . . . . 553

241. The roof of the mouth of a human embryo about two and a half months old, showing the formation of the palate. (After His).

242. The tongue and the floor of the mouth of a human embryo at the end of the second month. (After His) . . . . .

243. Human embryo lettered by Profesşor His, Bl, and estimated as twenty-three days old. The brain and spinal cord are exposed from the right side; and the body is dissected to show the heart, the blood-vessels, and the alimentary canal. (After His) 
24t. The dorsal half of the heart of a human embryo twenty-eight days old, seen from within. (After His) . . . . . . 569

245. The aortic arches of a human embryo thirty-two days old, from the left side. (After His). . . . . . . . . 576

246. The aortic arches of a human embryo thirty-five days old, from the left side. (After His) • . . . . . . . 577

247. The liver, and the veins in connection with it, of a human embryo twenty-four or twenty-five days old, seen from the ventral surface. (After His)

248. Transverse section across the body of a human embryo estimated as fourteen days old. (After Kollmann) . . . . . 589

24!). The adult ovary, parovarium and Fallopian tube. (From Quain's 'Anatomy,' after Kobelt) . . . . . . . . 595

250. The external genitalia of a human embryo of about the ninth week. (From Kölliker, after Ecker) . . . . . . . . 596

251. The external genitalia of a human embryo of about the tenth week. (From Kölliker, after Ecker) . . . . • • . 596

252. The external genitalia of a male human embryo towards the end of the third month. (From Kölliker, after Ecker). . . 597

25:3. The external genitalia of a female human embryo towards the end of the third month. (From Kölliker, after Ecker) . . . 597

25t. A diagrammatic section of the pregnant human uterus at the seventh or eighth week. (From Quain's 'Anatomy,' after Allen 'I'homson) • . . . . . . • . . .

255. A pregnant human uterus of about the twenty-fifth day. (From Quain's 'Anatomy', after Coste) . . . . . . . 608 

The connection between the formation of polar bodies and the process of fertilisation still remains to be explained. Such cases as those of the gipsy moth and the drone bee indicate that this connection is to be regarded rather as a normal than as a necessary one. Rapid cell division is an exhausting process, and Maupas has shown that in the Ciliate Infusoria the act of fission, which is the most frequent male of reproduction, although it commences and at first proceeds with great rapidity, after a certain number of generations becomes less rapid, then irregular, and finally ceases altogether. To set it going again, a process of rejuvenescence or constitutional invigoration is necessary; this is effected by conjugation, during which an interchange of nuclear matter is effected between the two individuals concerned in the act.

It seems very possible that the repeated cell division, which takes place in the formation of polar bodies, has a similar exhausting effect on the nucleus of the ovum, rendering a process of rejuvenescence desirable, and in most cases absolutely necessary, before any further division can take place; this rejuvenescence being effected by conjugation, or fusion, of the nuclei of the spermatozoon and of the ovum.

This view, as Hartog points out, is in accordance with Balfour's theory in so far as it regards the formation of polar bodies as a process the object of which is to prevent parthenogenesis; but differs from this theory in regarding the polar bodies, not as male elements extruded from an originally hermaphrodite egg, but as cells, the rapid formation of which has reduced the part of the nucleus still remaining in the egg, i.e. the [female pronucleus, to a condition of exhaustion which renders the stimulus of fertilisation necessary, or at least highly advantageous, if further cell-division is to take place.

\section{Segmentation of the Egg.}

The actual details of segmentation vary considerably in different cases, the differences depending chiefly on the relative amount of food-yolk present, and on its distribution within the egg.

The simplest form of segmentation is presented by alecithal eggs, such as those of Amphioxus. It is characterised by the almost geometrical regularity with which the successive divisions 

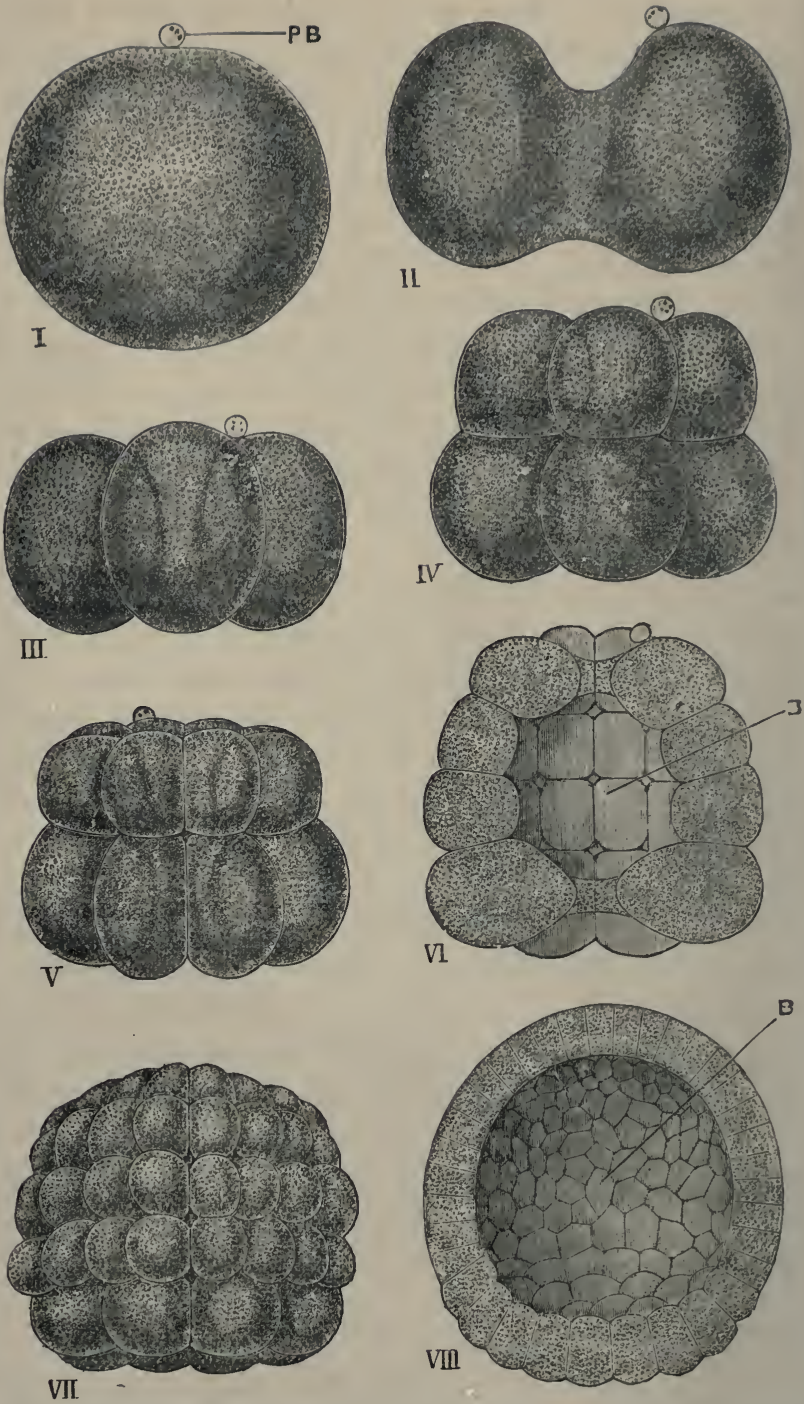

FIG. 2.-Segmentation of the egg of Amphioxus. $\quad \times 220$. (After Hatschek.)

I, the egg before the commencement of development : only one polar body, PB, has been seen, but from analogy with other animals it is probable that there are really two present. II, the ovum in the act of dividing, by a vertical cleft, into two equal blastomeres. III, stage with four equal blastomeres. IV, stage with eight blastomeres ; an upper tier of four slightly smaller ones, and a lower tier of four slightly larger ones. $\vec{V}$, stage with sixteen blastomeres, in two tiers, each of eight. VI, stage with thirtytwo blastomeres, in four tiers, each of eight : the embryo is represented bisected, to sliow the segmentation eavity or blastocel, B. VII, later stage : the blastomeres have increased in number by further division. VIII, blastula stage: bisected to show the blastocœl, B. 
occur, and by the fact that the cells, or blastomeres, into which the egg is divided are approximately equal to one another in size. The first cleft, Fig. 2, II, is a vertical one, and divides the egg into two perfectly similar halves. The second cleft is also vertical, but at right angles to the first one : on its completion the egg is divided into four cells or blastomeres of equal size, Fig. 2, IIr. The third cleft, Fig. 2, Iv, is a horizontal one, and divides each of the four blastomeres of the previous stage into two, of which the lower one is slightly the larger. Two vertical clefts next appear simultaneously, at angles of $45^{\circ}$ with the two first clefts: by these the number of the blastomeres is again doubled, giving sixteen in all, Fig. 2, v. Two new horizontal clefts double the number of blastomeres once more; the stage, with thirty-two blastomeres, being shown in Fig. 2, vi. From this time segmentation continues rapidly, but with less regularity: later stages are shown in Fig. 2, vII and virr.

Segmentation is said to be complete, or holoblastic, when, as in Amphioxus, the whole egg is divided up at once into blastomeres: it is further distinguished as equal when, as again in Amphioxus, the several blastomeres are from the first approximately equal in size.

In the frog's egg, Fig. 3, segmentation is holoblastic, but unequal. The first two clefts, which, as in Amphioxus, are vertical, divide the egg equally and symmetrically; but the third, or horizontal cleft, Fig. 3, II, is much nearer the upper than the lower pole, and throughout the later stages of segmentation, Fig. 3 , IV and $\mathrm{v}$, there is marked inequality in size between the blastomeres of the upper and lower halves of the egg. Unequal segmentation is due to food yolk, which, in a telolecithal egg like the frog's, is specially accumulated in the lower pole, and retards the developmental processes in this as compared with the upper half of the egg.

An exaggeration of this condition is seen in the hen's egg, in which food-yolk is present in such quantity as to absolutely stop the processes of development in all parts of the egg, except in a small circular patch on the surface, corresponding to the upper pole of the egg of Amphioxus or the frog. To this circular patch, or germinal disc, Fig. 4, BA, segmentation is restricted. Figs. 5 and 6 represent surface views of the germinal disc during the process of segmentation, and show the 
irregular manner in which the several clefts appear ; while Fig. 7 represents a vertical section of the germinal disc, with the I

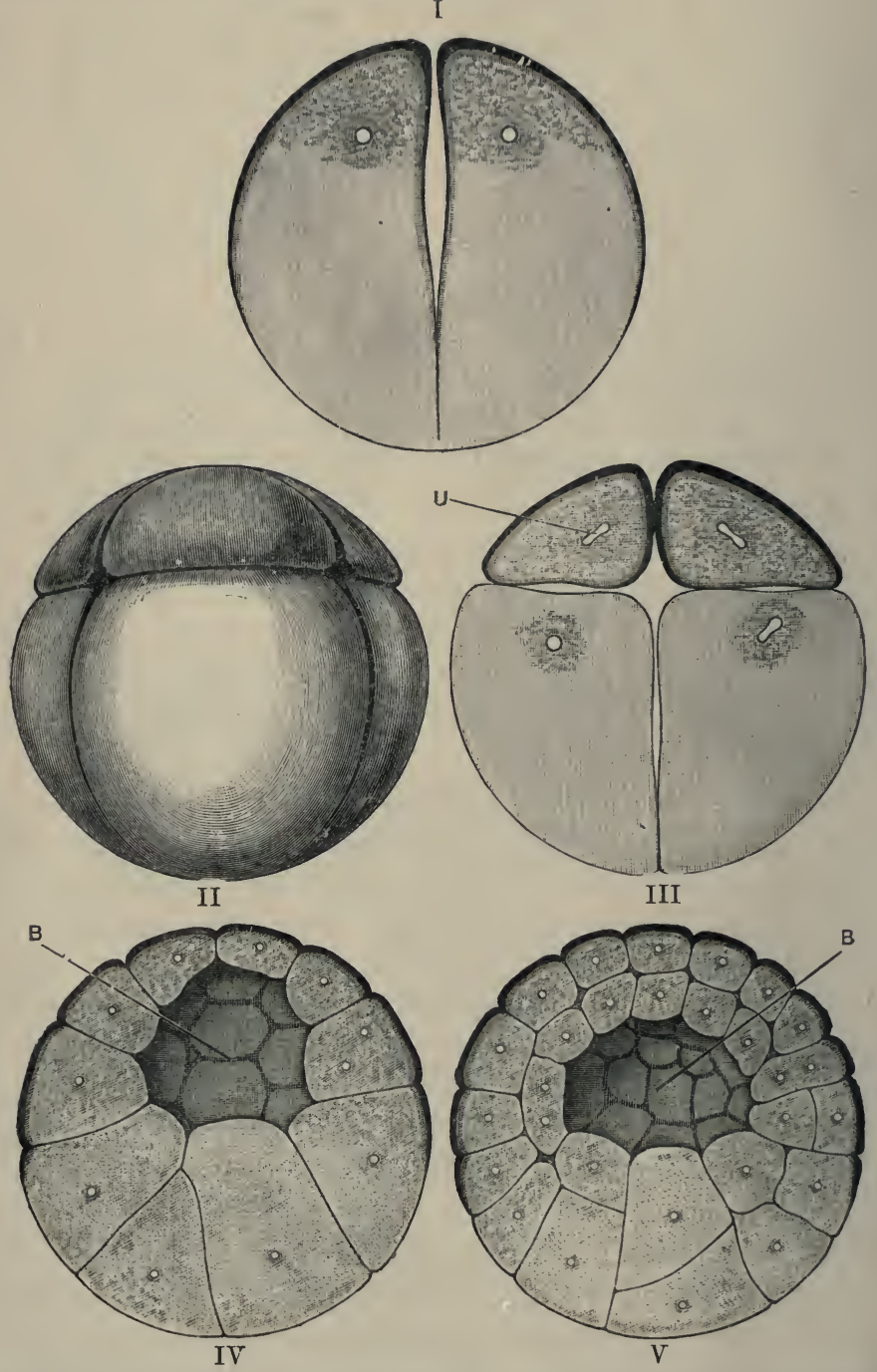

FIG. 3.- Segmentation of the Frog's Egg. The second figure is a surface view, the remaining four figures represent the egg in section. $\times 20$.

I, the ovum just before the completion of the first cleft, by which it is divided into two equal blastomeres. II, stage with eight blastomeres : an upper ticr of four small ones, and a lower tier of four much larger ones. III, the same stage, with eight blastomeres, in sectiou. IV, V, later stages, showing further increase in the number of the blastomeres, with great inequality in their size. $\mathbf{B}$, segmentation cavity or blastocoel. U, nucleus. 


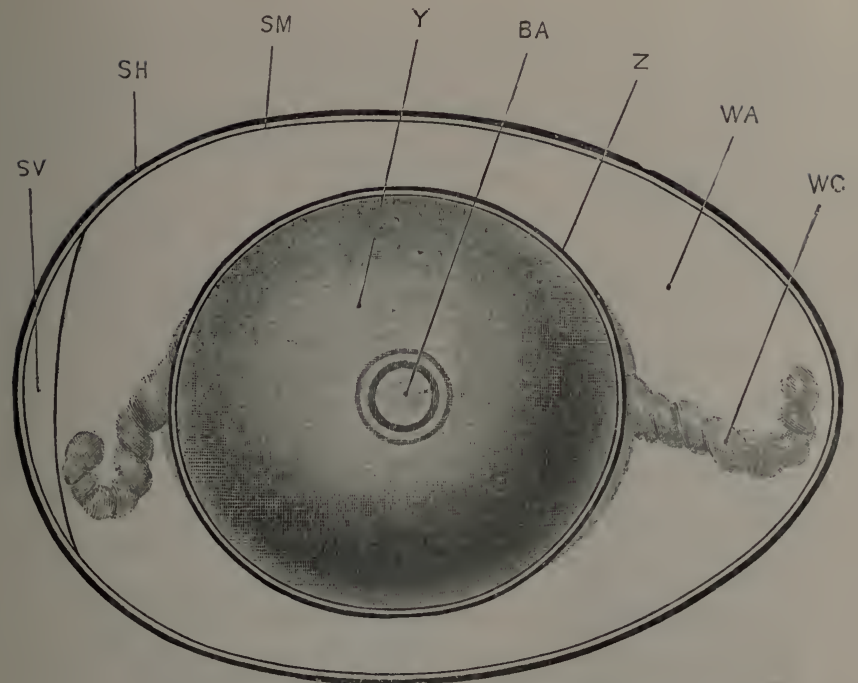

Fig. 4.-The Hen's Egg, freshly laid. $\times \frac{3}{2}$.

$\mathbf{B A}$, germinal disc. $\mathbf{S H}$, egg shell. $\mathbf{S} \mathbf{M}$, shell membrane. $\mathbf{S V}$, air chamber. WA, white or albumen. WC, chalaza. Y yolk. $\mathbf{Z}$, vitelline membrane.

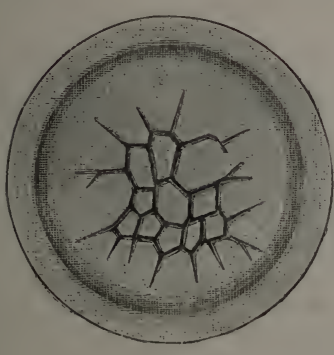

Fig. 5 .

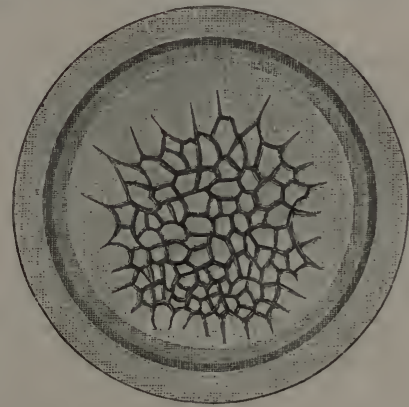

Fig. 6.

FIGS. 5, 6.- Stages in the segmentation of the germinal disc of the Hen's Egg. $\times 10$. (After Coste and Duval.)

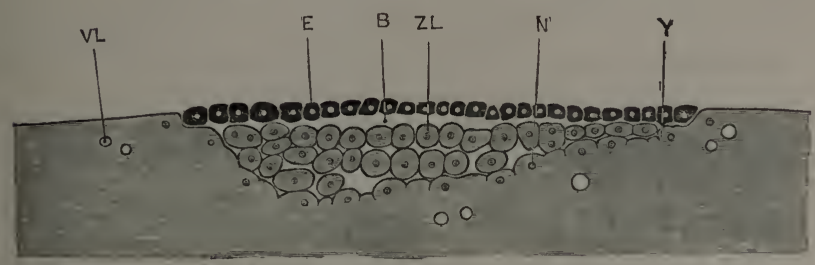

FIG. 7.-Vertical section of the germinal disc of the Hen's Egg at the close of segmentation. $\times 25$. (After Duval.)

B, segmentation cavity or blastocœl. E, upper layer of blastomeres, or epiblast. $\mathbf{N}^{\prime}$, nucleus of incompletely formed blastomere. VL, vacuole in jolk. $Y$, yolk ZL, lower layer of blastomeres. 
adjacent parts of the yolk, at the close of segmentation. Segmentation, when confined to part of the egg, is spoken of as meroblastic; and when, as in the hen's egg, it is limited to a circular patch on the surface of the egg it is further distinguished as discoidal.

Another type of meroblastic segmentation is presented by the centrolecithal eggs of Arthropods. Here, there is no localised germinal disc, but the whole surface of the egg consists of a layer of protoplasm free from yolk-granules, in which segmentation occurs almost simultaneously at all parts; such a mode of segmentation may be distinguished as superficial.

The principal types of segmentation, described above, may be tabulated as follows:-

I. Holoblastic or complete segmentation.

A. Equal : as in the alecithal egg of Amphioxus.

B. Unequal: as in the telolecithal egg of the frog.

II. Meroblastic or partial segmentation.

C. Discoidal : as in the telolecithal egg of the chick.

D. Superficial: as in the centrolecithal eggs of Arthropods.

\section{The Germinal Layers.}

At the close of segmentation the whole of the egg, or, in cases of meroblastic segmentation, a part only of it, is divided up into cells or blastomeres. These blastomeres very early become arranged in two layers; an outer layer, the epiblast, which covers the surface of the embryo; and an inner layer, the hypoblast, which lines a cavity within its interior. Epiblast and hypoblast form the two primary germinal layers of the embryo: the epiblast becomes ultimately the epidermis or outer layer of the skin; while the hypoblast becomes the epithelial lining of the alimentary canal; the cavity surrounded by the hypoblast, spoken of as the archenteron, forming the first commencement of the digestive tract. Figs. 8 and 9 represent early larvæ of Amphioxus which have reached the stage described.

The details of development of epiblast and hypoblast, and more especially the mode of appearance of the archenteric cavity, are subject to great modifications in different groups of animals, but the essential relations are in all cases as described above.

Between epiblast and hypoblast a third layer of cells, the mesoblast, appears at a later stage, usually derived, directly or 
indirectly, from the hypoblast. Though appearing after the other two germinal layers, the mesoblast grows very rapidly, and in the higher animals forms a larger part of the embryo than the other two layers together.

The two primary germinal layers, epiblast and hypoblast, occur, and with essentially similar relations, in all groups of Metazoa, from sponges up to mammals. The middle germinal layer, or mesoblast, presents far greater variations, and it is by

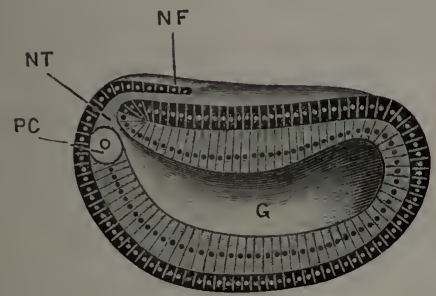

Fig. 8.

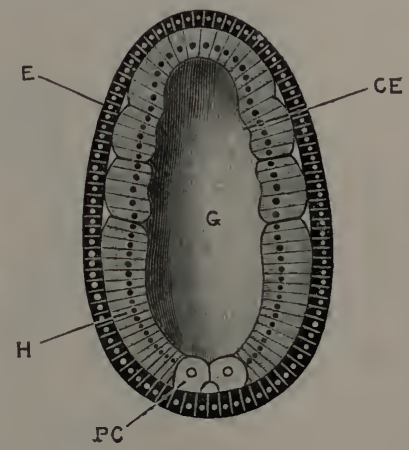

Fig. 9.

FIGs. 8, 9.-Vertical and horizontal sections of early larval stages of Amphioxus. $\times 220$. (After Hatschek.)

CE, commencing mesoblastic outgrowtl. E, epiblast. G, archenteron. H, hypoblast. NF, neural fold. NT, neurenteric canal, leading from neural tube to archenteron. PC, polar mesoblast cell.

no means clear that all the structures spoken of as mesoblastic in the different groups of animals have any real community of origin or relations. In Sponges and Colenterates a mesoblastic layer cannot be said to exist, but in all other groups of Metazoa it is present.

The three germinal layers together make up the whole of the embryo, and from them all parts of the adult animal are derived: the principal organs and parts to which the layers give origin respectively are as follows.

The epiblast, or outer layer, gives rise to the epidermis, covering the body generally; and to the various organs derived from the epidermis. Of these, the more important are:-the nervous system, both central and peripheral ; the olfactory and auditory epithelium, the retina and lens of the eye, and the other organs of sensation; the epithelial lining of the mouth and anus; the pineal and pituitary bodies ; the enamel of the teeth ; the hairs, 
nails, claws, and other epidermal mordifications; and the epithelial lining of the mammary, sweat, and other glands formed from the skin.

The hypoblast, or inner layer, gives rise to the epithelium lining the alimentary canal and its various diverticula; including the glands of the osophagus, stomach, and intestine, the lungs, the bladder, the bile ducts, gall bladder, and pancreatic ducts, the hepatic cells of the liver, and the secreting cells of the pancreas. The notochord also is formed from hypoblast.

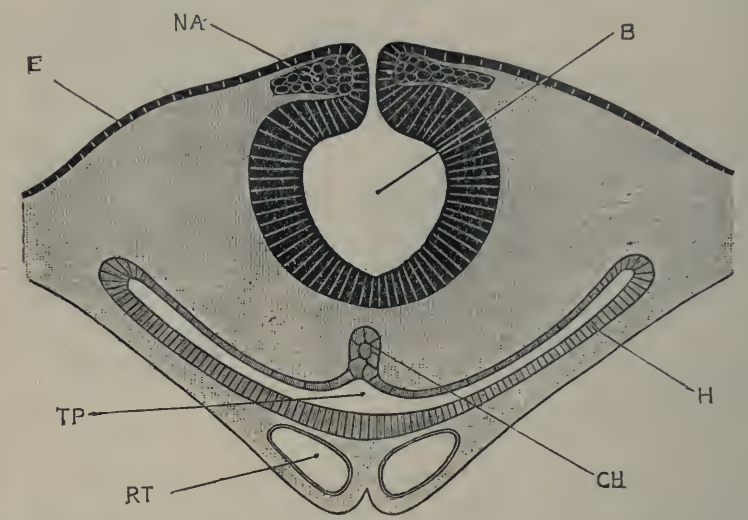

FIG. 10.-Transverse section through the head of a Chick Embryo at the end of the first day of incubation, showing the relations of the three germinal layers. $\times 100$.

B, cavity of the brain : the origin of the walls of the brain from the epiblast is well seen. $\mathbf{C H}$, notochord, arising from the hypoblast. $\mathbb{E}$, epiblast. $\boldsymbol{H}$, hypoblast. NA, root of one of the cranial nerves. TP, cavity of the alimentary canal, in the pharyngeal region. RT, blood-vessel. The whole of the part of the figure covererl by the lighter shading is mesoblast.

From the mesoblast, or middle layer, are derived all structures lying between the epiblast and hypoblast; i.e. the connective tissue, muscles, skeleton (except the notochord), bloodvessels, and lymphatics; and also the peritoneum, and the urinary and reproductive organs.

\section{The General History of Development: the Recapitulation Theory.}

It is a familiar fact that animals in the earlier stages of their existence differ greatly in form, in structure, and in habits from the adult condition.

In some cases, as, for example, in Amphioxus, the whole history of development is a steady upward progress towards the adult condition, the several organs and parts gradually approxi- 
mating towards the fully formed state, and each stage bringing the animal, not merely as a whole, but as regards each of its organs and parts, one step nearer to the perfect form.

In the great majority of animals, however, the course of development is not so straightforward. Even in Amphioxus there are features in the early embryonic stages, such as the communication between the neural tube and the digestive cavity, which completely disappear during development, and which have no relation to the adult condition of the animal.

In the higher Vertebrates, far more striking instances occur. In the embryo of a chick or of a mammal the structure and relations of the heart and blood-vessels are for a time those of a fish; and for the attainment of the adult condition it is necessary, not merely that new structures should appear and new relations be acquired, but that parts once present should actually become obliterated. The frog, again, commences its free exist= ence as a tadpole, which is really a fish, not merely as regards its breathing organs, but in all details of its organisation; and the change from the tadpole to the frog involves great modifica= tion in the shape, size, and relations of almost all its organs, with complete obliteration of parts such as gills and tail, which were essential to the tadpole but are absent from the frog.

It is to cases such as the frog, or as the butterfly, in which the transition from larva to adult is even more extensive and more abrupt, that the term metamorphosis is applied; cases in which the animal, instead of developing straight towards the adult condition, in place of aiming straight at its goal, deviates from the direct path, spends time and energy in developing and elaborating organs which, though in perfect keeping with its actual mode of existence, yet have no relation to the state it is ultimately to reach, and must indeed be got rid of before that final condition can be attained.

Cases of this kind forcibly illustrate the necessity for some explanation of the facts of development. Much attention has been given to the subject, especially of recent years, and it is now possible to frame a consistent theory which will explain the general history of development in all groups of animals, and which will also be in harmony with the accepted views concerning the mutual relations of these groups.

The doctrine of descent, or of evolution, teaches us that as 
individual animals arise, not spontaneously, but by direct descent from pre-existing animals, so also is it with species, with families, and with larger groups of amimals, and so also has it been for all time; that as the animals of succeeding generations are related together, so also are those of successive geologic periods; that all animals, living or that ever have lived, are united together by blood relationship of varying nearness or remoteness; and that every animal now in existence has a pedigree stretching back, not merely for ten or a hundred generations, but through all geologic time since life first commenced on the earth.

The study of development has in its turn revealed to us that each animal bears the mark of its ancestry, and is compelled to discover its parentage in its own development; that the phases through which an animal passes in its progress from the egg to the adult are no accidental freaks, no mere matters of developmental convenience, but represent more or less closely, in more or less modified manner, the successive ancestral stages through which the present condition has been acquired.

Evolution tells us that each animal has had a pedigree in the past. Embryology reveals to us this ancestry, because every animal in its own development repeats its history, climbs up its own genealogical tree.

This Recapitulation Theory, as it is termed, was obscurely hinted at by the elder Agassiz, and suggested more directly in the writings of Von Baer; but it was first clearly enunciated by Fritz Müller in 1863, and has since that date formed the foundation on which the explanation of the facts of embryology is based.

The fact that a frog commences its free existence as a tadpole, i.e. to all intents and purposes as a fish, is a very extraordinary one, but it becomes at once intelligible if we interpret it as meaning that frogs are descended from fish, and that every frog is constrained to repeat or recapitulate its pedigree in the course of its own individual development.

Similarly, the long-tailed condition of the young crab at the time of leaving the egg is to be viewed as an indication of the descent of the short-tailed or brachyurous crustaceans from macrurous ancestors; and the presence of gill clefts in the young stages of chicks or rabbits, which when adult are totally 
devoid of them, or of teeth in the embryo of the whalebone whale, are in like manner to be regarded as reminiscences of former ancestral conditions, and as indicating that the ancestors of chicks and rabbits breathed by gills, and that the toothless whalebone whales are descended from toothed progenitors.

It is on this fact of Recapitulation that the great value of embryology depends. The study of development acquires a new and striking interest when it is realised that through it we are enabled to obtain knowledge, in many cases unattainable by any other means, of the real or blood relationships between animals and groups of animals.

It is with animals as with men, the only natural classification is a genealogical or phylogenetic one, and the possibility of framing such a classification of animals depends very largely on the success with which we are able to reconstruct their pedigrees from a study of the stages through which they pass in their actual development or ontogeny.

Recapitulation must apply, not merely to the development of an animal as a whole, but to that of each one of its organs and parts : the formation of the ear, for example, as a pit of the skin, must be interpreted as meaning that the ear, like the other organs of sensation, was in its earliest commencement merely a specialised patch of skin.

The theory must also apply to the earliest stages of development equally with the later ones; and the fact that all Metazoa commence their existence as eggs-perhaps the most striking of all embryological facts-receives an entirely new significance when we interpret it as a reminiscence of a unicellular ancestry for all Metazoa, and as an indication that all the multicellular animals, or Metazoa, are descended from unicellular Protozoa.

From this point of view the earliest developmental stages of Mctazoa deserve special attention, as possibly indicating the actual lines of descent of Metazoa from Protozoa. Segmentation is simply cell-division; and the main difference between cell division in Protozoa and segmentation of the egg of a Metazoon is that, in the former case, the products of division separate from each other as independent unicellular animals, while in the latter they remain in close contact and become constituent units of one multicellular animal. The several stages of segmentation, Fig. 2, II to vil, may be compared with colonies of Protozoa; 
while the blastula stage, Fig. 2, vir, reached at the close of segmentation, bears a striking resemblance to such adult forms as Volvox or Pandorina.

There is, however, another side of the question which must not be overlooked. Although it is undoubtedly true that development is to be regarded as a recapitulation of ancestral phases, and that the embryonic history of an animal presents to us a record of the race history, yet it is also an undoubted fact, recognised by all writers on embryology, that the record so obtained is neither a complete nor a straightforward one.

It is indeed a history, but a history of which entire chapters are lost, while in those that remain many pages are misplaced, and others are so blurred as to be illegible; words, sentences, or entire paragraphs are omitted, and, worse still, alterations or spurious additions of later date have been freely introduced, and at times so cunningly as to defy detection.

Very slight consideration will show that development cannot in all cases be strictly a recapitulation of ancestral stages. It is well known that closely allied animals may differ markedly in their modes of development, which could not be the case if both recapitulated correctly. The common frog, for example, is at first a tadpole breathing by gills, a stage which is entirely omitted by the little West Indian frog, Hylodes. A crayfish, a lobster, and a prawn are allied animals, yet they leave the egg in totally different forms. Some developmental stages, as the pupa condition of insects, or the stage in the development of a tadpole in which the œsophagus is imperforate, cannot possibly be ancestral. Or again, a chick embryo, of say the third day, Fig. 113, is clearly not an animal capable of independent existence, and cannot therefore correctly represent any ancestral condition; an objection which applies to the earlier developmental histories of many, perhaps of most, animals.

Hæckel long ago urged the necessity of distinguishing, in actual development, between those characters which are really historical and inherited, and those which are acquired or spurious additions to the record. The former he terms palingenetic or ancestral characters, the latter cenogenetic or acquired. The distinction is certainly a true one, but an exceedingly difficult one to draw in practice. The causes which prevent development from being a strict recapitulation of ancestral 
history, the modes in which these came about, and the influence which they respectively exert, are problems which are as yet only partially solved.

Of these disturbing causes, the most potent and the most widely spread arises from the necessity of supplying the embryo with nutriment. This acts in two ways.

If the amount of nutritive matter within the egg be small, then, as we have already seen, the young animal must hatch early and in a very imperfectly developed condition. In such cases, as in Amphioxus or the frog, there is of necessity a long period of larval life, during which natural selection may act so as to introduce modifications of the ancestral history, spurious additions to the text. Of such ' larval organs,' the long spines that form conspicuous features in the young, free swimming larvæ of sea urchins, or of crabs, are good examples.

If, on the other hand, the egg contain within itself a considerable quantity of nutrient matter, then the period of hatching can be postponed until this nutrient matter has been used up. The consequence is that the embryo hatches at a much later stage of its development, and, if the amount of food material is sufficient, may even, as in the case of the chick, leave the egg in the form of the parent. In such cases the earlier developmental phases are often greatly condensed and abbreviated; and as the embryo does not lead a free existence, and has no need to exert itself to obtain food, it commonly happens that these stages are passed through in a very modified form, the embryo being, as in a three-day chick, in a condition in which it is clearly incapable of independent existence.

The effect of a greater or less amount of food-yolk on the recapitulation of ancestral characters has been summed up by Balfour thus: "There is a greater chance of the ancestral history being lost in forms which develop in the egg, and of its being masked in those which are hatched as larvæ.'

There are a number of other causes, besides food-yolk, which tend to modify the ancestral history as preserved in individual development. The following list gives a brief summary of the more important of these. 
Causes tending to falsify the ancestral history; or to prevent ontogeny from being a true record of phylogeny.

1. The general tendency to condensation of the ancestral history. Except perhaps in the lowest groups of Metazoa, such as sponges, no animal can possibly repeat, in its own development, all the ancestral stages in the history of the race. There is a tendency in all animals towards striking a direct path from the egg to the adult: a tendency best marked in the higher, the more complicated members of a group, i.e. those which have the longest and most tortuous pedigrees.

2. The tendency to the omission of ancestral stages. This has been already noticed as one of the commonest effects of abundance of food-yolk. The omission of the gill-breathing stage in Hylodes and in all Amniote Vertebrates is a typical example.

3. The tendency to distortion, either in time or space. All embryologists have noticed the tendency to anticipation, or precocious development, of characters which really belong to a later stage in the pedigree. Many early larvæ show it markedly, the explanation in this case being that it is essential for them to possess at the time of hatching all the organs necessary for independent existence.

Anachronisms, or actual reversals of the historical order of development of organs or parts, occur frequently. Thus the joint surfaces of bones acquire their characteristic curvatures before movement of one part on another is effected, and even before the joint cavities are formed.

4. The tendency to the accentuation or undue prolongation of certain stages. This is best seen in cases of abrupt metamorphosis, as of the caterpillar to the butterfly; or of the pelagic pluteus larva to the sea urchin, slowly crawling on the seabottom; or of the herbivorous aquatic tadpole to the terrestrial and carnivorous frog. In such cases there is usually a great difference between larva and adult in external form and appearance, in manner of life, and very usually in mode of nutrition; and a gradual transition is inadmissible, because in the intermediate stages the animal would be adapted neither to the larval nor to the adult conditions; a gradual conversion of the biting mouth parts of the caterpillar to the sucking proboscis of the moth would

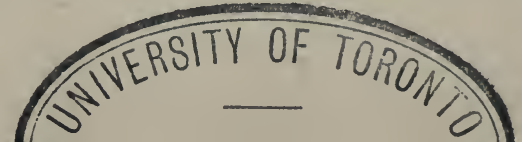


inevitably lead to starvation. The difficulty is evaded by retaining the external form and habits of one particular stage for an unduly long period, so that the relation of the animal to its surrounding environment remains unaltered, while, internally, preparations for the later changes are in progress.

5 . The tendency to the acquisition of new characters. This has been dealt with already; it arises from the fact that the larval forms of animals, like the adults, are exposed to the action of natural selection, and so are liable to acquire characters that do not belong to the ancestral history.

Before leaving the subject it is worth while inquiring whether any explanation can be found of recapitulation. A complete answer can certainly not be given at present, but a partial one may, perhaps, be found.

Darwin himself suggested that the clue might be found in the consideration that at whatever age a variation first appears in the parent, it tends to reappear at a corresponding age in the offspring; but this must be regarded rather as a statement of the fundamental fact of embryology than as an explanation of it.

It is probably safe to assume that animals would not recapitulate unless they were compelled to do so: that there must be some constraining influence at work, forcing them to repeat more or less closely the ancestral stages. It is impossible, for instance, to conceive what advantage it can be to a chick or a rabbit embryo to develop gill clefts which are never used, and which disappear at a slightly later stage; or how it can benefit a whale, that in its embryonic condition it should possess teeth which never cut the gum, and which are lost before birth.

Moreover, the whole history of development in different animals or groups of animals offers to us, as we have seen, a series of ingenious, determined, varied, but more or less unsuccessful efforts to escape from the necessity of recapitulating, and to substitute for the ancestral process a more direct method.

A further consideration of importance is that recapitulation is not seen in all forms of development, but only in development from the egg. In the several forms of asexual development, of which budding is the most frequent and most familiar, there is no repetition of ancestral phases; neither is there in cases of 
regeneration of lost parts, such as the tentacle of a snail, the arm of a starfish, or the tail of a lizard; in such regeneration it is not a larval tentacle, or arm, or tail that is produced, but an adult one.

The most striking point about the development of the higher animals is that they all alike commence as eggs. Looking more closely at the egg, and the conditions of its development, two facts impress us as of special importance : first, the egg is a single cell, and therefore represents morphologically the Protozoan, or earliest, ancestral stage; secondly, the egg, before it can develop, must, in the great majority of cases, be fertilised by a spermatozoon, just as the stimulus of fertilisation by the pollen grain is necessary before the ovum of a plant will commence to develop into the plant-embryo.

The advantage of cross-fertilisation in increasing the vigour of the offspring is well known, and in plants devices of the the most varied and even extraordinary kind are adopted to ensure that such cross-fertilisation occurs. The essence of the act of cross-fertilisation consists in combination of the nuclei of two cells, male and female, derived from different individuals. The nature of the process is of such a kind that two individual cells are alone concerned in it; and it may reasonably be argued that the reason why animals commence their existence as eggs, i.e. as single cells, is because it is in this way alone that the advantage of cross-fertilisation can be secured, an advantage admittedly of the greatest importance, and to secure which natural selection would operate powerfully.

The occurrence of parthenogenesis in certain groups, either occasionally or normally, is not so serious an objection to this view as it appears at first. There are strong reasons for holding that parthenogenetic development is a modified form, derived from the sexual method. Moreover, it is the very essence of the view advanced above, that it does not state that cross-fertilisation is essential to individual development, but merely that it is in the highest degree advantageous to the species; and hence room is left for the occurrence, exceptionally, of parthenogenetic development.

It may be objected that this is laying too much stress on sexual reproduction, and on the advantage of cross-fertilisation; but it must not be forgotten that sexual reproduction is the 
characteristic and essential mode of multiplication among Metazoa; that it occurs in all Metazoa; and that when asexual reproduction, as by budding, \&c., occurs, this merely alternates with the sexual process, which sooner or later becomes necessary.

If the fundamental importance of sexual reproduction to the welfare of the species be granted, and if it be further admitted that Metazoa are descended from Protozoa, then we see that there is a most powerful influence constraining every animal to commence its life history in the unicellular condition, the only condition in which the advantage of cross-fertilisation can be obtained; i.e. constraining every animal to begin its development at its earliest ancestral stage, at the very bottom of its genealogical tree.

On this view the actual development of any animal is strictly limited at both ends; it must commence as an egg, and it must end in the likeness of the parent. The problem of recapitulation becomes thereby greatly narrowed; all that remains being to explain why the intermediate stages in the actual development should repeat, more or less closely, the intermediate stages of the ancestral history. Although narrowed in this way, the problem still remains one of extreme difficulty, and no final solution can yet be given of it.

It is a consequence of the Theory of Natural Selection that identity of structure involves community of descent; a given result can only be arrived at through a given sequence of events. A negro and a white man have had common ancestors in the past; and it is through the long-continued action of selection and environment that the two types have gradually been evolved. You cannot turn a white man into a negro merely by sending him to live in Africa : to create a negro the whole ancestral history would have to be repeated, and it may be that it is for the same reason that the embryo must repeat, or recapitulate, its ancestral history in order to reach the adult goal.

Kleinenberg, in his ' Theory of the Development of Organs by Substitution,' has suggested that each historic stage in the evolution of an organ is necessary as a stimulus to the development of the next succeeding stage, and that the reason for the extraordinary persistence, in embryonic life, of organs which are rudimentary and functionless in the adult, may be that the 
presence of such organs in the embryo is indispensable as a stimulus to the development of the permanent structures of the adult. Should this theory prove to be well founded, it will afford a ready and welcome explanation of many perplexing facts in the development of animals.

\section{The Origin of Sex.}

The simplest mode of reproduction is a mere act of fission or' cell division, as seen in an Amœba or in an ordinary epithelial cell. Such a form of reproduction is characteristic of the simpler Protozoa, and of the component tissues of Metazoa. It may concern one individual alone, or may be preceded by the conjugation or fusion of two or more originally separate individuals or cells.

The higher Protozoa, or Infusoria, show considerable advance on this simple method. In Paramecium, or Stylonychia, reproduction is effected, as before, by fission, i.e. by division of the single animal into two separate animals; and under favourable circumstances this process may be repeated again and again with great rapidity. Sooner or later the rate slackens, and ultimately the process stops altogether; and it does not recommence until conjugation, usually temporary, has occurred between two individuals, which on the completion of the process begin to divide actively once more. Maupas' researches have shown that this conjugation is absolutely necessary, and that it must not take place between two closely allied individuals, but between ones of different broods.

In Vorticella there is further complication, for the conjugating individuals are in this case unlike; one being an ordinary large, stalked Vorticella ; the other a small free-swimming individual, of which a number, usually eight, are formed by simultaneous division of a large Vorticella. The conjugation is in this case a permanent one, the small Vorticella fusing completely with the large one; and the whole process corresponds singularly closely with the sexual reproduction of Metazoa, the small free-swimming Vorticella playing the part of the spermatozoon, while the large fixed one behaves as the ovum. This may be taken as the first definite establishment amongst animals of sexual differentiation, and the two Vorticellæ may not inappropriately be spoken of as male and female respectively. 
In the colonial Protozoa, such as Volvox, which take the form of hollow balls of cells, certain of the cells become large and stationary, forming the female cells or ova; these are fertilised by snall active male cells, derived from the same or from other colonies; and then, by division of the fertilised ova, new balls or colonies are formed.

This process is essentially the same as the sexual reproduction of Metazoa, and there can be little doubt that the process has been inherited by the Metazoa from their Protozoan ancestors.

The reason for the occurrence of sexual reproduction in all Metazoa is probably to be found, as suggested above, in the consideration that it is through sexual reproduction alone that the full advantage of cross-fertilisation can be obtained. This view, that sexual reproduction is to be regarded as highly advantageous rather than as absolutely essential to the species, is of great importance, as it leaves room for, and renders intelligible, the occurrence of other and asexual modes of reproduction such as are seen in so many groups of Invertebrates. It also affords a clue to the extraordinary condition of things described in certain of the pelagic Tunicates. Salensky has shown that in Salpa, and to a less marked degree in Pyrosoma, certain of the follicle-cells surrounding the ovum pass into its interior, and take an active part in the formation of the embryo; so that, although the egg is fertilised in the ordinary manner, the blastomeres resulting from its segmentation only give rise to certain of the component cells of the embryo, and not, as is usually the case, to all of them. This mode of development may be regarded as a combination of the ordinary sexual process with an asexual process similar to that by which the gemmules of sponges or the statoblasts of Polyzoa are formed.

\section{List of the more important Books and Memoirs bearing on the Subjects of Chapter $I$.}

Balfour, F. MI. : 'Treatise on Comparative Embryology.' Vol. i. chaps. i. ii. iii.; vol. ii. chap. xiii. $1880-81$.

Beneden, E. v.: 'Recherches sur la maturation de l'œuf et la fécondation.' Archives de Biologie, iv. 1884.

Beneden, E. v., et Neyt, A.: 'Nouvelles recherches sur la fécondation et la division mitotique chez l'Ascaride mégalocéphale.' Bulletin de l'Asadémie Royale des Sciences de Belgique, 3 sér., tome xiv. 1887. 
Blochmann, F.: 'Ueber die Richtungskörper bei Insekteneiern.' Morphologisches Jahrbuch, Bd. xii. 1887, und Bd. xv. 1889.

Boveri, 'T. : 'Zellenstudien,' Heft i. ii. iii. Jenaische Zeitschrift für Naturwissenschaft, $1887,1880,1890$.

Bütschli, O.: 'Gedanken über die morphologische Bedeutung der sogenannten Richtungskörperchen.' Biologisches Centralblatt, iv. 1884.

Carnoy, J. B. : 'Les globes polaires de l'Ascaris.' La Cellule, tcme ii. iii. 1887. Geddes and Thomson: 'The Evolution of Sex.' 1889.

Hartog, M. M. : 'Some Problems of Reproduction,' Qnarterly Journal of Microscopical Science, vol. xxxiii. 1891.

Hertwig, O. : 'Lehrbuch der Entwicklungsgeschichte des Menschen und der Wirbelthiere.' Dritte Auflage. 1890.

'Vergleich der Ei- und Samenbildung bei Nematoden.' Archiv für mikroskopische Anatomie, Bd. xxxvi. 1890.

Kleinenberg, N.: 'Die Entstehung des Annelids aus der Larve von Lopadorhynchus.' Zeitschrift für wissenschaftliche Zoologie, Bd. xliv. 1886.

Marshall, A. Milnes: 'Address to the Biological Section of the British Association.' British Association Report, 1890 ; and Nature, vol. xlij. 1890.

Maupas, E.: ' Recherches expérimentales sur la multiplication des Infusoires ciliés.' Archives de Zoologie Expérimentale, deuxième série, tome vi. 1888.

'Le rajeunissement karyogamique chez les Ciliés.' Archives de, Zoologie Expérimentale, deuxième série, tome vii. 1889.

Minot, C. S.: 'Theorie der Gonoblasten.' Biologisches Centralblatt, Bd. ii. 1882.

Nussbaum, MI.: 'Ueber die Veränderungen der Geschlechtsproducte bis zur Eifurchung:' Archiv für mikroskopische Anatomie, Bd. xxiii. 1884.

'Bildung und Anzahl der Richtungskörper bei Cirripedien.' Zoologischer Anzeiger, xii. 1889.

Salensky, W.: 'Beiträge zur Embryonal-Entwicklung der Pyrosomen.' Zoologische Jahrbücher; Abtheilung für Anatomie und Ontogenie, Bd. iv. u. v. $1890-91$.

Schultze, O.: ' Untersuchungen über die Reifung und Befruchtung des Amphibieneies.' Zeitschrift für wissenschaftliche Zoologie, Bd. xlv. 1887.

Waldeyer, W.: 'Karyokinesis and its relation to the process of Fertilisation,' (translation). Quarterly Journal of Microscopical Science, vol. xxx. 1889.

Weismann, A.: Essays upon Heredity and kindred Biological Problems. (translations). 1889 and 1892.

Weismann, A., und Ischikawa, C. : ' Ueber die Bildung der Richtungskörper bei thierischen Eiern.' Berichte der naturforschenden Gesellschaft zu Freiburg i. Br. Bd. iii. 1887.

Zacharias, O.: 'Neue Untersuchungen über die Copulation der Geschlechtsproducte und den Befruchtungsvorgang bei Ascaris megalocephala.' Archiv für mikroskopische Anatomie, Bd. xxx. 1887. 
Ch.ppter II.

\section{THE DEVELOPMENT OF AMPHIOXUS.}

\section{GENERAL ACCOUNT.}

\section{Structure of Amphioxus.}

Amphioxus is a small, semi-transparent, fish-like animal, about a couple of inches in length, found in shallow parts of the IIediterranean and other seas. It is of sluggish habits, and usually remains buried in the sand, either completely or with the anterior end alone protruding; but if disturbed it swims actively, by rapid lateral movements of the body.

In the general plan of its organisation Amphioxus agrees with the more familiar members of the group of Vertebrates, but in a large number of important respects it is far simpler than any of these.

The external appearance of Amphioxus is shown in Fig. 11. The body is elongated, laterally compressed, and pointed at both ends. There is no distinct head, and no trace of limbs.

A low dorsal fin runs along the middorsal line from end to end of the animal, becoming more prominent at the hinder end as the upper lobe of the caudal fin. The ventral surface bears a median fin along its posterior third, bat in front of this is flattened, so that the body is triangular in section. The sides of this flattened ventral surface are bordered by the lateral fins or metapleural folds. ( $O f$. Figs. 11, 12, 13.)

The skeleton is in an extremely simple condition. Neither cartilage nor bone is present, and the principal skeletal structure is an elongated elastic rod, the notochord (Fig. 11, K), which extends the entire length of the animal, lying dorsal to the alimentary canal and between this and the spinal cord. The notochord is surrounded by a thick sheath of dense connective tissue (Fig. 12, D), which is prolonged dorsalwards to form a tubuilar investment around the spinal cord. From these sheaths to the 
notochord and spinal cord, connective tissue partitions or septa arise, which, running outwards to the skin, divide the great lateral muscles of the body into muscle-segments or myotomes

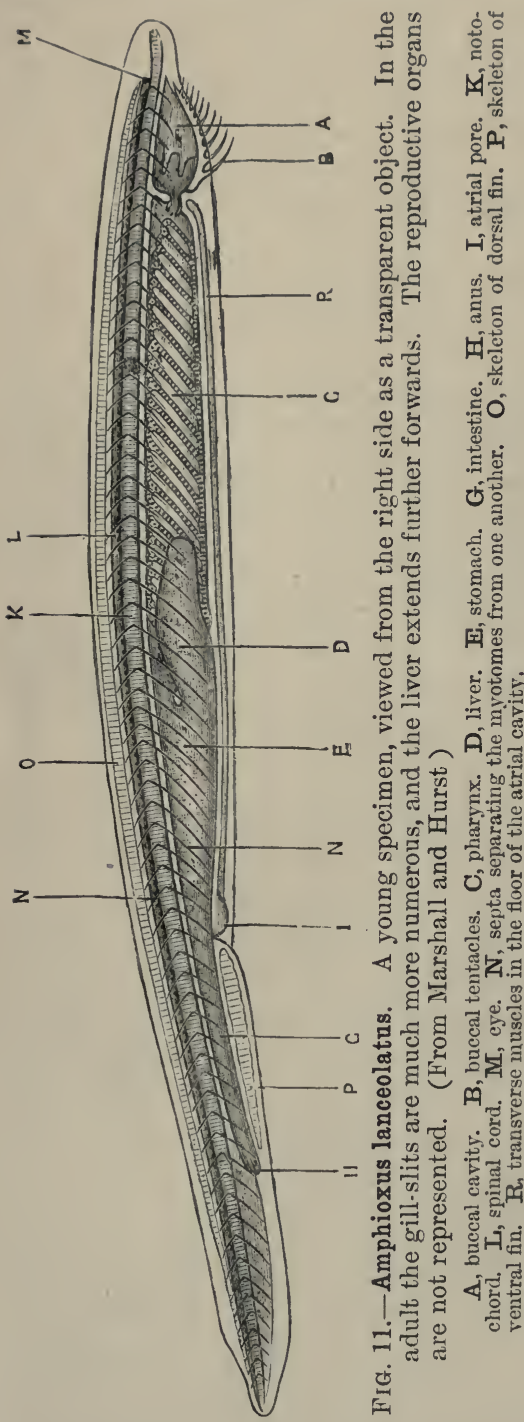

(Fig. 11, K, and Fig. 12, x.) The attachments of these septa to the skin are indicated by a series of $>-$ shaped markings, very clearly seen on the sides of the animal along its whole length (Fig. 11).

The only other skeletal structures of importance are a series of elastic chitinous rods, supporting the side walls of the pharynx; and an oval hoop, surrounding the mouth.

The great lateral muscles, noticed above, are the most important part of the muscular system. They form the side walls of the body along its whole length (cf. Figs. 12 and 13), and are divided, as already described, into muscle segments or myotomes by the connective tissue septa. Thè muscle fibres of each myotome run longitudinally, i.e. parallel to the axis of the body, the fibres taking origin from the connective tissue septa. The myotomes have been found to be sixty-one on each side of the body in a considerable number of specimens, and it seems probable that this number is constant. The myotomes of the two sides 
of the body are not arranged in pairs, but alternate with one another along the whole lengtl of the animal; and this lateral asymmetry, one of the most marked features of the adult Amphioxus, affects the nerves, blood-vessels, and other structures as well. The ventral surface of the body in the anterior two-

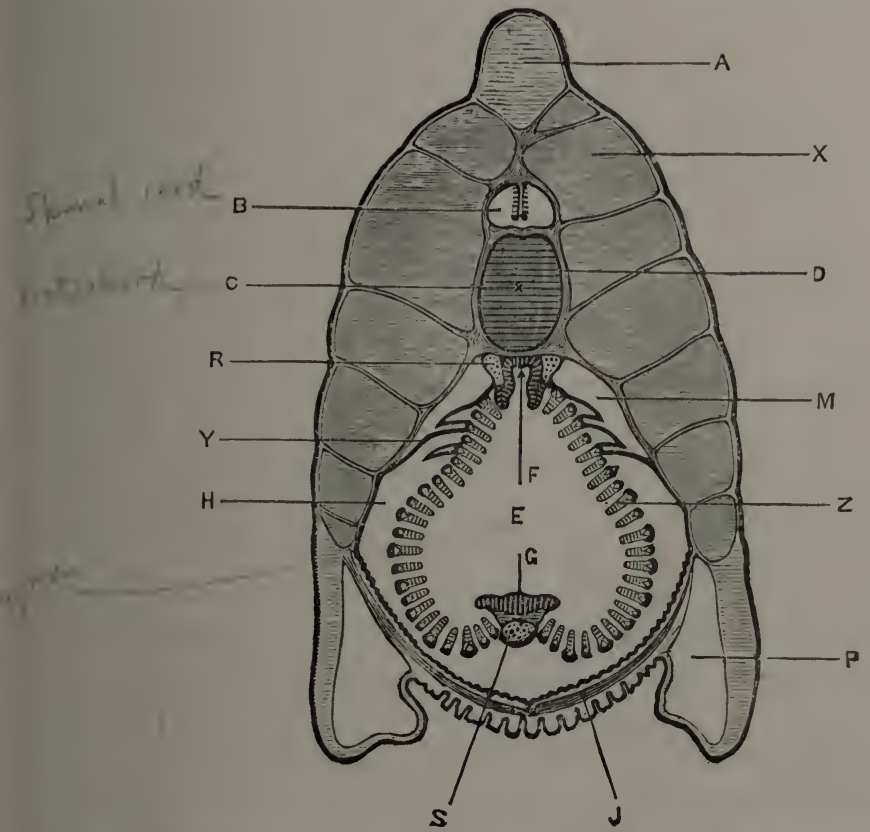

FIG. 12.-Amphioxus lanceolatus. Transverse section through the anterior part of the pharynx of an adult specimen. The boundary of the atrial cavity is indicated by a thick black line. The section is taken at about the level of the reference line $\mathbf{R}$ in Fig. 11. (From Marshall and Hurst.)

A, skeleton of dorsal fin. $\mathbf{B}$, spinal cord. C, notochord. D, connective-tissue sheath surrounding notochord. E, cavity of pharynx. F, epibranchial groove of pharynx. $G$, entostyle, which in this anterior part is flattened out or even convex. H, atrial cavity. $\mathbf{J}$, transverse muscles in floor of atrial cavity. $\mathbf{M}$, dorsal coelomic canal. $\mathbf{P}$, netapleural canal. $\mathbf{R}$, left dorsal aorta. $\mathbf{S}$, cardiac aorta. $\mathbf{X}$, myotome. $\mathbf{Y}$, suspensorv folil of pharynx, separating the dorsal colomic canal from the atrial cavity. $Z$, gill-arch or branchial bar; the white triangular spot represents the cut surface of the skeletal rod of the arch.

thirds of the animal is covered by a thin sheet of muscle (Fig. $12, \mathrm{~J})$, the fibres of which run transversely from side to side.

'The alimentary canal is a nearly straight tube, the anterior' part of which is modified for respiration, as in fish.

The buccal orifice (Fig. 11) is a large oval opening, on the rentral surface of the anterior end of the body; it is fringed on each side by a series of ciliated tentacles, but there are no 
jaws. The buccal orifice opens into a buccal cavity (Fig. 11, A), which is bounded laterally by the buccal hood, and posteriorly by a muscular diaphragm, the velum; a small perforation in the velum, a little way below its middle, is the true mouth and leads into the pharynx.

The pharynx (Fig. 11, c) is a wide sac, forming about half the length of the alimentary canal, and attached along its middorsal line to the under surface of the sheath of the notochord, (Fig. 12). The sides of the pharynx are perforated by a large number of slit-like apertures, the gill-slits, which run obliquely downwards and backwards, and of which in the adult animal there may be one hundred or more on each side. The parts of the pharyngeal wall left between successive slits are narrow bars, the gill-arches, each of which is strengthened by an axial rod of a chitinous substance. These arches are of two kinds, arranged alternately; the axial rods of the second, fourth, \&c., or primary arches, being forked at their ventral ends, while the rods of the alternate, or secondary arches, are unsplit. Each double gill-slit is originally a single one, but becomes divided in the course of development (vide p. 78), by the downgrowth of the unsplit bar, or tongue-bar as it is termed, from its dorsal end. The successive gill-arches are connected by horizontal bars, of which there are usually three or more crossing each slit, so that the pharynx has the character of an open meshwork.

Along themid-dorsal line of the pharynx is a deep epibranchial groove (Fig. 12, F), lined by a single layer of long columnar ciliated cells. A band of similar cells, the endostyle (Fig. 12, G), runs along the mid-ventral wall of the pharynx; it is folded longitudinally in its hinder part to form a groove (Fig. 13, G).

The intestine (Fig. 11) commences at the hinder end of the pharynx, close to the dorsal surface; and runs straight back to the anus, which is on the ventral surface, some little distance from the hinder end of the body, and slightly to the left of the median plane. The intestine is extremely narrow at its commencement; further back it dilates to form an expanded part or stomach, from which a large pouch-like outgrowth, the liver (Fig. 11, D), extends forwards some distance along the right side of the pharynx, ending blindly in front.

During life a stream of water passes through the mouth into the pharynx, and then out through the gill-slits in the sides 
of the pharynx, the stream being kept up by the action of columnar flagellate cells which clothe the gill-arches, and the water serving to aërate the blood in the vessels of the arches as it swills over them.

The water that has passed through the gill-slits escapes into a large space, the atrial or epipleural cavity (Fig. 12, H): this lies between the pharynx and the body wall, and into it the pharynx hangs freely, slung up to the body walls by suspensory folds (Fig. 12, y). 'The atrial cavity extends back some distance behind the pharynx, and along it the water passes, escaping finally by the atrial pore (Fig. 11, I), an aperture on the ventral surface of the body, bordered by prominent lips, and about onethird the length of the animal from its hinder end. The atrial cavity of Amphioxus is a very characteristic feature in its anatomy, and is apparently unrepresented in the higher Vertebrates.

The cœlom or body cavity is quite distinct from the atrial cavity, though its boundaries are not easy to follow. In the posterior part of the body, behind the atrial pore, the cœlom is a cavity of some width, surrounding the intestine and separating this from the body wall; in front of the atrial pore it becomes greatly reduced owing to the increased size of the atrial cavity; it is, however, readily recognisable as a narrow space immediately surrounding the intestine and the liver. Further forwards, in the region of the pharynx, the cœlom becomes much subdivided, and more difficult to trace; its principal divisions are a pair of dorsal cœlomic canals (Figs. 12 and 13, .r), lying at the sides of the dorsal part of the pharynx, between the body walls and the suspensory folds of the pharynx. From the dorsal cœlomic canals a series of tubular diverticula extend down the outer sides of the primary gill-arches, as far as their ventral ends. A series of spaces surrounding the reproductive organs (Fig. 13, ov) are also parts of the cœlom.

The large spaces, $\mathrm{P}$, in the metapleural folds do not belong to the cœlom, but are apparently lymphatic in nature.

In the circulatory system the more important features are the following. There is no heart, but the general course of the circulation is the same as in a fish. A median longitudinal vessel, the cardiac aorta or endostylar artery (Figs. 12 and 13, s), receives venous blood from the body at its hinder end, and carries it 
forwards along the floor of the pharynx: from the cardiac aorta the blood passes along a series of vessels in the gill-arches, becom ing aërated on the way, to the dorsal aortæ, a pair of longitudinal vessels (Figs. 12 and 13, R), lying just beneath the notochord :

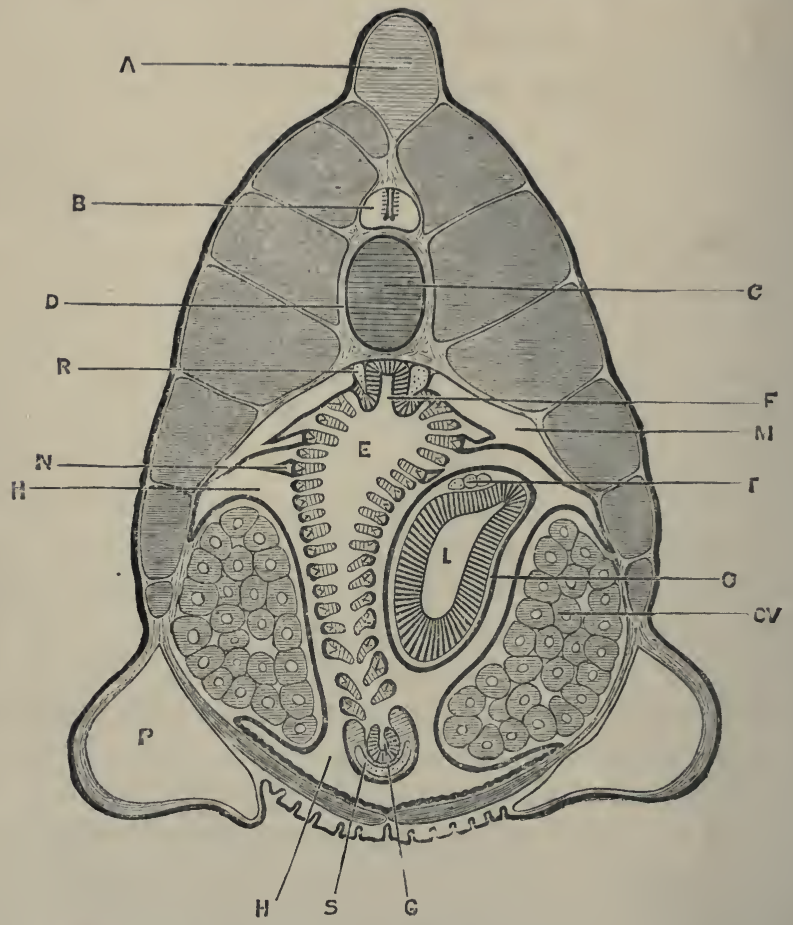

FIG. 13.-Amphioxus lanceolatus. Transverse section through the hinder part of the pharynx of an adult female, passing through the liver and the ovaries. The boundary of the atrial cavity is indicated by a thick black line. The section is taken at about the level of the reference line $\mathbf{K}$ in Fig. 11. (From Marshall and Hurst.)

A, skeleton of dorsal fin. B, spinal cord. C, notochord. D, comnectire-tissue sheath of notochord. E, cavity of pharynx. $\mathbf{F}$, epibranclial groove. $\mathbf{G}$, endostyle. H, atrial cavity. L, liver. IM, dorsal cœlomic canal. $\mathbf{N}$, branchial cœlomic canal. $\mathbf{O}$, colomic space surrounding liver. $\mathbf{O V}$, ovary. $\mathbf{P}$, metapleural canal. $\mathbf{R}$, left dorsal aorta. S, cardiac aorta. T, hepatic veins.

these unite behind the pharynx to form a single dorsal aorta, from which branches supply the various parts of the body.

The nervous system consists of a tube of nervous matter, the spinal cord, which lies immediately above the notochord, and extends almost the entire length of the body. It tapers slightly at its anterior end, and more markedly behind. The 
central canal of this tube is very small along the whole length of the cord, except at the extreme anterior end, where it expands to form a thin-walled chamber, or ventricle. This dilatation of the central canal constitutes the only indication, if indeed it can be regarded as such, of anything corresponding to the brain of higher Vertebrates. The nerves arise either by single roots from the dorsal surface of the cord, or by multiple roots from its ventral surface: the two sets of nerves, which are quite independent of each other, appear to correspond with the dorsal and ventral roots of the spinal nerves of other Vertebrates, although the dorsal roots have no ganglia and are both sensory and motor in function. Excepting the anterior three or four of each side, the nerves arise, not in pairs, but alternately from the right and left sides of the cord.

The sense organs are in a very simple condition, and can only doubtfully be compared with those of higher Vertebrates. From the anterior end of the ventricle of the central nervous system, a hollow outgrowth arises, which is in close relation with a ciliated pit on the dorsal surface and left side of the anterior end of the animal. This pit is commonly regarded as an olfac-

\section{tory organ.}

The 'eye' is a rounded pigment-spot in the anterior' wall of the ventricle; i.e. at the anterior end of the central nervous system (Fig. 11, M). There is no trace of an ear.

The sexes are distinct, but the male and female are similar, except as regards the microscopic structure of the reproductive organs. There are no genital ducts.

In the female, the ovaries (Fig. 13, or) are a series of saccular organs, arranged in a row along the inner surface of the body wall, on each side of the pharynx, in the segments from the tenth to the thirty-sixth. They lie in cavities, which are specialised portions of the coelom, and the true relations of which , will be described when the development of the reproductive organs is considered.

The ova, when ripe, are discharged into the atrial cavity by dehiscence of the proper wall of the ovary and of the atrial membrane. The discharged ova, together with the ovaries, form a bulky mass, which causes great distension of the atrial cavity, and distortion, through pressure, of the pharynx and other organs. 
The ova, which measure $0.105 \mathrm{~mm}$. in diameter, appear to escape from the atrial cavity, as a rule, through the atrial pore; but in some cases they have been seen to pass through the gillslits into the pharynx, and to make their exit through the mouth.

In the male, the testes are similar in form and position to the ovaries of the female; and the spermatozoa when ripe are discharged, like the ova, into the atrial cavity, from which they escape by the atrial pore.

\section{Morphological Importance of Amphioxus.}

It will be seen from the preceding account of its anatomy that Amphioxus, while clearly and undoubtedly a Vertebrate, yet differs from all ordinary Vertebrates, whether fish, amphibians, reptiles, birds, or mammals, in a number of points which are of great importance and affect almost every part of its body.

A closer examination shows that these points of difference between Amphioxus and the higher Vertebrates may be grouped under two chief heads.

(1) The atrial cavity, the large number of the gill-slits, the regular alternation of gill-arches of two kinds, the azygos character of the sense organs, the extension of the notochord to the extreme anterior end of the animal, and the curious lateral asymmetry shown by the myotomes, nerves, and other organs, are examples of a group of characters in which Amphioxus differs from the higher Vertebrates, not only in their adult condition, but at all stages of their existence.

(2) There is another and even more striking series of characters, in which Amphioxus differs from the adult forms of the higher Vertebrates, but resembles these in their early developmental stages. Thus in all the higher Vertebrates there is a stage in development when the notochord is the only skeletal structure present, neither cartilage nor bone having yet appeared; a stage in which the limbs are absent; and a stage in which the muscles of the body have the simple and definite segmental arrangement seen in Amphioxus throughout life. In all higher Vertebrates the heart is at first straight, like the cardiac aorta of Amphioxus; the liver arises as one or more outgrowths of the intestine; and the dorsal and ventral roots of the spinal nerves are at first independent of each other. In these and in 
many other points, Amphioxus remains throughout life in a condition characteristic of the early developmental phases of the higher Vertebrates. Amphioxus halts permanently at a stage through which all the higher Vertebrates pass during their development.

The Recapitulation Theory explains this as indicating that in these respects Amphioxus represents, more or less exactly, a phase through which the higher Vertebrates have passed in the history of their evolution; that, as regards the organs in question, Amphioxus may be viewed as figuring, with more or less exactness, an ancestral form from which the higher types of Vertebrates are descended.

From this standpoint Amphioxus is an animal of very special importance to morphologists; and the development of Amphioxus acquires peculiar interest from the consideration that, if the adult animal is far more primitive than any other existing Vertebrate, then the earlier stages in its life history may reasonably be expected, in accordance with the law of Recapitulation, to yield valuable evidence as to the relations of Vertebrates with the simpler groups of Metazoa.

The above considerations do not imply that Amphioxus itself stands in the direct line of ancestry of any of the higher Vertebrates, but that it is a surviving representative of a type of animals which preceded the higher Tertebrates in point of time, and from which type, though not necessarily from Amphioxus itself, the higher Vertebrates have arisen.

Amphioxus shows us that, in attempting to reconstruct the characters of the ancestors of Vertebrates, we are almost certainly justified in omitting such features as paired limbs, a cartilaginous or bony skeleton, jaws, a twisted or chambered heart, a highly specialised brain, and paired sense organs; characters which Amphioxus shows us are not necessary to an adult Vertebrate, and in the absence of which the embryos of higher Vertebrates agree with Amphioxus.

A different explanation of the peculiarities of Amphioxus has been offered by many zoologists, who consider that the simplicity that characterises so many of its organs, as the brain, heart, liver, \&c., is not primitive, but due to degeneration; that the immediate ancestors of Amphioxus were, in fact, animals higher in the zoological scale than itself. No distinct evidence of such 
degeneration has, however, been brought forward; and the theory of degeneration would leave altogether unexplained what is after all the most important fact, namely, the resemblance so often referred to above, and seen not in one organ only, but in almost every part of its structure, between the adult Amphioxus and the embryonic stages of development of the higher Vertebrates.

\section{General Account of the Development of Amphioxus.}

The development of Amphioxus has, as yet, been studied by a very limited number of investigators ; and many points, especially in the later stages, are still only imperfectly understood.

Our actual knowledge is due in the first instance to Kowalevsky, who published in 1867 an account of observations made by him at Naples in 1864. His descriptions, though brief, are exceedingly precise and well illustrated, and deal with both the earlier and later stages of development; they were supplemented by further papers in 1870 and 1876 .

In 1881, Hatschek published a detailed and admirably illustrated account of the earlier stages of development, from the laying of the eggs up to the formation of the mouth and first gill-cleft. His observations were made near Messina, the specimens being obtained from a small salt lake, communicating with the sea by a narrow channel two or three hundred yards in length.

The later stages of development, and more especially the mode of formation of the gill-clefts, the endostyle, and the atrial cavity were described very fully by Mr. Willey and Professor Lankester in 1890 and 1891, from observations on specimens obtained by Mr. Willey from the same locality as Hatschek. Quite recently, 1892, Boveri has described the mode of formation of the reproductive organs.

The spawning period, in the Mediterranean, begins early in spring, towards the end of March, and continues throughout the summer, up to September; June being apparently the month of greatest activity. The eggs are laid about sunset, usually between 7 and 8 P.M. ; they are very small, $0.105 \mathrm{~mm}$. in diameter, and consequently contain but little food-yolk. They are fertilised at once, by spermatozoa shed over them by the male, and begin to develop almost directly. The early stages are passed through with great rapidity, and early on the following morning, about 
eight hours after the eggs were laid, the little embryos work their way out of the egg-membranes and swim freely. Their condition at hatching is shown in Figs. 25 and 26, p. 59 .

After hatching, the embryos continue to develop rapidly, and in about thirty-six hour's from the time of spawning they reach the stage shown in Fig. 34, p. 74. The mouth is not formed until the end of this period, and development up to this stage is apparently effected at the expense of the small amount of food-yolk contained in the egg.

After the formation of the month, the embryo continues its pelagic life, but from this time develops slowly, increasing in length, and gradually acquiring the shape and characters of the adult. During this period the anterior part of the body presents an extraordinary asymmetry, by which the mode of formation of the gill-clefts, which appear in order from before backwards, is profoundly modified. The mouth is a large oval opening (Fig. 36) placed, not on the ventral surface, but on the left side of the pharynx. The gill-slits of the two sides appear, not simultaneously, but successively; those of the left side, which may be termed primary slits, being formed before those of the right side, or secondary slits. The primary slits, of which there are as a rule fourteen, are not at first on the left side, but in the midventral wall of the pharynx, and shift upwards so as to actually lie for a time on the right side of the pharynx. The secondary slits, usually eight in number, appear along the right side of the pharynx dorsal to the primary slits (Fig. 38); while between the two series of gill-slits, primary and secondary, the endostyle is formed at the anterior end of the pharynx.

During the later stages of pelagic life, the total duration of which is about three months, this curious asymmetry is gradually rectified. The mouth assumes its median position, the primary gill-slits shift across the median line, and take up their permanent position on the left side of the pharynx; the endostyle shifts from the right side to the mid-ventral wall; and, by disappearance of some of the primary gill-slits, the number of primary and of secondary gill-slits becomes equalised, eight being present on each side of the pharynx.

At the close of the pelagic period, which may be called the critical stage, the young Amphioxus, now about $3.5 \mathrm{~mm}$. in length, adopts the mode of life of the adult, burrowing in the sand, and 
gradually, by increase in the number of the gill-slits and in other ways, acquires the structure and size of the fully formed animal.

The whole developmental history of Amphioxus may, in accordance with the above account, be conveniently divided into periods, which will be dealt with in succession in the remainder of this chapter.

I. The Embryonic Period : including the stages from the commencement of development to the formation of the mouth. This lasts about thirty-six hours, and is characterised by the extreme rapidity with which the stages, especially the earlier ones, are passed through; and by the fact that throughout the period the embryo is dependent for nutrition on the yolk granules contained within the egg. The actual rate of development varies to a certain extent with the temperature. The times here given are those recorded by Willey during the summer months; in spring, Hatschek found the rate of development to be slower. The period may be subdivided into two parts.

1. Before hatching: from the commencement of development up to the hatching of the embryo; a period of about eight hours.

2. After hatching : from the hatching of the embryo to the formation of the mouth; a period of about twenty-eight hours, during which the embryo leads a pelagic life.

II. The Larval Period: from the formation of the mouth to the critical stage. This lasts about three months, and during it the larva is pelagic. Development takes place slowly; and the most notable events are the formation of the gill-slits and the atrial cavity; and the curious series of changes by which the symmetrical condition of the larva is re-established.

III. The Adolescent Period: during which the young Amphioxus, having adopted the mode of life of the adult, gradually acquires its full structure, by increase in the number of the gillslits, ripening of the reproductive organs, \&c.

\section{THE EMBRYONIC PERIOD.}

From the laying of the eggs to the formation of the mouth. Duration of the period, from thirty-two to thirty-six hours.

Part I. From the laying of the eggs to the hatching of the embryo: a period of about eight hours. 


\section{The Egg.}

The ripe egg of Amphioxus is a spherical mass of protoplasm, $0.105 \mathrm{~mm}$. in diameter on the average, and inclosed in an elastic vitelline membrane. 'The protoplasm is studded with uumerous yolk granules, which are sufficiently opaque to hide the nucleus. At one pole, which will be spoken of as the upper pole, there is a slightly flattened patch of protoplasm comparatively free from yolk granules; and on the top of this patch is a sharply defined polar body (Fig. 14, I, PB). A second polar body has not been seen.

The vitelline membrane, prior to fertilisation, adheres closely to the egg.

\section{Fertilisation.}

The male Amphioxus, as described above (p. 46), sheds spermatozoa over the eggs as these are laid by the female; and they may be seen adhering in numbers to the vitelline membranes. The details of fertilisation have not been studied, but shortly after the spermatozoa gain access to the egg the vitelline membrane, which previously invested the egg closely, swells up rapidly by imbibition of water, and becomes separated from the egg by a considerable space; the egg ultimately lying in the centre of a capsule three or four times its own diameter. The purpose of this swelling up of the vitelline membrane, and its separation from the egg, is probably to prevent the entrance of other spermatozoa after the egg has been fertilised.

\section{Segmentation.}

The process of segmentation commences at dusk, usually about 8 P.M., and is completed in about three hours.

The first cleft appears about an hour after the eggs are laid and fertilised. It commences as a depression at the upper pole of the egg, close to the polar body, extends rapidly across the upper pole, and then spreads quickly round the egg as a groove (Fig. 14, i1). The groove deepens rapidly, being always more prominent at the upper than the lower pole; and in about five minutes from its first appearance the cleft is completed, the egg being divided by it into two halves or blastomeres of equal size, and similar in all respects save for the presence of the polar body on the apex of one of them. 

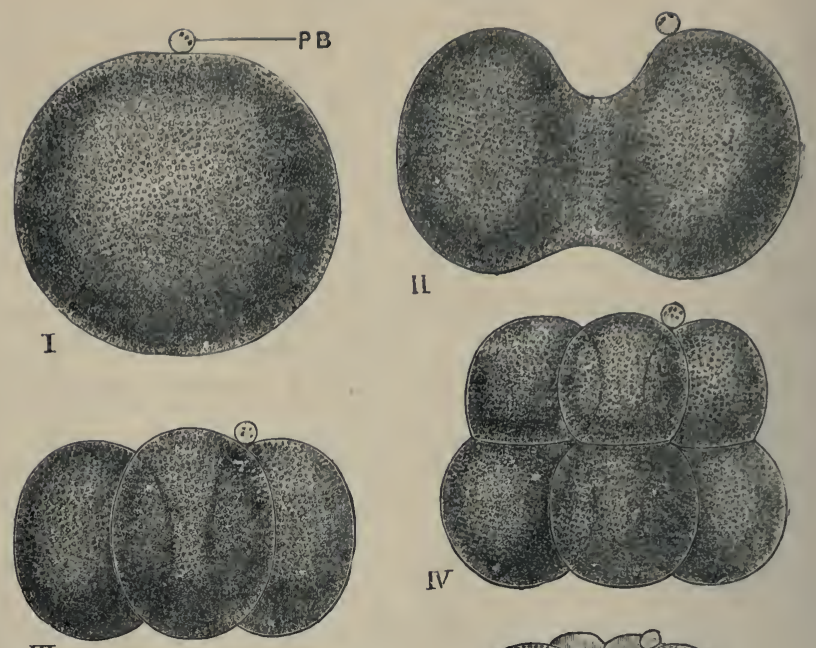

II

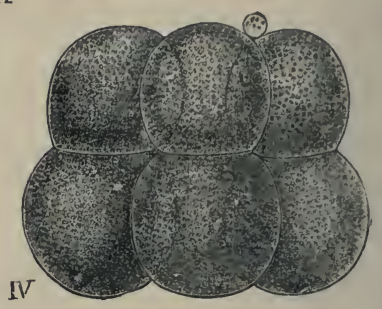

III
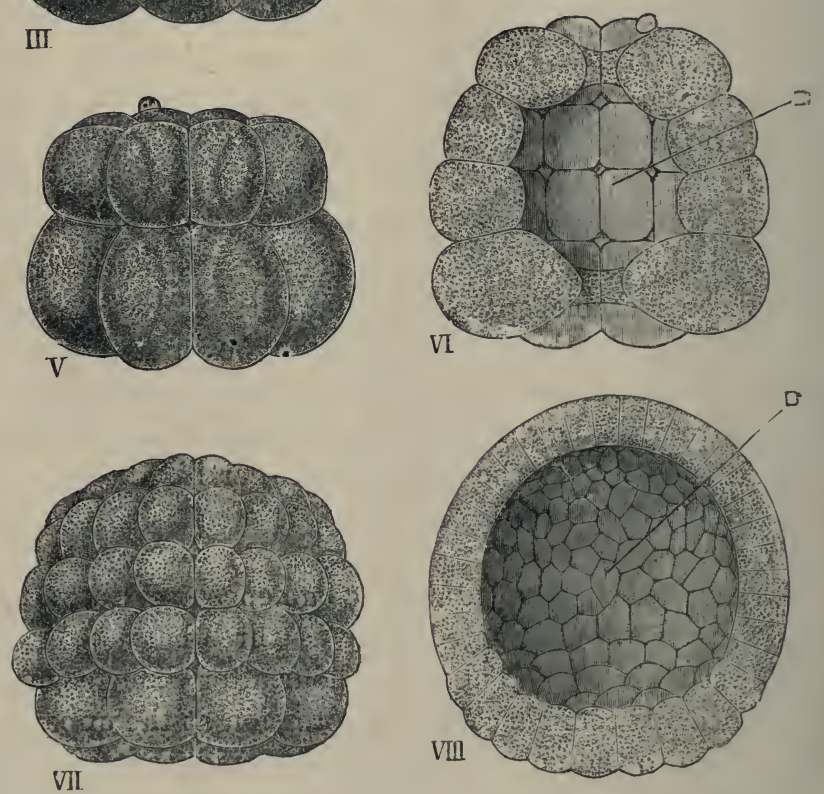

FIG. 14.-Segmentation of the egg of Amphioxus. $\times 220$. (After Hatschek.)

I, the egg before the commencement of development. PB, polar body. II, the egg in the act of dividing, by a vertical cleft, into two equal blastomeres; about one hour after fertilisation. III, stage with four equal blastomeres; about two hours after fertilisation. IV, stage with eight blastomeres : all upper tier of four slightly smaller ones, and a lower tier of four slightly larger ones. V, stage with sixteeu blastomeres, in two tiers, each of eight. VI, stage with thirty two-blastomeres, in four tiers, eacli of eight ; about three hours after fertilisation : the embryo is representerl bisecter vertically to show the segmentation cavity or blastocol, B. VII, later stage : the blastomeres have increased in number by further division. VIII, blastula stage: bisecterl to show the blistocol, $\mathbf{B}$; about four hours after fertilisation. 
A pause of about an hour now ensues, and then the second cleft is formed. This also is vertical, but in a plane at right angles to the first; it bisects each of the two first blastomeres, and so gives rise to four equal and similar blastomeres (Fig. 14, III); these are ovoid in shape, with their apposed surfaces slightly flattened by mutual pressure.

The third cleft, which appears about a quarter of an hour later, is a horizontal one, dividing each of the four blastomeres of the previous stage into two (Fig. 14, Iv). The cleft is a little above the equator, so that the four blastomeres of the upper tier are a little smaller than those of the lower tier. The blastomeres are in contact with one another laterally, but do not quite meet along the axis of the embryo. Hence the embryo is at this stage in the form of a ring, or short tube, with a central cavity, the segmentation cavity or blastocœl, which at present is open at both the upper and lower poles.

About a quarter of an hour later, the number of the blastomeres is again doubled by two new vertical clefts, which appear sinultaneously, in planes at right angles to each other, and at angles of $45^{\circ}$ with the first two clefts. The embryo now consists (Fig. 14, v) of sixteen blastomeres, arranged in an upper tier of eight rather smaller ones, and a lower tier of eight rather larger ones.

A little later, about three hours from the time of fertilisation, two more horizontal clefts appear simultaneously, dividing each of the tiers into two, and again doubling the number of the blastomeres. The embryo now (Fig. 14, vi) consists of four tiers, each of eight cells; the cells of the lowest tier, as shown in the figure, being decidedly larger than those of the other tiers. The blastocœl (Fig. 14, vI, B) still opens to the exterior at both poles, although the apertures are considerably narrowed by approximation of the cells of the upper and lower tiers respectively.

In the next stage (Fig. 14, vII) the lowest tier of blastomeres of the preceding stage has divided horizontally, giving five tiers in all; and each of the blastomeres of the four upper tiers has divided vertically into two. The embryo now, as shown in the figure, consists of five tiers of blastomeres, the four upper of which consist each of sixteen blastomeres, while the lowest tier consists of eight much larger blastomeres. The larva is nearly spherical in shape, and by approximation of the blastomeres of 
the top and bottom tiers the blastocœl is now completely closed.

From this time the blastomeres continue to increase in number by division, but in less regular fashion than before, so that the arrangement in tiers soon becomes lost : the blastomeres at the lower pole, however, remain throughout of larger size than those in other parts of the embryo. The polar body is often visible, resting on the upper pole of the egg, but it has sometimes disappeared by this stage. The blastomeres, which have hitherto been of somewhat irregular shape, rounded at their outer and inner ends, and flattened through mutual pressure at their sides, now begin to assume more definite form; and from this stage, which marks the close of segmentation, they may be more appropriately spoken of as cells.

\section{The Blastula.}

The embryo has now reached the stage to which the name blastula is given; a stage which occurs at corresponding periods in the development of a number of the lower animals, and which is therefore of interest as possibly representing a very early ancestral form of animal life. Pandorina and Volvox are examples of organisms in which the blastula stage forms the adult condition.

The blastula (Fig. 14, virI) is a spherical or ovoid embryo, consisting of a single layer of cells, inclosing a central segmen-. tation cavity or blastocol, filled with fluid. In the blastula of Amphioxus the cells are not all of equal size, those of the lower half, and especially those at the lower pole, being distinctly larger than those of the upper half; the greater size and more opaque appearance of these lower cells are due to the greater quantity of yolk granules which they contain. At first, the cells of the blastula, though flattened laterally where they press against one another, remain rounded at their ends, both inner and outer. These ends, however, soon become flattened; and the cells, in which the nuclei are now clearly visible, acquire the characters of columnar epithelial cells. These changes appear first at the upper pole of the embryo, and gradually extend to the lower pole. The blastula stage (Fig. 14, viII) is reached by the Amphioxus embryo at about the end of the fourth hour from the time of fertilisation of the eggs. 


\section{j. The Gastrula.}

On the completion of the blastula, as described above, the multiplication of the cells ceases for a time, and the embryo undergoes a great change in shape, whereby it becomes converted into the form to which the name gastrula is given. This change is brought about as follows.

The lower surface of the blastula, consisting of the larger cells, becomes flattened (Fig. 15, H), and then invaginated within the upper surface (Fig. 16). The embryo thus becomes cupshaped, its walls consisting of two layers: an outer layer, E, formed from the original upper part of the blastula; and an inner layer, $\mathrm{H}$, consisting of the invaginated cells, which originally formed the lower pole of the blastula.

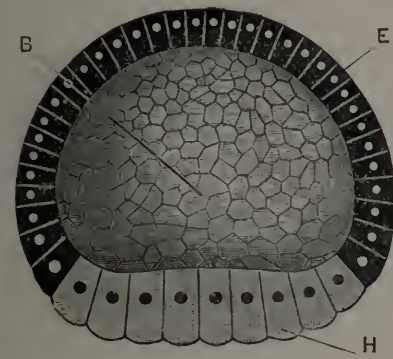

FIG. 15.

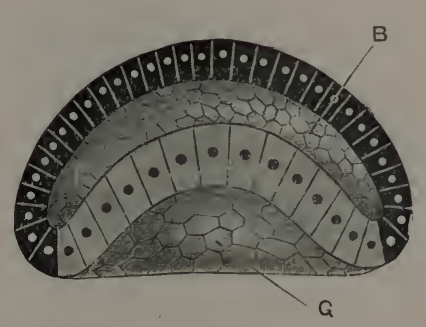

FIG. 16.

Ficis. 15 and 16.-Formation of the gastrula of Amphioxus. The embryos are bisected vertically, one half alone being represented. $\times 220$. (After Hatschek.)

Fig. 15.-Flattening of the lower pole of the blastula prior to invagination.

Fig. 16.-Commeneing invagination of the lower pole to form the gastrula.

B. blastocœl or segmentation cavity. E, epiblast. G, archenteron or gastrocœl. H, liypoblast.

As the invagination proceeds, the blastocœl becomes gradually diminished in size, and is ultimately completely obliterated, the inner and outer layers of the gastrula coming in contact with each other (Fig. 17, H, E).

The two layers of cells of which the wall of the gastrula consists are the two primary germinal layers. The outer layer is spoken of as the epiblast, E, and the cells of which it consists are called epiblast cells : the inner layer is the hypoblast, $\mathrm{H}$, and its cells, which originally were those forming the lower half of the blastula, are called hypoblast cells.

The cavity of the cup, formed by invagination of the hypo- 
blast, is called the archenteron or gastrocœl, G : it gives rise to the greater part of the alimentary canal of the larva and adult. The mouth of the cup is called the blastopore; it is at first (Fig. 17) a vary large aperture, but in the later stages becomes greatly reduced in size (Figs. 18 and 19).

Like the blastula, the gastrula is a very widely spread embryonic form, occurring not only in Vertebrates, but in a simple or modified condition in certain members of each of the great groups of Invertebrates as well. It has therefore, like the blastula, claims to be regarded as an ancestral form; claims which are greatly strengthened by the fact that some of the simpler sponges, and some of the Cœlenterates, such as Hydra, are closely comparable even in their adult condition to gastrulæ.

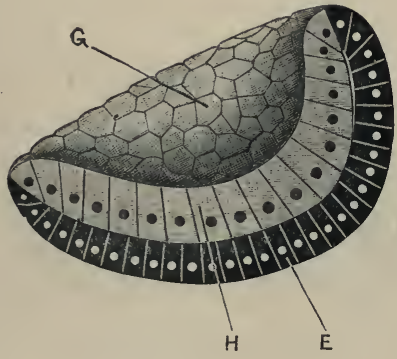

FIG. 17.

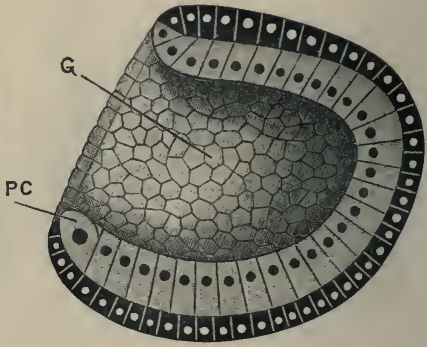

Fig. 18.

Figs. 17 and 18.-Completion of the gastrula of Amphioxus. The embryos are bisected vertically, and one half only of each is represented. $\times 220$. (After Hatschek.)

Fig. 17.-Completion of the process of invagination, and consequent obliteration of the blastocœl. Fig. 18.- Narrowing of the blastopore, through growth backwards of its dorsal lip. E, epiblast. G, archenteron or gastrocœl. H, lyypoblast. PC, polar mesoblast cell.

The mechanical causes that lead to invagination, i.e. that actually occasion the change from the blastula to the gastrula condition, are not easy to determine. The epiblast cells appear to take no part in the process, and to undergo no appreciable change or alteration during it; the active cells in the change are the hypoblast cells. By comparison of Figs. 15, 16, and 17, it will be seen that during invagination there is an increase in the number of the hypoblast cells ; and there is also, which is not so clearly brought out in the figures, an increase in the actual size of the individual cells. This increase in size is perhaps due to the cells absorbing the fluid of the blastocœl ; and this absorption 
of fluid may perhaps be one of the factors that determine or aid the process of invagination. It seems more probable, however, that invagination is due rather to inequality in the rates of growth of the cells at different parts, than to direct pressure from any cause on the surface of the embryo.

The later stages in the development of the gastrula show some features of importance. At its earliest formation, as shown in Fig. 16, the axis of the gastrula coincides with that of the blastula; and the blastopore or gastrula mouth is circular in outline. Later on, as shown by the careful observations of Hatschek, owing to unequal rates of growth in different directions, the blastopore becomes oval instead of circular in outline, and the shape of the embryo changes (Fig. 18) in such way that the axis of the gastrula no longer coincides with the original axis of the blastula, but forms a considerable angle with this.

At the stage shown in Fig. 18 there may be seen at the lower lip of the blastopore, and placed one on each side of the median plane, a pair of cells, $\mathrm{PC}$, which differ from the other hypoblast cells in their larger size and more rounded form, and in having very large nuclei. These two cells give rise at a later stage to important portions of the middle germinal layer or mesoblast: they may be named the polar mesoblast cells.

The further stages in the completion of the gastrula will be understood from a comparison of Figs. 18, 19, and 20. The embryo elongates, becoming ovoid or egg-shaped: at the same time the blastopore becomes still further reduced in size; the narrowing being effected, according to Hatschek, entirely by growth backwards of its anterior lip, the posterior lip, indicated by the pair of polar mesoblast cells, remaining quiescent throughout the process.

In the fully formed gastrula (Figs. 19 and 20), the ends and surfaces of the larva may be clearly recognised. The polar inesoblast cells, P C, mark the posterior end of the embryo; the blastopore, B P, now reduced to a small circular aperture, lies at the hinder end of the embryo, and slightly on the dorsal surface. The anterior end of the embryo is rounded and imperforate. The dorsal surface is flattened, and is further indicated by the blastopore; while the ventral surface is strongly convex.

If Hatschek is right in stating that the narrowing of the 
blastopore is effected entirely by growth backwards of its interior lip, then it is evident from a comparison of Figs. 17, 18, and 19 that the blastopore originally occupies almost the whole of what will afterwards be the dorsal surface of the larva; while the outer or convex surface of the young gastrula (Fig. 17) corresponds to the ventral surface, and perhaps also to the anterior end of the larva. If these determinations are correct, Figs. 15, 16, and 17 show that the lower pole of the blastula corresponds to the dorsal surface of the larva, and the upperpole to its ventral surface.

Before leaving the gastrula the cells of the two layers, epiblast and hypoblast, should be noticed more fully. The epiblast

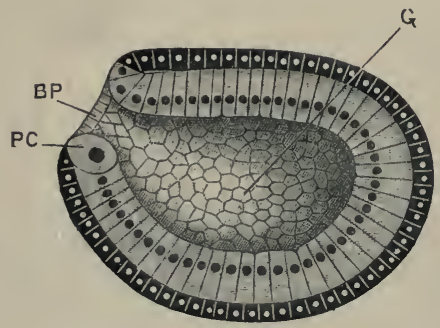

FIG. 19.

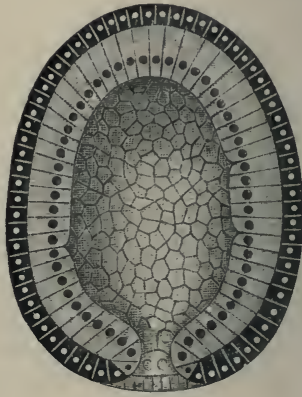

FIG. 20.

Figs. 19 and 20.-The fully formed gastrula of Amphioxus. $\times 220$. (After Hatschek.)

Fig. 19.-The gastrula bisected vertically : the left half is representerl, as sccn from the inner surface.

Fig. 20. - The gastrula bisected horizontally : the ventral half is represented, as seen from above.

BP, blastopore. $\mathbf{G}$, archenteron. PC, polar mesoblast cell.

(Figs. 19 and 20) is a single layer of very short columnar or almost cubical cells; at about the stage represented by Fig. 18 these cells develop on their outer surfaces flagella or lashlike processes, one from each cell, by which the embryo is caused to rotate within the vitelline membrane. These flagella persist. during the greater part of the pelagic existence of the embryo, but are not represented in the figures given here.

The hypoblast is a single layer of elongated columnar cells, with nuclei near their inner ends. At the lip of the blastopore the epiblast and hypoblast cells are necessarily continuous with one another; in the mid-ventral line the two polar mesoblast cells render the transition an abrupt one; but all round 
the rest of the lip, and especially at its dorsal or anterior border, the two layers pass gradually into each other. In the figures this transition has, for diagrammatic purposes, been represented as an abrupt one.

The fully formed gastrula stage, seen in Figs. 19 and 20, is reached, in the summer, in from seven to eight hours from the time of fertilisation of the eggs. In the spring, according to Hatschek's observations, the time taken to reach the same stage is about fourteen hours. A comparison of Figs. 14 and 19 will show that the gastrula, though of different shape, is approximately the same size as the egg itself.

\section{Development of the Embryo from the Completion of the Gas- trula to the Time of Hatching.}

The completion of the gastrula stage is followed by a short but well-marked and important period during which the rudiments of the nervous system, of the body cavity, and of the notochord are established, and at the close of which the embryo works its way out of the egg membrane, swims to the surface of the water by means of the flagella of the epiblast cells, and becomes a free living pelagic animal.

During this period it increases slightly in length but diminishes in breadth, so that at the time of hatching (Fig. 26) it is about twice as long as it is wide. Its bulk remains practically the same as before, for the mouth is not yet formed, and the embryo consequently cannot obtain food from without, but is still dependent for nourishment on the yolk granules contained in the cells, more especially in the hypoblast cells.

The nervous system is formed in the following manner. At the time of completion of the gastrula the epiblast is slightly flattened along the dorsal surface, as shown in Fig. 19, and still better in the transverse section, Fig. 21.

This flattened band of epiblast now becomes slightly depressed, and at the same time becomes marked off along its sides from the lateral epiblast (Fig. 22, xp). The lines of demarcation are at first indicated by slight modifications in the shape and arrangement of the cells, but soon become more pronounced, the edges of the lateral plates of epiblast overlapping the central depressed plate (Fig. 23), and ultimately meeting 
each other in the median plane so as to completely cover over the central plate (Fig. 24).

The central plate of epiblast, which thus becomes roofed over, is spoken of as the neural plate (Figs. 22-24, Nr), and becomes converted, later on, into the central nervous system. By longitudinal folding of the neural plate a groove is formed

Fig. 21.
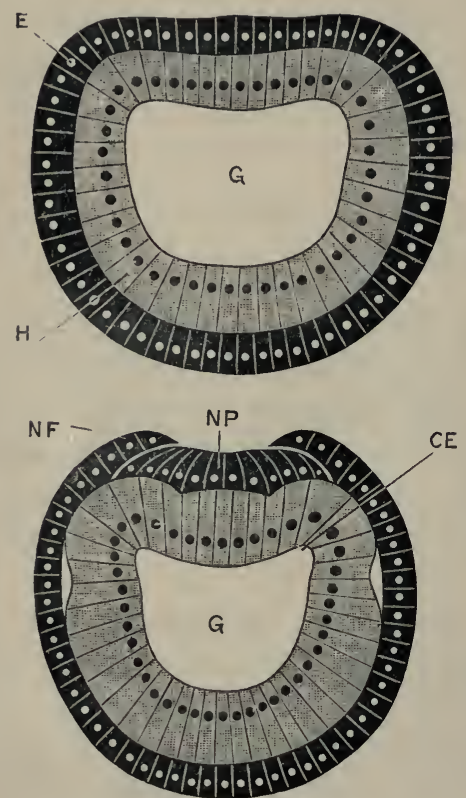

FIG. 23.
Fig. 22.
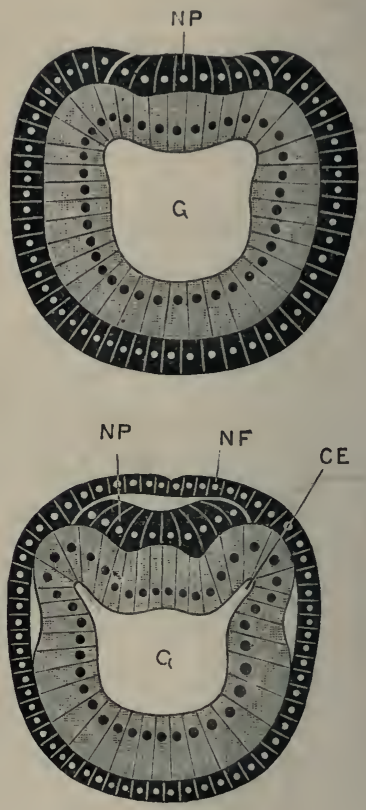

Fig. 24.

Figs. 21 to 24.--Transverse sections across the bodies of Amphinxus embryos, showing the mode of formation of the nervous system and of the mesoblastic somites. $\times 350$. (After Hatschek.)

Fig. 21.-Transverse section across the middle of the back of an embryo of the same age as those shown in Figs. 19 and 20. Fig. 22.-Transverse section across a slightly older embryo, with one pair of mesoblastic somites, and commencing nervous system. Fig. 23. - Transverse section across the same embryo as Fig. 22, but taken rather further back, the section passing throngh the middle of the first pair of somites. Fig. 24.-Transverse section through an embryo at the time of hatcling ( $c f$. Figs. 23 and 24): the section passes through the middle of the first pair of mesoblastic somites, and shows also the mole of formation of the neural tube. CE, enterocol or mesoblastic somite. E, epiblast. G, archenteron. H, hypoblast. NF', neural fold. NP, neural plate.

along its upper surface, and this groove, when roofed over by the lateral plates or neural folds, becomes the neural canal (Fig. 24).

The neural plate extends back to the blastopore, which, as already described, is situated on the dorsal surface of the hinder 
end of the embryo (Fig. 19, вг). The lateral plates, or neural folds, of the epiblast extend not merely along the edges of the neural plate, but round the sides and posterior lip of the blastopore as well; and by their fusion in the median plane the blastopore becomes roofed over, so that it no longer opens directly to the exterior, but into the hinder end of the neural canal ( $c f$. Figs. 19 and 25). The blastopore thus forms a short tubular channel of communication between the neural canal and the archenteron, and to this channel the name neurenteric canal is given (Fig. 25, NT).

It is a curious fact, and one the full meaning of which is not yet determined, that for a time the sole communication between the archenteron, or primitive alimentary canal, and the

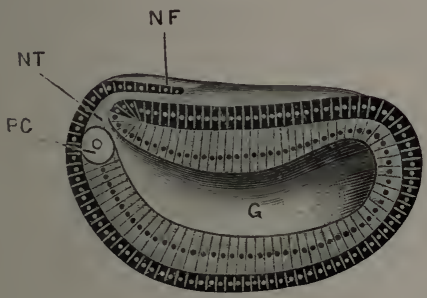

FIG. 25.

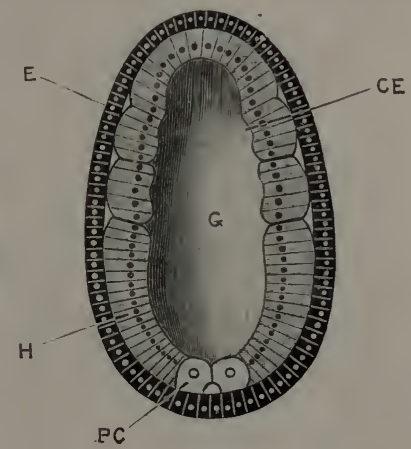

FIG. 26.

Figs. 25 and 26.-Amphioxus embryos at the time of hatching. $\quad \times 220$. (After Hatschek.)

Fig. 25.-The embryo bisected vertically : the left half is represented, as seen from the inner surface. Fig. 26.-The embryo bisected horizontally : the ventral half is represented, as seen from above. CE, enterocœel or mesoblastic somite. E, epiblast. $\mathbf{G}$, archenteron. H, hypoblast. NE, neural fold. NT, neurenteric canal. PC, polar mesoblast cell.

exterior should be through the central canal of the nervous system. Kowalevsky, who discovered the neurenteric canal in Amphioxus and in the Ascidians, suggested that these relations may possibly be ancestral, and that animals may have existed, or may still exist, in which the nerve-tube fulfilled a non-nervous function, and possibly acted as part of the alimentary canal. Comparative anatomy has not at present, however, given any support to this suggestion.

The closure of the neural tube, by meeting and fusion of the neural folds, proceeds from behind forwards, so that a section 
through the posterior part of an embryo (Fig. 23) will show a more advanced stage in the formation of the nervous system than one taken through the same embryo nearer its anterior end (Fig. 22).

At the time of hatching (Fig. 25), the closure of the neural tube is completed along about a third of the length of the embryo; the anterior opening of the tube, just in front of the reference line $\mathrm{NF}$ in the figure, is spoken of as the neuropore.

The mesoblastic somites. During the formation of the neural canal important changes take place in the hypoblast. The flattening of the dorsal surface of the embryo at the completion of the gastrula stage affects the hypoblast as well as the epiblast (cf. Figs. 19 and 21). As the neural plate becomes marked off and depressed, a pair of longitudinal folds of the wall of the archenteron are formed, one along each side, in the angle between its dorsal and lateral walls (Figs. 22 and 23, CE). These folds are at first very inconspicuous, but rapidly become more prominent, and especially so about the time of closure of the neural canal (Fig. 24, CE).

By the formation of these folds the archenteron becomes divided into three portions : a central division (Fig. 24, G), which is the alimentary canal itself, and a pair of lateral slit-like diverticula (Fig. 24, CE), which may be termed enterocœlic cavities, and which later on give rise to the body cavity or cœlom of the adult.

The cells composing the walls of these folds are clearly of hypoblastic origin. In the later stages (cf. Figs. 27, 28, and 29), they separate completely from the wall of the alimentary canal, and are then spoken of as forming a third germinal layer, or mesoblast, situated between the two primary layers, epiblast and hypoblast.

The mesoblastic folds extend the whole length of the embryo; they are most prominent near its anterior end, and gradually diminish posteriorly, becoming continuous at their hinder ends with the two large polar mesoblast cells (Figs. 25 and 26, p which have already been described as present in the posterior lip of the gastrula from an early stage in its formation (Fig. 18).

Soon after their appearance, the mesoblastic folds become divided by transverse constrictions into segments or compartments, the mesoblastic somites, arranged in pairs along the sides of the embryo. The anterior pair of somites, which are the first 
to be formed, lie a little way behind the anterior end of the embryo, and the remaining ones are formed in succession from before backwards as the embryo increases in length; at the time of hatching, two pairs of mesoblastic somites are usually present (Figs. 24, and 26, CE).

The notochord. The roof of the archenteron, between the mesoblastic folds, is formed by a band of hypoblast cells lying immediately below the neural plate, and in close contact with this (Figs. 21 to 24). The cells composing this band, up to the time of hatching, differ little if at all from the hypoblast cells of the sides or floor of the archenteron; but shortly after the time of hatching, they undergo changes and become converted into the notochord, the most important part of the skeleton of Amphioxus.

Condition of the embryo at hatching. At the time of hatching, which occurs about eight hours after fertilisation of the egg, the embryo (Figs. 25 and 26) is ovoid in form, abont twice as long as it is wide, and in bulk about equal to the egg from which it was developed (cf. Fig. 14, 1). The epiblast is a single layer of short, almost cubical cells, each of which bears a single flagellum, by which the swimming of the embryo is effected. The neural canal is roofed in for about the hinder third of its length; in front it opens to the exterior by a rather wide aperture, the neuropore; posteriorly, the neural canal communicates with the archenteron through the neurenteric canal, the former blastopore. The mesoblastic folds are present, and two pairs of mesoblastic somites are already constricted off from their anterior ends.

Immediately after working its way out of the egg membrane the embryo swims to the surface of the water, and enters on the second part of the embryonic period.

Part II. From the hatching of the embryo to the formation of the mouth: a period lasting from about twenty-four to twentyeight hours ( $c f \cdot$ p. 48).

The later stages of embryonic development consist chiefly in further elaboration of the organs which are already established at the time of hatching. The nervous system becomes more complex; the mesoblastic somites increase considerably in number, and undergo important changes whereby the muscular and other systems are formed; the notochord is definitely established; and at the close of the period the mouth and first 
gill-slit are formed. The embryo elongates very rapidly, and becoming much narrower and more slender, gradually acquires a shape and proportions resembling those of the adult. During the whole period the embryo is pelagic: swimming is effected at first by the flagella clothing the surface, but towards the close of the period the muscles of the body-walls become definitely established, and the young Amphioxus swims by means of muscular contractions, like the adult.

Although there is a great increase in length during the period, there is little if any change in bulk, and it is doubtful whether the embryo obtains any food from without until the formation of the mouth at the close of the period.

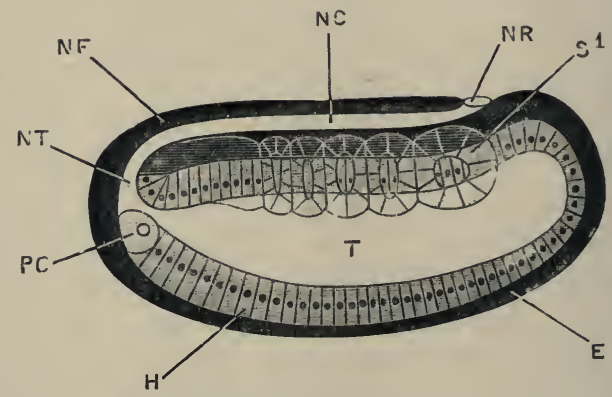

FIG. 27.-Amphioxus embryo shortly after hatching, with five pairs of mesoblastic somites; seen in optical section from the right side. $\times 224$ (After Hatschek.)

E, epiblast. H, hypoblast. NC, neural canal. NF, neural fold. NR, neuropore. NT, neurenteric canal. PC, polar mesoblast cell. S1, first mesoblastic somite of riglit side. T, archenteron.

In dealing with this period in the developmental history it will be convenient to describe the several systems one by one.

\section{The Nervous System.}

After hatching of the embryo, the closure of the neural canal. by fusion of the neural folds, proceeds rapidly forwards (Figs. 25 and 27), and soon reaches the anterior border of the first somite, beyond which level the nervous system does not extend.

From the mode of its formation (Figs. 23, 24, 26, and 27), the neural canal is, in its early stages, merely the space between the neural plate and the overlapping lateral plates of epiblast, and has at first no independent roof of its own. The canal is at first wide from side to side, but shallow dorso-rentrally. 
In the later stages the neural canal deepens, owing to longitudinal folding of the neural plate; at the same time the cells at the free margins of the plate grow in towards one another from the two sides, and meeting in the median plane complete the wall of the neural canal (Fig. 32).

The nervous system is now a tube (Figs. 30 and 33), with proper walls of its own, extending along the dorsal surface of the embryo. It opens in front to the exterior, at the neuropore, opposite the anterior border of the first somite; and it communicates posteriorly with the archenteron, through the neurenteric canal. The wall of the tube consists of a single layer of cells, which bear flagella at their inner ends.

The anterior end of the neural tube, close to the neuropore, has, almost from the first, thicker walls than the rest of the tube. This thickening, which affects especially the ventral wall of the tube (Fig. 33), becomes much more marked in the later stages; partly owing to actual increase in the thickness of the wall itself; and partly to a great diminution in the diameter of the hinder part of the tube, as the embryo becomes drawn out into the elongated form characteristic of the later larval condition.

In the ventral wall of the neural tube, opposite the fifth pair of somites, a black pigment spot, possibly a sense organ, appears at about the stage represented in Fig. 33; and much later, towards the end of the embryonic period, anotherpigment spot, the eye, is formed in the anterior wall of the brain swelling ( $c f$. Fig. 36 ).

\section{The Notochord.}

The notochord is developed, as already noticed, from the band of hypoblast cells which forms the dorsal wall of the archenteron, and lies between the two lateral mesoblast folds.

Its earliest appearance as a distinct structure is seen in a larva with three pairs of somites, i.e. immediately after the time of hatching; and the successive stages in its formation are shown in Figs. 28, 29, and 32, $\mathrm{cH}$.

The median plate of hypoblast cells, forming the roof of the archenteron, first becomes marked off, by a difference in mode of arrangement of the cells, from the lateral mesoblast folds, and 
then grooved ventrally along the median plane (Fig. 28). The ventral groove deepens, and at a stage with five pairs of mesoblastic somites the plate is completely folded on itself, so that its two halves are in contact with each other. The cells of the two halves now begin to grow across the median plane, interdigitating with one another (Fig. 29, $\mathrm{CH}$ ), and forming a solid ridge of cells along the mid-dorsal surface of the archenteron. At a slightly later stage, with eight or nine pairs of mesoblastic somites, this ridge begins to separate from the gut wall as a cylindrical rod of cells, the notochord (Fig. 32, CH).

Behind the first somite, i.e. along the greater part of its

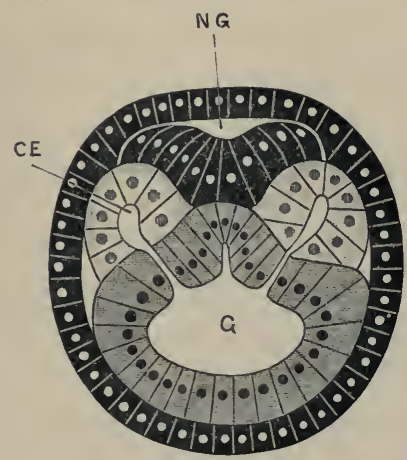

FIG. 28.

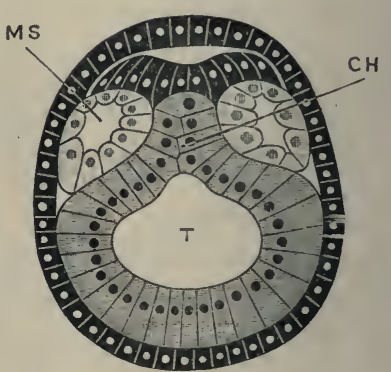

FIG. 29.

FIGs. 28 and 29.-Transverse sections through Amphioxus embryos shortly after the time of hatching ; showing stages in the formation of the notochord and mesoblastic somites. $\times 435$. (After Hatschek.)

Fig. 28.-Embryo with five pairs of somites : transverse section through the mirldle of the first pair. Fig. 29.-Embryo with six pairs of somites : transverse section through the hinder end of the first pair. $\mathbf{C E}$, enterocœlic pouch or mesoblastic somite. CH, notochord. G, archenteron. MS, mesoblastic somite. NG, neural cana!, $\mathrm{T}$, mesenteron.

length, the notochord develops from before backwards. Opposite the first somite the notochord forms more slowly, and is always a little behind the stage reached in the second somite. In front of the first somite the notochord is developed from behind forwards, but otherwise in the same manner as in the hinder part, though much more slowly; towards the close of the embryonic period, at the time when the pointed anterior end of the animal is forming, it grows much more rapidly (Fig. 33). This late development of the anterior end of the notochord will be referred to again further on. 
Opposite the neuropore, and corresponding to the marked thickening in the ventral wall of the neural tube already described, there is a distinct bending of the notochord (Fig. 33), traces of which persist even in the adult animal.

The histological development of the notochord presents some features of interest. The interdigitation of the cells of the two sides, the commencement of which is shown in Fig. 29, proceeds rapidly; and, at the time of its separation from the gut, the notochord consists (Fig. 32) of four or five rows of cells, arranged horizontally one above another, each cell extending across the whole of its width. Within the notochordal cells numerous small vacuoles now appear; these vacuoles are, from the first, most abundant in the two middle rows of cells, and in these they increase greatly in size; so that in its later stages, as in the adult, the notochord consists of a middle series of cells, enormously distended by vacuoles, and covered on its dorsal and rentral surfaces by rows of smaller and comparatively little modified cells.

\section{The Mesoblastic Somites.}

The mesoblastic ridges, as described above, are a pair of longitudinal folds of the dorso-lateral walls of the archenteron, inclosing slit-like diverticula of the archenteric cavity (Figs. 26, 28). By transverse constrictions these ridges become divided into somites, which, though separated from one another by the constrictions, still retain for a time their communications with the archenteron (Figs. 27, 28).

At the time of hatching, two pairs of these somites are present; and, as the embryo elongates, other pairs are added in succession from before backwards, the number of pairs of somites present affording a convenient basis for estimating the age of an embryo (Figs. 27, 30, 33).

The anterior somites, which are the first formed, are also the largest, and the remainder decrease in size towards the hinder end of the embryo (Figs. 27, 30); the hindmost pair passing into the, as yet, unsegmented mesoblast folds, which end posteriorly in the two polar mesoblast cells (Figs. 30, $31, \mathrm{PC})$.

At a stage when six pairs of somites are present, the cavities of the anterior ones become constricted off from the 
archenteron, and separate completely from this (Fig. 29). This separation rapidly extends backwards, involving the hincler somites in succession; and the somites now form (Figs. 27, 29) a series of squarish hollow bodies, arranged in a row along each side of the embryo, at the level of the notochord.

The somites are at first small, and lie above or dorsal to the alimentary canal (Fig. 29); but they rapidly increase in size, and, extending ventralwards (Figs. 30 and 32 ), make their way

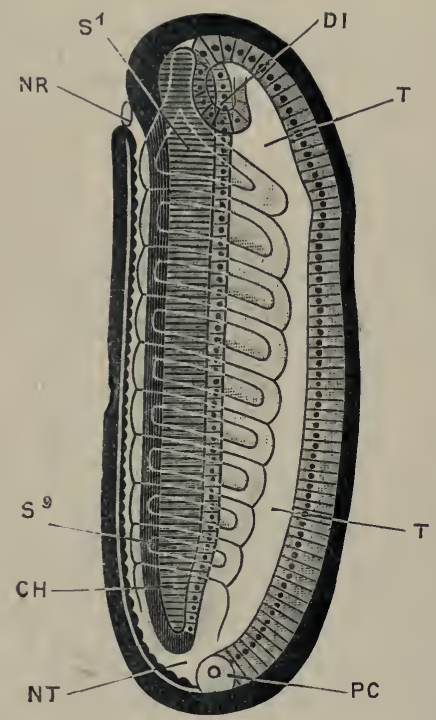

FIg. 30.

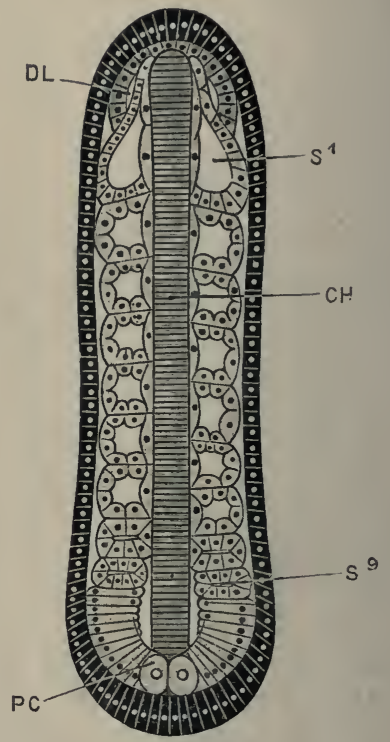

Fig. 31.

FIGs. 30 and 31.-Amphioxus embryos with nine pairs of mesoblastic somites. $\times 224$. (After Hatschek.)

Fig. 30.-Embryo seen in optical section from the right side. Fig. 31.-Embryo seen in horizontal section, at the level of the notochord. CH, notochord. DL, left anterior gut diverticulum. DR, right anterior gut diverticulnm. NR, neuropore. NT, neurcnteric canal. PC, polar mesoblast cell. S1, first mesoblastic somite of the right side. S9, ninth mesoblastic somite of the right side. $\mathbf{T}$, mesenteron.

round the sides of the embryo, between the gut wall and the external epiblast, ultimately reaching the mid-ventral line, where the somites of the right and left sides of the body become continuous with one another.

During their earlier stages (Figs. 27, 30), the long axes of the somites lie transversely, or slightly obliquely to the axis of the embryo; but towards the close of the embryonic period 
(Fig. 33) they acquire the $>$-like shape so characteristic of the adult (Fig. 11, s).

The walls of the somites soon undergo important changes. At the time of separation from the archenteron (Fig. 29, MS), the wall of each somite consists of a single layer of cells, somewhat irregular in shape and size, but showing no marked differences in different parts. As the somites extend down the sides of the body they become somewhat triangular in transverse section. In each somite there may now be distinguished (Fig. 32) an outer or parietal wall, next the external epiblast; a visceral wall, in contact with the hypoblast of the archenteron; and a notochordal wall, forming the base of the triangle, and in contact with the notochord and the nerve cord. The cells of the parietal and visceral walls are slightly flattened, but show no special peculiarities; those of the notochordal wall, on the other

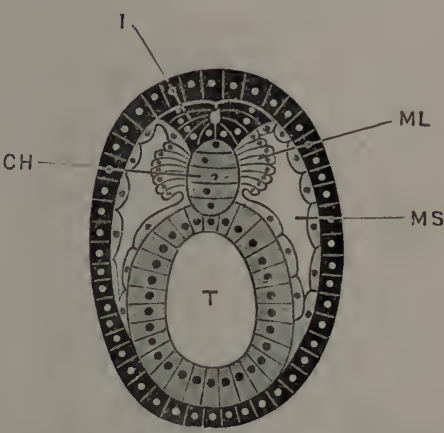

FIG. 32.--Transverse section through the middle of an Amphioxus embryo with nine pairs of mesoblastic somites. $\times 435$. (After Hatschek.)

$\mathrm{CH}$, notochord. I, spinal coril. MI, muscle layer. $\mathbf{M} S$, cavity of mesoblastic somite. T, mesenteron. hand, show marked changes. Each cell (Fig. 32, IIL) is much flattened dorso-ventrally, and elongated in a direction parallel to the axis of the embryo (Fig. 31) ; and is undergoing changes by which it becomes converted into a muscle cell or fibre. This differentiation of muscle cells begins at a stage with about nine pair of somites, and proceeds rapidly; the muscles, at a stage with eleven pairs of somites, beginning to contract and cause lateral undulations of the body. The mass of muscle cells, formed in this way by modification of the notochordal wall of a somite, is called a myotome: the myotomes, being formed from the somites, are, like these, arranged segmentally from their first appearance; they increase rapidly in size, and become the great lateral muscles or myotomes of the adult Amphioxus (Fig. 12, x). Each muscle cell extends the whole length of the somite to which it belongs.

In the higher Vertebrates it will be found that the earliest 
muscles to appear in the development of the embryo corresponit in mode of formation, and in relations, to the myotomes of $\mathrm{Am}$ phioxus. The formation of muscles, as indeed of all other tissues, by direct modification of epithelial cells, is a further point of very great and general interest, indicating that the epithelial cell is a more primitive type of structure than muscle, connective tissue, nerve tissue, or any of the other histological elements of which the body of an adult animal is composed.

The cavities of the somites give rise to the cœlom or body cavity of the adult. After their separation from the archenteron they are completely closed, and remain so for some time; the anterior and posterior walls of adjacent somites becoming closely applied to one another, and forming septa which separate the cavities of successive somites from one another (Fig. 31). Towards the close of the embryonic period, the ventral portions of these septa disappear, so that the somites open into one another; and the body cavity, which up to this time has been represented by a series of isolated chambers, now becomes continuous from end to end of the animal. The dorsal portions of the somites, however, remain separate from one another throughout life.

The first somite (Fig. 27, s 1) is a little distance from the anterior end of the body: from its anterior and dorsal border, at a stage with about nine pairs of somites, a hollow conical process is given off towards the anterior end of the embryo (Figs. 30, 31); the walls of this process undergo changes similar to those described above as occurring in the body of the somite itself.

At the time of their first appearance the somites are paired; the two somites of each pair being exactly opposite each other, and the whole ernbryo being bilaterally symmetrical. At a stage with nine pairs of somites this symmetry becomes disturbed (Fig. 31), the somites of the right side becoming situated a little behind the corresponding ones of the left side, and ultimately alternating with these. This curious lateral asymmetry is preserved in all the later stages, and in the adult animal as well. The fact that the somites are at first symmetrically arranged shows that it is a secondary and not a primitive feature, and the further fact that it appears just at the time when the great lateral muscles are being formed, and are coming into use for swimming, suggests that the explanation of the asymmetry is to be found in some mechanical advantage gained by the alternating arrangement of 
the muscles in an animal in which the skeleton is represented merely by an elastic notochord.

The development of new somites during the later stages of embryonic life occurs very slowly; and at the time of the formation of the mouth, marking the close of the period, there are not more than fourteen or fifteen pairs. The elongation of the body, which is so marked a feature of the later embryonic stages, is due, not so much to addition of new segments, as to lengthening of those already present; and this lengthening, as shown in Figs. 33 and 34 , principally concerns the anterior or oldest somites.

\section{The Alimentary Canal.}

After separation of the somites and the notochord, the archenteron, or, as it is usually termed from this time, the mesenteron, forms a straight tube (Figs. 30 and $33, \mathrm{~T}$ ), dilated at its anterior end, but narrow and cylindrical along the greater part of its length. It is closed in front, but at its hinder end it communicates through the neurenteric canal with the

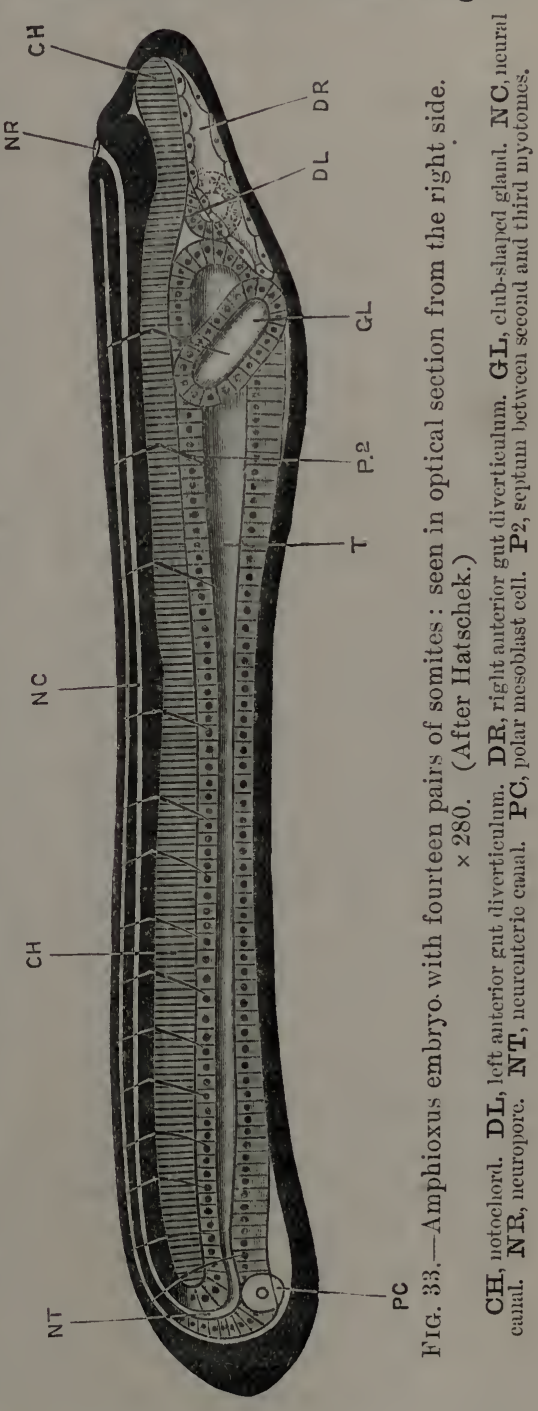
neural tube, and so, indirectly, through the neuropore, with the exterior. It is ciliated along its entire length, but no food particles have as yet been observed in it prior to the formation of the mouth. 
a. The anterior gut diverticula. At a stage with seven pairs of somites, a pair of lateral diverticula arise from the dilated anterior end of the mesenteron. These are situated (Figs. 30, 31 , DL, DR), near the dorsal surface of the mesenteron, just in front of the first pair of somites, and ventral to the anterior prolongations of these somites.

The two diverticula soon separate from the mesenteron, which then shrinks back from the anterior end of the body. They are at first of equal size, but from a stage with about ten pairs of somites, onwards, they develop very unequally.

The right anterior gut diverticulum (Fig. 33, DR) forms a thinwalled sac, which extends forwards so as to occupy a large space at the anterior end of the body, below the notochord; its walls become flattened epithelial cells, and the space which they inclose may be spoken of as the head-cavity.

The left anterior gut diverticulum (Fig. 33, DL) remains of small size, and forms a spherical thick-walled sac, lying on the left side of the head, just in front of the mesenteron and a little way behind the level of the neuropore; its wall consists of a single layer of columnar ciliated epithelial cells. Towards the close of the embryonic period it opens to the surface by a small pore on the left side of the head (Fig. 34, DL), and from this time is spoken of as the præoral pit.

The homologies of these anterior gut diverticula with organs of higher Vertebrates are very uncertain. They are probably to be regarded as parts of the body cavity or colom, though it must be admitted that their development differs in important respects from the rest of the cœlom. In the mode of their origin, in their asymmetry, and in the fact that the left diverticulum early acquires an opening to the exterior, they resemble the anterior cœlomic diverticula of Balanoglossus, and the enterocelic outgrowths of Echinoderms, with which they have by some observers been held to correspond.

b. The club-shaped gland. In embryos with nine or ten pairs of somites a shallow transverse groove appears across the floor of the mesenteron, and extending up its sides, opposite the septum between the first and second pairs of somites. The first commencement of this groove is seen in Fig. 30, opposite the ventral end of the first somite, but is not indicated by a reference letter. Towards the end of embryonic life the lips of the 
groove close to form a tube, which splits off along its whole length from the mesenteron, but remains in close contact with this. The limb of the tube which lies at the right side of the mesenteron expands slightly to form the club-shaped gland (Fig. 36, $\mathrm{GL})$; the rest of the tube forms a slender duct, which passing across the body, under the mesenteron, to its left side (Fig. 36, $\mathrm{GD})$, acquires an opening to the exterior just below the anterior border of the mouth, as soon as this latter is formed. The further development of the club-shaped gland will be described in the section dealing with the larval stages.

c. The mouth. At the close of the embryonic period, a disclike thickening of the epiblast forms on the left side of the head, opposite the first somite but ventral to its lower edge. The hypoblast of the mesenteron fuses with this patch of epiblast, and the mouth is formed as a perforation in the middle of the fused patch. The mouth is at first a minute circular aperture, but it rapidly increases in size, and at the end of the embryonic period is a large oval opening (Fig. 36, o), with a slightly thickened border, on the left side of the head.

d. The first gill-slit. Simultaneously with the formation of the mouth, a slight depression of the hypoblast of the ventral surface of the mesenteron appears, opposite the second pair of somites; this fuses with the epiblast, and then, by perforation, an opening is formed which is the first gill-slit (Fig. 34, I). The perforation is formed from within outwards: the gill-slit is at first very small, and situated in the mid-ventral wall; but it soon enlarges, and as it does so shifts upwards to the right side of the body (Fig. 36, HK 1). Like the mouth, it is bordered by long cilia.

e. The anus. This is formed shortly after the mouth and the first gill-slit (Fig. 34, U). It is at first much nearer the hinder end of the body than in the adult, and is placed slightly to the left of the median plane.

\section{The Blood-vessels.}

The development of the blood-vessels in Amphioxus has been but very imperfectly studied. The first vessel to appear is said to be the ventral or cardiac aorta, which is developed in a longitudinal strip of mesoblast, formed by fusion of the ventral edges of the somites of the two sides along the mid-rentral line, 
and extends along the whole length of the under surface of the intestine. The anterior end of the aorta, on reaching the level of the second somite, turns upwards, and runs obliquely forwards along the right side of the pharynx, passing dorsal to the first gill-cleft, and ending in close relation with the club-shaped gland.

\section{Structure of the Embryo at the Close of the Embryonic Period.}

The general appearance of the embryo at this stage is shown in Fig. 34. The embryo has a total length of about $1.3 \mathrm{~mm}$., and is of a glassy transparency in all its parts and organs, owing to the complete absorption of the yolk granules originally present in the egg. It is widest about the level of the mouth, in front of which it tapers rapidly, ending in a sharply pointed snout. The hinder part of the body tapers very gradually, and ends in a thin vertical fin of rather larger size than is shown in the figure.

The embryo swims actively, by alternating contractions of the myotomes of the two sides of the body. Of these myotomes there are fifteen pairs present; the myotomes of the first pair are opposite each other, those of the next two or three pairs are placed more or less obliquely, and behind the fourth pair the myotomes alternate regularly along the two sides of the body. The first pair of myotomes give off anterior prolongations, which extend along the sides of the notochord to the tip of the snout, and by their contractions bend the snout freely from side to side. Each muscle fibre is formed by elongation of a single cell, and the majority of the fibres show more or less evident transverse striation. The alimentary canal is divided into an anterior, dilated, pharyngeal region, lying opposite the first two myotomes; and a posterior, cylindrical, intestinal region which extends to the anus. In connection with the pharyngeal region are the mouth, the first gill-slit, and the club-shaped gland; there is as yet no trace of the liver.

The nervous system consists of a neural tube, with proper walls of its own, extending the whole length of the back of the animal, just above the notochord. The neural tube opens to the exterior at its anterior end through the neuropore, immediately behind which the tube presents a slight dilatation or 'brain.' The posterior end of the neural tube (Fig. 34, NE) bends downwards 
round the end of the notochord, and still communicates, though by a very minute aperture, with the hinder end of the intestine. Sense organs are represented by pigment spots in the wall of the neural tube; and a pair of small filaments, formed of elongated and adherent cilia, and situated on the under surface of the body behind the mouth, are very possibly taste organs.

One of the most interesting points to notice is that, up to this stage, all the various parts of the body, the epidermis, the walls of the neural tube and of the alimentary canal, the myotomes, \&c., all alike consist of single layers of cells, and cells which, at any rate in their earlier stages of development, are of epithelial origin.

\section{THE LARVAL PERIOD.}

This extends from the formation of the mouth to the critical stage, at which latter date the mouth assumes its median position, and the gill-slits become symmetrically arranged on the two sides of the pharynx. The duration of the period is about three months.

During the larval period, development proceeds far more slowly than in the earlier stages. An interval of about a fortnight is said to elapse between the formation of the first and the second gill-slits; and the close of the larval period, which indicates a very definite stage in development, is also marked by a pause of considerable duration. The chief events that occur during the larval period are the formation of the gillslits of both sides of the pharynx, the formation of the endostyle, the development of the atrial cavity, the shifting of the mouth to its adult position, the establishment of the full number of myotomes, together with certain important changes in their relations to other organs, and the disappearance of the clubshaped gland.

Until recently our acquaintance with these stages was very fragmentary, and due entirely to Kowalevsky's careful, but brief and incomplete descriptions. Now, owing to Hatschek's observations on the development of the myotomes, and those of Willey and Lankester on the formation of the gill-slits, atrial cavity, and endostyle, we have far more complete and satisfactory knowledge of the actual course of events, although there are many points that still require investigation. 
During the larval stages the young Amphioxus leads a pelagic life, and is found most abundantly, not at the surface,
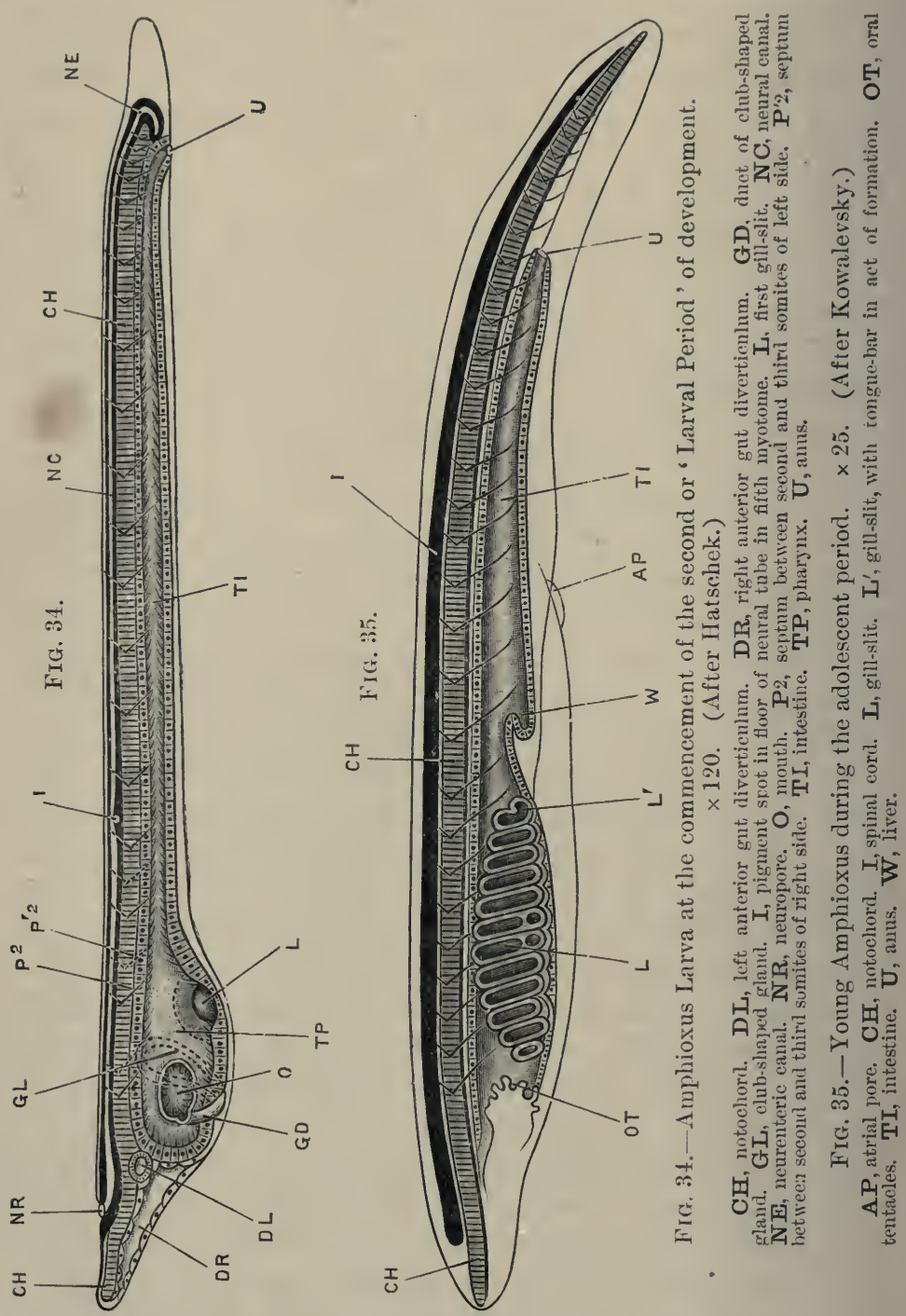

but at depths of from fifteen to twenty fathoms. At the close of the period, it usually abandons its pelagic life, and adopts the burrowing habits of the adult. The actual time, however, at 
which the larva takes to living in the sand varies greatly in different individuals. At the close of the larval period the larva measures about $3.5 \mathrm{~mm}$. in length.

\section{The Gill-slits.}

It has already been mentioned, in the general account of the development of Amphioxus, that the gill-slits of the two sides are not formed simultaneously; those of the left side, which may be termed primary slits, appearing before those of the right side, or secondary slits. The primary slits, of which there are as a rule fourteen, are formed, not on the left side, but in the mid-ventral wall of the pharynx, and, after their formation, shift upwards so as actually to be for a time on the right side of the pharynx. The secondary slits, usually eight in number, are formed at a later stage, along the right side of the pharynx, dorsal to the primary slits. Towards the close of the larval period, as the mouth assumes its median position, the primary slits shift across to their permanent position on the left side: at the same time, by an actual diminution in number, through disappearance of the slits at the two ends of the series, the primary slits become reduced to eight, and the critical stage is reached, at which the primary and secondary slits are equal in number, and symmetrically arranged along the left and right sides of the pharynx respectively.

a. The primary gill-slits, or the gill-slits of the adult left side of the pharynx, are formed in succession from before backwards. Like the first gill-slit, the development of which has already been described, each of the succeeding primary gill-slits lies at first in the mid-ventral wall, but, with the exception of the hindermost two or three, shifts almost at once to the right side of the pharynx. The full number of primary gill-slits is as a rule fourteen, but varies in different specimens from twelve to fifteen. The slits are at first metamerically arranged, corresponding, when they are fourteen in number, to the somites from the second to the fifteenth inclusive; this metameric arrangement is, however, entirely lost in the later stages of development.

The condition with three fully developed primary gill-slits, and $a$ fourth slit in the act of forming, is shown in Fig. 36 ; and the stage in which all fourteen primary gill-slits are present, in 
Fig. 37. The gili-slits are at first wide, window-like apertures in the wall of the pharynx; and, until the formation of the atrial

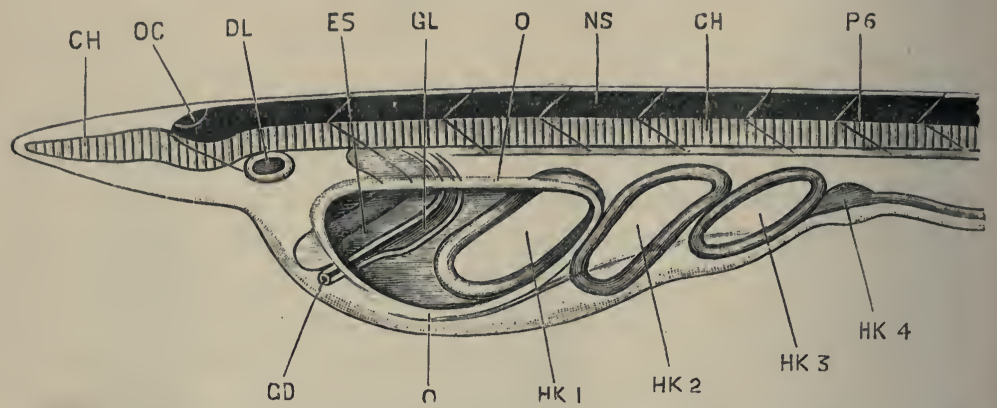

FIG. 36. - The anterior end of an Amphioxus Larva with four primary gill-slits, from the left side. (After Lankester and Willey.) $\times 200$.

CH, notochord. DL, præoral pit. ES, endostyle. GD, aperture of duct of eluhshaped glant. GL, club-shaped gland. HK 1, 2, 3, 4, first, second, third, and fourtl primary gill-slits. NS, spinal cord. $\mathrm{O}$, margin of mouth opening. OC, eye-sinut. P 6, sixtli myotome of the left side.

cavity, they open directly to the exterior. At a comparatively early stage (Fig. 37), the first primary gill-slit becomes markedly smaller than the succeeding ones.

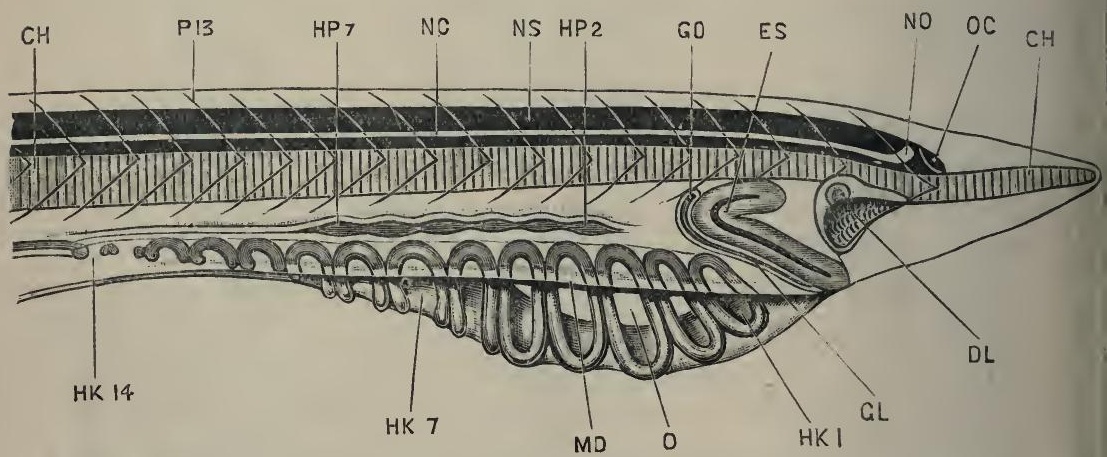

FIG. 37.-The anterior end of an Amphioxus Larva with fourteen primary gillslits, seen from the right side. (After Willey.)

CH, notochord. DL, præoral pit. ES, endostyle. GI, club-sliaperl gland. GO, opening from club-shaped gland into pharynx. HK 1,7, 14, first, seventli, and fourteenth primary gill-slits. HP 2, 7, thickenerl patches in which the second and seventh secondary gill-slits will be formed at a slightly later stage. MD, free elge of right metapleural fold. NC, neural canal. NO, anterior dilatation, or ventricle of neural canal. INS, spinal cord. O, mouth. OC, eye-spot. P 13, septum between thirteentl and fourteenth myotomes.

b. The secondary gill-slits, or the gill-slits of the adult right side of the pharynx, appear later than the primary slits, and in the following manner. At a stage (Fig. 37) when fourteen 
primary slits are present, of which the hinder three or four already open into the atrial cavity, a longitudinal ridge appears in the right wall of the pharynx, above the primary gill-slits. In this ridge six oval thickenings or enlargements appear simultaneously, formed by fusion of the hypoblastic wall of the pharynx with the external epiblast. These fuscd patches alternate with the primary gill-slits; the first patch (Fig. 37, HP 2) lying aboveand between the third and fourth primary slits, and the sixth patch, HP 7, above and between the eighth and ninth primary slits. Each patch now becomes perforated by a minute aperture, which by enlargement becomes one of the secondary gill-slits.

The most anterior of these six slits is usually formed a little later than the remaining five; and a little later still two more

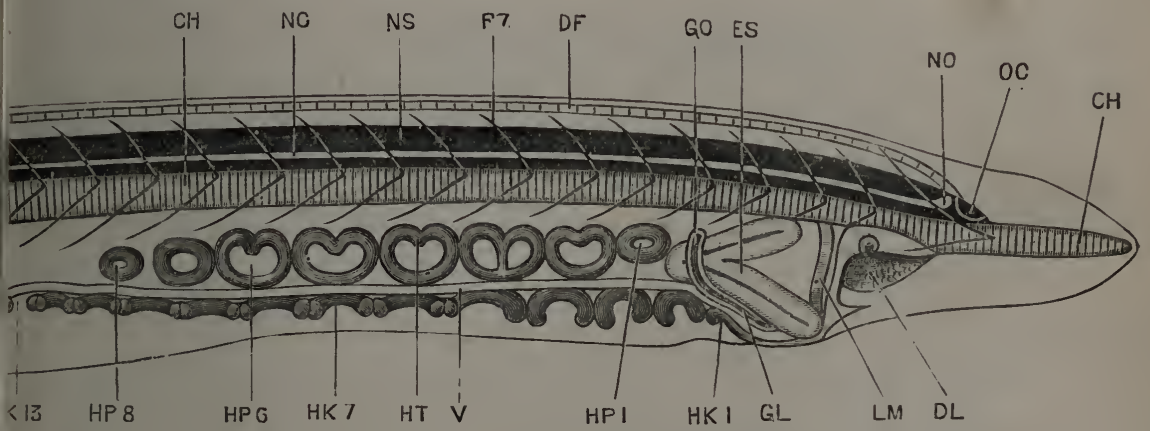

FIG. 38.-The anterior end of an Amphioxus Larva with thirteen primary, and eight secondary gill-slits, seen from the right side. (After Willey.)

CH, notochorl. DF, dorsal fin. DL, præoral pit. ES, endostrle. GL, clubshaped gland. GO, opening from club-shaped gland into pharynx. HK 1, 7, 13, first, seventh, and thirteenth primary gill-slits. HP 1, 6,8, first, sixth, and eiglith secondary gill-slits. HT, tongue-bar of the fourth secondary gill-slit. LM, velum. NC, neural canal. NO, anterior dilatation, or ventricle of neural canal. NS, spinal cord. OC, eye-spot. P 7, septum between seventh and eighth myotomes. V, eardiac aorta.

slits are formed in similar fashion, one at each end of the series. In this manner the full number of eight secondary gill-slits is acquired (Fig. 38); the first, HP 1, lying above and between the second and third primary slits ; and the eighth, HP 8 , above and between the ninth and tenth primary slits. A ninth secondary gill-slit is sometimes developed at the hincler end of the series.

c. Further development of the primary and secondary gillslits. The secondary gill-slits are at first very small, but they rapidly increase in size, extending down the right side of the pharynx; as they do so, the primary slits move downwards to the ventral wall of the pharynx, and then extend up its left 
wall, finally assuming their permanent position on the left side of the pharynx. During the process of shifting, the primary and secondary slits gradually become equal in size, and of similar shape. From the dorsal border of each slit a small process, the tongue-bar, grows downwards across the slit, dividing it into anterior and posterior portions; these tongue-bars (Fig. 38) appear rather earlier in the secondary than in the primary slits.

Of the fourteen primary slits, the first and the fourteenth close up and disappear; and at slightly later stages the thirteenth, twelfth, eleventh, and tenth similarly, and in succession, close and disappear ( $f f$. Fig. 39). In this way the primary gill-

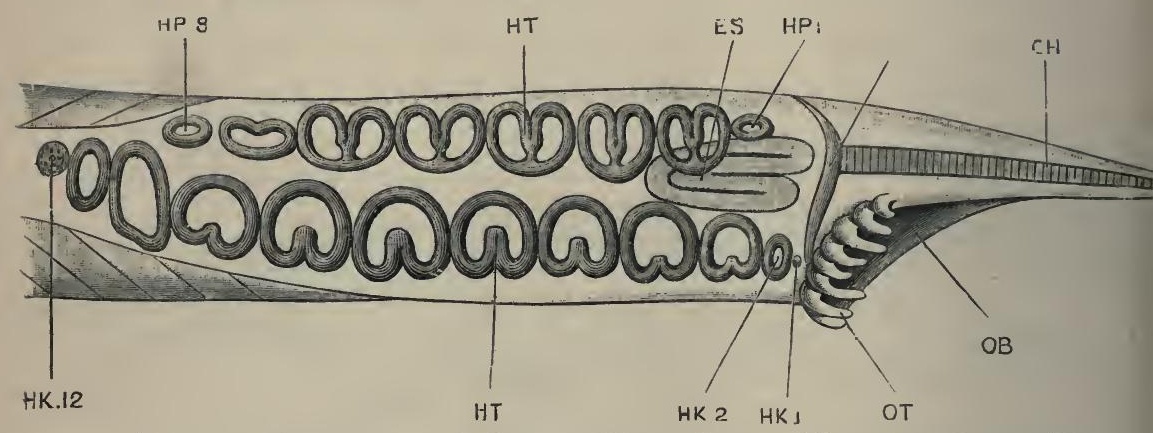

Fig. 39.--The anterior end of an Amphioxus Larva with twelve primary gillslits, of which the first and twelfth are disappearing, and eight secondary gill-slits; seen from the ventral surface. (After Willey.)

CH, notochord. ES, endostyle. HK 1, first primary gill-slit just before its final disappearance. HK2, second primary gill-slit. HK 12 , twelfth primary gill-slit, in the act of closing, prior to its disappearance. HP 1, 8, first and eighth secondary gillslits. HT, tongue-bar. LM, velum. OB, buccal cavity. OT, buccal tentacles.

slits become reduced to the same number, eight, as the secondary slits, the eight persisting primary slits being the second to the ninth inclusive.

The anterior persisting slits of both series, i.e. the second primary slit and the first secondary slit, differ from the others in their smaller size, and in the fact that they alone do not develop tongue-bars (Fig. 39, HK 2; HP 1).

The gill-slits have now reached the condition characteristic of the critical stage. Eight slits are present on each side of the pharynx, alternating with one another as in the adult; the anterior slit of the right side, i.e. the first secondary slit, HP 1, being opposite the interval between the first and second slits of the left side, i.e. the second and third primary slits. 


\section{The Endostyle.}

The endostyle appears at the commencement of the larval period, or towards the close of the embryonic period, as a band of columnar ciliated cells on the right side of the anterior end of the pharynx, immediately in front of the club-shaped gland, and in close contact with this. Its condition at an early stage of the larval period is shown in Fig. $36, \mathrm{Es}$, where it is seen as a broad >-shaped band, formed by modification of the hypoblast cells of the right side of the pharynx, opposite the anterior part of the mouth opening. The apex of the $>$ is directed backwards; the upper arm is much shorter than the lower; and the whole band is divided down its centre by a groove.

In the later stages (Figs. 37 and 38, Es), the endostyle extends backwards, its apex passing behind the duct of the clubshaped gland and making its way between the primary and secondary series of gill-slits. As the critical stage is approached, and the primary gill-slits shift across to the left side, the endostyle (Fig. 39) moves to its permanent position on the mid-ventral wall of the pharynx. At the same time it continues to extend backwards, and at the critical stage has reached to about the level of the fifth gill-slits. During the shifting of its position the two limbs of the $>$, which were originally upper and lower, become right and left respectively; and as it extends backwards along the floor of the pharynx the two limbs become closely applied, and fused together. From the anterior ends of the limbs, a pair of ciliated ridges of epithelial cells extend up the sides of the pharynx, and grow backwards along its dorsal surface to form the epibranchial band of the adult.

\section{The Club-shaped Gland.}

The early stages in the formation of the club-shaped gland have been already described, p. 70. The gland reaches its maximum development about the commencement of the larval period (Fig. 36), when it consists of a dilated sac, GL, lying on the right side of the pharynx, and continuous with a narrow tubular duct, which passes round the ventral surface of the pharynx and opens to the exterior on the left side, close to the anterior border of the mouth, GD.

The dilated part of the gland soon becomes narrower, and tubular, but according to Willey acquires an opening into the 
pharynx at its dorsal end. It does not shift its position in any way; but, about the stage represented in Fig. 38, when the secondary gill-slits are formed, and the primary slits are moving across to the left side, the club-shaped gland begins to atrophy, and by the stage shown in Fig. 39 has disappeared completely.

The function and the morphological meaning of the clubshaped gland are very doubtful. Willey has suggested that it may be the modified first gill-slit of the right side, adducing in support of the suggestion the fact that the first gill-slit of the left side is also a structure which disappears early; indeed, about the same time as the club-shaped gland itself. It is difficult, however, to understand, if the club-shaped gland is formed from a gill-slit of the right side, why its external opening shonld be on the left side of the head.

\section{The Mouth.}

The most striking features about the mouth, at the commencement of the larval period, are its position on the left side of the head, and its enormous size. As shown in Fig. 36, the mouth, $\mathrm{o}$, and the first gill-slit, HK 1, with the part of the pharyngeal cavity between them, form a huge opening, perforating the animal from side to side like the eye of a needle.

During the formation of the primary gill-slits the mouth remains on the left side of the head, and increases considerably in length ; extending, at the close of the stage (Fig. 37, o), from the second to the seventh myotome inclusive.

From the commencement of the formation of the secondary gill-slits the mouth gradually shifts its position, growing round the anterior end of the pharynx, and eventually attaining the median position and the shape characteristic of the adult. The shifting commences with the formation of a groove on the surface of the head, leading from the præoral pit to the upper and anterior angle of the mouth. By deepening of this groove the mouth opening becomes placed obliquely across the body, and by a continuance of the process, together with growth forwards of its posterior lip, it ultimately becomes median in position. The mouth is relatively much smaller in the adult than in the larva, but not actually so.

The margin of the mouth opening of the larva becomes the velum of the adult, from which the velar tentacles arise as out- 
growths; of these, there are four present at the critical stage, the remaining eight being developed later.

\section{J. The Buccal Hood and Buccal Tentacles.}

The true mouth of the adult Amphioxus, the development of which has just been described, is the small opening in the velum, or partition separating the buccal cavity from the pharynx (Fig. 11, p. 38).

The buccal cavity itself is formed by a pair of folds of integument, which appear about the time of formation of the secondary gill-slits. The two folds are at first upper and lower respectively ; the upper fold commencing above the præoral pit, and becoming continuous posteriorly with the upper margin of the mouth ; while the lower fold arises as a ridge along the lower and hinder border of the mouth, extending in front across the ventral surface to the right side.

As the mouth assumes its median position the upper and lower folds increase in size, and form the left and right halves of the buccal hood respectively.

The buccal tentacles appear early, as papilla-like outgrowths from the buccal folds (Fig. 39, o'T). They arise at first entirely from the lower, or future right fold, about the time the mouth commences to shift its position, and they do not extend into the left fold until a much later period. The median ventral tentacles are the first to be formed, and the others are added on in succession at either end of the series. Small cartilaginoid skeletal elements are present at the bases of the tentacles from their first appearance, and ultimately give rise to the buccal skeleton.

\section{The Præoral Pit.}

At the commencement of the larval period, the præoral pit, which, it will be remembered, is formed from the left anterior gut diverticulum (p. 70), is a small pit with thick ciliated walls, lying on the left side of the anterior part of the head, above and in front of the mouth, and opening to the exterior by a small aperture (Fig. 36, DL). When the mouth commences to shift towards the median plane, a ciliated groove is formed, comnecting its upper and anterior angle with the aperture of the præoral pit; and as the mouth sinks further and further towards the right side the præoral pit gradually becomes flattened out (Figs. 37, 38, DL), 
its walls becoming ultimately converted into the tract of columnar ciliated epithelium, which in the adult Amphioxus lines the posterior part of the buccal cavity.

\section{The Atrial Cavity.}

The atrial chamber begins to form in larvæ which have from nine to ten primary gill-slits, but in which the secondary gillslits have not commenced to develop. A narrow longitudinal groove appears along the ventral surface of the body of the larva,

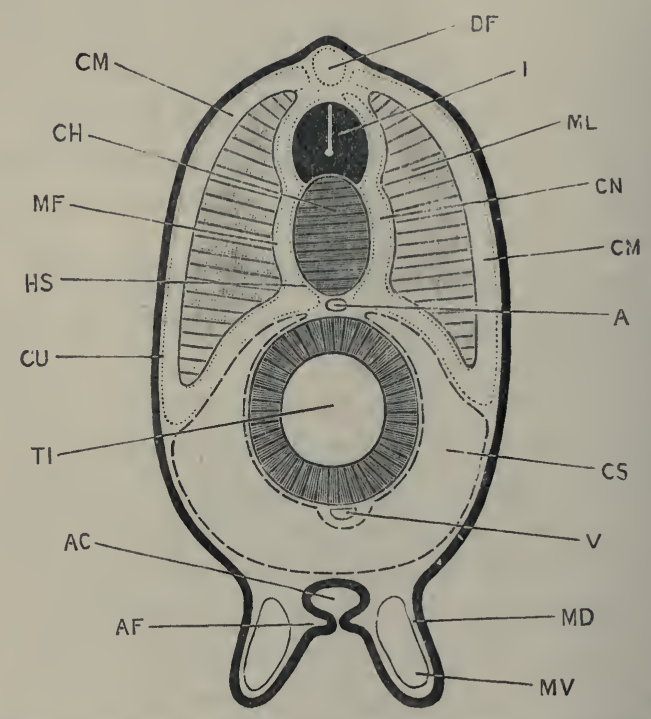

FIG. 40.-A diagrammatic transverse section across an Amphioxus Larva with eleven or twelve primary gill-slits, but no secondary ones. (Slightly modified from Lankester and Willey.)

A, aorta. AC, atrial cavity. AF, subatrial fold. $\mathbf{C H}$, notochori, CM, myocœl. CN, diverticulum of myocœl lying between notochord and myotome. CS, splanchnocosi. CU, cutis layer. DF, cavity of dorsal fin. HS, skeletogenons layer. I, spinal corr. MD, metapleural ridge. IMF, muscle-fascia layer. MI, myotomic muscle. MVV, metapletural canal. TI, intestine. V, subintestinal vessel.

behind the region of the pharynx. The groove is bordered by two folds, which become later the metapleural ridges of the adult (Fig. 40, MD) : on reaching the pharyngeal region, the two metapleural ridges are deflected towards the right side of the larva, and run forwards one on each side of the row of primary gill-slits.

From the inner side of each metapleural ridge a horizontal shelf-like outgrowth, the subatrial fold, arises; and the two sub- 
atrial folds meet and fuse, converting the groove into a tube (Fig. $40, \mathrm{AC})$. This tube, of which the roof is formed by the ventral wall of the body, the sides by the metapleural folds, and the floor by the fused subatrial folds, is the atrial chamber. The formation of the floor of the chamber proceeds from behind forwards. In the larva shown in Fig. 37, in which there are fourteen primary slits, and the secondary slits are just commencing to form, the

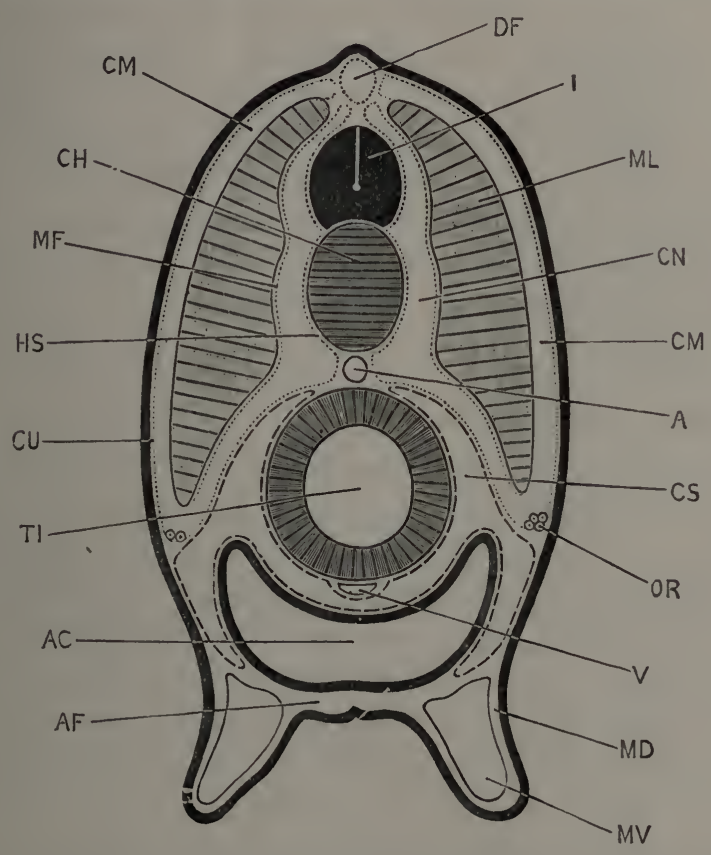

FIG. 41.-A diagrammatic transverse section through an advanced Amphioxus Larva with fully formed atrial cavity. (Slightly modified from Lankester and Willey, and from Boveri.)

A, aorta. AC, atrial cavity. AF, floor of atrial cavity, formed by fusion of the subatrial folds. $\mathbf{C H}$, notochord. $\mathrm{C} / \mathrm{M}$, myocel. $\mathrm{CN}$, liverticulum of myocœl lving between notochord and myotomic musele. CS, splanchnocol. CU, cutis layer. DF', eavity of dorsal fin. HS, skeletogenous layer. I, spinal cord. IMD, metapleural ridge. MF, muscle-fascia larer. ML, mrotomic muscle. MV, metapleural canal. OR, commencing reproductive organs. TI, intestine. V, subintestinal vessel.

atrial tube is completed to about the level of the ninth primary gill-slit; and at a stage shortly before that shown in Fig. 38 the tube is completed along the whole length of the pharynx. The anterior end of the tube ends blindly, but the posterior end remains open as the atrial pore.

The atrial tube is at first very narrow, and of nearly equal 
diameter along its whole length. Later on, it enlarges ver! greatly, and, pushing the ventral body-wall before it, encroaches on the space hitherto occupied by the colom, finally extending so far dorsalwards as nearly to surround the alimentary canal (Fig. 41, AC; cf. also Figs. 12 and 13).

The primary gill-slits at first open directly to the exterior, but, as they lie between the two metapleural folds, they become boxed in on the formation of the floor of the atrial tube, and from this time open into the atrial tube or chamber. The secondary gill-slits, which also lie between the two metapleural folds, very close to the base of the right metapleural fold, are not formed until the floor of the atrial chamber is completed, and consequently open into this chamber from the first.

The metapleural folds are at first solid ridges; large spaces soon appear in them, which become the metapleural canals of the adult (Figs. 12, 13, and 41, Mv).

\section{The Mesoblastic Somites.}

At the commencement of the larval period fourteen or fifteen pairs of somites are present; during the early part of this period the number steadily increases, and, shortly before the appearance of the secondary gill-slits, the full number of somites of the adult animal, which appears to be very generally sixtyone, is attained. The somites formed during the larval period differ from those developed in the embryonic stages in not communicating with the mesenteron at any time in their formation. In the development of these hinder somites it is probable that the polar mesoblast cells take an important share.

Concerning the further development of the somites some interesting details are given by Hatschek. At the commencement of the larval period, i.e. about the time of formation of the mouth, each somite ( $c f$. Figs. 32 and 42) becomes divided into a dorsal portion or protovertebra, and a ventral portion or lateral plate.

The protovertebræ retain the original segmental arrangement, i.e. the cavities of successive protovertebræ remain separate from one another; but in the ventral portions of the somites, or lateral plates, the septa become absorbed, and the cavities open into one another along the whole length of the body, forming a continuous body cavity or cœlom. 
The cavity of the protovertebra is spoken of as a myocœl (Fig. $42, \overline{C M}$ ); and at a stage when five primary gill-slits are present ( $c f$. Fig. 36) the myocols of each pair of protovertebræ communicate with each other above the spinal cord (Fig. 4.2). The outer or parietal wall of the protovertebra is very thin, and closely applied to the epidermis : it gives rise to the cutis, or connective tissue basis of the skin, and may be spoken of as the cutis layer (Fig. 42, CU). The inner or notochordal wall of the protovertebra, as already noticed (p. 67), thickens very greatly, and, though still remaining only one cell thick, becomes converted into the myotomic muscles (Fig. 42, ML). The lower or visceral wall of the protovertebra, like the parietal wall, is thin, and is in contact with the dor'sal wall of the alimentary canal.

The cavity of the lateral plates, ol splanchnocel (Fig. 42, CS), is continuous from end to end of the body, through absorption of the septa between the successive somites; it is also continuous from side to side across the mid-ventral plane. The walls of the splanchnocol are thin; the outer, or parietal layer, is in contact with

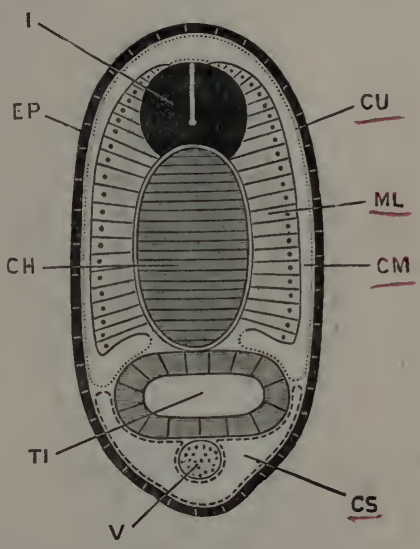

FIG. 42.-Diagrammatic transverse section across the intestinal region of an Amphioxus larva with five primary gill-slits : of. Fig. 36 . (After Hatschek.)

CH, notochord. CM, myocoel. CS, splanchnocœl. CU, cutis layer. EP. epidermis. I, spinal cord. MI, myotomic muscle. TI, intestine. $V$, subintestinal blood-vessel. the ventral epidermis, while the inner or splanchnic layer clothes the sides and ventral wall of the alimentary canal.

In the later stages important changes occur in these relations, and the condition immediately after the completion of the larval period is shown in Fig. 43.

The myocols now extend ventralwards much further than before, so that the parietal layer of the splanchnocœl (Fig. 43, cs) no longer touches the epidermis. The median dorsal and ventral parts of the myocœls have separated off as the compartments, DF and VF, of the dorsal and ventral fins, which are now prominent structures.

The ventral or splanchnic wall of each myocœl is folded to 
form a pouch, which extends upwards, between the myotome on the outer side, and the notochord and spinal cord on the inner side. The outer wall of this pouch (Fig. 43, MF) becomes the fascia covering the inner surface of the myotome; while the inner wall of the pouch (Fig. 4.3, Hs) gives rise to the

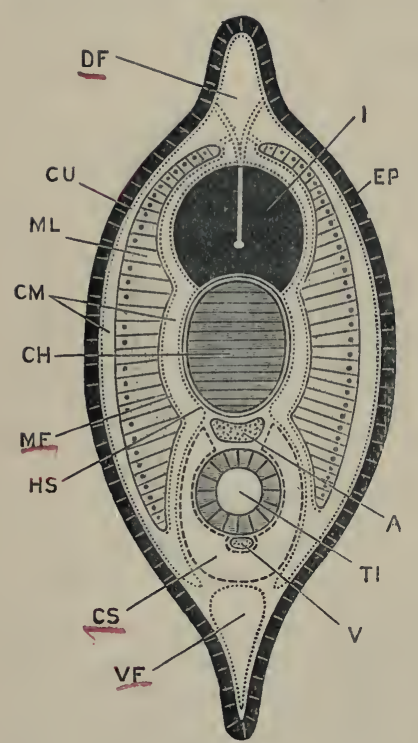

FIG. 43.-Diagrammatic transverse section across a young Amphioxus immediately after the completion of the larval period. The section is taken at a level between the atrial pore and the anus. (After Hatschek.)

A, dorsal aorta. $\mathbf{C H}$, notochord. CM, myocœl. CS, splanchnocel. $\mathbf{C U}$, cutis layer. DF, cavity of dorsal fin. EPP, epidermis. HS, skeletogenous layer. I, spinal cord. $\mathbf{M F}$, muscle-fascia laver. ML, myotomic muscle. V, subintestinal vessel. VF, cavity of ventral fin. (Compare also Figs. 40 and 41.) skeletal connective tissue, which invests the notochord and the spinal cord. The cavity of the pouch becomes ultimately obliterated by growth of the connective tissue, except in the anterior three or four segments of the body.

The splanchnocœl (Fig. 43, cs) undergoes but slight modification. It extends further dorsalwards than before, and almost completely surrounds the alimentary canal, cutting out the myocœl from its former share; while the myocol in its turn, owing to its ventral extension, shuts out the splanchnocœlic wall from all contact with the external epidermis. The splanchnocœl becomes the body cavity, or cœlom, of the adult.

It is interesting to note that even at this stage, when the larval development is completed, all the parts of the body are, as in the earlier stages already noticed in this respect, made up of epithelial layers, which in each case are but one cell thick; the complications in various regions being brought about by differences in the shapes of the cells at different places, together with foldings of the walls of the several cavities.

The origin of the connective tissue is not determined with certainty. Hatschek considers that it is at first of a gelatinous nature, probably formed by excretion from, and between, the 
several epithelial layers; any cellular elements it may obtain being derived by migration from these epithelial layers.

\section{The Asymmetry of the Larva.}

The asymmetry of the larva during its early stages is one of the most striking features in the development of Amphioxus. The fact that, but for the alternation of the myotomes on the two sides of the body, the embryonic stages are symmetrical; and the further fact that at the close of the larval period the symmetry is regained, indicate that the asymmetry of the earlier larval stages is a secondary or acquired character, and that the explanation of it is probably to be found in peculiarities of habit or environment of the larva during these stages.

The cardinal point in the asymmetry of the larva is the lateral position of the mouth, which, coupled with its huge size, is probably sufficient to explain the displacement of the gills of the left side.

Willey has suggested that the lateral position of the mouth is correlated with, or actually due to, the anterior extension of the notochord. The mode of developrnent of this front end of the notochord, and a comparison with other Vertebrates, strongly suggest that the prolongation forwards in front of all the other organs of the head is a secondary feature, associated not improbably with the burrowing habits of Amphioxus; and if we assume that the ancestral mouth was, as in the Ascidian tadpoles, dorsal in position, then the forward growth of the notochord would of necessity cause lateral displacement of the mouth. The suggestion is an ingenious one, and may be accepted as at any rate a provisional explanation.

\section{THE ADOLESCENT PERIOD.}

At the close of the larval period, i.e. at the completion of the critical stage, the young Amphioxus abandons its pelagic habits and burrows into the sand, where it passes the rest of its life; burying itself upright, with the tail downwards and the buccal hood alone projecting from the sand.

The further development takes place gradually. There is a steady increase in size, but no new myotomes are formed, the full number being present at the critical stage. The gill-slits, 
on the contrary, increase greatly in number, new ones being added on at the hinder end of the series, apparently throughout the life of the animal. Each new gill-slit (Fig. 35, L') becomes divided into two, at an early stage in its development, by the growth downwards of a tongue-bar from its dorsal border, just as in the earlier formed slits. The slits further become divided transversely by the horizontal bars characteristic of the adult (Fig. 35, L). Owing to this increase in number of the gill-slits, without any increase in the number of the myotomes, the correspondence between the two sets of structures is speedily lost; the alimentary canal, and the body generally, each acquiring a metamerism of its own.

\section{The Reproductive Organs.}

The reproductive organs are formed by proliferation of the epithelial walls of the septa which divide the successive somites from one another. Each of these septa ( $c f$. Fig. 31) is formed by the coalescence of the posterior and anterior walls of adjacent somites, and consists in the young Amphioxus of a thin connective tissue lamella, clothed on each surface by a single layer of flattened epithelial cells, these latter being really parts of the walls of the protovertebræ.

In young specimens of Amphioxus, of about $5 \mathrm{~mm}$. length, the epithelial layer becomes modified over a very small patch at the outer and lower corner of the septum, in the angle between the parietal wall or cutis layer, and the visceral wall of the protovertebra ( $c f$. Fig. 41, on): at this spot the cells become cubical or columnar in shape, while over the rest of the septum they remain flattened.

This modification does not occur along the whole length of the body, but is from the first confined to the somites in which the reproductive organs lie in the adult animal; i.e. the patches of modified epithelium are found on the septa forming the walls of the somites from the tenth to the thirty-sixth inclusive.

The modification affects the cells of both surfaces of each septum, but the cells of the posterior surface are almost from the first of larger size than those of the anterior surface, and, growing much more rapidly than these latter, push the septum forwards, and project into the segment in front of that to which they really belong, as a small stalked knob, to which the cells of 
the anterior surface of the septum form a follicular epithelial investment.

These knobs, each of which is a solid mass of enlarged epithelial cells, gradually increase in size, extending forwards until they ultimately occupy the whole length of the segments. The cavities in which they lie, really parts of the myocœl (Fig. 41, CM), widen considerably to allow for this increased size, and in specimens of about 15 or $16 \mathrm{~mm}$. length become shut off completely from the rest of the myocœl. Boveri has proposed the term gonotome for this portion of the somite, which is specially connected with the reproductive organs, and which is only found in the somites from the tenth to the thirty-fifth or thirty-sixth, in which these organs lie; he has further directed attention to the fact that the position at which the reproductive organs appear in Amphioxus, close to the line of separation between myocol and splanchnocœl, corresponds very nearly to that which they hold, in the earlier stages of their development, in the higher Vertebrates.

\section{List of the more important Publications dealing with the development of Amphioxus.}

Boveri, T.: ' Ueber die Bildungsstätte der Geschlechtsdrüsen und die Entstehung der Genitalkammern beim Amphioxus.' Anatomischer Anzeiger, vii. 1892.

Hatschek, B.: 'Studien über Entwicklung des Amphioxus.' Arbeiten aus dem Zoologischen Institute der Universität Wien, iv. 1881.

'Ueber den Schichtenbau von Amphioxus.' Anatomischer Anzeiger, iii. 1888.

'Die Metamerie des Amphioxus und des Ammocoetes.' Verhandlungen der Anatomischen Gesellschaft, 1892.

Kowalersky, A.: ' Entwickelungsgeschichte des Amphioxus lanceolatus.' Mémoires de l'Académie Impériale des Sciences de Saint-Pétersbourg, viie série, tome xi. No. 4.1867.

'Weitere Studien über die Entwicklungsgeschichte des Amphioxus lanceolatus, nebst einem Beitrage zur Homologie des Nervensystems der Würmer und Wirbelthiere.' Archiv für mikroskopische Anatomie, xiii. 1876.

Lankester, E. Ray: 'Contributions to the Knowledge of Amphioxus lanceolatus.' Quarterly Journal of Microscopical Science, New Series, xxix. 1889.

Lankester, E. Ray, and Willey, A.: 'The Development of the Atrial Chamber of Amphioxus.' Quarterly Journal of Microscopical Science, New Series, xxxi. 1890.

Willey, A. : 'TheLater Larval Development of Amplioxus.' Quarterly Journal of Microscopical Science, New Series, xxxii. 1891.

Wilson, E. B.: 'On Multiple and Partial Development in Amphioxus.' Anatomischer Anzeiger, vii. 1892. 


\section{Chapter III.}

\section{THE DEVELOPMENT OF THE FROG.}

Frogs belong to the class Amphibia, of which toads, newts, and salamanders are other well-known members, while less familiar examples are afforded by the axolotl of Mexico, the Proteus of the caves of Carniola and Dalmatia, the cryptobranch of Japan, which attains a length of three feet or more, and the curious snake-like Cœcilia of tropical countries.

As a group, Amphibia are characterised more especially by the double nature of their breathing organs. When adult, they all have lungs; but in the early stages of almost all genera, and throughout life in a large number, true gills are present, corresponding in structure and in mode of use to those of fish.

In the frog itself these gills are only present during the early, or tadpole, period of existence; in the later stages they are replaced functionally by lungs, and in the adult they have disappeared completely. The frog is thus, in the course of its own life history, transformed from a water-breathing to an airbreathing animal; and, in accordance with the principle of Recapitulation explained in the introductory chapter, this transformation is to be interpreted as indicating that frogs are descended from fish-like ancestors, each frog in its own development repeating the ancestral history.

The frog thus holds a position midway between Fish and the higher Vertebrates; and as frog's eggs can readily be obtained in large numbers, and the embryos and tadpoles develop well in captivity, the frog becomes a very convenient and instructive form for practical laboratory study.

\section{GENERAL ACCOUNT OF THE DEVELOPMENT OF THE FROG.}

Frogs' eggs are laid in water, usually during March or the early part of April.

During the act of oviposition, which may last several day's, 
the male frog clasps the female firmly, embracing her with lis arms; and as the eggs are passed out from the cloaca of the female into the water, they are fertilised by spermatozoa discharged over them by the male.

The eggs, which are very numerous, are small spherical bodies about $1.75 \mathrm{~mm}$. in diameter; they are invested by thin coatings of an albuminous substance, which sivell up very greatly in the water, and stick together to form the bulky masses we call frog's spawn. Such spawn consists of a transparent-gelatinous mass, formed by the swollen albuminous matter, in which the eggs are embedded; these latter appear as small spherical bodies, each presenting a black half and a white half.

If a number of hen's eggs were broken into a basin, care being taken not to rupture the yolks, a mass would be produced similar to frog's spawn; the yellow yolks corresponding to the frog's eggs, and the whites or albuminous investments of the rolks to the gelatinous matrix of the spawn.

'The frog's eggrs, laid in this way, and fertilised by spermatozoa shed over them by the male, begin to develop at once. The rate of development depends very largely on the temperature, and varies within very wide limits, warmth hastening development, and cold retarding it. Freezing of the water in which the eggs are kept merely retards development, and does not injure the eggs, provided the eggs themselves are not actually frozen. The times mentioned in this chapter may be taken as representing the average rate of development in this country.

Each egg is at first spherical, and remains so during the early stages of development; at the close of segmentation it becomes slightly ovoid, and then rapidly increases in length. A transverse constriction appears, separating the head from the trunk, and the tail buds out as a small process from the hinder end of the embryo. The embryo soon becomes fish-like in appearance, the tail growing very rapidly ; two pairs of branching tufts, the external gills, followed shortly by a third pair, grow out fiom the sides of the neck, and in about a fortnight from the time of laying of the egos the young tadpoles, now about $7 \mathrm{~mm}$. in length, wriggle their way out of the gelatinous mass of the sparn, and swim freely in the water (Fig. 44, 3, 4). 
At the time of hatching, the cloacal opening is already present; but the tadpole has no mouth, and is dependent for nutrition, as it has been during all the earlier stages, on the granules of food-yolk contained in the egg itself. A horse-shoe shaped sucker is present on the under surface of the head, by which the tadpole attaches itself, at first to the gelatinous mass of the spawn, and later on to weeds or other objects in the water.

A few days after hatching, the mouth appears, bordered by a pair of horny jaws, and fringed with fleshy lips studded with horny papillæ. The alimentary canal, which has hitherto been short and wide, rapidly increases in length, becoming tubular and convoluted; the liver and pancreas are formed; and the tadpole feeds eagerly on confervæ and other plants, especially on decomposing vegetable matter.

About the time of appearance of the mouth, i.e. shortly after hatching, a series of four slit-like openings, the gill-clefts, appear on each side of the neck, leading from the pharynx to the exterior. The margins of the slits become folded, and form the internal gills; the external gills at the same time decreasing in size and becoming shrivelled in appearance.

While the internal gills are developing, a fold of skin, the operculum, appears on each side of the head, in front of the gills. The two opercular folds, which soon become continuous with each other across the ventral surface of the head, grow backwards over the gills so as to inclose them in gill-chambers. Towards the end of the fourth week, the hinder edges of the opercular folds fuse with the body wall along the right side and across the ventral surface of the head. On the left side a spout-like opening remains, which communicates with the gillchambers of both sides; through this opening the water, taken in at the mouth for respiration, and passed out through the gillslits, makes its escape to the exterior ( $c f$. Fig. 83).

During this time the tadpole has been feeding freely, and has greatly increased in size. The body (Fig. 44, 8) is broad and round; the tail is much larger than before, and forms a powerful swimming organ; while the sucker on the under surface of the head, though still present, is small, and divided into two separate halves; and is but little used.

Very shortly afterwards, rudiments of the hind limbs can be seen as a pair of small papillæ at the root of the tail, one on 
considerably in size. The eyes, which as yet have been small, become larger and more prominent. The fore-limbs appear, the left one being pushed through the spout-like aperture of the gill-chamber, and the right one forcing its way through the opercular fold, in which it leaves a ragged hole. The abdomen shrinks; the stomach and liver enlarge, but the intestine becomes considerably shorter than before, and of smaller diameter; the animal, previously a vegetable feeder, now becomes carnivorous. The gill-clefts close up; the gills themselves are gradually absorbed; and important modifications, accompanying the change in the mode of breathing, occur in the blood-vessels of the pharynx. The kidneys undergo considerable changes; the bladder is formed; and sexual differentiation is definitely established. The tail, which is still of great length (Fig. 44, 11), now begins to shorten, and is soon completely absorbed; the hind legs lengthen considerably, and the animal leaves the water as a frog.

By preventing tadpoles from breathing air directly, as by placing a wire net an inch or so below the surface of the water in which they are living, the occurrence of the metamorphosis can be indefinitely deferred. Under these conditions tadpoles increase greatly in size, but do not become transformed into frogs.

In the remainder of this chapter the several stages in the development of the tadpole, and the formation of the various organs and systems, will be described in detail.

\section{THE FROG'S EGG.}

\section{Formation of the Egg.}

The early stages in the formation of the eggs cannot be seen in the adult frog, but must be studied in tadpoles.

In tadpoles of about $10 \mathrm{~mm}$. length, shortly after the opening of the mouth, a pair of longitudinal ridge-like thickenings of the peritoneum appear along the dorsal surface of the body cavity, close to the root of the mesentery. These genital ridges are found in all tadpoles alike, no difference of sex being established until a considerably later period.

Each genital ridge is at first due merely to a modification in shape of the peritoneal epithelial cells, which, elsewhere flattened, 
become here cubical or slightly columnar. The ridges soon become more prominent, especially at their anterior ends, their growth being due, partly to increase of the epithelial cells by repeated division, the epithelial layer becoming several cells thick; and partly to ingrowth of an axial core of connective tissue, from the basal membrane of the peritoneum, along which blood-vessels gain access to the ridge. The anterior third of each genital ridge undergoes degenerative changes at an early period (Figs. 85, 86), and ultimately becomes the fat body of the adult; the posterior two-thirds develop into the reproductive organ, or.

It an early stage, certain of the epithelial cells of the genital ridge become conspicuous by their larger size and more spherical shape; these are the primitive ova or gonoblasts. Round each primitive ovum the neighbouring cells become arranged so as to form a capsule or follicle; the follicles forming distinct projections on the surface of the genital ridge. New primitive ova are formed from the surface epithelium, and also by division of those already present; they, also, soon become inclosed in follicles formed by the neighbouring cells.

Sexual differentiation appears at the time of the metamorphosis. In the female, the changes consist essentially in a great increase in the size of the genital ridges, which now become the ovaries, and in the number of the contained follicles; and in the formation of the permanent ova or eggs. The permanent ova are formed from the primitive ova, but different accounts have been given of the details of the process, and it is possible that they are not the same in all cases. As a rule, each primitive ovum divides rapidly to form a nest of cells, one of which becomes a permanent ovum, while the rest form part of the follicle which surrounds it, and serves for its protection and nutrition. In other cases it is stated that a primitive ovum may become directly converted into a permanent ovum.

The permanent ovum, in whatever manner it is formed, differs from the primitive ovum:-(i) in its much greater size; (ii) in possessing a very large vesicular nucleus, or germinal vesicle; and (iii) in containing a number of yolk-granules, imbedded in the protoplasm of its cell-body.

The egg nucleus, or germinal vesicle, is a spherical capsule, with a diameter of from one-third to half that of the ovum itself. 
It consists of a thick elastic nuclear membrane, apparently perforated by fine radial pores, and inclosing a watery nuclear fluid; the latter is traversed by a finely granular protoplasmic network, enlarger at the nodes to form nucleoli, or germinal spots, of which one is usually larger than the others.

The yolk granules are small, sharply defined, spherical or ovoidal, yellowish particles of food-substance, which are elaborated by the follicle cells and passed on from them into the ovum. They are confined to the protoplasm of the cell-body, not penetrating into the nucleus. They increase rapidly in number as the egg approaches maturity, and it is to them that the size of the egg as well as its opacity are chiefly due.

When the egg has attained a diameter of about $0.5 \mathrm{~mm}$. an exceedingly thin structureless investment, the vitelline membrane, is formed immediately around it, and within the follicle. The mode of origin of the vitelline membrane is not clearly made out, but it seems to be formed from the egg itself rather than from the follicular epithelium.

A little later, and as the egg is approaching its full size, a layer of black pigment appears on its surface; this is at first irregularly distributed over the whole surface, but, as the egg ripens, the pigment becomes restricted to one half or hemisphere, and the distinction between the white and black poles of the egg is thus established. The pigment is contained, and apparently formed, within the egg itself; but it is not clear how it is formed, or what purpose it fulfils. The facts, that the pigment is confined to the pole of the egg which develops most rapidly, and that warmth greatly increases the rate of development, suggest that the pigment may facilitate development by promoting the absorption of heat.

\section{Maturation of the Egg.}

Our knowledge of the phenomena accompanying the maturation of the frog's egg is based almost entirely on the researche: of $\mathrm{O}$. Schultze, and is still in many respects imperfect. An account of these clianges has already been given in the introductory chapter, but will be repeated here in order that the developmental history of the frog may be given as fully as practicable.

The process of ripening or maturation commences in an egg 
while it is still in the ovary, shortly before it reaches its full size, and the successive stages are shown in Fig. 45.

The whole nucleus shrinks considerably, becoming reduced to less than half its former diameter. This shrinking is accompanied by exudation of part of the nuclear fluid, through the nuclear membrane, into the protoplasm of the cell-body (Fig. 4.5, A, UH), where it forms a fluid layer surrounding the nucleus: at the same time the nuclear membrane becomes wrinkled, its surface, which was previously smooth, becoming raised into little wart-like projections, so as to present an appearance something like a blackberry (Fig. 45, A).

Within the nucleus a number of the larger chromatin granules, or nucleoli, remain close to the nuclear membrane, often lying within the wart-like protuberances; a number of others, chiefly smaller ones, collect towards the centre, where they surround a clear region in which lie a number of exceedingly minute chromatin granules. These latter are at first scattered irregularly, but soon run together to form moniliform threads of extreme slenderness, which interlace and unite to form a minute nuclear skein (Fig. 45, A).

About the time of discharge of the egg from the ovary further changes occur, which are apparently stimulated by the act of copulation. The nuclear membrane disappears completely; and its contents, the nuclear fluid and nucleoli, become distributed through the yolk. The only part of the egg nucleus which persists is the minute nuclear skein: this moves towards the surface of the egg, and takes up a position at the upper or black pole of the egg, immediately below its surface (Fig. 45, B); here it lies in a lenticular patch, which is rather more fluid and more transparent than the rest of the yolk, and is separated from this by an ill-defined capsule of pigment, prolonged towards the centre of the egg in the manner shown in the figure.

The nuclear skein (Fig. 45, B, UG), now assumes a spindle form, and lies at first with its long axis tangential to the surface of the egg. Shortly afterwards the spindle turns so that its axis becomes radially situated, one of its poles being at the surface of the egg, and the other directed towards the interior; it then divides transversely into two parts, of which one (Fig. 45, C, UG), remains within the egg, while the other (Fig. 45, C, PB), is extruded as the first polar body. Shortly before the formation 
of the polar body the black pole of the egg becomes slightly flattened, leaving a space between the egg and the vitelline membrane (Fig. $45, \mathrm{C}$ ); this space is occupied at once by a perivitelline fluid, exuded from the eggr, and in this fluid the polar
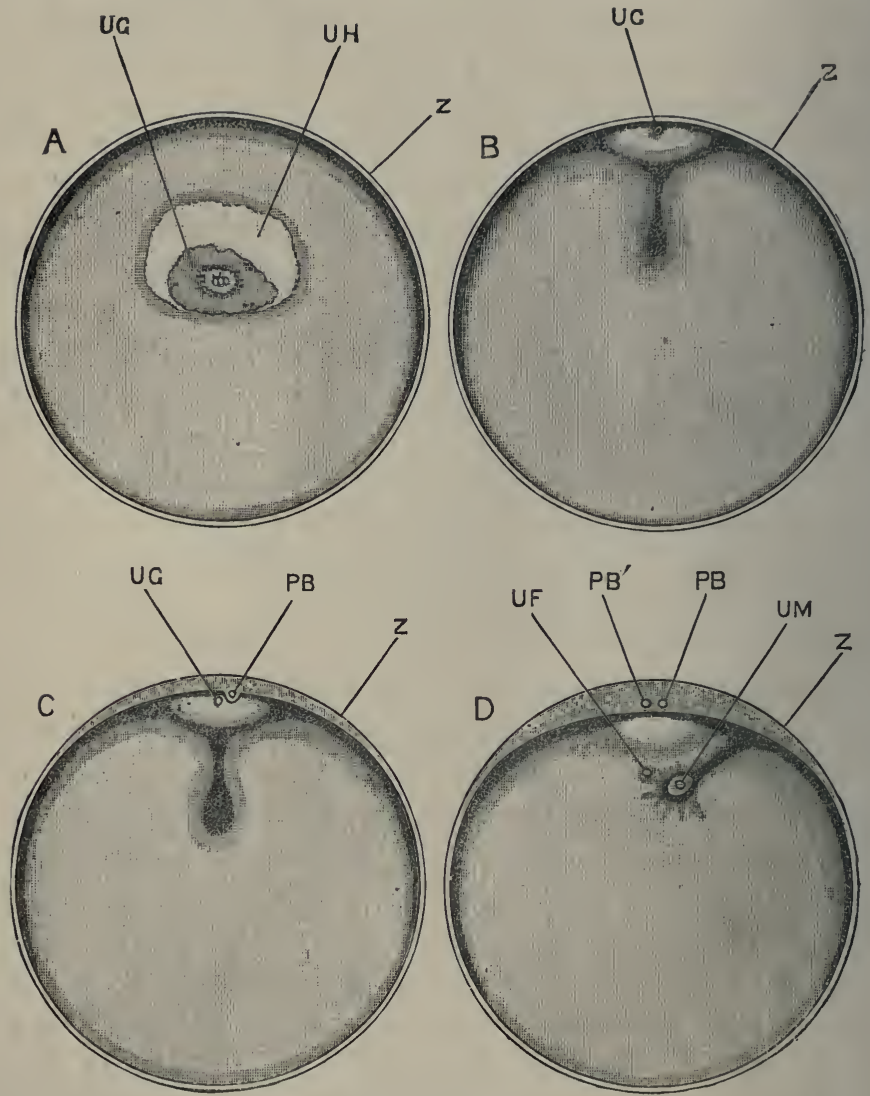

FIG. 45.-Successive stages in the maturation of the egg of the Frog. The eggs are represented as bisected vertically. $\times 25$. (After O. Schultze.)

$A$, stage in which the nucleus has commenced to shrink, and the nuclear skein is formed in its centre. $B$, stage in which the nuclear skein has moved to the surface of the egg, just prior to the formation of the first polar body. C, stage in which the first polar body has been formed, by division of the nuclear skein, and extruded. $D$, stage in which the second polar body has been extruded, and the remaining part of the nuclear skein, or female pronucleus, has retreated from the surface of the egg, and is about to unite with the male pronucleus or head of the spermatozoon.

PB, first polar body. PB', second polar body. UF', female pronucleus. UG, egg nucleus, or germinal vesicle. UH, peri-vitelline fluid exuded from germinal vesicle. UM, male pronucleus. Z, vitelline membrane.

body may be seen as a minute ovoidal white body, usually lying in a small depression on the surface of the egg (Fig. 45, C, PB). 
The formation of the second polar body in the frog has not been seen, but there can be little doubt that it is due, as in other animals, to a further division of the part of the nuclear epindle which remains within the egg, after extrusion of the first polar body. According to Schultze, the extrusion of the second polar body from the egg does not take place until about half an hour after fertilisation of the egg; i.e. after the entrance of the spermatozoon, but before the completion of the act of fertilisation.

The two polar bodies are of about equal size; they lie freely on the yolk, in the peri-vitelline fluid, and shift about with this latter if the eggs are rotated.

\section{Laying of the Eggs.}

The eggs when ripe are discharged from the ovary, and fall into the body cavity; along this they pass forwards, directed partly by contraction of the muscular body walls, partly by the action of the cilia of the peritoneum, to the mouths of the oviducts, which are situated at the extreme anterior end of the body cavity, opposite the roots of the lungs. Within the first or thick-walled part of the oviduct the eggs acquire gelatinous investments secreted by glands in its walls: the terminal part of each oviduct is a thin-walled pouch, capable of great distension, within which the eggs accumulate in large numbers. Finally, at the time of copulation, the eggs are passed out through the cloacal opening into water, in which the albuminous investments of the eggs speedily swell up to form the gelatinous mass of the frog's spawn.

\section{Fertilisation of the Egg.}

The spermatozoa, after being shed over the spawn by the male frog, swim actively by means of their long tails, work their way into the gelatinous mass of the spawn, bore through the vitelline membranes, and so penetrate into the eggs themselves, which they enter at, or close fo, their upper or black poles.

A single spermatozoon is sufficient to fertilise an egg, and it is doubtful whether more than one is ever concerned in the process. About an hour after the spermatozoon has entered, a pigmented process may be seen projecting into the egg from the point of entry (Fig. 45, D), and in the centre of the process a clear spot. This spot (Fig. 45, D, UM), is the nucleus of the sperma- 
tozoon, or male pronueléus; it penetrates further into the egg, carrying the pigment with it, and soon meets the female pronucleus, or part of the nuclear skein which remains within the egg after extrusion of the two polar bodies.

The two pronuclei come into close contact with each other, and, after having increased considerably in size, fuse together to form the segmentation nucleas. This fusion, which occurs about two and a half hours after the spermatozoon first entered the egg, completes the act of fertilisation.-

Almost immediately after the spermatozoon enters the egg a considerable extrusion of peri-vitelline fluid takes place, between the egg and the vitelline membrane (Figs. 45, C and D). This separates the egg from the vitelline membrane, and greatly facilitates the rotation of the egg within the membrane; from this time, in whatever position the spawn be placed, the black poles of the eggs will always, from their less specific gravity, be uppermost, and the white poles, which are of higher specific gravity owing to the greater abundance of yolk-granules in them, will be undermost. The extrusion of the peri-vitelline fluid, and the consequent separation of the egg from the vitelline membrane, may possibly serve further to prevent or hinder the entrance of a second spermatozoon.

\section{THE EARLY STAGES OF DEVELOPMENT OF THE FROG'S EGG.}

\section{Segmentation of the Egg.}

Segmentation of the frog's egg is, like that of Amphioxus, a process of cell-division; but although the processes in the two animals are essentially similar, there are important differences in detail, due to the much larger amount of food-yolk present in the egg of the frog, and its unequal distribution.

Food-yolk consists of small granules of highly nutritious matter, imbedded in the substance of the egg; but although it forms a store of readily assimilated nutriment, at the expense of which the development of the embryo can be effected, it must be remembered that until it has been so assimilated the yolk granules will be foreign bodies, and, like any other foreign bodies, will be a hindrance rather than an aid to development. The direct influence of food-yolk is to mechanically impede the activity of 
the protoplasm in which it is imbedded, acting in exactly the same way as so many grains of sand or other foreign matter would do, and actually checking the processes of development.

The frog's egg is a telolecithal egg; i.e. one in which the foodyolk is not uniformly distributed throughout the yolk, being more abundant in the lower or white hemisphere than in the upper or black one. The passage from one pole to the other is a

FIG. 46.

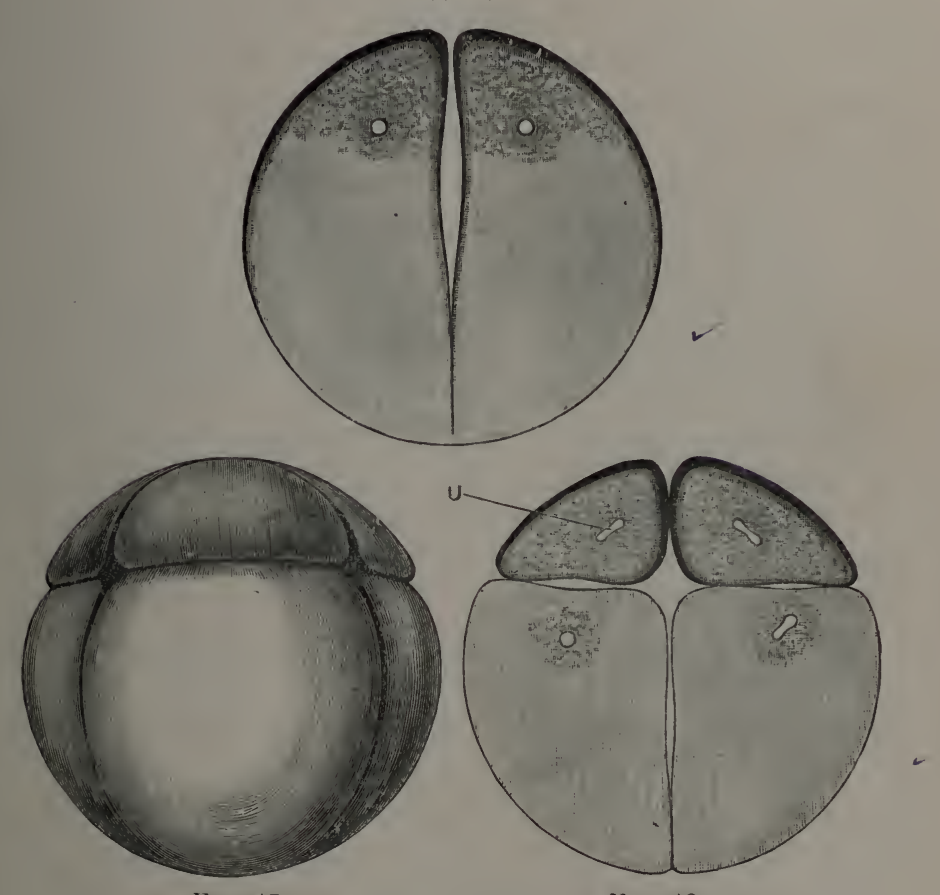

FIG. 47 .

Fig. 48.

Figs. 46, 47, and 48. Segmentation of the Frog's Egg. $\times \simeq 0$.

Fig. 46.-The egg just before the completion of the first cleft, by which it is divided into two equal blastomeres : the egg is represented in vertical section.

Fig. 47.-A surface view of an egg at the completion of the third cleft : the egg is now divided into eight blastomeres, an upper tier of four small ones, and a lower tier of four mucli larger ones.

Fig. 48.-A vertical section of an egg at the same stage as Fig. 47. In the middle, between the inner ends of the blastomeres, is the commencing segmentation cavity.

gradual, not an abrupt one; there is no line of demarcation between the two; still, the black pole is much less encumbered with food-yolk than the white or lower pole, and it is to this fact that the relatively rapid development of the black pole is due.

Very shortly after the completion of the act of fertilisation 
and the formation of the segmentation nucleus, this latter loses its spherical form and becomes spindle-shaped, the yolk granules at the same time showing a tendency to arrange themselves along lines radiating outwards from the ends of the spindle.

The nucleus now divides into two halves, which move away from each other; the yolk granules tend to aggregate themselves around the two nuclei, and a thin vertical plate of finely granular protoplasm, almost free from yolk granules, is left, dividing the egg into two halves. This plate, which soon becomes pigmented, splits vertically into two, the split appearing near the centre of the egg, and at first not reaching to its surface.

At the upper or black pole of the egg a depression now appears, at first as a small pit, and then elongating to form a groove, which rapidly extends all round the egg. The groove deepens, and, meeting with the split already present in the interior of the egg (Fig. 46), divides the whole egg into two completely separate and equal parts, the plane of division corresponding with the vertical pigmented plane mentioned above.

This first plane of division is stated to correspond to the median sagittal plane of the future embryo and adult; i.e. the two cells into which the egg is divided by the first segmentation plane are said to correspond respectively to the right and left halves of the body of the frog.

Each of the two nuclei soon becomes spindle-shaped, and then divides into two; and a second cleft is then formed in a similar manner to the first. This second cleft is also a vertical one, but in a plane at right angles to the first one ; on its completion the egg is divided into four similar and equal cells, or blastomeres.

The third cleft is horizontal, but not equatorial, lying (Fig. 47) much nearer the upper than the lower pole. It divides each of the four cells or blastomeres into two, an upper smaller and a lower larger one.

From this stage segmentation proceeds rapidly, but according to no definite rule, the several cells dividing independently of one another. Throughout the process the upper cells divide more rapidly, and are consequently always of smaller size than the lower cells, the latter being hampered by the large number of yolk-granules they contain: in all cases division of the cells is preceded by division of their nuclei, as in the earlier stages. 
At the stage represented by Figs. 47 and 48 , when eight cells are present, i.e. on the completion of the third cleft, a small cavity appears in the centre of the egg, between the inner ends of the cells (Fig. 48). This is the segmentation cavity or blastocœl. From its first appearance it is situated nearer the upper than the lower pole of the egg. It is filled with fluid, and during the later phases of segmentation it increases considerably in size (Figs. 49, 50).

At the close of segmentation the egg has the structure shown in section in Fig. 50. It is a hollow ball, the same size as the original ovum, with a small, excentrically-placed cavity, and with walls of very unequal thickness. The cells of the upper half

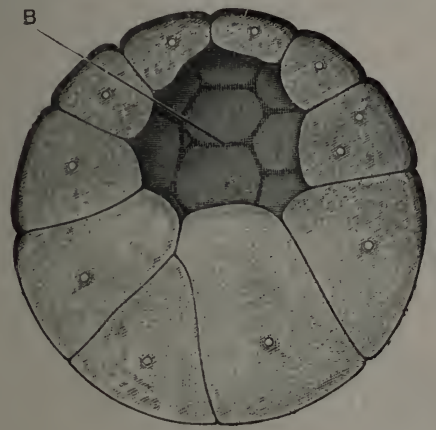

FIG. 49.

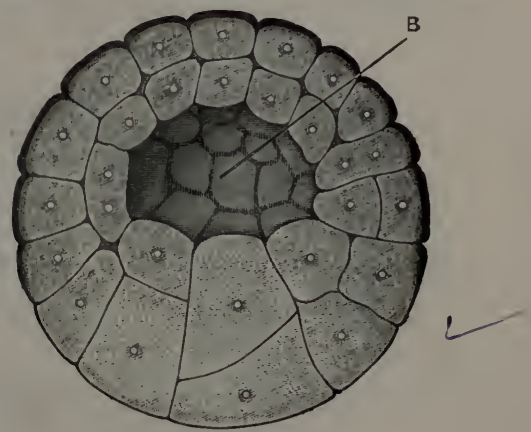

FIG. 50.

FIG. 49.-The blastula stage in the development of the Frog's Egg, bisected vertically. $\times 20$.

F'Is. 50.-The Frog's Egg at the close of segmentation, bisected vertically. $\times 20$.

B, segmentation cavity or blastoceel.

are small, approximately uniform in size, and arranged more or less definitely in two layers, outer and inner; while the cells of the lower half are larger, and much more irregular in shape, size, and arrangement: furthermore, the superficial cells of the upper half are deeply pigmented at their outer ends, while those of the lower half are nearly colourless.

The distinction between upper and lower cells is, however, not an absolute one, the cells at the equator being intermediate in all respects between those of the upper and lower poles.

The stage represented in Fig. 49 is the one which corresponds most closely with the blastula stage of Amphioxus (Fig. 14, viII). There are, however, important differences between the two. In the blastula stage of the frog there are fewer component cells; 
the cells differ more markedly from one another in shape and size; and the segmentation cavity is much smaller relatively to the entire ovum, and is excentric instead of central in position. From the description given above it will be seen that all these differences may be attributed to the greater amount of food-yolk present in the frog's egg.

\section{The Epiblast.}

Of the two kinds of cells of which the egg consists at the close of segmentation (Fig. 50), the smaller pigmented cells of the upper half are the epiblast cells, while the larger unpigmented cells of the lower half, in which the yolk-granules are mainly contained, may be spoken of as the lower layer cells or yolk-cells.

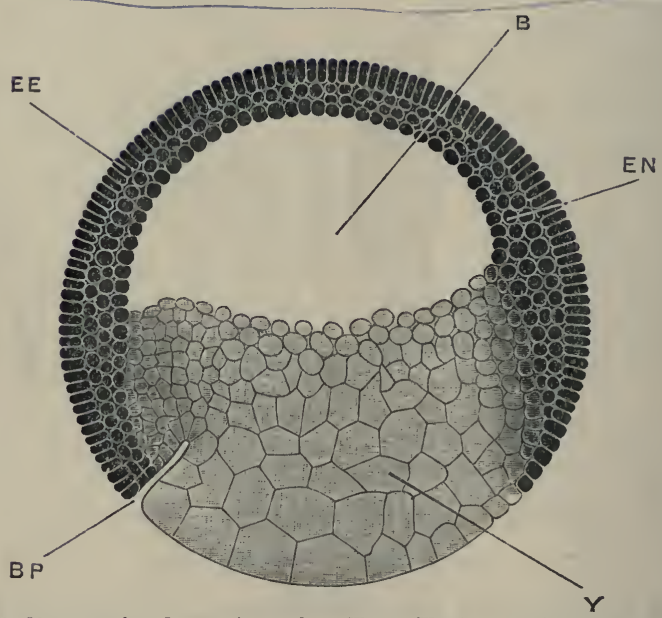

FIG. 51.-Median sagittal section of a Frog Embryo, showing the spreading of the epiblast and the commencing formation of the mesenteron. $\times 25$.

B, blastocœl or segmentation cavity. BP, lip of blastopore. FE, outer or epidermic layer of epiblast. $\mathbf{E N}$, inner or nervous lajer of epiblast. $\mathbf{Y}$, lower layer or yolk cells.

The distinction between the two is not an absolute one, the cells at the equator of the egg being intermediate in all respects between the epiblast and the yolk-cells. As seen from the surface, the limit is indicated by the boundary line between the black and the white areas of the egg, and at the close of segmentation these two areas are approximately equal in extent. In the succeeding stages the black area increases rapidly at the expense of the white area (Figs. 51, 52, 54), and in a few hours the pigmented epiblast cells have covered the whole of the egg 
with the exception of a small circular patch at the lower pole (Figs. 52, y, and 54 YP), where alone the white yolk-cells come to the surface.

This extension of the epiblast occurs all round its margin, and is effected by the addition of cells cut out from the superficial layer of yolk-cells. This superficial layer first becomes pigmented, and then divides into, (i) a surface stratum of small epiblast cells, which from the first are similar to the original epiblast cells, and are added on round their margin; and (ii) a deeper mass of larger and non-pigmented yolk-cells.

During this extension of the epiblast, the process of cell division has been continuing rapidly in all parts of the embryo. The epiblast now consists of two very definite layers of cells : an outer or epidermic layer (Fig. $51, \mathrm{EE}$ ), formed by a single stratum of short columnar cells, which are deeply pigmented, and packed close together side by side; and an inner or nervous layer (Fig. 51, EN), consisting of smaller, more spherical cells, less strongly pigmented than those of the epidermic layer, and arranged two or three deep. The cells that are added on round the margin of the epiblast, during its spreading, are similar in shape and size to the epiblast cells derived from the upper pole of the egg, and, like these, soon become arranged in epidermic and nervous layers.

\section{The Mesenteron.}

The alimentary cavity, or mesenteron, is formed as a narrow slit, opening to the surface at the lower pole of the egg and extending a certain distance into its interior (Fig. 51, BP). The slit rapidly deepens, spreading concentrically with the surface of the egg, and lying near to what will subsequently become the dorsal surface of the embryo ; it is at first exceedingly shallow, its two walls being almost in contact (Figs. 52, 53, T); but very shortly, by depression of the lower wall or floor (Figs. 54, $55,56, \mathrm{~T})$, the cavity becomes of considerable size, and forms the alimentary tract of the embryo.

This slit-like mesenteron was formerly described as arising by a process of invagination, the epiblast cells being said to grow into the interior of the egg to form the wall of the mesenteron cavity. Later investigations have shown that this description is incorrect, and that the cavity is formed, not by invagination from 
the surface, but by splitting apart of the yolk-cells as described above, this splitting being preceded by the formation of pigment in the adjacent surfaces of the cells between which the split is to appear.

The mesenteric slit appears first as a slightly crescentic groove on the surface of the egg (Fig. 51, BP), at the margin of the spreading epiblast, and about midway between the equator and the lower pole of the egg. It is very conspicuous, because the pigmented epiblast cells stop sharply at its upper or convex border, so that the boundary between the epiblast and yolk-

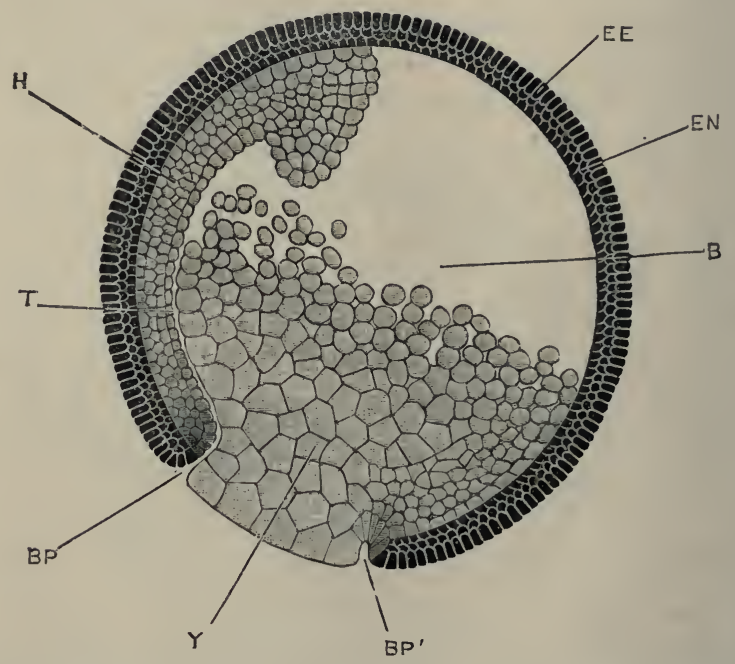

FIG. 52.-Sagittal section of a Frog Embryo during the formation of the mesenteron. $\times 25$.

$\mathbf{B}$, blastocœl or segmentation cavity. BP, upper or dorsal lip of blastopore. $\mathbf{B P}^{\prime}$, lower or ventral lip of blastopore. $\mathbf{E E}$, outer or epidermic layer of epiblast. $\mathbf{E} \mathbf{N}$, inner or nervous layer of epiblast. $\mathbf{H}$, hypoblast. T, mesenteron. $\mathbf{Y}$, jolk-plug.

cells is here an abrupt one, while round the rest of the circumference, as shown on the right-hand side of Fig. 51, the transition is more gradual.

The groove rapidly extends at its extremities, becoming semicircular, then horse-shoe shaped, and finally, by meeting of its limbs, a complete circle. This circular groove separates the epiblast, which now ends sharply against it round its entire margin, from a circular patch of yolk-cells (Fig. 52, Y, and Fig. 58, A), which still remains at the surface of the egg. The 
circular aperture in the epiblast, defined by this groove, is spoken of as the blastopore, or anus of Rusconi; and the mass of yolkcells which fills up the aperture, as the yolk-plug.

The blastopore lies at first at the lower pole of the egg. Reference to Figs. 54, 55, and 58 will show that this lower pole becomes subsequently the hinder or tail end of the embryo, so that the lips of the blastopore, $\mathrm{BP}$ and $\mathrm{BP}^{\prime}$, may be spoken of as dorsal and ventral respectively.

From the figures, and from the above description, it will be

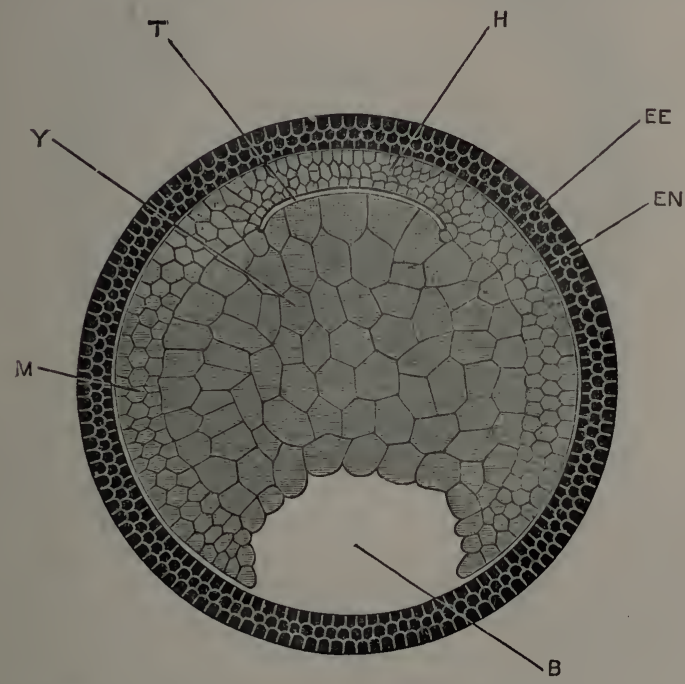

FIG. 53.-Horizontal section across a Frog Embryo of the same age as that shown in Fig. 52, the section being taken along a line joining the reference letters T and B in Fig. 52. $\times 25$.

B, blastocœl or segmentation cavity. FE, outer or epidermic laver of epiblast. $\mathbf{E N}$, inner or nervous lajer of epiblast. $\mathbf{H}$, liypoblast. $\mathbf{M}$, mesoblast. ' $\mathbf{T}$, mesenteron. $\mathrm{Y}$, yolk-cells.

seen that the groove which limits the blastopore appears first at its dorsal margin, BP, and spreads round the sides to the ventral margin, $\mathrm{BP}^{\prime}$. The slit extends at first radially inwards, towards the centre of the egg (Figs. 51, BP, and 52, BP'); but along the dorsal surface the slit, after a short radial course (Fig. 52, BP), turns sharply at right angles (Fig. 52, T), and spreads forwards concentrically with the surface of the embryo.

The whole embryo, which up to this stage has been spherical, now begins to elongate, becoming ellipsoidal, with the blastopore 
marking the posterior pole (Figs. 54, 55). By an alteration in the position of the cells of its floor, the mesenteric slit (Fig. 52, T) becomes widened out into a large cavity (Fig. 54, T); the roof or dorsal wall of which is formed by a well-defined layer of small cells, arranged three or four deep, and lying in close

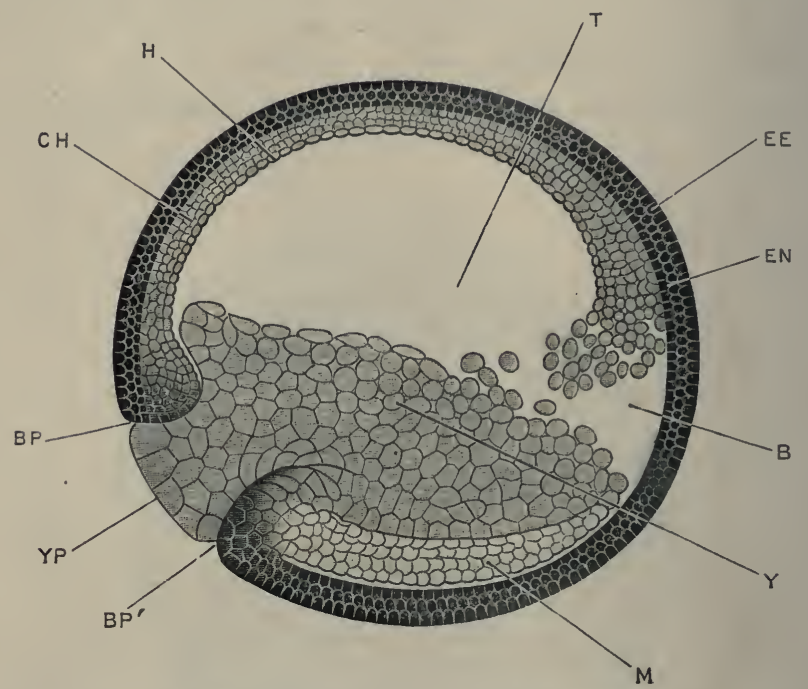

F'rG. 54.- Sagittal section of a Frog Embryo just before the disappearance of the segmentation cavity. $\times 25$.

B, blastocœl or segmentation cavity. BP, upper or dorsal lip of the blastopore. BP', lower or ventral lip of the blastopore. $\mathbf{C H}$, notochord. $\mathbf{E E}$, outer or epidermic lajer of epiblast. $\mathbf{E N}$, inner or nervous layer of epiblast. $\mathbf{H}$, hypoblast forming dorsal wall of meseuteron. M, mesoblast. T, mesenteron. $\mathbf{Y}$, yolk-cells. Y $\mathbf{Y}$, yolk-plug.

contact with the epiblast, while the floor and sides consist of yolk-cells (Fig. 54, y).

During this change the segmentation cavity, B, gradually becomes reduced in size, and ultimately disappears altogether. It can always be distinguished from the mesenteron by the fact that it lies between the epiblast and the yolk-cells, and that its wall is therefore formed on one side by epiblast cells only (Figs. 52 and $53, \mathrm{~B}$ ); while the mesenteron, $\mathrm{T}$, always has walls formed by both epiblast and lower layer cells.

Figs. 52, 54, and 55 show that the segmentation cavity becomes reduced and obliterated, partly by the growth forwards of the cells which form the roof of the mesenteron; and partly by a shifting in the position of the yolk-cells forming the floor 
of the mesenteron, which accompanies the elongation of the embryo and the enlargement of the mesenteric cavity. The mesenteron and the segmentation cavity may, as shown in Figs. 52 and 54, communicate with each other for a time during these changes.

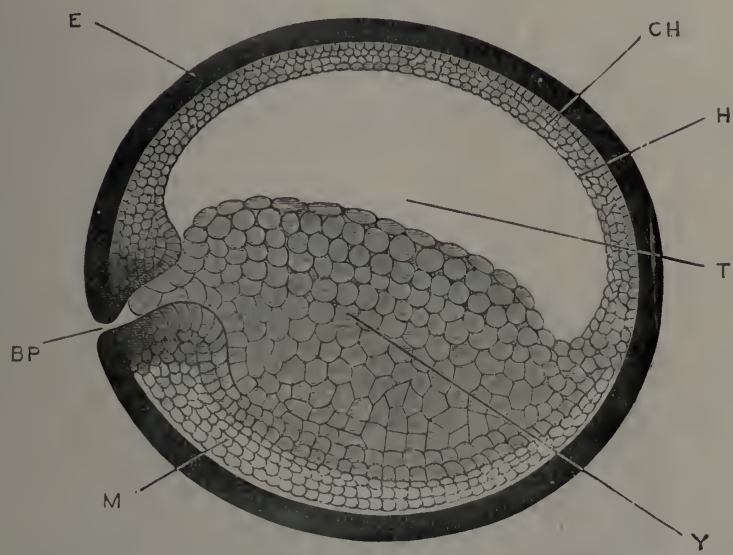

FIG. 55.--Sagittal section of a Frog Embryo after the disappearance of the segmentation cavity and completion of the mesenteron. $\times 25$.

BP, blastopore. CH, notochord. E, epiblast : the cell outlines and the distinction between the epidermic and nervous layers are not shown. $H$, lyppoblast. $\mathbf{M}$, mesoblast. T, mesenteron. Y, yolk-cells.

\section{Formation of the Hypoblast, the Notochord, and the Mesoblast.}

During the formation of the mesenteron, the cells forming its walls (Figs. 54 and 56) become arranged in two concentric layers:- an inner layer, the hypoblast, which forms the true wall of the mesenteron; and an outer layer, the mesoblast (Fig. 56, M), which lies between the hypoblast and the epiblast.

The splitting off of the mesoblast commences in the dorsolateral walls of the mesenteron, and spreads towards the median plane, both dorsally and ventrally. Before this splitting reaches the mid-dorsal plane, a pair of longitudinol clefts appear along the dorsal surface, by which a median longitudinal rod of cells (Fig. 56, CH) is cut off from the two laterally placed mesoblast sheets, M. This rod, CH, remains attached to the liypoblast for a short time after the mesoblast sheets are completely separated; but very shortly afterwards the rod in its turn splits off from the hypoblast, and becomes the notochord. 
The mesoblast (Fig. 56, $\mathrm{M}$ ) thus arises in the frog as two lateral sheets of cells, split off from the outer surface of the hypoblast and yolk-cells. The two sheets very early become continuous with each other in the mid-ventral plane, but are separated dorsally by the notochord, which is formed, independently, from the hypoblast in the mid-dorsal region.

At intervals along their length, the mesoblast sheets remain for a time attached to the hypoblast along the dorsal surface of the mesenteron, not far from the median plane; and, at these

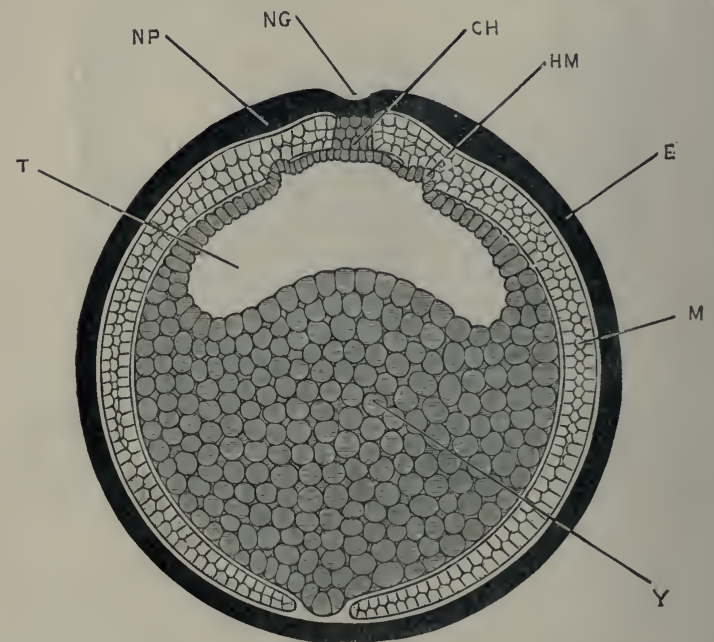

FIG. 56.-A transverse section through the middle of the length of a Frog Embryo at about the stage represented in Fig. 55. $\times 25$.

$\mathbf{C H}$, notochord. E, epiblast. HM, pouch-like diverticulum of the hypoblast into the mesoblast. $\mathbf{M}$, mesoblast. $\mathbf{N}$ G, neural groove. $\mathbf{N P}$, neural plate. $\mathbf{T}$, mesenteron. $\mathbf{Y}$, yolk-cells.

places, slight pouch-like diverticula from the mesenteron (Fig. $56, \mathrm{HM})$ may be seen extending into the mesoblast, sheets. It has been suggested by Hertwig that these diverticula are possibly indications of a mode of origin of the mesoblast as hollow diverticula from the mesenteron, such as occurs in Amphioxus (cf. Figs. 24 and 28, CE).

\section{The Blastopore and the Primitive Streak.}

The blastopore, or anus of Rusconi, has been defined above as the circular aperture in the epiblast which is filled up by the yolk-plug (Figs. 52 and $58, \mathbf{A}$ ); the lip of the blastopore and 
the yolk-plug being separated from each other by the narror circular groove which leads into the mesenteron. In the immediately succeeding stages the blastopore becomes greatly reduced in size, though still retaining its circular outline (cf. Figs. 52, 54, 55). This reduction is effected, not by contraction of the whole circumference of the blastopore, but by a folding together, or concrescence, of its lips in the median plane, beginning at the lower or ventral margin and proceeding upwards towards the dorsal margin, the line of fusion being marked by a faint vertical groove on the surface of the embryo (cf. Fig. $58, \mathbf{A}$ and $\mathbf{B})$.

At the lip of the blastopore, round its entire circumference, the three germinal layers, epiblast, mesoblast, and hypoblast, are indistinguishably fused together (Figs. 54, 55); the separation between the layers first appearing a little distance beyond the margin of the blastopore. As the lips of the blastopore meet and unite from below upwards, in the manner described above, a vertical band is produced by their union, at the hinder end of the embryo, in which the three germinal layers are fused. This band is spoken of as the primitive streak; and the faint median groove, already described (Fig. 58, B, C), which runs along it, and marks the line of union of the right and left lips of the blastopore, is named the primitive groove.

The primitive streak and primitive groove are comparatively inconspicuous features in the frog embryo, but are much more prominent in the chick and the rabbit. They are probably to be regarded as secondary rather than as essential characters, and as associated with the great distension which the egg has undergone in consequence of the number of yolk-granules imbedded in its substance.

The further development of the primitive streak, and the ultimate fate of the blastopore, will be described in a later part of this chapter.

The reduction in size of the blastopore, caused by the concrescence of its lips, gives rise to a corresponding diminution of the yolk-plug ( $c f$. Figs. 52, 54, YP); and at the close of the period now being described this withdraws completely from the surface of the embryo (Fig. 55). 


\section{Comparison of the Early Stages in the Development of the Frog with those of Amphioxus.}

'The frog's egg is more than 5,000 times the bulk of that of Amphioxus: this large size is due mainly to the much greater amount of food-yolk present in the frog's egg, and it is chiefly owing to this food-yolk that the development of the two forms is so different. In the earliest stages the differences are less marked than in the succeeding ones. The first two segmentation clefts divide the frog's egg in the same way as they do that of Amphioxus; the third cleft is in both cases a horizontal one, but while in Amphioxus it is nearly equatorial, in the frog it lies much nearer the upper pole. The stage shown for the frog in Fig. 49 corresponds fairly closely, in essential respects, with the blastula stage of Amphioxus; but from this point the development of the two forms becomes widely different.

There is no stage in the frog which exactly corresponds to the gastrula stage in Amphioxus; for at the stage shown in Fig. 52, which most nearly approaches to this, both epiblast and hypoblast are already three or more cells thick, instead of being, as in Amphioxus, single layers of cells. Moreover, the primitive digestive cavity of the frog (Fig. 52, T) is formed, not by invagination, as in Amphioxus, but by a process of splitting, or separation, among the yolk-cells occupying the interior of the embryo. The history of development in some allied animals, notably in the newt, suggests that the process of splitting is a secondary modification, which has arisen in consequence of the hindrance offered by the large mass of yolk-cells to the occurrence of invagination.

The early establishment of the two-layered condition of the epiblast is another point in which the frog presents a modified and specialised condition: in the corresponding stages of the newt the epiblast consists, as in Amphioxus, of a single layer of cells.

\section{DEVELOPMENT OF THE NERVOUS SYSTEM.}

It will be convenient from this point to deal with the several systems one by one, following each up to its condition in the adult. The order in which the systems are taken is chiefly a matter of convenience, but for several reasons the nervous 
system is the most suitable to commence with. It is formed from the epiblast, which is the earliest of the germinal layers to be definitely established; it appears at a very early stage; and it plays a prominent part, especially in the younger embryos, in determining the shape and proportions of the body.

\section{General History of the Central Nervous System.}

The epiblast of the frog, as already described, consists, almost from the first, of two layers, the distinction between which is established before the close of the period of segmentation. Of these, the upper or epidermic layer is a single stratum of closely fitted, short columnar or cubical cells; while the lower or nervous layer (Figs. 51, 52) consists of spherical or ovoid cells, more loosely arranged, and two or three deep : it is from this lower layer that the nervous system is developed.

The first trace of the nervous system appears at a. stage immediately succeeding that shown in Fig. 55, when the embryo is ellipsoidal in shape, and the blastopore has become much reduced, and less conspicuous owing to the yolk-plug having withdrawn from the surface.

The dorsal surface of the embryo now flattens slightly, and along the flattened area the deeper or nervous layer of the epiblast thickens to form the neural plate, a triangular area extending along the back of the embryo, wider in front but narrowing posteriorly towards the blastopore. Slightly raised ridges, the neural folds (Fig. $57, \mathrm{NF}$ ), soon appear, bordering the neural

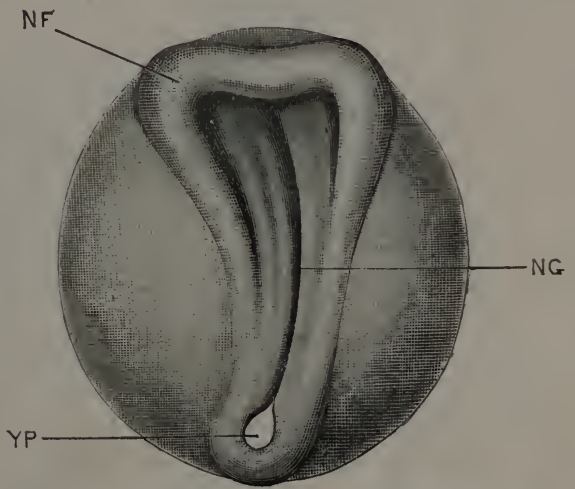

FI(x. 57.-A Frog Embryo at the time of appearance of the neural folds: seen from the dorsal surface. $\times 20$.

$\mathrm{NF}$, neural fold: the reference line points to the junction of the anterior and the left lateral folds. $\mathbf{N G}$, nemral groove. $\mathbf{Y P}$, yolk plug, greatly reduced in size, but still visible through the blastopore.

plate laterally; and a shallow neural groove (Figs. 56, 57, $\mathrm{NG}$ ) is formed along its dorsal surface in the median line, extending forwards from the blastopore. 
Anteriorly, the two neural folds are connected by a transverse fold (Fig. 57), which runs across the anterior end of the neural plate, and slightly raises it above the level of the surrounding parts; while at their hinder ends the two neural folds are continuous with the lateral lips of the blastopore.

The neural folds rapidly increase in height and thickness : the groove between them deepens; and the folds, becoming more and more prominent (Figs, 58, 59), approach each other, and finally meet in the median plane and fuse together, converting the neural groove into a tube.

The neural folds first meet about the junction of the head

A

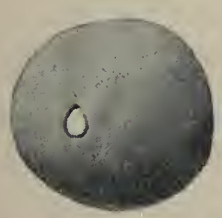

B

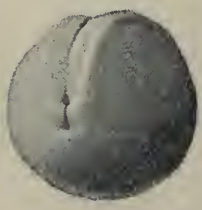

C

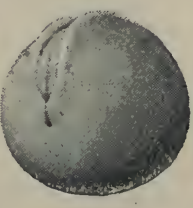

D

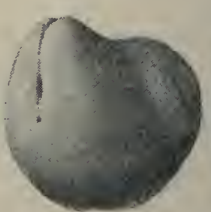

$\mathbf{E}$

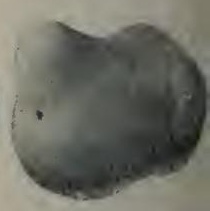

FiG. 58.-Stages in the early development of the Frog Embryo, seen obliquely from the hinder end. (From a series of wax models by Dr. F. Ziegler, of Freiburg i/B.)

A, stage in which the blastopore is nearly circular, and is occupied by the white yolk-plng. B. stage in which the lateral lips of the blastopore have met and fused to form the primitive streak ; the short vertical line, corresponding to the position of the blastopore in $\mathbf{A}$, is the primitive groove; the depression at the upper end of the primitive groove is the greatly reduced blastopore, and the depression at the lower end of the primitive groove is the commencing proctodæal or anal invagination. Above the blastopore is seen the commencing neural groove, bordered by the neural folds. C, later stage, in which the neural groove has deepened, while the neural folds are more promineut and are growing inwards to meet each other. $\mathbf{D}$, stage in which the neural folds lave met and the tail is commencing to form. Both blastopore and proctodæum are still present. $\mathrm{E}$, later stage, in which the neural tube is completed and the tail has increased in size. The blastopore lias finally closed, and the black spot below the tail is the proctodrenm.

and neck of the embryo; and from this point the fusion extends rapidly backwards, and more slowly forwards. The point at which fusion last occurs is a little distance behind the anterior end of the neural tube, at the spot where the pineal body is formed later, the part of the tube in front of this point being roofed in by growth backwards of the anterior or transverse neural fold seen in Fig. 57.

The neural groove extends back as far as the blastopore (Figs. 57 and 58, B), and the neural folds, as noticed above, becorne continuous at their hinder ends with the lips of the blastopore. For a short time, after completion of the neural tube, the blastopore still remains open, communicating, as seen in Fig. 60, both with the mesenteron and with the cavity of the 
neural tube. Very shortly, however, the fusion, by which the neural tube is closed, extends further back, so as to involve the lips of the blastopore, and the external opening of the blastopore becomes finally closed (Fig. 58, E, and Fig. 61). The communication between the neural tube and the mesenteron still persists, however, as a narrow tubular passage, the neurenteric canal (Fig. 61), passing round the hinder end of the notochord.

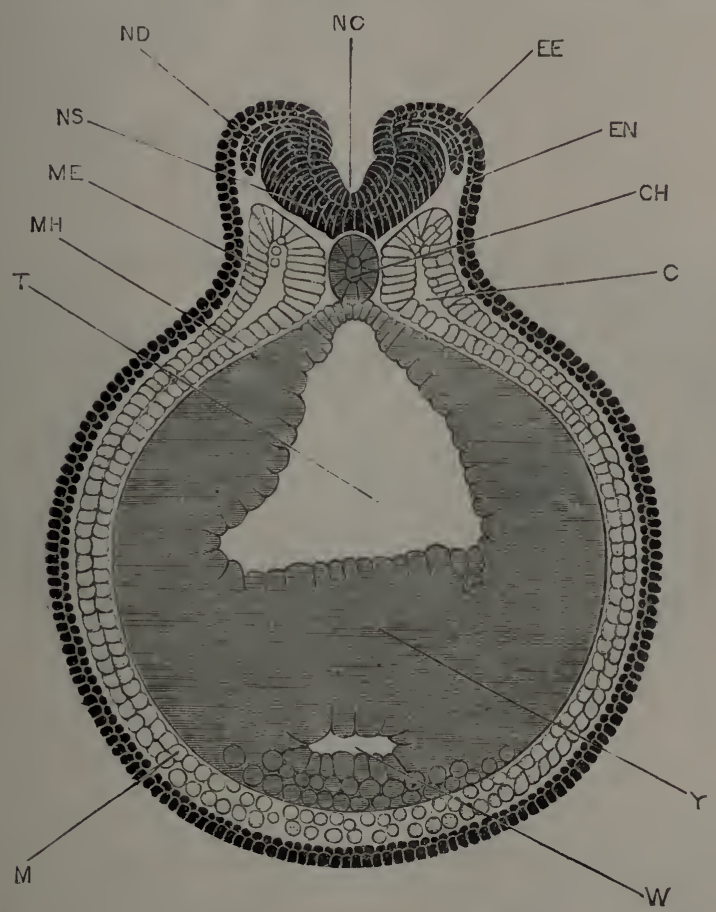

L'IG. 5\%.- T'ransverse section through a Frog Embryo, at a stage corresponding to Fig. 58, C, and showing the neural folds shortly before they meet each other to complete the neural tube.

C, colom or body cavity. CH, notochord. $\mathrm{EE}$, outer or epidermic layer of epiblast. EN, inuer or nervous larer of epiblast. $\mathbf{M}$, mesoblast. $\mathbf{M E}$, outer or somatopleuric layer of mesoblast. MH, inner or splanchnopleuric layer of mesoblast. NC, neural groove. ND, dorsal root of a spinal nerve. NS, spinal cord. T, mesenteron. W, liver diverticulum. Y, yolk.

The neurenteric canal persists only for a very short time. In the immediately succeeding stages the tail begins to lengthen rapidly, carrying the hinder end of the neural tube far' away from the mesenteron, and the channel of communication between the two becomes speedily obliterated. At the time of hatching (Fig. 69, p. 146), the hinder end of the neural tube 
curves slightly downwards, round the end of the notochord, but ends blindly a long distance from the mesenteron. A string of cells, connecting the two structures, is at this stage the sole indication of the former communication between them.

The neural tube, formed in the way described above, by fusion of the neural folds, soon separates along its entire length from the external epiblast, and by thickening of its walls and various histological changes becomes converted into the central nervous system; the anterior part forming the brain, and the posterior part the spinal cord. The lumen or cavity of the neural tube persists throughout life as the central canal of the

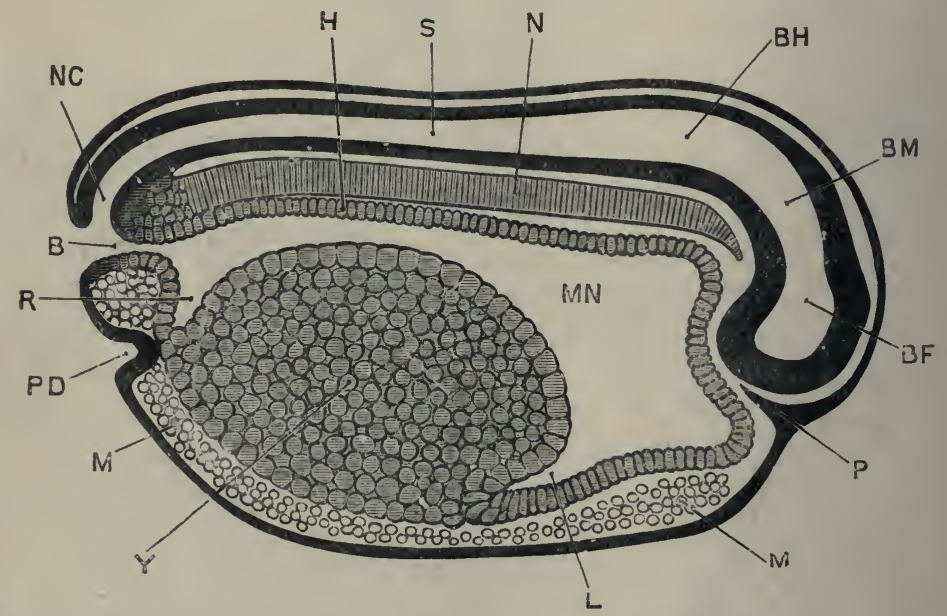

Fig. 60.-Sagittal section of a Frog Limbryo shortly before closure of the blastopore, and of the same age as the embryo shown in Fig. 58, D. $\times 30$.

B, blastopore. BF, fore-brain. BH, hind-brain. BM, mid-brain, H, hypoblast. L, liver. M, mesoblast. $\mathbf{M N}$, mesenteron. $\mathbf{N}$, notochord. $\mathbf{N C}$, neurenteric canal. $\mathbf{P}$, ingrowth of epiblast to form the pituitary body. $\mathbf{P D}$, proctodæum. $\mathbf{R}$, rectal diverticulum of mesenteron. $\mathbf{S}$, central canal of spinal cord.' $\mathbf{Y}$, yolk cells.

spinal cord and the ventricles of the brain (cf. Figs. 60, 61, 64, and 65$)$.

The further changes undergone by the spinal cord are comparatively slight, and will not be described in detail. Almost from the first (Fig. 70, p. 147), the spinal cord is oval in transverse section, the central canal being a vertical slit. The layer of cells lining the central canal, derived ( $c f$. Fig. 59) from the outer or epidermic layer of the epiblast, remains throughout life as a layer of columnar ciliated epithelial cells; while the outer wall of the neural tube, formed from the deeper or nervous layer 
of the epiblast, gives rise directly to the nervous elements, i.e. to the nerve cells and nerve fibres, of the adult spinal cord. The histological changes by which the nervous elements are formed will be described in the chapters dealing with the chick and the rabbit, in which animals they have been investigated more completely than in the frog.

The spinal cord extends to the extremity of the tail, which in the later stages of tadpole life is of great length (Fig. 44, 9, 10, 11). During the absorption of the tail, at the time of the metamorphosis, fully two-thirds of the length of the spinal cord are lost.

\section{The Development of the Brain.}

The brain is merely the specialised anterior part of the

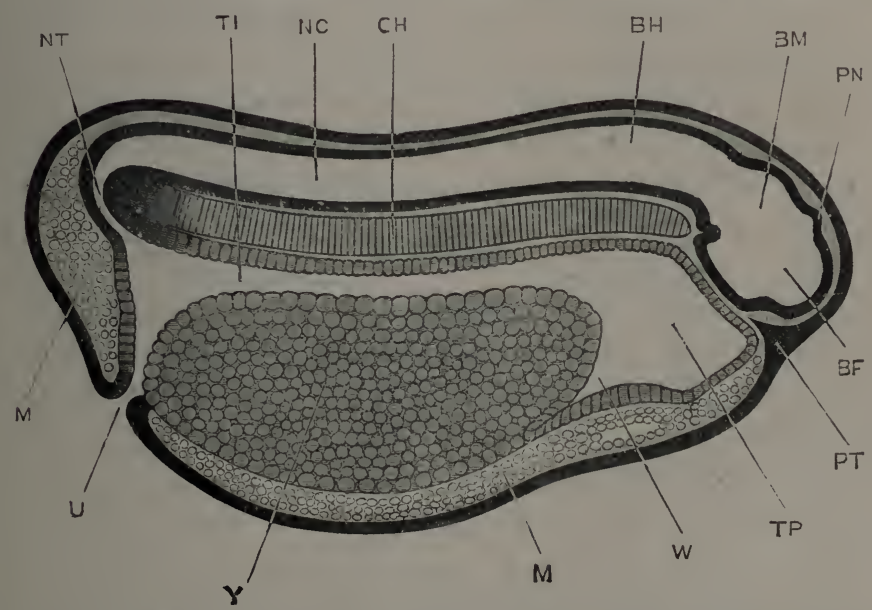

FIG. 61.- Sagittal section of a Frog Embryo, shortly after closure of the blastopore and formation of the anus, and of the same age as the embryo shown in Fig. 58, E. $\times 25$.

$\mathbf{B F}$, fore-brain. $\mathbf{B H}$, hiud-brain. $\mathbf{B M}$, mid-brain. $\mathbf{C H}$, notochord. $\mathbf{M}$, mesoblast. iNC, cavity of neural tube. NT, neurenteric canal. PN, pineal body. PT, ingrowth of epiblast which gives rise to the pituitary body. $\mathbf{T} \mathbf{I}$, intestinal region of mesenteron. TP, pharyngeal region of mesenteron. U, proctodæal or cloacal aperture. W, liver, $Y$, yolk-cells.

neural tube, and is directly continuous posteriorly with the spinal cord.

While the spinal cord is straight, or nearly so, the brain is from its first appearance bent rather sharply, and nearly at right angles, about the middle of its length; the axis of the posterior part being horizontal and continuous with that of the spinal cord, and the axis of the anterior part vertical. The 
whole central nervous system may be compared to a retort (Fig. 60 ), the bulb of the retort being formed by the anterior and vertical part of the brain, BF, and the neck by the posterior horizontal part of the brain, together with the spinal cord.

This bending of the brain is spoken of as cranial flexure. It takes place, as shown in Fig. 60, round the anterior end of the notochord, and is due, in the first instance, to the spherical shape of the surface of the egg on which the neural plate is formed. A similar ventral flexure of the hinder end of the neural tube is present at first, but becomes early obliterated by the outgrowth of the tail ( $c f$. Fig. $58, \mathbf{B}, \mathbf{C}, \mathbf{D}, \mathbf{E}$ ). The ventral flexure of the brain, round the anterior end of the notochord, persists throughout life.

Very shortly after the closure of the brain-tube is completed, a slight transverse constriction appears, at the bend between the horizontal and vertical portions of the brain, and a little later a second constriction is formed rather further forwards. By these constrictions the brain (Figs. 60 and 61) becomes divided into three portions, named fore-brain, mid-brain, and hind-brain respectively.

The fore-brain (Figs. 60 and 61, BF) is the terminal vertical portion, corresponding to the bulb of the retort; the midbrain, BM, which is the smallest of the three divisions, forms the angle of the bend, opposite the anterior end of the notochord; and the hind-brain, $\mathrm{BH}$, is the horizontal portion, continuous posteriorly with the spinal cord. This division of the embryonic brain into three regions, anterior, middle, and posterior, is a convenient one, as it obtains throughout the higher groups of Vertebrates, from fishes to mammals, each of the divisions giving rise to important and characteristic parts of the adult brain.

The walls of the brain-tube are at first of approximately uniform thickness in all parts, excepting the roof of the hindbrain, which from the first is thin. By mnequal thickening of various parts, especially of the sides, and by outgrowths, either median or paired, with accompanying histological changes, the adult brain is gradually built up.

In these changes the most important share is taken by the fore-brain. The fore-brain itself becomes the part known in the adult as the thalamencephalon, its cavity persisting as the 
third ventricle; from it the pineal body and the infundibulum are developed as median diverticula, dorsal and ventral respectively; while the optic vesicles and cerebral hemispheres arise as paired lateral and anterior outgrowths.

The mid-brain undergoes comparatively little change; from its roof the optic lobes of the adult are formed.

The hind-brain becomes the medulla oblongata of the adult : from the roof of its anterior part the cerebellum is formed.

Before considering the development of the several parts of the brain in detail it will be well to notice the general propor-

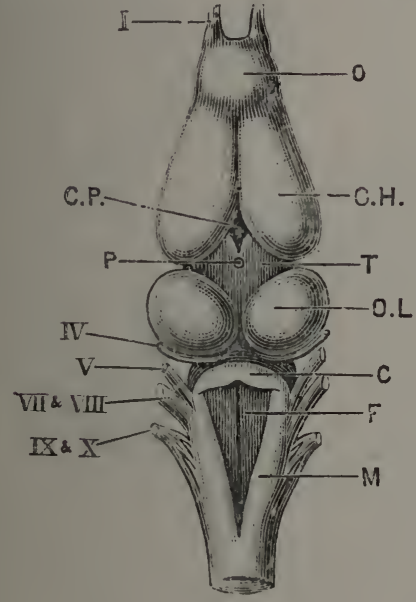

FIG. 62.

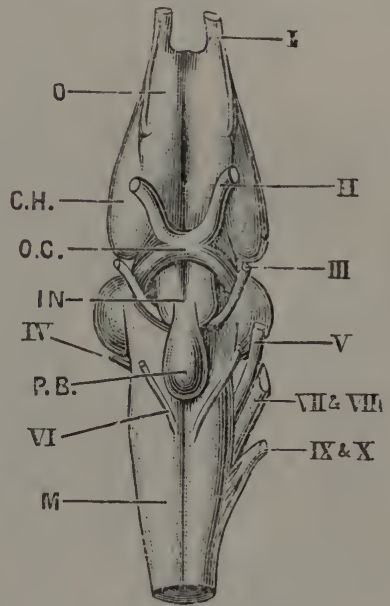

Fig. Ris.

FIG. (22. - The brain of the adult Frog: dorsal surface. $\times 4$.

Fic. 63.-The brain of the adult Frog: ventral surface. $\times 4$.

C, cerebellum. CH, cerebral hemisplere. CP, choroid plexus of third ventricle. F, fourth ventricle. IN, infundibulum. M, medulla oblongata. $O$, olfactory lobe. OC, optic chiasma. OL, optic lobe. P, stalk of pineal borly. PB, pituitary body. T, thalamencephalon. I, olfactory nerve. II, optic nerve. III, third or motor oculi nerve. IV, fourth nerve. $\mathrm{V}$, fifth or trigeminal nerve. $\mathrm{V} \overline{\mathrm{I}}$, sixth nerve. VII and VIII, combined root of facial and autitory nerves. IX ani $\mathbf{X}$, combined root of glossopharyngeal and pneumogastric nerves.

tions and relations of the brain during the successive stages of its formation. These will be readily understood from comparison of Figs. 60, 61, 64, 65, and 89 .

At the time of the first formation of the brain-tube, before the hatching of the tadpole (Figs. 60,61), cranial flexure is very strongly marked, and the fore-brain, BF, projects far in front of all other organs of the body. Later on (Figs. 64, 65), both these relations are changed; the brain appears to become 
straightened out, and it also recedes some distance from the anterior end of the head.

The straightening of the brain, or rectification of the cranial flexure as it is sometimes termed, is apparent rather than real, and is brought about principally by the formation of the cerebral hemispheres (Figs. 64, 65, BC), which grow forwards from the

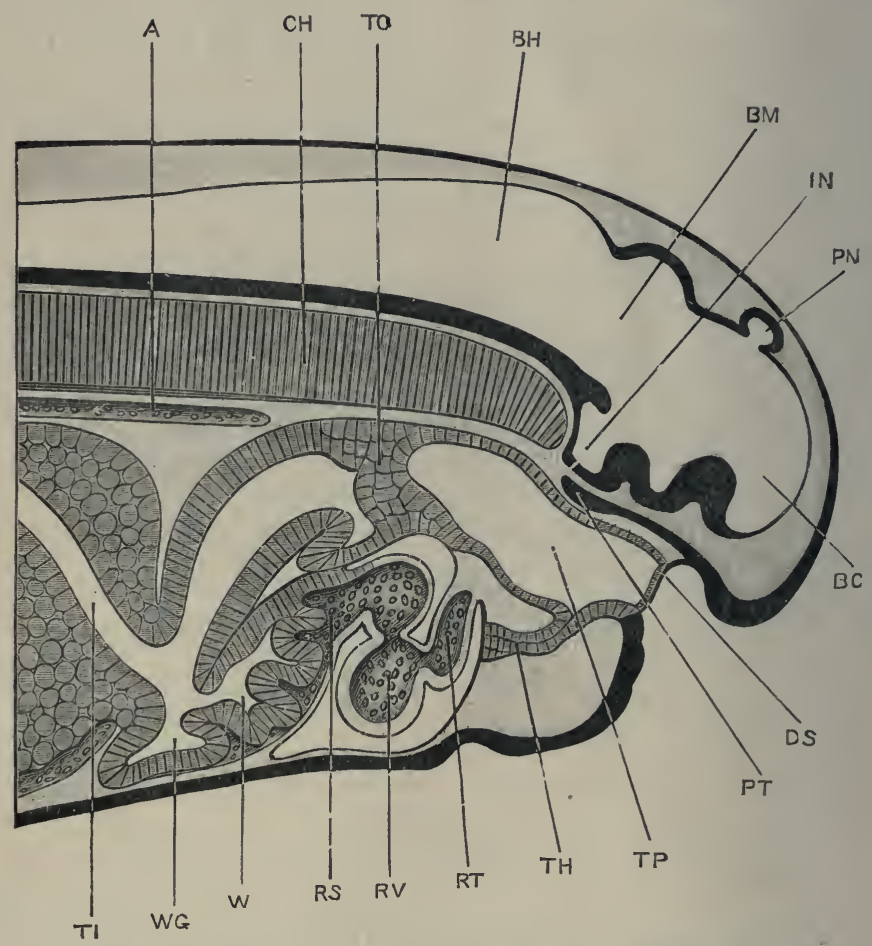

FIG. 64.- Sagittal section of the head end of a Tadpole just before the opening of the moutb.

A, dorsal aorta. BC, vesicle of the hemispheres. BH, hind-brain. BM, mid-brain. CH, notochord. DS. septum separating stomatodæum and pharynx. IN, infundibulum. PN, pineal body. PT, pituitary body. RS, sinus venosus. $R T$, truncus arteriosu. RV, ventricle. TH, thyroid body. TI, intestine. TP, pharynx. TO, plug of epithelial cells blocking up the œsophagus. 'W, liver. W W $\mathbf{W}$, gall-bladiler.

fore-brain, and speedily attain so large a size relatively to the other parts of the brain as to alter the direction of the axis of the brain as a whole, and to completely obscure the original flexure, which really persists throughout life. The receding of the brain from the anterior end of the head is due to the more rapid growth of the surrounding parts, and more especially of 
the face and lips, which causes the brain to take a much less prominent share in determining the shape of the hear.

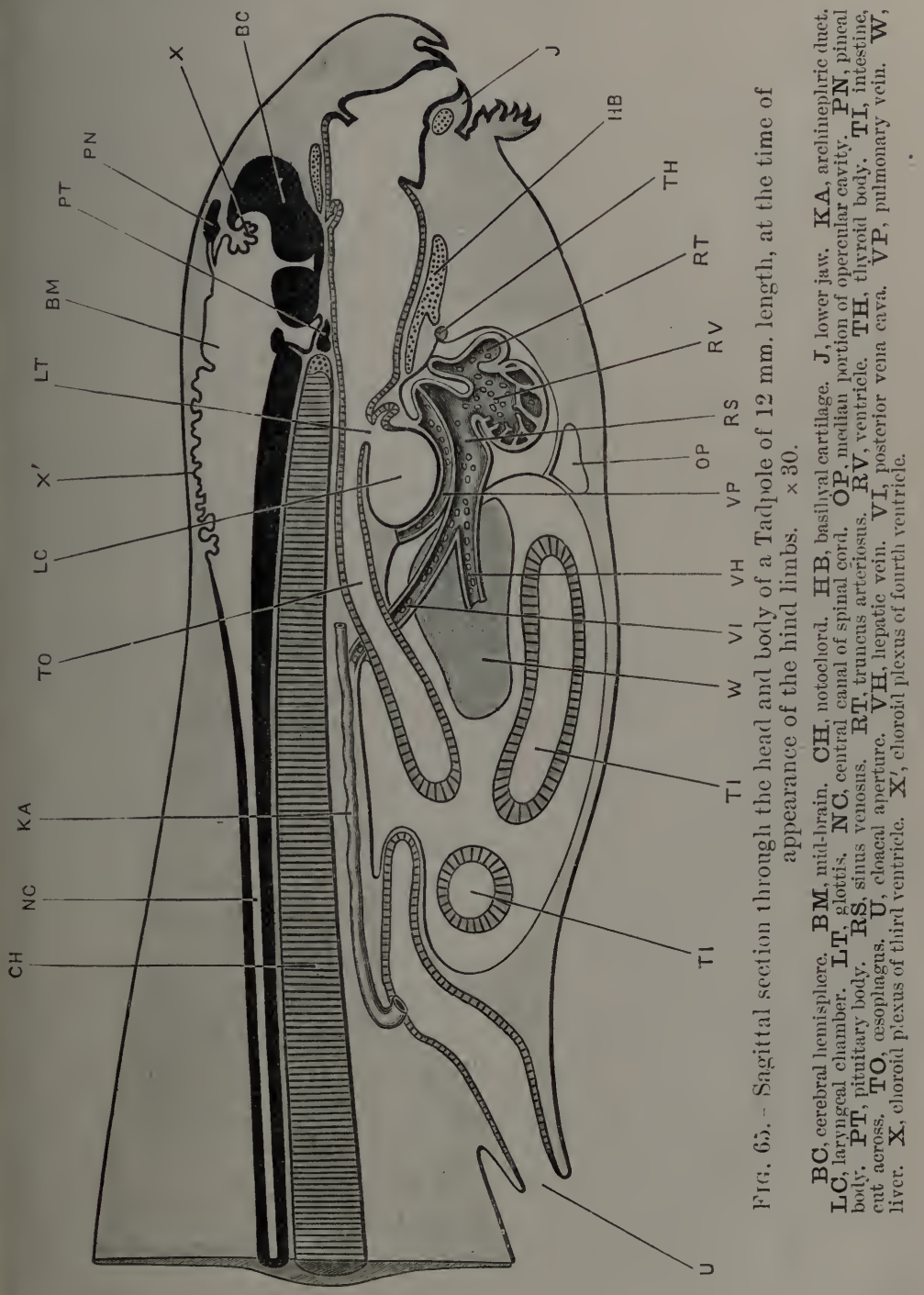

In describing the development of the brain in detail it will be convenient to take the several parts in order, from behind forwards, commencing with the medulla oblongata. 
'The medulla oblongata undergoes less change than any other part of the brain. In the early stages, up to about the time of formation of the mouth, it is the widest part of the brain, but afterwards it is exceeded by both the optic lobes and the cerebral hemispheres. It is continuous posteriorly, without any line of demarcation, with the spinal cord; while anteriorly it is separated from the mid-brain by a well-marked constriction, deepest dorsally and at the sides.

From the first, the roof of the medulla oblongata is thin; in the later stages the sides and floor thicken very considerably, while the roof (Figs. 65 and 84) widens out and becomes reduced to an extremely thin membrane, consisting of a single layer of pigmented and ciliated epithelial cells, without nervous elements of any kind.

This thin roof is at first smooth and level; but about the time of formation of the mouth opening, i.e. in tadpoles of about $9 \mathrm{~mm}$. length, the roof becomes thrown into folds (Fig. 65, $\mathrm{x}^{\prime}$ ), which become deeper and more pronounced as the tadpole increases in size. Lying on this thin roof, and in very close contact with it, is a rich network of blood-vessels, the choroid plexus, which extends between the folds of the roof, and so appears to hang down into the cavity of the medulla, though always in reality separated from this by the thin epithelial roof.

The cavity of the medulla oblongata, or fourth ventricle, is of considerable size: it is wide in front, and tapers gradually towards its hinder end, where it passes into the central canal of the spinal cord.

The cerebellum is an inconspicuous structure throughout the early stages of tadpole life. Up to the time of the opening of the mouth it can hardly be said to exist (Fig. 64); but shortly after this event it appears as a thickening of the roof of the fourth ventricle, in the form of a transverse band, immediately belind the constriction separating the medulla oblongata from the mid-brain. In the later stages of development it increases gradually in size (Fig. 89, BL), but even in the adult frog it is very small as compared with its condition in most other Vertebrates.

The mid-brain does not undergo very great changes. Its floor remains thin in the actual median plane; but immediately 
to the right and left of this the sides become thickened by the formation of the crura cerebri, two longitudinal bundles of nerve fibres which connect the mid-brain with the fore-brain. The roof of the mid-brain is thin in the early stages; but shortly after the opening of the mouth the two halves of the roof thicken considerably, and, bulging upwards, form a pair of rounded swellings, the optic lobes (Fig. 62, oL), separated by a median groove.

The optic lobes continue to increase in size, and about the time of the metamorphosis become, as in the adult frog, the widest portion of the brain. The cavity of the mid-brain persists as a fairly wide passage, the Sylvian aqueduct.

The thalamencephalon is the original fore-brain of the embryo; and in connection with it important changes occur. Its cavity, the third ventricle, is at first large; but, owing to thickening of its walls to form the optic thalami, the cavity becomes early reduced to a vertical cleft, very narrow from side to side.

The roof of the thalamencephalon is rery thin, consisting, like that of the medulla oblongata, of a single layer of epithelial cells, devoid of nervous elements. About the middle of its length, and at the place where the final closure of the neural tube was effected, the pineal body is formed. This appears in embryos of about $3 \mathrm{~mm}$. length (Fig. 61, Py) as a median hollow diverticulum, which at the time of hatching of the tadpole (Fig. 64, Pr), forms a small round knob on the top of the brain, immediately beneath the surface epiblast. This grows forwards, and becomes dilated distally. At the time of opening of the mouth it forms a small rounded vesicle, connected with the brain by a tubular stalk; it is of a glistening white appearance, owing to the presence of small snow-white particles imbedded in its substance, and stands in this respect in marked contrast to the rest of the brain, which is pigmented rather strongly. In tadpoles of $12 \mathrm{~mm}$. length the pineal body itself is solid (Fig. 65, PN), but its stalk is still tubular. Shortly after this, on the formation of the skull, the pineal body becomes cut off from its stalk, and lies outside the skull, just beneath the skin of the top of the head. It persists throughout the tadpole stages, but disappears at the time of the metamorphosis. The stalk of the pineal body persists throughout the life of the 
frog, retaining its tubular character, and its communication with the third ventricle.

About the time the roof of the fourth ventricle is becoming folded, and the choroid plexus established in connection with it, a similar change is going on in relation with the third ventricle. Immediately in front of the pineal body, the thin roof of the thalamencephalon becomes thrown into folds which hang down into the ventricle (Fig. 6r, x). A dense plexus of blood-vessels lies on the roof and grows in between its folds, giving rise to at choroid plexus similar to that of the fourth ventricle, but more restricted in its extent. The vascular plexus on the surface of the thalamencephalon forms also a dorsally projecting process, the supra-plexus, with which the distal end of the stalk of the pineal body is in very close relation (Fig. 89, PN).

The infundibulum (Figs. 64, 65, IN) is a depression of the floor of the thalamencephalon, with which the pituitary body comes into close relation at an early stage. The infundibulum is already recognisable at the time of closure of the neural tube: its hinder wall is in close relation with the anterior end of the notochord, and it is in fact the infundibular depression (Fig. 61) which causes the brain to appear to be bent round the end of the notochord, one of the most striking features of cranial flexure. The infundibulum is separated from the mid-brain (Figs. 61, 65) by a deep transverse groove, running across the ventral surface of the brain, and very conspicuous when the brain is seen from below. On the appearance of the optic vesicles, a second transverse groove (Fig. 64) is formed, further forwards, between the optic vesicles and the infundibulum. The infundibulum retains the same character and relations throughout all the later stages of development: it appears as a wide thin-walled sac, forming a conspicuous projection on the under surface of the brain, and having the pituitary body in close relation with it posteriorly (Fig. $89, \mathrm{Ir}$ ).

The pituitary body, although not really a part of the brain, may conveniently be described here. It arises (Figs. 60, r, and 61, PT) as a plug-like ingrowth of the deeper or nervous layer of the epiblast, immediately below the anterior end of the brain. It appears very early, and may be recognised as a slight thickening of the epiblast even before the neural tube is closed; and at 
the time of completion of the tube (Fig. 60) it projects inwards as a small, solid, tongue-like process beneath the brain, between this and the dorsal wall of the pharynx.

The projection continues its growth inwards, and expands at its end into a somewhat flattened mass of cells, which lies immediately beneath the infundibulum, close to the anterior end of the notochord, and which becomes the pituitary body itself (Fig. 64, PT); the rest of the process forms a slender stalk, which connects the pituitary body with the surface epiblast. About the time of opening of the mouth, the pituitary body becomes hollow and separates from the stalk, which atrophies and soon disappears completely. The pituitary body (Fig. 6J, PT), which is now all that remains of the original ingrowth, acquires close relations with the hinder end of the infundibulum, which it retains throughout life (Fig. 63, PB). It becomes partially divided into anterior and posterior portions, of which the latter forms a complicated mass of convoluted tubes.

The optic vesicles. From the sides of the fore-brain, about the time that closure of the neural tube is effected, a pair of hollow lateral outgrowths, the optic vesicles, arise. Each optic vesicle soon becomes constricted at its base, so as to form a bulb, opening by a tubular stalk into the fore-brain. From the bulbs, the eyes are developed in a manner that will be described later on; while the optic stalks form paths along which the fibres of the optic nerves pass from the eyes to the brain.

About the time of opening of the mouth (Fig. 64), a transverse groove runs across the floor of the fore-brain, in front of the infundibulum, 1s, and between this and the vesicle of the hemispheres, BC. This groove is bounded in front and behind by transverse ridges, and is produced outwards at its two ends into the tubular optic stalks. At a slightly later stage, about the time of appearance of the hind limbs (Fig. 65), the stalks become solid along their whole length; the further changes in commection with them will be described in the section dealing with the development of the eye.

The cerebral hemispheres. The hemispheres, although the largest part of the adult brain (Figs. 62, 63), are- the last to appear. About the time of hatching of the tadpole, the anterior 
end of the fore-brain begins to grow forwards as a median thinwalled vesicle of the hemispheres; this steadily increases in size, but, up to the time of the formation of the mouth, remains undivided. At this stage (Fig. 64, BC), it is approximately spherical, and about equal in size to the mid-brain: its roof and anterior wall are both extremely thin, but its side walls are much thickened, so that the central cavity is compressed laterally.

The vesicle continues to increase in size, but remains single and undivided up to a stage slightly later than that shown in Fig. 65, when a division between the two hemispheres appears. This is effected by the roof and anterior wall becoming folded vertically along the median plane; the fold, which is continuous posteriorly with the choroid plexus of the third ventricle, projects into the cavity of the vesicle, and partially divides this into right and left halves, which become the lateral ventricles of the hemispheres.

By further growth forwards of the hemispheres, with thickening of their walls, the proportions of the adult brain are gradually acquired; the brain at the time of the metamorphosis being practically identical with that of the fully formed frog. The anterior or distal extremities of the hemispheres become the olfactory lobes; these are at first separate from each other, but ultimately become fused together along their inner surfaces.

\section{The Development of the Peripheral Nervous System.}

It will be convenient to give first a general description of the early stages of development of the peripheral nervous system of the frog, and then to deal separately with the cranial and the spinal nerves in regard to the later phases of their formation. There are still many points on which our knowledge of the development of the nervous system in the frog is imperfect and unsatisfactory.

The early stages of development of the nerves. The dorsal roots of the spinal nerves, and the majority of the cranial nerves, arise in closely similar manner, and at a very early period. The first commencements are seen in embryos which are still almost spherical, and in which the neural plate and neural ridges are just commencing to form, but have not yet begun to fold in to inclose the neural tube (Fig. 57).

The neural plate is formed, as described above, by thickening: 
of the deeper or nervous layer of the epiblast along the dorsal surface of the embryo. Along the sides of the neural plate, where it passes into the unmodified epiblast of the body wall, and on its inner or deeper surface, opposite to the commencing neural ridges, a pair of longitudinal bands of epiblast appear. 'These are at first merely the lateral edges of the neural plate, but they soon become separated by lines of demarcation from the neural plate, and rather later from the epiblast along their outer sides. In transverse sections they appear as a pair of small triangular wedges, at the sides of the neural plate, continuous with the epiblast above, but separated by divisional planes, often indistinctly marked, from the neural plate on the inner side and the general epiblast on the outer side ( $c f$. Fig. 59, xD).

These bands of epiblast cells, cut out from the inner or nervous layer of the epiblast, are at first continuous structures, extending the whole length of the embryo. They are spoken of as the neural ridges, and from them the dorsal roots of the spinal nerves, and the majority of the cranial nerves, are derived.

As the neural folds grow upwards to inclose the neural tube, the neural ridges get carried up with them; and at a time when the lips of the tube are about to unite, the neural ridges form a pair of longitudinal bands (Fig. 59, ND), projecting outwards on either side from the angle between the external epiblast, EE, and the wall of the neural tube, Ns.

On the closure of the neural tube, the neural ridges separate completely from the external epiblast, and the ridges of the two sides become continuous with each other across the median plane, forming a plate of cells, the neural crest, attached to the dorsal surface of the brain and spinal cord (Fig. 70, xD).

As the several divisions of the brain are formed, the originally continuous neural ridge of each side becomes discontinuous, growing outwards so as to become more prominent at certain parts of its length, and disappearing in the intervening regions; it thus becomes broken up into a series of outgrowths, which are the rudiments of the nerves.

The neural crest, formed by the fusion of the two neural ridges, and therefore also the nerves into which the crest becomes cut up, are at first connected with the dorsal surface of the neural tube. The permanent attachments of the nerres to the sicles of the neural tube are acquired at a later stage, by 
the growth of processes from the cells of the nerves into the substance of the brain, or spinal cord.

Up to this point the development of the cranial and the spinal nerves is practically the same; the cranial nerves appear at an earlier stage in the formation of the neural tube than do the spinal nerves, and are from the first of much larger size than these latter, but the history of the early stages is essentially the same in the two cases.

The spinal nerves. After reaching the neural crest stage, the development of the spinal nerves proceeds for a time very slowly. The nerve rudiments, after a rather long pause, grow slowly down between the myotomes and the sides of the spinal cord. The permanent attachment to the side of the cord is acquired in the manner described above, by growth of nerve fibres from the nerve rudiment, or ganglion, into the cord. The ganglion itself enlarges, and the nerve fibres continue their course beyond it to form the trunk of the dorsal or sensory root of the spinal nerve.

The ventral or motor root arises quite independently: the details of its development have not been determined so accurately in the frog as in other animals, but each ventral root appears to arise as a number of outgrowths from the lower part of the side of the spinal cord, which from the first occupy their permanent positions in regard to the cord, and which very early become connected distally with the muscles of the body.

The dorsal and ventral roots of each nerve lie close alongside each other, and become bound together by a common connective-tissue sheath to form the trunk of the spinal nerve, from which branches soon arise supplying the various parts to which the nerve is distributed in the adult (cf. Figs. 87 and 88).

The cranial nerves. The development of the cranial nerves of the frog has not been very thoroughly studied; and there are several points on which our knowledge is still in an unsatisfactory condition. The nerves which are undoubtedly derived from the neural ridges are the trigeminal, the facial and auditory, and the sensory branches of the glosso-pharyngeal and pneumogastric; i.e. the fifth, seventh, eighth, ninth, and tenth cranial nerves according to the ordinary nomenclature. The olfactory 
nerve is perhaps to be added to these. The optic nerve develops in a very special manner; and the mode of development of the third, fourth, and sixth nerves in the frog has not yet been determined with accuracy.

The trigeminal, facial, glosso-pharyngeal, and pneumogastric nerves, although arising from the neural ridges in the same way as the dorsal roots of the spinal nerves, yet differ from these, and agree amongst themselves, in certain important features, of which the following are the principal :-

i. The nerves in question, in place of growing downwards, like the spinal nerves, alongside the central nervous system, grow outwards, close to the surface of the embryo, between the epiblast and the mesoblast.

ii. Each of these four nerves acquires a new connection with the surface epiblast some considerable distance beyond the root of origin from the brain, and at about the horizontal level of the notochord; at this place, and at any rate in part from the surface epiblast itself, the ganglion of the nerve is formed.

iii. The nerves have special relations to the gill-slits, each nerve dividing into two main branches, which embrace between them one of the gill-slits.

iv. A special system of cutaneous nerves is developed from the surface epiblast in connection with these four nerves, forming the lateral line system of nerves.

In dealing with the several cranial nerves individually it will be convenient to consider them in order from behind forwards.

$\mathrm{X}$. The pneumogastric, vagus, or tenth cranial nerve. This grows rapidly in the early stages, and soon attains an enormous size. In embryos of about $3 \mathrm{~mm}$. length (cf. Figs. 58, C, and 59 ), when the neural folds have not quite met in the hinder part of the head, and the neural groove is, therefore, still open, the pneumogastric nerves are already present as a pair of winglike expansions of the neural ridges. The root of attachment of the nerve, in the re-entering angle at the top of the brain, between the epiblast and the brain wall, is slender; but the rest of the nerve is of great thickness. It extends more than half way down the side of the pharynx, lying between the mesoblast and the surface epiblast, very close to the latter but distinct from it along its entire length ( $c f$. Fig. $79, \mathrm{x}$ ). The nerves of the two sides are in some cases unequally developed at this stage. 
In embryos of about $4 \mathrm{~mm}$. length ( $c f$. Fig. 61 ), the nerves themselves have undergone but little further change. At the level of the notochord the external epiblast presents, on each side, a very distinct and localised thickening of its inner or nervous layer. This thickening projects inwards, and lies very close to the pneumogastric nerve, a little below the middle of its length, but as yet the two structures are independent. The thickening is well marked, and extends horizontally backwards a little distance beyond the nerve.

At the time of hatching, i.e. in tadpoles of about $7 \mathrm{~mm}$. length ( $c f$. Figs. 69,72 , and 73), the epiblastic thickening and the nerve have fused, and together form the ganglion of the pneumogastric: the horizontal extension backwards of the thickening, which forms the lateral line nerve, has grown enormously, reaching now almost to the hinder end of the body of the tadpole. The mode in which this lateral line nerve grows has not been determined with certainty in the frog; at its first appearance it is clearly a ridge-like thickening of the inner surface of the epiblast, but it is difficult to decide whether the extension backwards, which is effected with great rapidity, is due to a splitting off from the epiblast, or to growth backwards of a solid rod of cells from the ganglion of the pneumogastric. Such evidence as is forthcoming rather favours the latter view. In transverse sections at this stage (Fig. 82, NL), the lateral line nerve has the appearance of a solid rod of cells, lying in a groove along the inner surface of the epiblast, at the level of the lower part of the spinal cord. The lateral line nerve is of large size throughout the whole period of tadpole life ; it is present during the metamorphosis, but disappears completely at its close. During the later tadpole stages it separates from the skin, and becomes more deeply placed among the muscles of the body wall. Besides the main lateral line nerve described above, other similar cutaneous branches are formed in connection with the pneumogastric ganglion; a more slender nerve is developed nearer the mid-dorsal line; and a stout nerve runs at first ventralwards from the ganglion, and then backwards along the sides of the ventral surface of the abdominal region.

Concerning the further development of the pneumogastric nerve itself there are some points of interest. The root of attachment to the brain, which is acquired in the same manner

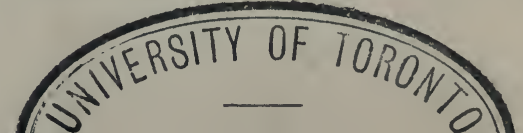


as that of the dorsal root of a spinal nerve, is from the first of considerable length horizontally. About the time of opening of the mouth the root divides into two; an anterior one, which runs nearly straight outwards from the brain; and a posterior one, which runs very obliquely forwards, to join the anterior root just before it reaches the ganglion. The ganglion lies immediately behind the ear; beyond it the nerve divides into a set of branches which supply the three hinder branchial clefts, and a set of visceral branches, which run to the heart and alimentary canal. All these branches are well established by the time the mouth of the tadpole is formed.

IX. The glosso-pharyngeal, or ninth cranial nerve, is formed from the part of the neural ridge immediately in front of that from which the pneumogastric nerve is developed, the roots of the two nerves being at first continuous with each other. The nerve is very similar to the pneumogastric, but of smaller size : in the upper part of its course it lies immediately in front of the pneumogastric; it then runs forwards and outwards, round the hinder border of the auditory vesicle, to the upper edge of the first branchial cleft, where it expands to form the ganglion. The ganglion, like that of the pneumogastric, is formed in part from an independently arising thickening of the external epiblast, which fuses with the nerve rudiment about the time of hatching of the tadpole. The ganglion of the glosso-pharyngeal nerve is separated from that of the pneumogastric by the anterior cardinal vein. Beyond the ganglion the glosso-pharyngeai runs downwards, as a slender nerve, along the anterior erlge of the first branchial arch, giving a small præbranchial branch to the hyoid arch. All the main branches are present at the time of opening of the mouth of the tadpole.

VIII. The auditory, or eighth nerve, axises from the neural crest, in common with the seventh nerve, opposite the middle of the auditory vesicle; the two nerves being absolutely continuous with each other up to the time of formation of the mouth. The auditory portion of the combined nerve forms a large ganglionic swelling, which is continuous with the inner wall of the auditory vesicle from its very earliest appearance. In the later stages, as the various parts of the ear become differentiated, the auditory nerve divides into separate branches supplying its several parts. (Cf. Fig. 75, viri.) 
VII. The facial, or seventh nerve, as noticed above, is, in its early stages, continuous with the auditory nerve. Beyond the auditory vesicle the facial nerve runs downwards and forwards, close to the surface epiblast. Shortly before the time of hatching of the tadpole, the nerve becomes connected with an ingrowth of the epiblast at the level of the upper border of the notochord, and at this place the ganglion is formed. Beyond the ganglion the nerve divides into three principal branches: (i) a small cutaneous branch which appears to develop in connection with the epiblastic ingrowth, and to belong to the lateral line series of nerves; (ii) a stout post-branchial branch, which runs downwards and forwards along the hyoid arch, close to its surface; and (iii) a small palatine nerve, which runs forwards in the roof of the pharynx, not far from the median plane.

VI. The development of the sixth, or abducent nerve, has not been determined in the frog. From its general relations, and from what is known concerning its mode of formation in other animals, it is probably comparable to a ventral root of a spinal nerve.

V. The trigeminal, or fifth cranial nerve, is the largest of the whole series; it lies immediately in front of the facial nerve, with which it is in close relation from the first.

The trigeminal nerve, like the pneumogastric, early attains a large size, and in $4 \mathrm{~mm}$. tadpoles ( $c f$. Figs. 58, $\mathbf{E}$, and 61 ) extends half way down the side of the pharynx. At or shortly before this stage, a thickening of the external epiblast occurs at the level of the upper border of the notochord, immediately behind the eye, and in front of the auditory vesicle; this meets and fuses with the nerve, the two together forming the ganglion. The thickening of the epiblast extends forwards a short distance in front of the ganglion, and gives rise to a cutaneous nerve, similar to the lateral line nerve formed in connection with the pneumogastric nerve. Shortly after hatching of the tadpole, the ganglion of the trigeminal nerve recedes somewhat from the surface, and becomes more deeply placed, though still remaining connected with the surface by the cutaneous branch.

Before the hatching of the tadpole, the trigeminal nerve divides distally into ophthalmic and mandibular branches, of which the former runs horizontally forwards, and the latter downwards and forwards, the eye lying in the fork between the two. 
At the time of opening of the mouth, in tadpoles of about $9 \mathrm{~mm}$. length, the condition of the trigeminal nerve is as follows:-The nerve arises on each side, by a single root, from the side of the medulla oblongata ; and, running downwards and forwards, expands to form the Gasserian ganglion, which lies midway between the eye and ear, and immediately in front of the ganglion of the facial nerve. From the ganglion three branches arise: (i) a small but well-marked cutaneous branch runs directly outwards, behind the eye, to the skin, along which it continues forwards for a short distance. (ii) A large ophthalmic branch runs horizontally forwards between the eye and the brain, parallel to the outer margin of the brain, and dorsal to the optic nerve and to the nose ; in front of the nose it turns slightly downwards, and ends in branches supplying the skin of the snout: the hindmost or proximal part of the ophthalmic nerve is very thick and ganglionic, the distal part is thin. (iii) A very thick mandibular branch, which is also ganglionic at its proximal end, runs downwards and forwards below the eye, close to the ganglion of the facial nerve, but separated from this by the anterior cardinal vein; it runs through the jaw muscles, and ends in the floor of the mouth. From the mandibular branch a slender maxillary branch runs forwards, beneath the eye, and along the upper jaw to the anterior end of the head, where it ends in the skin of the upper lip.

IV. The mode of development of the fourth cranial nerve of the frog has not been determined.

III. The third cranial nerve is also very imperfectly known. Its early development has not yet been ascertained. At the time of opening of the mouth, in tadpoles of about $9 \mathrm{~mm}$. length, it is present as a slender nerve, arising from the lower part of the side of the mid-brain, not far from the median plane, and having already the course and relations of the nerve in the adult.

II. The optic, or second cranial nerve, will be best dealt with in the description of the development of the eye. The nervefibres arise in connection with the retina, and grow inwards along the optic stalk to the brain.

I. The olfactory, or first cranial nerve. The early stages in the development of the olfactory nerve in the frog have not been seen; there are reasons for suspecting that it is developed in part from the epithelium of the olfactory pit itself, and perhaps 
also in part from the anterior end of the neural ridge. 'The nerve develops early, and is recognisable before the hatching of the tadpole as a short thick trunk, connecting the side of the brain with the thickened epithelium of the olfactory pit.

The nerve remains short, up to the time of opening of the mouth, or rather up to the time when the cerebral hemispheres. begin to grow forwards. This anterior growth of the cerebral hemispheres is accompanied, as already. noticed, by still more rapid growth of the anterior part of the head, in consequence of which the olfactory pits are carried forwards from their original position at the sides of the brain, and become situated in front of it. This causes lengthening of the olfactory nerves, and a change in their direction; in place of running outwards from the brain, they now run almost directly forwards : the roots of the nerves, however, still arise from the ventral surface of the brain, some distance from its anterior end, as in the adult.

The sympathetic nervous system. The sympathetic system develops as a series of outgrowths from certain of the cranial, and from all the spinal nerves. These develop ganglionic swellings, which, in the body region (Figs. 84 and $87, \mathrm{NY}$ ), lie beneath the notochord, and alongside the dorsal aorta. At an early stage, shortly after the formation of the mouth, the several ganglia of each side become connected together by a longitudinal nervecord, but whether this cord arises independently of the ganglia, or, as is more probable, by the formation of outgrowths from the ganglia, has not been definitely determined.

\section{DEVELOPMENT OF THE SENSE ORGANS.}

The organs of special sensation, like the nervous system itself, are developed from the deeper or nervous layer of the epiblast, and are continuous with their respective nerves from a very early stage in their formation. The derivation of the sense organs from the epiblast is explained by the fact that they are concerned in the appreciation of the presence and nature of external objects, and are therefore necessarily formed on the surface of the body. They are in all cases to be regarded as specially modified parts of the epidermis. 
1. The Nose.

The olfactory organs appear at a very early period of develop-

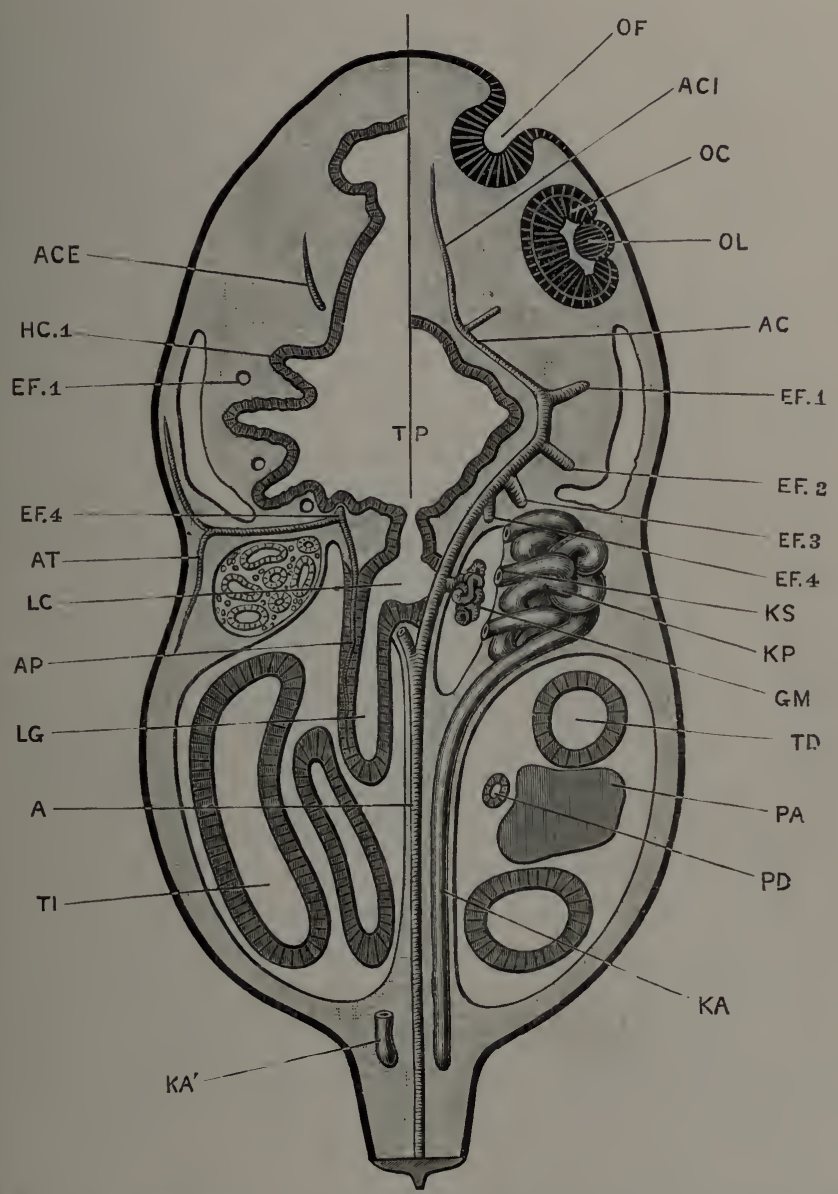

FIG. 66. - Diagrammatic horizontal section of a $12 \mathrm{~mm}$. Tadpole, at the time of appearance of the hind limbs. The plane of section of the right side of the head is taken at a more dorsal level than that of the left side. $\times 30$.

A, aorta. AC, carotid artery. ACE, pharyngeal artery. ACI, anterior cerebral artery. AP, pulmonary artery. AT. cutaueous artery. FF.1, FF.2, EF.3, EF.4, efferent branchial vessels of first, second, third, and fourth branchial arches. GMM, glomerulus. HC.1, first branchial cleft. KA, right segmental duct. KA', hinder end of left segmental duet. KP, head kinney. KS, nephrostome. IC, laryngeal chamber. LG, lung. OC, optic cup. OF, olfactory pit. OL, lens. PA, pancreas. $\mathrm{PD}$, pancreatic duct. TD, duodenum. TI, intestine.

ment, about the time of closure of the brain, as a pair of thickenings of the nervous layer of the epiblast at the anterior end of 
the head, lying at the sides of the fore-brain, and in front of, and slightly dorsal to, the position in which the mouth will afterwards appear.

The two layers of epiblast soon lose their distinctness in these patches; and a pitting in of the surface, involving both layers, appears in each of the patches. The pits so formed become the nasal sacs; the mouths of the pits forming the nostrils or anterior nares, and the epiblastic lining of the pits becoming converted into the olfactory epithelium. The condition of these pits at the time of hatching of the tadpole is shown from the surface in Fig. 72, oc, and in horizontal section in Fig. 74, oF.

The olfactory pits rapidly deepen (Fig. 66, oF), rather by the upgrowth of folds of skin round their margins than by depression of the floors of the pits themselves; the result of this process being the formation of a pair of deep pits, of which the inner walls are derived from the original patches of olfactory epithelium.

A short time after hatching of the tadpole, a solid rod of epithelial cells is formed by proliferation of the cells of the floor of each olfactory pit. These rods of cells grow downwards and inwards towards the roof of the pharynx, meeting and fusing with this immediately behind the septum between the pharynx and the stomatodæum (Fig. 64). Shortly after the mouth opening is established, by perforation of this septum, these rods of cells become tubular; and in tadpoles of $12 \mathrm{~mm}$. length, in which the hind limbs are just appearing, the tubes open into the roof of the mouth as the posterior nares (Fig. 76, ZI).

By further folding of the walls, and by the formation of cæcal outgrowths from each sac, the complicated olfactory labyrinth of the adult is developed. A special diverticulum of the ventral wall of each sac gives rise to the organ of Jacobson.

\section{The Eye.}

The eye differs from the other sense organs inasmuch as an accessory part, the lens, is alone formed from the surface epiblast; while the sensitive part of the eye, or retina, arises as an outgrowth from the brain, and thus is only indirectly derived from the epidermis.

The optic vesicles have already (p. 125) been described as a pair of hollow outgrowths, which arise from the fore-brain about 
the time that closure of the neural tube is effected; they project outwards at right angles to the axis of the head, their outer walls being in close contact with the epidermis of the sides of the head. Each of the vesicles becomes constricted at its base, so as to form a spherical optic bulb, connected with the fore-brain by a hollow tubular stalk. The outer wall of the bulb, which is in contact with the external epidermis, soon becomes flattened, and then thickens so greatly as almost to obliterate the cavity of the vesicle (Fig. 67, oc).

The lens. About this time a thickening of the inner, or nervous, layer of the surface epiblast takes place opposite to the

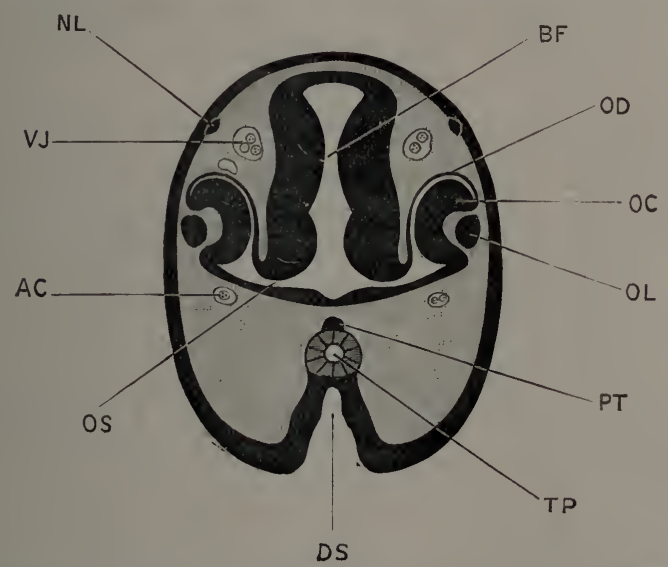

FIG. 67.-Transverse section through the head of a Tadpole of $6 \frac{1}{2} \mathrm{inm}$. length, about the time of hatching: the section passing through the fore-brain and the developing eyes. $\times 45$.

AC, carotid artery. BF, fore-brain. DS, stomatodæal invagination. NL, cutaneous or lateral line branch of the trigeminal nerve. OC, inner wall of optic cup. OD, outer wall of optic cup. OL, lens. OS, optic stalk. PT, pituitary body. TP, pharynx. VJ, jugular vein.

centre of each optic vesicle; this thickening increases rapidly, and at the time of hatching of the tadpole forms a solid spherical body projecting inwards from the surface; this soon becomes hollow, by breaking down of the cells in its centre, and then separates from the surface epiblast. It may now be spoken of as the lens vesicle (Fig. 66, oL) ; in the later stages, after the formation of the mouth opening, the lens vesicle becomes solid once more (Fig. 67, OL), mainly through lengthening of the cells of its inner wall; and by further increase in size it becomes the lens of the adult eye. 
The optic cup. Partly in consequence of the ingrowtl of the lens vesicle, but mainly through active growth of the walls of the optic vesicle itself, this latter becomes pitted on its outer surface, and so converted into a cup (Figs. 66, 67). This optic cup, as it is termed, has double walls: the inner wall (Figs. 66 and $67, \mathrm{oc}$ ) is very thick, and consists of cells arranged three or four deep; the outer wall (Fig. 67, OD) is thin, and consists of a single layer of flattened cells, in which pigment is early developed. (Cf. Fig. 76, oc.)

In the later stages of tadpole life the optic cup slowly enlarges ; it remains in contact with the lens at its edge or lip, but elsewhere is separated from this by a space, which becomes the posterior chamber of the eye, and in which the vitreous body is formed.

The inner, or thicker, wall of the optic cup gives rise to the retina; the molecular and nuclear layers, and the layers of nerve cells and nerve fibres, being formed by modification of the cells of the wall itself; while the rods and cones of the bacillary layer arise as outgrowths from its outer surface, which grow towards, and become imbedded in, processes developed from the pigmented cells of the outer wall (Fig. 67, oD) of the optic cup.

If the mode of development of the brain be called to mind, it will be seen that the layer of epithelial cells which lines the cavity, or ventricle, of the fore-brain (Fig. 67, BF) is morphologically equivalent to the outer or epidermic layer of the surface epiblast, and was originally directly continuous with this, before closure of the neural groove was effected. As the optic vesicle is an outgrowth from the fore-brain, the cells lining its cavity, i.e. the cells lining the space between the inner, oc, and outer, oD, walls of the optic cup, will be of the same nature as those lining the cavity of the fore-brain itself.

The optic nerve. The fibres of the optic nerve are developed on the inner surface of the inner layer of the optic cup, i.e. the surface next to the vitreous body, and grow inwards along the optic stalk to the brain. It follows from what has been said in the previous paragraph that this inner surface of the optic cup is morphologically equivalent to the deeper or nervous layer of the epidermis, from which we have seen that all the other nerves are developed, directly or indirectly.

Up to the time of hatching there is no trace of the optic 
nerve-fibres; but shortly after this period (Fig. 66) certain of the epithelial cells at the inner surface of the optic cup become pyriform in shape, forming what are termed neuroblasts. From the narrower ends of the neuroblasts, nerve-fibres grow out which spread over the ventral edge of the optic cup, and grow back as a bundle of nerve-fibres along the ventral and posterior wall of the optic stalk, and towards the brain. The optic stalk itself apparently takes no direct part in the formation of the nerve-fibres ; its cavity becomes obliterated shortly after the mouth opening is established, except at the end next the brain, where the carity persists, as the optic recess, throughout life. The rest of the stalk gradually becomes broken up, as the distance between the brain and the eye increases with growtl of the tadpole.

The optic fibres reach the under surface of the brain shortly after the mouth opens, and cross over almost at once to the opposite side of the brain to form the optic chiasma.

The onter coats of the eye, choroid, sclerotic, and cornea, are formed from the mesoblast surrounding the optic cup.

The eye develops very slowly, and during the greater part of the tadpole stage of existence is in an imperfect condition ; at the time of the metamorphosis it moves nearer to the surface, and becomes a functionally more perfect organ.

\section{The Ear.}

General account. The ears are developed as a pair of pitlike invaginations of the deeper or nervous layer of the epiblast, at the sides of the hind-brain. The invaginations do not involve the epidermic or outer layer of the epiblast, which is continued across the mouths of the pits. The auditory pits, therefore, do not, in the frog, open at any time to the exterior.

The mouths of the pits very early narrow and close, and the auditory vesicles so formed separate completely from the epiblast, and lie imbedded in the mesoblast at the sides of the head. $\mathrm{By}$ folding of its walls, and by the ingrowth of septa, the vesicle, from being a simple, almost spherical sac, becomes divided up into the complicated auditory vestibule of the adult.

The auditory nerve becomes connected with the inner wall of the vesicle at a very early stage, indeed almost from its first appearance ; the relations of the nerve to the wall of the vesicle being essentially similar to those between the other cranial 
nerves and the special patches of epiblast with which they become fused.

Certain of the accessory organs of hearing, especially the Eustachian tube and the tympanic cavity, may conveniently be described here, although they are essentially independent of the auditory apparatus, and only become secondarily connected with this.

The early development of the ear. About the time of closure of the neural groove, the auditory epithelium can be recognised as a pair of thickened circular patches of the deeper layer of epiblast, one at each side of the hind-brain, with which patches the auditory nerves are already continuous.

Soon after closure of the neural tube, in embryos of about $3 \mathrm{~mm}$. length (Fig. 60), each of these patches becomes depressed, forming a shallow pit, semicircular in transverse section, and covered at its mouth by the outer layer of epiblast, which is continued over it without interruption. The pit deepens, and the mouth gradually closes by ingrowth of its lips. Shortly before the hatching of the tadpole the closure is completed, and the auditory vesicle separates from the surface epiblast.

At the time of its separation the vesicle is a closed sac, somewhat pyriform in shape; its lower or ventral portion being spherical, and lying opposite the notochord, and its dorsal wall being prolonged upwards into a short blind diverticulum lying at the side of the hind-brain. The wall of the vesicle consists of a single layer of cubical or columnar cells; those of the inner wall, with which the auditory nerve is continuous, being rather more elongated and more deeply pigmented than the rest.

The internal ear or labyrinth. After closure of its mouth the vesicle increases considerably in size, and becomes further separated from the surface by ingrowth of mesoblast between its outer wall and the external epiblast. Up to the time of the formation of the mouth it undergoes no further change of importance, remaining as a spherical sac with a blind dorsal diverticulum.

Shortly after the opening of the mouth, i.e. in tadpoles of from 10 to $12 \mathrm{~mm}$. in length, the various parts of the internal ear become gradually differentiated, the chief process by which the changes are brought about being the formation of septa, by folding of the wall of the vesicle, which project inwards into the cavity and partially subdivide it. Mesoblast soon grows in 
between the two layers of each fold, the septa thereby acquiring increased thickness.

The first septum which appears divides the vesicle into its two main cavities, sacculus and utriculus. It arises in tadpoles of about $11 \mathrm{~mm}$. length as a fold of the outer wall of the vesicle, which projects somewhat obliquely across the cavity, dividing it into an upper and inner division, the utriculus; and a lower and outer portion, the sacculus. The septum is at first confined to the hinder part of the vesicle, but soon extends all round it; and, growing inwards, separates the two divisions almost completely from each other, a very small aperture of communication alone persisting between them.

From the utriculus, the semicircular canals are formed. Each canal is really a portion of the utriculus, which becomes partially shut off from the main cavity by the formation of a septum along the middle portion of its length; remaining, however, in communication with the cavity at each end. Each septum is formed by two separate folds, which grow towards each other from opposite sides of the vesicle, meet along their edges, and fuse to complete the septum (Fig. 75, p. 162). The septum soon thickens, through the ingrowth of mesoblast between its layers; it also elongates, and so causes lengthening of the canal, which gradually acquires the adult shape and relations.

Of the three semicircular canals, the anterior vertical and the horizontal are formed simultaneously, and first appear in tadpoles of about $11 \mathrm{~mm}$. length. The posterior vertical canal arises in the same way, but at a slightly later stage, in tadpoles of about $15 \mathrm{~mm}$. length.

The ampullø of the semicircular canals are formed later than the canals themselves, not as dilatations of the canals, but by constriction of parts of the utriculus, at the places where the canals open into it.

The second division of the vesicle, or sacculus, grows downwards, and soon acquires the pouch-like character it has in the adult. From its upper and hinder portions three small bulgings or pouch-like outgrowths appear, which together form the cochlea. Of these, the lagena cochler is the largest and the earliest to appear, arising in tadpoles of about $15 \mathrm{~mm}$. length; the pars neglecta appears shortly afterwards, and the pars basilaris last of all. 
The inner wall of the auditory vesicle, facing the brain, is from the first composed of cells which are more columnar in shape than those of the rest of the vesicle (Fig. 75, Ev); and it is with these elongated cells that the auditory nerve is connected. As the vesicle grows, and as the septa form, by which it is divided up into its various portions, the patch of epithelium with which the nerve is continuous also divides, giving rise to all the sensory patches present in the adult ear. Of these there are eight:- one in each of the three ampulla of the semicircular canals, three in the cochlea, one in the wall of the sacculus, and one in that of the utriculus.

The dorsally directed diverticulum, to which the pyriform shape of the vesicle in its early stages is due, persists in the adult, and undergoes a rather remarkable development.

On the formation of the septum, dividing the vesicle into sacculus and utriculus, the diverticulum remains in connection with the inner side of the sacculus. It elongates considerably, growing upwards close alongside the brain as the recessus vestibuli (Fig. 75, ER). In tadpoles of about $20 \mathrm{~mm}$. length, the distal blind end of the recessus vestibuli dilates to form i thin-walled vesicle, lying on the roof of the fourth ventricle; while the rest of its length forms a narrow tubular duct with rather thick walls, which connects the dilated end with the sacculus.

At the time of the metamorphosis the distal thin-walled dilatation, or saccus endolymphaticus, has increased greatly; it lies within the skull, between this and the brain, as a large sac with thin but very vascular walls, covering the roof of the hind-brain for a considerable length, and extending downwards along the sides of the brain and beneath its floor as well. The sacs of the two sides meet, both above and below the brain, and apparently open into each other; in their cavities abundant calcareous concretions are found.

The stalk, or ductus endolymphaticus, persists as a narrow tube, which passes through a hole in the skull wall, and connects the saccus endolymphaticus with the sacculus of the internal ear. These relations of the saccus and ductus endolymphaticus are retained in the adult frog.

In the mesoblast surrounding the internal ear the perilymph spaces are formed; and beyond these the cartilaginous and 
o-seous walls of the auditory capsule are laid down. ( $C f$. Figs. 75 and 68.)

The accessory auditory apparatus. It will be convenient to consider here the development of the Eustachian tube, and the tympanic cavity and membrane, which, though only secondary parts of the organ of hearing, are exceedingly characteristic of terrestrial Vertebrates, as contrasted with the truly aquatic Vertebrates, or Fishes.

The Eustachian tube and tympanic cavity. The details of development of these parts are not thoroughly determined.

The Eustachian tube appears first in tadpoles of about $25 \mathrm{~mm}$. length, as a solid rod of epithelial cells, rumning forwards from the anterior and dorsal edge of the first branchial cleft.

At the time of the metamorphosis, when the fore legs are protruded, the Eustachian tube is a rod of cells with a very illdefined lumen, starting from the dorsal and anterior part of the pharynx, and extending straight forwards beneath the eye; it is slightly dilated at its distal end, which lies opposite the anterior border of the eye.

During the metamorphosis, the Eustachian tube separates from the pharynx, and divides into a variable number of short lengths; these gradually shift backwards to the position occupied by the Eustachian tube in the adult frog; by the time the tail of the tadpole is completely absorbed, the several lengths unite together, and with a diverticulum from the pharynx, to form the definite Eustachian tube of the adult, which now runs almost directly outwards beneath the ear. The tympanic cavity is merely the dilated outer end of this tube, lying just beneath the surface; and the layer of skin closing its outer end is the tympanic membrane. ( $C f$. Fig. 68, E, D.)

From this account it appears that the tympanic cavity does not at any period open on the surface of the head; and it is loubtful whether the Eustachian tube in the frog has any definite relation to a gill-cleft. It is very probable that in this, as in many other features of its embryological history, the frog shows a modified rather than a primitive type of development.

The tympanic cartilage. In tadpoles of about $40 \mathrm{~mm}$. length, shortly before the fore leg's emerge, the tympanic cartilage appears as a dense mass of cells, surrounding the anterior 
end of the Eustachian tube at a time when this lies below the eye. During the metamorphosis, this ring of cells preserves its relation with the outer end of the Eustachian tube, or tympanic

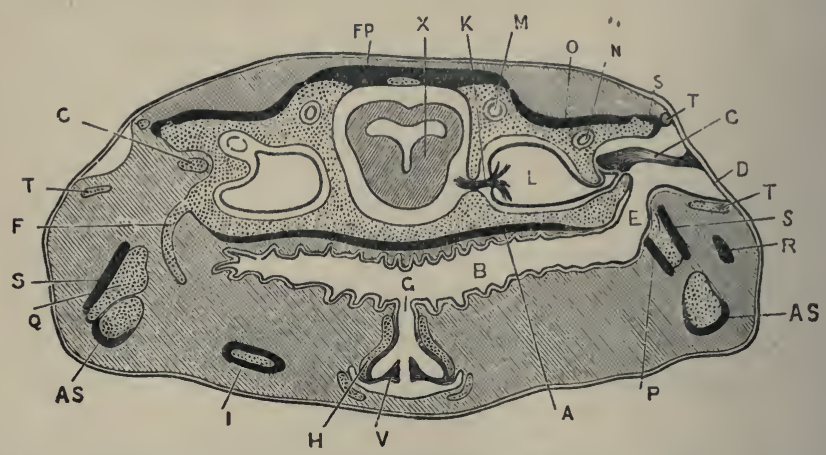

FIG. 68.-A transverse section across the posterior part of the head of an adult Frog, showing the position and relations of the auditory organs, Eustachian tube, and hyoid apparatus. On the right side the section passes through the tympanic cavity and the columella; on the left side through the anterior cornu of the hyoid. The cartilage is dotter, and the bones, except the columella, represented black.

A, parasphenoid. AS, angulosplenial. B, buccal cavity. C, columella. D. tympanic membrane. $\mathbf{E}$, Eustachian tube. $\dot{F}$, anterior cornu of the hyoid. F'P, frontoparietal. G, glottis. H, arytenoid cartilage. I, posterior cornu of the hyoid. K. auditory nerve. $L$, vestibule of the ear. $M$, anterior vertical semicircular canal. $\mathbf{N}$, horizontal semicircular canal. O. pro-otic. $\mathbf{P}$, pterygoid, $\mathbf{Q}$, quadrate cartilage.

$\mathbf{R}$, quadratojugal. $\mathbf{S}$, squamosal. $\mathbf{T}$, tympanic cartilage. $\mathbf{V}$, vocal cord. $\mathbf{X}$, mid-brain.

cavity, and gradually shifts back with this latter to its adult position. A bar of cartilage appears in its ventral portion, which gradually extends at its ends until it forms the complete annular tympanic cartilage.

The development of the auditory ossicle, or columella (Fig. $68, \mathrm{c})$, will be described in the section dealing with the development of the skull (p. 209).

\section{The Cutaneous Sense Organs.}

During the tadpole stage, while the animal is leading an aquatic life, special sense organs in the form of small epidermal papillæ are present, arranged in rows along the body, round the eyes, and on other parts of the head. They are supplied by the lateral line series of branches of the trigeminal and pneumogastric nerves, which have already been described (pp. 130, 132); they are lost completely at, or shortly after, the time of the metamorphosis.

The mouth of the tadpole is also provided with special 
papillæ, probably gustatory in function, which are lost at the time of the transformation to the frog.

\section{DEVELOPMENT OF THE ALIMENTARY CANAL.}

\section{General Account.}

The alimentary canal of the frog, like that of other Vertebrates, is developed in three lengths: (i) the mesenteron (Fig. 69 , T), which is formed, as already described, by a process of splitting amongst the yolk-cells, and which corresponds to the mesenteron or gastrula cavity of Amphioxus: the mesenteron of the frog gives rise to almost the whole length of the alimentary canal, from the pharynx to the rectum; and from it are developed the gill-clefts, the thyroid, the thymus, the lungs, the liver, the pancreas, and the bladder. (ii) The stomatodæum (Fig. 69, Ds) is a pitting in at the anterior end of the body, from which the mouth opening and buccal cavity are formed, and in connection with which the lips and teeth are developed. (iii) The proctodæum (Fig. 60, PD) is a pocket-like depression at the hinder end of the body, which gives rise to the anal or cloacal opening.

The mesenteron. The mode of development of the mesenteron, up to the stage shown in Fig. 55, has already been described. At its first appearance, and throughout the early stages, the mesenteron has walls of very unequal thickness; the roof or dorsal wall (Fig. 56) being thin ; and the floor or ventral wall being of great thickness, owing to the large size of the yolkcells which form it.

After separation of the mesoblast cells as a distinct layer, and the definite formation of the notochord, this difference becomes still more marked, the roof of the mesenteron (Fig. $56, \mathrm{~T}$ ) consisting of a single layer of hypoblast cells, while the floor is formed by the thick mass of yolk-cells; at the sides the transition from the thin roof to the thick floor is a somewhat abrupt one.

As the central nervous system is formed, and the shape of the embiryo becomes more clearly established, the mesenteron acquires more definite character's ( $c f$. Figs. 55 , т; 60, мN). Вy enlargement of its anterior end a wide pharyngeal cavity 
(Fig. 61, TP) is formed, of which the floor and sides, as well as the roof, are formed of a single layer of hypoblast cells. The hinder or intestinal region of the mesenteron (Fig. 61, TI), has much the same relations as before, its roof being thin, but its

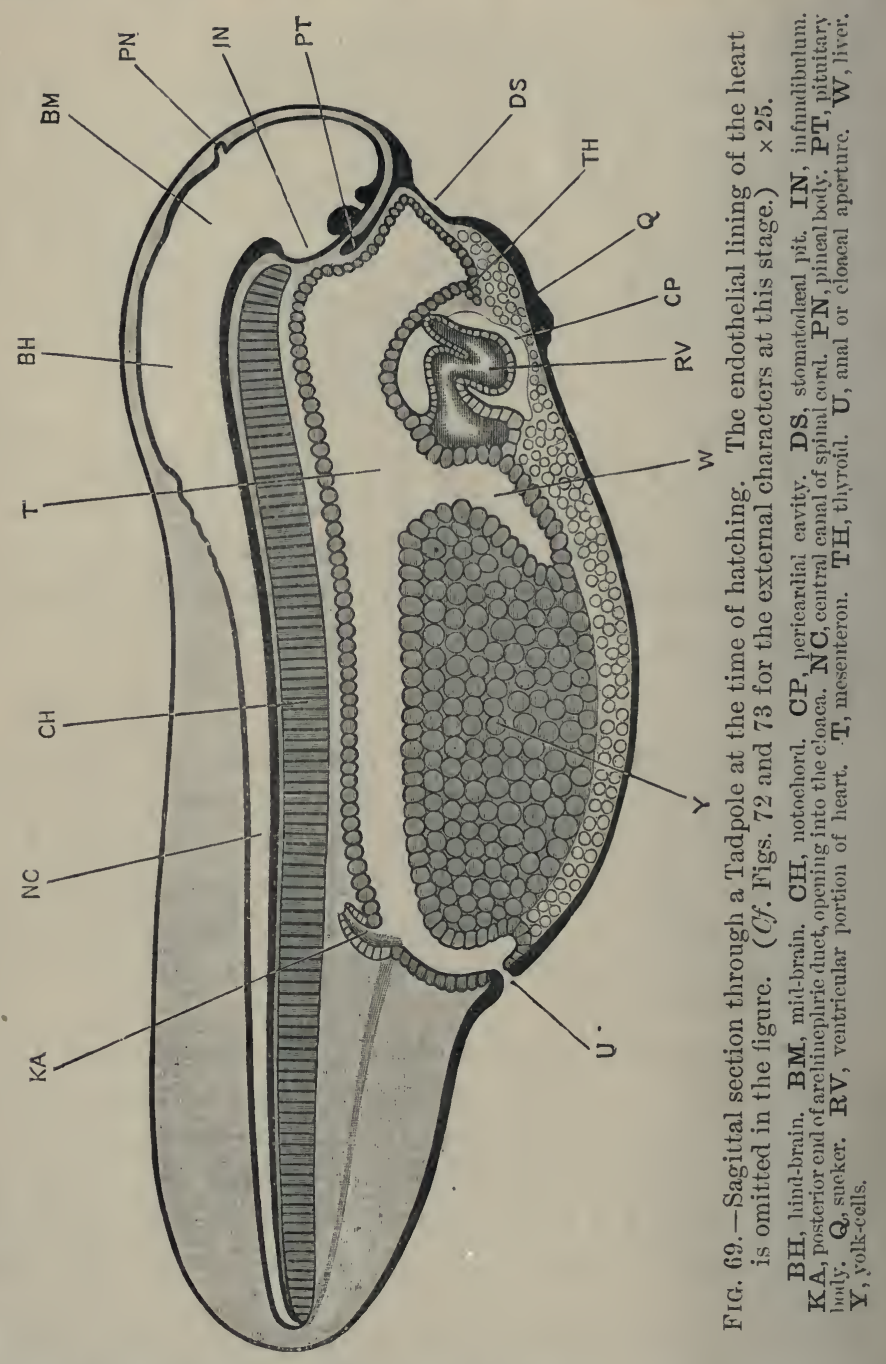

floor and sides (Fig. 70) of great thickness. The mass of yolkcells, forming the floor of the intestinal region, becomes more compactly arranged and more definitely restricted ; in front it is 
sharply marked off from the pharyngeal region by a backwardly directed diverticulum (Fig. 60, L), which forms the first commencement of the liver; while at the hinder end of the body, by withdrawal of the yolk-plug from the surface of the embryo ( $c f$. Figs. 55 and 60 ), the posterior limit of the yolk-mass becomes clearly defined, and the short rectal diverticulum (Fig. 60, R) opened out.

At the time of hatching of the tadpole (Figs. 69 and 74), this distinction between a wide, thin-walled pharyngeal region,

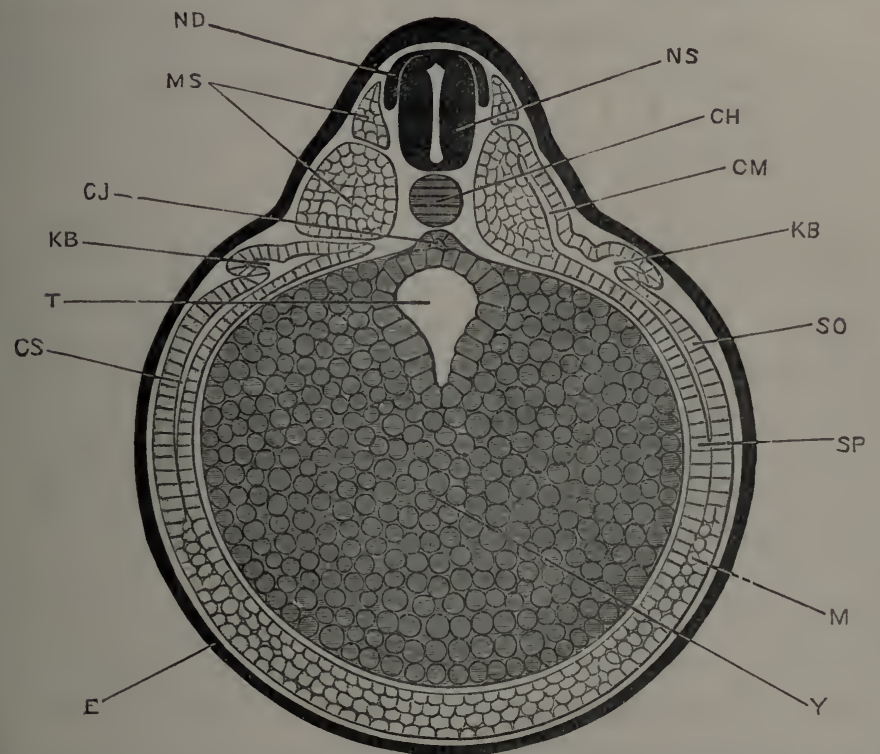

FIG. 70.-Transverse section across the middle of the length of a Frog embryo $3 \frac{1}{2} \mathrm{~mm}$. in length. (Cf. Figs. $58, \mathrm{D}$, and 60 for other views of embryos of the same age.) $\times 52$.

CH, notochord. CJ, subnotochordal rod. CMI, myocœl. CS, splanchnocœl. E, epiblast. KB, arclinepliric duct. $\mathbf{M}$, mesoblast. IMS, mesoblastic somite. ND, dorsal root of spinal nerve. NS, spinal cort. SO, somatopleurie layer of mesoblast. SP, splanchnopleuric layer of mesoblast. $T$, intestinal region of mesenteron. $Y$, yolkcells.

and a narrow, thick-walled intestinal portion is very well marked, the passage from one region to the other (Fig. 74) being an abrupt one. Up to this time the alimentary canal has been perfectly straight, but shortly after hatching, and especially after the formation of the mouth, the intestinal region elongates very rapidly; the food-yolk is speedily absorbed, and the intestine becomes a long tube, coiled in a characteristic spiral manner, and 
of approximately uniform diameter along its whole length (Fig. 65). Owing to this rapid elongation, and the convolutions into which it necessarily becomes thrown, the intestine, which at first is closely attached to the dorsal wall of the body cavity, immediately beneath the notochord, shifts ventralwards, remaining, however, suspended from the mid-dorsal wall of the body cavity by the mesentery.

At the time of the metamorphosis the alimentary canal shortens rapidly and very considerably; and the distinction in diameter between the stomach, small intestine, and large intestine becomes much more pronounced. During these changes the entire alimentary canal is in a condition of active inflammation, and no food is taken, nutrition being effected by the gradual absorption of the tadpole's tail.

The stomatodæum. At the time of hatching (Fig. 69, Ds), the stomatodæum is a well marked though shallow pit on the under surface of the head; its floor is in close relation with the anterior wall of the pharynx, the epiblast of the stomatodxal pit and the hypoblast of the pharyngeal wall being in contact with each other, without any intervening mesoblast. From the dorsal border of the stomatodæum, the pituitary body (Fig. 69, PT) projects inwards between the brain and the pharynx.

The stomatodæal pit rapidly deepens, not by depression of its floor, but by uprising of its walls (Fig. 64), the margins of which give rise to the lips. The septum between the stomatodæum and the pharynx gradually becomes thinner, and in tadpoles of from 9 to $10 \mathrm{~mm}$. length is perforated; the mouth opening is thus established, and the pharynx placed in direct communication with the exterior.

In the later stages the limits of the original stomatodæal invagination can be fairly accurately determined. In the section of a $12 \mathrm{~mm}$. tadpole given in Fig. 65 the boundary is indicated by a difference in the mode of shading employed; the epiblastic lining of the stomatodæum is represented by a thick black line, while the hypoblastic wall of the pharynx is shown by a double, cross-hatched line. The posterior nares mark the boundary between the two regions exactly; they open ( $c f$. Fig. 76, ZI) into the pharynx immediately behind the septum, so that a line drawn across the roof of the mouth, through the anterior borders 
of the narial openings, divides the stomatodral from the pharyngeal portion.

After the mouth opening is established, the lips of the stomatodæum grow forwards rapidly, and in connection with them the powerful horny jaws of the tadpole, by which it crops its food, are speedily developed (Fig. 65, J).

The proctodæum. The mesenteron, from its first appearance, and throughout the early stages of development, communicates with the exterior through the blastopore (Fig. 60, B). It also communicates, through the neurenteric canal, NC, with the central canal of the spinal cord and brain; this communication persists for some time after the blastopore has closed (Fig. 61), but is lost when the tail begins to lengthen (Fig. 69).

The proctodeal invagination appears as a pit-like depression at the ventral end of the primitive streak (Figs. 58, B, C, D, and $60, \mathrm{PD}$ ). In embryos of about $4 \mathrm{~mm}$. length (Fig. 61), this invagination reaches and opens into the rectal portion of the mesenteron, i.e. the portion which lies posterior to the mass of yolk-cells.

The closure of the blastopore usually occurs before the anal perforation is completed; but it may happen that the two openings into the mesenteron are present for a time simultaneously.

In the frog this proctodreal invagination is a new opening into the mesenteron, and is not a persistent part of the original communication of the mesenteron with the exterior, through the blastopore. If it be borne in mind, however, that the proctodixal invagination appears in the primitive streak, and as an actual deepening of the ventral end of the primitive groove (Fig. 58, B); and further, that the primitive streak is formed by concrescence of the lips of the blastopore, then the formation of the proctodæal invagination may be viewed, not as an entirely independent depression of the surface, but as a re-opening of the ventral portion of the blastopore. This view is strongly supported by the development of other Amphibians, in some of which the blastopore actually persists as the anus.

It will be noticed that the proctodæal, or anal, opening is established some time before the embryo hatches, while the stomatodæal or mouth opening is not formed until a consider- 
ably later period. 'This early appearance of the proctodxal opening is perhaps to be associated with the early formation of the kidneys, which are already present, and have ducts opening into the hinder end of the mesenteron (Figs. 69, KA, and 74, KP, $\mathrm{KA}$ ), shortly before the time of hatching of the tadpole.

The development of the several regions of the alimentary canal, and the structures arising in connection with them, will now be described in more detail, with the exception of the gillclefts and gills, which form the subject of the next section of this chapter.

\section{The Lips.}

The mouth of the tadpole is very small compared with that of the frog ( $c f$. Figs. 85 and 86, p. 193). It is surrounded by prominent frill-like lips, which form a short conical proboscis (Figs. 83 and 85, LI, LJ). The inner surfaces of the lips bear rows of minute teeth, and at the bottom of the funnel, separating the proboscis, or labial cavity, from the buccal cavity, is the beak, formed by the two powerful horny jaws (Fig. 6.5, J).

There are two lips, upper and lower, which are continuous with each other at the angles of the mouth, so as to completely surround the opening. The upper lip (Figs. 65 and 83, LI) is a crescentic fold of integument bounding the labial cavity in front; it is smaller and less mobile than the lower lip, and bears along its free edge a row of minute horny teeth. 'The lower lip (Figs. 65 and 83, LJ) is both longer and deeper than the upper; it is also softer and much more mobile. It is separated behind by a well-marked transverse groove from the under surface of the head, and is produced at its free edge into a series of small fleshy papillæ. These papillæ, which are probably tactile in function, are more numerous at the angles of the mouth, where they are arranged in groups.

The inner surfaces of the lips, between their free edges and the beak, bear transverse ridges or folds, which support along their crests comb-like rows of minute black horny teeth. Of these rows, the upper lip, in addition to the row round the margin already mentioned, has three incomplete rows, interrupted in the middle by a considerable interval. The lower lip bears four similar but complete rows of teeth.

Each of these teeth is formed by modification of a single 
epithelial cell. In shape it is a hollow cone, produced at its apex into a spoon-shaped process, notched at its free edge. These horny epithelial teeth are easily rubbed off during use, and are speedily replaced by other similar ones formed beneath them. Each tooth is in fact the top member of a column of specially modified epithelial cells, imbedded in the general epithelium of the lip. In each column the deepest cells are ordinary epithelial cells, scarcely distinguishable from those in which they are imbedded: the succeeding cells of the column, nearer the surface, become first flattened, then cup-shaped, and finally conical, the apex of the cone fitting into the cavity of the cell next above it.

The deeper cells of the column are soft, and have distinct nuclei; nearer the surface the cells have their outer layers converted into horny matter, while their shape gradually approaches that of the fully formed teeth. The nucleus becomes less distinct, and finally disappears, as the cornification extends deeper and deeper into the substance of the cell.

Each tooth is thus formed by cornification of a single epithelial cell, which commences its career in the deeper layer of the epidermis, at the base of the column, and gradually approaches the surface through loss of the teeth above it, acquiring, as it does so, the characters of the fully formed tooth. On reaching the surface it comes into functional use for a time, and then in its turn becomes rubbed off and lost.

\section{The Beak.}

The beak consists of the two jaws, upper and lower, and is in shape not unlike that of a bird or turtle (Figs. 65 and 71). Each jaw is a strong, curved band of cornified epithelium, supported at its base by the labial cartilages (Fig. 90, LU, LL), and ending at its free surface in a sharp biting edge. The upper or maxillary jaw (Fig. 65) is longer and less sharply curved; the lower or mandibular jaw, which bites behind the upper jaw, is shorter, stronger, and almost horse-shoe shaped in outline.

The minute structure of the two jaws is the same, each consisting of modified epithelial cells. The cutting edge of the jaw is formed by a row of horny teeth, very similar to those of the labial rows, but placed so closely side by side as to form a contintous blade. Each of these teeth is, as in the case of the labial 
teeth, the uppermost of a column of cells, the more deeply placed members of which are indifferent epithelial cells, but which as they approach the surface become first flattened, then cupped, and finally hollow cones fitting into one another. As in the labial teeth, the hardness is due to cornification of the cells, invading first the outer surface and ultimately the entire cell. As the biting edge of the jaw gets worn away by use, it is constantly renewed by the more deeply placed cells.

The rest of the jaw consists of a dense mass of flattened and cornified epithelial cells, which become firmly fused together, and which, like the cells of the cutting edge, are renewed from the indifferent epithelial cells of the deeper layers. Into this deeper layer vascular papillæ of the dermis project, increasing the extent of the nutritive surface of the jaw.

At the metamorphosis the horny jaws are cast off, and lost.

\section{The Pharynx.}

The characteristic feature of the pharynx, both in the tadpole and in the adult frog, is its great width from side to side ( $c f$. Figs. 74 and 68); and this is acquired, as already described, at a very early developmental stage. In horizontal section the pharynx of the tadpole is somewhat lozenge-shaped (Fig. 74), narrowing rather gradually in front to open into the buccal cavity, and much more abruptly behind, where it passes back into the œsophagus.

The roof of the pharynx may be divided into two regions: an anterior part, clothed by a flattened pavement epithelium, and bearing taste bulbs and sensory papillæ; and a posterior part, covered by a ciliated epithelium, and containing numerous multicellular glands.

The gills, which are the most important structures in connection with the sides of the pharynx, will be described in the next section (pp. 157 to 163 ).

The tongue is formed on the floor of the pharynx, but does not appear until shortly before the metamorphosis; it then grows rapidly and soon attains its adult shape and proportions (Fig. 89, TN).

\section{The Thyroid Body.}

About the time of hatching of the tadpole, or a little earlier, 
a short median longitudinal groove appears along the floor of the pharynx (Fig. 69, тн). The groove is shallow anteriorly, but deepens at its hinder end, where it leads into a small, conical, pitlike depression of the hypoblast forming the pharyngeal floor, just in front of the pericardial cavity (Fig. 69, CP.)

At a later stage, shortly before the opening of the mouth, the median groove is still present. The pit at its hinder end has deepened slightly, and the hypoblast cells, forming the floor of the pit, have grown back as a solid rod of cells (Fig. 64, тH), closely connected at its hinder end with the anterior wall of the pericardium ; this solid rod of cells becomes the thyroid body.

Soon after the mouth opens, the thyroid body separates completely from the floor of the pharynx, remaining as a solid rounded mass of pigmented cells, in close contact with the anterior wall of the pericardium. A little later, in tadpoles of about $12 \mathrm{~mm}$. length (Fig. 65, тH), the thyroid body becomes divided into right and left halves by the growth downwards of a median keel from the basihyal cartilage (Fig. 65, HB). The two halves remain connected by a narrow bridge of cells below the cartilage for a short time, but soon separate and become the paired thyroid bodies of the adult frog.

After their separation the thyroid bodies increase considerably in size ; they are at first solid, but the component cells soon become arranged in strings, which become hollowed out along their axes, and so form a series of rounded or oval vesicles, which communicate freely with one another, and are filled with fluid.

The thyroid bodies are very vascular; they lie in the floor of the mouth, a short way in front of the glottis, immediately to the inner sides of the lingual arteries, which supply them, and along the course of the lingual veins.

\section{The Esophagus.}

The osophagus is formed from the most anterior part of the narrow or intestinal region of the mesenteron, and leads directly from the pharynx. It is at first tubular, but in tadpoles of about $8 \mathrm{~mm}$. length, shortly before the opening of the mouth, the cavity of the œsophagus becomes completely blocked up, by proliferation of the cells forming its walls (Fig. 64, тo). This solid portion of the œsophagus lies immediately behind the pharynx, and has a length of about $0.15 \mathrm{~mm}$. The solid con- 
dition lasts for a little time after the opening of the mouth; and then, in tadpoles of about $10 \frac{1}{2} \mathrm{~mm}$. length, the lumen is gradually re-established, though it is for a time exceedingly narrow.

This blocking up of the œsophagus, which prevents any food getting into the digestive part of the alimentary canal until some little time after the mouth opening is established, is a curious developmental feature; it occurs also in the chick and in many other Vertebrates, but its meaning has not yet been explained satisfactorily.

\section{The Lungs.}

The lungs arise as a pair of pouch-like diverticula of the side walls of the œsophagus, shortly before the hatching of the tadpole; they are at first exceedingly small, and have strongly pigmented walls. After hatching, the lungs increase slowly in size, growing backwards along the sides of the œsophagus; in $9 \mathrm{~mm}$. tadpoles, at the time when the osophagus is solid, the lungs are present as a pair of lateral outgrowths immediately behind the œsophageal plug (Fig. 64, To), but sometimes arising from the solid part itself. After the re-opening of the œsophagus, the part of the ventral wall from which the lung sacs arise becomes depressed to form the laryngeal chamber: the mouth of the depressed portion narrows to form the glottis, and the lungs themselves rapidly increase in size.

In $12 \mathrm{~mm}$. tadpoles, in which the hind limbs are just appearing (Figs. 65 and 75), the glottis is a narrow slit-like opening, guarded in front by a well-developed epiglottis, and leading into a large laryngeal chamber (Fig. $65, \mathrm{LC}$ ), from which the two lungs arise; these latter are thin-walled vascular sacs (Fig. 76, LG), which now reach to the hinder end of the body cavity, lying along the sides of the alimentary canal.

From their mode of development as outgrowths of the œsophagus, it follows that the lungs are lined by an epithelium which is of hypoblastic origin ; the connective tissue and vascular elements of the lung wall are, like those of other parts of the body, mesoblastic.

\section{The Liver.}

About the time of first appearance of the nervous system, the yolk-mass becomes marked off in front by a deep, backwardly projecting depression (Fig. 60, L), from the thin-walled anterior 
region of the mesenteron. This depression becomes still more marked in the later stages (Fig. 69, w); and from its anterior wall the liver is developed.

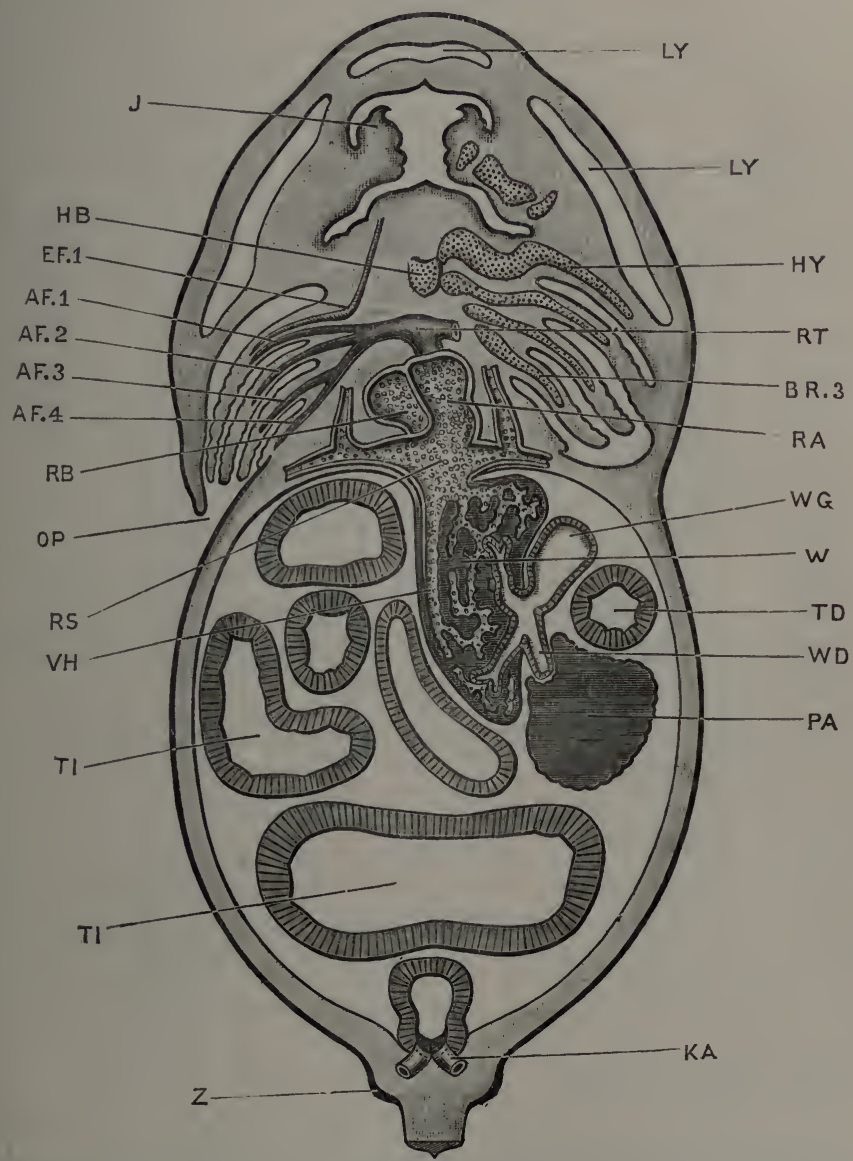

FIG. 71.-Horizontal section of the heat and body of a $12 \mathrm{~mm}$. Tadpole; drawn from the dorsal surface. $\times 27$.

AF.1, 2, 3, 4, afferent branchial vessels of first, second, thirr, and fourth bramclial arches. BR.3, cartilaginous bar of third branchial arch. EF.1, efferent branchial vessel of first branclial arch. HB, basilyyal cartilage. HY, cartilaginous bar of hyoid arch. J, jaw. KA, posterior end of archincphric duct, opening into cloaca. TY, subcutaneous lymphatic spaces. OP, aperture leading from opercular chamber. $\overrightarrow{R A}$, right auricle of heart. RB, left auricle. RS, sinus venosus. $R T$, trunens arteriosus. TD, duodenum. TI, intestine. VH, nosterior vena cava. W, liver. WD, bile duct. WG, gall bladder. Z, commencing lind limb.

This anterior wall becomes early invested by mesoblast on its outer surface, and in this mesoblast numerous blood-vessels 
of large size are developed. The wall now becomes thrown into folds (Fig. 64, w); the blood-vessels following in between the folds. By a continuation of this process, accompanied by the formation of outgrowths from the hypoblast cells, and ingrowth of the blood-vessels, the liver rapidly increases in size and acquires the structure shown in Fig. 71, w; consisting of a trabecular framework of solid rods of hypoblast cells, the meshes of the framework being occupied by the hepatic blood-vessels. As the liver attains definite shape and increased size, it separates more distinctly from the intestine, remaining, however, connected with this by the bile-duct, which is formed by lengthening out of the original diverticulum from the mesenteron. The gallbladder is a lateral outgrowth from the bile-duct; it develops at an early period (Fig. 64, WG), and is of large size during the whole of tadpole life (Fig. 71, wG).

\section{The Pancreas.}

The pancreas develops as a pair of hollow outgrowths from the mesenteron, behind the liver. In the later stages (Fig. 71, PA), the ducts shift so as to open into the bile-duct instead of, as at first, directly into the intestine.

The secreting cells of the pancreas, like those of the liver, are of hypoblastic origin.

\section{The Bladder.}

The bladder is absent during the greater part of the tadpole period; but shortly before the metamorphosis it arises as a median ventral outgrowth from the hinder end of the mesenteron, which soon becomes bifid distally (Fig. 89, тв).

\section{The Post-anal Gut.}

Post-anal gut is the name given to an extension of the hinder end of the mesenteron into the base of the tail, which appears as this latter is developed.

The mode of formation of the neurenteric canal as a tubular communication between the hinder end of the neural canal and the mesenteron has already been described ( $c f$. Fig. 61, NT). As the tail lengthens, the notochord and spinal cord grow backwards with it, and the neurenteric canal becomes drawn out into the post-anal gut. This is an evanescent structure, disappearing completely at a very early stage: at the time of hatching of 
the tadpole (Fig. 69), the only trace of the post-anal gut is a solid cord of cells, running in a slightly irregular course beneath the notochord, from the hinder end of the spinal cord to the mesenteron.

\section{DEVELOPMENT OF THE GILL-CLEFTS AND THE GILLS.}

The gills and gill-clefts, which form the main respiratory apparatus of the tadpole, are developed in connection with the side walls of the pharynx. The gill-clefts are a series of slit-like

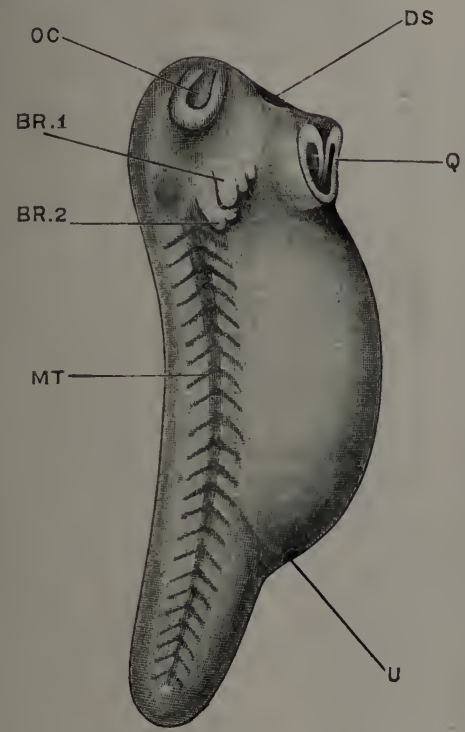

FIG. 72.

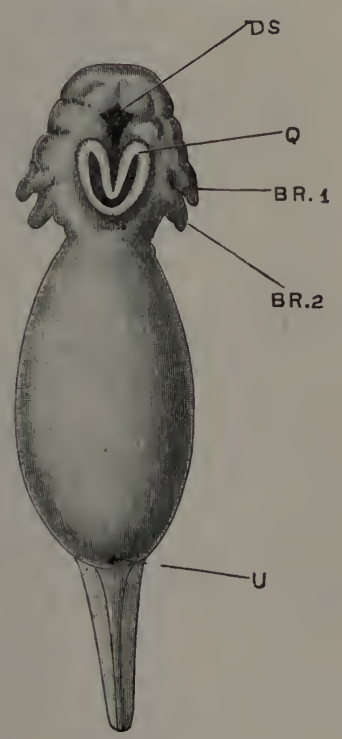

FIG. 73.

FIG. 72.-Side view of a Tadpole at the time of hatching. $\quad \times 16$.

FIG. 73.-Ventral view of the same Tadpole.

BR.1, external gill of first branchial arch. BR.2, exterual gill of second brnuchial arch. DS, stomatodeal pit. MT, mesoblastic somites seen through the skin. OC, olfactory pit. $\mathbf{Q}$, sucker. U, proctodæal or cloacal aperture.

perforations in these walls, leading from the pharynx to the exterior ; while the gills themselves are vascular tufts developed on the gill-arches, i.e. on the parts of the pharyngeal wall between the successive gill-clefts.

\section{The Gill-clefts.}

The gill-clefts are formed as vertical, pouch-like foldings of 
the side walls of the pharynx (Fig. 74, HM, HC), which grow outwards towards the exterior. They appear first at a very early stage, while the blastopore is still open (Fig. 60), and even

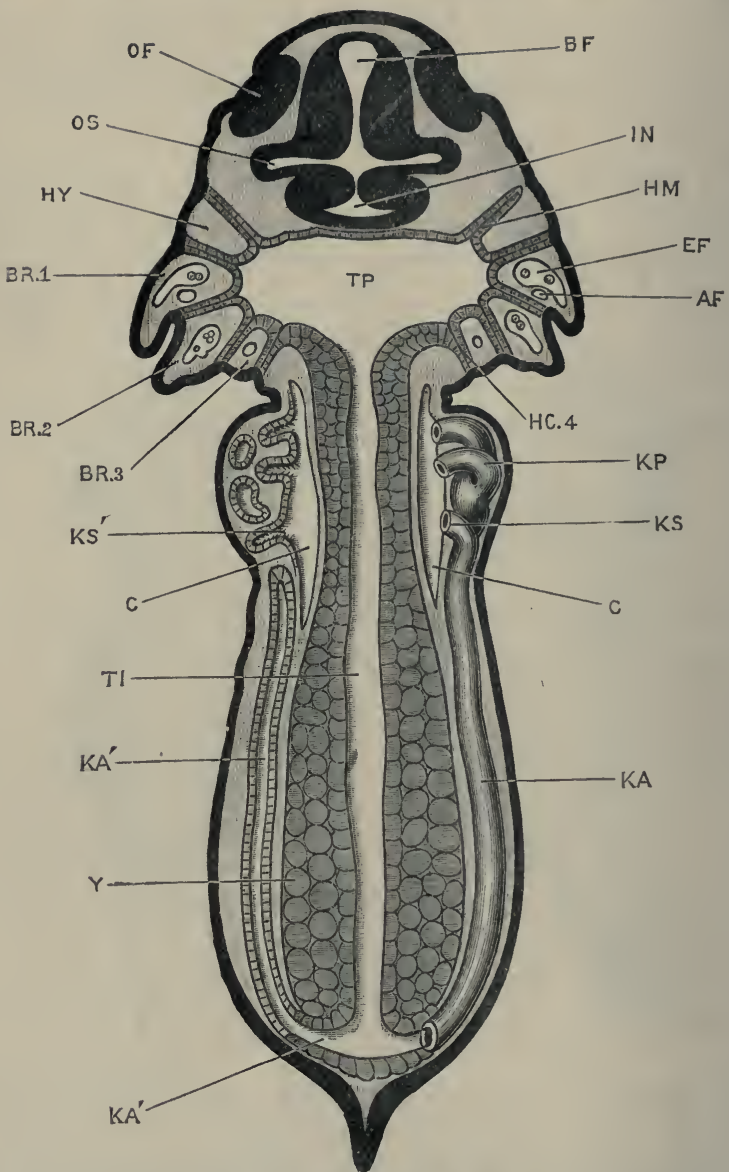

FIG. 74.-Horizontal section of a Tadpole at the time of hatching. $\quad \times 40$.

AF, afferent branchial vessel of first branchial arch. BF', fore-brain. BR.1, first branchial arcl. BR.2, second branchial arch. BR.3, third branchial arch. C, body cavity or cœlom. EF, efferent branchial vessel of first branchial arch. HM, hyomandibular gill-pouch. HY, hyoid arch. IN, infundibulum. KA, archinephric duet of right side. $\mathbf{K} \mathbf{A}^{\prime}$, archinephric duct of left side, seen in section. $\mathbf{K P}$, head kidney or pronephros. KS, third nephrostome of right pronephros. $\mathbf{K S}^{\prime}$, third nephrostome of left pronepliros, seen in section. OF', olfactory pit. OS, optic stalk. TP, pharyngeal region of mesenteron. TI, intestinal region of mesentcron. Y, yolk-cells.

before the closure of the neural canal is completed; they develop rapidly, reaching the external epiblast, and fusing with it, at an early stage. 
In tadpoles of $3 \mathrm{~mm}$. length there are three pairs of gillpouches present, which appear almost simultaneously; and by the time of hatching of the tadpole two additional pairs are formed behind these, making five pairs in all.

The condition at this stage is well shown in the horizontal section given in Fig. 74. The gill-pouches form vertical partitions, radiating outwards from the pharynx to the surface epiblast. Each pouch is formed of a double fold of hypoblast, the two layers of which are in close contact with each other. The outer ends of all five pairs of gill-pouches reach the epiblast, and fuse with its inner or nervous layer.

Of the five pouches of each side, the most anterior one is the hyomandibular pouch or cleft (Fig. 74, HM), and the succeeding ones are named first, second, third, and fourth branchial pouches respectively: the hindmost or fourth branchial pouch (Fig. 74, HC.4) is smaller than the others, and is often imperfectly developed at this stage.

The parts of the wall of the pharynx between the successive gill-pouches are spoken of as the visceral or gill arches. The arch between the hyomandibular and the first branchial pouches is named the hyoid arch (Fig. 74, HY); and then in succession come the first branchial arch, BR.1; second branchial arch, BR.2, and third branchial arch, BR.3. Behind the fourth branchial pouch, HC.4, is an imperfectly defined fourth branchial arch.

The pharynx is widest opposite the first branchial arches; and between the pair of fourth branchial arches it passes back into the narrow œsophagus.

About the time of formation of the mouth, the two hypoblastic lamellæ, of which each gill-pouch consists, separate from each other, so as to form a narrow vertical slit, or chink, leading: from the pharynx to the exterior. These slits are the gillclefts.

The first clefts to open in this way are the second and third branchial clefts, i.e. the ones immediately behind the first and second branchial arches respectively. At a slightly later stage the first branchial cleft, between the hyoid and first branchial arches, also opens in a similar way; and later still the fourth, or hindmost branchial cleft opens.

The hyomandibular pouch, although it is in its early stages exactly like the hinder branchial clefts, and is fused in similar 
manner with the external epiblast, yet does not open to the exterior. Shortly before the mouth opening is established, the hyomandibular gill-pouch separates from the external epiblast and recedes somewhat from the surface. The two hypoblastic lamella separate from each other, so as to form a saccular diverticulum from the pharynx, and this gradually opens out into the cavity of the pharynx, and in tadpoles of about $20 \mathrm{~mm}$. length ceases to be recognisable as a distinct pouch.

The Eustachian tube and tympanic cavity are formed near to the hyomandibular pouch, but independently of it, and in a manner which has already been described in the section dealing with the development of the ear (p. 143).

\section{The Gills.}

There are two sets of gills in the tadpole, external and internal respectively; the former being branching processes projecting outwards from the first three branchial arches on each side, while the internal gills are formed later as vascular tufts on the sides of all four branchial arches. The two sets of gills differ in some important respects, and it is generally considered that they are independent series of structures.

The external gills appear shortly before the time of hatching, as two pairs of small, backwardly directed processes from the first and second branchial arches. They are at first somewhat conical in shape, with rounded or very slightly notched borders: the gill of the first arch overlaps that of the second arch, and is placed rather more ventrally than this latter.

By the time of hatching (Figs. 72 and 73, BR.1, BR.2), the external gills have increased in size. The first one is notched at its free posterior border into three blunt lobes; and the second into two or three similar ones.

In the succeeding stages the external gills grow rapidly, and the lobes into which they are divided become larger and more numerous. A third external gill appears on the third branchial arch of each side (Figs. 73, 74) : it is very small, and is overlapped and almost concealed by the two anterior gills.

The external gills attain their maximum development about the time of opening of the mouth. At this stage (Figs. 44, 5 , and 77), they form much-branched plumose tufts, exceeding 
in length the transverse diameter of the head. Each of the two anterior gills consists of from five to seven main lobes, decreasing in size from above downwards; and each main lobe gives off minol lobes along its posterior border. The third or posterior gill (Fig: 77) is much smaller than the other two, and only slightly subdivided.

The external gills are usually carried projecting outwards and backwards from the head, at an angle of about $45^{\circ}$ with the axis of the body. Each gill has, however, muscles of its own, by means of which the entire gill or its individual lobes can be moved freely and independently.

The course of the circulation in the external gills can be well studied in the living animal. Each main lobe, and each of its minor lobes, contains two blood-vessels, afferent and efferent, which lie alongside each other and are directly continuous at the tip of the lobe ; the afferent vessel being posterior, and in part ventral to the efferent vessel (Fig. 77, AF and EF).

Before the mouth opens, the opercular folds arise, as a pair of folds of skin from the posterior edges of the hyoid arches, which soon become continuous with each other across the ventral surface of the head. Shortly after the formation of the mouth, the opercular fold begins to grow back rapidly, covering over the gills like a hood. The posterior border of the fold fuses with the body wall along the right side, and across the ventral surface: on the left side of the body it remains free, and is prolonged backwards as a short tubular spout (Fig. 71, oP), through which the opercular cavity opens to the exterior. After completion of the opercular fold the external gills rapidly slirink up, those of the left side persisting longer than those of the right side, and often protruding for a time through the opercular spout.

External gills occur in the adult or in the larval stages of most, though not of all Amphibians. Their morphological value has been much discussed, and it is commonly held that they are to be regarded as secondarily acquired or larval organs, essentially different in their nature to the internal gills.

The internal gills. In tadpoles of from 9 to $10 \mathrm{~mm}$. length the mouth opening is formed, by perforation of the oral septum (p. 148, and Fig. 64, Ds); and about the same time the gill-clefts 
open to the exterior. Almost directly after the opening out of the gill-clefts, the internal gills begin to form, as a series of small papilla along their margins, ventral to the external gills: the tadpole now begins to breathe in the typical fish-like manner, taking in water at its mouth, and passing it through the gill-clefts, and so over the internal gills, into the opercular cavity, from which it escapes by the opercular spont.

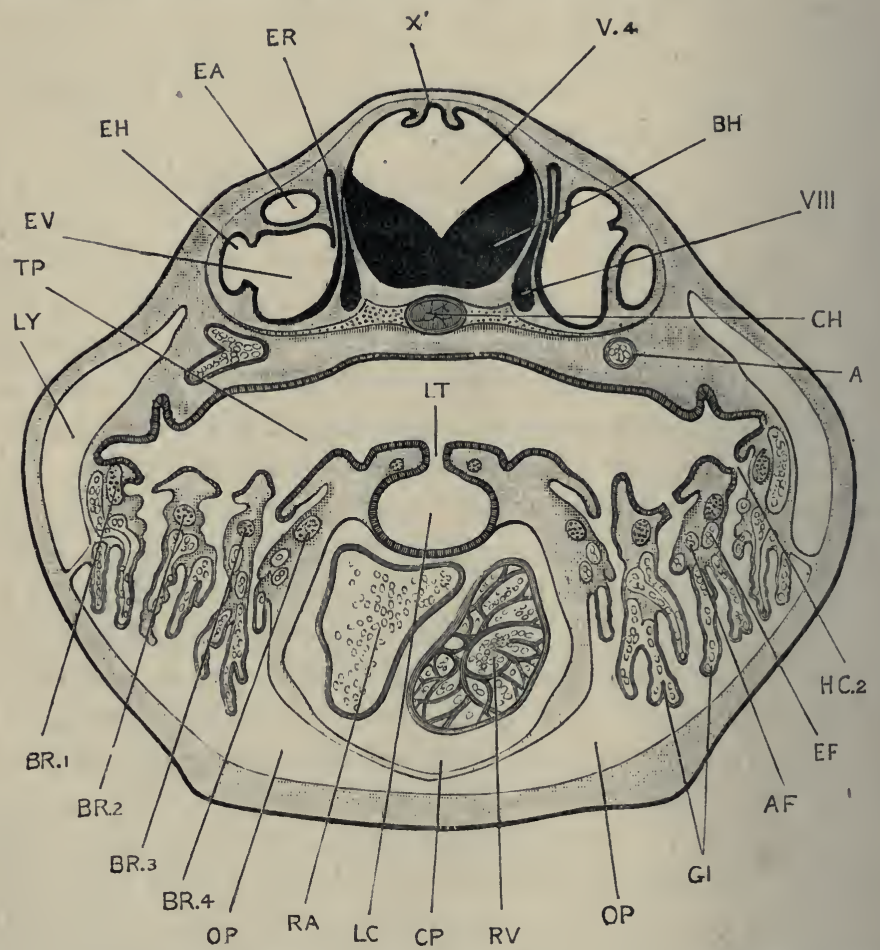

FIf. 75. - Transverse section through the head of a $12 \mathrm{~mm}$. Tadpole; the section passing through the auditory organs, the pharynx and internal gills, the glottis and laryngeal chamber, and the heart. $\quad \times 40$.

A, aorta. AF, afferent blood-vessel of second branchial arcl.. BH, hind-brain. BR.1, .2, .3, .4, first, second, third, and fourth branchial arches. $\mathbf{C H}$, notochoril. $\mathbf{C P}$, pericardial cavity. $\mathbf{E A}$, anterior vertical semicircular canal. $\mathbf{E F}$, efferent bloorvesscl of second branchial arch. EH, liorizontal semicircular canal. FR, recessns vestibuli. EV, vestibule of ear. GI, internal gills. HC.2, second branchial cleft. LC, laryngeal chamber. LT, glottis. LY, lymplatic space. OP, opercular eavit:. $\mathbf{R A}$, auricle of heart. $\mathbf{R V}$, ventricle. TP, pharynx. V.4, fourth ventricle. $\mathbf{X}^{\prime}$, choroid plexus of fourth ventricle. VIII, auditory nerve.

The internal gills rapidly increase in size, and branch so as to form plumose tufts arranged in a double row along the ventral half of each of the first three branchial arches, and a 
single row along the fourth arch ( $c f$. Figs. 75 and 83). From their first appearance the internal gills are very vascular, receiving branches from the afferent and efferent branchial vessels, which are connected by capillaries in the gill-tufts themselves.

The relations remain much the same up to the time of the metamorphosis, the gills forming a series of vascular tufts arranged in double rows along the ventral surfaces of the gill arches (Fig. 75, GI), and hanging down into the opercular cavity, which they in great part fill. The dorsal or pharyngeal borders of the gill arches develop a complicated system of tooth-like processes, which form a filtering or straining apparatus, preventing the passage of food from the pharynx through the gillclefts. This is still furtlier obviated by a pair of velar plates, anterior and posterior, on each side of the floor of the pharynx, which cover over the gill-arches, and separate them from the pharyngeal cavity ; a rather narrow slit is left between the edge: of the two plates of each pair, for the passage of water from the mouth to the gill-clefts, for the purpose of respiration.

The disappearance of the gills. Towards the end of the tadpole period of existence, large numbers of lymph follicles form on the inner surface of the opercular membrane; and at the same time a great proliferation of epithelial cells takes place from the epithelium of the opercular membrane, and from the gills themsebves. On the gills the cells become cubical, and then by rapid division form layers several cells thick. In this way, by thickening of its walls, the opercular cavity becomes greatly reduced in size, and ultimately completely blocked up. The gillclefts become closed, by fusion of their walls with one another; and the gills themselves, with the branchial cartilages, and the entire gill apparatus, degenerate and are rapidly absorbed.

Portions of the ventral ends of the gills persist, even in the adult, as a pair of soft, lymphoid bodies, reddish in colour, which lie at the sides of the larynx, just behind the thyroid bodies, and a little further apart than these. They are sometimes spoken of as tonsils.

Remnants of the dorsal ends of the gills also persist for a time as a pair of compact lymphoid masses, lying immediately beneath the skin, and just behind the ears ; they usually disappear in the course of the second year. 


\section{The Thymus.}

The thymus arises in tadpoles of about $8 \mathrm{~mm}$. length, shortly after hatching, as a pair of epithelial buds from the wall of the pharynx, opposite the dorsal ends of the first branchial clefts. Soon after the opening of the mouth, these buds separate from the epithelium as a pair of solid rounded bodies, formed of deeply-staining epithelial cells, which lie imbedded in the roof of the mouth, below the anterior ends of the auditory vesicles, and between the ganglia of the facial and glosso-pharyngeal nerves.

In each thymus a distinction early appears between an outer cortical layer of small deeply pigmented cells, and a central medullary portion consisting of large pale granular cells. At a later stage the distinction becomes less evident, owing to the cortical cells extending inwards through all parts of the thymus.

The thymus lies behind the quadrate cartilage, and is carried backwards by the rotation of this cartilage which accompanies the widening of the mouth at the time of the metamolphosis. The thymus is larger in the tadpole than in the frog, and undergoes degenerative changes after the metamorphosis. In the frog it lies behind the ear and the tympanic membrane, and slightly ventral to these.

Buds similar to those from which the thymus is formed are developed opposite the dorsal ends of the hyomandibular clefts, simultaneously with the thymus buds; and at a slightly later stage opposite the second and third branchial clefts as well. These all disappear before the metamorphosis and take no part in the formation of the adult thymus.

\section{The Post-branchial Bodies.}

A pair of small diverticula of the floor of the pharynx arise, in tadpoles of about $8 \mathrm{~mm}$. length, behind the last gill-clefts, and at the sides of the glottis. These soon separate from the epithelium as a pair of small vesicular bodies, lined by cylindrical epithelium; they disappear shortly after the metamorphosis. It is possible that they represent, in a modified form, a fifth pair of branchial clefts. 


\section{DEVELOPMENT OF THE HEART AND BLOOD. VESSELS.}

\section{Preliminary Account.}

The blood-vessels arise in the mesoblast. In most parts of the body of the tadpole they appear first as irregular spaces or lacunæ, formed by separation of the mesoblast cells from one another. These lacunar spaces are at first independent, but soon extend so as to meet and open into one another as irregular channels. The cells surrounding these channels assume more definite arrangement and character, and in this way the channels become converted into blood-vessels. The blood corpuscles are either cells which are inclosed from the first within the lacunar spaces, or more usually are cells budded off at a later stage from the walls of the vessels into their cavities.

Each blood corpuscle is a single cell. In the early stages of revelopment all the blood corpuscles of the frog embryo are alike, consisting of spherical cells in which are imbedded numerous yolkgranules. These yolk-granules are gradually used up for the nutrition of the embryo, and shortly after the hatching of the tadpole the corpuscles begin to acquire the shape and characters of the red blood corpuscles in the adult frog.

The chief point of interest in the development of the bloodvessels of the frog is afforded by the changes which occur during the transition from the gill-breathing to the lung-breathing condition.

While the tadpole is breathing by means of gills its circulation is in all essential respects that of a fish. The venous blood, returned from the body at large, enters the posterior end of the beart, or sinus venosus: from this it passes into the second or auricular chamber, thence to the rentricle, and from that to the truncus arteriosus (Fig. 64). The blood passes through the several cavities in succession, there being as yet no division between the right and left sides of the heart.

The truncus arteriosus divides distally into right and left branches, from each of which four afferent branchial vessels (Fig. $76, \mathrm{AF}$ ) arise. The four vessels of each side run outwards along the hinder borders of the four branchial arches, giving off along their whole length numerous branches to the gill-tufts 
on these arches. From the gills the blood, now aërated, passes into the efferent branchial vessels (Fig. 76, wr). 'These lie

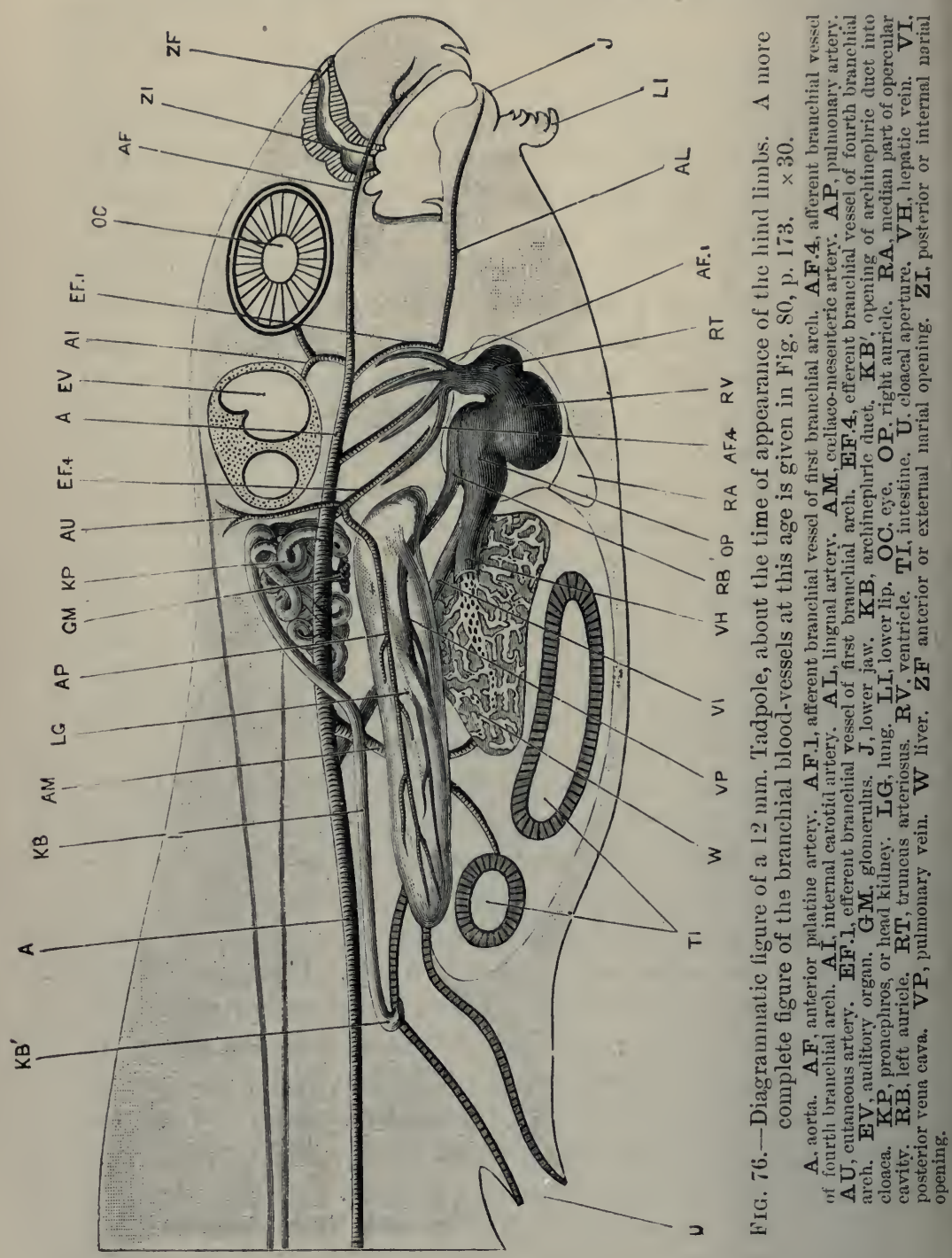

alongside the afferent branchial vessels, and just in front of them, but do not communicate with them except through the capillary loops of the gills. 
The four efferent branchial vessels of each side unite in the rlorsal wall of the pharynx to form the aorta: the two aorte are continued forwards to the head as the carotid arteries, while posteriorly they unite to form the single dorsal aorta, from which branches arise supplying all parts of the body.

The lungs arise at a very early stage, but are for a long time extremely small and of little functional importance. Each lung receives blood from a branch of the fourth efferent branchial vessel (Fig. 76, AP), and returns it directly to the auricle by a pulmonary vein, vP. As the tadpole increases in size, and the lungs become of greater importance, a septum appears, dividing the auricle into systemic or venous, and pulmonary or arterial cavities. Simultaneously with this, valves are formed in the truncus arteriosus, by which the venous and arterial streams of blood are kept apart to a certain extent.

At the time of the metamorphosis the gill circulation is cut off', by the establishment of direct communications between the afferent and efferent branchial vessels, and the pulmonary circulation becomes of much greater importance than before.

\section{The Heart.}

The heart lies at first (Fig. 69, p. 146) on the uncler surface of the head, below the floor of the pharynx, above and slightly behind the sucker, and immediately in front of the commencing liver.

In this region the mesoblast, as in the body generally (Fig. 70, so, sP), is split into somatic and splanchnic layers, separated by a distinct space. This space becomes the pericardial cavity; the outer or somatic layer of mesoblast forming the wall of the pericardial cavity; and the inner or splanchnic layer giving rise to the muscular wall of the heart.

The endothelial lining of the heart is derived from a number of scattered cells, which appear below the floor of the pharynx, and which are formed partly, if not entirely, by direct proliferation of the hypoblast cells of the pharyngeal wall and of the liver (cf. Fig. 69). These cells are at first irregularly arranced, but soon become disposed so as to form a tubular lining to tho heart, which is for a time closed in front, while its posterior wall is formed by the anterior surface of the liver diverticulum (Figs. 64 and 69 ). 
The heart remains attached at its hinder end to the liver, and in front to the floor of the pharynx; but along the rest of its length it becomes free, and increasing rapidly in length becomes twisted on itself in a letter $\mathbf{S}$ shape. At the same time, constrictions appear, partially dividing the cavity into chambers, the first loop of the $\mathbf{S}$ forming the auricular, the second the ventricular portion of the heart; while the posterior and anterior limbs become the sinus venosus and truncus arteriosus respectively (Fig. 64).

At the time of opening of the mouth the heart is still more markedly twisted on itself, and the successive chambers more sharply separated from one another; and a little later a septum grows down from the dorsal wall of the auricle, dividing its cavity into a small left auricle and a much larger right auricle.

The condition of the heart in tadpoles of $12 \mathrm{~mm}$. length is shown from the right side in Fig. 76, p. 166; in sagittal section in Fig. $6 \breve{5}$, p. 121 ; and in horizontal section in Fig. 71, p. 155. The sinus venosus, or proximal division of the heart, is a wide transverse vessel (Fig. 71, RS), which runs across the hinder and dorsal part of the pericardial cavity, and receives the blood returning from the body generally.

The sinus venosus opens in front by a large round median aperture into the right auricle (Figs. 65 and 71, RA). From the auricle the blood passes through a narrow auriculo-ventricular aperture (Fig. 65) into the rentricle, which receires also the blood from the smaller left auricle. The cavity of the ventricle is much subdivided by muscular trabeculæ, which, growing inwards from its walls (Fig. 65 and $75, \mathrm{Rv}$ ), branch and unite to form a kind of spongework, in the meshes of which lie the blood corpuscles.

From the ventricle a small aperture leads into the trumcus arteriosus. This latter is divided into proximal and distal parts, by a pair of valve-like folds, just before the point where it bifurcates into right and left branches; of these, the proximal part, which becomes the pylangium of the adult, is partially subdivided by a longitudinal fold, which runs along its interior in a somewhat spiral course. It is difficult to imagine that these valves can play any part in directing the blood into one pair of afferent branchial vessels rather than another; but it is significant that they should appear just at the time when the 
auricular septum is being completed and the lungs are coming into use.

At the time of the metamorphosis the condition of the heart is practically that of the adult. The proximal and distal parts of the truncus arteriosus, or pylangium and synangium, are now separated by three pocket ralves in place of the two simple valves originally present; the spiral valve of the pylangium is more strongly developed than before; and the synangium is divided internally into anterior and posterior portions, the former communicating with the first and second pairs of aortic arches, and the latter with the third and fourth pairs.

The mode in which the thickening of the wall of the ventricle is effected, by the formation of a muscular reticulum within the cavity, and not by a simple increase in thickness of the wall itself, is of interest, inasmuch as it explains why the ventricle of the frog's heart has no nutrient blood-vessels. 'The blood within the ventricle occupies the meshes of the muscular reticulum and so comes in close contact with all parts of the ventricular walls, a condition which render's special nutrient vessels unnecessary.

The pericardial cavity, in its early stages, communicates freely with the general body cavity, of which it is merely the anterior portion. Owing to the great bulk of the mass of yolk cells, the body cavity in the abdominal region of the embryo is at first merely a narrow chink (Fig. 70, CS); while the pericardial cavity is alrearly a space of considerable size.

Later on, and especially as the large veins opening into the sinus venosus increase in size, the opening between the pericardial cavity and the general body carity becomes much reduced; but up to the time of the metamorphosis there is free communication between the two cavities, through a pair of apertures at the clorsal and posterior border of the pericardial carity, close to the sides of the laryngeal chamber.

\section{The Branchial Blood-vessels.}

The blood-vessels of the pharynx have close relations with the visceral arches, into which the side walls of the pharynx are divided by the gill-clefts.

The first vessels to appear in the body, with the exception of the heart and the great veins opening into it, are the dorsal aortæ. These arise on eacli side as a series of isolated lacunar 


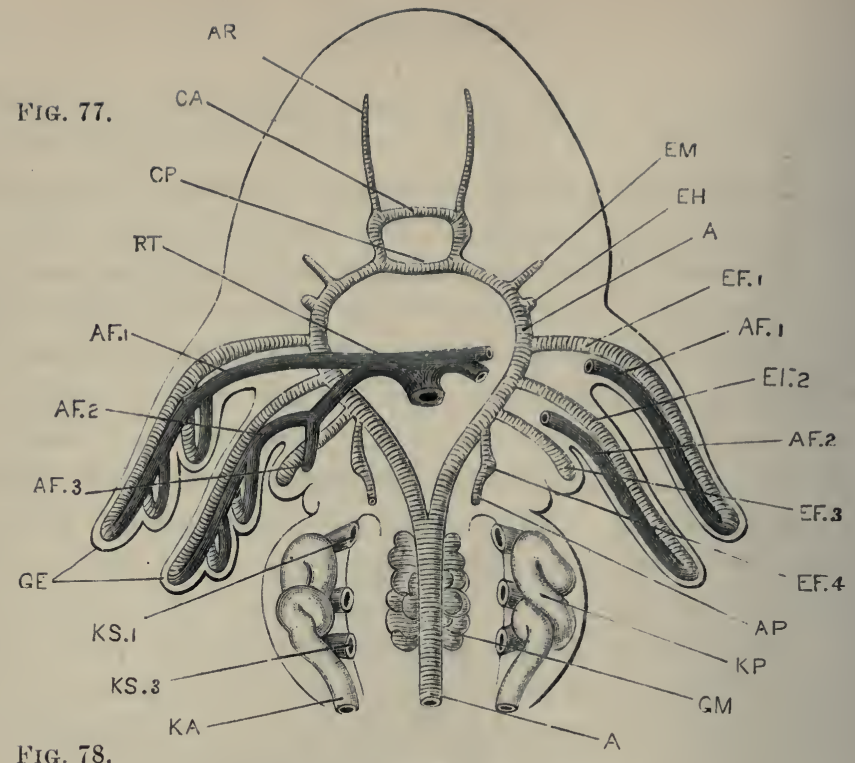

Fig. 78.

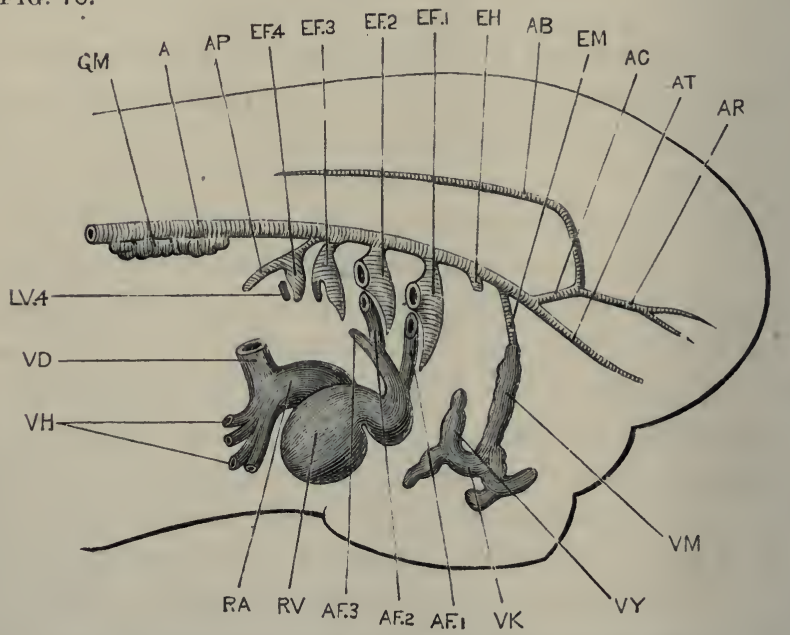

FIG. 77.-Diagrammatic figure of the head and anterior part of the body of a $7 \mathrm{~mm}$. Tadpole, shortly after hatching; showing the branchial blood. vessels from the ventral surface. The heart has been removed. $\times 35$.

FIG. 78. -Diagrammatic figure of the same embryo from the right side. The heart is represented in situ, but the external gills of the first and second branchial arches have been cut off short at their bases. $\times 35$.

A, dorsal anrta. AB, basilar artery. AC, earotid artery. AF.1, AF.2, AF.3, afferent branchial vessels of first, second, and third branchial arehes. AP, pulmonary urtery. AR, anterior cerebral artery. AT, anterior palatine artery. $\mathbf{C A}$, anterior commissural vessel. CP, posterior commissural vessel. $\mathrm{EF} \cdot \mathbf{1}, \mathrm{FF} \cdot \mathbf{2}, \mathrm{FF} \cdot \mathbf{3}, \mathrm{F} \cdot \mathbf{4}$ efferent branchial vessels of first. second, third, and fourth branchial arches. EH, efferent vessel of hyoid arch. $\mathbf{E M}$, efferent vessel of mandibular areh. $\mathbf{G E}$, external gills. GM, glomerulus. KA, segmental duct. KP, head kithey or pronepluros. KS.1, KS.3, first and third nephrostomes of head kirnev. LV.4, efferent lacmar vessel of fourth branchial arch. RA, auricle. $\mathbf{R V}$, ventricle. $\mathbf{R T}$, truneus arteriosus. VD, Cuvierian vein. VH, hepatic veins. VK, vein of sucker. VM, mandibular vein. VY, hyoilean vein. 
spaces along the roof of the pharynx, which by opening into one another form a pair of longitudinal vessels; these soon extend backwards along the body, but remain for some time distinct from each other.

In the four branchial arches, blood-vessels are formed on a definite plan. In the first and second branchial arches these ressels appear immediately after the dorsal aorta, and are well established at the time of hatching; in the third and fourth bran-

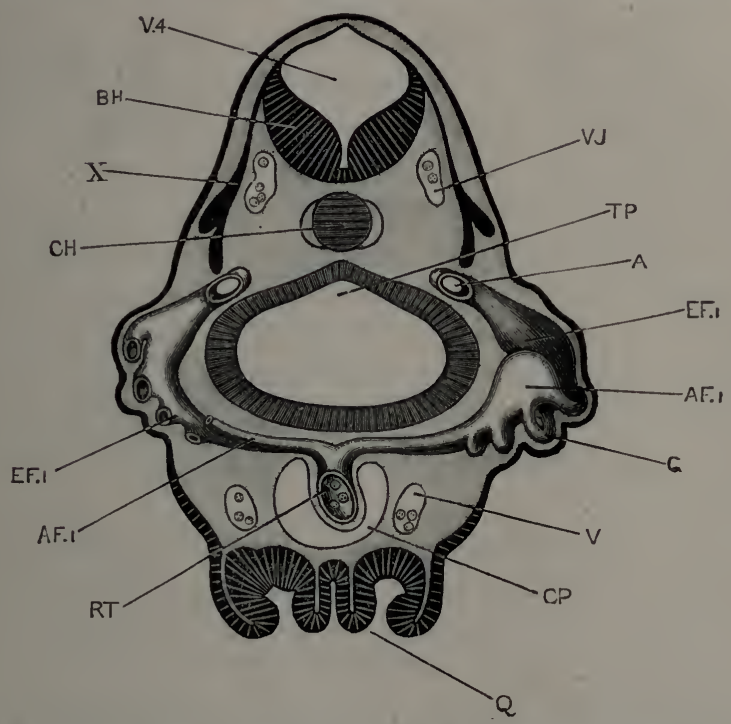

FI(. 79.-A diagrammatic transverse section throngh the head of a $7 \mathrm{~mm}$. Tarlpole, seen from behind; the section is taken just behind the auditory vesicles, and passes through the first branchial arch on each side. On the right side both the afferent and efferent vessels of this arch are shown: on the left side the greater part of the afferent vessel has been removed in order to expose the efferent vessel more thoroughly. $\times 50$.

A, aorta. A.F.1, afferent vessel of first branchial arch. BH, medulla oblongata. CH, notoeliord. CP, pericarlial eavity. EF.1, efferent vestel of first branchial arel. G. eapillary loop of gill, comecting the afferent and efferent vessels together. $\mathbf{Q}$, sucker. RT, truneus arteriosus. TP, pharyx. V, inferior jugnlar vein. VJ, anterior curdinal vein. V.4, fourth ventricle. $\mathbf{X}$, pnemmogastric nerve.

chial arches they arise in a similar manner, but at a somewhat later stage. In the hyoid and mandibular arches, vessels comparable to those of the branchial arches appear at an early stage: these, however, never quite conform to the type seen in the branchial arches, and early undergo degenerative changes.

a. The vessels of the first branchial arch may conveniently 
be taken as typical of the series. The vessels proper to the arch are derived from four factors: (i) an efferent lacunar vessel, which appears in the mesoblast opposite the base of the external gill, and may be recognised in tadpoles some little time before hatching; (ii) an efferent diverticulum from the dorsal aorta; (iii) an afferent diverticulum from the truncus arteriosus; (iv) an afferent lacunar vessel, which lies opposite the base of the external gill, immediately behind the efferent lacunar vessel.

These four vessels appear in the order given above; they are at first quite independent of one another ; and, for some time after their first appearance, the lacunar vessels, afferent, and efferent, have no connection with any other vessels.

The efferent lacunar ressel grows rapidly; it extends dorsally until it meets with, and opens into, the efferent diverticulum from the aorta; and it extends ventrally towards, but not to meet, the truncus arteriosus. It is widest in the middle part of its course, opposite the external gill ; and here it becomes connected with the afferent lacunar vessel by capillary loops in the substance of the gill. This is the condition reached at the time of hatching.

Shortly after this, the afferent lacunar vessel and the diverticulum from the truncus arteriosus grow towards each other and unite. The circulation in the gill is now definitely established (Figs. 77 and 78); the blood passes from the heart to the truncus arteriosus, RT, and from this along the afferent diverticulum and the afferent lacunar vessel, which now form one continuous afferent branchial vessel, AF, to the gill loops, in which it becomes aërated; from the gill loops it passes along the efferent lacunar vessel and efferent diverticulum, which form a continuous efferent branchial vessel, EF.1, to the dorsal aorta.

As the external gill increases in size, and becomes fimbriated or lobed at its margin, the original capillary loops become lengthened out, and additional ones are developed; but the afferent and efferent vessels remain connected by capillaries alone, and it is only by passing through the gill capillaries that blood can get from the heart to the aorta.

At a later period of tadpole life, shortly after the moutl opening is established, the internal gills are developed on the 
gill arch as a double row of branching tufts, ventral to the external gill. Capillary loops soon appear in these tufts, forming a series of capillary connections between the afferent and efferent branchial vessels, similar to those in the external gill, but situated more ventrally. At the same time the external gill diminishes considerably in size.

'The next change of importance is the establishment of a

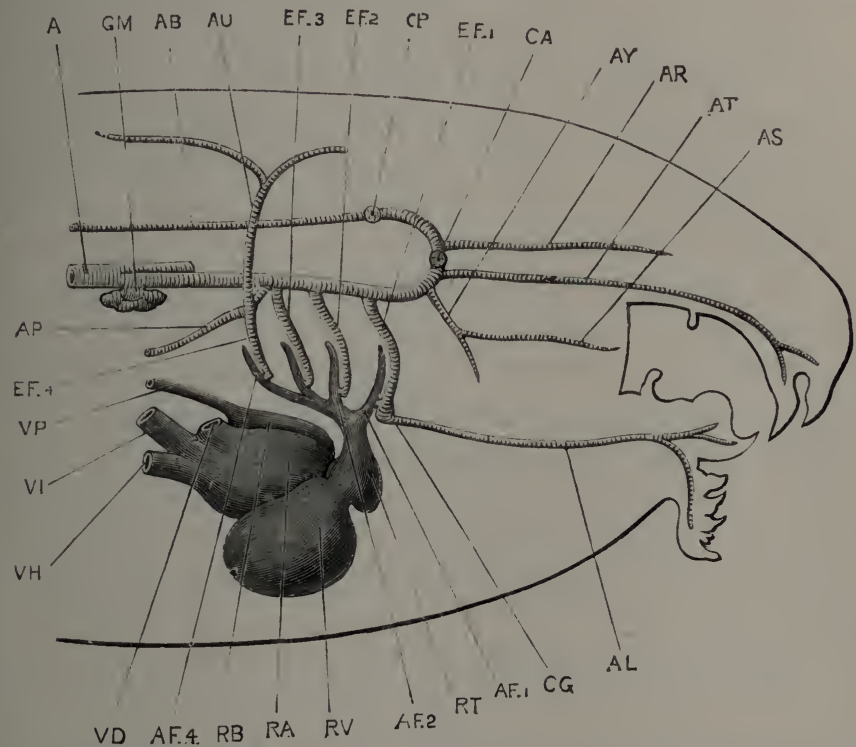

FIG. S0.-Diagrammatic figure of the head of a $12 \mathrm{~mm}$. Tadpole from the right side, showing the heart and branchial blood-vessels. The capillary loops of the gills are omitted. $\times 3$.

A, aorta. AB, basilar artery. AF.1, AF.2, AF.4, afferent branchial vessels of first, second, and fourth branchial arehes. A., lingual artery. AP, pulmonary artery. $\mathbf{A R}$, anterior cerebral artery. A. , posterior jalatine artery. $\mathbf{A} \mathbf{T}$, anterior palatine artery. AU, cutaneous artery. AY, pharyngeal arterv. CA, anterior commissural vessel, seen in section. $\mathbf{C G}$, bulb-like clilatation on the lingual artery. $\mathbf{C P}$, posterior commissural vessel, seen in section. EF.1, FF.2, EF.3, FF.4, efferent branchial vessels of first, second, third, and fourth branchial arches. GM, glomerulus. RA. right anricle. RB, left auricle. RT, truneus arteriosus. $\mathbf{R V}$, ventricle. VD, Cuvierian vein. VH, hepatic rein. VI, posterior vena cava. VP, pulmonary vein.

direct commection between the afferent and efferent branchial vessels. The two vessels (Figs. 77 and 79, AF.1 and EF.1) lie alongside each other in the arch, the afferent being the posterior of the two. In tadpoles of about $12 \mathrm{~mm}$. length, in which the sucker is disappearing, and the hind limbs are present as a pair of small rounded papillæ at the base of the tail, a direct communication is established between the ventral ends of the 
afferent and efferent vessels, close to the truncus arteriosus, (Fig. 80). The precise mode in which the communication is established will be described in the section dealing with the development of the carotid gland (p. 181). As the communication is ventral to the gills, both external and intemal, any blood which passes across it will get from the heart direct to the aorta, without passing through any part of the gill circulation, i.e. without being aërated; and the efficiency of the gill respiration will consequently be impaired in direct proportion to the amount of blood which takes this short cut in preference to the circuitous route through the gill capillaries.

The subsequent changes in the vessels of the first branchial arch may conveniently be considered after the vessels of the other arches have been described.

b. The vessels of the second branchial arch (Figs. 77, 78, and $80, A F .2, E F .2$ ) develop in exactly the same way as those of the first branchial arch, and almost simultaneously with these.

c. The vessels of the third branchial arch (Figs. 77, 78, and 80, AF.3, EF.3) are formed rather later. They are of smaller size than those of the first and second branchial arches, but in other respects are similar to these. The external gill, and the vessels supplying it, are considerably smaller than those of the two arches in frout.

d. The vessels of the fourth branchial arch (Figs. 77, 78, and $80, \mathrm{AF} .4, \mathrm{EF} .4$ ) arise still later, but in essentially the same manner, except that no external gill is formed on this arch. The vessels are well established before the mouth opening appears. From the dorsal end of the efferent vessel of this arch the pulmonary artery, AP, arises as a diverticulum which grows backwards along the outer side of the lung (cf. Fig. 76, p. 166).

It should be noticed that the pulmonary artery arises, and acquires its relations with the lung, before the afferent branchial vessel of the arch has joined the diverticulum from the truncus arteriosus; indeed, before this latter has commenced to develop (cf. Fig. 78). Consequently, the only blood that can at this stage reach the lung is blood from the dorsal aorta, and not blood from the heart; in other words, the lung in the early stages of its development receives arterial and not venous blood.

The afferent ressels of the fourth branchial arch develop very late; and the afferent diverticulum from the truncus 
arteriosus (Fig. 80, AF.4), really a branch from that of the third arch, does not commence to form until after the mouth of the tadpole is established.

e. The vessels of the hyoid arch. In the lyoid arch, at an early stage of development, vessels are present which agree closely in relations and in arrangement with those of the branchial arches; but which, after developing up to a certain point, undergo degenerative changes, and in the later stages of tadpole existence lose all trace of their original disposition.

In tadpoles of $5 \mathrm{~mm}$. length, not long before hatching, the hyoid vessels consist of :-(i) an elongated efferent lacunar vessel, lying parallel to, and in front of, the efferent vessel of the first branchial arch; and (ii) a very small diverticulum from the dorsal aorta, which lies opposite the upper end of the lacunar vessel, but does not quite meet this.

In newly hatched tadpoles two further changes have occurred:-(i) a small blind diverticulum arises from the truncus arteriosus, just in front of the diverticulum for the first branchial arch; and (ii) the efferent lacunar vessel has become obliterated about the middle of its length, and so divided into two separate portions, dorsal and ventral. Of these, the dorsal one has no communication with any other vessel, although it lies very close to the diverticulum from the aorta; while the ventral portion, which may be spoken of as the hyoidean vein, opens below into an irregular longitudinal venous sinus lying just above the sucker.

In tadpoles shortly after hatching (Fig. 78), the diverticulum from the truncus arteriosus has disappeared, as has also the dorsal portion of the efferent sinus; so that the only vessels remaining are the small diverticulum, EH, from the aorta, and the ventral portion of the efferent sinus, or hyoidean vein, ry, which opens below into the veins of the sucker, vk. By the time the mouth opening is established, the diverticulum from the aorta has also vanished, and the hyoidean vein is the only persistent part of the series of hyoidean vessels.

It thus appears that in the hyoid arch vessels are developed which are essentially similar to those of a branchial arch; the chief difference being that no afferent lacunar vessel is formed in the hyoid arch, a difference which may clearly be correlated with the absence of gills, both external and internal, from the 
arch. The arrangement and mode of development of the vessels which are actually present in the liycid arch, agree so closely with those seen in the vessels of the branchial arches as to strongly suggest that frogs must be descended from ancestors in which gills were present on the hyoid arch as well as on the branchial arches.

f. The vessels of the mandibular arch. These appear later than the vessels of the hyoid arch, and depart even more markedly from the typical branchial arrangement.

Up to the time of hatching there are no vessels at all in the mandibular arch. Shortly after hatching there appears in the lower or ventral part of the arch a lacunar vessel, which lies parallel to and in front of the similar vessel in the hyoid arch, and, like this, opens into the venous sinuses above the sucker; it may be spoken of as the mandibular vein. There is also present a very small diverticulum from the dorsal aorta.

A little later (Fig. 78), both these factors have grown considerably. The mandibular vein, vis, has extended dorsalwards, and the aortic diverticulum, EM, ventralwards; and the two ressels are now continuous with eacl other. Shortly after the mouth opens, the two again separate; the mandibular vein gradually shrinks up, as the sucker degenerates, and the aortic diverticulum grows forwards as the pharyngeal artery of the adult (Fig. 80, AY).

From the above account it appears that the vessels of the mandibular arch, though still referable to the type of the branchial vessels, are even more modified than those of the hyoid arch; the afferent lacunar vessel and the diverticulum from the truncus arteriosus are completely absent, and at no time have the vessels any connection with the heart.

g. The changes in the branchial vessels at the metamorphosis. For some time before the metamorphosis the tadpole breathes by lungs as well as by gills, though the main part of the respiratory work is performed by the latter.

The condition of the blood-vessels during this period of double respiration is as follows. The mandibular and hyoid vessels may be omitted, as, although these are formed on the type of the branchial vessels, they have no connection with the heart, and no gills are developed in relation with them.

Gills are present on all four branchial arches, and the 
arrangement of the vessels is practically the same in all. In each arch (Fig. 80) there are two main vessels, afferent and efferent, which lie side by side close to each other. Of these, the afferent vessel is a branch of the truncus arteriosus, and lies in the posterior part of the arch; while the efferent vessel lies immediately in front of the afferent, and opens at its dorsal end into the aorta. The afferent vessel is confined to the ventral half of the arch; while the efferent extends along its whole length, its ventral termination lying in the floor of the mouth, close to the origin of the afferent vessel from the truncus arteriosus.

The afferent and efferent vessels of each arch are connected together in two ways: (i) by the capillary loops of the gills, of which the most dorsally placed belong to the external gill, and the ventral series to the internal gills; (ii) by each afferent vessel opening directly into the corresponding efferent vessel, the communication (Fig. 80) being ventral to the gills, and of very small size. This direct connection between the afferent and efferent vessels is present in all four branchial arches, though its position and relations are not easy to determine. The blood entering an afferent vessel from the heart has thus two courses open to it: it may either continue along the afferent vessel, and pass through the gill capillaries to the efferent vessel, and so reach the aorta; or it may pass across at once, through the aperture of communication, to the efferent vessel, and reach the aorta without having passed through the gills.

At the commencement of the metamorphosis these direct communications enlarge, so that an increasing quantity of blood passes from the heart to the aorta without going through the gills. The gills thus receive less and less blood, and gradually diminish in size and in efficiency. Increased work is thereby thrown on the lungs; and an increasing supply of blood is sent to the lungs and skin by enlargement of the pulmonary and cutaneous arteries, which are branches of the efferent vessel of the fourth branchial arch (Fig. S0), close to its dorsal end.

By further enlargement of the direct communications between the afferent and efferent vessels, the definite aortic ar'ches are formed, leading directly from the heart to the aorta; each aortic arch ( $c f$. Figs. 80 and 81 ) consisting of the basal or proximal end of the afferent branchial vessel, and the whole 
length of the efferent vessel; while very nearly the whole length of the afferent vessel, and all the gill capillaries, disappear. completely.

Very slight changes will now convert the branchial system of the tadpole to the aortic system of the adult frog. Of the four aortic arches, the first, in the first branchial arch, becomes the carotid arch of the frog (Fig. 81, 1). The portion of the dorsal aorta between the points of opening of the first and second aortic arches remains an open tubular vessel for some time, and may even retain its lumen in the adult. More usually, however, the cavity becomes obliterated, and the walls of the

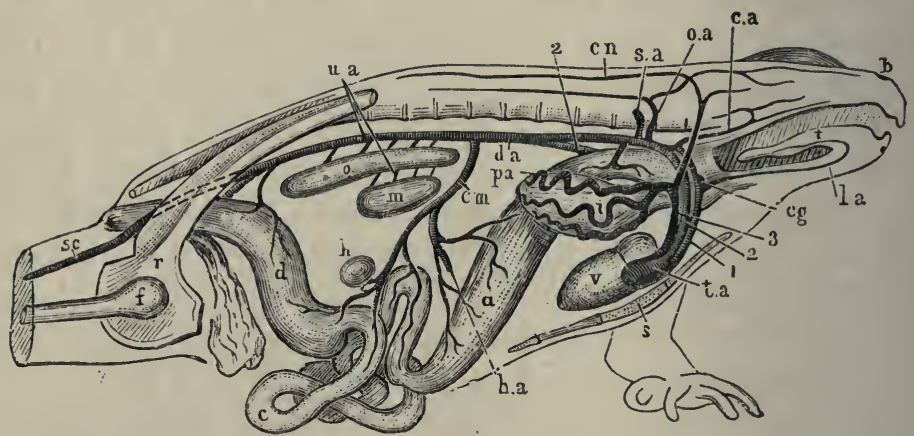

FIG. 81.-Diagrammatic figure of the arterial system of an adult male Frog, from the right side. $\times 1$.

$c$, stomach. $b$, nostril. $c$, small intestine. $c a$, carotid arters. $c q$, carotid glani . $\mathbf{c m}$, cœliaco-mesenteric artery. $c n$, cutaneous artery. $d$, large intestine. $d a$, lorsal aorta. $\rho$, femur. $h$, spleen. $h a$, hepatic artery. $i$, right lung. $l a$, lingual arters. $m$, testis. o, kidner. oa, occipito-vertebral artery. $p a$, pulnonary artery. $r$, pelvic girdle $s$, sternum. $s a$, subclavian artery. $s c$, sciatic artery. $t$, tougue. $t a$, truncus arteriosus. $u a$, urinogenital arteries. $v$, ventricle. 1, carotid arch. 2, systemic arch. 3 , pulmo-cutaneous arch.

vessel persist merely as a pigmented band, connecting the dorsal ends of the carotid and systemic arches with each other. After the obliteration of this part of the aorta, the blood in the carotid arch is distributed exclusively to the head.

The second aortic arch, in the second branchial arch of the tadpole, becomes the systemic arch of the frog (Fig. 81, 2).

The third aortic arch, in the third branchial arch of the tadpole, disappears altogether. In young frogs of the first year it loses its connection with the aorta, and then gradually shortens up, the distal part becoming a solid cellular cord, and the proximal or cardiac part retaining for a time its lumen. Before the end of the first year this vessel has entirely disappeared. 
'The fourth aortic arch, in the fourth branchial arch of the tadpole, becomes the pulmo-cutaneous arch of the frog. It retains its communication with the aorta for some time after the dorsal part of the third arch has atrophied; but before the end of the first year, the part of the fourth arch above the origin of the cutaneous artery loses its cavity and becomes solid, so that the pulmo-cutaneous arch no longer opens into the aorta.

The condition of the artic arches is now that of the adult fiog (Fig. 81).

Before leaving the branchial circulation, it shuuld be noticed that in other species of frogs the development of the bloodvessels differs in some important respects from that described above for the common English frog, Rana temporaria. In Rana esculenta, according to Maurer, the efferent lacunar vessel of each arch becomes connected at its ventral end with the truncus arteriosus, and at its dorsal end with the aorta, before the afferent branchial vessel is formed; so that in this species of frog there is a stage, prior to the formation of the gills, in which there is a direct passage from the heart to the aorta. As the gills appear, the afferent gill-vessel arises as an outgrowth from the ventral end of the efferent vessel, with which it becomes connected, more dorsally, through the gill-capillaries. During the time that the taclpole is breathing by gills, the direct connection between the ventral ends of the afferent vessel and the efferent vessel is lost, but it is re-established at the time of the metamorphosis, after which time the further changes are the same as in Rana temporaria. A similar mode of development has been observed as an exceptional occurrence in Rana temporaria itself, and there are reasons for thinking that this plan of development, in which there is, from the first, direct connection between the heart and the aorta, through the aortic arches, and in which the whole gill circulation is secondarily derived from this condition, is more primitive than the mode of development usually seen in Rana temporaria. This more primitive type of larval vessels is comparable to the condition obtaining throughout life in the branchial blood-vessels of Amphioxus.

\section{The Dorsal Aorta and its Branches.}

There are at first two aortæ, one on either side of the body. These appear, as described above, as a number of isolated 
lacunar spaces in the roof of the pharynx, which run together to form a pair of continuous vessels. These are widest apart opposite the first branchial arches (Fig. 77, A); further back, behind the pharyngeal region, they lie close alongside each other, and soon fuse to form the definite dorsal aorta.

a. The arteries of the head are derived from the anterior ends of the dorsal aortæ.

The carotid arteries (Fig. 78, AC), which are the direct continuations forwards of the dorsal aortæ, become early connected by two transverse vessels, the anterior and posterior commissural vessels (Fig. 77, CA, CP), which, with the carotid arteries themselves, form an arterial circle surrounding the infundibulun. In front of the anterior commissural vessel the carotid arteries are continued forwards as the anterior cerebral arteries (Figs. 78 and $80, \mathrm{AR})$.

The basilar arteries (Figs. 78 and 80, AB), appear shortly before the mouth opens, as a pair of vessels which arise from the outer ends of the posterior commissural vessel, and run backwards along the ventral surface of the brain and spinal cord, not far from the median plane.

The anterior palatine arteries (Figs. 78 and 80, AT) arise as branches from the carotid arteries, just before these enter the skull, and run forwards in the mucous membrane of the roof of the mouth às far as the nose ; they appear very shortly after the tadpole hatches.

The pharyngeal artery (Fig. 80, AY) is formed, as already described, from the dorsal part of the efferent vessel of the mandibular arch (Fig. 78, Em). From it the posterior palatine artery (Fig. 80, As) arises as a small, anteriorly directed branch, which runs forwards and outwards in the roof of the pharynx.

The lingual artery (Fig. 80, $\mathrm{AL}$ ) is of considerable interest. It appears shortly before the mouth opens, and in tadpoles of about $9 \mathrm{~mm}$. length is present on each side of the head as a very short vessel, lying in the floor of the mouth immediately in front of the truncus arteriosus. In the middle of its course it presents a swollen bulb-like dilatation, from which arise :-(i) an anterior branch, the lingual artery proper, which runs forwards a short distance, giving off a small thyroid artery to the thyroid body; and (ii) a posterior branch, which runs a short distance outwards and backwards towards the ventral end of the efferent branchial 
vessel of the first branchial arch, EF.1, but does not quite meet this. At this stage, therefore, the lingual artery is a closed vessel, having no communication with any other blood-vessel.

Slightly later, in tadpoles of about $12 \mathrm{~mm}$. length, the posterior end of the lingual artery and the ventral end of the first efferent branchial vessel become directly continuous with each other (Fig. 80). The bulb-like swelling is still present, and it is immediately dorsal to this that the direct connection between the afferent and efferent branchial vessels is effected, as described above. 'The anterior end of the lingual artery has by this time grown forwards considerably (Fig. 80), extending to the lower jaw and lower lip.

The lingual artery thus arises in the floor of the mouth independently of any other vessel, but soon acquires connection with the ventral end of the first efferent branchial vessel, of which in the later stages it appears to be a direct continuation.

b. The carotid gland of the frog is formed by elaboration of the direct passage between the afferent and efferent vessels of the first branchial arch. At $12 \mathrm{~mm}$., as just noticed, the lingual artery and the first efferent branchial vessel are continuous; the lingual artery has at its base (Fig. 80) a small bulb-like swelling, C $(\dot{*}$, and immediately dorsal to this swelling the afferent and efferent branchial vessels are in direct communication with each other. This direct passage is at present a single, and a narrow one : it traverses a small plate of epithelial cells, which is budded off from the epithelium of the first branchial cleft, and wedged in between the afferent and efferent vessels.

In the later stages of development this passage becomes plexiform, there being now three or four openings into the afferent vessel, and about the same number into the efferent vessel, one or more of the latter leading directly into the bulblike swelling at the base of the lingual artery.

This plexiform communication forms the carotid gland; the history of its formation shows that it is not to be regarcled as a persistent or modified part of a gill, as was formerly held to be the case, but that it is a specially acquired structure, formed by elaboration of the direct passage between the afferent and efferent branchial vessels, a passage which is present in a simpler form in the hinder arches as well.

Solid epithelial plates, of a similar character to that in which 
the carotid gland is formed, are developed, at a slightly later stage, between the ventral ends of the afferent and efferent vessels of the second and third branchial arches, and perhaps in the fourth arch as well; and it is by perforation of these plates that the direct communications between the afferent and efferent vessels are established. After the metamorphosis the epithelial plates persist, and even increase in size, forming solid epithelial bodies, lying in the floor of the mouth, in the angles between the carotid and systemic, and systemic and pulmocutaneous arches respectively. They acquire connective tissue capsules, and have been spoken of as accessory thyroid bodies.

c. The hinder part of the dorsal aorta. The union of the two aortæe to form the definite dorsal aorta occurs almost immediately behind the pharyngeal region (Fig. 77).

At, or close to, their point of union each aorta has, at the time of hatching, a small bulging of its ventral wall. Later on, these bulgings increase in size and become sacculated, forming a pair of prominent, pigmented, thick-walled swellings, the glomeruli of the head kidneys (Figs. 77 and 78, GM), which hang down into the dorsal part of the body cavity, lying opposite the head kidneys, $\mathrm{KP}$, along almost their entire length.

In their further development, the glomeruli keep pace with the head kidneys; they remain large up to about $23 \mathrm{~mm}$., i.e. so long as the head kidneys remain functional (cf. Figs. 83, 84, and $85)$; but in the later stages, when the head kidneys begin to degenerate, the glomeruli also become smaller. They are still present, though of very small size, up to the end of the first year, but disappear completely in the second year. Certain further points in connection with the glomeruli will be noted in the section dealing with the kidneys.

d. The pulmonary arteries arise, as described above, shortly after the time of hatching (Figs. 77, 78, AP), as diverticula from the dorsal ends of the efferent vessels of the fourth branchial arches. As the connection of the afferent vessel of this arch with the truncus arteriosus is not acquired until some time after the mouth opening is established, there is a considerable period during which the supply of blood to the lungs is derived from the aorta and not from the heart, i.e. is arterial and not venous; a condition which suggests that the lungs had originally some function other than respiration to fulfil. 
The cutaneous artery (Fig. 80, $\mathrm{AU}$ ) is a branch of the fourth efferent vessel, which arises very close to the pulmonary artery, but independently of this, and at a rather later stage.

\section{The Veins.}

The veins, in the earlier stages of their development, are chiefly characterised by their large size, and irregular lacunar character.

a. The vitelline veins are the first veins to be formed in the body. They appear as irregular lacunz in the mesoblast of the splanchnopleure, along the sides of the yolk-mass and of the liver diverticulum, and unite in front to form the sinus venosus or most posterior part of the heart; they carry to the heart the food matter absorbed from the yolk-mass.

About, or shortly after, the time of hatching, the liver diverticulum becomes more definitely bounded, and the vitelline and hepatic veins become distinct from one another; later still, by folding of the wall of the hepatic diverticulum, the hepatic veins are carried deeply into the substance of the liver (Figs. 64 and $76)$, as already described in the section dealing with the development of the liver (p. 154).

b. The sinus venosus (Fig. 71, Rs) is at first formed merely by the union of the vitelline veins, but very early becomes a definite transverse vessel, running across the body in close contact with the anterior wall of the liver, and opening into the auricular portion of the heart by a large anterior aperture.

c. The anterior cardinal veins are paired, and return blood from all parts of the head, except the floor of the mouth. Each is formed by the union, belind the ear, of two principal veins :the jugular vein, which returus blood from the brain and dorsal part of the head; and the facial vein, which runs more superficially along the side of the head, ventral to the eye and ear.

d. The posterior cardinal veins (Fig. 84, vc) are also paired, and are in special relation with the head kidneys, which they completely surround. They are of enormous size during the early stages of tadpole life, when the head kidneys are functionally active, forming vascular networks which occupy the spaces between the tubules of the head kidneys. Each posterior cardinal vein receives somatic veins from the hinder part of the body wall, and unites, in front, with the anterior cardinal 
vein, at the level of the hinder border of the pericardial cavity, to form the Cuvierian vein (Fig. 71). The two Cuvierian veins, right and left, run almost vertically downwards to open into the outer ends of the sinus venosus (Fig. 71). The Cuvierian veins persist as the anterior venæ cavæ of the frog.

e. The inferior jugular veins collect the blood from the floor of the mouth, including the large sinuses above the sucker into which the mandibular and hyoidean veins open (Fig. 78): they then run back in the side walls of the pericardial cavity, and open into the right and left Cuvierian veins respectively, just before these reach the sinus venosus.

f. The posterior vena cava is a large median vein (Fig. 76, vI), which develops shortly after the mouth opening is established; it is continuous posteriorly with the hinder portions of the two posterior cardinal veins, which unite together; and, further forwards, it runs in a deep groove along the left side of the liver (Fig. 71, vH), joins with the hepatic veins, and then opens into the sinus venosus. The blood from the hinder part of the body can now return to the heart either along the posterior cardinal and Cuvierian veins, or else by the posterior vena cava. In the later stages of tadpole life more and more of the blood follows the latter course, and the anterior ends of the posterior cardinal veins gradually diminish in size, and during the metamorphosis disappear completely.

g. The renal portal veins are formed by longitudinal anastomotic communications between the transverse, or vertebral, veins of the hinder part of the body ; they are joined posteriorly by the iliac veins, and with these form the afferent renal system of veins.

h. The anterior abdominal vein is at first paired, and is in connection, not with the liver, but the heart. The pair of vessels appear first in the ventral body wall, extending backwards a short distance from the sinus venosus; they soon extend further backwards, and acquire communications with the veins of the hind legs and of the bladder. At a later stage, the two anterior abdominal veins unite at their hinder ends, in front of the bladder, while further forwards the vein of the right side disappears, the left one alone persisting. Later still, the anterior abdominal vein loses its direct communication with the sinus venosus, and acquires a secondary one with the hepatic portal veins, or afferent veins of the liver. 
i. The pulmonary veins (Fig. 76, vr) develop early, but not quite so soon as the pulmonary arteries. They appear, about the time of establishment of the mouth opening, as a series of irregular lacunar spaces along the inner or median walls of the lungs, and at first do not reach the heart. Very shortly afterwards, they are completed along their whole length, about the time of formation of the inter-auricular septum in the heart.

\section{(j. The Lymphatic System.}

The most striking point about the lymphatic system in the tadpole stages is the great development of the subcutaneous lymph spaces. In tadpoles of about $20 \mathrm{~mm}$. length, and upwards, these form sacs of literally enormous size, filled with a coagulable fluid, and almost completely surromding the sides and ventral surface of the body and head ( $f f$. Figs. 71, 75, and 88, $\mathrm{LY})$; in the living tadpole they give rise to the appearance of a semi-transparent border surrounding the head and trunk.

The early development of the lymphatics appears to take place in the same manner as that of the blood-vessels; the lymphatics appearing first as intercellular lacunar spaces, to which proper walls are formed later by the surrounding tissues.

The lymph-cells appear rather late, after the internal gills are well established; according to Maurer, they are derived from the hypoblast cells of the wall of the mesenteron, and wander thence into the connective tissue.

The spleen arises as a spherical bud on the mesenteric artery; it consists of cells similar to those of the lymphatic tissues, and, like these, is said to be derived originally from the hypoblast cells of the mesenteron.

\section{DEVELOPMENT OF THE URINARY AND REPRODUCTIVE ORGANS.}

\section{Preliminary Account.}

The excretory organs of the tadpole are the head kidneys, a pair of globular bodies (Fig. 83, KP), imbedded in the dorsal body-wall, immediately behind the pericardial cavity. Each head kidney, or pronephros, consists of a convoluted and complex mass of tubules, with glandular walls, opening into the body 
cavity by three ciliated mouths or nephrostomes; the tubules are surrounded by, or rather imbedded in, the large posterior cardinal veins, and it is from the blood in these veins that the excretory matters are separated. The excretions are carried away from the head kidneys by a pair of tubes, the segmental ducts, $\mathrm{KA}$, which run along the dorsal wall of the body to its hinder end, where they open into the cloaca, or hinder part of the alimentary canal.

The head kidneys and their ducts are well developed in the tadpole at the time of hatching; they subsequently increase considerably in size, and are the sole excretory organs of the tadpole during the earlier stages of its existence. About the time the hind legs appear, the adult kidneys, or IVolffian bodies, begin to form in the hinder part of the body, as a series of tubes which grow towards, and open into, the segmental ducts (Fig. 83, KM); these ducts carrying away the excretory matters separated by both the larval and adult kidneys. The Wolffian bodies rapidly increase in size and in complexity, especially at their posterior ends, and by the time of the metamorphosis (Figs. 85 and 86) have attained considerable dimensions. The head kidneys at the same time undergo degenerative changes, and gradually disappear, while the Wolffian bodies, growing still larger, become the kidneys of the frog.

The genital ducts are formed in close relation with the segmental ducts, or actually from these ducts; and the Wolffian bodies become so closely related to the reproductive organs in both sexes, that it is desirable to describe the development of the two systems, urinary and reproductive, together.

\section{The Head Kidney and Segmental Duct.}

The segniental duct (Fig. 70, $\mathrm{KB}$ ) first appears, in embryos of between $3 \frac{1}{2}$ and $4 \mathrm{~mm}$. length, as a longitudinal ridge of the somatopleuric mesoblast, immediately below the ventral borders of the myotomes, MS. This ridge lies immediately beneath the epiblast, but is, in the frog, distinct from this at all stages of its development. It is difficult to determine whether or not it is solid at its first appearance, but it very soon becomes grooved along its inner surface (Fig. 70); the groove communicating with the body cavity, and the entire ridge having, in transverse section, the appearance of a fold of the mesoblast, with a 
well-defined ventral or outer lip, and a less distinct dor'sal boundary.

In the hinder part of the body the lips of the groove meet, so as to form a tube, lying between the mesoblast and the epiblast; and this tube is the segmental duct. At the anterior end, the lips remain separate for a time, so that the duct opens in front into the body cavity by a longitudinal slit-like mouth.

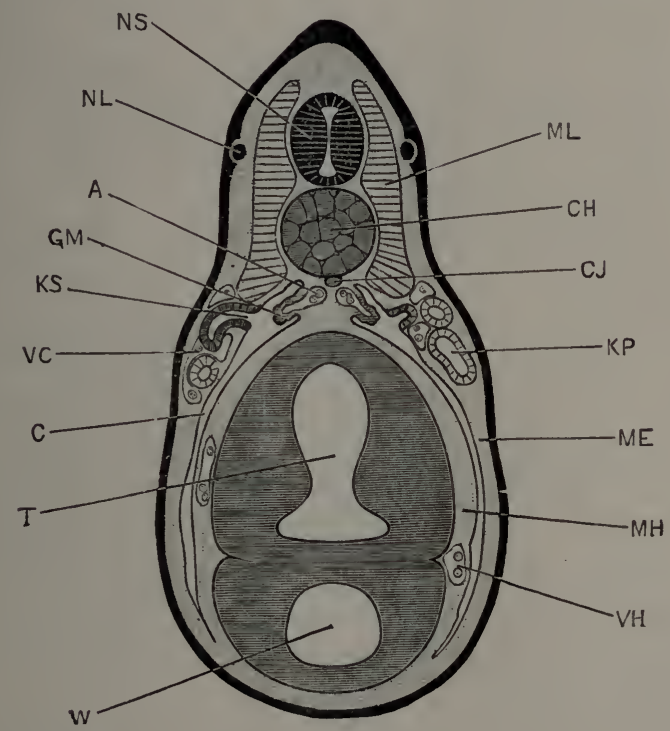

FIG. 82.-Transverse section through the body of a Tadpole at the time of hatching; the section passing through the second pair of nephrostomes, and the third pair of myotomes. $\times 50$. A, anrta. C, cœlom, or body cavity. CH, notochord. CJ, subnotochordal rod.
GMM, glomerulus. KP, segmental duct. KS, second nephrostome of left sile.
ME, somatopletric layer of mesoblast. MH, splanchnopleuric larer of mesoblast.
ML, myotome. NL, lateral line braneh of pmeumogastric nerve. NS, spinal coril.
T, intestinal region of mesenteron. VC, posterior carlinal vein. VH, hepatic vein.
W, liver diverticulum.

By meeting of the lips at two places this slit becomes divided into three openings or nephrostomes, lying one behind another, and immediately below the ventral edges of the second, third, and fourth myotomes respectively ( $c f$. Figs. 74 and 82).

The anterior end of the duct, and especially the part between the second and third nephrostomes, now increases rapidly in length, becoming twisted on itself; and at the same time the nephrostomes become drawn out into short tubes. At its 
hinder end the duct grows backwards, and, shortly before the hatching of the tadpole, acquires an opening into the cloaca.

The condition at the time of hatching is shown in Figs. 74 and 82. The head kidney, $\mathrm{KP}$, is a slightly convoluted tube, opening into the body cavity by the three nephrostomes, $\mathrm{Ks}$; of which the anterior one is at a level slightly ventral to that of the other two. Opposite the nephrostomes, and projecting into the dilated dorsal portion of the body cavity, is the glomerulu. (Fig. 82, GM), a sacculated diverticulum of the aorta, the development of which has been described above. Behind the nephrostomes, the segmental duct is continued backwards in an almost straight course to the hinder end of the body, where it opens into the cloaca (Fig. 74, $\mathrm{KA}$ ).

After the tadpole has hatched, the head kidney increases considerably in size (Figs. 76, 83, and 84). The tubules of which it consists become more markedly convoluted; and further complication is caused by the formation of lateral diverticula, of which there are three principal ones, directed outwards, and themselves branched. The three nephrostomial openings persist; their walls are formed of pigmented cells, bearing long flagella, directed inwards. The head kidney reaches its full development in tadpoles of about $12 \mathrm{~mm}$. length (Figs. 83 and 84). when it is almost spherical in shape, and formed of an intricately coiled mass of tubes, imbedded in the greatly dilaterl anterior cardinal sinus, rc.

In tadpoles of about $20 \mathrm{~mm}$. length, the head kidneys commence to degenerate. The first step in the process consists in irregular dilatation of the tubules, more especially of the blind lateral diverticula. This dilatation may be so great that a single tubule may nearly equal in diameter the entire head kidney of the earlier stages; but as the dilatation of. some tubules is accompanied by compression of others, the size of the head kidney as a whole remains practically unaltered.

This irregular dilatation is accompanied by, and perhaps due to, partial or complete obstruction of the segmental duct behind the head kidney. The epithelium of the kidney tubules soon shows degenerative changes, the cells becoming flattenerl out in an irregular manner, their outlines indistinct, the cell contents cloudy, and the inner surfaces of the cells ragged. From this time degeneration proceeds steadily, and the whole organ 
diminishes in size. In $4: 0 \mathrm{~mm}$. tadpoles (Fig. 85) the head kidneys are less than half their former size, and the tubules are completely collapsed. By the time of the metamorphosis, the head kidneys

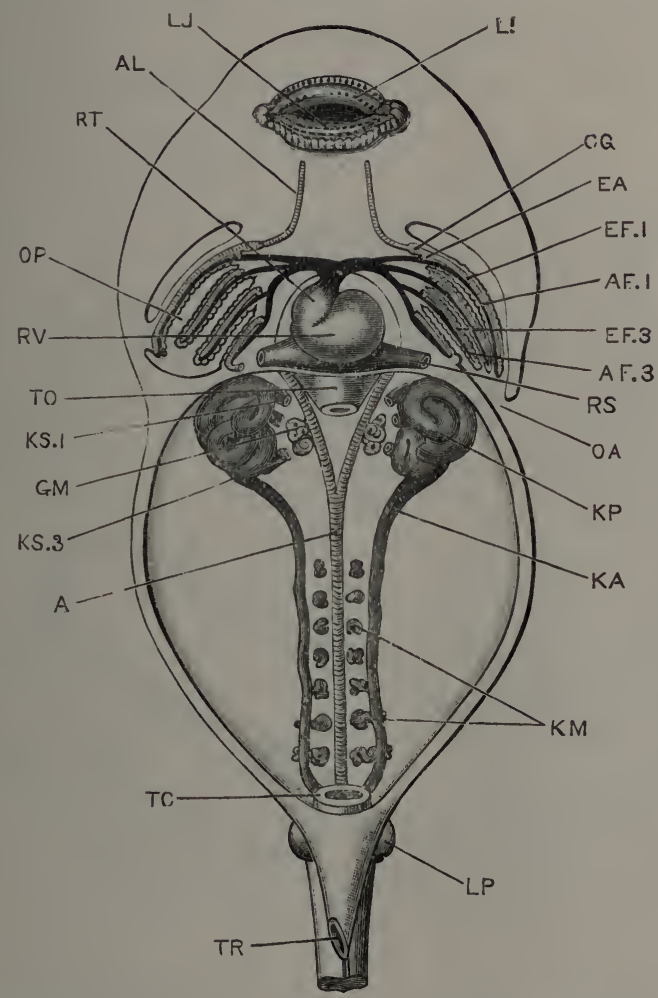

FIG. 83.-Diagrammatic figure of a $12 \mathrm{~mm}$. Tadpole, dissected from the ventral surface, to show the heart and branchial vessels, and the head kidneys and commencing Wolffian bodies. The alimentary canal, from the osophagus to the rectum, has been removed. $\times 22$.

A, aorta. AF.1, AF.3, afferent branchial vessels of first and third branchial arches. AL, lingnal artery. CG, dilatation at the base of the lingual artery. EA. communication between the afferent and efferent vessels of the first branchial arch, by further elaboration of which the carotid gland will be formed. EF.1, EF.3, efferent branchial vessels of first aml third branchial arehes. GM, glomerulus. $\mathbf{K} \mathbf{A}$, segmental luet. KM, Wolffian tubules. KP, head kidney. KS.1, first nephrostome. KS.3, thiri nephrostome. LI, upper lip. LJ, lower lip. IP, commeneing hind limb. OA, spont-like aperture of opercular cavity. OP, opercular cavity. RS, simns venosus. RT, truneus arteriosus. RV, ventriele. TC, cloaca. TO, œsophagus. TR, aperture of rectal slout.

have almost disappeared (Figs. 86 and 88), a few pigmented and slightly twisted tubules alone remaining.

All three nephrostomes disappear: the anterior one is the first to close, and the middle one soon follows ; the third or most 
posterior one persists for a short time longer, and then in its turn closes, loses its connection with the peritoneum, and disappears. Shortly before the closure of the third nephrostome, the head kidney separates completely from the segmental duct, which then ends blindly in front.

The glomerulus of the head kidney (Figs. 83, 84, GM) arises, as

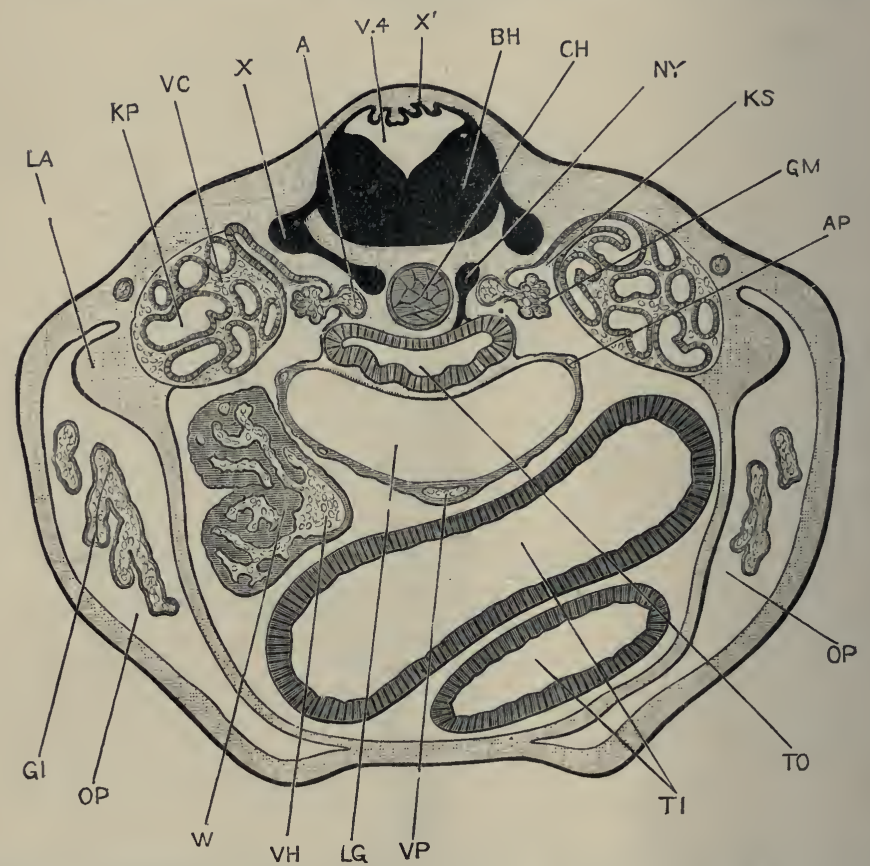

FIG. 84.--Transverse section across a $12 \mathrm{~mm}$. Tadpole; the section passing through the middle of the length of the head kidney. $\times 44$.

A, aorta. AP, pulmonary artery. BH, medulla oblongata. $\mathbf{C H}$, notochori. GI, portion of a gill. GM, glomerulus. $\mathbf{K} \mathbf{P}$, tubule of head kidnev. $\mathbf{K S}$, secoud nephrostome. LA, commencing fore limb. LG, root of lungs. NY, sympathetic ganglion. OP, opercular eavity. TI, intestine. TO, œsophagus. V.4, fourth ventriele. VC, posterior cardinal vein. VH, posterior vena cava. VP, pulmonary vein. $\mathbf{W}$, liver. $\mathbf{X}$, ganglion of spinal nerve. $\mathbf{X}^{\prime}$, thin roof of fourth ventricle.

described above (p. 182), as a sacculated outgrowth from the ventral and outer wall of the aorta, bulging outwards into the body cavity opposite the nephrostomes. At the time of hatching of the tadpole (Fig. 82) the dorsal portion of the body cavity is practically the only part present; for although the splitting of the mesoblast extends down the sides of the body to the ventral surface, yet the two layers, somatic and splanchnic, 
are in contact with each other, owing to the distension of the body by the mass of food-yolk, except at the upper or dorsal angle. The glomerulus, therefore, appears at this stage to lie in a special cavity, which later on opens into the general body cavity, as the yolk becomes absorbed and the abdominal viscera acquire more definite form.

At a later stage still, the part of the body cavity in which the glomerulus lies becomes partially boxed in, by fusion of the outer wall of the lung with the peritoneal covering of the head kidney ( $c f$. Fig. 84). This inclosure is only an incomplete one, as the part of the cœlom lodging the glomerulus still opens into the general body cavity, both in front of the root of the lung and behind this.

The position of the glomerulus, opposite the nephrostomes of the head kidney, and the fact that its development, both progressive and retrogressive, keeps pace with that of the head kidney, point to the existence of some close physiological connection between the two organs, though it is not easy to imagine of what precise nature this connection can be.

The glomerulus, with the part of the body cavity in which it lies, may be compared to one of the Malpighian bodies of the adult kidney; the glomerulus itself corresponding to the capillary knot of vessels; the localised part of the colom in which it lies corresponding to the capsule of the Malpighian body; and the nephrostonial tube leading from the colom answering to the neck of the Malpighian body.

\section{The Wolffian Body.}

The development of the Wolffian body, or kidney, commences in tadpoles of about 10 to $12 \mathrm{~mm}$. length, by the formation of the Wolffian tubules (Fig. 83, KM). 'These appear as a series of small, paired masses of mesoblast cells, lying along the inner sides of the segmental ducts, between these and the aorta. The Wolffian tubules develop from behind forwards; the hindmost, and at first the largest pair, being a short distance in front of the cloaca, and the most anterior pair being about three segments behind the head kidneys. They are at first segmentally arranged, one pair corresponding to each pair of muscle segments or myotomes; but this definite arrangement is early lost in the hinder region. 
The Wolffian tubules are, at first, solid masses of spherical cells; these arise in the mesoblast of the body wall, and are for a time independent of both the peritoneum and the segmental ducts. The cellular masses soon become elongated into solid rods; by separation of the component cells along their axes, the rods become tubes; and these tubes, growing outwards and towards the dorsal surface, meet with the segmental ducts and open into these.

At the opposite end of each tubule, a Malpighian body is formed, the end of the tubule being dilated into a bulb-like enlargement, which becomes doubled up, to form the Malpighian capsule, by the ingrowth of a knot of blood-vessels derived from a branch of the dorsal aorta ( $c f$. Fig. 87, A).

From the neck, or part of the tubule immediately beyond the Malpighian body, a short solid rod of epithelial cells arises, which grows towards the peritoneum and fuses with this. By separation of the cells the rod becomes tubular, and opens at its outer or peritoneal end into the body cavity. The peritoneal opening is a nephrostome (Fig. $87, \mathrm{Ks}$ ), and the tube into which it leads may be called the nephrostomial tubule.

Although the walls of the nephrostomial tubules are at first continuous with the necks of the Malpighian bodies, it is very doubtful whether the nephrostomial tubules ever open into the Wolffian tubules; and, shortly after their first appearance, in tadpoles of 18 to $20 \mathrm{~mm}$. length, the nephrostomial tubules break away completely from the Wolffian tubules, and acquire openings at their inner ends into the renal veins, on the ventral surface of the kidney. This curious arrangement persists throughout life. On the ventral surface of the kidney of an adult frog there are as many as two hundred or more of these nephrostomes present, as funnel-like depressions or mouths: these lead into short tubes, lined by flagellated epithelial cells, and running obliquely inwards into the substance of the kidney, where they end by opening into the renal veins.

The anterior three or four pairs of Wolffian tubules never complete their development, but early undergo fatty degeneration. The hinder tubules are at first segmentally arranged, but, owing to the formation of new tubules, soon become much more numerous than the segments in which they lie. They increase greatly in length, become markedly convoluted, and soon 
become massed together, with the blood-vessels in relation with them, to form the definite Wolffim bodies or kidneys (Figs. 85, 86 , and $87, \mathrm{kM})$.

\section{The Wolffian Ducts or Ureters.}

The segmental duct is at first the duct for the head kidney

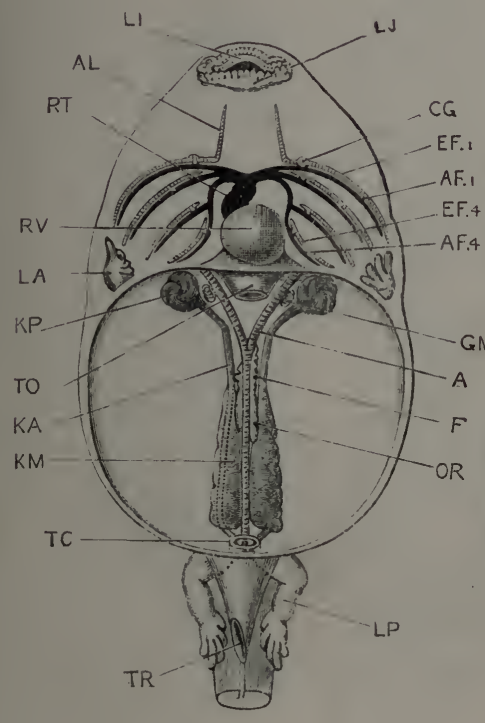

FIG. S.).

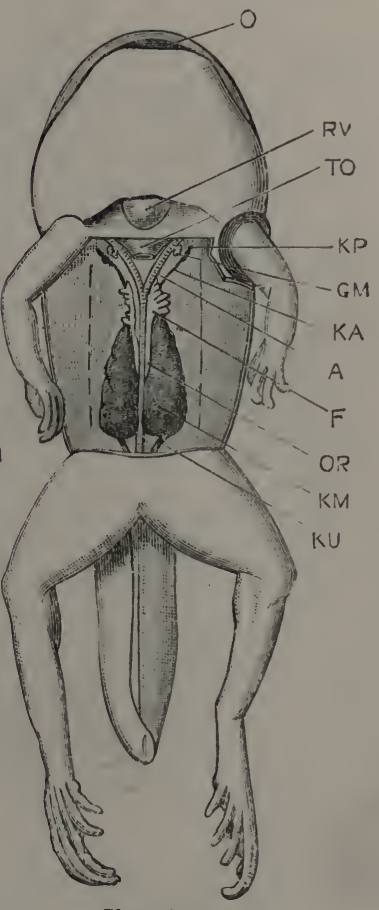

FIG. 86 .

FIG. 85. - A $40 \mathrm{~mm}$. Tadpole, dis-ected from the rentral surface to show the heart, the branchial ressels, and the urinary and reproductive organs. The tail, and the alimentary canal, from the osophagus to the rectum, have been removed. $\times 4$.

FIG. 86.-A Tailed Frog, cluring the metamorphosis, dissected from the ventral surface to show the urinary and reproductive organs. The alimentary canal lias been removecl. $\times 3 \frac{1}{2}$.

A, aorta. AF.1. AF.4, afferent vessels of first anl fourth branehial arches. AL, lingual artery. CG. earotil gland. EF.1, EF.4, efferent vessels of first and fourth hranchial arehes. F, fat borly: GM. glomerulus. KA, segmental duct. KM. Wolttian boly. KP, heal killiey. KU, Wolffian duet or ureter. IA, fore limb. LI, upper lip. LJ, lower lip. LP, lime limb. O mouth. OR, genital ridge. RT, trunchs arteriosus. $R V$, ventricle. TC. cloaen. TO, œsophagus. TR, aperture of rectal $\leqslant$ prout.

alone (Fig. $71, \mathrm{kA}$ ); in the later stages (Figs. 83 and 85 ), it serves for both the head kidney and the Wolffian body. 
During the metamorphosis, and shortly before the complete disappearance of the tail, the head kidney separates from the segmental duct. For a short time, a portion of the segmental duct still persists in front of the Wolffian body; but this soon

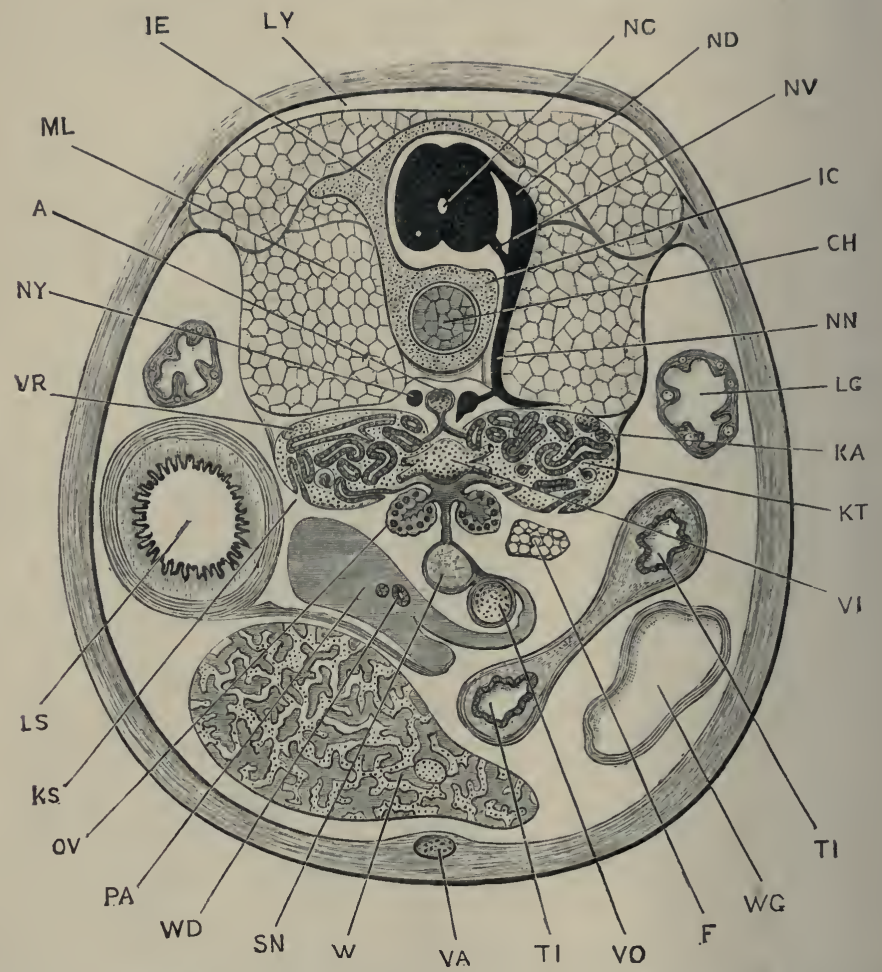

FIG. 87.-Transverse section through the hinder part of the body of a Tailed Frog during the metamorphosis ( $c f$. Fig. 86 ). On the right side a spinal nerve is shown, on the left side the vertebral column. $\times 17$.

A, aorta. CH, notochord. F, fat body. IC, centrum of vertebra. IE, neural arch of vertebra. KA. Wolffian duct. KS, nephrostome. KT, Wolffian boily. LG, lung. LS, stomach. LY, subcutaneous lrmph space. ML, myotome. NC, spinal cord. ND, dorsal root of spinal nerve. NN, spinal nerve, main trunk. NV, ventral root of spinal nerve. NY, sympathetic nerve ganglion. OV, ovary. PA, pancreas. SN, spleen. TI, intestine. VA, anterior abrlominal vein. VI, posterior vena cava. VO, hepatic portal vein. VR, renal portal vein. W, liver. WD, bile duct. WG, gall bladiler.

loses its lumen, undergoes degenerative changes, and disappears : the remaining, or posterior part of the segmental duct, which is now connected with the Wolffian body alone, is from this time spoken of as the Wolffian duct or ureter.

The vesicula seminalis is a glandular body developed in con- 
nection with the hinder end of the Wolffian duct, close to the cloaca. It attains a large size in the male frog, but is absent or ruclimentary in the female.

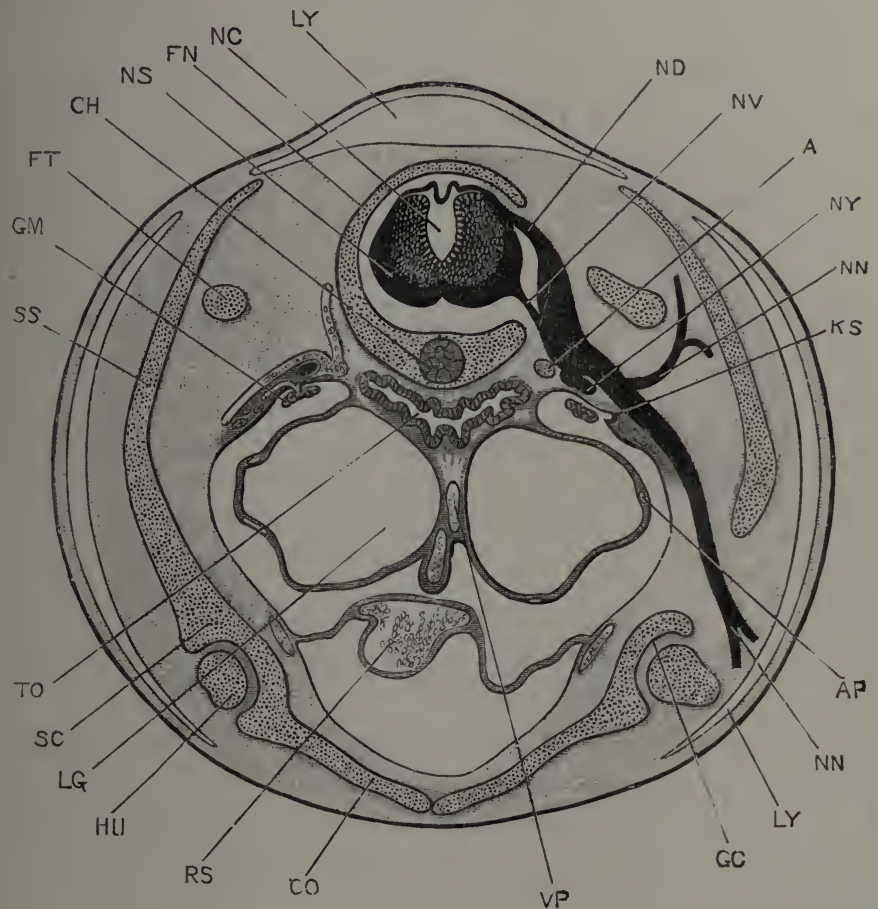

FiG. 88.-Transverse section through the anterior part of the body of a Tailed Frog during the metamorphosis ( $f$. Fig. 86). The section passes through the second spinal nerve on the right side, and on the left side immediately in front of this nerve. $\times 14$.

A, aorta. AP, pulmonary artery. CH, notoclioril. CO, coracoid portion of shoulder girlle. $\mathbf{F} \mathbf{N}$, neural areh of vertebra. FT, transverse process of third vertebra. GC, glenoid cavity of shoulder girdle. GM, glomernlus. HU, hearl of humerus. KS, third or posterior nephrostome of head kidney. LG, lung. LY, subcutaneous lympli space. IN , spinal canal. ND, dorsal root of second spinal nerve. NN , dorsal and ventral branches of second spinal nerve. NS, spinal coril. NV, ventral root of second spinal nerve. NY, sympathetic nerve ganglion. RS, siuns venosus. SC, scapular fortion of shouliler girlle. SS, supra-seapular portion of shoulder girdle. TO, osophagus. VP, pulmonary vein.

\section{j. The Muillerian Ducts.}

The Müllerian ducts form the oviducts of the female frog; they are present in the male as well, but are of very small size, and apparently of no functional importance. The early stages in the development of the Müllerian ducts are difficult to investigate, and have not yet been determined with absolute certainty; 
but there is no doubt that the greater part of the length, and perhaps the whole, of the duct arises independently of the Wolffian duct; and apparently from a modified strip of peritoneal epithelium which runs parallel to the Wolffian duct, along its outer side.

'Towards the close of the metamorphosis, and during the absorption of the tail, a patch of modified peritoneal epithelium appears on the ventral surface of the now rapidly disappearing head kidney, below and to the outer side of the nephrostomes, of which, as a rule, only one persists at this stage (Fig. 88, $\mathrm{ks}$ ). This patch of epithelium differs from the general peritoneal epithelium in its cells being columnar instead of squamous in shape. A longitudinal groove forms on the surface of this patch of columnar epithelium, to the outer side of the nephrostome; and, by fusion of its lips, the groove becomes a tube, opening in front into the body cavity, and ending blindly behind. This tube is the first formed part of the Müllerian duct; it lies very close to the anterior end of the segmental duct, but it is not clear that there is any connection between the two; it is quite independent of the nephrostome, which closes up and disappear's very shortly after this stage.

The Müllerian duct extends forwards, as an open groove, some distance in front of the point at which the duct is first formed; and, by closure of the lips of the groove from behind forwards, the mouth of the duct is carried forwards, outwards, and downwards, round the anterior end of the body cavity, to its adult position at the root of the lung.

The growth backwards of the Müllerian duct is effected by a longitudinal band of cells, which appears a little to the outer side of the Wolffian duct, but quite independent of this, and apparently derived directly from the peritoneal epithelium. This band, which is continuous with the hinder end of the Müllerian duct, ultimately becomes tubular, and acquires posteriorly an independent opening into the cloaca.

In the male frog the Müllerian duct stops at this stage. In the female it undergoes further changes by which it becomes converted into the oviduct. It increases in length, becoming sinuous, and ultimately convoluted along the greater part of its length; its walls thicken greatly, and the epithelium lining them gives rise to the gelatinous secretion which is poured ont orer the 
egg's as they pass down the duct, and which, on reaching the water, swells up to form the mass of the frog's spawn. 'The hincler end of the oviduct remains thin-walled, but dilates very greatly, forming a capacious sac in which the eggs accumulate before being laid.

\section{The Reproductive Organs.}

The development of the reproductive organs has already been described (p. 94), but a few further details may be given here, more especially with regard to the relation between the reproductive organs and the Wolffian bodies, and the development of the fat bodies, or corpora adiposa.

The reproductive organs appear in tadpoles of about $10 \mathrm{~mm}$. length, as a pair of ridge-like folds of the peritoneum, lying along the inner borders of the Wolffian bodies, close to the root of the mesentery (Fig. 85, or, and 87, ov). These genital folds soon become more conspicuous, and undergo changes which have already been described in detail, by which the germinal cells or gonoblasts are formed.

As the Malpighian bodies develop on the tubules of the Wolffian body, those lying nearest to the genital ridge give off hollow tubular diverticula, which, arising from the capsules of the Malpighian bodies, grow towards the median plane, and into the substance of the genital ridge ( $c f$. Fig. 87), where they form the so-called tubuliferous tissue.

So far, the processes of development are the same in both sexes, but from this point differences occur. In the ovary of the female the tubules of the tubuliferous tissue expand very greatly, and give rise to the large axial cavities of the ovary, of which there are usually about fifteen in the adult.

In the male, the outgrowths from the Malpighian capsules acquire still more intimate relations with the reproductive organs than in the female, and become the vasa efferentia of the adult, which convey the spermatozoa from the testis to the kidney. The Malpighian bodies with which the vasa efferentia are connected are at first perfectly normal; but later on they undergo retrogressive changes, and by the end of the first year their glomeruli have disappeared, and the Malpighian bodies themselves merely remain as slight ampulliform enlargements of the tubules, which have now completely lost their kidney structure 
and function. 'The tubules of these Malpighian bodies do not join those of the other parts of the kidney, but open at once into the terminal or collecting tubules of the Wolffian body, so that there is practically a sharp separation of the kidney into two portions, excretory and reproductive respectively.

\section{The Fat Bodies.}

The fat bodies, which are conspicuous and characteristic structures in the adult frog, are formed by fatty degeneration of the anterior ends of the genital ridges. In tadpoles of about $40 \mathrm{~mm}$. length (Fig. 85) each genital ridge becomes divided by a slight constriction into two parts of about equal length. Of these, the posterior part, or, forms the smooth genital ridge; while the anterior part, $F$, is irregularly notched along its ventral surface, and is already commencing to undergo fatty degeneration. In the later stages (Fig. 86, F) the fat bodies increase greatly in size, and are produced at their margins into the characteristic finger-like lobes of the adult.

Histologically, the changes by which the fat body is formed are due to the accumulation of fat within cells, which are at first indistinguishable from those of the hinder, or reproductive, part of the genital ridge.

\section{DEVELOPIIENT OF THE SKELETON AND THE TEETH.}

\section{The Vertebral Column.}

The earliest skeletal structure, and for a time the only one, is the notochord, the development of which, from the mid-dorsal wall of the mesenteron, has already been described (p. 109).

The notochord (Fig. 61, CH) is a cylindrical rod, extending from the blastopore to the pituitary body; and growing backwards along the tail as this is formed (Fig. 69, CH). It consists of vacuolated cells, filled with fluid : the cells are all alike, and their nuclei, which at first are conspicuous, disappear in the later stages. Around the notochord a delicate structureless elastic sheath is formed at an early period; this is really double, consisting of a thicker inner, and an outer thinner layer; both layer's are homogeneous, and cuticular in nature.

About the time of appearance of the hind limbs as bucts, a 
delicate skeletal tube, at first soft, but soon becoming cartilaginous, is formed from the inesoblast surrounding the notochord (Fig. 84, CH). This grows upwards at the sides of the spinal cord as a pair of longitudinal ridges, with which a series of cartilaginous arches, which appeared at the sides of the spinal cord at a slightly earlier stage, soon become continuous (Figs. $87,88)$.

By the appearance of transverse lines of demarcation, this axial skeleton becomes cut up into a series of nine vertebræ, followed by a posterior unsegmented portion, which later on gives rise to the urostyle. The vertebræ are cut off in order, from before backwards, and the division at first involves the cartilaginous tube alone, the notochord remaining as a continuous structure until the complete absorption of the tail, at the end of the metamorphosis (Fig. 89).

Shortly before the metamorphosis, thin rings of bone, slightly constricted in their middles so as to be hour-glass shaped in section, are developed in the membrane investing the cartilaginous sheath of the notochord; these from their first appearance correspond with the nine vertebræ, to the bony centra of which they give rise. Like the cartilaginous vertebræ, they develop in order from before backwards.

In the intervertebral regions, between the successive bony rings, annular thickenings of the cartilaginous sheath occur, which grow inwards so as to constrict, and ultimately obliterate the notochord. Each of these intervertebral rings, after the metamorphosis, becomes divided into anterior and posterior portions, which fuse with the bony centra of the adjacent vertebre, and ossify to form their articular ends.

From the circumference, and from the articular ends of each vertebra, ossification gradually spreads inwards; but a small portion of the notochord persists in the middle of each centrum for a long time, or even throughout life.

The vertebræ do not lie opposite the muscle segments or myotomes, but alternate with these; so that each vertebra is acted on by two myotomes on each side, one pulling it forwards, and the other backwards.

The transverse processes arise independently of the corresponding vertebræ, but very early fuse with these. They extencl along the connective-tissue septa between the successive 
inyotomes, and very probably correspond to the ribs of other Vertebrates.

The urostyle is the hindmost part of the axial skeleton, which
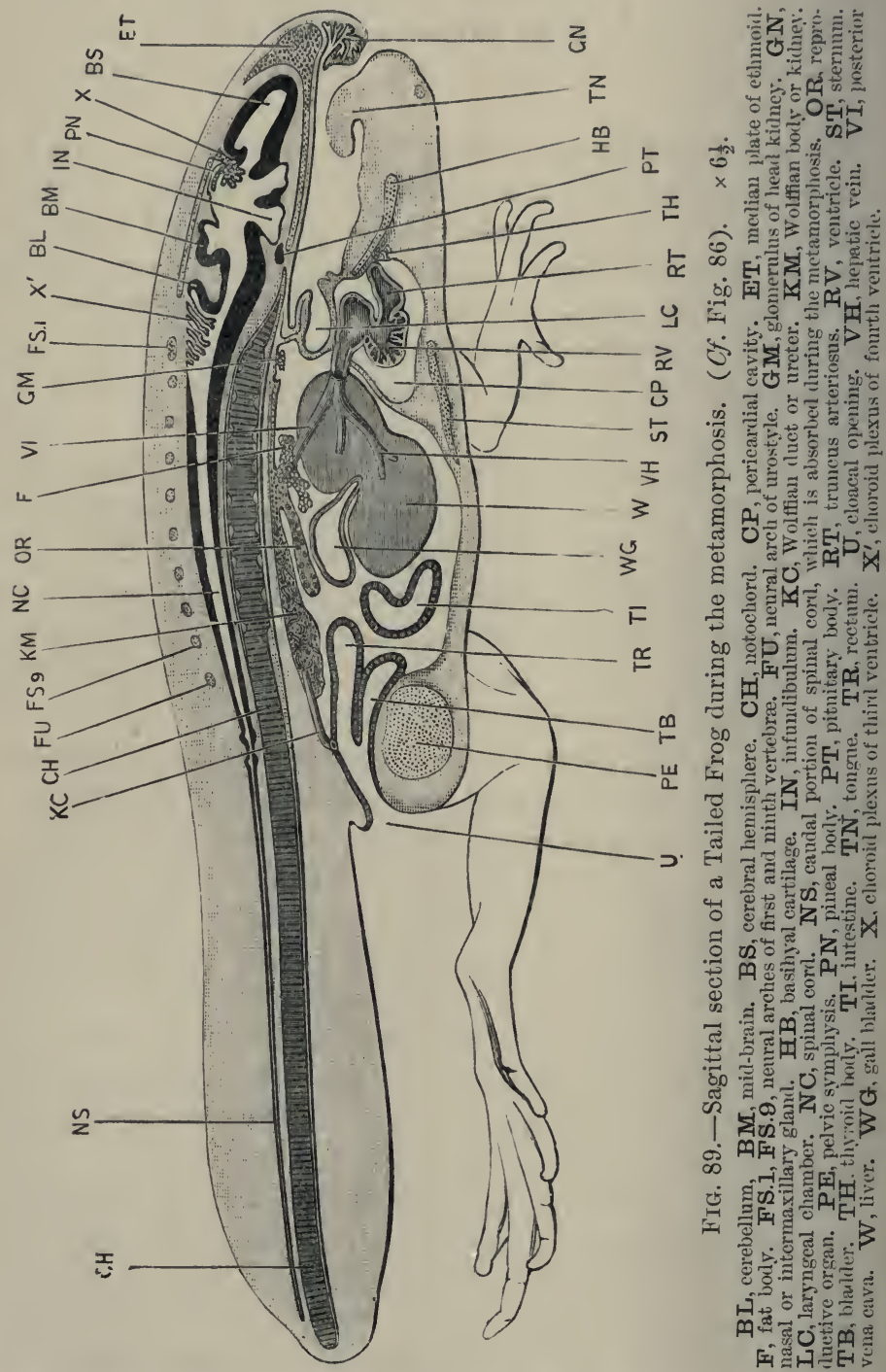

does not become segmented into vertebræ. The cartilage of the urostyle forms a continuous tube in fiont, surrounding the noto- 
chord: posteriorly, it extends back as a flattened epichordal plate above the notochord, and a thick median hypochordal rod below it. During the shortening of the tail, on the completion of the metamorphosis, the epichordal and hypochordal cartilages extend and meet, so as to completely surround the notochord. Later still, the cartilage becomes in great part replaced by bone; but the hinder end persists as a terminal plug of cartilage throughout life.

The anterior end of the notochord, imbedded in the base of the skull, is gradually encroached upon by the cartilage and bone around it; and ultimately, in half-grown frogs, is completely absorbed.

\section{The Skull.}

a. The skull of the tadpole. The skull of the tadpole consists almost entirely of cartilage; none of the bones of the skull, with the exception of the parasphenoid, appearing until a short time before the metamorphosis.

In the cartilaginous skull of the tadpole two main elements may be distinguished:

(i) The cranium, with the olfactory and auditory capsules, which may conveniently be taken together.

(ii) 'The visceral skeleton, which consists of a series of cartilaginous bars developed in the visceral arches, and encircling the pharynx.

Speaking generally, the skull as a whole develops from before backwards; the first formed parts being at the anterior end, in connection with the jaws; and the hinder end of the skull being very imperfect in the early stages of tadpole life.

True cartilage does not appear until the tadpole is about 10 to $11 \mathrm{~mm}$. in length; i.e. when the opercular covering of the gills is almost completed, and the hind limbs are visible as minute buds at the base of the tail. Long before true cartilage is present, however, the skeletal elements can be readily distinguished, as tracts of condensed and modified mesoblast.

The first stage in the histological differentiation, by which cartilage is formed, consists in a number of mesoblast cells becoming more closely compacted together; these cells have large nuclei and very scanty protoplasm, and are further distinguished from the surrounding mesollast cells by having 
only a slight tendency to develop processes, and by being almost completely devoid of yolk-granules.

FIG. 90

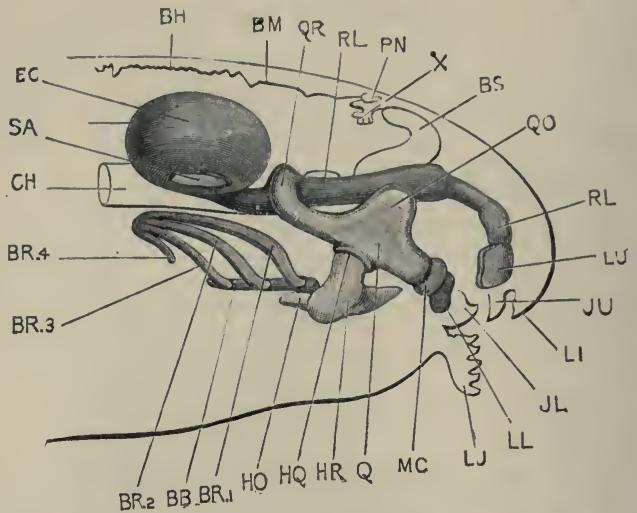

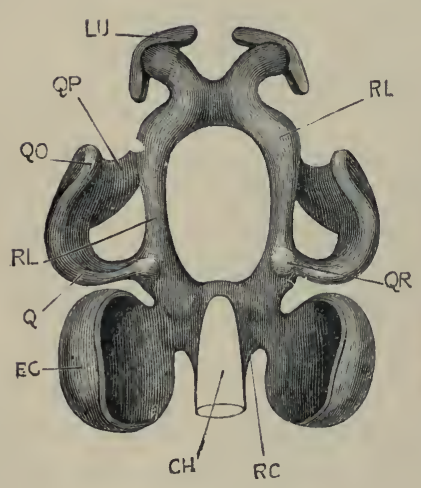

FIG. 91.

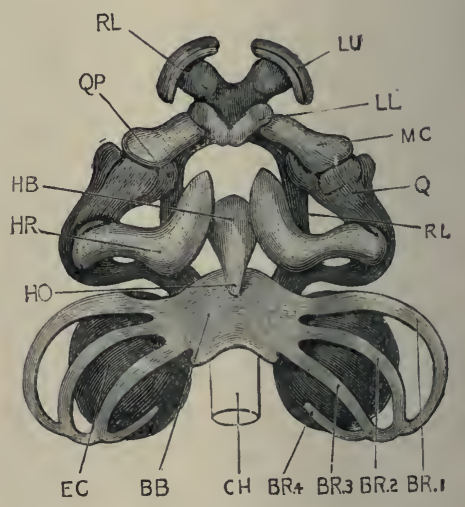

FIG. 92.

FIG. 90.-The skull of a $12 \mathrm{~mm}$. Tadpole, seen from the right side. The notochord, the brain, and the entire head are represented in outline, in order to show the relations of the skull to them. $\times 30$.

FIG. 91. -The same skull from the dorsal surface. The lower jaw, and the hyoidean and branchial bars are omitted. $\times 30$.

FIG. 92. - The same skull from the ventral surface. $\times 30$.

BB, basi-branchial. BH, roof of hind-brain. BM, roof of mid-brain. BR.1. BR.2, BR.3. BR.4, first, second, third, and fourtl branchial bars. BS, cerebral hemisphere. CH, notochort. EC, auditory capsule. HB, basihyal. HO, urohyal. HQ, articulation of ceratohyal with quadrate. HR, ceratohyal. JL, lower jaw. JU, upper jaw. LI, upper lij). LJ, lower lip. IL, lower labial cartilage. LU, upper labial cartilage. MIC, Meckel's eartilage. PN, pineal bodv. Q, quadrate. QO orbital process of quadrate. $\mathbf{Q P}$, palato-pterygoid process. $\mathbf{Q R}$, commection of quadrate with trabecula. RC, parachordal cartilage. RL, trabccula eranii. SA, membranous patch in the outer wall of the auditory capsule, in which the stapes is dereloperl at a slightly later stage. $\mathbf{X}$. choroil plexus of third ventriele.

In the next stage the cells become still more closely com- 
pacterl, so that the nuclei of adjacent cells lie very close to one another, while the protoplasm of the several cells becomes fused into a continuous plasma or matrix. Very slight further changes convert this into true cartilage.

(i) The cranium or brain case. This, in its fully formed condition (Fig. 93), is an unsegmented cartilaginous tube, incomplete dorsally, and inclosing the brain. It is developed in the following manner.

Shortly after hatching of the tadpole, a pair of longitudinal rods of condensed mesoblast, the trabeculæ cranii, appear, one on either side of the anterior end of the brain. 'These are at first entirely in front of the notochord ( $c f$. Fig. 64): their posterior ends lie alongside the fore-brain; while anteriorly they are Hattened dorso-ventrally, and fused to form a horizontal plate lying between the two nasal sacs; the extreme anterior ends of the trabeculæ separate again, and bend downwards into the upper lip, which they support.

As the tadpole grows, the trabecula rapidly extend backwards along the sides of the infundibulum, and soon reach the anterior end of the notochord; they continue backwards alongside the notochord, and in close contact with this, as a pair of horizontal rods, the parachordals, which with the notochord form the floor of the brain case.

In tadpoles of $12 \mathrm{~mm}$. length (Figs. 90, 91, and 92) the trabecula have become cartilaginous; their extreme anterior ends chondrify independently as a pair of thin curved plates, the upper labial cartilages, LU, which support the upper lip. Behind the labial cartilages the two trabeculæ remain separate for a short distance, but soon fuse to form a median horizontal plate of cartilage, lying between the two nasal sacs, and supporting the extreme anterior end of the brain ; behind this they are continued backwards as two parallel bars of cartilage, lying at the sides of, and slightly ventral to the brain; behind the infundibulum, and just in front of the anterior end of the notochord (Fig. 91), the two trabecula are again connected by a narrow transverse bridge of cartilage, beyond which they continue backwards, as two narrow parachordal bands, RC, along the sides of the notochord, to the hinder end of the skull.

In the later tadpole stages the trabecula extend horizontally 
inwards to form the floor of the skull, while their outer edges grow upwards, forming low ridges along the sides of the brain in the ethmoidal region, in front of the eye, and in the occipital region behind the ear. Opposite the eyes, the side walls of the cranium are formed by a pair of cartilaginous plates, which appear independently, in the membrane at the sides of the brain, and soon extend ventralwards to meet, and fuse with, the trabeculæ.

'T'he side walls and roof of the hinder' part of the cranium are mainly formed by the auditory capsules. At an early period the mesoblast surrounding each auditory vesicle forms a definite capsule around it: in this a thin shell of cartilage is formed, first in the outer wall, but soon spreading round to the floor, where it becomes continuous with the outer edge of the parachordal cartilage (Figs. 70, 90, 91, EC). This cartilaginous auditory capsule spreads more slowly over the dorsal surface of the anditory vesicle, and then extends downwards, between the brain and the ear, to form the side wall of this part of the skull. Later still, in tadpoles of about $20 \mathrm{~mm}$. length, the cartilage of the dorsal borders of the auditory capsules spreads inwards across the top of the brain case, and so completes its roof in this region.

'The hindmost end, or occipital region of the skull, is formed by upgrowth of the edges of the parachordal cartilages. This occurs very slowly, and quite independently of the auditory capsules; it is not completed until shortly before the metamorphosis.

In the outer wall of each auditory capsule, a large oval or circular patch remains unchondrified: this is the fenestra ovalis (Fig. 90, sA). In the middle of this membranous patch the stapes appears, in tadpoles of about $16 \mathrm{~mm}$. length, as a cartilaginous nodule ( $c f$. Fig. 93, sa), the further development of which will be considered later.

At the anterior end of the cranium, the nasal or olfactory organs become roofed in by cartilage, which arises as a vertical crest from the upper surface of the median internasal plate formed by the trabeculæ, and spreads out right and left as a pair of thin horizontal plates, covering over the olfactory organs, and forming the cartilaginous olfactory capsules (Fig. 93, of).

It thus appears that the trabeculæ, with their posterior continuations, the parachordals, give rise to the floor of the cranium along its whole length; they also, by upgrowth of their edges, 
form the sides and the roof of the extreme anterior and posterior ends of the skull. In the orbital region the side walls of the skull arise independently, but grow down to, and fuse with, the trabeculæ; while the roof remains membranous. Finally, in the auditory region, both the sides and roof are formed as extensions of the auditory capsules.

(ii) The visceral skeleton. This consists of a series of cartilaginous hoops or bars (Figs. 90, 92), developed in the mandibular, the hyoidean, and the four branchial arches, which tend to encircle the pharynx. Each hoop consists of right and left halves, which are independent at their dorsal ends, but fused or closely connected ventrally. Like the cranium itself, the visceral skeleton develops from before backwards.

The mandibular bar is one of the very earliest parts of the skull to be developed; it may be recognised at the time of hatching of the tadpole as a curved band of condensed mesoblast, lying transversely across the floor of the mouth, close to its anterior end. The outer ends of the bar are enlarged, and produced upwards into rertical processes, which lie at the sides of the mouth cavity, and are continuous above with the trabecula cranii at a level between the nose and eye; a connection which afterwards gives rise to the palato-pterygoid bars.

After hatching, the mandibular bar grows rapidly, and undergoes important changes. It becomes divided on each side into three parts :-(i) a small anterior segment, which later on becomes the lower labial cartilage, and which is continuous across the median plane with its fellow of the opposite side; (ii) a small middle segment, which becomes later the basis of the lower jaw, or Meckel's cartilage; and (iii) a hindmost or quadrate segment, which is much the largest of the three, and which is connected with the trabecula by the palato-pterygoid bar mentioned above, while from its outer side a dorsally directed. leaf-like, orbital process projects upwards.

On the appearance of cartilage, in tadpoles of about $12 \mathrm{~mm}$. length, the condition of the mandibular bar is as shown in Figs. 90, 91, and 92. The hinder part of the bar forms the quadrate cartilage, Q, a stout horizontal subocular bar, which lies parallel to the trabecula, but some distance from this, and along the under and outer surface of the eyeball. The hinder end 
of the quadrate turns sharply inwards, QR, and is comnected with the trabecula just in front of the ear capsule, Ec. From the onter edge of the quadrate the prominent, vertical, orbital process, $\mathrm{QO}$, projects upwards; and the anterior end of the quadrate, like its posterior end, turns sharply inwards, to form the palato-pterygoid bar, QP, which is fused with the trabecula, between the nose and the eye. The quadrate may thus be described as a horizontal bar of cartilage, lying to the outer side of the trabecula, and connected with this by two struts, one in front of the eye and one behind it.

The second segment of the mandibular bar, or Meckel's cartilage, MC, is a short, stout bar of cartilage, which l'uns inwards and forwards across the floor of the mouth. The third segment, or lower labial cartilage, LL, is a much smaller bar at the inner end of Meckel's cartilage, which meets, or is actually fused with, its fellow of the other side in the median plane.

'The chief further changes that occur in the mandibular bar during the later periods of tadpole life (cf. Fig. 93) :- are (i) a progressive diminution in size of the orbital process, QO ; and (ii) the development of an additional connection between the hinder end of the quadrate and the auditory capsule, by means of an otic process (Fig. 93, QE).

At the time of the metamorphosis, very considerable and important changes take place, which will be described later on.

The hyoid bar appears directly after the mandibular bar, and immediately behind this. At first the right and left bars are separate ventrally, but they soon become connected by a median plate. The hyoid bar thickens rapidly, and at $12 \mathrm{~mm}$. (Figs. 90,92 ) forms a broad, somewhat $\mathbf{S}$-shaped plate of cartilage, the ceratohyal, HR, which lies across the floor of the mouth. The outer end of the ceratohyal is enlarged, and turned upwards, and articulates with the ventral surface of the quadrate about the middle of its length (Fig. 90, $\mathrm{HQ}$ ). The inner ends of the two ceratohyals are connected, across the floor of the mouth, by a median basihyal cartilage, $\mathrm{HB}$, the posterior end of which grows back as a keel-like urohyal process ( $c f$. Fig. 65, нв), which separates the two halves of the thyroid body from each other.

The branchial bars are a series of four pairs of cartilaginous rods, developed in the four branchial arches of each side. They appear in order, from before backwards, as slender curved bands 
of cartilage (Fig. 92, BR.1 to BR.4). 'The dor'sal ends of the four bars of each side very early become connected together. The ventral ends are at first independent, but the ends of the first branchial bars early unite to form a broad median basibranchial plate (Fig. 92, BB), with which the ventral ends of the hinder branchial bars soon join.

The chief characteristic of the skull of the tarlpole is the early development, and large size, of the cartilages of the extreme anterior end, in connection with the jaws. 'The early formation and great size of the anterior ends of the trabeculæ, the large labial cartilages, the early attachment of the mandibular arch to the anterior end of the trabecula by the palato-pterygoid bar, the enormous and singularly shaped orbital process of the quadrate, and the massive character of the hyoid arch in the floor of the mouth, are all connected with the need for a firm supporting skeleton for the horny jaws, and an extensive surface of origin for the muscles by which these jaws are worked. All these are characters which concern the tadpole, and not the frog; and they are all lost, or greatly modified, in the adult animal.

b. The skull of the frog. About the time of the metamorphosis, the skull undergoes important changes, by which the adult condition and proportions are attained.

A complete cartilaginous floor is formed to the skull, between the two trabeculæ. The hinder end of the skull acquires definite shape: the parachordals increase greatly in size, growing inwards so as to encroach upon, and ultimately to obliterate the notochord; they fuse with the auditory capsules and grow up behind these to complete the occipital ring. The side walls and roof of the skull are formed in the manner already described (p. 204). The nasal capsules are formed by expansion of the anterior ends of the trabeculæ; the upper labial cartilages persisting as the pro-rhinal cartilages, which bound the anterior nostrils in front. A thin cartilaginous shell is formed in the sclerotic coat of each eye, but remains throughout life free from the skull proper.

In the mandibular arch great modifications occur, associated with the change from the small ventral mouth of the tadpole to the wide, slit-like, and terminal mouth of the frog. The anterior' end of the quadrate rotates downwards and backwards, causing great lengthening of the palato-pterygoid bar (Figs. 90 and 93, 
QP), which becomes drawn out to form the framework of the upper jaw. At the same time, Meckel's cartilage, Mc, forming the basis of the lower jaw, becomes also greatly lengthened, while the lower labial cartilages disappear. At the time of the metamorphosis, the quadrate still slopes downwards and forwards (Fig. 93); but the rotation continues until the quadrate becomes vertical, and finally, before the end of the first year, acquires the inclination downwards and backwards so characteristic of

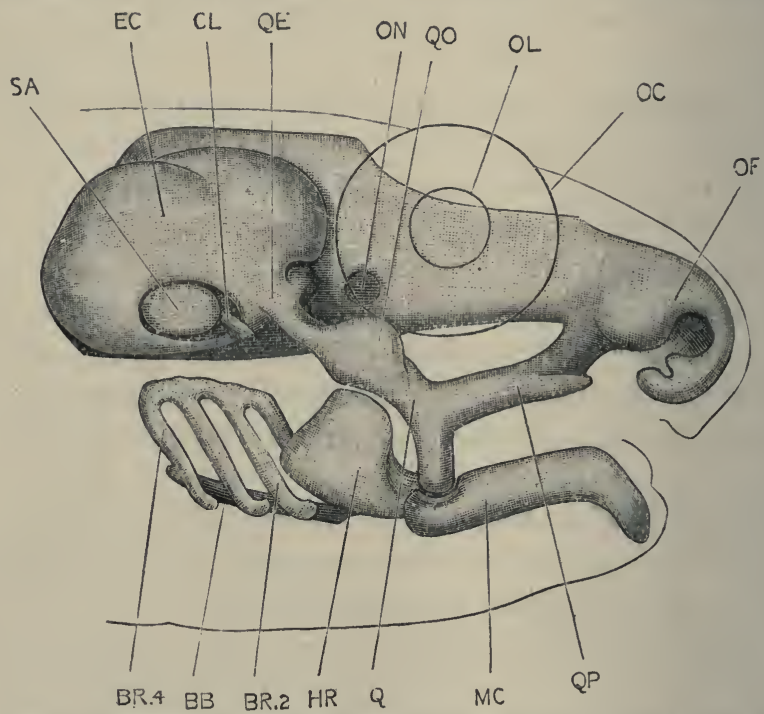

FIG. 93.- Skull of a Tailer Frog towards the close of the metamorphosis, from the right side. The head and eye are represented in outline. $\times 13$.

BB, basibranchial. BR.2, BR.4, secont and fourth branchial bars. CL, columella. EC, auditory capsule. HR, ceratolyal. MC, Meckel's cartilage. OC, outline of eve. OF, olfactors eapsule. OL, outline of lens. ON, foramen for optic nerve. $Q$. quairate. QE, conmection of quadrate with auditory capsnle. QO, orbital process of quarirate. QP, palato-pterygoid process. SA, stapes.

the adult (Fig. 94). The orbital process of the quadrate early becomes inconspicuous (Fig. 93, Q0), and finally disappears.

The hyoid bar, which is massive in the tadpole, becomes very slender in the frog. It gradually elongates, extending dorsalwards until it meets, and fuses with, the ventral surface of the auditory capsule (Fig. 94, B).

'The branchial bars become more and more slender' as the gills begin to shrink, and ultimately disappear almost completely. The basihyal and basibranchial cartilages give rise to the body of the hyoid (Fig. 94, H) : from the ventral ends of the fourth 
branchial bars, the backwardly directed thyrohyals (Fig. 94, T) are formed; while the rest of the fourth branchial bar, and the whole of the first three bars disappear.

The fenestra ovalis has been described above as a large aperture, left in the outer and ventral wall of the auditory capsule (Fig. 90, SA). This aperture is closed by membrane, and in this membrane, shortly before the metamorphosis, the stapes is formed as a disc-like plug of cartilage (Fig. 93, sA).

The columella appears, in tadpoles of about $40 \mathrm{~mm}$. length (cf. Fig $93, \mathrm{CL})$, as a minute bar of cartilage immediately in front of the stapes, and with its outer end directed forwards. At the time of the metamorphosis the columella grows rapidly,

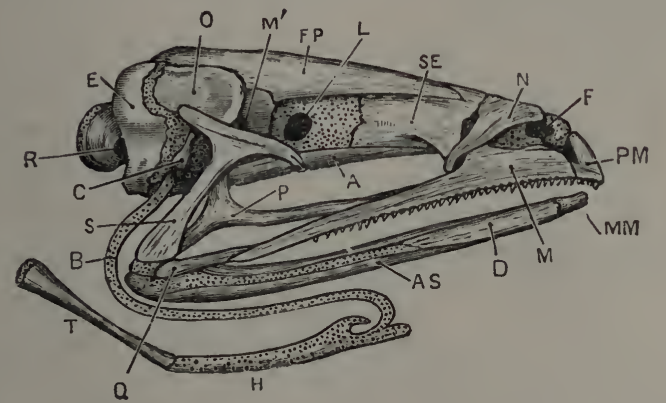

FIG. 94.-The skull of an adult Frog, from the right side. $\times 2$.

A. parasphenoid. AS, angulosplenial. B, anterior cornu of lyyoil. C, colnmellin D, ilentary. $E$, exoccipital. F, nostril. FP, fronito-parietal. $H$, body of hyoil. $L$ aperture for exit of optic nerve. IM. maxilla. 'M.M, mento-meckelian. $\mathbf{M}^{\prime}$. aperture for exit of fifth anl seventh nerres. $\mathbf{N}$, nasal. $\mathrm{O}$, pro-otic. $\mathbf{P}$, pterygoil. $\mathrm{PM}$, premaxilla. $Q$, quadratojngal. $R$, aperture for exit of ninth anil tenth nerves. $S$. squamosal. SE, sphen-etlimoin. T, posterior cornu of hyoid, or thyroliyal.

and becomes rotated outwards, so that its outer end comes into close relation with the tympanic membrane, while its inner end fuses with the stapes. At first, the columella lies in the dorsal wall of the tympanic cavity, but this latter gradually extend: upwards around it, until the columella acquires its adult relations, and appears to cross the tympanic cavity ( $c f$. Fig. 68, c, p. 144). In its actual development, the columella of the frog shows no sign of any connection with either the hyoid or mandibular bars, but comparison with other vertebrates renders it very possible that the frog is in this, as in so many other respects, in a modifierl rather than a primitive condition.

The bones of the skull are of two kinds:-(i) cartilage bones, which are formed in direct connection with the prinary 
cartilaginous skeleton; and (ii) membrane bones, which arise independently of the cartilaginous skeleton, although they may in their later stages become firmly grafted on to this.

The membrane bones of the skull appear in a comnectivetissue layer, very rich in cells, which is found immediately outside the cartilaginous cranium. In this connective tissue, trabeculæ and spicules of calcified substance appear, and soon interlace to form a network; surrounding the spicules are osteoblasts or bone cells, by which the further growth of the framework

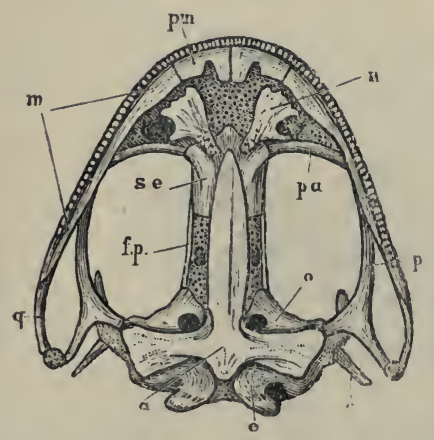

FIG. 95.-The skull of an adult Frog, from the ventral surface. $\times 1 \frac{1}{2}$.

$a$, parasphenoid. $c$, columella. e, exoccipital. f.p, fronto-parietal. $m$, maxilla. $n$, vomer. $o$, pro-otic. $p$, pterygoill. pa, palatine. $p m$, premaxilla. q, quailrato-jugal. se, sphen-ethmoil.

is effected, a consistent bony lamella with imbedded bone cells being ultimately formed.

The first bone to be developed is the parasphenoid, which appears, in tadpoles of about $20 \mathrm{~mm}$. length, in the comnective tissue underlying the floor of the skull. The exoccipitals, and the frontals and parietals, which are at first separate from one another, soon follow. The premaxilla, maxilla, dentary, and angulare are formed at the commencement of the metamorphosis; and towards its close the vomer, palatine, pterygoid, and other bones appear.

By the time the metamorphosis is completed, and the tail absorbed, all the bones of the skull are present except the sphen-ethmoid, which appears rather late, in the course of the first summer, as a narrow transverse splinter of bone, crossing the roof of the skull near its anterior end.

\section{The Teeth.}

In the frog the teeth are confined to the premaxillæ, maxillæ, and vomers, the lower jaw being edentulous.

The upper jaw bears a single row of small conical teeth arranged along its inner border, each tooth being attached to the bone at its base and along its outer surface, and only a very sniall part of the tooth projecting freely. 
Lach tooth is a hollow cone ; the central or pulp-cavity containing the blood-vessels and nerves. The basal part of the cone consists of bone, the apical part of dentine, capped at the tip by a very thin layer of enamel. 'The teeth are readily lost, and are replaced by new ones developed below them.

The teeth do not begin to form until the time of the metamorphosis. Round the border of the jaw, a solid ridge-like ingrowth of the deeper layer of the epidermis takes place into the underlying connective tissue ; and opposite the edge of this ridge a series of small processes, the dentinal papillæ, are formed in the dermis. These papillæ, which are very rich in cells, grow into the epidermal ridge, which thus forms a cap over each of them. The inner lining of each cap, immediately covering: the papilla, consists of a single layer of short columnar cells, the enamel cells; while the rest of the cap, which is three or four cells thick, consists of indifferent cells, the outermost layer of which forms a more or less definite capsule.

Of the hard tissues of the tooth, the thin cap of enamel is formed by calcification of the ends of the enamel cells next to the papilla. The dentine is formed by calcification of the surface layer of the papilla itself, the cells of the papilla sending processes into the dentinal substance while it is forming.

The young tooth now separates from the epithelial ridge, and moves towards the surface ; it lengthens, by formation of bony matter at its base, but does not acquire its definite attachment to the bones of the jaw until some time after the completion of the metamorphosis, usually during the autumn of the first year. The dermal papilla persists as the pulp of the tooth.

The replacing teeth are developed in precisely similar fashion, and from the original epidermal ridge. They lie at first to the inner side of the first row of teeth, but during their development shift their positions so as to lie directly above these. By further growth downwards, accompanied by absorption of the bony bases of the teeth in use, the new teeth move towards the surface; they often lie for a time partly within the pulp cavities of their predecessors, and, as these latter fall out, speedily grow into their places.

The vomerine teeth are straighter than those of the margin of the jaw, but are otherwise similar to these, both in structure and in mode of development. 


\section{The Appendicular Skeleton.}

The limbs arise about the time of completion of the opercular fold, and shortly after the opening of the mouth, i.e. in tadpoles of about 11 or $12 \mathrm{~mm}$. length.

The hind limbs (Fig. 83, LP) appear as a pair of small rounded buds from the ventral surface of the hinder part of the body wall, at the base of the rectal spout.

The fore limbs (Fig. 84, LA) are similar buds, which arise about the same time, from the sides of the body wall at its anterior end, opposite the head kidneys. They lie in the dorsal angle between the body wall and the opercular fold, and, being covered by this latter, are not visible from the surface.

The limbs grow from the somatopleure alone; each is a solid mass of compact mesoblast, covered by a cap of epidermal cells, which differ in their cubical or columnar shape from the flattened cells of the general surface of the body.

The limbs at first grow slowly. They gradually elongate, become segmented, and then divided distally into fingers and toes: (Fig. 85, LA, LP). Up to the time of the metamorphosis the hind limbs are small, while the fore limbs remain concealed within the opercular cavity. During the metamorphosis (Fig. 86), both pairs of limbs grow rapidly, more especially the hind limbs.

The skeleton of the limbs, and of the limb-girdles by which the limbs are attached to the body, does not assume definite form until a short time before the metamorphosis.

a. The pectoral girdle develops as two half-rings of cartilage, one on each side of the body, which they encircle at the level of the second or third vertebra. The dorsal ends of the half-rings (Fig. 88, ss) lie superficially to the transverse processes of the vertebræ, FT, and are connected with these by muscles and ligaments; the rentral ends, $\mathrm{CO}$, meet each other in the median plane.

Each half-ring has on its outer surface, rather below the middle of its length, a cup-shaped depression, the glenoid cavity, GC, with which the head of the humerus, HU, articulates. The part of the ring above the glenoid cavity is the scapular portion, the part below it the coracoid portion.

The scapular portion is divided, shortly before the metamor- 
phosis, into a dorsal blade-like part, the suprascapula, ss, which remains in great part cartilaginous throughout life; and a ventral, more slender, and shaft-like part, the scapula, Sc, round which a ring of bone soon forms.

The coracoid portion is, from the first, split into two diverging processes; an anterior or pre-coracoid portion, and a posterior or coracoid proper. The ventral ends of the coracoid and pre-coracoid of each side grow towards each other and meet, forming a longitudinal band of cartilage, the epi-coracoid; the two epi-coracoids lie in close contact with each other in the median plane, but do not fuse. Along the anterior border of the pre-coracoid cartilage, a bony rod, the pre-coracoid bone or clavicle, is formed; and around the coracoid cartilage a tubular sheath of bone, the coracoid bone, is developed. There is thus at first no trace of a sternum, either as a median or paired structure.

During and after the metamorphosis further changes occur. The bones increase in size, especially the scapula and coracoid. The two epi-coracoid cartilages, in place of merely meeting in the median plane, overlap each other to a certain extent, the left epi-coracoid lying dorsal to the right one. From the anterior ends of the epi-coracoid cartilages a pair of small processes grow forwards ; these soon fuse to form the omosternum, which rapidly increases in size. Behind the epi-coracoids, and in close contact with them, a pair of cartilaginous bands appear, which fuse together to form a flat median plate of cartilage; this gives rise in front to the sternum, round which a ring of bone soon forms, and behind to the xiphi-sternum, which remains permanently cartilaginous.

b. The pelvic girdle also consists at first of two half-rings of cartilage, encircling the hinder part of the trunk. The ventral ends of the half-rings, which are flattened and expanded, are in contact in the median plane, and very early fuse firmly together to form the pelvic symphysis. The dorsal ends are more slender: at first they lie fiee in the muscles of the body wall; later on they become commected with the transverse processes of the ninth or sacral vertebra.

Each half-ring has on its outer surface, close to its ventral end, a depression, the cotyloid or acetabular cavity, for articulation with the head of the femur. The part above the acetabulum, 
which corresponds with the scapular portion of the pectoral girdle, ossifies as the ilium; in the part below the acetabulum, the anterior or pubic portion remains cartilaginous, while the posterior portion ossifies as the ischinm.

The pelvic girdle is, in its early stages, and until shortly

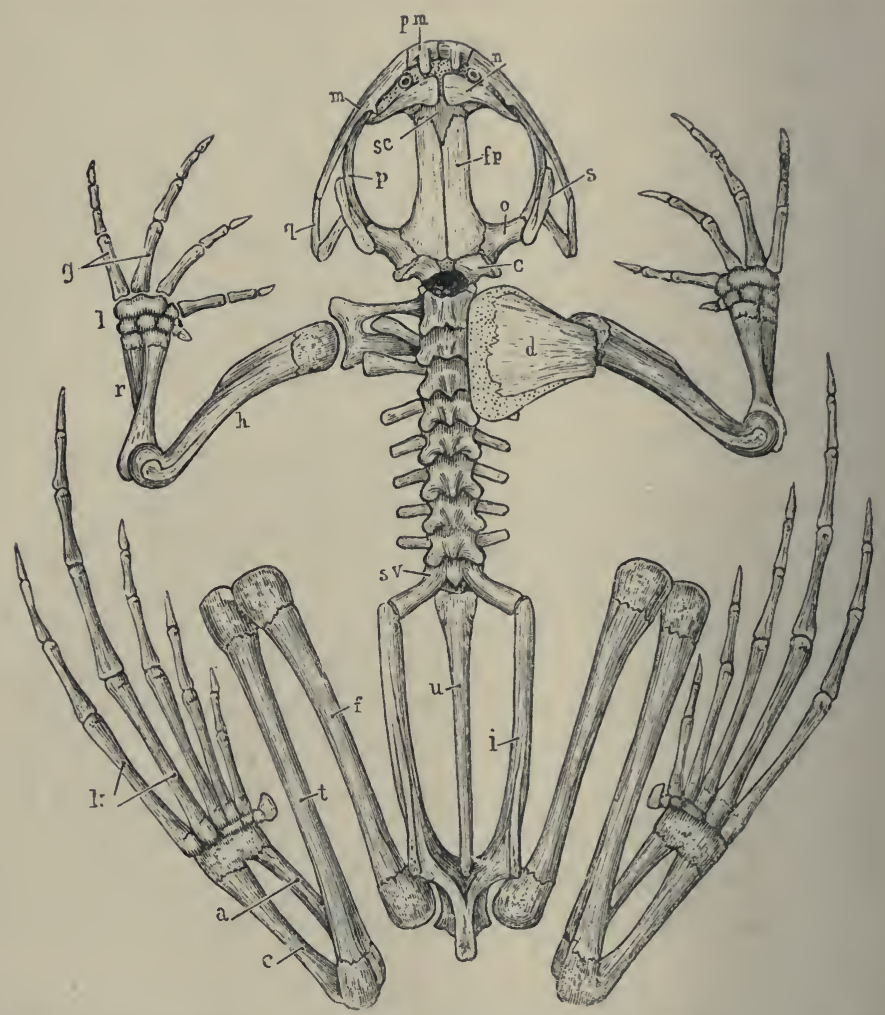

FIG. 96.-The skeleton of the Frog, seen from the dorsal surface. The left supra-scapula and scapula have been removed.

$a$, astragalus. $c$, calcaneum. $d$, supra-scapula. e, exoccipital. $f$, femur. $f p$, frontoparietal. $g$, metacarpals. $h$, humerus. $i$, ilium. $k$, metatarsals. $l$, carpus. $m$, maxilla. $n$, nasal. $\quad o$, pro-otic. $p$, pterygoil. $p m$, premaxilla. $q$, quadrato-jugal. $r$, radio-ulna. $s$, squamosal. se, sphen-ethwoid. $s v$, sacral vertebra. $t$, tibio-fibula. $u$, urostyle.

before the metamorphosis, about the same length as the pectoral girdle; and, like this, lies at right angles to the vertebral column. As the hind limbs lengthen, during and after the metamorphosis, the rentral or acetabular end of the girdle moves backwards, so that the ilium, which lengthens considerably at 
the same time, now lies, as in the adult, almost parallel to the backbone; and the hip joint is shifted to the extreme hinder end of the body.

c. The limbs. The skeletal framework of each limb develops from the proximal towards the distal end ; the humerus and the femur being the first bones to be differentiated. The radius and ulna, and the tibia and fibula, are at first independent of each other, but the cartilages of each pair soon fuse. Of the digits, the two preaxial, radial or tibial, of each limb are establisherl first; the others budding out in succession. There is some doubt as to which of the normal five digits is absent in the hand of the adult frog; and the embryological evidence, though not conclusive, is rather in favour of the view that it is the fifth, and not, as is commonly held, the first digit or pollex that is wanting.

List of the more important P'ublications dealing with the Development of l'rogs.

Alcock, T.: 'On the Development of the Common Frog:' Memoirs of the Manchester Literary and Philosophical Society, series iii., vol. viii. 1883. Assheton, R.: 'The Development of the Optic Nerve of Vertebrates.' Quarterly Journal of Microscopical Science, vol. xxxiv. 1892.

Balfour, F. M.: 'A Treatise on Comparative Embryology,' vol. ii. 1881.

Bambeke, C. van: 'Recherches sur le Développement du Pélobate bruu.' 1867.

'Nonvelles Recherches sur l'Embryologie des Batraciens.' Archives de Biologie, i. 1880.

Barfurth, D.: 'Versuche über die Verwandlung der Froschlarven.' Archiv für mikroskopische Anatomie, xxix. 1887.

'Die Rückbildung des Froschlarvenschwanzes und die sogenannten Sarkoplasten.' Archiv für mikroskopische Anatomie, xxix. $188 \pi$.

Bedot, M. : 'Recherches sur le Développement des Nerfs Spinaux chez les Tritons.' Archives des Sciences Phys. et Nat., xi. 1881.

Boas, J. E. V.: 'Ueber den Conus arteriosus und die Arterienbogen der Amphi bien.' Morphologisches Jahrbuch, vii. 1881.

'Beiträige zur Angiologie der Amphibien.' Morphologisches Jahrbuch, viii. 1882 .

Bourne, A. G.: 'On Certain Abnormalities in the Common Frog.' Quarterly Journal of Microscopical Science, xxiv. 1884.

Cope, E. D.: ' On the Relations of the Hyoid and Otic Elements of the Skeleton in the Batrachia.' Journal of Morphology, ii. 1888.

Dugès, A.: ' liecherches sur l'Ostéologie et la Myologie des Batraciens a leur différents Ages.' Mémoires de l'Académie cles Sciences. Paris. 1835.

Duval, M. : 'Sur le Développement de l'Appareil Génito-urinairẹ de la Grenouille.' Revue des Sciences Naturelles. 188:. 
Ecker, A.: 'Icones Physiologicx.' Leipzig. 1851-1859.

'The Anatomy of the Erog.' English translation by G. Haslam. Oxford. 1889.

Erlanger, R. von: 'Ueber den Blastoporus der anuren Amphibien, sein Schicksal und seine lBeziehungen zum bleibenden After.' 'Zoologische Jahrbücher : Abtheilung für Anatomie und Ontogenie der 'Thiere, iv. 1890.

Field, H. F.: 'The Development of the Pronephros and Segmental Duct in Amphibia.' Bulletin of the Museum of Comparative Anatomy at Harvard College, xxi. 1891.

Fïrbringer, M.: 'Zur Entwickelung der Amphibienniere.' Heidelberg. 1877. Gatehouse, J. W.: 'The Development ancl Life History of the Tadpole.' Journal of Microscopical and Natural Science, i. ii. 1888 and 1889.

Goette, A.: 'Die Entwickelungsgeschichte der Unke.' Leipzig. 187 j.

Göppert, E. : 'Die Entwicklung und das spaitere Verhalten des Pankreas der Amphibien.' Morphologisches Jahrbuch, xvii. 1891.

Héron-Royer and C. van Bambeke: 'Le Vestibule de la Bouche chez les Têtards des Batraciens Anoures d'Europe.' Archives de Biologie, ix. 1889.

Hertwig, O.: ' Ueber das Zahnsystem der Amphibien und seine Bedeutung für die Genese cles Skelets der IIunthöhle.' Archiv für mikroskopische Anatomie, xi. Supplement. 1874.

'Beiträge zur Kenntniss der Bildung, Befruchtung und Theilung des thierischen Eies.' Morphologisches Jahrbuch, iii. 1877.

'Die Entwicklung des mittleren Keimblattes der Wirbelthiere.' Jenaische Zeitschrift für Naturwissenschaft, xvi. 1883.

Higginbotham, ..: 'Influence of Physical Agents on the Development of the Tadpole of the Triton and the Frog.' I'hilosophical Transactions of the Royal Society. 1850.

Hinckley, Mary H.: 'Notes on the Development of Rana sylvatica.' Proceedings of the Boston Society of Natural History. 1882.

Hochstetter, F.: 'Beiträge zur' vergleichenden Anatomie und Entwickelungsgeschichte des Venensystems der Amphibien und Fische.' Morphologisches Jahrbuch, xiii. 1887.

Hoffmann, C. K. : 'Zur Entwicklungsgeschichte der Urogenitalorgane bei den Anamnia.' Zeitschrift für wissenschaftliche Zoologie, xliv. 1886.

Bronn's Klassen und Ordnungen des Thierreichs: Amphibia. $1873-$ 1878.

Houssay, F. : 'Etudes d'Embryologie sur les Vertébrés.' Archives de 'Loologie Expérimentale, 18.90; and Bulletin Scientifique de la France et de la Belgique, 1891.

Howes and Ridewood: ' On the Carpus and Tarsus of the Anura.' Proceedings of the Zoological Society. 1888.

Huxley, T. H.: Article 'Amphibia.' Encyclopædia Britannica, 9th edition. 1875 .

Kölliker, A.: 'Note sur le Développement des Tissus chez les Batraciens.' Annales des Sciences Naturelles. 1846.

Lambotte, M.: 'Observations Anatomiques et Physiologiques sur les Appareils Sanguins et Respiratoires des Batraciens Anoures.' Bruxelles. 1837.

MacBride, E. W.: 'The Development of the Oviduct in the Frog.' Quarterly Journal of Microscopical Science, xxxiii. 1892. 
Malbranc, M.: 'Von rler Seitenlinie und ihren Sinnesorganen bei Amplibien.' Leipzig. $187 \tilde{5}$.

Marshall, A. Milnes: 'On Certain Abnormal Conditions of the Reproductive Organs in the Frog.' Journal of Anatomy and Physiology, xviii. 1884.

'The Frog: an Introduction to Anatomy, Histology, and Embryology. 4 th edition. 1891.

Iarshall, A. Milnes, and Bles, E. J.: 'The Development of the Kidneys and Fat Bodies in the Frog.' Studies from the Biological Laboratories of the Owens College, ii. 1890.

'The Development of the Blood-vessels in the Frog.' Studies from the Biological Laboratories of the Owens College, ii. 1890.

Maurer, F.: 'Schilddrïse, Thymus und Kiemenreste der Amphibien.' Morphologisches Jahrbuch, xiii. 1887.

'Die Kiemen und ihre Gefässe bei Urodelen und Anuren.' Morplologisches Jahrbuch, xiv. 1888.

'Die erste Anlage der Milz und das erste Auftreten von lynphatischen Zellen bei Amphibien.' Morphologisches Jahrbuch, xvii. 1890.

Metschnikoff, E.: 'The Ancestral History of the Inflammatory Process.' Quarterly Journal of Microscopical Science, xxiv. 1884.

IIeuron, P. de: 'Recherches sur le Développement du Thymus et de la Glande Thyroïde.' Recueil Zool. Suisse, iii. 1886.

'Sur le Développement de l'GEsophage.' Comptes Rendus, cii. 1886. Moquin-Tandon, G. : 'Recherches sur les Premières Phases du Développement des Batraciens Anoures.' Annales des Sciences Naturelles. 1876.

Morgan, T. H. : 'On the Amphibian Blastopore.' Studies from the Biological Laboratory of the Johns Ilopkins University, iv. 1887.

'Some Notes on the Breedirg Habits and Embryology of Frogs.' American Naturalist. August, 1891.

Nussbaum, M. : 'Ueber den Bau und die 'Thätigkeit der Drüsen.' Archiv für mikroskopische Anatomie, xxvii. 1886 .

(1)r, H.: 'Note on the Development of Amplibians.' Quarterly Journal of Microscopical Science, xxix. 1888.

Osborn, H. F.: 'A Contribution to the Internal Structure of the Amphibian Brain.' Journal of Morphology, ii. 1888.

Parker, W. K.: 'On the Structure and Development of the Skull of the Common Frog.' Philosophical Transactions, 1871.

' On the Structure and Development of the skull of the Batrachia.' Philosophical Transactions, 1876 and 1881.

'A Monograph on the Structure and Development of the Shoulder Girdle and Sternum.' Ray Society. 1867.

l'erényi, J.: 'Die Entwickelung der Keimblätter und der Chorda in neuer Beleuchtung.' Anatomischer Anzeiger, iv. 1889.

Rabl, C.: 'Theorie des Mesorlerms.' Morphologisches Jahrbuch, xv. 1859.

Rauber, A.: 'Primitivrinne und Urmund.' Morphologisches Jahrbuch, ii. 1876 .

Tiemak, R. 'Untersuchungen ïber die Entwickelung der Wirbelthiere. Berlin. 1855 .

Robinson, A., and Assheton, R.: "The Formation and Fate of the Primitive Streak, with Observations on the Archenteron and Germinal Layers of liana temporaria.' Quarterly Journal of Ilicroscopical Science, xxxii. 1891 . 
Rossi, U.: "Contributo alli maturazione delle uove degli $\Lambda$ mfibii. Anato. mischer Anzeiger, $v$. 1890.

Rusconi, M.: 'Descrizione anatomica degli organi della circolazione delle larve delle Salamandre aquatiche.' Pavia. 1817.

Schoebel, E.: 'Zur postembryonalen Ent wickelung des Auges der Amphibien.' Zoologische Jahrbücher : Abtheilung für Anatomie und Ontogenie rler Thiere, iv. 1891.

Schultze, F. E. : 'Ueber die inneren Kiemen der Batrachierlarven.' Berlin. 1888.

Schultze, O.: ' Untersuchungen über die Reifung und Befruchtung des Am. phibieneies.' Zeitschrift für wissenschaftliche Zoologie, xlv. 1887.

'Die Entwicklung der Keimblätter und der Chorda dorsalis ron Rana fusca.' Zeitschrift für wissenschaftliche Zoologie, xlvii. 1888.

' Ueber die Entwicklung der Medullarplatte des Frosches.' Verhandlungen d. Würzburg. phys.-med. Gesellschaft, xxiii. 1889.

Schivartz, D.: 'Untersuchungen des Schwanzendes bei den Embryonen der Wirbelthiere.' 'Zeitschrift für wissenschaftliche Zoologie, xlviii. 1889.

Scliwink: 'Untersuchungen ïber die Entwicklung des Endothels und der Blutkörperchen der Amphibien.' Morphologisches Jahrbuch, xvii. 1891.

Sedgwick, A. : ' On the Early Development of the Anterior Part of the Wolffian Duct and Body in the Chick, together with some Remarks on the Excretory System of the Vertebrates.' Quarterly Journal of Microscopical Science, xxi. 1881.

Selenka, E. : 'Der embryonale Excretionsapparat der kiemenlosen Hylodes Martinicensis.' Sitzungsberichte der königlichen Akademie der Wissenschaften zu Berlin. 1882.

Shore, T.: 'Notes on the Origin of the Liver.' Journal of Anatomy and Physiology, xxv. 1891.

Sidebotham, H.: 'Note on the Fate of the Blastopore in Rana temporaria. Quarterly Journal of Microscopical Science, xxix. 1888.

Spencer, W. B. : 'Some Notes on the Early Development of Rana temporaria.' Quarterly Journal of Microscopical Science, xxv. Supplement. IS85.

Spengel, J. W.: 'Das Urogenitalsystem der Amphibien.' Arbeiten aus dem zoologisch-zootomischen Institut in Würzburg, iii. 1876.

Stöhr, P.: Zur Entwicklungsgeschichte der Anurenschädels.' Zeitschrift für wissenschaftliche Zoologie, xxxvi. 1881.

Tomes, C. S.: 'On the Development of the Teeth of the Newt, Frog, Slowworm, and Green Lizard.' Philosophical Transactions. 1875.

Villy, F. : 'The Development of the Ear and Accessory Organs in the Common Frog.' Quarterly Journal of Microscopical Science, xxx. 1890.

Vogt, C.: 'Untersuchungen über die Entwicklungsgeschichte der Geburtshelferkröte (Alytes obstetricans).' Solothurn. 1842.

Wittich, W. H. von: 'Beiträge zur morphologischen und histologischen Entwickelung der Harn- und Geschlechtswerkzeuge der nackten Amphibien.' Zeitschrift für wissenschaftliche Zoologie, iv. 1853.

Ziegler, F.: 'Zur Kenntniss der Oberflächenbilder der Rana-Embryonen.' Anatomischer Anzeiger, vii. 1892. 


\section{CHAPTER IV. \\ 'THE DEVELOPMENT' OF THE CHICК.}

\section{GENERAL ACCOUNT.}

\section{Historical Sketch.}

The development of the chick has attracted great attention on account of the ease with which embryos of any desired age may be obtained, and of the shortness of the period within which the embryonic development is completed. Almost all the earlier investigations into the development of animals were made on chick embryos, and it is round the chick that the most famous embryological controversies have centred. Even at the present day, on account of their great convenience for laboratory purposes, chick embryos usually afford the material from which the student derives his first lessons in practical embryology.

Embryology as a science is barely three centuries old; the earliest descriptions and figures of the development of the chick within the egg, that are of any real value, are contained in two treatises published by Fabricius, professor at Padua, in 1600 and 1604. Half a century later, Harvey added important details in his 'Theoria Generationis ;' and towards the close of the seventeenth century, in 1687, Malpighi published the first accounts of chick embryos based on microscopical examination.

During the eighteenth century facts accumulated rapidly, but the theories quite ontpaced them; and the current doctrine throughout the century, supported by many, and notably by Haller, was that of Preformation, according to which the chick was stated to be present in the egg at the time it is laid ; all its parts and organs being there from the beginning, but in an extremely minute and unexpanded condition; the development of the embryo being regarded as a process comparable to the unfolding and enlargement of the several parts of a bud to form the perfect flower. 
This theory of Preformation was vigorously combated by Caspar Friedrich Wolff, who in 1759, when only twenty-six year's old, published as a thesis for the doctor's degree his theory of Epigenesis, which offered an entirely new explanation of the mode of development of the chick and other animals. Wolff showed conclusively that in the hen's egg, as laid, there is no trace whatever of the embryo, or of any of its parts or organs; and that the formation of the embryo does not commence until after the egg is laid and incubation has begun. He noted further, and described accurately, the manner in which the embryo is formed by folding of the germinal layers or membranes.

Wolff was too far ahead of his age, and his conclusions, though perfectly sound, did not obtain acceptance until towards the middle of the present century, when their correctness was demonstrated, not merely for the chick, but for many other groups of animals as well, by von Baer, Remak, Bischoff, Kölliker, and others.

Although the chick has thus played a more important part in the history of embryology than any other animal, it must be borne in mind that birds are one of the most highly specialised groups of animals, and that their development is, more particularly in the early stages, very greatly modified. It is practical convenience alone that justifies the great attention they have received.

\section{The Egg.}

The hen's egg is of large size, and ovoid in shape. It consists (Fig. 97) of a calcareous shell, lined by a fibrous shell membrane; and inclosing a quantity of a viscid albuminous fluid, the 'white of the egg,' WA, in the centre of which lies the 'yolk,' y, a spherical mass of a yellow colour, rather more than an inch in diameter, and inclosed in an elastic vitelline membrane, to which the preservation of its shape is due.

Of these parts, the yolk is the egg proper; it corresponds to the egg of Amphioxus, or of the frog, and from it the embryo is developed directly.

The white of the egg corresponds to the investment of the egg of Amphioxus, which swells up so greatly on reaching the water, or to the jelly of the frog's spawn. 'The egg-shell and 
shell membrane are protective envelopes, which are not represented in the eggs of Amphioxus or of the frog.

The yolk or ovum is, as in other animals, a single cell; its great size being due to the enormous quantity of food yolk accumulated within it, and distending it. As regards the quantity of food-yolk contained within it, the hen's egg is at the opposite extreme to that of Amphioxus; the frog's egg being midway between the two.

It is in consequence of the abundance of food material

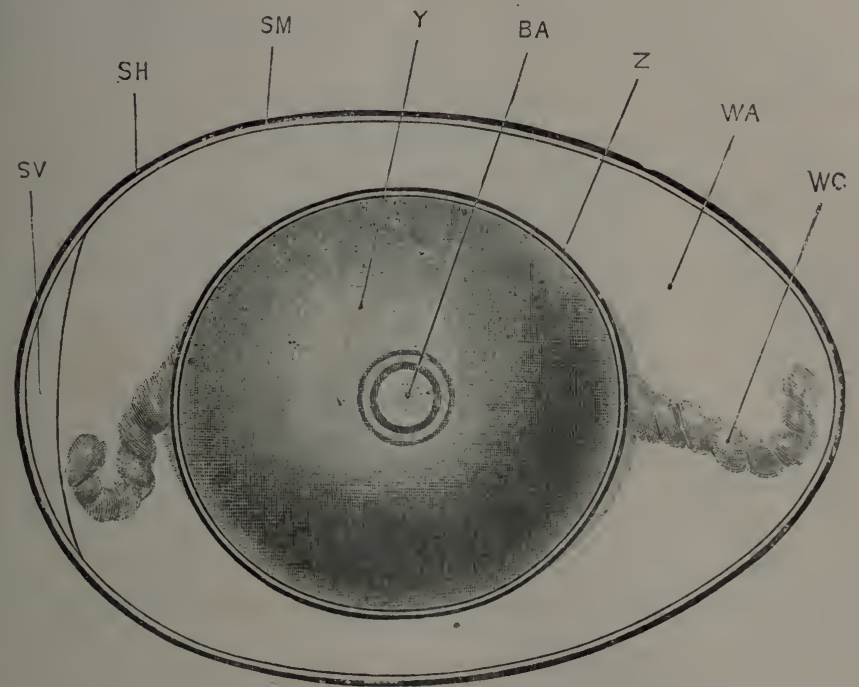

FIG. 97.-The Hen's Egg at the time of laying. $\times \frac{3}{2}$.

BA, blastoderm. SH. egg shell. SMI, shell membrane. SV, air chamber. WA, white or albumen. WC, chalaza, or twisted cort of denser albumen. Y, yolk. Z, vitelline membrane.

present in the egg itself, that the chick embryo is enabled to complete its development within twenty-one days, while the frog requires three months or more, and Amphioxus an even longer time. The Amphioxus larva hatches in about eight hours, but in an extremely immature condition (Figs. 25, 26, p. 59); the frog hatches in about a fortnight, in a form utterly unlike the parent, and devoid of mouth and limbs (Figs. 72, 73, p. 157); the chick does not leave the egg until the tiventy-first day, but is already a fully developed bird.

$\Lambda$ large amount of food-yolk is undoubtedly an advantage, 
inasmuch as it enables the embryo to develop rapidly and securely, and frees it from the necessity of obtaining food from without. However, it has also its disadvantages. Food-yolk is itself inert, as already noticed in the opening chapter. It is present in the egg as a number of granules of various shapes and sizes, embedded in the living protoplasm of the egg; and the immediate effect of these inert, inactive, yolk-granules is, not to aid, but to mechanically impede the processes of development; an effect which will necessarily be most marked in the early stages, when the food-yolk is most abundant. Hence the early strges of development of the chick, and especially the processes of segmentation, occur more slowly than those of the frog, and much more slowly than those of Amphioxus.

Moreover, the amount of food-yolk in the hen's egg is so great that serious distortion of the shape would occur, were the whole mass contained within the body of the embryo. To avoid this difficulty, the yolk, at a very early stage of development, becomes constricted into two parts, embryonic and vitelline respectively, which remain connected by a stalk. Of these (cf. Figs. 99 and 100), the embryonic portion, EM, is formed from the part of the egg comparatively free from food-yolk, and becomes converted directly into the embryo; while the vitelline portion or yolk-sac, Ys, which contains the bulk of the food-yolk, does not give rise directly to any part of the embryo, but forms a store of nutriment at the expense of which the development of the embryo is effected.

At first, the embryonic portion is very much smaller than the vitelline portion or yolk-sac (Fig. 98); but, inasmuch as the embiryo grows by absorption of the food-yolk, the yolk-sac diminishes as the embryo increases in size (cf. Figs. 99, 100, 101). A time comes when the two are about equal in bulk, and in the later days of incubation the yolk-sac is much smaller than the embryo. By the twenty-first day of incubation the yolk-sac is almost completely absorbed, and the chick pecks its way out of the shell, and hatches.

\section{The Embryo.}

The hen's egg is fertilised before it is laid, indeed before the egg-shell is formed, for no spermatozoon could possibly make its way through the shell. At the time the egg is laid, not only 
has fertilisation been effected, but the egg has already been developing for a period which varies in different cases, but amounts on an average to about eighteen hours.

When the egg is laid, development stops. To set it going again, to start development afresh, all that is necessary is that the egg should be kept at a temperature about equal to the blood-heat of the parent bird. This is normally effected by incubation, the hen sitting on the egg, and so keeping it warm; but it may be effected equally well by artificial means. A certain amount of moisture, and free access of air, are necessary to insure normal development. The rate of development

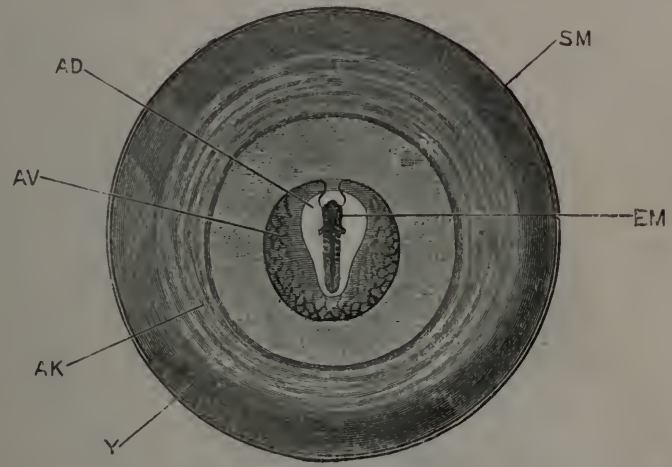

F1G. 95.-The yolk of a Hen's Egg at the thirty-sixth hour from the commencement of incubation. The structure of the embryo at this stage is shown on a larger scale in Figs. 111 and $112 . \times \frac{3}{2}$.

$\mathrm{AD}$, area pellncida of the blastolerm. $\mathrm{AK}$, area opaca. $\mathrm{AV}$, area vasculosa. EM, embryo. SM, vitelline membrane. Y, yolk-sac.

varies to a slight extent according to the season of the year, autumn eggs developing more slowly than spring eggs; or according to the temperature, if an artificial incubator is employed. The length of time the egg takes to travel down the oviduct, during the whole of which time it is developing, varies considerably, and individual variations may occur from other causes; but, as a rule, the chick hatches on the twenty-first day from the commencernent of incubation. The age of an embryo is always calculated from the commencement of incubation, or from the time of placing the egg in the incubator; to obtain the true age there must be added to this the time during which the egg was developing, in its passage down the 
oviduct, a period averaging, as we have seen, about eighteen hours.

Owing to the enormous amount of food-yolk, and the mechanical hindrance which this offers to the processes of development, the entire yolk, i.e. the egg proper, does not divide, but segmentation is restricted to a small circular patch (Fig. 97, BA), on the surface of the yolk, which is comparatively free from yolk-granules, and in which development can readily take place. This patch, the germinal disc, segments to form the blastoderm, a membrane composed of cells (Fig. 106), which lies like an inverted watch-glass on the surface of the yolk. The blastoderm rapidly increases in diameter, by growth all round

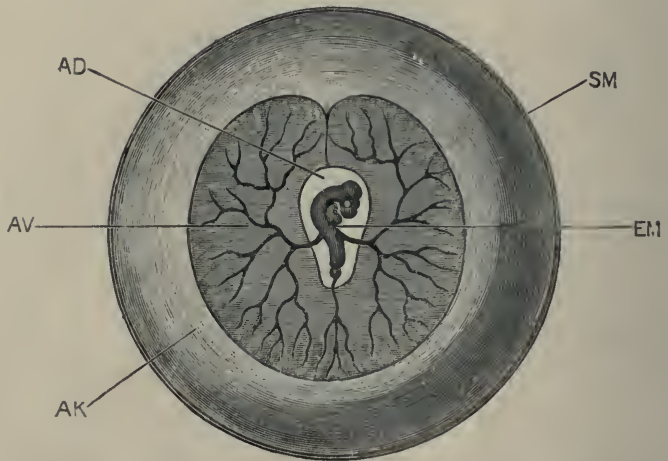

F'IG. 99.-The yolk of a Hen's Egg at the end of the third day of incubation. The structure of the embryo at this stage is shown on a larger scale in Figs. 113 and $114 . \times \frac{3}{2}$.

AD. area pellncila of the blastolerm. AK, area opaca. $\mathbf{A V}$, area vasculosa. EM, embryo. SM. vitelline membrane.

its margin, and spreads so as to cover more and more of the surface of the yolk, which it ultimately incloses completely (Figs. $98,99,100,101)$. Owing, apparently, to its less specific gravity, the germinal disc, and consequently the embryo, which is formed from its central part, lies at the top of the egg, and nearest to the body of the hen, however much the egg be rolled over.

The central part of the blastoderm is thin and translucent, and is spoken of as the area pellucida (Fig. 98, AD); the marginal portion is thicker and less transparent, and is called the area opaca, $\mathrm{AK}$; the inner rim of the area opaca, bordering the area pellucida, is the seat of an abundant formation of bloodvessels, and is called in consequence the area vasculosa, $\mathrm{Av}$. 
'The first trace of the embryo appears in the centre of the area pellucida, about the twentieth hour of incubation; the formation of the embryo consisting essentially in a process of folding off, or constriction, of the central part of the area pellucida from the rest of the yolk. By the middle of the second day the embryo (Fig. 98) measures about $5 \mathrm{~mm}$. in length, and has acquired definite shape; the brain, spinal cord, heart, and other organs being already established (cf. Figs. 111 and 112).

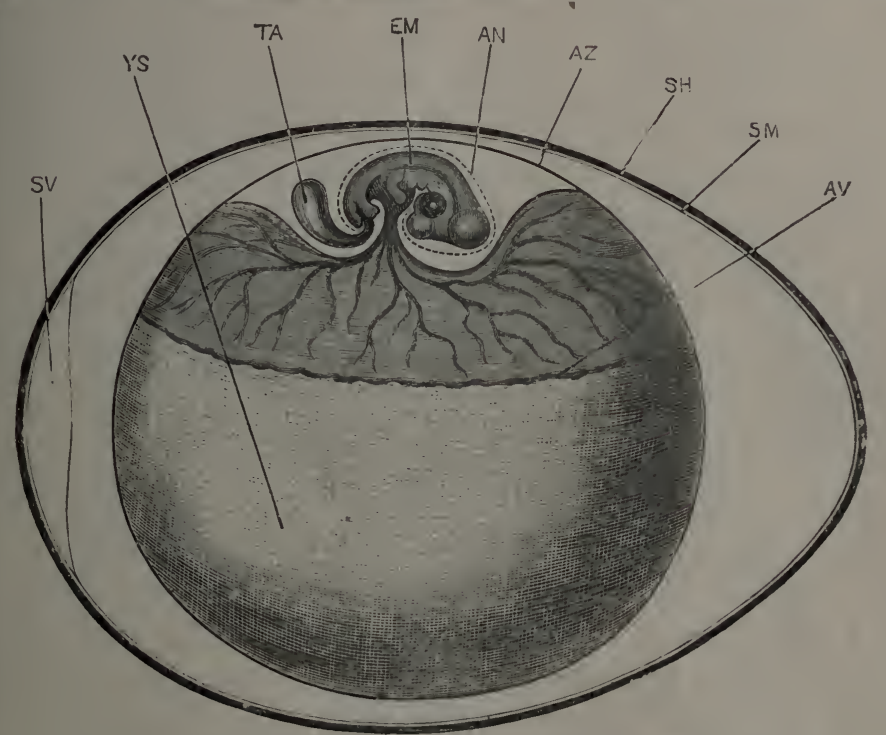

FIG. 100.-The Hen's Egg at the end of the fifth day of incubation, seen from the side. The embryo, which naturally lies with its left side on the yolk-sac and its right side towards the egg-shell, has been lifted up, in order to show its shape more clearly. The structure of the embryo at this stage is shown on a larger scale in Figs. 115 and 123. $\times \frac{3}{2}$.

$\mathbf{A N}$, inner or 'true' amnion. AV, outer margin of area vasculosa. AZ, outer or 'false' ammion, together with the vitelline membrane. EM, embryo. SH, egg-shell. SM, shell membrane. SV, air chamber. TA, allantois. Y Y , yolk-sac.

The embryo, at this stage, lies with its dorsal surface towards the shell, and its ventral surface towards the yolk. The axis of the body is straight, and is usually directed across the axis of the egg; the head end of the embryo, in the majority of cases, pointing away from the observer if th $\rightarrow$ egg is placed before him with the broader end to his left. There are, however, great variations in this respect, and the axis of the embryo may form almost any angle with that of the egg. 
At thirty-six hours, the folding off of the embryo from the yolk-sac has only made slight progress; the head of the embryo (Fig. 112) is lifted up above the yolk-sac by an anterior constriction or head fold, but the sides and tail end are as yet only very imperfectly defined.

By the end of the third day great advance has been made. The embryo (Fig. 99) has increased considerably in size. In the head, which has grown much faster than the body, and is now disproportionately large (Fig. 113), the nose, eye, and ear, and the several divisions of the brain, are well established. The head is no longer straight, but is strongly flexed, owing to the dorsal surface growing much more rapidly than the ventral. The heart and blood-vessels have acquired definite and characteristic arrangement. The folding off of the embryo from the yolk-sac has made considerable progress (Fig. 114); the head and neck are now quite free from the yolk-sac; the hinder end of the embryo is lifted up from the yolk by a definite tail fold (Fig. 114, TL), and the side walls of the embryo are much more clearly defined. The yolk-stalk, connecting the embryo with the yolk-sac, is now a short tube, the diameter of which is about a third of the length of the embryo. The hinder end of the embryo still lies with its dorsal surface facing the egg-shell, and its ventral surface resting on the yolk-sac; but the head and neck have rolled over, so as to lie with their left side on the yolk-sac and their right side towards the egg-shell; the axis of the body becoming spirally twisted in consequence (Fig. 113).

On the fourth day the folding off of the embryo makes further progress, and the yolk-stalk becomes greatly narrowed. The whole embryo becomes strongly flexed, the dorsal surface being convex along its entire length. The body, as well as the head, of the embryo now lies with its left side on the yolk-sac; and the rudiments of the limbs have appeared as two pairs of small, ill-defined buds from the sides of the body.

By the end of the fifth day the embryo has acquired the shape and proportions shown in Fig. 100. In the natural condition, it lies with its left side on the yolk-sac, with which it is connected by the narrow tubular yolk-stalk. 'The whole embryo is strongly flexed, the convex dorsal surface being about four times the length of the concave ventral surface. The head is of relatively enormous size, chiefly orving to the great development 
of the brain vesicles and of the eyes. The limbs are still small, but liave increased considerably in size as compared with the earlier stages, and already show indications of their division into segments (Fig. 115). From the under surface of the tail of the embryo a saccular diverticulum, with thin but very vascular walls, arises as an outgrowth from the alimentary canal : this is the allantois (Fig. 115, TA), a structure which grows very

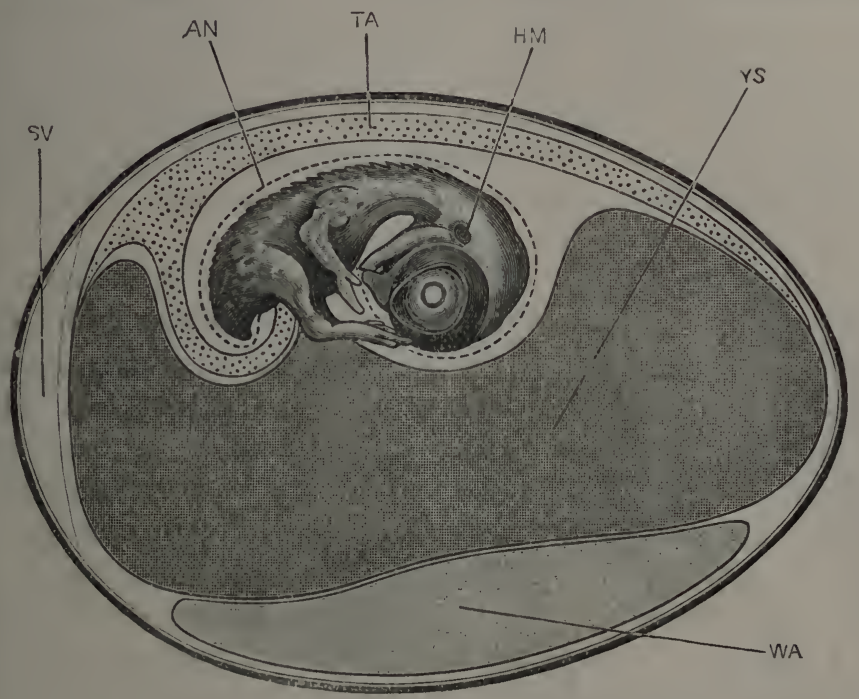

FI(1. 101.-The Hen's Egg at the end of the ninth day of incubation, seen in vertical section. The embryo naturally lies with its left side on the yolksac, but has been lifted up in order to show its shape more clearly. $\times \frac{3}{2}$.

AN, inner or 'true' ammion. HM, hyomantibular cleft. SV air cliamber. TA allantois. WA, wlite or albumen. $\mathbf{Y} \mathbf{S}$, yolk-sac.

rapidly during the succeeding days, and forms the respiratory organ of the embryo.

By the end of the ninth day the embryo has grown consilerably, and has attained the shape and proportions shown in Fig. 101. The body walls are now definitely formed, and rudiments of the feathers are already present. The head is still disproportionately large, and the eyes are of enormous size. The beak, which was absent in the earlier stages, has now grown out from the front of the face, and at once gives the head a characteristic avian appearance. The neck is long and slender. The body is much more bulky than before, largely owing to the great sizo of 
the heart and the liver. The limbs have greatly increased in length; their several segments are well established, and the division of the distal ends into fingers and toes is very evident. The white of the egg has almost disappeared, a thick and very viscid mass, WA, alone remaining at the lower surface of the egg. The yolk-sac, ys, is still large, but its walls are flabby, owing to the absorption of a large part of its contents as food by the embryo. 'The allantois, TA, has grown enormously, and has spread over the back of the embryo, and quite half way round the interior of the egg-shell. It lies close to the shell, so that respiratory interchanges can readily take place, by diffusion through the porous shell, between the gases of the blood, in the vessels of the allantois, and the air outside the egg. In this way the respiration of the embryo is effected.

During the second half of the period of incubation the changes are of less interest. The young chick steadily increases in size at the expense of the yolk-sac, and gradually acquires the proportions and characters which it has on hatching. About the fourteenth day it shifts its position so as to lie lengthways in the egg, rather than across it. On the twentieth or twentyfirst day the yolk-sac is nearly absorbed, and what remains of it is drawn into the body of the chick, the body walls closing over it at the umbilicus. The chick thrusts its beak through the shell membrane into the air chamber at the broader end of the egg, and for the first time draws air directly into its lungs. Invigorated in this way, it breaks through the shell, by means of a hard knob on the tip of its beak, and steps out into the world.

\section{THE EGG.}

\section{Formation of the Egg.}

In the embryo fowl there are two ovaries, but in the course of development the right ovary disappears, and in the adult hen the left ovary is alone present. This is a large, irregularlyshaped body, suspended in a fold of peritoneum from the dorsal body-wall, opposite the anterior part of the left kidney. Numerous ova in different stages of development project from its surface, varying in size from dust shot up to spherical bodies an inch or more in diameter.

Of the two oviducts, the right one is rudimentary; the left 
one, which alone is functional, forms in the adult hen a wide convoluted tube, which commences in front with a long, oblique, funnel-like mouth, bordered by a fimbriated edge, and lying in close contact with the ovary. Behind this mouth comes a long, convoluted, but thin-walled part of the oviduct, and then a short terminal part with very thick walls, which opens into the cloaca, and through this to the exterior.

The ovum at the time of its discharge from the ovary consists of the yolk alone, inclosed in the vitelline membrane. The albuminous investment, or 'white of the egg', is formed around the yolk by the walls of the first, or thin-walled, part of the oviduct; and the shell membrane and egg-shell are added while the egg is in the thick-walled terminal part of the oviduct, just before being laid.

The ovaries can be recognised in chick embryos during the third day of incubation, as a pair of slightly modified tracts of the peritoneal epithelium which clothes the dorsal wall of the bodly cavity, close to the root of the mesentery. This germinal epithelium is at first merely a longitudinal strip of peritoneum, of which the component cells are columnar instead of squamous in shape. By multiplication of the cells, to form a layer several cells thick, the strip becomes a prominent ridge. Vascular connective tissue soon grows in along the axis of this genital ridge, and renders it still more conspicuous.

Almost from the first, certain of the epithelial cells of the genital ridge differ from their fellows in their greater size and more spherical shape, and in possessing nuclei of unusual dimensions; these larger cells are the primitive ova or gonoblasts. The primitive ova rapidly increase in size, and move from the surface, where they all take their origin, into the deeper parts of the genital ridge; the smaller, indifferent epithelial cells at the same time becoming arranged so as to form follicles around them.

The follicular epithelial cells serve to nourish the ova, drawing nutriment from the blood-vessels of the genital ridge, and passing it on, probably after elaborating it, into the ovum. Within the ovum the food matter undergoes further changes, and is deposited in the form of granules, from which the definite yolkgranules of the fully-formed egg are finally derived.

During these changes the nucleus of the primitive orum increases greatly in size, and acquires a distinctly vesicular 
structure, with one or more nucleoli: it is now spoken of as the germinal vesicle; and the establishment of the germinal vesicle, together with the marked increase in size of the ovum, owing to the accumulation of yolk-granules within it, mark the conversion of the primitive ovum, which occurs in both sexes alike, into the permanent ovum characteristic of the ovary of the hen bird.

As the egg increases in size it forms a swelling on the surface of the ovary, which rapidly becomes more prominent. A vitelline membrane is formed round the egg, between it and the follicular epithelium, and apparently derived from the egg itself. The follicular epithelium, with the outer wall of the ovary, form a vascular capsule, investing the egg.

The accumulation of yolk-granules within the egg continues until this has reached its full size. From an early stage, a difference may be noticed between white yolk-spheres, and yellow yolk-spheres; the former consisting of minute vesicles, each containing a highly refracting body; while the latter, which are apparently derived from the white yolk-spheres, are much larger bodies, filled with numerous bright, highly refracting granules.

In the fully-formed egg the white and yellow yolk-spheres are arranged in a very definite manner. The yellow yolk makes up the greater part of the bulk of what we call the yolk of the egg; the white yolk-spheres forming, (i) a somewhat flask-shaped plug in the centre of the yolk, with a neck reaching to the surface at the germinal disc; (ii) a thin superficial layer investing the whole exterior of the yolk, immediately below the vitelline membrane; (iii) a series of thin concentric shells between the surface and the central plug, the spaces between the successive shells being occupied by the yellow yolk.

\section{Maturation of the Egg.}

The ripening of the egg is accornpanied by changes in the nucleus, which are as yet only imperfectly known.

The nucleus, or germinal vesicle, during the growth of the egg, is large and vesicular, and occupies a position at or close to the centre of the egg. As the egg ripens, the nucleus moves towards the surface, where it lies just beneath the vitelline membrane, in a small lenticular patch, the germinal disc, which

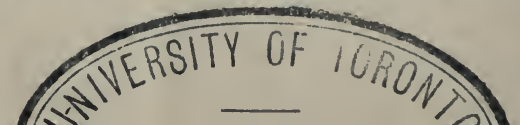


is comparatively free from yolk-granules. The nuclear membrane disappears; the chromatin elements form a reticular network, which then becomes distributed through the whole nuclear substance in the form of very fine granules; and finally, these granules run together to form six chromatin rods. The further changes have not been followed with certainty in the hen's egg; and neither the formation nor the extrusion of polar bodies has as yet been seen.

The egg, which is now ripe, is discharged from the ovary by rupture of the capsule at its most prominent part. The egg is received at once into the open mouth of the oviduct, which is closely applied to the ovary at the time, and then begins its passage down the oviduct to the exterior.

As it travels along the first or thin-walled part of the oviduct, the albumen, or 'white of the egg,' is poured out around it from the walls of the oviduct. The albumen is cleposited as a continuous sheet, which is wrapped spirally round the yolk, owing to the egg being caused to rotate, in its downward passage, by spirally arranged folds on the inner wall of the oviduct.

'This rotation of the egg causes the spiral twisting of the cords of denser albumen, at the ends of the egg, which are spoken of as the chalaza (Fig. 97, wC).

On reaching the lower part of the oviduct, or ' uterus,' the shell membrane, and finally the shell, are deposited on the outside of the egg, which is then passed into the cloaca, and laid.

The egg takes about three hours to travel along the thinwalled part of the oviduct; in the uterus it remains for a variable time, estimated by different authorities as usually lasting from twelve to eighteen hours.

\section{Fertilisation of the Egg.}

The details of the fertilisation of the hen's egg have not yet been determined. The large size of the egg offers great difficulties to the investigation of minute changes in connection with the nucleus, and these difficulties have not yet been surmounted.

All that is known with certainty is that fertilisation is effected, either in the upper part of the oviduct, or possibly, as stated by Coste, before the egg leares the ovary; so that during the whole time of the passage down the oviduct development is 
taking place. The spermatozoa are received by the hen some time before the laying of the eggs, and retain their vitality and functional activity for about a fortnight.

\section{THE EARLY STAGES OF DEVELOPMENT.}

\section{Segmentation of the Egg.}

Segmentation commences about the time the egg arrives in the lower, thick-walled part of the oviduct, or uterus ; it is continued actively during the stay of the egg in the uterus, and is completed about the time the egg is laid.

Segmentation, as already noticed, does not concern the whole egg, but is confined to the germinal disc; and the hen's egg is therefore spoken of as meroblastic, inasmuch as only a portion of it takes part in the process of segmentation, in contradistinction to the holoblastic eggs of Amphioxus and the frog, in which the entire egg is divided by the first cleft into two equal parts.

In the hen's egg, segmentation commences with the formation of a vertical groove or furrow, which runs across the middle of the germinal disc, but does not quite reach its edge at either end. This is very shortly followed by a second furrow, crossing the first one almost at right angles. Four radial furrows soon appear, about midway between the two first ones; and then by cross furrows each segment becomes divided into a central and a peripheral portion. Additional furrows soon appear, both radial and concentric, and by these the germinal disc becomes cut up into a mosaic of segments of irregular shape and size, separated from one another by the furrows or grooves (Figs. 102, 103).

The segmentation is slightly excentric almost from its first commencement, the furrows extending nearer to the edge of the disc, and the segments being smaller, at one side (the lower side of Fig. 103), which corresponds to the future posterior end of the embryo; while at the opposite, or anterior part, of the germinal disc the furrows stop short further from the edge, and the segments are of larger size.

Sections of the germinal disc at the stage represented in Fig. 103 show that, in addition to the vertical furrows by which the moșaic pattern is produced on the surface, horizontal clefts 
are also forming, by which the segments become completely isolated from one another, and from the underlying yolk (Fig. 104, ZA). These horizontal clefts, like the vertical ones, appear first in the centre of the germinal disc, and do not reach its margin until a later stage.

In each segment, or cell as it may now be termed, a nucleus is present from the first. The precise mode of origin of these nuclei has not been determined with certainty, but the history of the segmentation of the egg in Amphioxus, the frog, and other

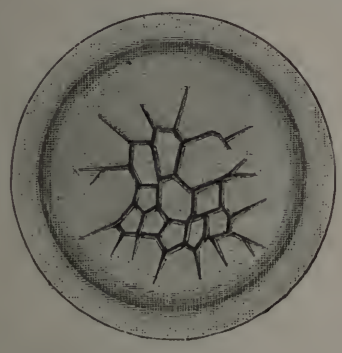

FIG. 102.

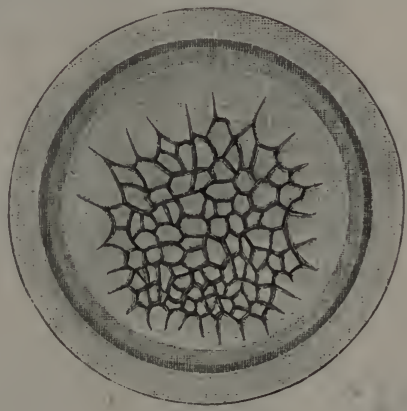

Fig. 103.

FIG. 102.-An early stage in the segmentation of the germinal disc of the Hen's Egg. (After Coste, and Duval.) $\times 10$.

FIG. 103.-A later stage, in which the germinal disc has increaserl in size, and the segments have, by further division, become smaller and more numerous. (After Coste, and Duval). $\times 10$.

Both these figures are from eggs taken from the lower part of the oviduct of the hen.

animals, leaves little doubt that the nuclei of all the cells are derived, by division, from the single segmentation nucleus of the fertilised egg.

The result of the process of segmentation, up to the point shown in Figs. 103 and 104, is the formation of a cap, occupying the centre of the germinal disc, and consisting of a single layer of nucleated cells : of these, the central ones, $z_{A}$, are small, and completely isolated from their neighbours, and from the underlying yolk; while the marginal ones, zB, are larger, and are only imperfectly marked off from the yolk, the horizontal clefts having not yet appeared.

The process of segmentation soon extends into the leeper part of the germinal disc; and by a further series of clefts, in different planes, this deeper part of the disc becomes cut up into 
cells, which from the first are nucleated, and are arranged in at layer two or three cells deep) (Fig. 10.5, ZL).

In this way, shortly before the time of laying of the egg, the germinal disc becomes converted into a cap of cells, spoken of as the blastoderm (Fig. 10.5). Of these cells the uppermost or

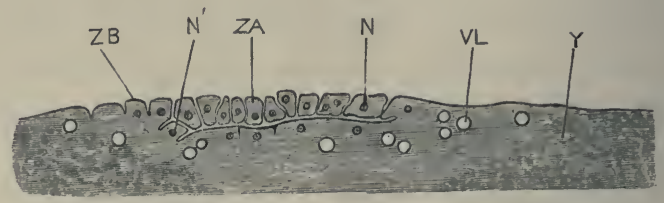

FIG. 104. - Section through the germinal clisc and adjacent parts of the yolk of a Hen's Egg about the middle of its stay in the uterus. The plane of section corresponds to a vertical line drawn through the centre of Fig. 103 ; the right-hand end of Fig. 104, which is the future anterior end, corresponding to the upper border of Fig. 103; and the left-hand end of Fig. 104 to the lower or posterior border of Fig. 103. (After Duval.) $\times 25$.

$\mathbf{N}$, nucleus of completed segment. $\mathbf{N}^{\prime}$, mucleus of segment not yet completely separateil from the yolk. VL, vacuole. $\mathbf{Y}$, yolk. ZA, completed blastomere. ZB, incompletely separatel blastomere.

most superficial layer (Fig. 10こ, E), which was the first to be definitely established, constitutes the epiblast; it consists of a single layer of cells, and is separated by a very shallow space, the blastocol or segmentation cavity, $B$, which appear's in section

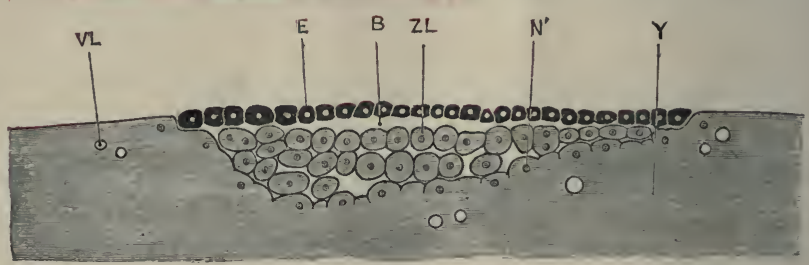

FIG. 105.--Vertical section of the blastoderm and adjacent part of the yolk of a Hen's Egg towards the close of segmentation. The anterior edge is to the right, the posterior edge to the left hand. (After Duval.) $\times 25$.

B, blastocol or segmentation cavity. E, epiblast. $\mathbf{N}^{\prime}$, nucleus of blastomere, which as yet is only incompletely separated from the yolk. VI, vacuole. $\mathbf{Y}$,yolk. ZI, one of the lower-layer cells or blastomeres.

as a mere chink or split, from the deeper mass of cells which may be spoken of collectively as lower layer cells, zL.

During the rest of the time that the egg stays in the uterus, while the egg-shell is forming, the process of segmentation continues actively. The clefts extend to the edge of the germinal disc, which becomes sharply marked off from the yolk beyond it; 
and, by rapid division, the cells become of nearly uniform size in all parts of the blastoderm.

The lowèr-layer cells become more sharply separated from the yolk. a space, filled with fluid, appearing beneath them, between the blastoderm and the yolk. This space, the subgerminal cavity (Fig. 106, Bv), is sometimes spoken of as the segmentation cavity; a name, however, which ought to be restricted to the narrow chink between the epiblast and the lower-layer cells (Fig. 10テ, B), which is clearly visible in the early stages, but becomes practically obliterated before the egg is laid.

Round its margin, new cells are still being cut out of the yolk, and added on to the blastoderm. Some of the cells which arise in this way, and lie between the edge of the blastoderm and the yolk, are markedly larger than any of the others, and are spoken of as formative cells (Fig. 106, ZF).

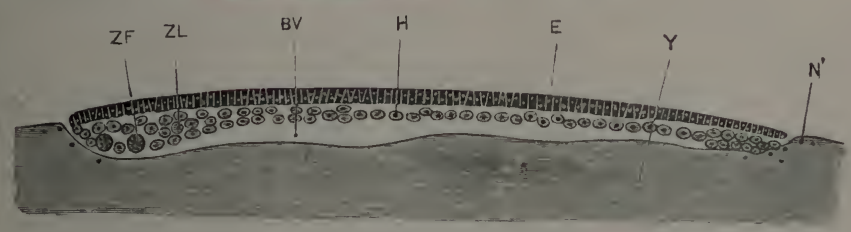

FIG. 106.--Vertical section of the blastoderm and adjacent parts of the yolk of a Hen's Egg at the time of laying, but before the commencement of incubation. The anterior edge of the blastoderm is to the right, the posterior edge to the left sicle of the figure. (After Duval.) $\times 25$.

BV, subgerminal cavity. E, epiblast. H. liypoblast. $\mathbf{N}^{\prime}$, uncleus in yolk, roum which a cell will be formel later. $\mathbf{Y}$, yolk. $\mathbf{Z} \mathbf{F}$, formative cell. $\mathbf{Z L}$, lower-layer cells.

\section{The Blastoderm.}

\section{a. The condition of the blastoderm at the time of laying of} the egg. The actual stage of development reached when the egg is laid depends on the length of time the egg remains in the uterus; and this we have seen is subject to considerable variation. The following description will apply to an average case.

Naked-eye examination shows the blastoderm (Fig. 97, BA) to be a small circular patch, about $3.5 \mathrm{~mm}$. in diameter, on the surface of the yolk: owing to its less specific gravity, the blastoderm is always uppermost, however much the egg be rolled over. The blastoderm consists of a marginal white rim, the area opaca, thickest at the posterior edge of the blastoderm (Fig. 106, ZF); and a central, circular, and more translucent portion, the area 
pellucida. Beyond the edge of the blastoderm (Fig. 97) the yolk shows one or more broad concentric bands, alternately darker and lighter in appearance.

Sections of the blastoderm at this stage (Fig. 106) show that it consists of two distinct layers of cells. (i) The upper layer, or epiblast, $\mathrm{E}$, is a continuous membrane, formed of small, short columnar cells, varying very little in size, and packed closely together side by side.

(ii) The lower layer consists of cells which are more loosely arranged, and which vary a good deal in shape and size in different parts. In the area pellucida, or middle portion of the blastoderm, they form a thin layer of somewhat flattened cells, $\mathrm{H}$, only one, or at most two cells in thickness. At the margin of the blastoderm, or area opaca, the cells become more numerous and more spherical in shape, forming a thickened rim which rests on the underlying yolk, and in which the large formative cells, $Z F$, are found, especially near the posterior margin. In the yolk, on which the edge of the blastoderm rests, nuclei (Fig. $\left.106, \mathrm{~s}^{\prime}\right)$ are present, round which cells are formed at a later stage, and added on to the margin of the blastoderm.

Beneath the area pellucida, and separating it from the bed of yolk, $\mathrm{Y}$, is the subgerminal cavity, $\mathrm{BV}$; a well-marked space, filled with fluid.

b. The growth of the blastoderm. Round the margin of the blastoderm the epiblast and the lower-layer cells are at first continuous with each other, but shortly before the laying of the egg this continuity is lost, except at the posterior border, where, as shown on the left-hand side of Fig. 106, the two layers are still continuous with each other at the time the egg is laid.

After incubation has commenced, the blastoderm spreads rapidly, retaining its circular shape. By the end of the first day of incubation it is about the size of a sixpence; and by the end of the second day it has extended nearly half way round the egg; after this it proceeds more slowly, the complete inciosure of the yolk not being effected until about the seventeenth day.

In this spreading of the blastoderm (cf. Figs. 98 and 99) the peripheral part, or area opaca, grows much more rapidly than the central area pellucida; the area opaca retains its circular outline, but the area pellucida (Figs. 98 and 99,AD) very early 
becomes oval, and then pyriform in shape, the broader end corresponding to the anterior end of the embryo.

The two layers of the blastoderm grow independently. 'The epiblast, after it has become free from the lower layer, extends slightly beyond this, so that its margin rests directly on the yolk; its further spreading is effected mainly by division of the already formed cells, stimulated, no doubt, by absorption of nutriment from the yolk on which they are lying. The lower-layer cells, after separation from the epiblast, become directly continuous at their margin with the yolk, forming a thickened rim, spoken of as the germinal wall: the extension of the lowerlayer cells is effected principally by the addition of new cells cut out from the yolk, but partly also by division of the already formed cells, as in the epiblast.

\section{The Hypoblast.}

A few hours after the commencement of incubation, the lower-layer cells undergo important changes, by which the hypoblast and mesoblast become established.

In the area pellucida, the majority of the lower-layer cells become flattened horizontally, and unite at their edges so as to form a continuous cellular membrane, the hypoblast; a few isolated lower-layer cells are left between the epiblast and the hypoblast, which take part, as will be noticed immediately, in the formation of the mesoblast.

In the area opaca, or marginal part of the blastoderm, the differentiation of the hypoblast as a distinct cellular membrane occurs somewhat later; and the hypoblast cells of this region, which are large, and cubical or slightly columnar in shape, differ markedly from the thin, parement, hypoblast cells of the area pellucida.

\section{The Primitive Streak.}

At the posterior border of the blastoderm, as noticed above, the fusion of the epiblast and the lower-layer cells persists longer than it does round the rest of the blastodermic rim; and in the egg, at the time of laying, a crescentic opacity is visible at the posterior edge of the blastoderm, marking this line of fusion.

As the blastoderm grows, during the earlier hours of incu- 
bation, this opacity becomes lengthened out into a linear band, the primitive streak, which, starting from near the centre of the blastoderm, extends backwards across the area pellucida towards its margin. The increase in length of the primitive streak is effected almost entirely by growth backwards of its hinder end, the anterior end lengthening very little, if at all.

The area pellucida grows more rapidly in its posterior than in its anterior part, and from about the fifteenth hour becomes pyriform in outline. The primitive streak keeps pace with the growth of the area pellucida; and about the twentieth hour, when the area pellucida is markedly pyriform in shape (Fig. 107, AD),

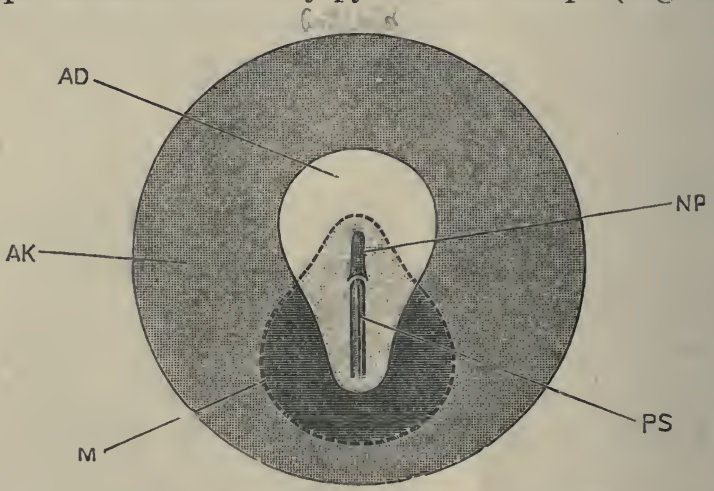

ErG. 107. -A diagrammatic figure of the blastoderm of a Hen's Egg about the twentieth hour of incubation. (In part after Duval.) $\times 8$.

\footnotetext{
AD, area pellucida : the part left white consists of epiblast and hypoblast alone ; in the linder part of the pyriform area, covered by the light sliading, mesoblast is present as well. AK, area opaca. IMI, dotterl line inilicating the boumlary of the mesoblast. N P, neural plate, the first commencement of the central nervous system. PS, prinitive streak.
}

the primitive streak, PS, forms a well-defined opaque band stretching about two-thirds of the way across the area pellucida. The anterior end of the primitive streak is sharply defined; the posterior end is less distinct, is often irregularly bent, and usually dies away a short distance before reaching the edge of the area pellucida. A shallow median furrow, the primitive groove, runs along the whole length of the primitive streak.

Transverse sections of the blastoderm (Fig. 108) show that the primitive streak is formed by proliferation of cells from the under surface of the epiblast, in the median line. The cells grow downwards as a solid keel, which spreads out right and left as a horizontal sheet of cells, PS; these are spherical in 
shape, rather closely packed together, and situated between the epiblast, $\mathrm{E}$, and the hypoblast, $\mathrm{H}$.

The primitive streak appears before any trace of the nerrous or other systems of the embryo has commenced to form. The meaning of the primitive streak has been much discussed, but it is now generally agreed that it corresponds, at any rate in part, to the lips of the blastopore in the frog, which have become lengthened out, and fused together'; the primitive groove marking the line of concrescence of the lips of opposite sides of the blastopore. The anterior end of the primitive streak in the chick certainly corresponds to the anterior or dorsal lip of the blastopore in the frog; but it is not quite clear whether the entire length of the primitive streak is to be compared to an elongated and drawn out blastopore, or whether the hinder part

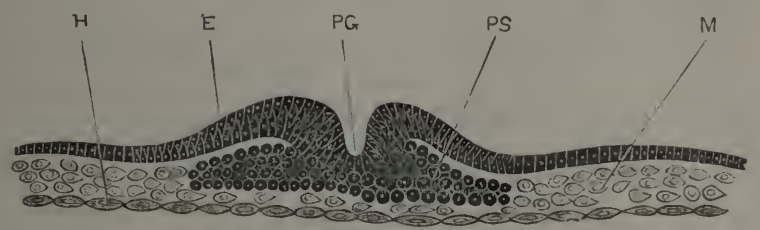

Fig. 108.-Transverse section across the blastoderm of a Hen's Egg about the twentieth hour of incubation, the section passing through the primitive streak about the middle of its length (of. Fig. 107). $\times 200$.

E, epiblast. $\mathbf{H}$, lyypoblast. $\mathbf{M}$, mesoblast. PG, mimitive groove. PS, primitive streak.

of it is not rather due to the peculiar method of spreading of the blastoderm, imposed on the chick embryo in consequence of the distension of the egg by the enormous mass of food-yolk which it contains.

\section{j. The Mesoblast.}

The middle germinal layer, or mesoblast, gives rise in the chick, as in Amphioxus, in the frog, and in other animals generally, to all the connective tissue, vascular, muscular, and skeletal structures, as well as to the urinary and reproductive organs.

In the chick, the mesoblast cells have a less clearly defined origin than in Amphioxus or in the frog, and are derived from three distinct sources.

(i) In the hinder part of the blastoclerm, some of the cells 
of the original lower layer are left, lying between the epiblast and hypoblast, on the establishment of the latter as a distinct and continuous membrane; and these cells become mesoblast cells (Fig. 108, M).

(ii) In the middle and lateral portions of the area pellucida. about the time of appearance of the primitive streak, mesoblast cells are budded off freely from the upper surface of the hypoblast, and form a layer between the epiblast and hypoblast in this region.

(iii) The horizontal sheets of cells (Fig. 108, PS), which spread out right and left as the wing-like expansions of the primitive streak, and which, it will be remembered, are of epiblastic origin, also take part in the formation of the mesoblast.

As regards the cells themselves, those of groups (i) and (ii) agree with one another in being usually of an irregular stellate shape (Fig. 108, M), and in being very loosely arranged. The origin of these two groups is very similar, though not identical; the cells of the first group being derived from the lower-layer cells, formed by segmentation of the germinal disc; while those of the second group arise directly from the hypoblast, after this is established as a distinct cellular membrane. It is not possible to draw a sharp line between the two groups, nor to determine in all cases to which group a given cell belongs. Speaking generally, the mesoblast of the body of the embryo itself is derived from group (ii), the cells of group (i) lying almost entirely in the extra-embryonic parts of the blastoderm.

The cells of group (iii) are derived directly from the epiblast, and are therefore of totally different origin to those of groups (i) and (ii). They also differ from these latter in their spherical form and more compact arrangement. They are at first (Fig. 108, ps) sharply marked off from the cells of groups (i) and (ii), but as the primitive streak spreads laterally, the cells composing it come into close relation with those of the other groups, and becoming at the same time less compactly arranged, and less regular in form, can no longer be distinguished from those of groups (i) and (ii). The cells of group (iii), or primitive streak mesoblast cells, lie almost entirely behind the embryo, and take but little share in its formation.

The mesoblast cells of all three groups soon become con- 
timuous, forming a sheet of somewhat loosely arranged and usually stellate cells, which at the twentieth hour of incubation has a shape and extent indicated by the strong dotted line, $\mathrm{M}$, in Fig. 107. The two halves of the sheet are continuous with each other across the median line in the region of the primitive streak, rs, and behind it; but in front of the primitive streak,

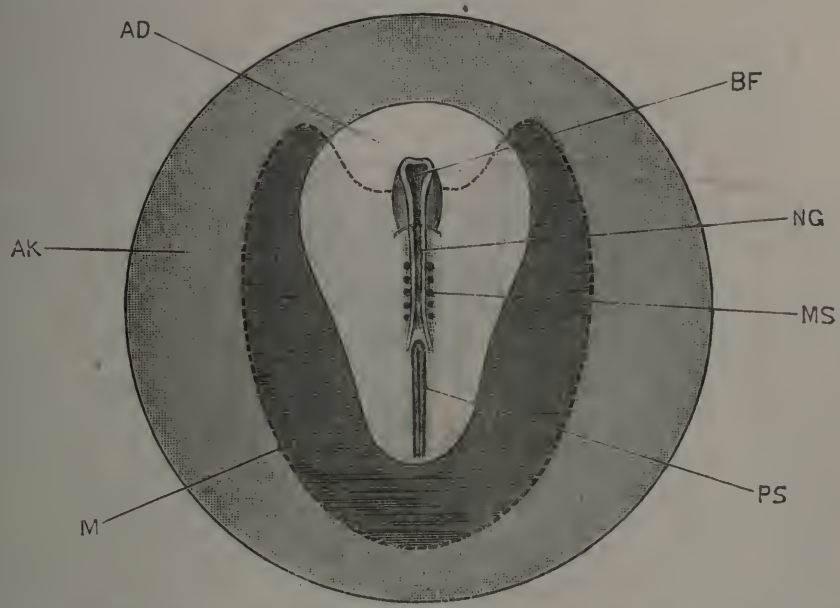

FIG. 109.-A diagrammatic figure of the blastoderm of a Hen's Egg about the twenty-fourth hour of incubation. (In part after Duval.) $\times 8$.

AD, area pellucida : the part left white is the proamnion, and consists of epiblast and hypoblast alone; in the hiniler part of the pyriform area pellucida, covered by the light shading, mesoblast is present as well. AK, area opaca. BF, commencing fore-brain. M, dottell line indicating the limit to which the mesoblast has spreal. MS, mesoblastic somite or protovertebra. $\mathbf{N G}$, neural groove. PS, primitive streak.

For a more exact view of an embryo of this age see Fig. 110.

in the region where the embryo is formed, NP, the two halves are separated in the middle line by the notochord, the description of which is given on the next page.

In the later stages, as the embryo appears, the mesoblast sheet spreads rapidly. It does not extend directly in front of the embryo, but grows forwards as two lateral horns (Fig. 109), so that for a considerable time there is, immediately in front of the embryo, a transparent area of the blastoderm, AD, which consists of epiblast and hypoblast only, without any middle layer or mesoblast. This area, the proamnion, remains twolayered until about the middle of the third day of incubation, when the two lateral horns of mesoblast gradually grow inwards to meet each other in front of the embryo. 
As shown in Figs. 107 and 109, the mesoblast very early extends outwards beyond the area pellucida so as to underlie the inner zone of the area opaca; this three-layered zone of the area opaca, represented by the dark shading in Figs. 107 and 109 , is known as the area vasculosa, because the blood-vessels which absorb the yolk and carry it to the embryo are very early developed in it (cf. Figs. 98, 99, Av).

\section{The Notochord.}

Before the sheet of mesoblast cells, spoken of above as group (ii), separates completely from the hypoblast, a distinction may be noticed in it between a median longitudinal rod of cells, and two lateral tracts. This median rod is the notochord (Fig. 117, $\mathrm{CH}$ ); and the cells of which it consists are, from the first, more closely compacted than those of the lateral tracts.

The notochord sometimes remains attached to the hypoblast after the lateral mesoblastic sheets have completely separated from this ; in other specimens the entire sheet of cells separates as one continuous layer, which then divides into the median rod, or notochord, and the two lateral mesoblastic tracts.

The notochord of the chick has, accordingly, been described by some authorities as of hypoblastic, by others as of mesoblastic origin; the component cells are, however, in all cases derived directly from the. hypoblast, and the difference is merely in the relative times of separation of the notochord from the lateral sheets of mesoblast, and from the mnderlying hypoblast respectively.

The notochord lies entirely in the part of the blastoderm in front of the primitive streak; its posterior end is, however, directly continuous with the anterior end of the primitive streak. Inasmuch as the primitive streak cells are continuous with the epiblast, and the notochord is, at any rate at first, continuous with the hypoblast, it follows that the three germinal layers, epiblast, mesoblast, and hypoblast, are directly continuous and fused with one another at this point, which marks the hinder end of the chick embryo, and corresponds to the anterior or dorsal lip of the blastopore in the frog ( $c f$. Fig. 60, B).

\section{The Mesoblastic Somites and the Cœlom.}

The mesoblast of either side forms at first a continuous sheet of loosely arranged cells, which in transverse section is somewhat 
wedge-shaped, being thickest next to the notochord and gradually thinning as it passes outwards towards the margin of the blastoderm (cf. Fig. 117, M).

About the twenty-first hour of incubation, the mesoblast cells become arranged more or less clearly in two layers, upper and lower, with a slight space between them. This splitting of the mesoblast, as it is termed, first appears in the part of the mesob]ast beyond the embryo, but soon spreads inwards to the embryonic region, extending almost up to the notochord.

The cavity, formed in this way, by splitting of the mesoblast, becomes the colom or body cavity of the chick. Of the two layers into which the mesoblast is split, the upper or outer is spoken of as the somatic layer, and the lower or inner as the splanchnic layer. From a very early period the somatic layer (Fig. 129, ME) becomes closely connected with the surface epiblast, forming with this the somatopleure or body wall; while the splanchnic layer becomes similarly related to the hypoblast, and forms with this the splanchnopleure or wall of the alimentary canal (Fig. 129, мH).

A body cavity that is formed in this way, by splitting of the mesoblast into somatic and splanchnic layers, is spoken of as a schizocœl, in contradistinction to the enterocœl of Amphioxus, which arises as a series of hollow outgrowths from the enteron or primitive alimentary canal. Inasmuch as the mesoblast of the embryo is derived almost entirely from the hypoblast, as described above, the distinction between an enterocœl and a schizocœl may be said to consist in this:-in the enterocœl the mesoblast arises as hollow outgrowths from the hypoblast, which subsequently become shut off from the gut, while the cavities of the outgrowths open into one another and become the cœlom of the adult. In the schizocœl, on the other hand, the mesoblast arises as two solid sheets, budded off from the hypoblast, in which the cœlom is formed at a later stage by splitting of the sheet into two layers, with a space between them. Of these two methods of formation of the cœlom there can be little doubt that the enterocœlic is the more primitive one, the schizocœlic the more modified.

Almost immediately after the splitting of the mesoblast is effected, about the twenty-second hour, a series of clear transverse lines, really vertical clefts through the mesoblast, appear in the embryo, extending outwards a short distance each side of the 
notochord; these are quickly followed by a pair of similar but longitudinal clefts, which appear one along each side of the body, a little distance from the middle line. By these clefts the mesoblast of each side of the body becomes divided into a vertebral plate, alongside the notochord; and a lateral plate, inore peripherally placed; the vertebral plate being further cut up by the transverse clefts into a series of somewhat cubical blocks, the mesoblastic somites or proto-vertebræ (Fig. 110, Ms).

The mesoblastic somites appear first in the neck region, and increase rapidly in number during the last two hours of the first day, and the following two or three days. One or perhaps two pairs are formed in front of the pair which appears first; the remainder are added on in succession at the hinder end of the series, as the embryo increases in length. At the twenty-fourth hour of incubation there are usually five or six pairs present (Fig. 110, MS); by the thirty-sixth hour (Fig. 111) these have increased to about fifteen pairs; at the end of the second day there are twenty-seven or twenty-eight pairs, after which date the further increase takes place more slowly until, during the fourth day, the full number is established. The increase takes place in a very regular manner, and the number of somites present affords a convenient basis for estimating the age, and the grade of development, of embryos during the earlier stages of their formation.

The somites extend along the whole length of the neck, trunk, and tail, but are not formed in the head, in which no segmentation of the mesoblast occurs. In the case of the first three or four somites, the splitting of the mesoblast extends up to the notochord before the somites become marked off from the lateral plates; and consequently the cavities of these somites communicate for a time with the colom or cavity of the lateral plate, though this communication is lost as soon as the longitudinal cleft is formed which separates the vertebral and lateral plates from each other.

The remaining somites, behind the first three or four pairs, do not communicate at any stage with the cœlom, their cavities appearing independently, and after the separation of the vertebral from the lateral plates.

The further stages in the development of the mesoblastic somites will be described in a later section (p. 322). 


\section{The Amnion.}

The amnion is a fold of the somatopleure which rises up as a wall all round the embryo, a little distance from it, and, spreadng over its back, forms a thin double membrane between the embryo and the egg-shell. Though a very characteristic structure, it is of only secondary importance, and gives rise to no part of the embryo itself.

The first trace of the amnion appears about the thirty-third hour, as a small crescentic fold immediately in front of the head of the embryo. This grows rapidly, and by the thirty-sixth hour (Figs. 111, 112, Ax), has extended back over the anterior end of the head as a transparent cap, formed by a double membranous fold.

This first formed part, or head fold, of the amnion consists at first of epiblast only, inasmuch as it arises from the proamnion, or part of the blastoderm immediately in front of the embryo, into which the mesoblast has not yet spread (cf. Fig. 109). During the latter part of the second, and the third day, the mesoblast gradually grows in from the sides, forming a thin lining to the amnion, which from this time is two-layered.

The head fold of the amnion extends backwards rapidly, and before the end of the second day covers over the whole of the head and neck region of the embryo. At the hinder end of the embryo a similar tail fold is formed during the second day ; and, a little later, side folds appear, connecting the head and tail folds together. The embryo is now completely surrounded by the amnion, which forms a low wall round its sides and tail, and extends backwards over the head and neck as a thin membranous. cap.

Unlike the hearl fold, the side and tail folds of the amnion ( ff. Fig. 129, Ax) consist from the first of both epiblast and mesoblast; i.e. are folds of the somatopleure, beyond the margin of the embryo.

During the third day the ammion grows rapidly on all sides, and by the close of the day (Fig. 114, Ax, $A x^{\prime}$ ) has covered over the whole of the embryo, except a small patch near the hinder end. During the fourth day the side folds meet each other over the back of the embryo, which thus becomes completely covered by the amnion. As the amnion folds meet, they coalesce, the inner layers of the folds forming a continuous membrane, the inner or true amnion (Fig. 100, AN), which 
closely invests the embryo, and is continuous with the margin of its body wall (Fig. 129). The outer layers of the amnion folds also form a continuous membrane, the outer or false amnion (Fig. 100, Az), which lies close beneath the vitelline membrane, and soon fuses with this, while peripherally it passes into the layer of somatopleure investing the yolk-sac.

The space between the inner or true amnion and the embryo is called the cavity of the amnion. It is filled with fluid, and is at first very small, the true amnion on the fourth and fifth days investing the embryo very closely (Fig. 100). During the following days, owing to accumulation of fluid within it, the amnionic cavity increases very considerably, forming a waterbath in which the embryo can move freely in any direction. During the later stages of incubation, muscle fibres are developed in the mesoblast of the amnion, which by their contractions rock the embryo to and fro within the egg.

The space between the inner and outer layers of the anmion (Fig. $100 \mathrm{AN}$ and $\mathrm{AZ}$ ) is, from the mode of formation of the amnion (Figs. 114, 129), continuous with the cœlomic space which lies between the two layers of the mesoblast, both within the embryo and in the extra-embryonic region of the blastodern. By the sixth day the splitting of the mesoblast (cf. Fig. 100) has extended about half-way round the yolk-sac. 'The further extension of the splitting takes place much more slowly, and does not reach the lower pole of the yolk-sac until within a few days of the time of hatching.

About the tenth day (cf. Fig. 101), when the splitting of the mesoblast has extended about three-fourths of the way round the yolk-sac, a circular fold of somatopleure arises from near its ventral edge, and grows over the dense mass of albumen, WA, at the lower surface of the egg, inclosing this in much the same way as the amnion incloses the embryo at an earlier stage, and aiding in the absorption of this mass of albumen.

The formation of an amnion is a very characteristic feature in the development of the three higher groups of VertebratesReptiles, Birds, and Mammals. These same three groups are also characterised by the presence, during the later stages of development, of an allantois, which plays an important part in the respiration of the embryo, and, in mammals, in its nutrition as well. The two structures, amnion and allantois, are associated 
to this extent, that. the space between the two layers of the amnion gives the allantois a ready opportunity for free and rapid growth, and enables it to obtain a position close to the inner surface of the egg-shell (Figs. 100, 101, TA), where its respiratory efficiency is greatest.

It would, however, not be right to regard the amnion as merely a provision to insure free growth of the allantois, for this would not explain how the amnion originated in the first instance; and it must be remembered that all the characteristic stages in the development of the amnion are completed while the allantois is still in a very rudimentary condition. The amnion has probably to be explained quite irrespectively of the allantois.

The most satisfactory explanation of the formation of the amnion is that it is due, in the first instance, not to uprising of a fold of somatopleure, but to depression of the embryo into the yolk-sac; the sinking of the embryo being due partly to its own weight, partly to the downward growth of the front part of the head caused by cranial flexure; and perhaps in part to the resistance of the vitelline membrane, aided by the liquefaction of the yolk as this becomes absorbed for the nourishment of the embryo. The main purpose effected by the depression of the embryo is to remove it from the danger of pressure against the egg-shell, a consideration which has more weight in the case of Reptiles, in which group the amnion was first acquired, and in which the yolk often completely fills the egg-shell, than in their descendants, the Birds.

\section{THE DEVELOPMENT OF THE NERVOUS SYSTEM.}

\section{General Account.}

The development of the nervous system of the chick is effected in practically the same mamner as that of the frog. About the nineteenth or twentieth hour, almost immediately after the notochord has appeared, the epiblast in front of the primitive streak becomes thickened along the median line to form the neural plate (Fig. 107, NP).

During the next four or five hours, the anterior part of the area pellucida grows rapidly (Fig. 109) : the neural plate lengthens with it, and soon becomes considerably longer, and more promi- 
nent, than the primitive streak. A longitudinal neural groove (Fig. 117, NG) forms along its dorsal surface; this is at first shallow, but rapidly deepens by uprising of its borders as a pair of longitudinal ridges, the neural folds.

At their hinder ends (Fig. 110), the two neural folds diverge from each other, and embrace between them the anterior end of the primitive streak. In front, the neural folds rapidly increase

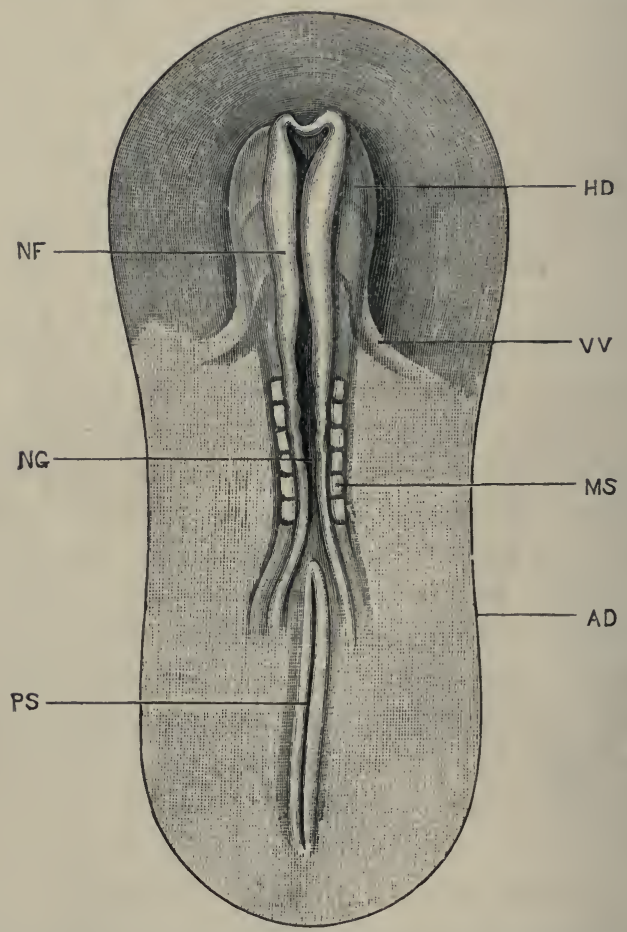

FIG. 110.-A Chick Embryo at the twenty-fourth hour of incubation ; seen from the dorsal surface. $C f$. Fig. 109, which shows the relations of an embryo of this age to the blastoderm. $\times 20$.

- AD, margin of area pellueida. HD, head of embryo. MSS, mesoblastic somite or protovertebra. NF, neural fold. NG, neural groove. PS, primitive streak. VV, vitelline vein.

in height, the neural groove between them becoming deeper in consequence. About the end of the first day (Fig. 110, sF), the two neural folds meet, in the region of the future hind-brain, converting the open groove into the closed neural tube ( $c f$. Fig. 118); and this closure of the tube rapidly extends both forwards and backwards. 
The anterior end of the head of the embryo is lifted up above the blastoderm by the head fold ( $c f$. Fig. 112); and the neural folds are continued round this uplifted head to its under surface (Fig. 110), where they become continuous with each other in the median plane. A transverse section across the extreme anterior end of an embryo at this stage (Fig. 110) will cut the projecting neural folds, but no other part of the embryo, and will consist of two completely separate halves.

By the middle of the second day (Fig. 111) the neural folds have met and fused, so as to complete the neural tube, along the whole length of the brain region; the last point to close being in the position afterwards occupied by the pineal body. The fusion has also extended backwards along the greater part of the region of the spinal cord, but at the hinder end of the embryo the two neural folds are still a little distance apart.

\section{The Spinal Cord.}

The spinal cord, in the earlier stages of its development, is oval in transverse section (Fig. 129, Ns): its roof and floor, in the mid-dorsal and mid-ventral planes, remain thin; but its side wails thicken, so as to reduce the central cavity to a narrow vertical slit.

In the side walls of the spinal cord a distinction is present, almost from the first, between (i) an inner layer of columnar ciliated epithelial cells, lining the central canal; and (ii) the cells composing the rest of the thickness of the wall. These latter apparently do not give rise to either nerve-cells or nervefibres, but become modified to form a supporting framework to the cord. The cells of this second group are from the first radially arranged, and during the second day they branch at their outer ends, the branches anastomosing with those of adjacent cells to form a delicate reticular framework.

In the meshes of this reticulum certain other cells, the neuroblasts, appear during the third day; these are apparently derived, by direct modification, from certain of the columnar epithelial cells lining the central canal, which migrate outwards into the reticulum. Each neuroblast is at first bipolar, having a shorter process, directed inwards towards the central canal; and a longer process which is directed outwards, and which by further growth becomes the axis cylinder of a nerve fibre. The axis cylinder's thread their way through the meshes of the 
reticulum and reach the surface of the spinal cord, where someleave it to form the roots of the spinal nerves, while others rum longitudinally along its outer surface to form the layer of white matter of the spinal cord.

From the third, or fourth, to about the tenth day, this process of development of neuroblasts and of nerve fibres proceeds rapidly. The neuroblasts become the nerve cells of the spinal cord, the first cells to be established being those of the ventral cornua: their inner processes disappear, and from the bodies of the cells fine branching protoplasmic outgrowths arise at a later stage, which anastomose with those of neighbouring cells. As the nerve fibres increase in number, the layer of white matter on the surface of the spinal cord necessarily gains in thickness, and the spinal cord rapidly approaches the shape characteristic of it in the adult.

The central cavity of the spinal cord is at first a narrow vertical cleft (Fig. 129). The side walls of the dorsal half of this cleft come in contact with each other and fuse, so as to obliterate the cavity; the ventral half of the cleft persists throughout life as the central canal of the spinal cord.

Of the two longitudinal fissures of the adult spinal cord, the ventral fissure is a median groove left between the ventral columns of white matter, as these increase in thickness; it may be recognised on the sixth or seventh day, and by the tenth day is a conspicuous feature in transverse sections of the spinal cord.

The dorsal fissure is formed in quite different fashion. The white matter of the dorsal surface grows down into the spinal cord, about the ninth day, as a pair of vertical plates; these are at first separated by a thin median lamina of grey matter ; and it is by absorption of this median lamina that the dorsal fissure is formed. The absorption is a gradual one, and for some time the fissure remains bridged across by slender fibres, derived from the grey matter.

The neurenteric passages. In the floor of the neural canal, at the hinder end of the body, two or three pit-like depressions appear in the early stages of development, which, although they are usually incomplete, and only rarely open into the mesenteron, still appear to be homologous with the neurenteric passage in Amphioxus or in the frog. 
Three of these depressions have been observed in chick embryos. They appear in succession; the first one shortly before

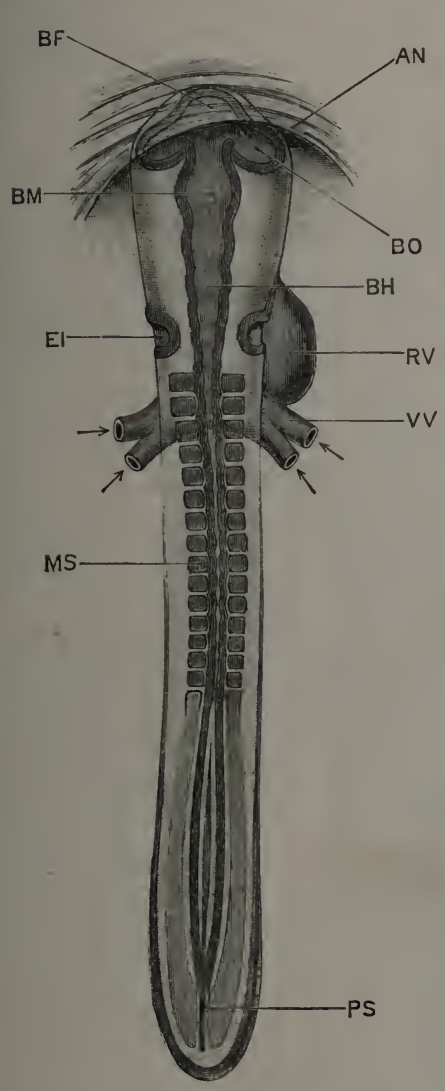

FIG. 111.

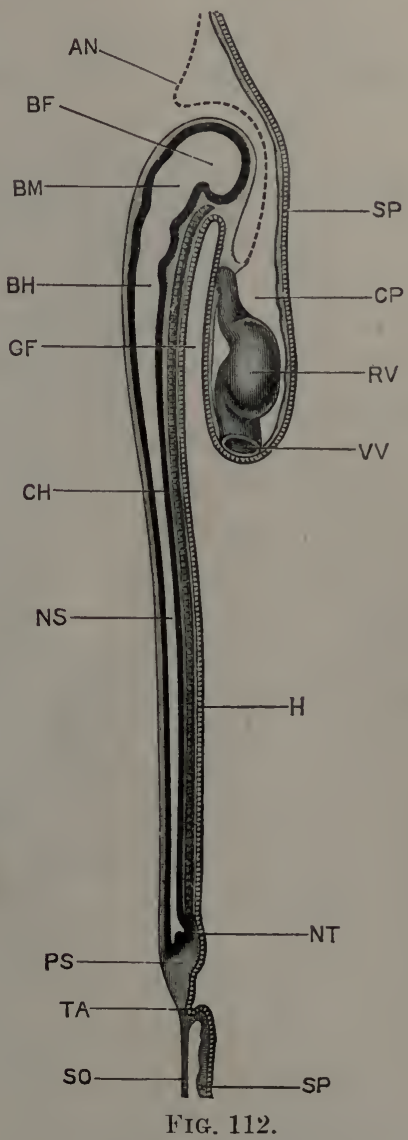

FIG. 111.-A Chick Embryo at the thirty-sixth hour of incubation; seen from the dorsal surface. $\times 20$.

FIG. 112.-A median longitudinal, or sagittal, section of a Chick Embryo at the thirty-sixth hour of incubation. $\times 20$.

A N, head fold of the amnion. BF, fore-brain. BH, hind-brain. BM, mid-brain. BO, optic vesicle. CH, notochord. CP, perieardial eavity. FI, auditory pit. GF, fore-gut, or anterior nortion of the mesenteron. H, hypoblast. MS, mesoblastic somite or protovertebra. NS, suinal corc. NT, neurenteric canal. PS, primitive streak. RV, ventricular portion of the heart. SO, somatopleure. SP, splanchnopleure. TA, allautois. VV, vitelline veins.

the end of the first day; the second one (Fig. 112, vT) about the middle of the second day; and the third one in the course of the third day. 
They are all three blind pockets, extending somewhat obliquely from the floor of the hinder end of the neural tube into a fused mass of cells just behind the notochord: this mass is really the anterior end of the primitive streak, and therefore corresponds to the anterior lip of the blastopore in the frog (cf. Fig. 60).

\section{The Brain.}

The general history of the development of the brain in the chick is very closely similar to that already described in the frog.

At the commencement of the second day, and before actual fusion of the neural folds has taken place at any part of their length, the neural canal becomes dilated at its anterior end to form the anterior cerebral vesicle or fore-brain (Fig. 111, BF), from which the optic vesicles, $\mathrm{BO}$, arise almost at once as lateral outgrowths. Immediately behind the fore-brain, and separated from it by a slight constriction, is a second and rather smaller dilatation, the middle cerebral vesicle or mid-brain, BM.

The part of the brain behind the mid-brain, about half its entire length, is the hind-brain, BH; this consists of a series of vesicles, separated by slight constrictions, decreasing in size from before backwards, and passing without any limiting boundary into the spinal cord posteriorly. The vesicles of the hind-brain vary considerably in different specimens; they are usually four or five in number, of which the two anterior ones, at any rate, appear to possess considerable constancy. Their mode of development, and their relations to the nerves and other structures, strongly suggest that they are each equivalent to a single vesicle, such as the mid-brain.

By the middle of the second day (Figs. 111 and 112) the brain is closed, by fusion of the neural folds, along its entire length ; the point where the folds last meet being at the summit of the fore-brain, in the position subsequently held by the pineal body.

The walls of the brain are at first of nearly uniform thickness in all parts; and transverse sections of the brain are approximately circular in outline at all parts of its length.

In the following account the several parts of the brain will be considered in order from behind forwards, and the leading points in their development described. 
The medulla oblongata is formed from the hind-brain, the central canal of this part of the brain becoming the fourth ventricle of the adult.

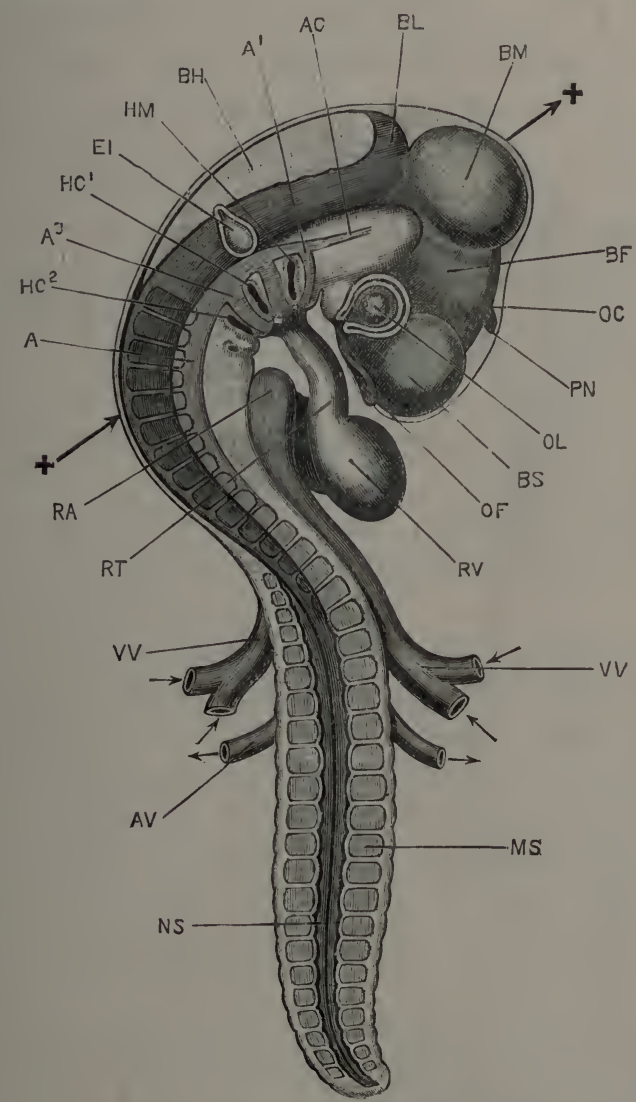

FIG. 113.-A Chick Embryo at the end of the third day of incubation. Owing to the twisting of the fore part of the embryo, the head and neck are seen from the right side, and the hinder part of the body from the dorsal surface. The amnion has been removed. $(C f$. Fig. 99.) $\times 20$.

\footnotetext{
A, dorsal aorta. A1, first or mandibular aortic arch. A3, third aortic arel, in the first branchial arch. AC, carotid artery. AV, vitelline artery. BF, thalamenceplialon or fore-brain. $\mathbf{B H}$ medulla oblongata. $\mathbf{B I}$, cerebellum. $\mathbf{B} \mathbf{M}$, mid-brain. $\mathbf{B S}$, cerebral hemisphere. EI, auditory vesicle. HC1, first branchial cleft. HC2, seconil branchial cleft. HM, hyo-mandibular eleft. MS, mesoblastic somite or protovertebra. NS, spinal cord. OC, optic cup. OF, olfactory pit. OL, lens. PN, pineal body. RA, auricle of heart. RT, truncus arteriosus. $\mathrm{RV}$, ventricle of heart. VV, vitelliue veins.

The two arrows anl crosses indicate the plane along which tle section shown in Fig. 124 is taken.
}

The walls of the medulla oblongata are at first of nearly equal thickness all round; but before the end of the second day 
(Fig. 121, BH) the dorsal wall or roof becomes very much thinner than the sides and floor. In the later stages this difference becomes increasingly marked; and before the end of the third day (Figs. 113 and 114) the roof, which is now very wide, becomes reduced to a single layer of epithelial cells, entirely devoid of nervous matter; a condition in which it remains throughout life. This thin roof soon becomes thrown into folds, which appear about the seventh day, and rapidly increase in depth, hanging down into the cavity of the medulla. Between the layers of these folds a network of vessels, which early appears on the outer surface of the roof, grows in to form the choroid plexus of the fourth ventricle (Fig. 116, xв).

The division of the hind-brain into a series of vesicles, which is very noticeable about the thirty-sixth hour (Fig. 111), becomes less evident as the side walls thicken, through the formation of the white nervous matter; and from the middle of the third day onwards it is barely perceptible.

The cerebellum is developed from the roof of the anterior vesicle of the hind-brain, immediately behind the well-marked constriction which separates the hind-brain from the mid-brain.

It appears towards the end of the second day, as a slightly marked transverse thickening of the roof of the hind-brain; it becomes more conspicuous during the third, fourth, and following days (Figs. 113, 114, and 115, BL), but remains as a simple transverse band until a comparatively late stage of development.

About the eighth day, the cerebellum (Fig. 116, BL) becomes doubled transversely on itself; and at the same time it thickens considerably, its outer surface becoming slightly folded. From this time it steadily increases in thickness, and by further folding of its surface becomes more complicated in structure; but up to about the sixteenth day it lies completely behind the optic lobes.

During the last few days of incubation the cerebellum enlarges considerably, growing forwards over the top of the mid-brain and between the optic lobes : by the time of hatching it has almost met the cerebral hemispheres, and has acquired the shape and proportions characteristic of the cerebellum in the adult bird. 
The fact that the cerebellum remains for so long a time in the condition of a mere transverse thickening of the roof of the medulla oblongata, becomes of considerable interest when it is

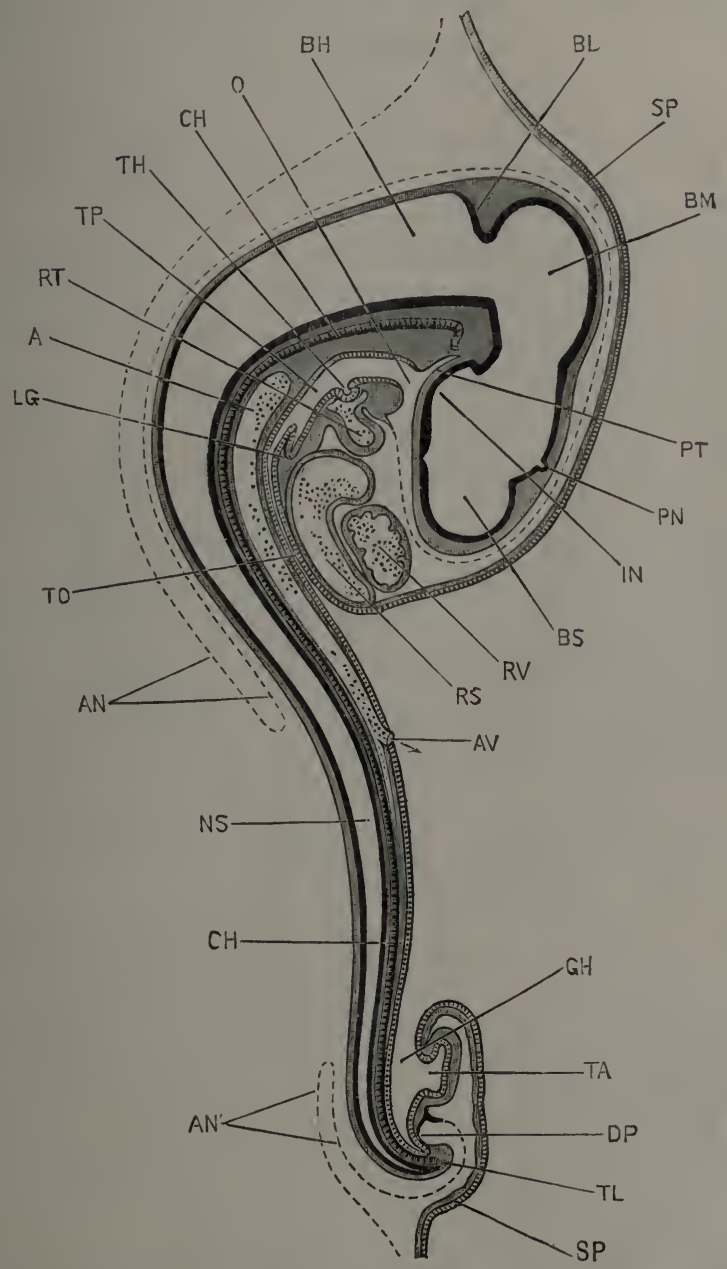

Fit. 114.-A median longitudinal, or sagittal, section through a Chick Embryo at the end of the third day of incubation. The amnion is represented by a dotted line. (Cf. Fig. 113.) $\times 20$.

A, dorsal aorta. AN, hear fold of the amnion. AN', tail fold of the amnion. AV, vitelline artery. $\mathbf{B H}$, fourth ventricle, or cavity of medulla oblongata. $\mathrm{BL}$, cerebellum. BM, cavity of mill-braiu, the future Sylvian aquelluct. BS, lateral ventricle, or eavity of the cerebral hemisphere. CH, notochord. DP, proctodæal pit. GH, hind-gut, or posterior portion of the mesenteron. IN, infunlibulum. LG, lung. NS, central eanal of spinal cord. $\mathrm{O}$, mouth. $\mathbf{P N}$, pineal boily. PT, pitnitary body. RS, sinus venosus. RT, truncus arteriosus. $\mathbf{R V}$, ventricle. $\mathbf{S P}$, splanclmopleure. TA, allantois. TH, thyroid botly. TL, tail. TO, œsophagus. TP, pliaryux. 
borne in mincl that this is the condition in which it remains throughout life in the frog, and in many fish.

The mid-brain undergoes comparatively slight changes. Up to the end of the fourth day it is approximately spherical in shape; and, owing to its great size and the position which, through cranial flexure, it occupies at the apex of the head, it plays a prominent part in determining the shape of the embryo (Figs. 113 and 115, Bur).

On the fifth day, the optic lobes begin to grow out as a pair of rounded swellings from the roof of the mid-brain, separated by a median longitudinal groove. These steadily increase in size during the following days; up to the sixteenth day they remain in close contact with each other, but during the last few days of incubation they become pushed apart by the forward growth of the cerebellum, and take up the position at the sides of the brain characteristic of the optic lobes in the adult bird.

The floor of the mid-brain, and the sides, ventral to the optic lobes, become greatly thickened by the formation of the crura cerebri. The cavity of the mid-brain becomes greatly reduced by this thickening of its floor and sides, and forms the Sylvian aqueduct of the adult.

The thalamencephalon is formed from the original anterior cerebral vesicle, or fore-brain (Fig. 111, BF).

The roof and floor of the thalamencephalon remain thin throughout life, but the sides thicken very greatly to form the optic thalami, reducing the central cavity to a narrow vertical cleft, the third ventricle of the adult ( $c f$. Fig. 116, BF).

The anterior wall of the thalamencephalon forms a thin and narrow band, the lamina terminalis (Fig. 116, BT), which lies between the roots of the two cerebral hemispheres: in connection with this, the anterior commissure is developed as a narrow transverse band of nerve fibres, running across between the basal parts of the hemisplieres.

The roof of the thalamencephalon, like that of the fourth ventricle, becomes early reduced, along the greater part of its length, to a single layer of epithelial cells, devoid of nervous elements. About the middle of its length, the pineal body arises, at the commencement of the thisd dar, as a hollow, rounded, 
median diverticulum : this is at first directed slightly backwards, but by the end of the third day becomes inclined forwards (Figs. 113 and 114, PN), and lies close beneath the external epiblast. In the later stages, the pineal body increases in size, becomes dilated at its distal end, and gives off a number of branching

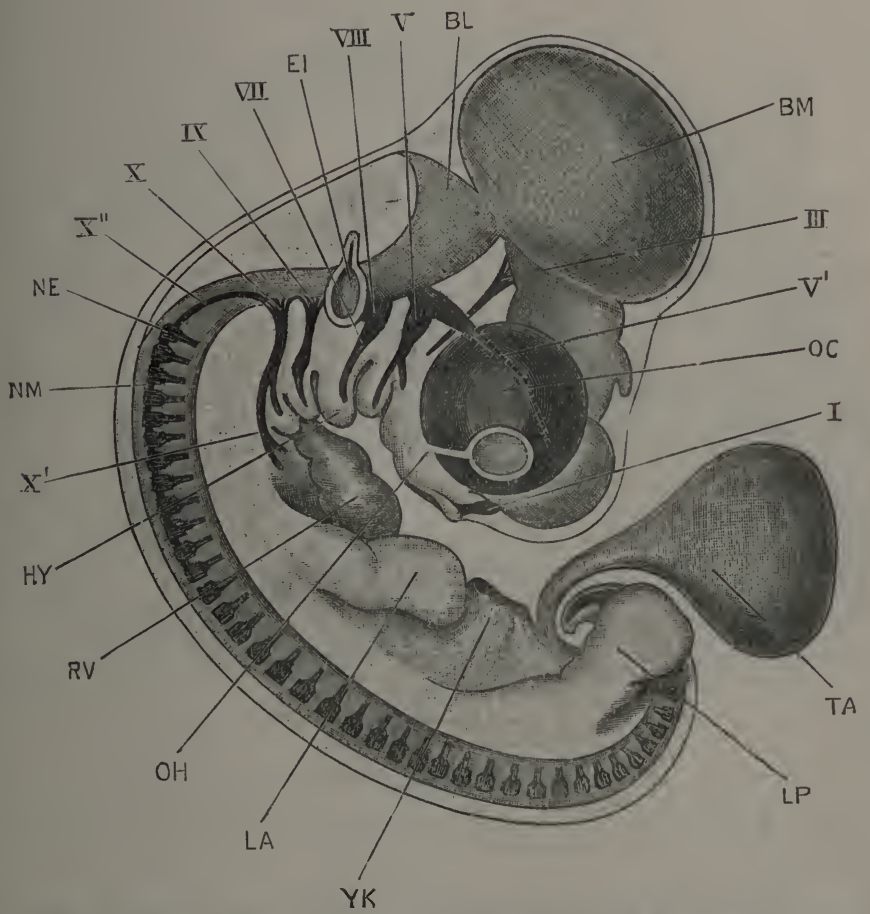

FiG. 115.-A Chick Embryo at the end of the fifth clay of incubation, seen from the right side. The amnion has been removed. $\times 20$.

BI, cerebellnm. BM, optic lobe, formed from the mid-brain. EI, auditory vesicle. HY, hyoil arcl. LA, fore-limb or wing. LP, lind-limb or leg. NE, ganglion of first spinal nerve. NM, commissure connecting first and second spinal ganglia. $\mathrm{OC}$, eye. $\mathrm{OH}$, choroidal fissure. $\mathrm{RV}$, ventricle of lieart. TA, allantois. YK, yolk-stalk, cut short. I, olfactory nerve. III, thiril nerve, or motor oculi. V, fifth or trigeminal nerve. $V^{\prime}$, ophthalmic branoh of trigeminal nerve. VII, seventh or facial nerve. VIII, eighth or auditory nerve. IX, ninth or glossopharyngeal nerve. $\mathbf{X}$, teuth or pneumogastric nerve. $\mathbf{X}^{\prime}$, visceral branch of pneumogastric nerve. $\mathbf{X}^{\prime \prime}$, commissure comecting pneumogastric nerve with the ganglion of the first spinal nerve.

tubular diverticula. Its condition on the eighth day is shown in Fig. 116, PN.

In front of the pineal body the roof of the thalamencephalon is very thin, and becomes thrown into folds which hang down into the ventricle: between the layers of these folds numerous 
blood-vessels penetrate, to form the choroid plexus of the third ventricle (Fig. 116, xA).

Immediately behind the stalk of the pineal body, the posterior commissure is developed in the roof of the thalamencephalon, as

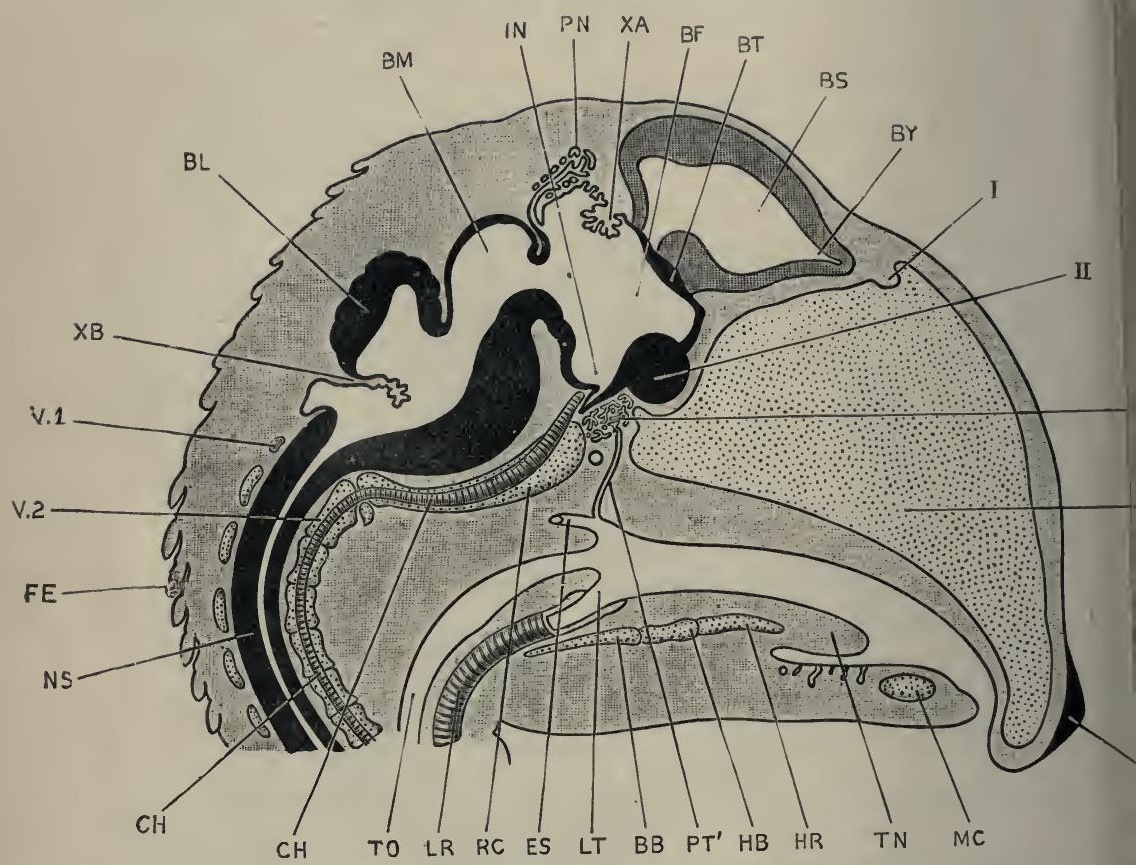

FIG. 116.-A median longitudinal, or sagittal, section of the head and anterior part of the neck of a Chick Embryo at the end of the eighth day of incubation. $\times 10$.

BB, basibranchial cartilagc. BF, third ventricle, or cavity of the thalamencephalon. BL, cerebellum. BM, Sylvian aqueduct, or cavity of the mid-brain. BS, lateral ventricle, or cavity of the cerebral hemisphere. BT, lamina terminalis. BY, olfactory lobe of the cerebral hemisphere. CH, notochord. ES, aperture of Eustachian tube. ET, mesethmoid cartilage. FE, rudimentary feather. HB, basihyal cartilage. HR, ceratohyal. IN, infundibulum. K, epithelial knob on beak. IR, trachea. $\mathbf{L}$, glottis. MC, Meckel's cartilage. NS, spinal cord. PN, pineal borly. PT, pituitary body. PT', stalk of pituitary body. RC, parachordal cartilage. TiN, tongue. TO, cesophagus. V1, neural arch of first or atlas vertebra. V2, centrum of second or axis vertebra. XA, choroid plexus of third ventricle. $\mathbf{X B}$, choroir plexus of fourth ventricle. I, notch in mesethmoid cartilage for olfactory nerve. II, optic chiasma.

a transverse band of nerve fibres connecting the two optic thalami; it is shown, though not lettered, in Fig. 116.

The floor of the thalamencephalon is depressed ventralwards to form the infundibulum, which lies very close to the anterior end of the notochord, and early acquires intimate relations with the pituitary body. The infundibulum is already present 
on the second day (Fig. 112): during the third and following days it becomes much more clearly defined (Figs. 114, 123, IN); and about the eighth day (Fig. 116, Is), a pocket-like diverticulum arises from its floor, which is directed backwards, and becomes wedged in between the anterior end of the notochord and the pituitary body.

In front of the infundibulum the floor of the thalamencephalon becomes greatly thickened, in the later stages, by the development of the optic chiasma (Fig. 116, II).

The pituitary body, though not really a part of the brain, is so intimately connected with this that it may conveniently be described here.

The pituitary body appears, towards the end of the second rlay, as a pocket-like diverticulum of the anterior angle of the stomatodæum, or mouth invagination ( $c f$. Fig. 114, PT); it lies wedged in between the anterior end of the mesenteron and the floor of the infundibulum, and its blind extremity is in close contact with the anterior end of the notochord.

On the formation of the mouth perforation, which places the stomatodæum in communication with the mesenteron, the pituitary body (Figs. 114, 123, P'T) persists as a diverticulum from the roof of the mouth, with the same relations as before to the infundibulum and to the notochord.

During the succeeding days, while the face is being established and the beak is growing forwards prominently, the pituitary body, retaining its relations with the brain and the notochord, becomes left further and further back in the roof of the mouth.

At the eighth day its position and relations are shown in Fig. 116. The upper blind end, PT, has given off a number of branching tubular diverticula, which together form a rounded vascular mass, lying immediately below the infundibulum, IN, and in the pituitary foramen at the base of the skull, between the trabeculæ cranii. The stalk of the pituitary body is still present as a narrow tube, $\mathrm{PT}^{\prime}$, which opens into the roof of the mouth in the median plane, opposite the glottis, LT, and just in front of the opening of the Eustachian tubes, ES. By the twelfth day the stalk has become a solid rod of cells, and the communication between the pituitary body and the mouth is finally cut off. 
The optic vesicles arise, early on the second day, as a pair of lateral outgrowths from the fore-brain (Fig. 111, Bo). They give rise, as in the frog, to the retina and the retinal pigment of the eye, and their developmental changes will be described in the section dealing with the formation of the eye (p. 275).

The cerebral hemispheres. About the middle of the second day, the fore-brain (Fig. 111, BF) begins to grow forwards, in front of the optic vesicles, as an anterior, median outgrowth, the vesicle of the hemispheres. At the same time cranial flexure becomes pronounced (Fig. 112), owing to the dorsal surface of the head growing faster than the ventral surface; the axis of the brain becoming a curved instead of a straight line, and the fore-brain being carried round to the ventral surface of the head. The curvature of the brain progresses rapidly; the fore-brain (Fig. 113) becoming placed at right angles to the rest of the brain, and the mid-brain growing forwards so as to lie at the extreme anterior end of the head.

The vesicle of the hemispheres grows rapidly, both in length and width : during the third day the paired cerebral hemispheres arise from its anterior end as thin-walled outgrowths, separated by a median furrow. The hemispheres (Figs. 113, 115, and 123, BS) enlarge rapidly, growing upwards and forwards, and forming a pair of prominent rounded swellings at the anterior end of the head, very conspicuous in embryos of the third to the seventh or eighth day. From the ventral surface of their anterior ends the olfactory nerves arise at a very early stage.

From the eighth day onwards the hemispheres, though still increasing in size, become less conspicuous from the surface, owing to the forward growth of the face, and especially of the beak, which elongates rapidly and completely alters the shape of the head (Fig. 116). As the beak extends forwards, the anterior ends of the hemispheres, from which the olfactory nerves arise, grow out as a pair of small hollow buds, the olfactory lobes (Fig. 116, BY), from the ends of which the olfactory nerves run forwards to the nose. The walls of the hemispheres are at first thin; in the later stages they thicken considerably, especially on the outer side of their hinder ends, where they form the corpora striata. The cavities of the hemispheres persist throughout life as the lateral ventricles of the brain, which retain their communication with 
the third ventricle, or cavity of the fore-brain, through a pair of narrow apertures, the foramina of Monro.

\section{The Peripheral Nervous System.}

a. General Account. The nerves, both cranial and spinal, which compose the peripheral nervous system are entirely of epiblastic origin, and develop in a manner closely similar to that already described in the frog.

The nerves fall under two categories :-

(i) The ganglionated nerves. These arise directly from the imner surface of the epiblast, as a pair of longitudinal neural ridges, along the margins of the neural plate. They appear before the neural tube is closed (Fig. 117, MA), and by the folding of its walls to complete the tube they get carried on to its dorsal surface, where they form a pair of bands (Fig. 118, NA), projecting

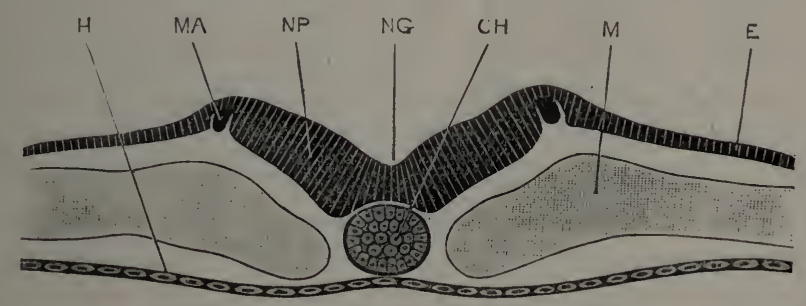

FIG. 117.-Transverse section across the body of a Chick Embryo at the twentyfourth hour of incubation. (Cf. Fig. 110.) $\times 200$.

CH, notochorrl. E, epiblast. H, lyppoblast. M, mesoblast. MA, commeneing neural ridge. $\mathbf{N G}$, neural groove. $\mathrm{NP}$, neural plate.

outwards from the angles between the external epiblast and the walls of the neural tube. On the completion of the neural tube by fusion of its lips, the neural ridges separate from the surface epiblast, but remain in close contact with the dorsal surface of the tube (Fig. 119, NB).

The neural ridges are at first continuous structures, from which the nerve ganglia arise as paired outgrowths; these grow rapidly, extending outwards and downwards, and acquire their permanent roots of attachment by outgrowth of nerve fibres from the ganglion cells into the brain or spinal cord.

To this category belong the fifth, the seventh and eighth, the sensory roots of the ninth and tenth, with perhaps one or two of the other cranial nerves; and the dorsal or sensory roots of the spinal nerves. 
(ii) The non-ganglionated nerves. These arise as direct outgrowths from the nerve cells of the brain or spinal cord. The nerves of this category develop at a rather later period than those of the former one ; they are all motor in function, and to them belong the sixth, and perhaps some of the other cranial nerves, and the ventral or motor roots of the spinal nerves.

Certain of the cranial nerves cannot at present be referred with certainty to either category ; but in their cases our knowledge of the developmental history is incomplete, and further research is necessary before any definite statement can be made concerning their real nature.

With regard to the nerves definitely included in the first category, a distinction must be made between the cranial and the spinal nerves, similar to that already described in the frog. The cranial nerves, in their growth outwards, lie at first very superficially, just beneath the external epiblast. Near their distal ends they early acquire connection with localised thickenings of the external epiblast, situated about the horizontal level of the notochord, and just above the dorsal borders of the gill-clefts. From these thickened patches of epiblast, which are probably to be regarded as sense organs, cells are budded off into the nerves, which appear to take a direct part in their further development.

The spinal nerves, on the other hand, are from the first more deeply situated. They lie between the spinal cord and the muscle plates (Fig. 124, NE), and do not acquire the connections with the external epiblast which are characteristic of the cranial nerves.

b. The Cranial Nerves. The first trace of the cranial nerves appears, in the chick, in the region of the mid-brain, about the twenty-second hour. At this stage, slightly younger than that shown in Figs. 110 and 118, the neural folds have nearly met, in the region of the head and neck, but have not yet coalesced at any part of their length; while in the body region the central nervous system is still a widely open groove. Only one or two pairs of mesoblastic somites are as yet present.

At the lips of the neural groove there is on either side a ridge-like outgrowth of epiblast cells, from the angle between the external epiblast and the wall of the neural canal. This 
outgrowth (Fig. 118, NA), which consists of cells mole spherical in shape than those of the surface epiblast, or of the brainwall, appears first in the region of the mid-brain, but rapidly extends both forwards and backwards: forwards as far as the anterior part of the fore-brain; backwards along the whole length of the hind-brain, and a certain distance down the spinal cord.

These outgrowths (Fig. 118, NA) are the neural ridges. As they arise before the lips of the neural canal have met, the neural ridges of the two sides are at first completely independent of each other. A few hours later, when closure of the neural

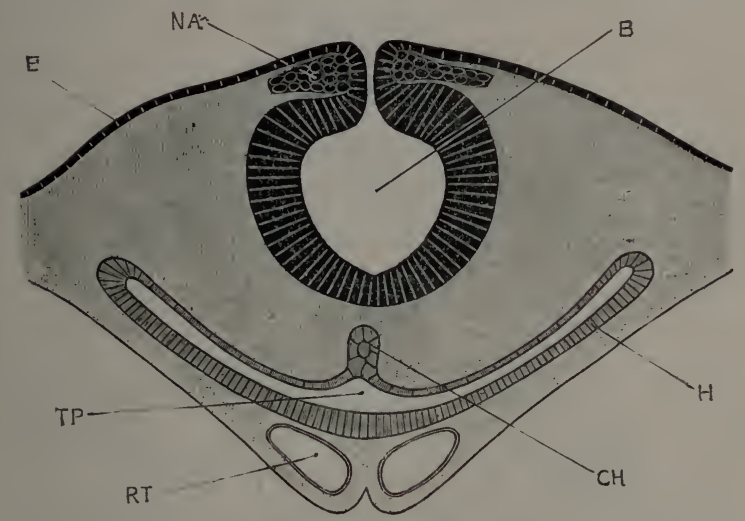

FIG. 118. - Transverse section across the head of a Chick Embryo at he twentyfourth hour of incubation, passing through the region of the mid-brain. (Cf. Fig. 110.) $\times 100$.

$\mathbf{B}$, cavity of the mill-brain. $\mathbf{C H}$, notochorl, not ret separated from the hypoblustic wall of the pharynx. $\mathbf{E}$, epiblast. $\mathbf{H}$, hypoblast. $\mathbf{N} \mathbf{A}$, neural ridge. $\mathbf{R T}$, commeneing heart. TP, pharynx.

canal is effected, the neural ridges separate completely from the external epiblast, but remain closely attached to the brain; the ridges of the two sides at the same time coalescing with each other to form a continuous longitudinal band, the neural crest (Fig. 119, NB), extending along the dorsal surface of the brain.

Almost from its first appearance, and before the neural tube is closed, the neural crest becomes more prominent at certain places. These more prominent parts form paired outgrowths of the crest, and are situated opposite the widest parts of the cerebral vesicles. They are the rudiments of the cranial nerves (Fig. 119, NB), while the intervening narrower parts of the crest (Fig. 120, NB), opposite the constrictions 
between the cerebral vesicles, form commissural bands, which for a time connect together the successive pairs of nerve outgrowths.

In a typical cranial nerve, such as the facial or glosso-pharyngeal, the further changes are as follows. The nerve rudiment rapidly extends outwards, lying close beneath the external epiblast, but independent of this. Opposite the nerve, but at some little distance beyond the brain, and about the horizontal level of the notochord, a proliferation of the cells of the external epiblast takes place, forming a small, inwardly projecting knob.

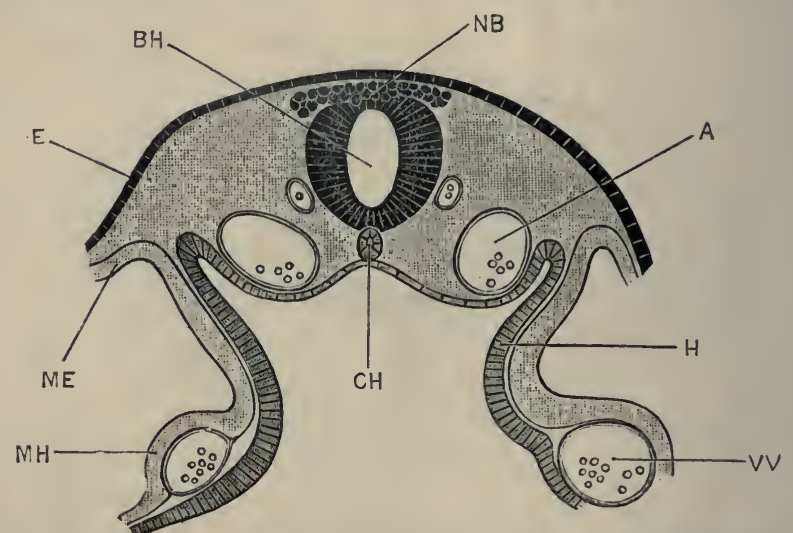

FIG. 119.- Transverse section across the head of a Chick Embryo at the fortythird hour of incubation. The section is taken immediately behind the auditory pits and the heart, and passes through the rudiments of the glossopharyngeal nerves. $\times 100$.

A, aorta. BH, cavity of hind-brain. CH, notochord. E, epiblast. H, hyloblast. $\mathrm{MF}$, somatopleuric laver of mesoblast. $\mathrm{MH}$, splanclmopleuric layer of miesoblast. NB, neural crest; the part shown in the figure gives rise later on to the granglia of the glosso-pharyngeal nerves. VV, vitelline vein.

The nerve soon comes in contact with this knob, and fuses with it, close to its distal end. Cells are budded off from the lnob into the nerve, which thus becomes reinforced from the epiblast. The exact fate of these cells is uncertain, but it is probable that they take part in the formation of the ganglionic thickening on the nerve.

The inner or proximal end of the nerve thins rapidly, and loses its connection with the dorsal surface of the brain, a connection which from the first has been one rather of close contact than of actual continuity. A little way beyond this point, however, the nerve acquires its permanent attachment to the brain, 
about half way down its side ; this attachment being effected by the outgrowth of processes from the cells of the nerve, into the substance of the brain.

This attachment is acquired by the seventh nerve about the end of the second, or early in the third day (Fig. 121, VII). Owing to the part of the brain dorsal to the nerve growing more rapidly than its ventral part, the root of attachment of the nerve becomes apparently shifted further downwards, towards the

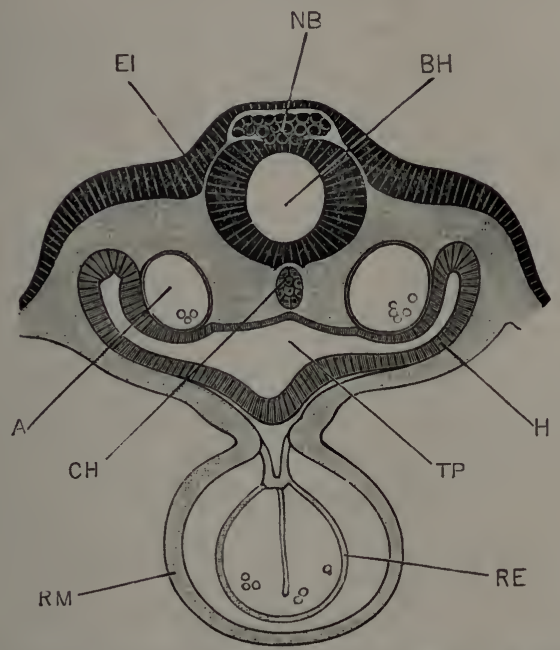

FIG. 120.-Transverse section across the head of a Chick Embryo at the fortythird hour of incubation. The section passes through the commencing auditory pits, and through the heart. $\times 100$.

A, aorta. BH, cavity of hind-brain. CH, notochord. EI, commencing auditory pit. H, hypoblast. NB, neural crest; the section passes through the narrow commissural part of the crest, which connects the ruliments of the facial aul aulitory nerves with those of the glosso-pharyngeal nerves. RE, endothelial lining of lieart. RM, museular wall of heart. TP, pliarynx.

ventral surface of the brain, and by the end of the third day has acquired the position characteristic of the nerve-root in the adult.

At the same time, changes occur in the trunk of the nerve. Owing to intrusion of mesoblast between the sirface epiblast and the nerve, the latter becomes more deeply placed than in the early stages. The connection with the sensory patch of the surface epiblast persists, but becomes drawn out, as the nerve recedes from the surface, into a cutaneous branch of greater or less length. Beyond the origin of this cutaneous branch the nerve continues its growth, and by the end of the third day, or 
early on the fourth day, its main branches of distribution become definitely established.

These branches, in the case of the seventh or facial nerve, are closely connected with the hyo-mandibular cleft. They consist of a large hyoidean or post-branchial branch (Fig. 115, vII), which runs along the hyoid arch; and a smaller mandibular or prebranchial branch, which runs forwards over the dorsal end of the hyo-mandibular cleft, and then downwards a short distance along the mandibular arch.

The above account will apply to any one of what may be termed the typical cranial nerves. It will now be convenient to take the several cranial nerves one by one, and note the chief points in their individual development.

I. The olfactory, or first cranial nerve. Our knowledge of the development of the olfactory nerve in the chick is still incomplete in some respects. At the twenty-ninth hour the neural ridges extend forwards along the brain as far as the anterior end of the fore-brain, i.e. in front of the optic vesicles (cf. Fig. 111). There are reasons for thinking that it is from the anterior ends of the neural ridges that the olfactory nerves are, at any rate in part, developed; but the point has not been proved by actual observation.

At the fiftieth hour, before the paired cerebral hemispheres have commenced to appear, the olfactory nerves may be recognised as a pair of short outgrowths, arising from the dorsal surface of the unpaired vesicle of the hemispheres, and running downwards and outwards towards a pair of slightly thickened patches of epiblast, on the under surface of the head, which form the earliest rudiments of the olfactory pits.

During the third day the cerebral hemispheres arise. These are, from the first, situated dorsally to the roots of the olfactory nerves; and, growing rapidly forwards and upwards (Fig. 113), they appear to drive the olfactory nerves down to the base or ventral surface of the brain. By the further growth of the cerebral hemispheres the original unpaired vesicle of the hemispheres becomes obliterated, or rather absorbed into the hemispheres, and the olfactory nerves from this time arise directly from the hemispheres. During the third day the olfactory pits deepen rapidly, and the distal ends of the olfactory nerves 
become continuous with the olfactory epithelium. The mode in which this connection is acquired is closely similar to that in which the typical cranial nerve acquires connection with the sensory patch of the surface epiblast, and it has been suggested, with much reason, that the olfactory epithelium may be homologous with one of these sensory patches.

The condition of the olfactory nerve at the end of the fifth day is shown in Fig. 115, I. The nerve, which is still very short, runs downwards and backwards from the under surface of the hemisphere to the olfactory pit.

On the seventh day, as already noticed, the beak begins to form; and cluring this and the following days it grows forwards with great rapidity. The olfactory sacs become imbedded in the sides of the beak (Fig. 131, ok), and are carried forwards with the beak as it lengthens. This causes a change in the direction and in the relations of the olfactory nerves, which, previously quiescent and inactive, have now to elongate rapidly, in order to maintain the connection between the olfactory organs and the brain. This elongation is effected mainly by growth of the nerves themselves, but partly, as already explained, by pulling out of the anterior ends of the hemispheres, from which the olfactory nerves arise, to form the olfactory lobes (Fig. 116, BY).

It is very possible, therefore, though not yet proved, that the olfactory nerve is really comparable to a typical cranial nerve, such as the facial, in which the sensory cutaneous branch is the only one developed.

II. The optic, or second cranial nerve. The optic nerves in the chick are very generally described as being formed directly from the constricted necks, or stalks, of the optic vesicles, which connect these with the brain. If this be correct, the optic nerve is in no way comparable with the other nerves, cranial or spinal, but must be contrasted with all of these as being formed by direct modification of part of the brain walls.

There are, however, strong grounds for suspecting that, as in the frog (p. 139), the fibres of the optic nerve really arise in the retina, and grow inwards to the brain; the optic stalk affording the path along which they grow, but not itself taking any direct part in their formation.

'The neural ridges, as already described, extend forwards 
along the whole length of the fore-brain, but they do not appear to take any part in the development of the optic nerves.

III. The motor oculi, or third cranial nerve. The third nerve is the only one which, in the adult bird, arises from the mid-brain. 'The neural ridges appear first of all on the top of the mid-brain, and early attain a great size in that position (Fig. 118, NA), but it is not yet clear what happens to these ridges in the later stages. It is possible that they take part in the formation of the third nerve, but this has not been proved to be the case.

The actual date of the first appearance of the third nerve has not been determined. About the middle of the third day it is clearly visible as a nerve of rather large size (Fig. 124, III), arising from the base of the mid-brain, not far from the middle line, and running backwards and downwards towards the hinder border of the eye.

By the fifth day (Fig. 115, III), the third nerve has the characteristic course of the adult nerve, arising from the floor of the mid-brain and running downwards and backwards immediately behind the eye.

There are strong reasons for regarding the third nerve as corresponding to at any rate a part of a typical cranial nerve, but until its early development is more clearly ascertained it is impossible to speak definitely with regard to it. Its origin from the base of the brain, close to the median plane, its distribution to muscles, and the fact that its root in the early stages (Fig. 115, III) is multiple, have led most investigators to compare it with the ventral root of a spinal nerve rather than with the dorsal root.

The ciliary ganglion is stated to be formed in the chick in connection with a knob-like thickening of the surface epiblast, similar to the sensory patch of a typical cranial nerve.

IV. The fourth cranial nerve. The fourth nerve in the adult is peculiar, inasmuch as it is the only nerve which arises from the dorsal surface of the brain, and also, so far as is known, the only nerve which arises from a constriction betrreen two brain vesicles instead of from the middle of a vesicle.

.In a chick embryo of the fifth day the fourth nerve is easily recognised. It is very slender, but has already the course and 
relations characteristic of the nerve in the adult bircl. Its development in the chick is unknown.

V. The trigeminal, or fifth cranial nerve. The trigeminal nerve arises from the neural ridge on the first or most anterior of the vesicles of the hind-brain, and its development accords exactly with that of a typical cranial nerve as described above. 'I'he ganglion of the trigeminal nerve, or Gasserian ganglion, is formed mainly from a portion of the neural ridge, reinforced from an independently arising knob of the surface epiblast. The permanent attachment of the nerve to the side of the hind-brain is acquired at the commencement of the third day; and about the same time the nerve divides distally into ophthalmic and mandibular branches, of which the former ( $c f$. Fig. 115, v') runs forwards along the inner side of the eyeball to the front of the head, while the latter, v, runs downwards and backwards in the mandibular arch. From the mandibular nerve, the maxillary nerve arises on the third day as a branch ( $c f$. Fig. 115), which runs forwards in the maxillary arch or upper jaw.

The development of the motor root of the trigeminal nerve in the chick has not been determined satisfactorily, and it is not yet certain whether this is a part of the original nerve, or whether, as seems more probable, it arises independently as an outgrowth from the brain itself.

VI. The sixth cranial nerve. The sixth nerve is of a very different nature to the trigeminal or facial nerves, and in its mode of origin and relations agrees more closely than any of the other cranial nerves with the ventral or motor root of a spinal nerre.

It appears during the fourth day, arising from the base of the hind-brain, near the median plane, by a number of very slender rootlets, the most anterior of which is on a level with the hinder part of the root of the trigeminal nerve, and the most posterior one opposite the root of the facial nerve. The rootlets unite together to form a slender nerve, which runs forwards below the base of the brain to the external rectus muscle of the eyeball, in which it ends.

VII. The facial, or seventh cranial nerve arises from the neural crest on the top of the second vesicle of the hind-brain; 
its development has already been described as that of a typical cranial nerve.

VIII. The auditory, or eighth cranial nerve (Fig. 115, VIII) is, in the chick, continuous with the facial nerve from its first appearance. It is a short stout nerve, which at a very early period, about the fiftieth hour, comes in contact with the auditory epithelium, and fuses with this. The subsequent development of the nerve consists mainly in its division, distally, into branches supplying the several special patches of the auditory epithelium, and will be described more fully in the section dealing with the development of the ear.

So far as the chick is concerned, there appears to be no reason for separating the facial and auditory nerves from each other. The two together make up a typical cranial nerve, of which the auditory nerve represents the cutaneous branch, greatly hypertrophied in consequence of the large size and importance of the sensory patch, i.e. the internal ear, which it supplies.

IX. The glosso-pharyngeal, or ninth cranial nerve (Figs. 115, IX, and $119, \mathrm{NB})$, is at first continuous with the pneumogastric or tenth nerve, a single elongated strip of the neural ridge on the roof of the hind-brain, immediately behind the ear, giving origin to both these nerves. The strip divides, before the end of the second day, into an anterior or glosso-pharyngeal portion, and a posterior or pneumogastric portion.

The glosso-pharyngeal develops as a typical cranial nerve; it early acquires connection with a sensory patch of the surface epiblast, and its main stem, beyond this point, runs downwards along the first branchial arch (Fig. 115). The root of attachment of the nerve to the brain early becomes multiple, consisting of four or five small rootlets, which spread out in a fan-like manner on entering the brain. The multiple character of the roots of the glosso-pharyngeal nerve is of interest, as showing that the similarly multiple nature of the roots of the third nerve is not incompatible with a possible origin of this latter from the neural ridge.

$X$. The pneumogastric, or tenth cranial nerve (Fig. 115, x) arises from the posterior part of the outgrowth from the neural 
ridge, common to it and the glosso-pharyngeal nerve. At first the pneumogastric is, if anything, the smaller of the two nerves, but it soon becomes distinctly the larger. Like the glossopharyngeal nerve, it early acquires multiple roots, the most anterior of which is directly continuous with the hindmost of the roots of the glossopharyngeal nerve, without entering the brain.

Beyond the roots of origin, the main stem of the pneumogastric nerve runs downwards and backwards, parallel to the glosso-pharyngeal nerve; it expands into a large fusiform ganglion, from which branches are given off to the second and third branchial arches, as well as large branches to the heart, lungs, and intestines.

From the hindmost root of origin of the pneumogastric nerve from the brain, a long commissural branch (Fig. 115, $x^{\prime \prime}$ ) runs backwards along the side of the medulla oblongata, and is continuous posteriorly with the ganglion of the first spinal nerve. This commissural branch is derived from the part of the neural ridge between the pneumogastric and first spinal nerves.

The mode of development of the spinal accessory or eleventh cranial nerve, and of the hypoglossal or twelfth cranial nerve, has not been satisfactorily determined in the chick. The hypoglossal nerve has, from the first, the relations characteristic of the ventral roots of the spinal nerves; though whether it correspouds to one, or to more than one, of such roots is not determined with certainty.

It is interesting to note that the definite relations of the fifth, seventh, ninth, and tenth cranial nerves to the visceral arches are as characteristically shown in an embryo chick of the fifth day (Fig. 115) as they are throughout life in a typical waterbreathing Vertebrate such as a dogfish.

\section{c. The Spinal Nerves.}

The dorsal roots of the spinal nerves develop, as already noticed, in a manner practically identical with the typical cranial nerves. Their first appearance is almost simultaneous with that of the cranial nerves; they may be recognised in embryos in which the first two or three pairs of mesoblastic 
somites are alone present ( $c f$. Fig. 110), and sometimes even prior to the definite formation of any of the somites.

In the anterior part of the spinal cord the neural ridges appear, just as in the brain, as cellular proliferations from the reentering angles between the external epiblast and the lips of the neural plate, which latter have already grown in towards each other a certain distance. The neural ridges of the spinal cord are directly continuous with those of the brain, and from them the ganglia of the spinal nerves are derived as paired outgrowths. The spinal ganglia differ, however, from the cranial ganglia in not acquiring any distal connection with sensory patches of the epiblast, and in being from the first much more deeply situated, growing downwards close alongside the spinal cord, between this and the muscle-plates (Fig. 124, NE).

During the third day, the spinal ganglia acquire their definite attachments to the sides of the spinal cord, these being effected by the outgrowth of nerve fibres from the inner sides of the ganglia into the cord. The parts of the ganglia above, or dorsal to, the points of attachment persist for some time as small pointed processes, but soon become inconspicuous, and are finally absorbed into the ganglia.

The spinal ganglia are of considerable width, more than half the width of the somites to which they belong (Fig. 115, NE), so that the intervals between successive ganglia are distinctly less than the width of the ganglia themselves. The ganglia lie, from the first, opposite the anterior parts of the somites to which they belong.

The ganglia of the anterior part of the body are connected together by short commissural bands (Fig. 115, NM), situated at the same level as the attachments of the ganglia to the spinal cord; and the most anterior spinal ganglion, as already noticed, is connected with the hindmost root of the pneumogastric nerve by a similar but much longer commissure (Fig. 115, $\mathrm{x}^{\prime \prime}$ ). These commissures appear to be formed from the parts of the originally continuous neural ridge which are left between the successive ganglion outgrowths; they are well developed and conspicuous structures on the fourth and fifth days, but after the latter date are difficult to detect.

The spinal nerves of the hinder part of the cord develop in slightly different fashion to those of the anterior part. They appear 
at a slightly later date, but at a relatively earlier stage in the formation of the spinal cord. At a time when the neural canal has hardly commenced to form, and the neural plate is only very slightly folded on itself in the middle line, the nerve rudiments may be recognised in transverse sections (Fig. 117, MA), as small conical masses of cells, cut out from the deeper part of the epiblast, at the edges of the neural plate. As the neural folds rise up, and grow in towards each other, the nerve rudiments are carried up with the folds to the dorsal surface of the spinal cord, and then complete their development in the manner already described.

In the posterior half, or so, of the body there appears to be no continuous neural ridge developed, the nerve. rudiments being, from the first, independent outgrowths. There are consequently no longitudinal commissures connecting these hinder nerves, similar to those in the anterior part of the body (Fig. 115). These commissures disappear in the anterior part of the body shortly after the fifth day, and it is possible that their absence in the hinder part of the body is to be explained as due to abbreviation of the developmental history, by omission of this stage.

The ventral roots of the spinal nerves arise later than the dorsal roots, during the latter part of the third day. They appear' as small outgrowths from the lower part of the sides of the spinal cord, and from the first occupy the position held by them in the adult. This position is indicated, before the actual appearance of the root, by a slight convergence of the cells at the side of the cord ; and the nerve root is apparently formed by the direct outgrowth of processes from these cells, which, passing out from the side of the spinal cord, become the axis cylinders of the nerve fibres.

Each ventral root arises by a number of separate rootlets, which leave the spinal cord in a longitudinal series, the total length of a root being about equal to half that of a somite; the root lies opposite the anterior half of the somite, and vertically below the corresponding dorsal root.

Towards the end of the third day (Fig. 124), the ventral roots, growing downwards and outwards, meet the dorsal roots, and with these form the trunks of the spinal nerves. Beyond the place of union of the roots the nerves continue their growth outwards and downwards, lying along the inner surfaces of 
the muscle plates. By the end of the fourth day the nerves have doubled in length, and the primary dorsal and ventral divisions are already established, each division including fibres from both the dorsal and ventral roots.

In the part of the body between the fore and hind limbs (cf. Fig. 115), the main branches of the nerves run in the body wall or somatopleure. In the segments opposite the limbs, the nerves enter the limbs and divide into dorsal and ventral branches, which unite with the corresponding branches of the nerves in front of, or behind, them to form broad plates of nerve fibres, from which the individual nerves of the adult limb arise.

d. The Sympathetic Nervous System. The origin of the sympathetic nervous system in the chick has been much debated, and is not yet satisfactorily determined. The most trustworthy observations are to the effect that the sympathetic nervous system arises at an early stage, the third or fourth day, as a series of outgrowths from the spinal nerves, apparently derived directly from the spinal ganglia. These grow inwards, at a level immediately above the cardinal veins, and close to the dorsal aorta: at their ends are ganglionic enlargements, the nervecells of which are apparently derived, by direct migration, from the spinal ganglia. These ganglionic enlargements soon become connected, along each side of the body, by longitudinal commissures, apparently formed by outgrowths of nerve-fibres from the ganglia themselves.

If this account is correct, the sympathetic nervous system of the chick is to be regarded merely as a specialised part of the spinal nervous system.

\section{DEVELOPMENT OF THE SENSE ORGANS.}

The general history of the development of the sense organs in the chick is very similar to that already described in the frog. In all cases the essential part of the organ, the actual sensitive surface itself, is derived directly or indirectly from the epiblast or epidermis.

\section{The Nose.}

The olfactory organs appear, about the fiftieth hour, as a pair of thickened patches of the external epiblast on the under 
surface of the fore part of the head; these soon become depressed, forming pits (Fig. 113, OF), with the bottoms of which the olfactory nerves very early become connected (p. 266).

The mouths of the olfactory pits narrow, and become slitlike, but remain open throughout life as the external nostrils (Figs. 125, 126, oK).

The epithelial lining of each olfactory pit becomes thrown into folds, to increase its surface; and gives rise directly to the olfactory epithelium, or Schneiderian membrane, of the adult nose.

The posterior narial passage is a secondary formation; it appears at first as a groove on the under surface of the head, leading from the edge of the olfactory pit to the anterior and outer angle of the stomatodæum. This groove is well marked on the fourth day, its inner lip being formed by the fronto-nasal process (Fig. 125, FP), or median part of the face, between the two olfactory pits; and its outer lip being formed by the maxillary arch (Fig. 125, Mx), or rudiment of the upper jaw.

On the fifth day (Fig. 125) the olfactory groove deepens, and its inner and outer lips, formed by the fronto-nasal process and maxillary arch respectively, meet and coalesce, so as to convert the groove into a tube, leading from the olfactory pit to the mouth. This tube is the posterior narial passage; it at first opens into the anterior end of the mouth cavity, immediately behind the upper lip; but as the mouth elongates, by growth forwards of the beak, a horizontal shelf-like partition is formed at the anterior end of the upper jaw on either side. By fusion in the median plane, the two horizontal partitions form the palatal septum, which stretches across the anterior part of the mouth, separating the olfactory or nasal region above from the buccal cavity below, and shifting backwards the communication between the posterior nostrils and the mouth.

\section{The Eye.}

As in the frog, and in Vertebrates generally, the retina or essential part of the eye is formed from the optic vesicle, while the lens is an independent invagination of the surface epiblast.

The optic vesicles arise, at the commencement of the second day, as a pair of hollow lateral outgrowths from the fore-brain; they grow rapidly, and attain some size before the lips of the 
neural folds fuse to complete the neural tube. The optic vesicles at first stand out at right angles to the head, but they soon become constricted at their bases, and directed somewhat downwards and backwards (Fig. 111, Bo). These constrictions rapidly deepen, so that by the end of the second day the optic vesicles

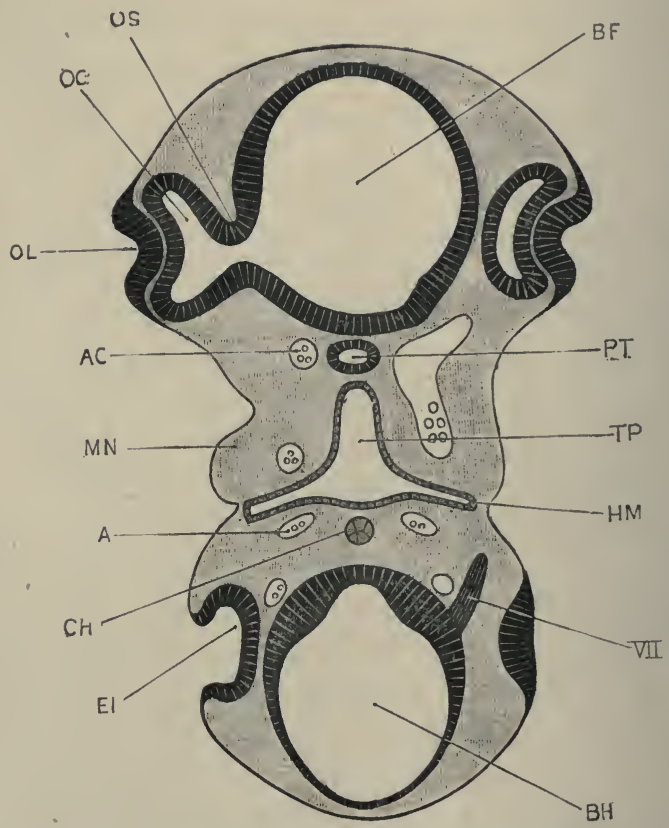

Frg. 121.-Transverse section across the head of a Chick Embryo at the fortyeighth hour of incubation. The section is taken along a line corresponding to one joining the reference letters EI and oL in the three-day embryo shown in Fig. 113. Owing to the cranial flexure, both fore-brain and mid-brain are cut by the section. The right side of the section is slightly anterior in position to the left side. $\times 60$.

A, aorta. AC, carotid artery. BF, cavity of fore-brain. BH, cavity of lind-brain. CH, notochord. 'EI, auditory pit. HM, hyo-mandibular cleft. MN, mandibular arch. OC, cavity of optic cup. OL, invagination of epiblast to form the lens. OS. optic stalk. PT, pituitary body. TP, pharyux. VII, facial nerve.

are connected with the floor of the fore-brain by narrow tubular stalks (Fig. 121, os).

Towards the end of the second day a circular patch of the external epiblast, opposite the outer wall of each optic vesicle, becomes thickened, and shortly afterwards pitted in to form the vesicle of the lens (Fig. 121, OL). The formation of this pit is accompanied by an infolding of the outer wall of the optic 
vesicle, which thus becomes doubled on itself to form the optic cup (Fig. 121, oc).

The lens. The pitting-in of the epiblast, to form the lens, rapidly deepens; the lips of the pit close in, and unite, so as to convert the pit into a closed sac, the lens vesicle, which separates completely from the external epiblast during the third day. After this separation, the outer wall of the lens vesicle remains thin, and is formed of a single layer of flattened epithelial cells; the inner wall thickens rapidly, by elongation of its component

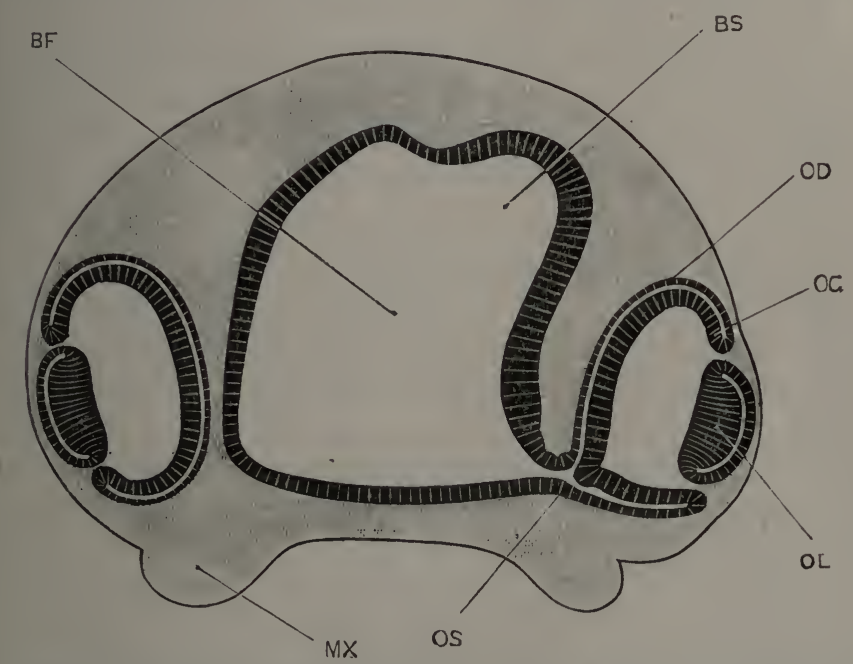

FIG. 122.-Transverse section across the fore-brain and eye of a Chick Embryo at the sixtieth hour of incubation. On the right side the section passes through the optic stalk; on the left side it passes just behind the stalk. $\times 45$.

BF, cavity of fore-brain. BS, cavity of commencing cerebral hemisphere. $\mathbf{M X}$, maxillary arch. OC, imer wall of optic cup. OD, outer wall of optic cup. OL, lens. OS, optic stalk.

cells (Fig. 122, oL); and by the fourth day it comes in contact with the outer wall, so as to obliterate the cavity of the vesicle entirely. From the epithelial cells of this thickened inner wall the whole of the substance of the adult lens is derived. The outer, thin wall of the lens vesicle becomes the epithelial lining of the lens capsule; while the lens capsule itself is apparently a cuticular membrane excreted by the epithelial cells of the lens vesicle.

The optic cup. In the optic cup important changes occur. The two layers of the cup soon come in contact with each other 
(Fig. 122, oc, oD), and by the end of the third day the original cavity of the optic vesicle is practically obliterated. The whole cup grows rapidly; its lip remains in contact with the margin of the lens the whole way round, except at one point on the under surface of the cup, below the reference line, OL, in Fig. 122, where a small chink is left between the lens and the lip of the cup. As the optic cup increases in size, this chink becomes lengthened out into a slit, the choroidal fissure (Figs. 113, 115 , and $125, \mathrm{OH})$, through which the mesoblast of the head gains admittance into the cavity of the cup.

From the wall of the optic cup the retina is developed, while the mesoblast which grows into the cavity of the cup, through the choroidal fissure, gives rise to the vitreous body. The choroid and sclerotic coats of the eye are formed from the mesoblast outside the optic cup, and the cornea from mesoblast which grows in between the lens and the surface epiblast.

The exact mode of formation of the choroidal fissure is difficult to determine. The first step in the doubling up of the optic vesicle to form the optic cup (Fig. 121) is intimately associated with the ingrowth of the surface epiblast to form the lens vesicle, and is perhaps due, in part, to mechanical in-pushing by this latter: the later stages of the doubling up, however, concern the optic cup alone, and must be regarded as due to unequal rates of growth of different parts of the wall of the cup. This unequal rate of growth in different directions probably plays an important, or even predominant, part in the formation of the choroidal fissure. The formation of the choroidal fissure has been recently shown to be closely associated with the growth of the fibres of the optic nerves; these fibres passing through the choroidal fissure on their way from the retina towards the brain.

The choroidal fissure only remains open for a short time. About the sixth day its lips come in contact, and very shortly afterwards they fuse together, so as to complete the closure of the optic cup; by the ninth day all trace of the fissure has disappeared.

The retina is formed directly from the wall of the optic cup. Of the two layers of which the doubled-up wall of the cup consists, the inner (Fig. 122, oc) is from the first much the thicker. It consists, on the third day, of elongated nucleated 
cells arranged side by side, and vertically to the surface. From the fourth day onwards it increases rapidly in thickness, and by a series of histological changes which have not yet been determined very accurately, it becomes converted into the several layers of the retina. The inner layers of the retina are the first to be established, and the last elements formed are the rods and cones; these latter growing outwards as processes from the outer nuclear layer, which until the appearance of the rods and cones is the outermost layer of the retina.

The outer wall of the optic cup (Fig. 122, oD) is, from the first, much thinner than the inner wall. By the middle of the fourth day it is reduced to a single layer of flattened cells, which soon become pignented, and ultimately give rise to the layer of special pigmented cells which lie in close contact with the outer ends of the rods and cones.

It appears, therefore, that the whole of the sensory part of the retina is derived from the inner layer of the optic cup. It is worthy of notice that the rods and cones, the only elements of the retina directly sensitive to light, are the last parts to be formed.

The optic nerve is very commonly said to be formed from the optic stalk; but it is more probable that it arises in the chick, as it is known to do in the frog (p. 138), by the formation of processes from cells in the retina, which grow inwards along the optic stalk to the brain, and become the fibres of the optic nerve.

The iris. The marginal part of the optic cup, nearest to the lens, does not become converted into the retina, but undergoes changes of a different character, the boundary between the retinal and non-retinal parts being indicated by the ora serrata. 'The inner and outer walls of this marginal, or non-retinal, part of the cup coalesce completely, and become pigmented throughout their whole thickness. They become closely connected with the choroid coat, on their outer surface; and the combined choroid and retina grow forwards, in front of the lens, to form the iris, which reduces the mouth of the optic cup to a comparatively narrow aperture, the pupil.

The pecten arises on the fifth day as a lamellar process of mesoblast, which grows into the cavity of the optic cup through the choroidal fissure, close to the optic nerve. It early becomes rery vascular; about the tenth day it becomes folded in the 
fan-like manner characteristic of the adult; and toward the close of incubation it becomes densely pigmented.

The cornea is formed from mesoblast, which grows in between the lens and the surface epiblast, at first as a ring, but soon becoming a continuous layer across the front of the eye. It is at first structureless, but cells from the mesoblast round its edge soon grow inwards into its substance to form the corneal corpuscles. These corpuscles are confined to the middle layer of the thickness of the cornea, the outer and inner surfaces remaining structureless as the anterior and posterior elastic membranes of the cornea respectively. The surface layer of epiblast persists as the conjunctival epithelium.

The anterior chamber of the eye forms as a space between the cornea and the lens; and in it a watery fluid, the aqueous humour, soon collects.

The accessory organs of the eye. The eyelids are folds of the integument round the eye: there are three of them, an upper and a lower eyelid, and the third eyelid or nictitating membrane (Fig. 126, CD), which arises on the inner or nasal side of the eye. The lacrymal glands are solid ingrowths of the conjunctival epithelium, which appear on the eighth day. The lacrymal duct is also at first solid; it appears as a ridge of epidermis, along the line of the lacrymal groove, extending from the eye to the olfactory pit (Fig. 125). This ridge sinks into the mesoblast, and soon splits off from the epiblast along the greater part of its length, but remains attached at its ends to the lower eyelid and to the wall of the olfactory pit respectively. About the twelfth day it acquires a central lumen, and becomes the tubular duct.

\section{The Ear.}

The ears appear, about the middle of the second day, as a pair of shallow depressions of the external epiblast at the sides of the hind-brain, just in front of the first pair of mesoblastic somites (Figs. 111 and 120, kI). The pits rapidly deepen (Fig. 121, EI) ; their mouths narrow, and by the end of the third day become completely closed, the pits thus becoming resicles imbedded in the mesoblast at the sides of the head (Fig. 113, EI). By a series of changes very similar to those already described in the frog, the vesicle gives rise to the various parts of the membranous labyrinth of the ear ; the epiblastic wall forming the epithelial lining of 
the labyrinth, and becoming specially developed at certain places, to form the anditory epithelium. The anditory nerve, as noticed above, very early comes in contact with the anterior and inner wall of the auditory vesicle, fusing completely with this by the fiftieth hour. This fused patch, by division and subsequent separation of the several portions, gives rise to all the special patches of auditory epithelium present in the adult labyrinth.

The accessory organs of hearing. The development of the Eustachian tube, tympanic cavity, and tympanic membrane will be described in the section dealing with the development of the pharynx and gill-clefts (p. 283). The development of the columella, or auditory ossicle, will be clescribed with the skeleton (p. 3:30).

\section{DEVELOPMENT OF THE ALIMENTARY CANAL.}

\section{General Account.}

The alimentary canal of the chick, like that of the frog, is developed in three portions, of independent origin, and of very unequal length.

(i) The stomatodæum, or mouth invagination, is formed by a pitting-in of the epiblast at the anterior end of the alimentary tract; from it the anterior part of the buccal cavity, and the pituitary body are developed.

(ii) The mesenteron gives rise to almost the entire length of the alimentary canal, from the hinder part of the buccal cavity to the cloaca; and from it the lungs, liver, pancreas, and other important structures arise as outgrowths. The mesenteron is the tubular cavity formed within the embryo, as the result of the process of folding or constriction by which the embryo becomes pinched off from the yolk-sac (Figs. 112, 114, and 123). It is lined by hypoblast along its whole length. Owing to the mode of its formation, it communicates freely, through the yolk-stalk, with the yolk-sac; and, so long as the yolk-stalk remains tubular, the mesenteron may be described as consisting of three lengths :- the fore-gut (Figs. 112, GF, and 114, TP, TO), which is the part included in the head-fold, and has complete roof, sides, and floor : the midgut (Figs. 114 and 123, ys), which opens into the yolk-stalk, and which therefore has roof and sides, but no floor ; and the hind-gut (Figs. 114 and 123, GH), which is the part included in the tail-fold, and has, like the fore-gut, complete roof, sides, and floor. Is the 
constriction of the embryo from the yolk-sac proceeds, the foregut and hind-gut lengthen at the expense of the mid-gut; and after about the seventh day, when the yolk-stalk is reduced to a

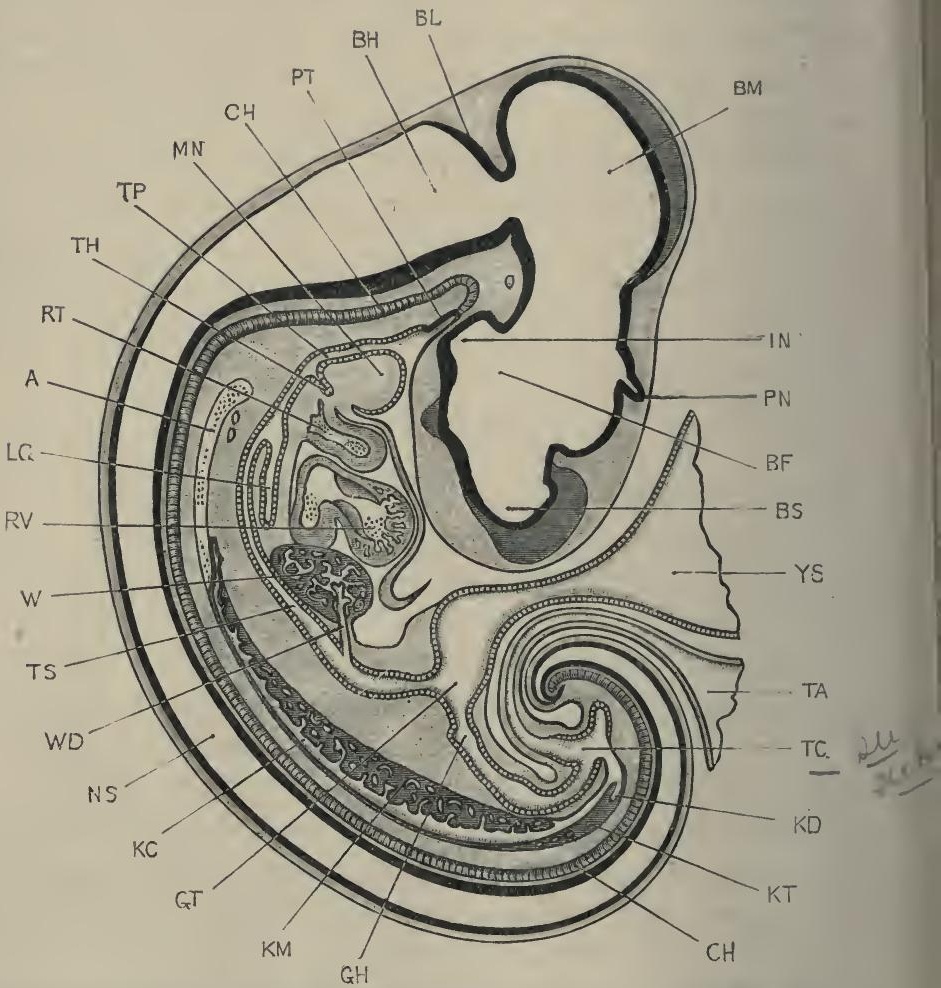

FIG. 123.-A median longitudinal, or sagittal, section through a Chick Embryo at the end of the fifth day of incubation; the section is taken strictly in the median plane, except as regards the Wolffian body and kidney, which are introduced in the figure in order that their relations to the alimentary canal may be shown. The optic lobe, cerebral hemisphere, and optic stalk of the left side are shown in perspective. The amnion has been removed, and the allantois and yolk-stalk cut short close to tbe embryo. (Compare Figs. 100 and 115 for surface views of embryos of the same age.) $\times 12$.

$\mathbf{A}$, dorsal norta. BF, third ventricle, or cavity of thalamencephalon. $\mathbf{B H}$, fourth ventricle, or cavity of medulla oblongata. BL, cerebellum. BM, cavity of mid-brain. BS, cavity of the vesicle of the hemispheres. $\mathbf{C H}$, notochord. GH, hind-gut. GT, mid-gut. IN, infundibulum. KC, Wolffian duct. KD, ureter. KM, Wolffian bodv. KT, killıey. LG, lung. IMN, mandibular arch. INS, cavity of spinal cord. $\mathbf{P N}$, pineal body. PT, pituitary boly. RT, truncus arteriosus. RV, ventricle of heart. TA, stalk of allantois, cut short. TC, eloaca. TH, thyroid body. TP, pharynx. TS, stomach. W, liver. WD, bile duct. Y YS, yolk-stalk, cut short.

very narrow tube, and the walls of the mesenteron are complete along its whole length, the mid-gut, as a distinct portion of the alimentary tract, ceases to exist. 
(iii) The proctodæum is a barely perceptible pitting-in of the epiblast, at the hinder end of the alimentary tract, which forms the anal or cloacal aperture.

$\mathrm{Up}$ to the end of the fourth day the alimentary canal is nearly straight; but from this time it grows more rapidly than the part of the body in which it lies, and soon becomes markedly convoluted; it retains its connection with the mid-dorsal wall of the body cavity by means of the mesentery.

The several regions of the alimentary canal, and the various organs formed in connection with it, will now be taken in order; and the more important points in their developmental history described.

\section{The Pharynx.}

Almost from the first, there is a great difference between the anterior or pharyngeal portion of the mesenteron, which is shallow dorso-ventrally but very wide from side to side (Fig. 118, TP); and the hinder part, from the œsophagus to the cloaca, which is narrow and cylindrical.

Towards the end of the second day, pouch-like folds of hypoblast grow out in pairs from the sides of the pharynx, towards the surface. These correspond exactly, in their relations and their mode of formation, to the gill-pouches of the tadpole; and like these, they grow outwards until they meet the external epiblast, with which they fuse. At a slightly later stage, the fused patches of epiblast and hypoblast become perforated to form the gill-clefts, which place the gill pouches, and therefore the pharynx, in direct communication with the exterior.

Of these gill-pouches, four are formed on each side of the neck, and are developed in order from before backwards. The most anterior one is the hyomandibular gill-pouch (Fig. 124, HM); and the succeeding three are the first, second, and third branchial pouches respectively.

The parts of the side walls of the pharynx between the successive gill-pouches are spoken of as the visceral arches; their boundaries are indicated on the surface of the neck by grooves, marking the lines along which the hypoblastic walls of the gillpouches meet and fuse with the external epiblast, as shown on the right-hand side of Fig. 124. The first or most anterior of these visceral arches is the mandibular arch (Figs. 124 and 125, 
MN), which forms the basis of the lower jaw. The second and widest arch is the hyoid arch, HY ; and behind this come the first, second, and third branchial arches, the hindmost or third branchial arch being immediately belind the last or third branchial cleft.

These visceral arches, and the gill-pouches separating them from one another, correspond exactly with the similarly named

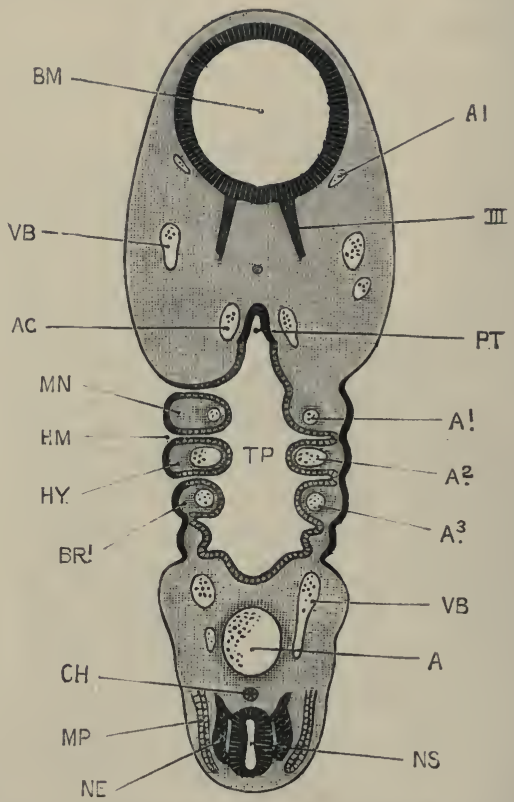

FIG. 124.-A section through the head of a Chick Embryo at the end of the third day of incubation, the section being taken along a plane indicated by the two arrows and crosses in Fig. 113, p. 253. The right side of the section is at a level slightly dorsal to that of the left side. $\times 30$.

A, dorsal aorta. A1, first aortic arch, in the mandibular arch. A2, second aortic arch, in the hyoid arch. A3, third aortic arch, in the first branchial arch. AC, carotil artery. AI, internal carotid artery. BM, cavity of mid-brain. BR1, first branchial arch. $\mathbf{C H}$, notochord. HM, hyomandibular cleft. $\mathbf{H} \mathbf{Y}$, hyoid arch. $\mathbf{M} \mathbf{N}$, mandibular arch. MP, muscle plate. NE, ganglion of spinal nerve. NS, spinal cord. TP, pharynx. VB, anterior cardinal vein.

structures in the tadpole; the sole difference of importance being that in the chick no gills are, at any period, developed in connection with them. The fact that these structures, which are only intelligible through their association with aquatic respiration, are present in the early developmental stages of the chick, must be held to prove the descent of birds from aquatic, gillbreathing ancestor's. 
The hyomandibular cleft opens to the exterior in the latter part of the third day (Figs. 113 and 12 l, HM); it remains open until about the end of the fourth day, when its walls come in contact, and the cleft becomes closed. The first branchial cleft, between the hyoid and first branchial arches, opens a little later, early on the fourth day; and closes again during the fifth day. The second branchial cleft, between the first and second branchial arches, is open only for a short time during the fifth day : and the third branchial cleft does not open to the exterior at any time.

It is stated by some observers that none of the visceral clefts in the chick open to the exterior at any stage, but the real condition appears to be as described above; it is possible that individual variations occur in respect to the dates of opening of the clefts, and the times during which they remain open.

The tympano-Eustachian passage. The branchial clefts close up and disappear completely at an early stage; but the most anterior, or hyomandibular, cleft appears to persist, and to give rise directly to the tympano-Eustachian passage of the adult bird. The cleft becomes closed at its outer end, about the end of the fourth day, by a fold of skin, which becomes directly the tympanic membrane. From the gill-pouch, on the inner side of the tympanic membrane, the tympanic cavity and Eustachian passage are formed; while the external auditory meatus is built up as a short tubular passage on the outer side of the tympanic membrane (Fig. 126, HM). The Eustachian passages of the two sides unite at their inner ends, and open into the mouth by a median aperture (Fig. 116, Es), nearly opposite the glottis.

According to some observers, the hyomandibular pouch does not open to the exterior at any period in the chick; and the tympanic membrane is formed directly from the thin double layer, consisting of both epiblast and hypoblast, which closes the pouch at its outer end; a layer of mesoblast growing in between the epiblast and hypoblast, which persist as the epithelial layers of the outer and inner surfaces of the tympanic membrane respectively. The whole history of the development of these parts stands in need of renewed and thorough investigation.

The thyroid body arises, towards the end of the second day, as a median longitudinal groove in the floor of the pharynx, 
opposite the first pair of branchial arches. The hinder end of the groove deepens during the third day to form a pit (Fig. 114, $\mathrm{TH})$. The walls of this pit soon join together, obliterating the cavity and giving rise to a solid plug of hypoblastic epithelium (Fig. 123, TH). About the end of the fifth, or early part of the sixth day this plug separates from the floor of the throat as a solid body, composed of epithelial cells, which lies embedded in the mesoblast, immediately in front of the truncus arteriosus.

The thyroid body soon becomes bilobed, and the lobes branch out as solid strings of cells, which later on become tubular. A sheath of vascular connective tissue early forms around the lobes, which, as development proceeds, gradually shift backwards along the neck to their adult position.

A pair of solid bodies, formed of epithelial cells, which separate from the hypoblast immediately behind the third branchial pouches, and take up a position at the sides of the larynx, are sometimes spoken of as accessory thyroid bodies.

The thymus arises, on each side, as a couple of epithelial buds from the walls of the second and third branchial pouches. The buds soon separate from the surface, and, the two buds of each side fusing together, give rise to a pair of elongated rod-like bodies, lying aloug the sides of the neck close to the carotid arteries.

The tongue is formed as an outgrowth from the floor of the pharynx, opposite the hyoid and first branchial arches. It first becomes conspicuous about the sixth day, and by the eighth or ninth day (Fig. 116, Tr) has attained a definite shape. It is formed behind the boundary line between the pharynx and stomatodæum, and its epithelium is therefore of hypoblastic origin.

\section{The Stomatodæum.}

The stomatodæum, or inouth invagination, is formed by pitting-in of the ventral wall of the pharynx from the exterior.

From the time of its first formation the ventral wall of the pharynx, in front of the heart, is very thin ( $c f$. Fig. 112). On the appearance of the visceral arches, as thickenings of the side walls of the pharynx, this thin-walled area on its ventral surface 
becomes more clearly defined, as a slightly depressed, transversely elongated patch, bordered by a thickened rim, which is formed partly by the ventral ends of the anterior visceral arches, and partly by the under surface of the head itself.

By further thickening of this rim, the depression which it surrounds becomes deepened; and the pit formed in this way, rather by building up of its walls than by lowering of its floor, becomes the stomatodæum.

Towards the end of the third day the floor of the stomatodxal pit thins away and becomes perforated, placing the pharynx for

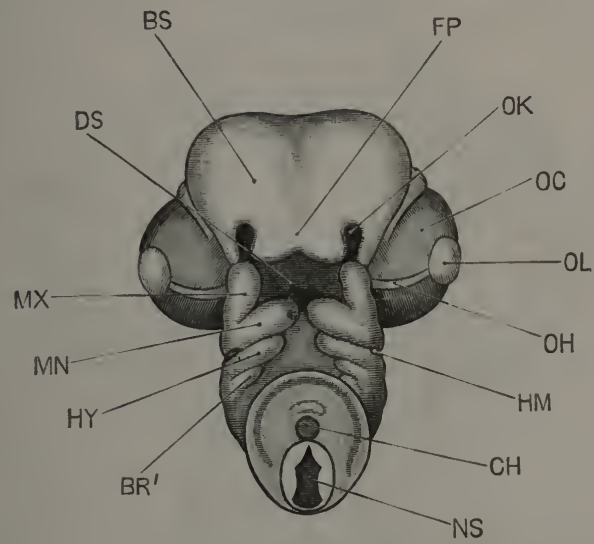

Fig. 125. - The head of an Embryo Chick at the end of the fifth day of incubation; seen from below. Compare Fig. 115 for a view of an embryo of the same age from the side. $\times 8$.

BR', 'first branchial arch. BS, cerehral hemisplere. $\mathbf{C H}$, notochoril. DS. mouth. FP, fronto-nasal process. $H M$, hyomandibular cleft. HY, hyoid arch. MN, mandibular arch. MX, maxillary arch. NS, spinal cord, seen in section where the neck has been cut across. OC, eye. OH, choroidal fissure. OK, olfactory pit. OL, lens.

the first time in direct communication with the exterior, and forming the permanent mouth opening (Fig. 114, o).

The Face. After the definite formation of the mouth opening, the borders of the stomatodæal pit continue to develop, and gradually give rise to the beak and the anterior part of the face of the bird. At the end of the fifth day the mouth opening (Fig. $125, \mathrm{DS}$ ) is oblong in shape. Its anterior border is formed by the fronto-nasal process, FP, a broad plate, notched in the median line, and forming, at this stage, the under surface of the head. The posterior border of the mouth opening is formed by the ventral ends of the mandibular arches, MN, which meet each 
other in the median plane at the chin; and the sides of the opening are bounded by the maxillary arches, mx, which grow forwards from the mandibular arches to meet the outer angles of the fronto-nasal process.

The olfactory pits, ok, lie just beyond the anterior and outer angles of the mouth: the inner border of each pit is formed by the side of the fronto-nasal process, or inner nasal process;

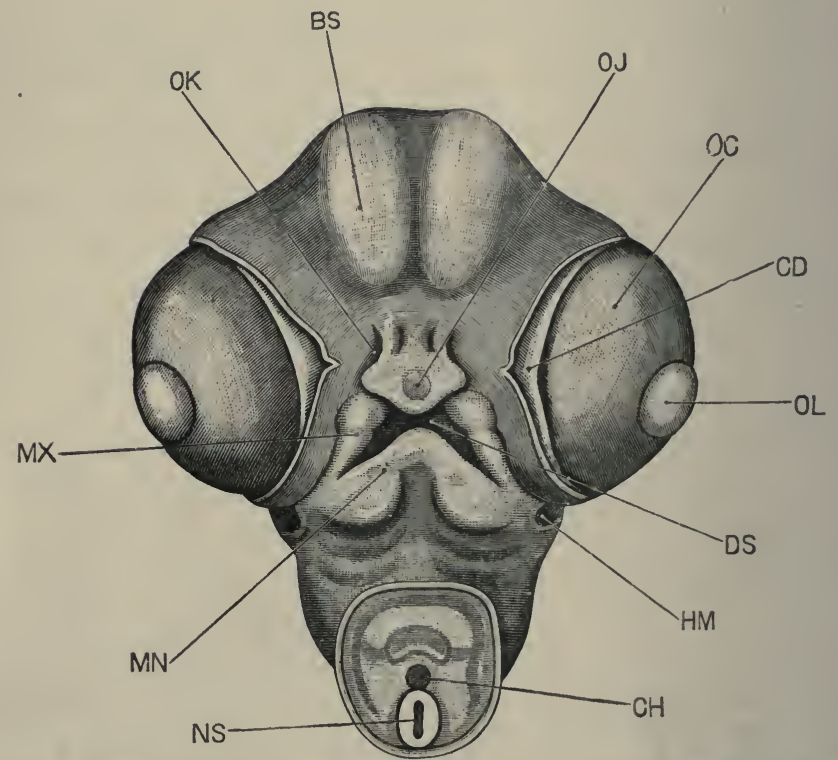

FiG. 126. - The head of an Embryo Chick at the end of the seventh day of incubation; seen from below. $\times 8$.

BS, cerebral hemisphere. CD, third eyelicl, or nictitating membrane. CH, notochord, seen in section where the neck has been cut across. DS, mouth. HM. external auditory meatus. MN, mandibular arch. MX, maxillary areh. NS, spinal cord, seen in section. OC, eyeball. OJ, epithelial knob on tip of beak. OK, external nostril. OL, lens.

the outer border is formed by a strip of the side of the head between the olfactory pit and the eye, which is spoken of as the outer nasal process.

Between the outer nasal process and the maxillary arch there is a slight depression, the lacrymal groove, which runs from the under surface of the eye to the outer border of the olfactory pit. Between the inner nasal process, or wing of the fronto-nasal process, and the anterior end of the maxillary arch there is a more conspicuous depression, the nasal groove, which becomes 
converted by fusion of its lips, as described on p. 275 , into the posteriol narial passage.

By the seventh day (Fig. 126) the parts of the face begin to assume more definite form. The mouth opening, DS, is more slit-like, and its boundaries are more clearly defined. The fronto-nasal process is narrower, and has begun to grow forwards as the upper beak, on the tip of which the small epithelial knob, oJ, which is used for breaking the egg-shell at the time of hatching, is already present. The maxillary arches have fused with the sides of the fronto-nasal process ; the nasal grooves are converted into the narial passages, and the lacrymal grooves have disappeared.

The two mandibular arches, mn, have fused in the median plane to complete the lower jaw, which is already beginning to grow forwards as the lower beak. Finally, the external nostrils, oK, have narrowed very considerably, and have acquired the slitlike form characteristic of them in the adult.

The pituitary body (Fig. 114, РT) is a pocket-like diverticulum from the anterior angle of the stomatodæum, which appears towards the end of the second day, and which early acquires its characteristic relations with the infundibulum, and with the anterior end of the notochord. Its development has already been described in the section dealing with the brain (p. 259).

\section{The Esophagus.}

Immediately behind the pharynx the alimentary canal suddenly narrows, becoming a very slender tube, the œsophagus, which runs back in a perfectly straight course through the neck (Figs. 114, To, and 123).

The œsophagus is at first very short; but, as the neck: lengthens, the osophagus grows rapidly, to keep pace with this. A curious point with regard to the œesophagus is that for a time, commencing about the middle of the sixth day, and lasting for two or three days, the lumen is completely lost, the cesophagus becoming solid along the greater part of its length. A little later, about the ninth day, the lumen is gradually re-established, from below upwards.

This temporary obliteration of the cavity of the œsophagus in the chick is perhaps to be associated with the rapid lengthening which the neck and the œsophagus are undergoing at this period; 
but the fact that a similar solidification of the œsophagus occurs in dogfish, frogs, reptiles, and mammals, as well as in birds, renders it possible that it has some further and deeper signification, not yet determined.

\section{The Stomach and Intestine.}

Up to the end of the fifth day (Fig. 123), the alimentary canal remains almost straight, except for a slight, ventrally directed loop, GT, at the place where the yolk-stalk, Ys, arises, connecting the intestine with the yolk-sac.

The stomach is recognisable as a slight, fusiform dilatation, TS, about the end of the fifth day; during the sixth day the gizzard becomes evident, as a thick-walled dilatation of the distal end of the stomach, which grows rapidly, and by the twelfth day has attained a great size.

From the sixth day onwards, the intestine lengthens rapidly; growth occurring most markedly at two parts of its length, and giving rise to two loops, both of which are directed ventralwards. Of these, the proximal or duodenal loop is formed from the part of the intestine immediately beyond the gizzard. The distal, or vitelline loop, which is much the longer of the two, is formed by elongation of the two limbs of the V-shaped loop which is already present on the fifth day, and from the angle of which the yolk-stalk arises (Fig. 123, ys).

Between the duodenal and vitelline loops there is a part of the intestine which undergoes hardly any elongation at all, but remains throughout life closely attached to the dorsal surface of the body cavity; it corresponds to the point in Fig. $123 \mathrm{im}-$ mediately beyond the opening of the bile-duct, wD, where the intestine bends ventralwards to form the proximal limb of the vitelline loop.

The further development of the intestine consists chiefly in great elongation of the vitelline loop, which gives rise to the whole length of the small intestine, beyond the duodenum. Both limbs of the loop lengthen very rapidly, and become twisted somewhat spirally. Up to about the seventeenth day the vitelline loop lies almost entirely in the yolk-stalk, and therefore outside the body of the embryo; about the eighteenth day the greater part of the loop becomes withdrawn into the body, and acquires the convolutions characteristic of the adult. 
The rectum, or terminal part of the intestine, grows very slowly, and remains nearly straight throughout the whole period of development. The boundary between the small intestine and the rectum is marked by the two rectal diverticula, which appear as a pair of small pouch-like outgrowths (Fig. 123, GH) abont the end of the fifth day; these grow rapidly, and by the eighth or ninth day have attained a considerable length. The rectum itself remains short; in the later days of incubation it dilates very greatly, and shortly before the time of hatching the bursa Fabricii arises as a dorsal outgrowth from its distal end.

The mesentery. The alimentary canal, along its whole length, is at first closely attached to the dorsal wall of the body cavity, immediately below the notochord.

The pharynx, or most anterior division of the alimentary canal, retains these relations throughout life. The œsophagus shifts ventralwards to a slight extent, owing to the intrusion of mesoblast between it and the notochord. Further back the ventral shifting is much more marked; and the whole intestinal region, from the stomach to the rectum, becomes situated some distance ventral to the notochord, remaining, however, connected with the dorsal wall of the body cavity by a vertical, laterally compressed sheet of mesoblast, the mesentery.

An exception to this statement must be made with regard to the short portion of the intestine between the duodenal and vitelline loops, which, as already noticed, remains in close connection with the dorsal body wall throughout life.

As the duodenal and vitelline loops of the intestine lengthen, the mesentery grows, keeping pace with them, and becoming still further reduced in thickness; it ultimately forms a thin sheet, consisting of two epithelial layers, derived from the peritoneum, and inclosing between them a very thin layer of mesoblast, along which the blood-vessels run to and from the alimentary canal.

The terminal part of the alimentary canal, or rectum, like the anterior part, remains closely connected with the dorsal body wall throughout life, the mesentery in this region only attaining a comparatively slight development.

\section{The Proctodæum.}

The proctodrum is a slight depression of the skin at the hinder end of the body, beneath the tail (Fig. 123). It develops 
very late, and does not open into the rectum until about the fifteenth day. The proctodæum in the chick is very shallow, and gives rise only to the outermost portion of the adult cloaca, and to the actual external opening.

\section{The Lungs.}

The lungs arise, during the third day, as a pair of small hollow outgrowths from the ventral surface of the anterior end of the cesophagus. By lateral constriction, the ventral part of the œsophagus, from which the lungs arise, becomes separated off as a median chamber (Fig. 114, LG) : this lies ventral to the ceso. phagus, and opens in front into the hinder end of the pharynx; while from its hinder end the lungs extend backwards as posteriorly directed outgrowths.

The lungs, after their first appearance, rapidly increase in size; they give off secondary diverticula, which branch again and again; and from the finest branches arborescent outgrowths arise at right angles, which become the ultimate spongy substance of the lungs.

The air sacs, which are structures very characteristic of birds, appear about the eighth day as thin-walled saccular diverticula from the hinder edges of the lungs; the alodominal air sacs are in the earlier stages the best developed.

The trachea (Fig. 116, LR) is formed by elongation of the median laryngeal tube, as the neck lengthens, and the lungs gradually shift backwards into the thorax.

From the mode of development of the lungs, as outgrowths from the alimentary canal, it follows that their lining epithelium, including the minutest passages, and that of the air sacs as well, is of hypoblastic origin : the rest of the thickness of the lung walls, including all the blood-vessels, is mesoblastic.

The lungs contain no air, and are not used for breathing, until immediately before the time of hatching; when the chick, breaking through the shell membrane into the air chamber at the larger end of the egg (Fig. 101, sv), draws air into its lungs for the first time, and, invigorated by the act, proceeds to peck its way out of the shell.

\section{The Liver.}

The liver arises, about the middle of the third day, as a tubular diverticulum from the posterior end of the fore-gut, in 
the angle between the two vitelline veins, and immediately behind their point of union. A second diverticulum arises from the same spot almost directly afterwards ; it is similar to the first, but of rather smaller size. Both these diverticula have hypoblastic walls, with thin mesoblastic investments.

Towards the latter part of the third day, as the folding off of the embryo from the yolk-sac proceeds, the liver diverticula are found to arise definitely from the part of the mesenteron which will later become the duodenum. At the same time they come into very close relation with a large median vein, the meatus venosus, which is formed by the union of the right and left vitelline veins behind the heart ( $f f$. Fig. 128, vE).

The two liver diverticula lie one at each side of the meatus venosus, and in very close contact with this. The hypoblastic cells forming the walls of the diverticula now begin to proliferate freely, growing out as solid strands of cells, which form an irregular reticulum closely surrounding the meatus venosus; the meshes of the reticulum being occupied by capillary blood-ressels, which develop in the mesoblast, and early acquire connection with the meatus venosus itself.

These processes proceed rapidly during the fourth and fifth days, and by the end of the fifth day (Figs. 123 and 128) the liver is an organ of considerable size, consisting of a network of solid rods of hypoblast cells, which branch and anastomose freely in all directions; the meshes of the network being occupied by blood-vessels, which penetrate all parts of the liver, and are in free communication with the meatus venosus, round which the liver is formed.

The liver continues to grow rapidly, and by the tenth day is the largest organ in the abdominal cavity. The trabecular network of hypoblast cells becomes the liver parenchyma; the tubular diverticula from the duodenum branch out freely in the substance of the liver, and become the two bile ducts of the adult bird; while the gall bladder arises on the fifth day as a saccular outgrowth from the right or larger of the two primary diverticula.

The early formation of the liver in the chick, and its large size during the greater part of the developmental history, indicate that it must be of considerable functional importance during embryonic life. Its relation to the blood system, and 
especially the fact that it intercepts the blood returning from the yolk-sac to the heart, suggest that its chief purpose is connected with the elaboration of the food material which is obtained from the yolk-sac, and at the expense of which the nutrition of the embryo is effected.

\section{The Pancreas.}

The pancreas arises, rather later than the liver, as a tubular outgrowth from the duodenum, just beyond the two liver diverticula, from which secondary outgrowths arise in much the same manner as in the liver itself. A second diverticulum arises from the duodenum about the eighth day, and gives rise to the greater part of the adult pancreas; and at a later period a third diverticulum is formed. The three diverticula persist as the three pancreatic ducts of the adult bird, while the three lobes of the pancreas, with which they are connected, soon fuse indistinguishably with one another.

\section{The Allantois.}

The allantois is really an appendage of the alimentary caual, arising as an outgrowth of its ventral wall, in front of the cloaca; it is therefore lined by hypoblast, like all other outgrowths of the mesenteron, while the rest of the thickness of its wall is formed by the splanchnopleuric mesoblast.

The allantois of the chick is homologous with the bladder of the frog (Fig. 89, TB). It differs mainly from this in the fact that, while arising in the same manner, it is not confined within the body of the embryo, but, growing rapidly, passes out beyond this as a thin-walled vascular sac (Figs. 100 and 101, TA), which spreads out in close contact with the inner surface of the eggshell, and acts as the respiratory organ of the embryo during the greater part of its development.

In the chick the allantois commences to form about the middle of the second day. At this time the tail fold is not yet established, so that the allantois (Fig. 112, TA) appears at first as a pocket-like fold of the splanchnopleure, lying a short way behind the embryo, and with its cavity opening ventralwards.

On the formation of the tail fold, early on the third lay, the part of the splanchnopleure from which the allantois arises becomes doubled forwards under the embryo to form the ventral 
wall of the gut, and the allantois now appears as a saccular depression of the ventral wall of the hind-gut (Fig. 114, TA).

During the third day the allantois increases considerably in size, projecting downwards and forwards, as a hollow, thickwalled bud from the ventral surface of the hind-gut, into the body cavity, or space between the somatic and splanchnic layers of the mesoblast.

During the fourth day, by its further growth, the allantois passes out beyond the embryo, and turns up, along its right side, into the space between the two layers of the amnion, which, from the mode of formation of the amnion, is directly continuous with the body cavity of the embryo ( $f$. Fig. 114).

On the fifth and following days the allantois grows rapidly; from the first it is very vascular, and the blood-vessels now increase greatly in size; the arteries, which lie in its superficial layer, are derived directly from the aorta (Fig. 128, AA); while the veins, va, which lie in its inner or deeper layer, join the vitelline veins from the yolk-sac, and, passing through the liver, reach the heart.

By the seventh or eighth day (Fig. 101, $\mathrm{TA}$ ), the allantois has spread all round the upper half of the egg, covering over the embryo, and extending half way round the yolk-sac as well. It is still saccular, and its cavity contains fluid. Its outer wall lies in very close contact with the outer layer of the amnion, or false amnion, and soon fuses with this completely, so that from this time the allantois lies in close contact with the shell membrane.

In its further growth the allantois does not follow the rolksac; but, keeping close to the egg-shell, and carrying the somatopleure before it, it extends so as gradually to inclose the mass of the white, wa, which still remains on the under surface and near the smaller end of the egg. The allantois, about the sixteenth day, completely incloses this plug of white or albumen, and from this time the absorption of the plug proceeds rapidly, the albumen being apparently carried by the allantoic vessels to the embryo, and aiding in its nutrition.

Towards the close of incubation deposits of urates occur in the cavity of the allantois, indicating that it serves as a receptacle for the excretory matters formed within the embryo itself, as well as a respiratory organ in the more restricted sense of the term. 
Shortly before the time of hatching, the allantoic vessels become constricted, by the closure of the body walls at the umbilicus. 'The allantois itself shrivels up, and is cast off' as the chick works its way out of the shell.

\section{DEVELOPMENT OF THE HEART AND BLOOD- VESSELS.}

\section{Preliminary Account.}

The general arrangement of the vascular system during embryonic life is strikingly similar to that of the tadpole. The heart is at first a straight, and later a twisted tube, lying beneath the pharynx, and driving the blood through a series of paired aortic arches (Fig. 128) to the dorsal aortæ, which distribute it to all parts of the body. From the body generally, and from the Wolffian bodies, the blood is returned by anterior and posterior cardinal veins on each side; these unite to form the Cuvierian veins, or anterior venæ cavæ, which open into the sinus venosus or posterior end of the heart. From the alimentary canal the blood is returned by the mesenteric or hepatic portal vein, which, passing through the liver, joins the posterior vena cava, the vein through which the blood is returned to the heart from the kidneys and other organs.

The chief differences between the chick and the frog as regards the arrangement of the blood-vessels are: (i) that the chick embryo has no gills, either external or internal, and therefore possesses no vessels corresponding to the gill loops of the tadpole; and (ii) that in the chick the vessels connected with the yolk-sac and with the allantois, both of which are structures outside the embryo itself, are enormously developed. These bloodvessels, vitelline and allantoic, are in direct connection with the vessels of the embryo: the afferent vessels, i.e. the vitelline and allantoic arteries, being branches of the dorsal aorta; while the efferent vessels, the vitelline and allantoic veins, on entering the embryo, join the mesenteric veins and run, through the liver, to the heart.

Throughout the greater part of the period of incubation, the vitelline and the allantoic vessels are of very large size; and inasmuch as the returning vessels, the vitelline and allantoic veins, bring to the embryo food matter from the yolk-sac, and 
oxygen from the allantois, it follows that the blood entering the heart by the posterior vena cava is arterial, and not venous, in character. 'The right understanding of the peculiarities in the circulation in the chick during embryonic life is mainly dependent on a full appreciation of this fact.

The aortic arches of the chick embryo undergo changes very similar to those which occur in the frog; the arches disappearing in part, and in part becoming modified into the arterial system of the adult. As in the frog, the pulmonary arteries are branches from the hindmost pair of aortic arches.

Histological development of the blood-vessels. The bloodvessels appear in the vascular area before they are formed in the embryo itself, and the mode of their development is easier to determine in the former situation.

Shortly before the end of the first day, when two or three pairs of mesoblastic somites are present in the embryo, a number of outgrowths from the upper surface of the hypoblast appear round the inner margin of the area opaca. These branch freely, and mite with one another to form a network, lying between the mesoblast and the hypoblast: the strands of the network are solid; they contain numerous nuclei, but cell outlines are difficult or impossible to determine in them. Within the strands, vacuoles soon appear at intervals: these enlarge rapidly, and, running into one another, convert the solid network into a system of anastomosing tubules with nucleated walls. These tubules are capillary blood-vessels; they are filled with fluid, but contain no blood corpuscles until a later stage.

This vascular network spreads rapidly, extending outwards as the vascular area widens, and inwards across the area pellucida to the embryo, which it invades on the second day. From their first appearance the vessels of the embryo are continuous with those of the area pellucida; but it is not quite clear how far they arise in situ, or how far by intrusion of vessels from the area pellucida.

This network of blood-ressels lies below the mesoblast, between this and the hypoblast; it is connected at places with the hypoblast, from which it arises in the first instance, but it is quite independent of the mesoblast. If this appears to contradict the general rule according to which the blood-vessels are derived 
from mesoblast, it should be remembered that the whole of the mesoblast in the chick, with the exception of the primitive streak mesoblast, is of hypoblastic origin; and the facts with regard to the formation of the blood-vessels might therefore be expressed by saying that the blood-vessels separate from the hypoblast at a stage later than that at which the othèr mesoblastic structures are formed from it. It is better, however, to accept the facts as they stand; namely, that in the chick many of the blood-vessels are derived directly and independently from the hypoblast; and to bear in mind that the middle germinal layer, or mesoblast, cannot be regarded as in any sense equivalent to either of the two primary germinal layers, epiblast and hypoblast; the term 'mesoblast' being used to include a number of very diverse structures, most if not all of which owe their ultimate origin to either hypoblast or epiblast.

The hypoblastic vascular network, formed in the way described above, gives rise directly to the capillaries and to the endothelial lining of the larger blood-vessels. The connective tissue and muscular walls of these latter are derived independently from the mesoblast, which grows round and envelopes them.

The blood-vessels within the embryo are at first, like those of the area pellucida and area vasculosa, reticular in their arrangement. The definite arteries and veins are formed by straightening and enlargement of certain of the strands of the network, with disappearance of other portions; the dorsal aortæ, for instance, arising early in the second day, in embryos with eight pairs of mesoblastic somites, as a pair of longitudinal trunks, lying along the outer and ventral borders of the somites, between the mesoblast and hypoblast, and communicating freely along the hinder part of their course with the reticular network of the area pellucida.

\section{The Heart.}

The heart is formed on the under surface of the fore-gut, at the commencement of the second day. It consists at first of two longitudinal vessels, which, though closely applied in the median plane, are for a time quite distinct, but which soon fuse to form a single median tube.

The walls of the heart consist, as in the frog, of an outer 
muscular coat (Fig. 120, RM), formed by the splanchnopleuric layer of mesoblast; and an inner endothelial lining, RE, concerning the origin of which it is difficult to speak with certainty, but which appears, like that of the other blood-vessels, to be derived from the hypoblast.

The muscular wall (Fig. 120, RM) is at first incomplete dorsally, but, after the two halves of the heart have united, the muscular walls grow in towards the median plane, above the heart,

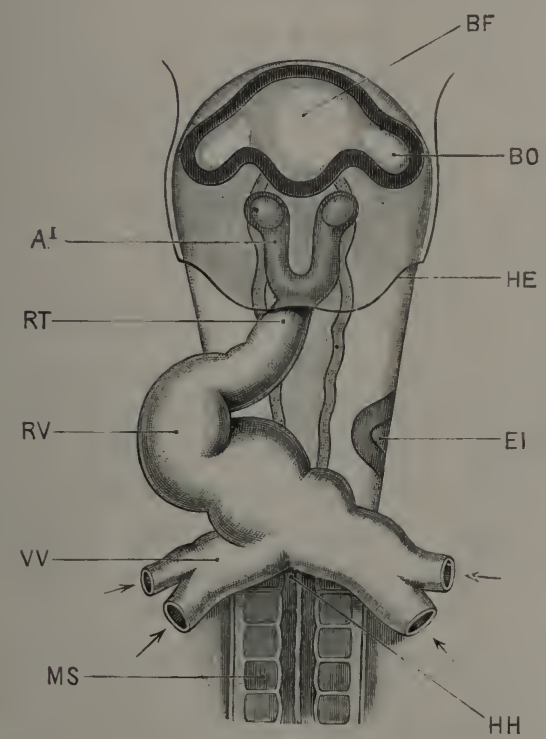

FIG. 127.-The anterior end of a Chick Embryo at the thirty-sixth hour of incubation, removed from the yolk-sac, and seen from the ventral surface. (Compare Figs. 111 and 112 for other views of an embryo of the same age.) $\times 30$.

A1, first aortic arch, in the mandibular areh. BF, fore-brain. BO, optic vesiele. EI, auditory pit. HE, posterior limit of head fold of somatopleure (compare Fig. 112). $\mathrm{HH}$, posterior limit of head fold of splanehnopleure, aul posterior boundlary of fore-gut. MS, mesoblastic somite or protovertebra. RT, truncus arteriosus. RV, rentricular portion of heart. VV, vitelline vein, eut short.

and coalesce so as to complete its wall. The endothelial tubes of the two halves of the heart remain distinct, though closely apposed, for some time after the muscular walls have coalesced, but ultimately they, also, become continuous. Between the muscular and endothelial walls there is at first a considerable space, filled with a mucous substance (Fig. 120).

The heart thus forms, about the thirtieth hour, a short and 
straight tube, lying below the fore-gut, and closely attached to its ventral surface, and with its walls consisting of an outer muscular tube and an inner endothelial tube.

The posterior end of the heart is, from the first, continuous with, or rather is formed by the union of, two large vessels, the vitelline veins (Fig. 127, vv), which collect and return to the embryo the blood from the network of capillaries in the area pellucida and area opaca ( $c f$. Fig. 98).

The heart is at first very short; but as the head fold becomes deeper, constricting the embryo more and more markedly from the yolk-sac, the vitelline veins remain at the edge of the fold, and so get carried back with it, causing thereby lengthening of the heart.

The heart begins to beat very shortly after its first formation, and before any distinct histological differentiation into muscle and nerve cells can be distinguished.

On its first formation, the heart is attached along its whole length to the uncler surface of the fore-gut. It remains attached at its two ends, but about the thirty-third hour becomes free along the middle portion of its length (Fig. 112, RV); and, growing more rapidly than the parts to which it is attached, becomes thrown into a loop (Fig. 127), with the convexity towards the right, and the concavity towards the left side of the embryo.

The loop, continuing to lengthen, projects downwards and backwards, so that the whole heart, towards the end of the second day, becomes twisted obliquely, into a letter $\mathbf{S}$ shape. Starting from the point of union of the vitelline veins, the heart runs forwards a certain distance, then makes a sharp bend downwards, backwards, and to the right side; then, making a second, equally sharp bend ( $c f$. Fig. 113, Rv) upwards, forwards, and to the left, reaches the median plane again, and is attached to the under surface of the pharynx opposite the first two pairs of gill-clefts.

The posterior end of the heart, into which the vitelline veins open, may be called the venous end of the heart; and the anterior end, the arterial end. The first bend or loop (Fig. $113, \mathrm{RA})$ marks the auricular part of the heart; and the second bend, RV, the ventricular part. The forwardly directed portion of the heart, in front of the second bend, is the truncus arteriosus, RT. 
During the third day (Fig. 113), the heart increases considerably in size; the S-like twisting becomes still more pronounced than before; and constrictions appear, separating the several chambers of the heart from one another.

On the fourth day, the auricular portion of the heart becomes widened laterally, and marked off by a sharp constriction from the ventricular portion, which, in its turn, is separated by a distinct though less pronounced constriction from the truncus arteriosus.

The most important event, however, that happens during the fourth day, so far as the heart is concerned, is the first appearance of the partitions by which the right and left sides of the heart become separated from each other. Up to the fourth day the heart is a single and continuous, though twisted tube, without any division whatever into right and left sides. The blood enters at the posterior or venous end of the heart, and passing through the several cavities in succession passes out in front, through the truncus arteriosus, into the aortic arches.

The internal division of the heart, into right and left sides, is effected by three septa or partitions, which appear within the cavity of the heart, and which arise perfectly independently of one another: (i) the interauricular septum, which divides the auricular chamber into the right and left auricles; (ii) the interventricular septum, which divides the ventricular chamber into the right and left ventricles; (iii) the septum of the truncus arteriosus, which divides the truncus arteriosus, or terminal chamber of the heart, into right and left halves. Of these septa, the first two commence to form on the fourth day; the third, or septum of the truncus arteriosus, does not arise until the fifth day.

Concerning the relative times of appearance of the interauricular and interventricular septa, there is some discrepancy in the published accounts. It is commonly stated that the interventricular septum develops the earlier of the two, but according to Masius it is the interauricular septum which is the first to be formed.

The interauricular septum appears, during the fourth day, as a septum projecting into the auricular chamber from its anterior and dorsal wall; it lies between the apertures of the sinus 
venosus and the pulmonary vein, and ends in a free posterior edge. Of the two cavities into which it partially divides the auricular chamber, the left auricle is for a time much the larger of the two.

The interventricular septum also appears during the fourth day, as a crescentic partition which arises from the ventral wall of the apex of the ventricular chamber, and gradually extends across towards the dorsal wall. It divides the ventricular chamber somewhat obliquely, and as yet imperfectly, into a left and more dorsally placed cavity, and a right and more ventrally placed one. The position of the septum is indicated by a slight groove on the surface of the heart.

On the fifth day the interventricular septum is completed, but the interauricular septum remains imperfect throughout the whole period of development, up to the time of hatching.

The septum of the truncus arteriosus appears on this day as a longitudinal fold, corresponding exactly to the similar one in the frog. The fold commences near the distal end of the truncus arteriosus, between the fourth and fifth pairs of aortic arches, and grows backwards with a somewhat spiral course, dividing the cavity of the truncus arteriosus into right and left halves. $\mathrm{By}$ the end of the fifth day this longitudinal septum has grown back to the base of the truncus arteriosus, and now meets with the upper edge of the interventricular septum and fuses with this. The effect of this fusion is that the right ventricle now communicates with the right division of the truncus arteriosus, and through this with the hindmost or fifth pair of aortic arches alone; while the left ventricle communicates, through the left division of the truncus arteriosus, with the anterior pairs of aortic arches, but no longer with the fifth or hindmost pair.

Before the completion of the septum of the truncus arteriosus three semilunar valves are formed at the base of each of the divisions of the truncus arteriosus, between these and the ventricles.

During the sixth day the shape of the heart as a whole approaches much more closely to that of the adult; the apex of the heart becomes more pointed, and the auricular appendices more prominent. 
Up to about the twelfth day the interauricular septum remains very imperfect, and there is free communication between the two auricles through a large aperture, the foramen ovale. About the twelfth day this communication narrows considerably.

On the sixteenth day the Eustachian valve is formed as a fold projecting into the right auricle, between the openings of the posterior vena cava and the right anterior vena cava. Up to this time the blood from both these vessels has passed from the right auricle, through the foramen ovale, into the left auricle and so to the left ventricle. The effect of the Eustachian valve is to direct the blood from the right anterior vena cava into the right ventricle, while still allowing the blood from the posterior vena cava to pass through the foramen ovale into the left auricle. From this time the two auricles are about equal in size.

Shortly before hatching, the foramen ovale becomes partially blocked up by a membranous, ralve-like fold; the completion of this stoppage is effected shortly after the time of hatching, from which time the structure of the heart is practically that of the adult bird.

'The thickening of the ventricular wall, which is a marked feature of the later stages of development, is effected by invardly projecting ridges of the muscular wall, which ultimately form a system of anastomosing muscular trabeculæ, from which, by further thickening, the columnæ carneæ and musculi papillares are developed.

The thickening of the wall of the auricles is effected in similar fashion, but is not carried to so great an extent.

The wall of the truncus arteriosus thickens by simple increase in the thickness of the muscular and other layers composing it.

\section{The Arteries.}

a. The Aortic Arches. The truncus arteriosus divides right and left, as in the frog, into the aortic arches, which run round the sides of the pharynx to its dorsal surface; here they open into the dorsal aortre, by which the blood is carried all over the body of the embryo, as well as to the yolk-sac and the allantois (cf. Figs. 113 and 128).

The aortic arches of the chick are developed in order, from before backwards. The first, or most anterior pair (Fig. 127, A1), 
is formed early on the second day, and lies opposite the anterior end of the fore-gut. The remaining arches are formed in succession behind the first pair.

By the end of the second day a second pair is present, and

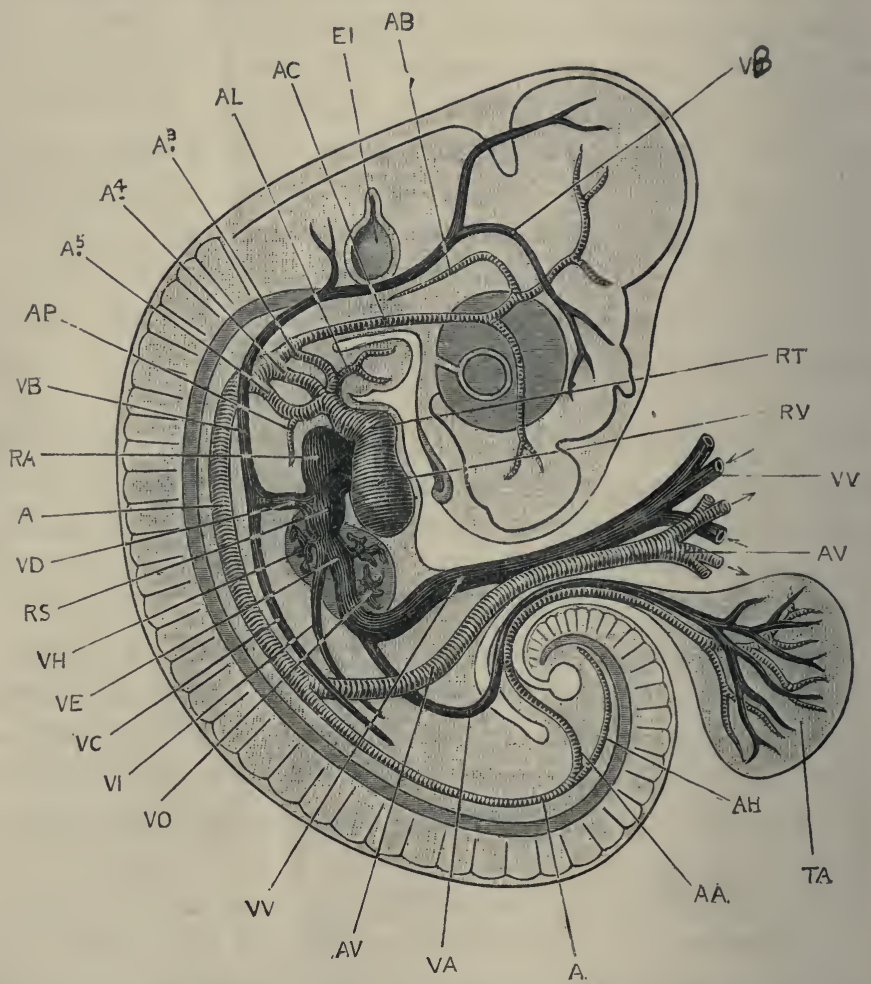

FIG. 128.-A diagrammatic figure showing the arrangement of the bloodvessels in a Chick Embryo at the end of the fifth day of incubation. The amnion has been removed, and the vitelline vessels cut short a little distance from the embryo. $\times 12$.

A, dorsal aorta. A3, A4, A5, third, fourth, and fifth aortic arches of the right sile, lying in the first, second, and third branchial arches respectively. A A, allantoic artery. A B , basilar artery. AC, carotid artery. $\mathbf{A H}$, candal artery, the terminal portion of the dorsal aorta. AL, lingual artery. AP, pulmonary artery. AV, vitelline artery. EI, auditory vesicle. RA, right auricle. $\mathbf{R S}$, sinus venosus. $\mathbf{R T}$, truncus arteriosus. $\mathbf{R V}$, right ventricle. TA, allantois. $\mathbf{V A}$, allantoic vein. $\mathbf{V} \mathbf{B}$, anterior cardinal vein. VC, posterior cardinal vein. VD, Cuvierian vein. VE, meatus venosus. VH, efferent hepatic vessel. VI, posterior vena cava. VJ, jugular vein. VO, afferent hepatic vessel. VV, vitelline vein.

by the commencement of the third day a third pair appears still further back (Fig. 113 A3).

On the establishment of the visceral clefts and arches, the 
aortic arches acquire definite relations with the latter; the first, or most anterior aortic arch of each side (Figs. 113 and 124, A1), lying in the first or mandibular arch; the second aortic arch, 12, lying in the hyoid arch; and the third aortic arch, A3, in the first branchial arch. During the fourth day, fourth and fifth pairs of aortic arches (Fig. 128, A4, A5) appear in the second and third branchial arches.

There are thus altogether five pairs of aortic arches in the chick, corresponding to the five anterior of the six pairs present in the tadpole. These arches, however, differ from those of the tadpole inasmuch as (i) they never have any gills developed in connection with them; (ii) they form from their first appearance direct connections between the truncus arteriosus and the aorta, there being no separation into afferent and efferent vessels. A further difference lies in the fact that the aortic arches in the mandibular and hyoid arches are complete in the chick, while they never become so in the tadpole.

The condition of the aortic arches in the chick is comparable to that of a frog after the metamorphosis; but it is at present a matter of doubt whether this indicates an entire omission of the earlier stages in the chick, owing to the absence of gills; or whether the continuous aortic arch from heart to aorta does not rather represent a still earlier ancestral condition, prior to the acquisition of gills.

From the five pairs of aortic arches of the embryo the adult arterial system is derived, in the following manner.

During the fourth, or fourth and fifth days, the first or mandibular, and the second or hyoidean aortic arches disappear along the middle portions of their lengths; their ventral and dorsal portions persist, the ventral portions remaining of comparatively small size as the lingual or mandibular arteries (Fig. 128, AL); while the dorsal portion, which is much larger, extends forwards into the head as the carotid artery (Fig. 128, AC), which divides into internal and external carotid arteries, supplying the brain and face respectively.

By the end of the fifth day the ventricular septum is completed, and has fused with the longitudinal or spiral septum of the truncus arteriosus ( $c f$. p. 302). This latter septum arises, in front, between the roots of the fourth and fifth pairs of aortic 
arches, and divides the truncus arteriosus in such manner that the right division of the truncus arteriosus, and consequently the right ventricle, from which this division arises, sends all its blood into the fifth pair of aortic arches; while the left ventricle and left division of the truncus arteriosus conduct blood to the third and fourth pairs of arches. It follows from this that the supply of blood to the head and anterior part of the body is derived from the left ventricle; while the right ventricle supplies the whole of the body behind the heart, as well as the yolk-sac and allantois.

About the seventh day the two divisions of the truncus arteriosus separate completely from each other at their bases; the right branch, or pulmonary trunk, remaining in connection with the right ventricle and the fifth pair of aortic arches; and the left branch, or systemic trunk, with the left ventricle and the third and fourth pairs of aortic arches.

The part of the aorta connecting the dorsal ends of the third and fourth pairs of aortic arches (Fig. 128) becomes very slender, and is finally obliterated altogether, while the branch of the truncus arteriosus from which the third aortic arch arises elongates very considerably, and carries this arch forwards some distance in front of the next, or fourth aortic arch. The subclavian arteries, supplying the fore-limbs, arise from the ventral ends of the third aortic arches, during the third day; they grow backwards, lying ventral to the other vessels, and reach the limbs during the fourth or fifth day.

The fourth pair of aortic arches is, from the fifth day onwards, much the largest of the three persistent pairs. The arches of the two sides of the body are at first of equal size; but the arch of the right side soon becomes much larger than that of the left side; and the latter ultimately becomes obliterated along the greater part of its length, while the arch of the right side persists as the arch of the aorta in the adult bird.

The pulmonary arteries appear, in the walls of the lungs, about the middle of the third day, before the two hinder pairs of aortic arches are formed. On the appearance of the fifth pair of aortic arches the pulmonary arteries (Fig. 128, AP) become connected with their ventral ends. Each fifth aortic arch thus consists of two parts : a proximal part running from the truncus arteriosus, i.e. from the right ventricle, to the lung; and a distal 
(1) dorsal part which connects the root of the pulmonary artery with the aorta. This distal portion is spoken of as the ductus Botalli, or ductus arteriosus; and so long as it remains open the blood from the right ventricle can avoid the lung circulation, and pass to the aorta direct. Shortly after the hatching of the chick, however, the ductus Botalli shrivels up, and its cavity becomes obliterated; from this time the fifth arch communicates with the lungs alone, and all the blood from the right ventricle must pass through the pulmonary capillaries.

b. The Dorsal Aortæ and their branches. The two dorsal aortæ are at first separate along their whole length, running parallel to the notochord and some little distance from the median plane. Each aorta is, from the first, continuous along its outer side at several places with the vascular reticulum of the area pellucida and area vasculosa. By coalescence of the vessels of the reticulum at one place, with shrinking and disappearance of the reticulum in front of and behind this spot, the definite vitelline artery of each side is formed (Figs. 113 and 128, Av): this is a large vessel running outwards from the aorta, between the splanchnopleuric mesoblast and the hypoblast, and passing out beyond the embryo to open distally into the vascular reticulum of the area vasculosa.

Before the end of the second day the two aortæ have met and fused for a short distance along the middle part of their course, separating again towards their hinder ends, and giving off at intervals along their length small arteries to supply the various parts of the body.

By the fourth day the union of the two aortæ has extended much further back than before, and involves the part from which the vitelline arteries arise. The two vitelline arteries have themselves coalesced at their proximal ends, and now spring from the aorta as a single trunk, which divides almost at once into the right and left vitelline arteries, of which the left one is much the larger.

The allantoic, or, as they are often called, umbilical arteries (Fig. 128, AA), arise from the aortæ just beyond their point of bifurcation, and run outwards to the allantois. The left allantoic artery is usually the larger of the two from the first, and becomes ultimately the sole one, the right allantoic artery disappearing. 


\section{The Veins.}

The chief peculiarities in the veins of the chick, as distinguished from those of the tadpole, consist in the large size and great importance of the vitelline and allantoic veins, which return to the embryo the blood from the yolk-sac and the allantois respectively; the blood of the vitelline veins being laden with food matter absorbed from the yolk, while that of the allantoic veins is charged with oxygen, and freed from its excess of carbonic acid.

The blood is returned to the heart by three chief veins, as in the tadpole: (i) and (ii) the right and left Cuvierian veins (Fig. 128 , vD), which return blood from the head and body of the embryo, and which afterwards become the right and left anterior venæ cavæ: and (iii) the meatus venosus (Fig. 128, VE), a median posterior vein, which is formed in the first instance by the union of the right and left vitelline veins, vv; is joined a little later by the allantoic veins, vA; and, later still, receives in addition the posterior vena cava, vi. At the time of hatching of the chick the vitelline and allantoic veins disappear, or become comparatively insignificant vessels, and the posterior vena cava acquires its adult relations.

\section{a. The System of the Anterior Venæ Cavæ.}

Towards the end of the second day a pair of longitudinal vessels, the anterior cardinal veins ( $c f$. Fig. 128, vB), are formed in the mesoblast of the sides of the head, slightly ventral to the level of the notochord. They collect the blood from the sides of the head, and carry it backwards.

A similar pair of vessels, the posterior cardinal veins, vc, appear about the same time in the trunk of the embryo. They return the blood from the hinder part of the body, and more especially from the Wolffian bodies or embryonic kidneys, as soon as these are formed.

The anterior and posterior cardinal veins of each side unite, opposite the heart, to form a short transverse vessel, the Cuvierian vein (Fig. 128, vD), sometimes called the ductus Cuvieri, which opens into the sinus venosus, RS, or most posterior division of the heart.

Throughout the earlier stages of development the two cardinal veins return the blood from practically the whole of 
the body of the embryo, excepting the alimentary canal and the liver. The anterior cardinal veins persist throughout life as the jugular veins, and are joined. at an early stage by pectoral veins from the wing, and vertebral veins from the head and neck. The posterior cardinal veins remain of large size so long as the Wolffian bodies are in functional activity; but when these begin to diminish in size, on the appearance of the permanent kidneys, the posterior cardinal veins also shrink up, and ultimately disappear.

The Cuvierian veins, as already noticed, persist and become the anterior venæ cavæ of the adult bird.

\section{b. The System of the Posterior Vena Cava.}

The meatus venosus. The heart, as already described, is formea by the union of the right and left vitelline veins (Fig. $127, \mathrm{vv})$, which lie along the edge of the fold of the splanchnopleure, marking the posterior limit of the fore-gut (cf. Fig. 112).

The vitelline veins remain at the edge of the splanchnopleuric fold, and consequently travel backwards with this fold as the embryo becomes more definitely constricted from the yolksac. This shifting backwards of the point of meeting of the right and left vitelline veins causes, first, a lengthening of the heart; and then, after the heart is definitely established, the formation of a median vitelline vein lying posteriorly to the heart, between the hinder end of the lieart and the point of meeting of the two vitelline veins.

This median vitelline vein may be divided into an anterior part or sinus venosus (Fig. 128, RS), which really forms the posterior chamber of the heart and receives the Cuvierian veins; and a posterior part or meatus venosus, vE.

The meatus venosus has, from the first, very close relations with the liver. The two primary liver diverticula lie one on each side of it, and as the liver increases in size it completely surrounds the vein. Blood-vessels appear in the substance of the liver mass, and soon acquire openings into the meatus venosus. At first these vessels are very irregularly disposed, but by about the fifth day a definite arrangement can be made out. The meatus venosus on entering the liver gives off afferent hepatic vessels (Fig. 128, vo), which open into the capillary 
plexus of the liver substance; from this plexus, efferent hepatic vessels (Fig. 128, vir) arise, which open into the meatus venosus shortly before this emerges from the anterior end of the liver. 'Thus, while the main stream of blood, entering the liver from the vitelline veins, passes along the meatus venosus direct to the heart, a small part of it is diverted through the capillary system of the liver, joining the meatus venosus again further on in its course.

The part of the meatus venosus in the substance of the liver, between the openings of the afferent and efferent hepatic vessels, is usually spoken of as the ductus venosus.

The vitelline veins return to the embryo the blood from the capillary network of the vascular area of the blastoderm ( $c f$. Fig. $99, \mathrm{Av})$. From the greater part of the area vasculosa the blood is returned directly by the right or left vitelline veins; but from the more peripheral part of the area the blood is collected by a circular marginal or terminal vein, which runs round the outer edge of the area vasculosa, and from which anterior and posterior veins carry the blood to the vitelline veins.

Of the two vitelline veins, the left is, almost from the first, larger than the right. After the embryo has turned so as to lie on its left side, the difference between the two veins becomes more pronounced, and ultimately the right one disappears.

The allantoic veins. As soon as the allantois is definitely formed, on the fourth day, two allantoic veins are developed, returning the blood from it to the embryo. These unite on entering the embryo to form a single allantoic vein, which runs forwards in the splanchnopleure of the left side to join the left vitelline vein. With growth of the allantois, the allantoic vessels increase greatly in size, but their relations remain practically the same. In the earlier stages (Fig. 128), the vitelline vein is much larger than the allantoic ; but later on, as the yolk becomes smaller through absorption of its contents, while the allantois continues to increase, the proportions are reversed, and the allantoic vein becomes the larger of the two.

The mesenteric vein is formed by the union of the veins which return the blood from the intestine of the embryo. It 
appears during the fourth day, and is at first very small, but it becomes larger and more important as the alimentary canal lengthens.

The mesenteric vein joins the vitelline vein just before this reaches the liver. The blood entering the liver by the meatus renosus is thus derived from three sources: (i) the vitelline vein returns blood, highly charged with nutritious matter, from the rolk-sac; (ii) the allantoic vein returns blood from the allantois, rich in oxygen and freed from carbonic acid; (iii) the mesenteric vein brings venous blood from the walls of the alimentary canal of the embryo. Of these, the vitelline and allantoic veins are, during the period of incubation, much larger than the mesenteric vein ; but inasmuch as the two former vessels return blood from parts outside the embryo, they become obliterated at the time of hatching; while the mesenteric vein, which alone persists of the three, retains its relations with the liver, and becomes the hepatic portal vein of the adult bird.

The posterior vena cava appears about the fourth day ( $c f$. Fig. 128, vi). It arises in the mesoblast between the hinder ends of the Wolffian bodies, and runs forwards in the median line, ventral to the aorta ; at its anterior end it joins the meatus venosus, as this emerges from the liver, and just before it reaches the heart.

As the permanent kidneys become definitely established, the posterior vena cava acquires special relations with them, and increases in size as they develop. At first (Fig. 128), the posterior vena cava appears as a comparatively unimportant tributary of the meatus venosus, but with growth of the embryo, and especially with the enlargement of the hind limbs, it steadily increases in size, and towards the close of incubation becomes the larger vessel of the two.

After the posterior vena cava has attained some size the hepatic efferent veins shift so as to open into it directly, instead of into the meatus venosus, and it is from this time that the vena cava becomes a larger and more important vessel than the meatus venosus.

\section{The Course of the Circulation.}

It will render the description of the development of the blood-vessels easier to follow if a connected account is given of 
the course of the circulation on the third day, and during the later stages of incubation.

a. The circulation at the end of the third day (Fig. 113). The heart, at the end of the third day, is a single twisted tube, slightly constricted at intervals which mark the boundaries of the successive chambers, but with no trace of a division into right and left sides. The blood enters the hinder end of the heart by the two vitelline veins, and passes out in front through the truncus arteriosus; this at once splits into right and left branches, each of which again divides into the three anterior aortic arches, which encircle the pharynx and open dorsally into the aortæ. The two aortæ are widely separate in the head, but approach each other further back, and are fused for a short distance in the body. Behind this point they separate again, and each aorta gives off a large vitelline artery (Fig. 113, Av), which, passing out beyond the embryo, opens into the capillary network of the area vasculosa; from this network the blood is collected again by vessels which unite to form the vitelline veins, and so returns once more to the heart.

The great purpose of the circulation at this stage is to insure the absorption of nutriment from the yolk-sac, and its conveyance along definite channels to the embryo. The vascular system of the embryo itself is as yet only imperfectly formed, and there is no special respiratory organ.

\section{b. The circulation during the latter half of the period of incuba-}

tion. The heart is now fully formed. The sinus venosus has become absorbed into the right auricle, of which it now forms part : the auricular septum is still incomplete, the large foramen ovale allowing blood to pass freely from the right auricle to the left auricle. The ventricular septum is complete; and the truncus arteriosus is divided into two entirely separate vessels, of which one, the pulmonary trunk, arises from the right ventricle, and the other or systemic trunk from the left ventricle.

Three pairs of aortic arches are present, but these are the third, fourth, and fifth of the complete series, the first and second having disappeared along the greater part of their length. The systemic trunk, arising from the left ventricle, leads to the third and fourth pairs of aortic arches, and through these to the 
head and fore-limbs. The pulmonary trunk, arising from the ight ventricle, leads to the fifth pair of aortic arches, which are directly continuous with the dorsal aorta of the body of the embryo, and from which also the small pulmonary arteries arise. From the arorta a vitelline artery carries blood to the yolk-sac; and a still larger allantoic artery runs from the aorta to the allantois.

The blool is brought back to the heart by three great veins:the right and left anterior venæ cavæ, and the posterior vena cava. The right and left anterior venæ cavæ return venous blood from the head and fore-limbs of the embryo. The posterior vena cavi returns blood from the hinder part of the body, the hind limbs, and the kilneys; just before reaching the heart it is joined by the ductus venosus, which returns the blood from the yolk-sac, from the allantois, and from the alimentary canal of the embryo, by the vitelline, allantoic, and mesenteric veins respectively. The blood in the vitelline vein is arterial as regards nutrient matter; the blood in the allantoic vein is arterial as regards its gaseous components; and the blood in the mesenteric vein is venous. The blood in the posterior vena cava is venous as regards nutriment, and as regards gaseous components, but, having just passed throngh the kidneys, is arterial as regards freedom from nitrogenous excretory matters.

The blood brought to the heart by the posterior vena cava may therefore be spoken of as arterial, and stands in this respect in marked contrast to the venous blood brought to the heart by the right and left anterior vena caræ.

All three venæ cavæ open into the right auricle of the heart; but, owing to the position and direction of the opening, and to the Eustachian valve, the arterial blood from the posterior vena cava is directed at once through the foramen ovale into the left auricle, while the venous blood from the right and left anterior venæ cavæ remains in the right auricle. The right auricle is thus filled with venous blood, and the left auricle with arterial blood.

On contraction of the auricles, the blood they contain is driven into the ventricles, so that the right ventricle will be filled with venous, and the left with arterial blood.

The left ventricle drives its arterial blood along the systemic trunk, and through the third and fourth pairs of aortic arches 
to the head and fore-limbs; while the right ventricle forces its venous blood through the pulmonary trunk and the fifth pair of aortic arches into the dorsal aorta, from which part goes to supply the body and hind limbs of the embryo, and part, in the earlier stages by far the larger part, passes out along the vitelline and allantoic arteries to the yolk-sac and allantois, where it takes up nutriment and oxygen.

The enormously disproportionate size of the head and anterior part of the embryo and the stunted condition of the hinder part during the earlier stages are to be ascribed, at any rate in part, to this arterial supply of the anterior half as contrasted with the venous supply of the posterior half of the embryo.

c. The changes in the circulation on hatching. The changes which occur in the blood-vessels at or about the time of hatching of the chick are comparatively slight, but suffice to convert the circulation into that of the adult bird. The more important of these changes are the following:-

(i) Closure of the ductus arteriosus of each side: i.e. obliteration of the distal parts of the fifth pair of aortic arches, beyond the points of origin of the pulmonary arteries ( $c f$. Fig. 128, A5). 'The effect of this change is that the blood from the right ventricle can no longer pass into the aorta, but is sent entirely, through the pulmonary arteries, to the lungs.

(ii) Obliteration of the vitelline and allantoic veins. This is a necessary consequence of the complete absorption of the yolksac, and the casting off of the allantois. Its effect is to reduce the blood supply of the liver to the venous blood brought by the mesenteric vein, or, as it may now be called, the hepatic portal vein.

(iii) Closure of the ductus venosus. The effect of this change is to render it impossible for the blood brought to the liver to pass straight through it to the heart. All the blood entering the liver by the portal vein must now pass through the capillaries of the liver in order to get to the posterior vena cava, and so to the heart. The short cut by which the liver capillaries could previously be avoided is now stopped. 
(iv) Closure of the foramen ovale. This is not completed until some little time after hatching. Its effect is to absolutely prevent the passage of blood from the right to the left auricle. From the time of its completion, the blood brought to the heart by all three venæ cavæ is discharged into the right auricle, and passes from this into the right ventricle; while the only blood entering the left auricle is that brought to it from the lungs by the pulmonary veins.

On the completion of these changes the circulation becomes that of the adult bird. The arterial and venous streams of blood are kept quite distinct, and the so-called double circulation is completely established.

\section{DEVELOPMENT OF THE URINARY ORGANS.}

\section{General Account.}

'The urinary organs of the chick, while agreeing in their general relations and mode of development with those of the frog, yet present considerable and important points of difference.

The head kidneys, which in the young tadpole are of large size, and for a considerable time are the sole excretory organs present, are in the chick extremely rudimentary structures, which appear later than the Wolffian bodies, and disappear again almost at once.

'The Wolffian bodies of the chick are developed early: they soon attain a large size, and form the functional excretory organs during embryonic life. Shortly after the time of hatching, they lose their kidney structure and excretory function completely, though parts of them persist as accessory portions of the reproductive apparatus.

The Wolffian and Müllerian ducts develop independently in the chick, at any rate so far as their anterior ends are concerned. As in the frog, the Müllerian duct becomes the oviduct of the female, while the vas deferens of the male is formed from the Wolffian duct.

\section{The Wolffian Duct.}

'The Wolffian duct, which is the first part of the kidney system to be developed, appears early in the second day, in 
embryos with about eight pairs of mesoblastic somites ( $c f$. Fig. 110). The duct arises on either side as a ridge-like projection of the mesoblast, immediately beneath the dorsal epiblast, and a little to the outer side of the mesoblastic somites. At the stage mentioned, it lies opposite the three hindmost of the eight somites.

As the embryo grows, the ridge lengthens, extending slowly forwards, and more rapidly backwards. It also becomes free from the mesoblast; and in embryos with fourteen pairs of somites, i.e. of about the thirty-sixth hour (cf. Fig. 111), the Wolffian duct is present along each side of the body as a solid rod of cells, lying free between the epiblast and the mesoblast, and extending from the fourth to the fourteenth somite.

The rod soon becomes tubular, by acquiring a central lumen ; and at the same time its position becomes changed, the mesoblast growing rapidly, and spreading over the duct, between it and the epiblast. The Wolffian duct (Fig. 129, Kc) consequently gets driven down into the mesoblast, where it lies, about the level of the dorsal aortæ, imbedded in the mass of mesoblast, sometimes spoken of as the intermediate cell mass, which projects into the dorsal part of the body cavity as a longitudinal ridge between the somatopleuric and splanchnopleuric folds.

The Wolffian duct continues its growth backwards during the succeeding stages, and towards the end of the fourth day (cf. Fig. 123, KC) reaches the cloaca, or terminal dilatation of the alimentary canal, and opens into its dorsal surface.

\section{The Wolffian Body.}

The Wolffian body is developed entirely from mesoblast, and agrees closely in its structure and mode of development with that of the frog. In its fully formed condition (Fig. 123, KM) it consists of a conıplicated mass of convoluted Wolffian tubules, each of which commences with a Malpighian body, and opens at its other end into the Wolffian duct.

The Wolffian body extends along the greater part of the length of the dorsal body-wall, as far back as the thirtieth somite. Its anterior end is imperfectly developed, or even rudimentary from the first, but from about the sixteenth to the thirtieth somite the Wolffian body remains of large size almost up to the time of hatching. 
In their mode of development the Wolffian tubules present certain differences in the anterior and posterior parts of the Wolffian body respectively, and must be described separately.

In front of about the sixteenth somite, a number of small funnel-like depressions of the peritoneal epithelium appear, towards the end of the second day (Fig. 129, Ks), below and a little to the inner side of the Wolffian duct. The bottoms of these funnels are in connection with slightly twisted cellular cords, which form in the mesoblast. These cords soon become tubular,

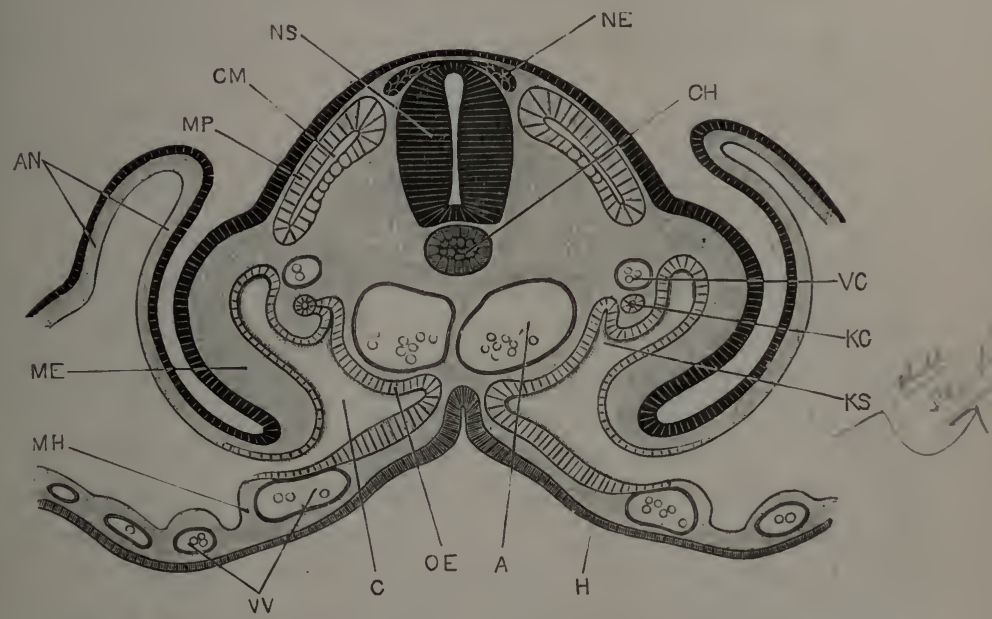

FIG. 129.-A transverse section across the body of a Chick Embryo at the forty-eighth hour of incubation. $\times 150$.

A, aorta. AN, amuion. C, borly cavity or cœlom. CH, notochord. CM, mrocœl, or cavity of mesoblastic somite. H, hypoblast forming roof of mid-gut. KC, Wolffian cuet. KS, nephrostome. ME, somatoplenric layer of mesoblast. MH, splanchnopleuric layer of mesoblast. $\mathbf{M P}$, muscle plate. NE, rudiment of spinal ganglion. NS, spinal corl. OE, genital epithelium. VC, posterior cardinal vein. VV, vitelline vein.

and acquire communications at one end with the funnel-like depressions, and at the other end with the Wolffian duct; so that the peritoneal funnels, or nephrostomes, now lead from the cœlom through these Wolffian tubules into the Wolffian duct. The nephrostomes, and the Wolffian tubules into which they open, are usually from the first rather more numerous than the somites in which they lie.

The anterior Wolffian tubules are imperfect from the first, and soon undergo degenerative changes. The mouths of the nephrostomes first dilate greatly. A vascular process, or glo- 
merulus, then arises from the side wall of each tubule, and projects into its cavity; by further enlargement, the glomeruli grow out through the expanded mouths of the tubules, or nephrostomes, so as to hang freely into the body cavity. These changes are probably of a degenerative character, for shortly afterwards both the glomeruli and the tubules disappear completely. Further back in the body, but still in front of the sixteenth somite, the Wolffian tubules develop differently; the nephrostomes close, the tubules separate from the peritoneum, and then become dilated to form Malpighian bodies, into which little vascular tufts or glomeruli, derived from the aorta, soon penetrate.

In the posterior part of the Wolffian body, from about the sixteenth to the thirtieth somites, there are no nephrostomes. The Wolffian tubules in this region have no connection with the peritoneal epithelium, but arise from the first in the mesoblast, appearing as oval vesicles, which by elongation become the Wolffian tubules. These acquire openings into the Wolffian duct, and dilate at their opposite ends to form Malpighian bodies. After the first-formed tubules are completed, others arise in the same manner, and usually nearer the dorsal surface. Partly owing to this increase in the number of the Wolffian tubules, and partly owing to each tubule increasing greatly in length and becoming much convoluted, the Wolffian body soon attains a considerable size, causing a marked ridge-like projection of the intermediate cell mass into the body cavity, along each side of the mid-dorsal line.

\section{The Head Kidney and the Müllerian Duct.}

Towards the end of the fourth day, three pit-like involutions of the peritoneal epithelium appear, one behind another, close to the outer side of the Wolffian duct, and three or four somites behind its anterior end. A ridge-like thickening of the peritoneal epithelium connects the three pits of each side with one another, and grows backwards behind the third pit as a solid rod of cells, lying along the outer side of the Wolffian duct, and very close to this.

This rod soon becomes tubular, ending blindly behind, but opening in front into the body cavity through the three pits. These three pits form the head-kidney of the chick embryo, and 
the tube into which they open is the commencement of the Miillerian duct.

Towards the end of the fifth day the two hinder pits close up and disappear. The anterior pit persists, and forms the peritoneal opening of the Müllerian duct or oviduct. The Muillerian duct itself grows rapidly backwards; it lies in close contact with the outer wall of the Wolffian duct, and in its hinder part appears to be formed from cells derived from the wall of the Wolffian duct.

About the end of the sixth day, the Müllerian duct has grown backwards as far as the cloaca. It remains blind at its hinder end in the male, but in the female opens, at a later stage, into the cloaca.

\section{J. The Permanent Kidney, or Metanephros ; and the Ureter.}

The cloacal opening of the Wolffian duct (Fig. 123, TC) is opposite the thirty-fourth somite, while the hinder end of the Wolffian body itself does not extend behind the thirtieth somite. The permanent kidney is formed in the mesoblast immediately behind the Wolffian body, and is at first confined to the somites from the thirty-first to the thirty-fourth inclusive, i.e. to the somites between the hinder end of the Wolffian body and the cloacal opening of the Wolffian duct.

Towards the end of the fourth day, the ureter arises on each side (cf. Fig. 123, KD) as a forwardly directed diverticulum from the dorsal surface of the hinder end of the Wolffian duct. From the ureter lateral outgrowths arise, which become continuous with masses of cells in the mesoblast around it ; and from these cells the tubules of the kidney, with their Malpighian bodies, are formed at a later stage. The kidney is at first very small as compared with the Wolffian body, but towards the close of incubation it increases very considerably in size, growing forwards dorsal to the Wolffian body. The ureters, which at first open into the dorsal wall of the cloaca, through the hinder ends of the Wolffian ducts (Fig. 123), acquire independent openings into the cloaca about the sixth day.

\section{The Genital Ducts.}

a. In the male, or cock bird, the Miillerian ducts, although they reach the cloaca, never open into it. In the later stages they 
undergo degenerative changes, and ultimately they become almost completely obliterated on both sides of the body.

The Wolffian body aborts in great part: a portion of it. however, persists as the epididymis; while outgrowths from the Malpighian bodies of this portion penetrate into the testis. and become the vasa efferentia. The Wolffian duct persist as the vas deferens, becoming bent on itself into short transverse folds along almost its entire length, in the manner so frequently seen in the male genital duct both of Vertebrates and of Invertebrates.

b. In the female, or hen bird, the Wolffian body atrophies, a small part alone persisting as the parovarium, a body lying in the mesentery between the ovary and the kidney, and in which the tubular structure of the Wolffian body remains recognisable even in the adult bird. The Wolffian duct-disappears.

The Müllerian duct of the right side, like the right ovary, disappears, though traces of it may persist in the adult. The left Müllerian duct becomes the oviduct : its peritoneal opening becomes the fimbriated mouth of the oviduct, and its walls thicken greatly, especially at the cloacal end.

\section{The Supra-renal Bodies.}

The development of the supra-renal or adrenal bodies is not very satisfactorily determined. Each consists of two parts, cortical and medullary. Of these, it is generally agreed that the medullary portion arises as a part of the sympathetic nervous system. The cortical portion is developed from groups of cells which appear, about the end of the fourth day, in the mesoblast along the inner side of the Wolffian body, between this and the aorta; and which increase during the fifth and sixth days so as to form cell masses of considerable size. On the seventh day these masses become closely attached to the Malpighian capsules of the Wolffian body, but whether this is to be interpreted as indicating any essential connection between the two bodies is very doubtful. 
THE DEVELOPMENT OF THE BODY CAVITY AND MUSCULAR SYSTEM.

\section{The Body Cavity or Cœlom.}

The body cavity or cœlom is formed, as described on p. 243, towards the end of the first day, by the splitting of the mesoblast, or rather by rearrangement of the mesoblast cells into two layers, upper or somatic and lower or splanchnic respec. tively; the body cavity being the narrow chink-like space between the two layers.

In the later stages, the body cavity enlarges considerably, by further separation of its walls. It extends the whole length of the body, but does not reach into the head, stopping at the first mesoblastic somite or protovertebra.

The two halves, right and left, of the body cavity are at first separate; but as the ventral body-wall of the embryo is completed, by constriction of the embryo from the yolk, the two halves meet and open into each other across the mid-ventral plane. From the mode of its formation, the body cavity is directly continuous with the space between the two layers, inner and outer, of the amnion (Figs. 112 and 129, Ar); and it is owing to this continuity that the allantois is able to pass out beyond the limits of the embryo, and spread over its back (Figs. 100 and 101).

The mesoblast cells lining the body cavity become the peritoneal epithelium; from this epithelium the genital organs, i.e. the ovary or testis, are developed; and from it, or in close relation with it, the tubules of the Wolffian body are formed.

\section{The Pericardial Cavity.}

There is at first no separate pericardial cavity; the heart lying in the anterior part of the general body cavity, ventral to the cesophagus and pharynx (Fig. 112). About the end of the second day a septum begins to form, which shuts off the ventral portion of the body cavity, in which the heart lies, at first partially and ultimately completely from the general body cavity; and this shut-off portion becomes the pericardial cavity. The septum is formed in the following manner.

Opposite the hinder end of the heart the Cuvierian veins 
cross the body cavity transversely, in order to reach the sinus venosus from the somatopleure; and it is from the walls of the Cuvierian veins that the pericardial septum arises, as a thin sheet of connective tissue which grows forwards and upwards to the under surface of the fore-gut, and obliquely downwards and backwards to the ventral body wall, which it meets a little way behind the heart.

The lungs lie in two pocket-like diverticula of the bodycavity which extend forwards along the sides of the œesophagus (cf. Fig. 163). These pleural cavities at first lie dorsal to the pericardial cavity, but, as they gradually enlarge to make room for the lungs, they spread ventralwards over the pericardial cavity, between it and the body walls. The pleural cavities in the bird remain throughout life continuous at their hinder ends with the general body cavity.

\section{The Muscular System.}

The mesoblastic somites or proto-vertebræ ( $c f$. p. 244) are paired, cubical blocks of mesoblast, arranged in series along the sides of the spinal cord, and formed by transverse division of the vertebral plates of the mesoblast (Figs. 110, 111, 113, Ms).

The somites are at first hollow; their cavities are to be regarded as parts of the cœlom; and in the case of the anterior three or four somites the cavities are for a time actually continuous with the general body cavity. The walls of the somites are at first of nearly uniform thickness on all sides, but during the second and following days they thicken very unequally, and undergo further changes, by which they give rise to the greater part of the voluntary muscles of the trunk, and to the elements from which the vertebral column is developed. These changes are effected as follows.

During the second and third days (Fig. 129) the ventral walls of the somites thicken very considerably, so that the whole embryo is increased greatly in depth, from the dorsal to the ventral surface, and the cavities of the somites, CM, become situated, not in their centres, but close to their dorsal surfaces. The dorsal part of each somite, inclosing the cavity, or myocœl, CM, now separates off from the underlying mesoblast, as the muscle-plate, MP : the walls of the muscle-plate consist of cells of an epithelial character, closely packed side by side, and contrast 
strongly with the loosely arranged stellate cells of the ventral part of the somite.

The cells forming the deeper, or ventral, walls of the muscleplates become elongated parallel to the axis of the embryo, and very soon become converted into a system of longitudinal muscle fibres. In this way a broad band of longitudinal muscle fibres is formed, stretching along the whole length of each side of the body of the embryo, each band being divided transversely, by the original protovertebral lines of segmentation, into paired muscle segments or myotomes. These muscle bands in the chick correspond to the great longitudinal body muscles of Amphioxus or of Fishes; and from them a great part of the voluntary muscular system is developed.

The muscle-plates at first lie nearly horizontally, their inner borders being at a level slightly dorsal to their outer edges. As the body of the embryo increases in thickness, and assumes more definite shape, the muscle-plates become placed more and more obliquely ( $c f$. Fig. 129); and by the end of the third day (Fig. 124, MP) they lie almost vertically, the original inner border becoming dorsal, and the original outer border ventral in position.

The outer, or ventral, edge of each muscle-plate rapidly extends into the somatopleure, and by the fifth day has spread half way down to the ventral surface of the embryo; the greater part of its component cells become converted into fusiform musclecells, from which the muscles of the back and trunk are developed. At the dorsal and ventral borders of the muscle-plates the cells retain their epithelial character so long as the plate continues to grow.

In the segments opposite the limb buds (Fig. 115), the muscle-plates stop at the bases of the limbs; the muscles of the limbs themselves are formed independently of the muscleplates, about the fifth or sixth day, an arrangement which is probably to be regarded as a modification of a more primitive one by which the musculature of the limbs is derived directly from that of the body. 


\section{THE DEVELOPMENT OF THE SKELETON.}

'The skeleton of the chick, like that of the tadpole, consists in its earliest stages of densely packed mesoblast cells, which soon give rise, by the formation of an intercellular matrix, to the primary or cartilaginous skeleton. This, in the adult, is almost completely replaced by the secondary or bony skeleton, but persists in places, especially in the skull.

As in the frog, bone may appear either in direct relation with the pre-existing cartilage, or independently of it, and hence a distinction may be drawn between cartilage-bones and membrane-bones ; the latter, which appear independently of the cartilage, being almost confined to the head.

\section{The Vertebral Column.}

The vertebral column is formed from the ventral portions of the mesoblastic somites or protovertebræ. After the muscleplates have separated off, the remaining or ventral portions of the somites of each pair, which consist of indifferent and rather loosely compacted mesoblast-cells, grow inwards towards the median plane, and meet both above and below the spinal cord, and below the notochord as well; so that by the end of the third day (Fig. 124) both the spinal cord and the notochord have mesoblastic investments, which during the fourth day increase considerably in thickness.

Early on the fifth day, the transverse lines of demarcation between the successive pairs of somites disappear, the mesoblast becoming a continuous mass the whole length of the body, and forming continuous investments to the notochord and the spinal cord. This fusion only concerns the ventral portions of the somites, the muscle-plates retaining their original distinctness.

In the course of the fifth day, the mesoblast immediately around the notochord becomes cartilaginous, forming a continuous unsegmented cartilaginous tube, ensheathing the notochord along the whole length of the body. At the sides of the spinal cord, paired cartilaginous bars appear, which soon fuse with the cartilaginous investment of the notochord, and become the neural arches of the vertebræ.

A little later, but before the close of the fifth day, further histological changes occur in the cartilaginous tube surrounding 


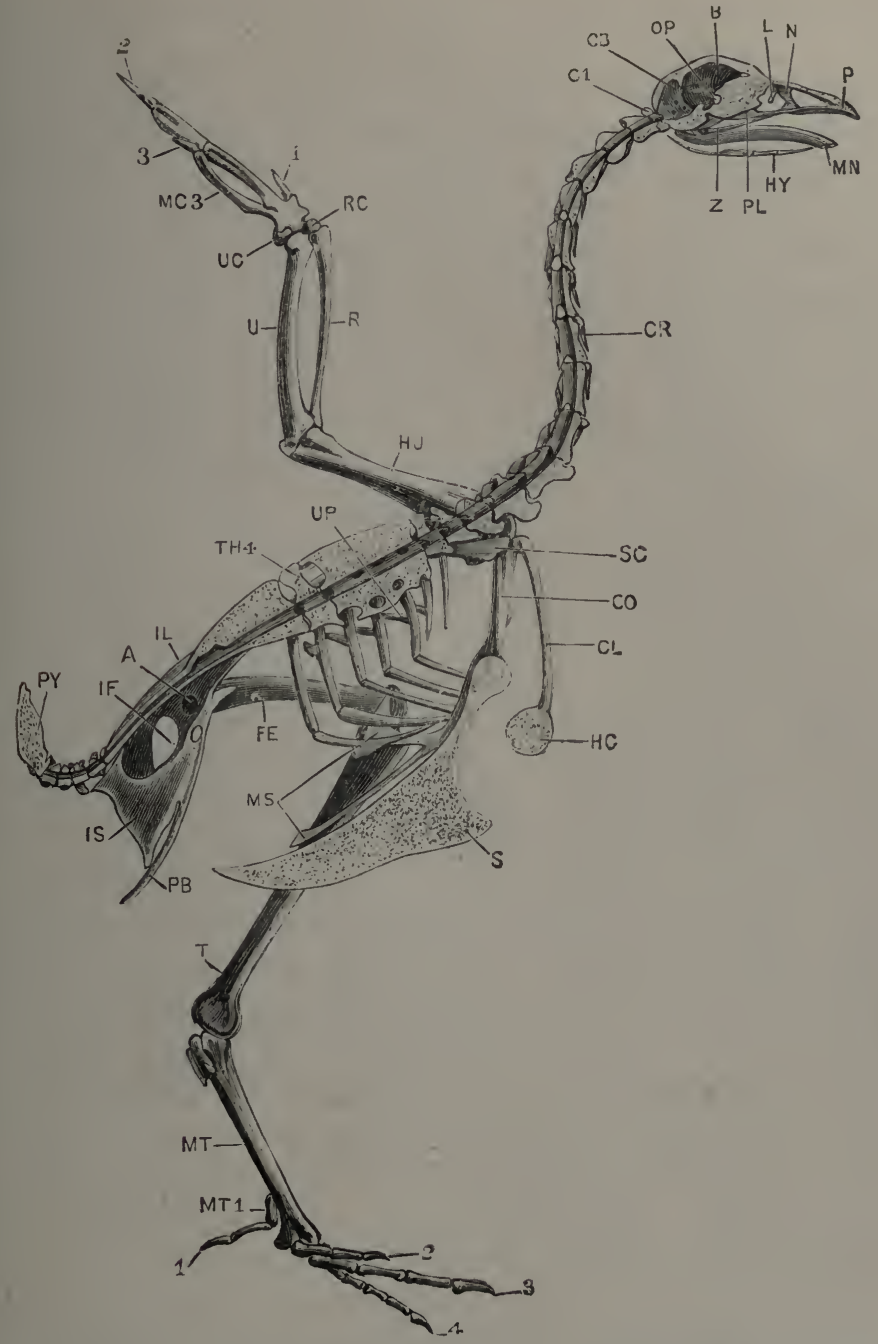

FIG. 130.-The left half of the skeleton of the Common Fowl. The skull, vertebral column, and sternum are bisected in the median plane. (From Marshall and Hurst.)

A, acetabulum. B, cerebral fossa. CB, cerebellar fossa. CL, claviele. $\mathbf{C O}$ coracoil. CR, cervical rib. Cl, first cervical vertebra. FE, femur. HC, hypoeleirlium. HU, lumerns. HY, hyoil. IF, ilio-sciatic foramen. IL, ilium. 'IS, isehium. I, lacrsmal bone. MiC3, metacarnal bone of third digit. MN, mandible. MS, metosteon. MT, tarso-metatarsus. MT1, metatarsal bone of first digit. $\mathbf{N}$, nasal bone. OP, optic foramen. $\mathbf{P}$, premaxilla. PB, pubes. $\mathrm{PL}$, palatine bone. PY, wygostyle. $\mathbf{R}$, radius. $\mathbf{R C}$, ratial carpal bone. $\mathbf{S}$, keel of the sternum. $\mathbf{S C}$ seapula. T, tibio-tarsus. TH4, fourth thoracio vertebra. U, ulna. UC, ulnar carpal bonc. UP, uneinate process of rib. Z. infra-orbital har.

1, 2, 3, 4, the first, second, third, and fourth digits. 
the notochord. Opposite the places of attachment of the neural arches, the matrix becomes inore abundant, and the cartilage cells fewer; while between the successive neural arches the matrix remains comparatively scanty, and the cartilage cells more numerous. In this way the cartilaginous tube round the notochord, while still remaining a continuous unsegmented structure, becomes marked into alternate vertebral and intervertebral rings, the vertebral rings being the parts to which the neural arches are attached, and in which the cartilage is of a more mature type ; and the intervertebral rings being the parts between successive neural arches, in which the cartilage remains of a mole embryonic character.

Each intervertebral ring, about the end of the fifth day, divides into two portions, anterior and posterior, which attach themselves to the vertebral rings in front of, and behind, them respectively. By this division the originally continuous cartilaginous sheath of the notochord becomes cut up into a series of segments; each segment consisting of a vertebral ring, fused with the posterior and anterior halves of successive intervertebral rings, and fused also with a cartilaginous nemal arch. The segments so formed become the adult vertebræ (Fig. 116).

The original vertebral rings, and the neural arches, lie opposite the intervals between successive pairs of muscle-plates; while the intervertebral rings lie opposite the muscle-plates themselves. As the division into vertebræ takes place across the centres of the intervertebral rings, it follows that the planes of division between the vertebræ do not coincide with the planes of division between the muscle-plates, i.e. with the original protovertebral planes of division, but are midway between these. Hence the division, or segmentation, of the vertebral column has been spoken of as secondary or permanent segmentation, in contrast to the primary or protovertebral segmentation which is retained by the muscle-plates or myotomes.

It must be borne in mind, however, that the permanent segmentation is the only one ever shown by the skeletal elements themselves. The primary segmentation is essentially a division into myotomes or muscle segments, and occurs at a time when the notochord is the only skeletal structure present. The cartilaginous skeleton is at its first appearance unsegmented, and 
the only segmentation it ever' shows is the permanent or 'secondary' one.

The reason why the permanent or vertebral segmentation alternates with the primary or myotomic segmentation is probably to be found in mechanical considerations. The longitudinal muscle fibres of the myotomes are attached at their ends to the vertebræ or to their processes, and the strain on the axial skeleton, and consequent tendency to lateral bending, caused by alternate contractions of the muscles of the two sides of the body, will be greatest, not opposite the attachments of the muscles, but midway between these; and it is at these midway points that the intervertebral joints are formed when the axial skeleton becomes too rigid to allow of free bending of the body, without segmentation. The mechanical advantage of the arrangement, by which each vertebra is acted on by two myotomes on each side, one pulling it forwards and one backwards, is sufficiently clear ; and the actual segmentation is probably due, in the first instance, to the direct action of the muscles themselves, causing bending, and subsequently jointing, of the originally continuous cartilaginous tube at points midway between the attachments of the muscles.

Up to the sixth or seventh day ( $c f$. Fig. $116, \mathrm{CH})$, the motochord remains of full size and nearly uniform diameter'; but from this time it becomes gradually encroached on by the vertebral centra surrounding it, which grow inwards, constricting it, and causing its gradual absorption and final disappearance. Ossification of the vertebra begins about the twelfth day, in the centrum of the second or third cervical vertebra, and gradually extends backwards along the column; the neural arches ossify rather later than the centra, and independently of them; each having two centres of ossification.

At an early stage, about the seventh day, the true centrum of the first, or atlas, vertebra separates from the outer ring, and becomes attached to the second, or axis vertebra, as its odontoid process. The atlas and axis vertebræ have no rib elements, but these are present in the remaining cervical vertebræ; they lie to the outer sides of the vertebral arteries, and they are from the first continuous with the centra of the vertebræ to which they belong, except in the case of the hindmost two or three cervical vertebræ, in which the rib elements are for a time independent. 
In a seven-day chick embryo there are forty-five vertebres present, of which the hindmost five or six fuse at a later stage to form the pygostyle.

Of the 'sacral' vertebræ, the first four have ribs, which in the first are long', and in the remaining three are much shorter. 'The fifth, sixth, seventh, and eighth vertebræ have no ribs; the ninth and tenth have ribs, and are for this reason regarded by many zoologists as the true sacral vertebræ. The remaining five, or ' urosacral,' vertebræ have no rib elements. Up to the seventh day these latter are quite distinct from the ilia, which stop at the tenth vertebra of the sacral series: in the later stages the ilia gradually extend further backwards, and ultimately overlap and fuse with all five ' urosacral' vertebræ.

\section{The Skull.}

The skull of the chick consists of the same morphological elements as that of the frog, viz. :

(i) The cranium, or brain case.

(ii) 'The sense capsules, olfactory and auditory.

(iii) The visceral skeleton.

The sense capsules and the cranium are, however, so closely united from their earliest appearance that it will be convenient to describe them together.

The main factors of the skull may be recognised, in the form of tracts of condensed mesoblast, as early as the fourth day, but it is not until the sixth day that cartilage is definitely established.

\section{a. The Cartilaginous Skull.}

(i) and (ii) The Cranium and the. Sense Capsules.

At the end of the sixth day, when cartilage first appears in definite form, the structure of the skull is as follows ( $c f$. Figs. 116 and 123). The notochord extends forwards in the median plane, beneath the brain, as far as the hinder end of the pituitary body, where it stops. At the sides of the notochord, and in close contact with it, are a pair of horizontal cartilaginous plates, the parachordal plates, which, with the notochord, form a broad floor to the hinder part of the skull, underlying the hind- and-midbrains. Imbedded in the parachordal plates, and continuous with them from their first appearance, are the cartilaginous auditory capsules, inclosing the auditory organs. 
In front of the notochord, the parachordals are continued forwards as a pair of short and rather slender rods, the trabeculæ cranii : these lie at the sides of the pituitary body, and unite in front of this to form the ethmoidal plate, which underlies and supports the fore-brain.

By the eightl day (Figs. 131 and 116) important changes

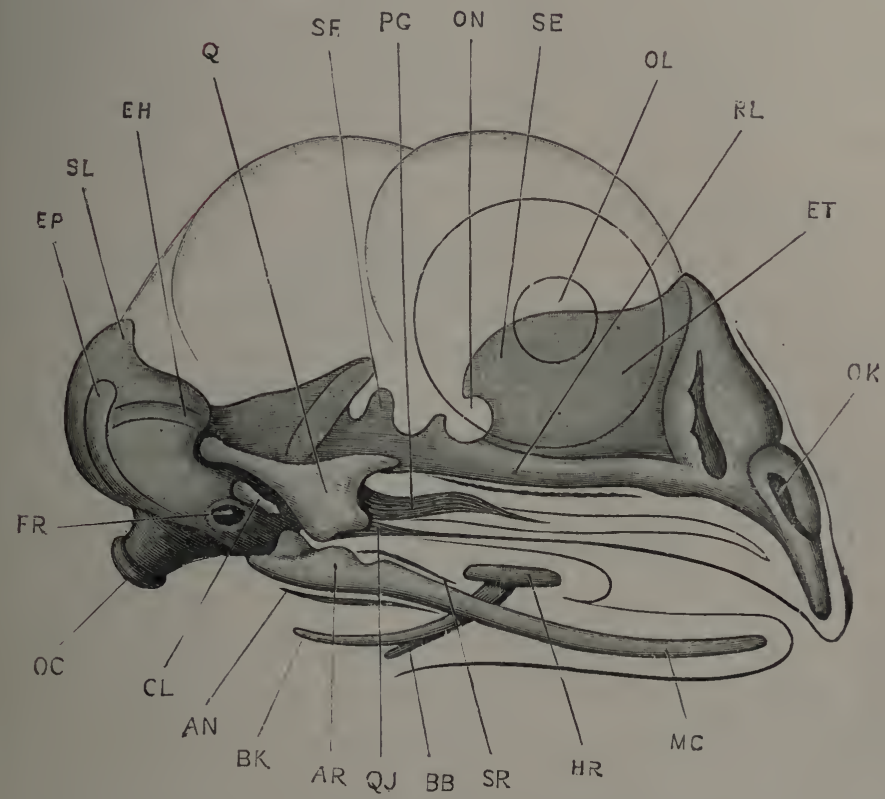

FIG. 131.-The skull of a Chick Embryo at the end of the eighth day of incubation; seen from the right side. The head and eye are represented in outline. $\times 10$.

AN, angulare. AR, articular portion of mantibular bar. BB, basi-branehial eartilage. BK, cerato-branchial cartilage. CL, columella. FH, external or horizontal semicirenlar canal. EP, posterior vertical semicircular canal. ET, mesethmoir cartilage. FR, fenestra ovalis. HR, ceratohyal cartilage. MC, Meckel's cartilage. OC, oceipital condyle. OK, slit-like aperture of olfactory capsule. OL, outline of lens. ON, outline of eyeball. PG, pterygoid. Q Q qualirate cartilage. QJ, quarratojngal. RI, trabeculi cranii. SE, pre-sphenoidal region. SF, ali-sphenoidal region. SL, supra-occipital region. SR. supra-angular.

have occurred in the skull, mainly associated with the growth forwards of the beak.

At the linder end of the skull the two parachordal cartilages (Fig. 116, RC) have united, above and below the notochord, to form the basilar plate; and the sides of the basilar plate, including the auditory capsules which are fused with them, have grown up- 
wards to form the side walls of the skull (Fig. 131); the exoccipital, ali-sphenoidal, and orbito-sphenoidal regions being already established.

In front of the pituitary body the ethmoidal plate (Fig. 131, ET) has grown enormously; it extends forwards to the tip of the beak, and is fused in front with the cartilaginous capsules of the olfactory organs, ok. From the dorsal surface of the ethmoidal plate, along its whole length, a huge vertical crest, the interorbital plate, has arisen, which supports the fore part of the brain (Fig. 116) along its upper edge, and is notched in front for the passage of the olfactory nerves, I.

\section{(iii) The Visceral Skeleton.}

In the chick embryo, cartilaginous elements, corresponding to the cartilaginous bars of the tadpole's skull, are developed in the mandibular, hyoidean, and first branchial arches.

The mandibular arch. In the mandibular arch two cartilages appear, proximal and distal respectively, which are from the first independent. The proximal, or dorsal, one (Fig. 131, Q) is the quadrate cartilage, a stout tri-radiate cartilage of which the longest arm, or otic process, is directed backwards, and articulates with the auditory or periotic capsule; while the ventral, and stoutest limb furnishes the articular surface for the mandible. The distal cartilage of the mandibular arch is a slender rod, Meckel's cartilage, Mc, which forms the basis of the lower jaw ; its hinder end, AR, which articulates with the quadrate, is expanded and thickened.

The hyoid arch. 'The bar of cartilage belonging to the hyoid arch is imperfect or absent along the greater part of its length, its dorsal and ventral ends alone being present. The uppermost or dorsal end is believed to be represented by the columella (Fig. 131, CL), a slender rod of cartilage which very early fuses with the stapes, a small plug of cartilage formed in the membrane closing the fenestra ovalis. The ventral end of the hyoid bar forms the cerato-hyal, or lesser cormu of the hyoid, HR; and the median element of the hyoid, or basihyal, appears also to belong: to this arch.

The first branchial arch. In the ventral part of the first branchial arch a slender cartilaginous bar, the cerato-branchial, or greater cornu of the hyoid (Fig. 131, BK), is formed; and in the

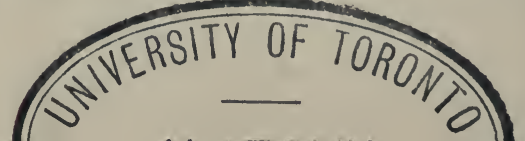


mid-ventral plane, where the arches of the two sides meet, a median basibranchial cartilage, BB, is developed.

In the hinder branchial arches no skeletal elements are formed in the chick.

b. The Osseous or Bony Skull.

(i) The cartilage-bones developed in connection with the skull are as follows:

From the parachordal cartilages are formed the basi-occipital, ex-occipitals, and supra-occipital.

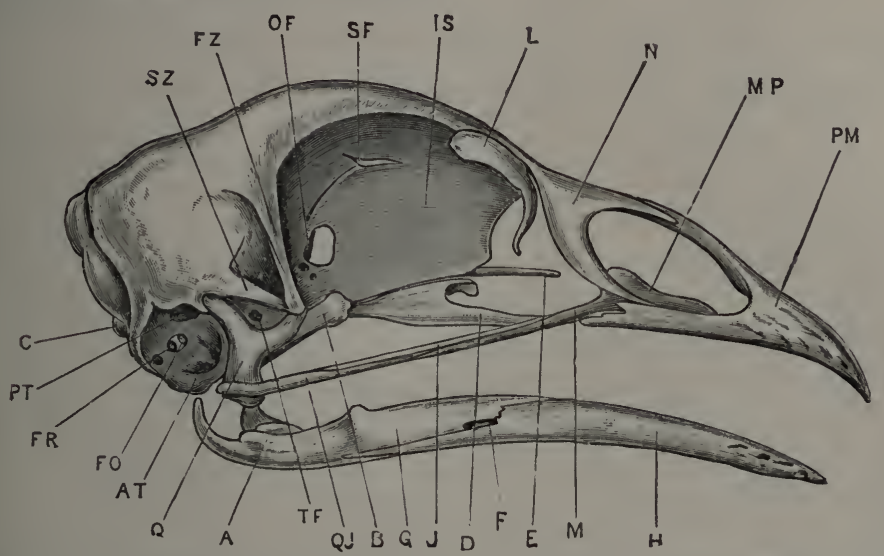

FI(: 132.-The skull of the Fowl, from the right side. (From Marshall and i. Hurst.)

A, artieular surface of the mandible. AT, anterior tympanic reess, lealing to Enstaclian tube. B, pterrgoid. C, oceipital conlyle. D, palatine. E, rostrum. F', mandibular foramen. FO, fenestra ovalis. F'R, fenestra rotunda. FZ, zygomatic process of frontal bone. G, supra-nngular. H, tentary. IS, inter-orbital septum. J, ingal. L, lacrymal. MT, maxilla. MP, maxillo-palatine proeess of maxilla. $\mathrm{N}$, nasal. OF, optic foramen. PM, premaxilla. $P T$, posterior tympanic recess. $Q$, quadrate. QJ, qualrato-jugal. SF, olfactory foramen. SZ, zygomatic process of squamosal. TF, foramen for fifth nerve.

From the periotic capsules are formed the prootics, epiotics, and opisthotics.

From the trabeculie cranii, the ethmoidal cartilages and the olfactory capsules, are formed the alisphenoids, orbitosphenoids, presphenoid, and mesethmoid.

In the mandibular arch of each side are formed the quadrate, and the articulare.

In the hyoid arch are formed the columella, the ceratohyal, and basihyal.

In the first branchial arch are formed the ceratobranchial, and basibranchial. 
(ii) 'The membrane-bones are less elosely connected with the cartilaginous skull than are the cartilage-bones, and can only be grouped somewhat arbitrarily according to the primary divisions of the cartilaginous skull.

In connection with the cranium and the sense capsules are formed the parietals, squamosals, frontals, lacrymals, nasals, vomer, basitemporal, and parasphenoid.

In connection with the upper jaw are formed the pterygoids, palatines, quadratojugals, jugals, maxillce, and premaxilla.

In connection with the lower jaw are formed on each side the dentary, angulare, supra-angulare, and splenial.

\section{The Pectoral Girdle and Sternum.}

The sternum develops as two separate halves, apparently formed by fusion of the ventral ends of the ribs, which meet and unite in the median plane on the ninth or tenth day. Both halves contribute to the formation of the keel, which is formed by fusion of their adjacent edges. The keel is very small until towards the close of development. The manubrium of the sternum is formed rather later, and is apparently a secondary outgrowth.

The sternum shows, in its development, evidence of having been originally of greater length, and associated with a larger number of ribs than in the adult fowl. In embryos of the sixth day the two hindmost cervical ribs are attached to the sternum ; on the seventh day they have lost their sternal attachments, but are still greatly elongated. At the hinder end of the thoracic series there is, during the sixth and seventh days, a rudiment of an eighth rib, which disappears shortly afterwards.

In the shoulder girdle, the scapula and coracoid are almost at right angles to each other on the seventh day, the scapula being long and blade-like, and the coracoid short and stout. At the beginning of the sixth day the scapula and coracoid are continuous with each other, but before the end of the day they separate.

The clavicles are membrane-bones; the median part of the furcula has been compared to an interclavicle, but the embryological evidence does not support this view.

\section{The Fore-limb or Wing.}

The humerus, and the radius and ulna, present no points of special importance in their development; but the carpus and the 
manus show peculiar modifications in all birds, and are of much greater interest (Fig. 130).

a. The carpus. On the seventh day the carpus consists of :(i) a proximal row of two cartilages, of which the larger one is situated opposite the end of the radius, and is regarded by Parker as corresponding to the radiale and intermedium of the typical carpus; while the smaller one, placed opposite the end of the ulna, is commonly regarded as the ulnare, but, according to Parker, corresponds to the ulnare and centrale of the typical carpus. (ii) A distal row of two cartilages, a larger one on the radial side and a smaller one on the ulnar side. A little later, about the tenth day, the radial cartilage divides into two, giving three cartilages in the distal row of the carpus, of which the middle one is the largest.

The carpus remains in this condition until some time after the hatching of the chick. The two proximal carpals persist as the two free carpals of the adult bird; they begin to ossify in chicks about five weeks after hatching. The distal carpals remain free for some time, but ultimately, in chicks eight or nine months old, they unite with the metacarpals.

b. The digits. In the manus of the fowl there are three wellformed digits, which at first are quite independent of one another, and which correspond to the three radial digits, pollex, index, and medius, of the typical Vertebrate manus. The fourth digit is rather doubtfully represented by a small rudiment.

On the seventh day the first three metacarpals are welldeveloped cartilaginous rods, completely separate from one another, and from the carpus. The first metacarpal is short; the second long and thick; the third about the same length as the second, but much thinner. The first digit or pollex has two short phalanges; the second digit or index has three; and the third digit or medius has two, of which the terminal one ultimately disappears.

During the tenth day a small nodule of cartilage, the prepollex, appears on the radial side of the first metacarpal, with which it ultimately fuses. Another small bar of cartilage appears, about the same time, on the outer side of the third metacarpal, at its proximal end; this, which perhaps represents the fourth metacarpal, remains distinct until some time after hatching, ultimately fusing with the base of the third metacarpal. 
The further changes in the manus are effected very slowly. 'The three metacarpals begin to ossify about the tenth or' twelfth day, but remain distinct from one another until about a month after hatching, when they begin slowly to unite together; the fusion of the metacarpals with the distal row of carpals does not occur until some time later.

\section{The Pelvic Girdle.}

The pelvic girdle, about the sixth day, consists of a somewhat squarish plate on either side of the body, the central part of which is at first directly continuous with the femur. The dorsal border of the plate corresponds with the iliac region, which at this stage does not extend over more than about three somites, differing in this respect very markedly from its condition in the adult. From the ventral and anterior border of the plate two processes, prepubic and pubic, project downwards and forwards ; and from the ventral and posterior border a broad ischiatic process projects downwards and inwards. .

During the seventh day, the femur becomes separated off from the hip girdle; the ilium extends rapidly backwards along the vertebral column, and more slowly forwards; the ischium grows backwards; and the pubis also begins to grow backwards as a slender bar, lying parallel to the ventral border of the ischium, and a little way below this. The prepubic process is relatively much less conspicuous than in the earlier stages.

During the later stages of development these changes become more and more marked, and the pelvis gradually acquires its adult shape. The ilium elongates, both anteriorly and posteriorly, becoming ultimately attached to no less than fifteen vertebræ. The ischium also lengthens greatly: its hinder end becomes expanded, and, shortly before the time of hatching, fuses with the posterior end of the ilium, to complete the boundary of the ilio-sciatic foramen. The pubes elongates still more than the ischium, and forms a long slender rod, lying parallel to the ventral edge of the ischium and projecting backwards some distance beyond this. The prepubic process, which, both in its cartilaginous condition and when ossified, appears to belong to the ilium rather than to the pubes, forms in the adult a small blunt process, projecting from the anterior and ventral border of the acetabulum. 


\section{The Hind-limb or Leg.}

The femur, and the tibia and fibula, present no special points of interest in their development.

The tarsus consists, on the seventh day, of a proximal row of two tarsal cartilages, of which one represents the tibiale and intermedium, and the other the fibulare of the typical tarsus; and a single distal cartilage, which shows indications of its formation from three centres. At a later stage the proximal tarsal cartilages fuse together; and about the fourteenth or fifteenth day the proximal tarsals fuse with the distal end of the tibia to form the tibio-tarsus, while the distal tarsal cartilage fuses with the metatarsals to form the tar'so-metatarsus.

In the pes, the first digit, or hallux, is represented by a short metatarsal, of which the proximal end is never present; and two phalanges. The second, third, and fourth digits are approximately equal in size, having well-formed metatarsals, and three, four, and five phalanges respectively. The fifth digit is represented by a small nodule of cartilage on the outer, or fibular, side of the proximal end of the fourth metatarsal.

The three fully-developed metatarsals, i.e. the second, third, and fourth, remain distinct, though closely apposed, until about the beginning of the third week of incubation, when they fuse with one another and with the distal tarsal cartilage.

\section{DEVELOPMENT OF THE FEATHERS.}

The feathers are formed by special modification of the epidermal coverings of papillæ, which appear as projections of the skin about the eighth day of incubation.

Each of the permanent feathers is preceded by an embryonic or down feather, the mode of development of which is as follows. About the eighth day small conical projections of the skin appear, the feather papillæ, each consisting of a central core of vascular connective tissue, covered by a cap of epidermis. As the papillæ increase in height, their bases become depressed below the general surface of the skin. This depression is most marked on the surface of the papillæe towards the tail end of the embryo, and gives rise to the characteristic backward slant of the feathers.

The epidermal cap of each papilla consists of a superficial, or epitrichial, layer of flattened pavement cells, and a deeper or 
Malpighian layer of short cylindrical or cubical cells. At first the epidermal cap is of uniform thickness all over the papilla; but, as the papilla lengthens, the inner surface of the epithelium thickens along certain lines, so as to form ridges projecting into the papilla. The thickening is due to the formation of a third or intermediary layer of cells, spherical or polygonal in shape, and lying between the epitrichial and Malpighian layers; and it is from the ridges formed in this way that the feather is developed.

The thickest and best marked of the ridges runs longitudinally, along the upper or anterior surface of the papilla, from base to apex; while the other ridges, which develop in order from the apex towards the base of the papilla, arise from the sides of this main ridge, and run very obliquely round the papilla to its lower or posterior surface, the ridges of the two sides not quite meeting on the lower surface of the papilla.

In each ridge, which is thus a solid rod of epithelial cells, the outer cells become elongated and cornified, while the central or axial cells remain comparatively soft. The vascular connective tissue, forming the core of the papilla, now shrinks away from its apex. The outer, or epitrichial, layer of the epidermis, which merely forms a sheath inclosing the papilla, is cast off; and the epithelial ridges or rods, which now alone remain, spread out to form the feather; the main longitudinal ridge becoming the shaft, and the diverging lateral ridges the barbs; while minor or tertiary ridges, which arise from the barbs, give rise to the barbules.

Towards the base of the papilla the epithelial ridges die out, and the entire epithelial investment of the papilla, including both epitrichial and Malpighian layers, becomes converted, by cornification of its cells, into the quill of the feather, which remains open at its lower end for the admission of blood-vessels.

At the lower end of the shaft, immediately above the quill, the main epithelial ridge widens, and the two sides bend inwards towards each other, and ultimately meet to form a tube, which is continuous below with the quill, and the upper aperture of which persists as the superior umbilicus of the feather.

The down feathers do not involve the entire length of the papillæ, but only their distal or apical portions. The basal portions of the papillæ sink deeply below the skin; a second set 
of epithelial ridges is formed on them, and gives rise to the permanent feathers, the development of which is essentially similar to that of the down feathers. The permanent feathers, as they are developed, gradually make their way to the surface, replacing the down feathers, the nutrition of which is cut off by further narrowing of the opening, or inferior umbilicus, at the base of the quill.

Feathers do not develop uniformly over the entire surface of the body, but along certain definite lines or tracts spoken of as pterylia, and separated from one another by naked areas or apteria ; the actual arrangement of these feathered and featherless patches varying considerably in different groups of birds.

\section{List of the more important Publications dealing with the development of the Chick.}

Von Baer, K. E.: ' 'Ueber Entwickelungsgeschichte der Thiere.' Königsberg, $1828,1837$.

Balfour, F. M.: 'The Development and Growth of the Layers of the Blastoderm ; and on the Disappearance of the Primitive Groove in the Embryo Chick.' Quarterly Journal of Microscopical Science, vol. xiii. 1873.

'A Treatise on Comparative Embryology.' 1880-81.

Balfour, F. M., and Sedgwick, A. : 'On the Existence of a Head-kidney in the Embryo Chick, and on Certain Points in the Development of the Müllerian Duct.' Quarterly Journal of Microscopical Science, vol. xix. 1879.

Balfour, F. M., and Deighton, F.: 'A Renewed Study of the Germinal Layers of the Chick.' Quarterly Journal of Microscopical Science, vol.xxii. 1882.

Beard, J.: 'The Development of the Peripheral Nervous System of Vertebrates.' Quarterly Journal of Microscopical Science, vol. xxix. 1888.

Brandt, A. : 'Ueber den Zusammenhang der Glandula suprarenalis mit dem Parovarium resp. der Epididymis bei Hühnern.' Biologisches Centralblatt, ix. 1889.

Budge, A. : 'Untersuchungen über die Entwickelung des Lymphsystems beim Hühnerembryo.' Archiv für Anatomie und Entwickelungsgeschichte. 1887.

Cajal, R.: 'A quelle Époque apparaissent les Expansions des Cellules Nerveuses de la Moëlle Épinière du Poulet?' Anatomischer Anzeiger, v. 1880.

Cazin, M. : 'Recherches Anatomiques, Histologiques, et Embryologiques sur l'Appareil Gastrique des Oiseaux.' Annales des Sciences Naturelles, série 7, tome iv. 1888.

Corin, G., et Bérard, E. : 'Contributions à l'Étude des Matières Albuminoïdes du Blanc d'Cuf.' Archives de Biologie, ix. 1889.

Coste, M. : 'Histoire Générale et Particulière du Développement des Corps Organisés.' Paris, 1847-59.

Davies, H. R.: ' Die Entwicklung der Feder und ihre Beziehungen zu anderen Integumentgebilden.' Morphologisches Jahrbuch, xv. 1889. 
Dexter, S.: 'The Somites and Cœlome in the Chick.' Anatomischer Anzeiger, vi. 1891.

Duval, M.: 'Etudes Histologiques et Morphologiques sur les Annexes des Embryons d'Oiseau.' Journal de l'Anatomie et de la Physiologie, xx. 1884.

'De la Formation du Blastoderme dans l'Cuf d'Oiseau.' Annales des Sciences Naturelles, série 6, tome xviii. 1885.

'Atlas d'Embryologie.' Paris. 1889.

Fabricius ab Aquapendente: 'De Formato Fotu,' 1600. 'De Formatione Fœtus,' 1604. The earliest descriptions, with figures, of the Development of the Chick and other Vertebrates.

Felix, W.: 'Zur Entwickelungsgeschichte der Vorniere des Hühnchens.' Anatomischer Anzeiger, v. 1890.

Foster, M., and Balfour, F. M.: 'The Elements of Embryology.' Second edition, by Sedgwick and Heape. 1883.

Gadow, H.: 'On the Modifications of the First and Second Visceral Arches, with especial Reference to the Homologies of the Auditory Ossicles.' Philosophical Transactions, vol. 179. 1888.

Gasser, E. : 'Der Primitivstreifen bei Vogelembryonen.' Marburg, 1878.

'Beiträge zur Kenntnis der Vogelkeimscheibe.' Archiv für Anatomie und Entwickelungsgeschichte.' 1882.

Gerlach, L. : ' Ueber die entodermale Entstehungsweise der Chorda dorsalis.' Biologisches Centralblatt, i. 1881.

Golowine, E.: 'Sur le Développement du Système Ganglionnaire chez le Poulet.' Anatomischer Anzeiger, v. 1890.

Holl, M.: 'Ueber die Reifung der Eizelle des Huhns.' 'Sitzungsberichte d. k. Akademie d. Wiss. in Wien,' xcix. 1890.

Johnson, Alice: 'On the Development of the Pelvic Girdle and Skeleton of the Hind Limb in the Chick.' Quarterly Journal of Microscopical Science, xxiii. 1883.

Kastschenko, N.: 'Das Schlundspaltengebiet des Hühnchens.' Archiv für Anatomie und Entwickelungsgeschichte. 1887.

Kölliker, A.: ' Entwicklungsgeschichte des Menschen und der höheren Thiere.' Leipzig, 1879.

Kowalevsky, R. : 'Die Bildung der Urinogenitalanlage (des Wolff'schen Ganges) bei Hühnerembryonen.' Warsaw, 1875.

Lahousse, E. : 'Recherches sur l'Ontogenèse du Cervelet.' Archives de Biologie, viii. 1888.

Liessner, E.: ' Untersuchungen betreffend die Entwicklung der Kiemenspalten bei Vertretern der drei oberen Wirbelthierklassen.' Sitzungsberichte d. Nat.-Gesellschaft. Dorpat. Band viii. 1887.

Lindsay, Beatrice: 'On the Avian Sternum.' Proceedings of the Zoological Society of London. 1885.

Mackay, T. J.: 'The Development of the Branchial Arterial Arches in Birds, with special Reference to the Origin of the Subclavians and Carotids.' Philosophical Transactions, vol. 179. 1888.

Mall, F. P. : 'Entwickelung der Branchialbogen und Spalten des Hühnchens.' Archiv für Anatomie und Entwickelungsgeschichte. 1887.

'Development of the Eustachian Tube, Middle Ear, Tympanic Membrane, and Meatus of the Chick.' Studies from the Biological Laboratory of the Johns Hopkins University, vol. iv. 1888. 
Marshall, A. Milnes: 'On the Early Stages of Development of the Nerves in Birds.' Journal of Anatomy and Physiology, vol. xi. 1877.

'The Development of the Cranial Nerves in the Chick.' Quarterly Journal of Microscopical Science, xviii. 1878.

'The Morphology of the Vertebrate Olfactory Organ.' Quarterly Journal of Microscopical Science, xix. 1879.

Masius, J.: 'Quelques Notes sur le Développement du Cœur chez le Poulet.' Archives de Biologie, ix. 1889.

Mehnert, E.: 'Untersuchungen über die Entwickelung des Os pelvis der Vögel.' Morphologisches Jahrbuch, xiii. 1887.

Meuron, P. de: 'Sur le Développement de l'CEsophage.' Comptes Rendus, tome 102.1886 .

'Recherches sur le Développoment du Thymus et de la Glande Thyroïde.' Genève, 1886.

Mihálkovics, G. V. von: 'Untersuchungen ïber die Entwicklung des Harnund Geschlechtsapparates der Amnioten.' Internat. Monatsschr. Anat. Histol., Bd. ii. 1885.

Moldenhauer, W.: 'Die Entwicklung des mittleren und des äusseren Ohres.' Morphologisches Jharbuch, iii. 1877.

Onodi, A. D. : 'Ueber die Entwickelung des sympathischen Nervensystems.' Archiv für mikroskopische Anatomie, xxvi. 1885.

Pander, C.: 'Beiträge zur Entwicklungsgeschichte des Hühnchens im Eie.' Würzburg. 1817.

Parker, W. K.: 'On the Structure and Development of the Skull of the Common Fowl.' Philosophical Transactions. 1869.

'On the Structure and Development of the Bird's Skull.' Transactions of the Linnean Society, series 2, Zoology, vol. i. 1875.

'On the Structure and Development of the Wing in the Common Fowl.' Philosophical Transactions, 1888.

'On the Morphology of the Gallinacere.' Transactions of the Linnean Society, series 2, Zoology, vol. v. 1891.

'On the Vertebral Chain of Birds.' Proceedings of the Royal Society, vol. xliii. 1888.

Rathke, H.: 'Abhandlungen zur Bildung und Entwicklungsgeschichte des Menschen und der Thiere.' Leipzig, 1833.

Ravn, E.: 'Ueber die mesodermfreie Stelle in der Keimscheibe des Hühnerembryos.' Archiv für Anatomie und Entwickelungsgeschichte. 1886.

Remak, R.: 'Untersuchungen über die Entwickelung der Wirbelthiere.' Berlin, 1855.

Sedgwick, A.: 'Development of the Kidney in its Relation to the Wolffian Body in the Chick.' Quarterly Journal of Microscopical Science, xx. 1880 .

' On the Development of the Structure known as the Glomerulus of the Head-kidney in the Chick.' Quarterly Journal of Microscopical Science, $\mathrm{xx} .1880$.

' On the Early Development of the Anterior Part of the Wolffian Duct and Body in the Chick, together with some Remarks on the Excretory System of the Vertebrata.' Quarterly Journal of Microscopical Science, xxi. 1881.

Semon, R.: 'Die indifferente Anlage der Keimdrüsen beim Hühnchen und 
ihre Differenzirung zum Hoden.' Jenaische Zeitschrift für Naturwissenschaft, Band xxxi. 1887.

Shore, T. W.: 'Notes on the Origin of the Liver.' Journal of Anatomy and Physiology, vol. xxv. 1891.

Shore, T. W., and Pickering, J. W.: "The Proamnion and Amnion in the Chick.' Journal of Anatomy and Physiology, vol. xxiii. 1889.

Studer, T. : 'Beiträge zur Entwicklungsgeschichte d. Feder.' Zeitschrift für wissenschaftliche Zoologie,' xxx. 1878.

Uskow, N. : ' Die Blutgefässkeime und deren Entwickelung bei einem Hühnerembryo.' Mémoires de l'Académie Impériale des Sciences de St. Pétersbourg, série 7, tome xxxv. 1887.

Vialleton, L. : 'Développement des Aortes chez l'Embryon du Poulet.' Journal de l'Anatomie et de la Physiologie, xxviii. 1892.

Wijhe, J. W. van: 'Ueber Somiten und Nerven im Kopfe von Vögel- und Reptilien-embryonen.' Zoologischer Anzeiger, ix. 1886.

Wolff, C. F.: 'Theoria Generationis.' Halle. 1759.

'De Formatione Intestinorum.' Halle. 1768-69. 


\section{Chapter V.}

\section{'THE DEVELOPMENT OF 'THE RABBI'T.}

\section{PRELIMINARY ACCOUNT.}

The rabbit may conveniently be taken as a typical member of the Mammalia, the highest group of Vertebrates.

Mammals differ notably from the animals dealt with in the previous chapter in being viviparous; that is, in bringing forth their young alive, instead of laying eggs. 'This difference, though at first sight a striking one, is really of but secondary importance; and the actual processes of development of the Mammal are effected in the same way as in the oviparous Vertebrates.

Amphioxus and the frog lay their eggs in water, and the eggs are not even fertilised until they have left the body of the mother. In the Bird the eggs are fertilised as they leave the ovary and enter the oviduct, and the earliest stages of development are effected during the passage of the egg down the oviduct; the egg at the time of laying having already been developing for eighteen hours or more. However, although development, in the case of the bird, commences while the egg is still within the parent, it is only the earliest phases-segmentation of the egg and the establishment of the germinal layers-that are effected in this position; the formation and development of the embryo itself not commencing until after the egg has been laid.

In the Mammal, on the other hand, the ovum is retained within the oviduct and uterus of the mother for a very much longer time; and the whole embryonic development is completed before leaving the parent; the young Mammal at the time of birth being fully formed, and of considerable size, though not yet fit for independent existence.

The rabbit embryo, like that of other animals, is developed from an ovum or egg, which, as in other animals, is a single 
nucleated cell, of epithelial origin. 'This ovum, after being fertilised by a spermatozoon from the male, undergoes cell division or segmentation, which, as in the bird, is effected during the passage of the ovum along the oviduct. On reaching the uterus, which is simply the distal, enlarged end of the oviduct, the ovum halts, becomes fixed to the wall of the uterus, and there remains during all the further stages of development, up to the time of birth.

The actual details of development of the rabbit embryo, the mode of formation of its various organs, and even the proportions of the several parts and the order of their appearance, are essentially the same as in the chick. The most noteworthy point of difference is the exceeding slowness with which the earlier stages are passed through in the rabbit.

The entire period of development of the rabbit, from the discharge of the ovum from the ovary, to the birth of the young animal, occupies thirty days. Of this time the first three days are spent by the egg in passing down the oviduct, and in undergoing segmentation; and it is not until the seventh day that the first trace of the embryo appears; the rabbit's ovum at the end of the seventh day being in a condition closely corresponding to that of the chick about the end of the first day of incubation ( $c f$. Figs. 107 and 143). From this time, development proceeds much more rapidly, at about the same rate as in the chick, a twelve-day rabbit embryo (Fig. 161) corresponding closely in structure, and in actual size, with a five-day chick embryo (Fig. 115).

The eggs of the three animals described in the previous chapters differ very greatly in size. That of Amphioxus has a diameter of only $0.1 \mathrm{~mm}$; the frog's egg measures about $1.75 \mathrm{~mm}$.; and the yolk, or true orum, of the hen's egg about $30 \mathrm{~mm}$. ; i.e. in round numbers the frog's egg is 5,000 times, and the hen's egg 27,000,000 times the bulk of that of Amphioxus. It has been already shown that the dimensions of the egg are chiefly governed by the amount of food-yolk which it contains, and that the size of the young animal on leaving the egg is directly dependent on the amount of this food material ; the tadpole on hatching being far larger than the Amphioxus larva, while the chick on leaving the egg vastly exceeds the tadpole in bulk. 
From the large size of the young rabbit at birth it might be inferred that the rabbit's ova or eggrs were large. This, however, is not the case ; the egg of the rabbit is really very small indeed, and, at the time it leaves the ovary, measures only $0.116 \mathrm{~mm}$. in diameter, i.e. is practically the same size as the egg of Amphioxus, and only a fifteenth the diameter of the frog's egg.

It is clear that this very small egg could not develop into an animal the size of a rabbit at birth unless it received a very plentiful supply of nutriment from without; and inasmuch as the whole increase in bulk takes place while the embryo is within the uterus, it follows that there must be some arrangement in the uterus for supplying it with food.

This is effected by means of a special organ known as the placenta, in which blood-vessels, derived from the embryo and from the mother respectively, lie side by side in very close and extensive contact with one another. The two sets of blood-vessels, foetal and maternal, are distinct, but interchange of fluid and gaseous contents takes place readily, by diffusion through their thin walls, and in this way the blood of the embryo receives nutrient matter from the blood of the mother during the whole period of gestation.

The placenta is formed practically by the allantois, which develops as an outgrowth from the alimentary canal of the embryo, and which at an early period becomes closely attached to the wall of the uterus of the mother. The blood-vessels of the allantois are, as in the chick, directly continuous with those of the embryo; and, by the outgrowth of vascular processes of the allantois into the walls of the uterus, the vessels of the embryo are brought into intimate relation with the large, dilated, and very thin-walled blood-vessels of the uterus itself.

Although the eggs of the rabbit, like those of nearly all other Mammals, are very small indeed, yet in the mode of their development they agree in several respects with the large eggs of the bird, rather than with the smaller ones of the frog, or of Amphioxus. A large yolk-sac, for instance, is formed from the rabbit's ovum, although it contains no food material whatever; the embryo appears in the middle of the blastoderm, and there is a well-marked primitive streak present; while the formation of the amnion and allantois are further points of resemblance with the chick, and of difference from the frog or Amphioxus. 
These and other features in the development of Mammals, which will be described more fully later on in this chapter, are best explained by supposing that existing Mammals are descended from forms in which a greater amount of yolk was present, and in which the eggs were consequently of larger size; and that, in accordance with the Recapitulation Theory, existing Mammals have consequently an inherited tendency to develop after the manner of forms with large eggs. The lowest group of Mammals now living, the Monotremes of the Australian region, afford strong evidence of the truth of this view, as, unlike other Mammals, they are oviparous, laying eggs about the size of olives, and closely resembling the eggs of many Reptiles.

The young rabbit at birth has not yet completed its development. Its eyes are closed, like a kitten's ; and it is quite incapable of obtaining food for itself, being dependent for a time on the supply of milk afforded it by the mammary glands of the mother. Using the word gestation for the whole period of development, from the first appearance of the embryo to the time when the young animal becomes capable of independent existence, two stages may be distinguished in it:-(i) uterine or placental gestation, which comprises the period prior to birth, during which the embryo is nourished by osmotic interchanges between its blood and that of the mother; and (ii) mammary gestation, which embraces the period after birth, during which the young animal is nourished by the milk yielded by the mother.

The relative lengths of these two periods vary greatly in different Mammals. In the Marsupials, a lowly organised group, the uterine gestation is very short, the young animal, as in the kangaroo or opossum, being born at an early stage, of small size, and very imperfectly developed; while in the higher Mammals the period of uterine gestation is a much longer one, and the young animals at birth are of larger size, and more completely formed.

\section{Formation of the Egg.}

THE EGG.

To study the mode of formation of the ova in the rabbit it is necessary, as in the chick and frog, to take, not adult animals nor even new-born young, but embryos at an early stage of development. 
In a rabbit embryo of about the eleventh day, a pair of ridge-like thickenings of the peritoneal epithelium appear, close to the mesentery, and along the inner sides of the Wolffian bodies. These genital ridges (Fig. 165, GR) constitute the earliest stage in the development of the ovaries, and are at first caused merely by an alteration in the shape of the epithelial cells of the peritoneum, which, elsewhere flattened, become here columnar.

The genital ridges soon become more prominent, partly through an increase in the thickness of the epithelium, which becomes two or three cells deep; and partly through the ingrowth into each ridge of an axial core of connective tissue.

'The genital ridge (Fig. 165) lies very close to the Wolffian body ; and, about the fourteenth day, solid columns of cells grow out from the Malpighian bodies of the anterior end of the Wolffian body into the ridge. These columns of cells form what is called the tubuliferous tissue of the ovary; in the early stages they occupy almost the whole of the axial part of the genital ridge, but as development proceeds they gradually become less conspicuous, and withdraw more or less completely from the ridge. They have nothing whatever to do with the formation of the ova.

In a rabbit embryo of the eighteenth day the genital ridges have grown considerably, and project into the body cavity as a pair of longitudinal folds, close to the attachment of the mesentery to the dorsal body wall. Each ridge is covered by the germinal epithelium, which consists of two or three layers of cells, the outermost of which are columnar in shape, while the more deeply placed ones are more or less spherical. The central part, or core, of the genital ridge is made up almost entirely of the tubuliferous tissue, with numerous blood-ressels and a small amount of connective tissue.

By the twenty-second day the germinal epithelium has increased considerably in thickness; and among the cells of which it consists are some of larger size than the rest, these larger cells being the primitive ova. By the twenty-eighth day, i.e. about two days before birth, the genital ridges are still larger; the germinal epithelium covering their surface is much thicker than before, and its deeper layers are honeycombed, or broken up into irregular columns, by ingrowth of the vascular connective tissue from below. 
A thin layer of connective tissue, the tunica albuginea, extends parallel to the surface of the ovary, and divides the germinal epitheliuin into two almost completely separate layers; a thin outer layer of columnar cells, investing the outer surface of the ovary; and a much thicker, deeper layer of spherical cells, broken up into nests by the connective tissue partitions. In the germinal epithelium, and more especially in its outer layer, the primitive ova are conspicuous, as individual epithelial cells, much larger than their neighbours, and usually spherical or polygonal in shape, with large granular nuclei. The tubuliferous tissue is still present along the axis of the ovary, but now occupies a relatively much smalier space than before.

The permanent ova. Four or five days after birth of the young rabbit, the germinal epithelium undergoes further changes, marking the establishment of sexuality, and the conversion of the genital ridge of the embryo into the definite ovary.

The changes consist essentially in the formation of permanent ova from the primitive ova, and occur in the rabbit in much the same way as in the chick or tadpole. The nucleus, or germinal vesicle, increases in size and becomes vesicular, acquiring a very distinct nuclear membrane; the nuclear contents collect to one spot, where they form a granular mass, from which, by branching, a definite reticulum is established; and, finally, one or more of the nodal points of the reticulum enlarge, to form the nucleoli or germinal spots.

The other cells of the germinal epithelium have small nuclei, and soon arrange themselves in a more or less definite manner, so as to form follicles surrounding the permanent ova (Fig. $133, \mathrm{GA})$.

At first (Fig. 133, oz), each nest of epithelial cells may contain several permanent ova, but as development proceeds, and as the follicle cells become more definitely arranged around the ova, the nests are broken up by further ingrowth of the vascular connective tissue, and the separate follicles isolated from one another.

Although it appears to be the rule in the rabbit's ovary that each primitive ovum should become a permanent ovum, yet this is by no means always the case. Sometimes two or more primitive 
ova fuse together to form a bi-nuclear or poly-nuclear mass, in which all the nuclei but one may disappear, the fused mass with the remaining nucleus becoming a permanent ovum; while in other cases it is stated that after a follicle has been formed round the fused mass, the entire mass, with the follicle, may divide into two or more permanent ova.

The development of the permanent ova proceeds from the surface of the ovary towards its deeper parts. In young

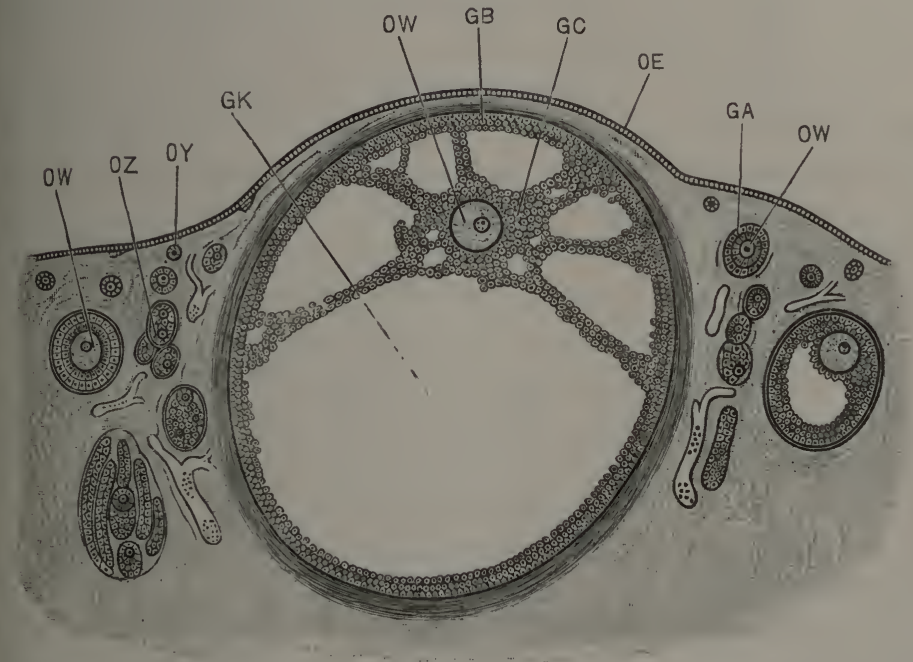

FIG. 133.- Section through part of the ovary of an adult Rabbit. The section is taken vertical to the surface of the ovary, and shows one fully formed Graafian follicle, and others in various stages of development. $\times 50$.

GA, follicle cells surrounding an ovum. GB, outer layer of Graafian follicle, or 'tunica granulosa.' GC, inner layer of Graafian follicle, or 'discus proligerus.' GK, cavity of Graafian follicle. OE, outer layer of columnar epitlielial cells, investing the ovary. OW, ovum. OY, primitive ovim. OZ, nests of epithelial cells derived fronı the deeper layers of the genital epithelinm.

rabbits, about a week after birth, the surface epithelium of the ovary contains numerous primitive ova in process of formation ; deeper down, beneath the tunica albuginea, are nests of epithelial cells in which are permanent ova in the early stages of their formation, surrounded by imperfectly formed follicles; while still lower, in the deepest parts of the germinal epithelium, the nests are in many places broken up into isolated follicles, each containing a large and well-formed permanent ovum. 


\section{The Graafian Follicles.}

Each follicle consists, at first, of a single layer of cells surrounding an ovum, the cells being derived directly from the germinal epithelium, and being therefore morphologically comparable with the ova themselves. Very shortly, each follicle becomes two-layered (Fig. 133, GA), the second layer appearing within the first one, between this and the ovum. As to the origin of this second layer of follicular cells opinions differ ; it is generally held to be formed by division of the cells of the originally single layer; but some investigators maintain that it is derived from the ovum itself.

Immediately arouid the ovum, between it and the inner layer of follicle cells, a thick non-cellular layer, with faint radial striations, the zona radiata, is formed, apparently as a cuticular secretion from either the ovum or the follicle cells. Yolkgranules, elaborated by the follicle cells, accumulate within the ovum, which consequently increases in size.

The follicle cells increase rapidly by cell-division, so that the follicle soon becomes several cells thick. The outer layer of cells now grows much more rapidly than the inner layer, so that a space, somewhat crescentic in section, and filled with fluid. appears between the two layers (Fig. 133).

By further growth of the two layers, the fully-formed Graafian follicle (Fig. 133, GK) is established. This consists of, (i) an outer layer of follicular cells, GB, arranged three or four cells deep, and invested by the vascular connective tissue of the ovary; and (ii) an inner layer of similar cells, GC, which closely invests the ovum, ow. This inner layer is attached to the inner' surface of the follicle, with which it is also connected by irregular radiating strands of follicular cells, well shown in Fig. 133. The cavity of the Graafian follicle is filled with fluid.

The riper egg-follicles lie at first, as noticed above, in the deeper parts of the ovary; but as the Graafian follicles enlarge they gradually extend nearer and nearer to the surface, and when fully formed cause rounded projections on the surface of the ovary (Fig. 133). This enlargement of the Graafian follicles, with accompanying ripening of the ova, occurs successively in different parts of the ovary, so that there are never more than a limited number, half a dozen or so, of ripe Graafian follicles at any one time in a given ovary. 
Having reached its full size, the Graafian follicle ruptures at its most prominent part, and the ovum is clischarged on the surface of the ovary, from which it is normally taken up at once by the fimbriated mouth of the oviduct. After discharge of the orum, the walls of the Graafian follicle undergo a series of curious changes, which will be more fully described in the chapter dealing with the human embryo, and which result in the formation of the corpus luteum, a body which disappears early if the ovum is not fertilised; but which, if the ovum is fertilised and develops into an embryo, persists in the ovary during the whole period of development, and is even recognisable at the time of birth of the young rabbit.

Of the two layers of the Graafian follicle, the outer (Fig. 133, $(\mathrm{BB})$ is sometimes spoken of as the tunica granulosa; and the inner, GC, as the discus proligerus ; these names, however, and especially the latter one, are badly chosen, and it will be well if they drop out of use altogether.

The ovum, surrounded by the inner layer of the Graafian follicle, may be attached to any part of the outer layer of the follicle: it not uncommonly lies at the side nearest the surface of the ovary, but it may occur at the opposite or deepest part of the follicle, or at any other part of its inner surface.

The meaning of the Graafian follicle has been much debated: the most probable explanation seems to be that it is in some way associated with the great diminution in size which there is strong reason for thinking that the ovum has undergone during the evolution of the existing types of Mammals.

\section{Maturation of the Egg.}

The nucleus of the ovum is at first centrally placed : but some time before the Graafian follicle reaches its full development, the nucleus moves towards the surface of the ovum. The exact changes that then occur have not been determined with certainty in the case of the rabbit: so far as they are known, they agree closely with those already described in the case of the frog.

A thin, homogeneous vitelline membrane is formed within the zona radiata, and apparently from the egg itself : the nucleus of the egg becomes inconspicuous; the yolk retracts slightly from the vitelline membrane, and the first polar body is extruded from the egg. 
At this stage the egg is liberated; by rupture of the Graafian follicle, and is taken up by the mouth of the oviduct. It is invested by the thin vitelline membrane, outside which is the much thicker zona radiata. More or fewer of the cells of the

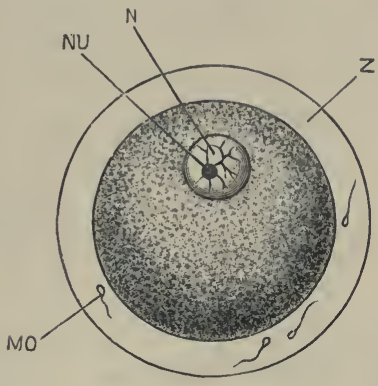

FIG. 134.

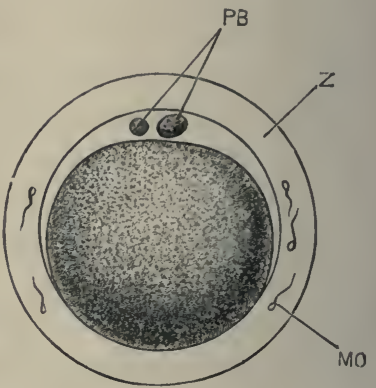

FIG. 135.

FiG. 134. - A fully formed ovum of a Rabbit, shortly before its discharge from the ovary. (After Bischoff.) $\times 200$.

FIG. 135.-A Rabbit's ovum, from the upper end of the oviduct, after extrusion of the two polar bodies. (After Bischoff.) $\times 200$.

MO, spermatozoon. N, nucleus or germinal vesicle. NU, nucleolus or germinal spot. PB, polar bodies. Z, zona radiata.

inner layer of the follicle usually remain adhering to the zona radiata.

After entering the oviduct, but before fertilisation is effected, a second polar body, apparently not more than half the size of the first one, is extruded from the egg (Fig. 135, PB).

\section{Ovulation.}

Throughout the warmer part of the year, there is a periodically recurring ripening and discharge of ova from the ovaries of the doe rabbit. From April to July this periodic discharge, which is spoken of as ovulation, occurs regularly, and at monthly intervals : after July it usually takes place with less regularity.

The total period occupied in the development of the young rabbit, from fertilisation of the egg to the time of birth, is thirty days: that is to say, the total period of development is in the rabbit of the same length as the interval between two successive acts of ovulation.

The ovary of the doe rabbit, at the time she gives birth to young, usually contains fully formed Graafian follicles, with ripe ova ready for discharge. As a rule the doe is impregnated by 
the buck immediately after giving birth to young; and at a period, estimated by different observers at from eight to twelve houl's after impregnation, the ova are liberated from the ripe follicles.

At each period of ovulation, from three to nine ova are as a rule discharged from each ovary; the several ova being set free, not absolutely at the same moment, but within a very short time of one another.

Although ovulation, or the discharge of ova from the ovaries, usually occurs a few hours after impregnation, and is probably stimulated by this, it should be regarded as an essentially independent act, a point of view that will be more fully considered in the next chapter.

\section{J. Fertilisation.}

Fertilisation appears to occur in the rabbit, as a rule, from eight to twelve hours after copulation; the interval being due, not to the time taken by the spermatozoa to travel up the uterus and oviduct, for this is effected, according to Hensen, in from a quarter of an hour to two hours; but to the fact that the discharge of ova from the ovary does not take place until eight to twelve hours after copulation.

The act of fertilisation is effected, as a rule, directly after the eggs enter the oviduct: and, in eggs taken from the upper part of the oviduct, spermatozon may be seen in considerable numbers imbedded in the zona radiata, or lying in the space between the vitelline membrane and the egg, formed by the shrinking of the latter.

The details of the process of fertilisation have not been accurately determined in the rabbit. Fusion of a spermatozoon with the female pronucleus has been seen by Van Beneden; and there is no reason for supposing the process to differ from what is known to occur in other animals.

\section{THE EARLY STAGES OF DEVELOPMENT.}

It will be convenient to deal, in the present section, with the changes undergone by the egg up to the end of the seventh day. During the first three days the egg is travelling dorwn the oviduct, and passing through the stages of segmentation; at the end 
of the third day it passes into the uterus, and undergoes further changes, consisting chiefly in the establishment of the germinal layers, and in preparations for the attachment of the ovum to the uterus. Up to the end of the seventh day the ovum lies freely in the uterus, and there is no trace of the embryo, which does not commence to form until the early part of the eighth day.

In estimating the age of rabbit ova, or embryos, it is customary to date from the time of copulation, which can always be determined with precision ; and this method of computation will be adopted here. As the eggs are not discharged from the ovary, or fertilised, until from eight to twelve hours after this event, the actual time during which developmental changes have been proceeding will be less than the stated periods by this amount.

\section{Segmentation of the Egg.}

Segmentation is effected while the egg is travelling down the oviduct towards the uterus. During its passage it becomes surrounded by a thick layer of albumen, formed of concentric layers secreted by the walls of the oviduct. The egg itself, on entering the uterus at the close of segmentation, is practically the same size as the unfertilised egg, in - reality slightly smaller than this; but owing to the layer of albumen, which may be thicker than the diameter of the egg itself, it appears on a superficial examination to have increased considerably in size.

Segmentation commences, according to Van Beneden, some ten or twelve hours after fertilisation is effected, i.e. from eighteen to twenty-four hours after copulation, and is continued during the next two days. About the seventieth hour, or the end of the third day from the time of copulation, segmentation is completed, and the ovum enters the uterus.

In segmentation, the first cleft (Fig. 136) divides the egg into two ovoid cells, which are nearly, but according to Van Beneden not absolutely, equal in size.

After a pause of about four hours, each of these cells again divides into two, giving in all four cells, which from the first are approximately spherical in shape. Each of these four then divides, giving eight in all, of which the four derived from the smaller of the first two cells are slightly smaller than the other four. 
'Lhe larger cells now become grouped together in the centre, while the smaller cells form a cap lying on these, and partially inclosing them. In the later stages, the smaller outer cells divide rather more rapidly than the larger cells, and inclose these more completely; and at the close of segmentation, about the seventieth hour', when the ovum passes from the oviduct into the uterus, it

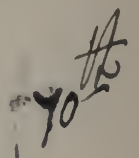
consists of a central solid mass of rather larger and more granular

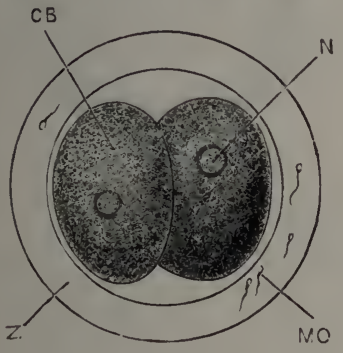

FIG. 136.

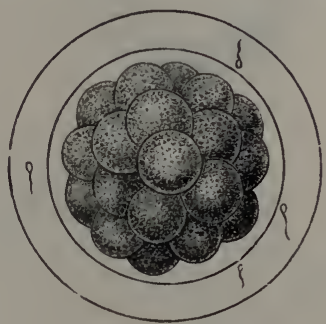

FIG. 137.

FIf: 136.-A Rabbit's Ovum from the middle of the length of the oviduct, about twenty-two hours after copulation, showing division of the ovum into two cells. (After Bischoff.) $\times 200$.

CB, blastomere or segmentation cell. MO, spermatozoon imbedted in the zona ralliatil. $\mathbf{N}$, nucleus. $\mathbf{Z}$, zona radiata.

FIG. 137.-A Rabbit's Ovum from the lower end of the oviduct, about the midclle of the third day; showing the morula stage, shortly before the completion of segmentation. (After Bischoff.) $\times 200$.

cells (Fig. 138, CD), almost completely surrounded by a layer of rather smaller and more transparent cells, slightly flattened at their outer ends (Fig. 138, cc); the larger cells being visible on the surface at one spot only.

In the size of the eggs there is a close agreement between the rabbit and Amphioxus ; the rabbit's ovum measuring on an average $0.116 \mathrm{~mm}$. in diameter, and that of Amphioxus measuring $0.104 \mathrm{~mm}$. The two eggs agree also in undergoing complete or holoblastic segmentation, and in the blastomeres, or cells formed by segmentation, differing very little from one another in size.

The comparison, however, must not be pushed too far. The actual arrangement of the cells is entirely different in the two cases : the rabbit's ovum does not pass through a gastrula stage (cf. Figs. 15 and 16); and there is no stage in the development of Amphioxus similar to that represented for the rabbit in Fig. 138. 
In the spreading of the smaller cells over the larger ones, the rabbit and the frog appear to agree; but the details of the process

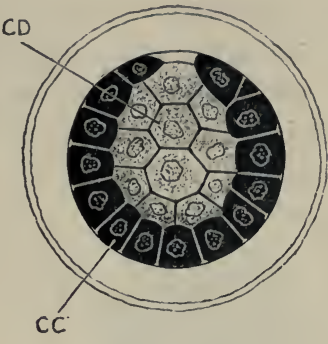

Fig. 138 .

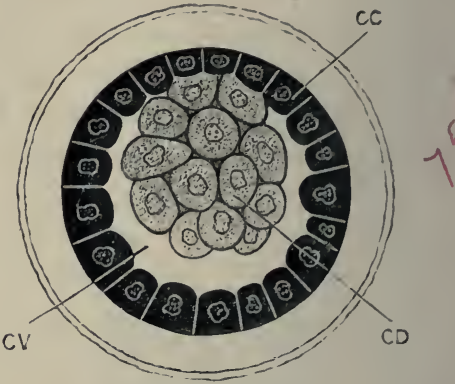

Fig. 139.

FIG. 138.-A Rabbit's Orum seventy hours after copulation, taken from the lower end of the oviduct just before entering the uterus, and showing the condition at the close of segmentation. (After Van Beneden.) $\times 200$.

FIg. 139.-A Rabbit's Ovum seventy-five hours after copulation, taken from the uterus, and showing the first stage in the formation of the blastodermic vesicle. (After Van Beneden.) $\times 200$.

CC, outer layer of cells. CD, inner mass of cells. CV, cavity of blastodermic vesicle.

have not been accurately determined in the rabbit's ovum, and it is doubtful how far the correspondence is a real one.

\section{The Blastodermic Vesicle.}

On entering the uterus, at the end of the third day, the ovum has the structure shown in Fig. 138 and described above. It is spherical in shape, with a diameter averaging $0.09 \mathrm{~mm}$., i.e. is slightly smaller than the unfertilised egg. It is surrounded by the vitelline membrane and zona radiata as before; and outside the latter are the concentric layers of albumen, which are deposited round the egg during its passage along the oviduct, and which have a total thickness of about $0 \cdot 1 \mathrm{~mm}$.

From three to nine ova are usually discharged from the ovary at each period of ovulation. These enter the uterus almost simultaneously, and at first lie close together at its proximal end. As development proceeds they gradually become spread out along the uterus, at approximately equal intervals; each ovum lying in a special dilatation of the uterus, to the wall of which it becomes attached during the eighth day.

Very shortly after the egg enters the uterus, and in some cases before it leaves the oviduct, the smaller outer cells grow 
completely round the larger inner cells, which from this time they surround on all sides.

The outer layer of cells now begins to grow rapidly; the central or inner cells remaining attached to the outer layer at one spot, but becoming separated from it at all other parts. By about the seventy-fifth hour, i.e. four or five hours after entering the uterus, the ovum has acquired the structure shown in Fig. 139 : the outer layer of cells, CC, forms a hollow ball, about $0.12 \mathrm{~mm}$. in cliameter, to the inner surface of which the mass of inner cells,

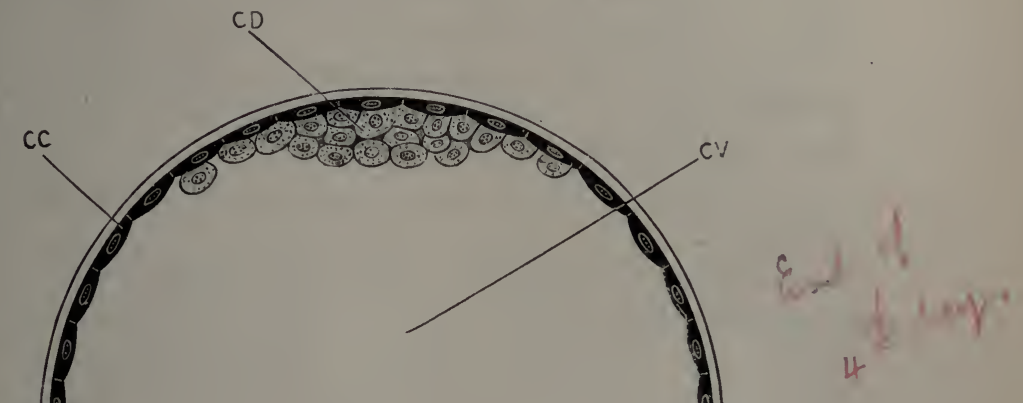

FIG. 140.-Section of the blastodermic vessel of a Rabbit at the end of the fourth day. (After Van Beneden.) $\times 250$.

CC, outer layer of cells. CD, imner lenticular mass of cells. CV, cavity of the blastodermic vesicle.

$\mathrm{CD}$, is attached at one spot, the rest of the cavity of the ball, $\mathrm{CV}$, between the outer and inner cells, being filled with fluid.

The growth of the ball, or blastodermic vesicle, as it is now termed, proceeds rapidly; and by the end of the fourth day, i.e. about twenty-four hours after entering the uterus, the structure and proportions are as represented in Fig. 140. The vesicle is still spherical, measuring on an average about $0.28 \mathrm{~mm}$. in diameter. It consists of an outer wall of flattened polygonal 
cells, Cc, formed from the smaller, outer cells of the previous stages, to the inner surface of which is attached at one pole the mass of inner cells, CD. 'This mass of inner cells is now flattened out into a lenticular shape; thicker and more compact in the middle, where the cells are two or three deep, and polygonal from mutual pressure; and thinning towards its margins, where the cells are in a single layer, less closely apposed to one another, and irregular or even amœboid in form. The vitelline membrane is no longer recognisable: the zona radiata is still present, but, like the outer albuminous investment, is greatly reduced in thickness.

During the fifth and sixth days, the blastodermic vesicle remains spherical or nearly so in shape, and continues to increase rapidly in size. By the end of the fifth day it measures about $1.5 \mathrm{~mm}$. in diameter; and by the end of the sixth day, 3 to $3.5 \mathrm{~mm}$.

On the seventh day it becomes ellipsoidal in shape, and by the end of this day (Fig. 143) it measures from 4.5 to $5 \mathrm{~mm}$. in length by 3.5 to $4 \mathrm{~mm}$. in width. Up to the end of the seventh day the several blastodermic vesicles lie quite freely within the uterus, but become gradually spaced out along this, and take up the positions they will retain during the remainder of their development.

The measurements given above must be regarded as approximate only; the several blastodermic vesicles in the same uterus vary within certain limits, those lowest down being the largest and most advanced in development; and one or two at the proximal end of the uterus, nearest to the oviduct, being almost invariably smaller and less developed than the others.

\section{The Germinal Layers.}

During the fifth, sixth, and seventh days, important changes occur in the structure of the wall of the blastodermic vesicle, leading to the establishment of the three germinal layers, epiblast, hypoblast, and mesoblast, from which the several parts of the embryo are formed.

These changes more especially concern the part of the wall of the vesicle to which the lenticular mass of inner cells (Fig. $140, \mathrm{CD})$ is attached; and to this part, which at the end of the fourth day is the only portion in which the wall of the vesicle is 
more than one cell thick, the name embryonal area may be given, as it is from the central portion of this that the embryo is developerl.

The mode of formation of the germinal layers in the rabbit has been very differently described by different observers, and there are several points, even of primary importance, that are as yet imperfectly understood. The following description is based on the independent observations of Rauber and of Kölliker, which appear to be the most exact; but the account, though consistent in itself, makes it very difficult to establish any comparison between the mode of formation of the germinal layers in the rabbit and that occurring in other Vertebrates, or even in other Mammals; and it seems not at all improbable-that further investigation may necessitate considerable modification in the interpretation to be put upon the appearances described.

The great length of time that is occupied in the process, as compared with the chick or frog, for example, is remarkable, and may perhaps be taken as an indication that the actual mode of development is a much modified one.

The fourth day. At the end of the fourth day, as already described, the wall of the blastodermic vesicle is one cell thick, except in the embryonal area, where cells of two kinds are present (Fig. 140).

The fifth day. During the fifth day, the cells of the outer layer become thinner and larger; they also increase in number, by division, as the blastodermic vesicle grows larger. In the embryonal area the cells of the outer layer have the same character's as in other parts of the vesicle.

The granular cells forming the inner layer of the embryonal area, on the other hand, undergo important changes. They increase in number by repeated division; they become smaller in size; and they extend further round the interior of the vesicle. But the most important change is that they become arranged in two layers:-(i) an upper layer of cells with large nuclei, rather wider than they are long, and closely fitted together at their edges so that the outlines of the cells are difficult to determine; (ii) a lower layer of very thin flat pavement cells, similar to those of the outer layer of the vesicle, but 
slightly smaller; this lower layer extends at its margin some distance beyond the edge of the upper or thicker layer.

Three regions may, therefore, be distinguished in the wall of the blastodermic vesicle of the rabbit on the fifth day.

(i) The embryonal area is a circular patch about $0.48 \mathrm{~mm}$. in diameter, in which three layers of cells are present (Fig. 14.1); an upper layer, Cc, of thin pavement cells; a middle layer, E, of much larger, almost cubical cells; and a lower layer, $\mathrm{H}$, of thin pavement cells, very similar to those of the upper layer. Each of these three layers is one cell thick; and the threelayered condition is brought about by the splitting of the inner mass of cells of the fourth day (Fig. 140, CD) into two, which become respectively the middle and lower layers of the fifth day.

(ii) Surrounding the embryonal area is a border, varying in width in different specimens, in which the wall of the vesicle consists of two layers, as seen at the margin of Fig. 141. These two layers coirespond to the upper and lower layers of the three present in the embryonal area; and the two-layered condition is brought about by the lower or innermost layer, $\mathrm{H}$, extending beyond the margin of the embryonal area.

(iii) All the rest of the blastodermic vesicle, at this stage about four-fifths of the whole periphery, consists of a single layer of cells, the outermost layer, Cc, of the embryonal area (cf. Fig. 140).

With regard to the ultimate fate of these layers, it may be mentioned at once that in the embryonal area, according to Rauber and Kölliker, the uppermost layer of cells, CC, often spoken of as Rauber's layer, disappears altogether; the middle layer, $\mathrm{E}$, becomes the epiblast; and the lower layer, $\mathrm{H}$, becomes the hypoblast; so that, according to these observers, both epiblast and hypoblast are formed from the inner mass of cells of the fourth day.

This interpretation involves very considerable difficulties, and will not improbably require revision. The disappearance of Rauber's layer from the embryonal area, and its persistence as the outer wall of the vesicle in other parts, together with the derivation of both epiblast and hypoblast from the original inner layer of cells, are difficult to reconcile with the course of development in other Mammals; and further investigation is much needed on these points. 
The sixth day. By the end of the sixth day, the blastodermic vesicle has a diameter of 3 to $3.5 \mathrm{~mm}$., and the embryonal area, which is still approximately circular in outline, measures 0.75 mm. across.

In the embryonal area the upper layer of cells, or Rauber's layer, is thinner than before, and very difficult to recognise in
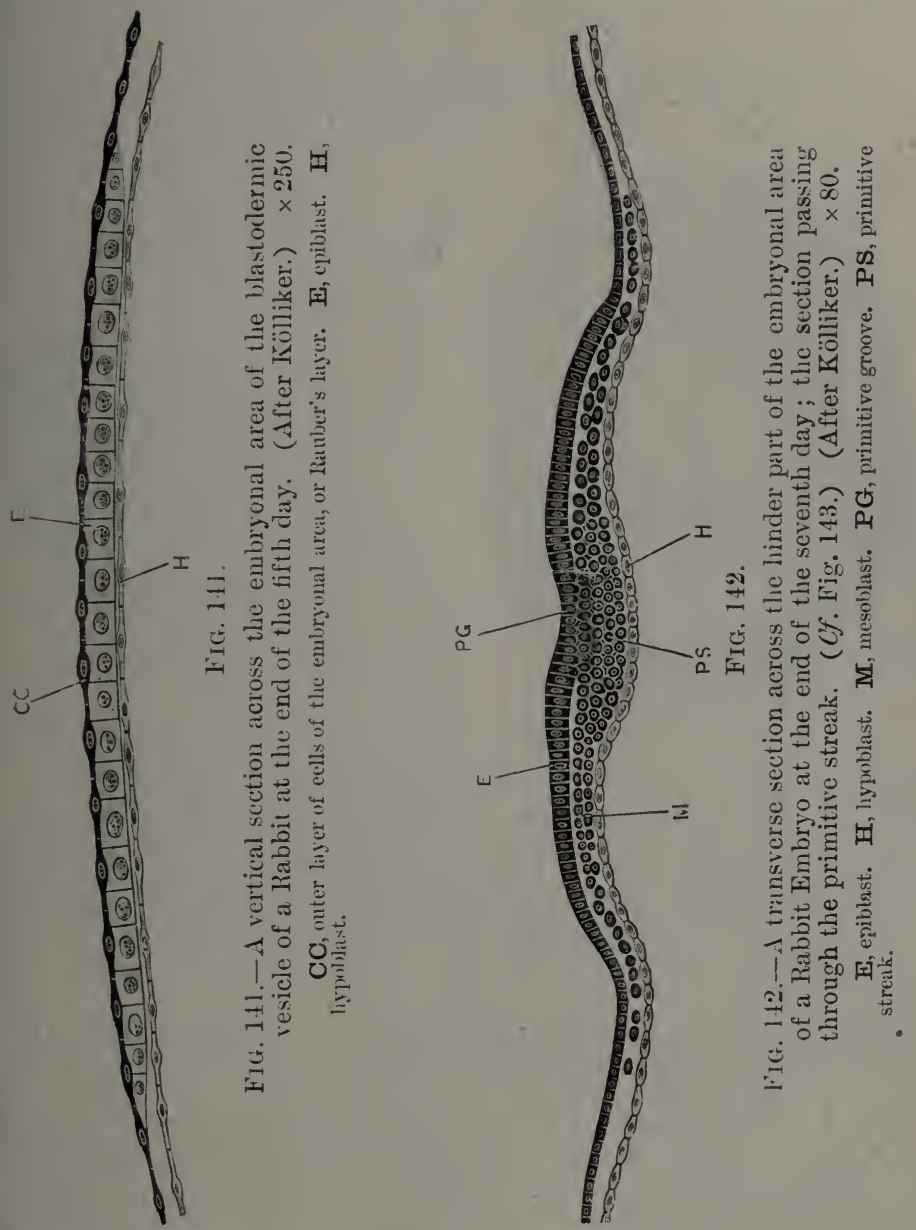

sections. 'The middle layer' of cells, or epiblast ( $c f$. lig. 1.1, E), is rather thicker than before, owing to a change in the shape of the indiviclual cells, which are now columnar in place of being cubical. The lower layer, or hypoblast, consists, as before, of a 
single layer of flattened pavement cells, thickened in their centres by the nuclei, but very thin at their margins.

Berond the embryonal area, the lower layer, or hypoblast, has extender further round the vesicle than before, so that it now lines about a third of the entire vesicle; the wall of the remaining two-thirds still consists of a single layer of flattened cells. continuous with those forming Rauber's layer.

The seventh day. During the serenth day, and often before the close of the sixth, the blastodermic vesicle loses its spherical

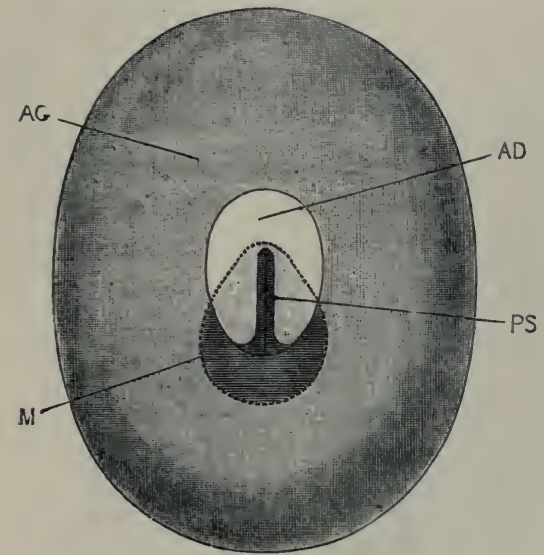

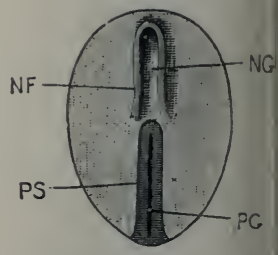

F16. 144 .

Fig. 143.

FIG. 143.-The blastodermic vesicle of a Rabbit at the end of the seventh day, seen from above. (Modified from Kölliker.) $\times 12$.

AD. embryonal area. A G, wall of blastodermic vesicle. $\mathbf{M}$, dutterl line inlienting the boundary of the mesoblast. PS, primitive streak.

Fig. 144. - The embryonal area of a Rabbit at the middle of the eighth day. (Modified from Kölliker.) $\times 12$.

NF, neural foll. NG, neural groove. PG, primitive groove. PS, primitive

shape, and becomes ellipsoidal (Fig. 143). The average dimensions of the entire resicle at the end of the seventh day are from $4 \cdot \check{\jmath}$ to $5 \mathrm{~mm}$. in length, by $3 \cdot \tilde{5}$ to $4 \mathrm{~mm}$. in width; but individual specimens may considerably exceed these limits.

The embryonal area (Fig. 143, AD) is now distinctly pyriform in outline, measuring on an average $1.5 \mathrm{~mm}$. in length, by $1 \mathrm{~mm}$. in width. Its longer diameter corresponds to the axis of the blastodermic resicle, and, as a rule, to that of the uterus as well. From their relations to the embryo at a later stage, 
the hroader end of the embryonal area mas be called the anterior end, and the narrower one the posterior end.

As regards the structure of the embryonal area, Rauber's layer has disappeared almost completely; a few individual cells may still be recognised here and there, but there is no longer a continuous stratum of cells. In consequence of the disappearance of Rauber's layer (cf. Fig. 141), the embryonal area now consists of only two layers of cells:-(i) the epiblast, or former middle layer. which now becomes the superficial layer, consists, as before, of a single layer of short columnar cells; it thins towards the margin of the embryonal area, and at its margin is said to become continuous with the outer layer of cells, or epiblast cells of the rest of the blastodermic resicle: (ii) the hypoblast, in the embryonal area, has the same characters as before: beyond the embryonal area, it has now extended about half way round the inner surface of the blastodermic resicle.

'The blastodermic resicle at the end of the seventl day is, therefore, an ellipsoidal sac filled with fluid. Its wall consists, in the upper half of the vesicle, of two layers of cells, epiblast and hypoblast: in the lower half, of a single layer, the epiblast alone. In the middle of the upper half of the resicle is the embryonal area, which is also two-layered, but in which the epiblast differs from that of the rest of the resicle in consisting of columnar instead of parement cells.

\section{The Primitive Streak and the Mesoblast.}

Towards the close of the serenth day, the primitive streak appears. This structure, which in the mode of its formation, and in its relations to other parts, agrees closely with that of the chick. is at first an axial thickening of the epiblast at the posterior; or narrower, end of the embryonal area. It rapidly lengthens, and by the end of the seventh day (Fig. 143, PS) it extends, as a linear opacity, along about two-thirds of the length of the area, having a faint longitudinal groore, the primitice groove, along its dorsal surface.

Transrerse sections at this stage (Fig. 142, Ps) show that the primitive streak is formed by proliferation of cells from the uncler surface of the epiblast, in the median plane.

The mesoblast. The cells of the primitive streak spread out, beyond the margins of the thickened streak itself, as two 
thin lateral sheets of cells (Fig. 142, i), which lie between the epiblast and hypoblast, and which give rise to the middle germinal layer or mesoblast. In the primitive streak itself the cells are spherical and closely compacted; but in the lateral mesoblast sheets the cells are more loosely arranged, and are stellate in shape.

The layer of mesoblast spreads rapidly, both laterally and posteriorly; at the end of the seventh day, its limits are indicated by the shaded area bounded by the dotted line, M, in Fig. 143, a figure that may with advantage be compared with Fig. 107, which shows the corresponding stage in the development of the chick.

While it is certain that the mesoblast in the posterior part of the embryonal area of the rabbit, i.e. in the region of the primitive streak, arises in the manner just described, by proliferation of cells of epiblastic origin, it is by no means clear that the whole of the mesoblast is formed in this way; and, although further observations are wanted on the point, it seems probable that in front of the primitive streak, in the part of the embryonal area in which the embryo will appear, the mesoblast arises, as in the chick, by budding off of cells fiom the hypoblast.

\section{GENERAL HISTORY OF THE DEVELOPMENT OF THE RABBIT EMBRYO.}

In the preceding section the development of the rabbit's ovum has been followed up to the end of the serenth day, that is, up to a point corresponding to that reached by a hen's egg about the sixteenth hour of incubation. At this stage all three germinal layers, epiblast, mesoblast, and hypoblast, are established; a primitive streak and primitive groove are present ; but there is as yet no trace of the embryo itself.

It will be convenient to give, in the present section, a brief summary of the mode of formation, and of the general course of development of the embryo, before considering in detail the history of the several systems and organs.

\section{The Formation of the Embryo.}

The embryonal area of the blastodermic vesicle of the rabbit at the end of the seventh day (Fig. 143, AD) corresponds very 
closely, as just noticed, with the area pellucida of a hen's egg about the sixteenth hour of incubation.

The formation of the rabbit embryo is also effected in very similar fashion to the chick. The embryonal area increases in size, especially by growth at its anterior end. Immediately in front of the primitive streak a neural groove (Fig. 14t, NG) is

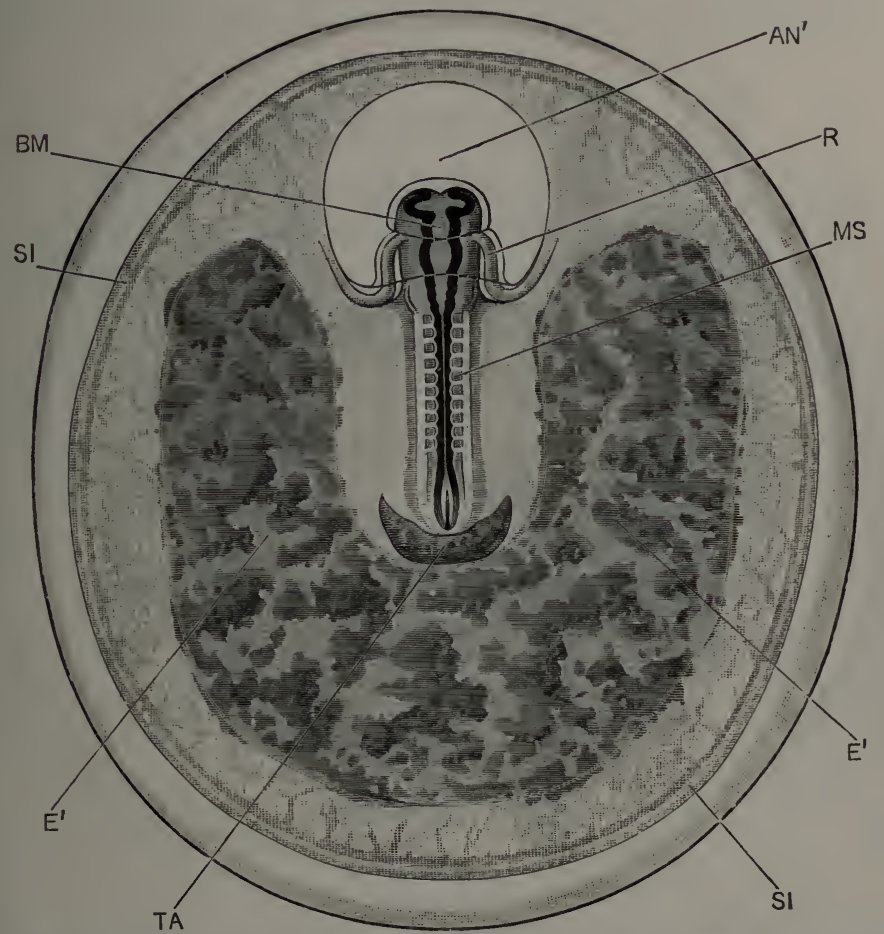

FIG. 145.-A Rabbit Embryo at the end of the ninth day. The entire blastodermic vesicle is represented, with the embryo in situ, as seen from the dorsal surface. (Cf. Fig. 146, which represents an embryo of the same age in sagittal section.) $\times 10$.

A.N', proamnion. BM, mid-urain. E', horse-shoe shaped pateh of thiekenerlepiblast, hy which the blastodermie vesicle is attached to the wall of the uterus (cf. Fig. 169). MS , mesoblastic somite or protovertebra. $R$, right half of heart. SI, sinus terminalis. TA, allantois.

formed, bordered by neural folds, NF, which speedily unite, conrerting the groove into a tube. This tube becomes the central nervous system, and in its anterior or cerebral part the sereral brain vesicles are early established (Fig. 115).

By means of head, tail, and side folds the embryo is con- 
stricted off from the rest of the blastodermic vesicle, in a manner practically identical with that in which the embryo chick is constricted off from the yolk-sac ( $c f$. Figs. 145, 146, and 110, 112).

By the end of the ninth day the rabbit embryo (Figs. 14.5, 146) has acquired shape, structure, and proportions agreeing very closely with those of a chick embryo of about the twenty-sixth hour, with which it also corresponds almost exactly in size.

Up to this stage the embryo has been practically straight

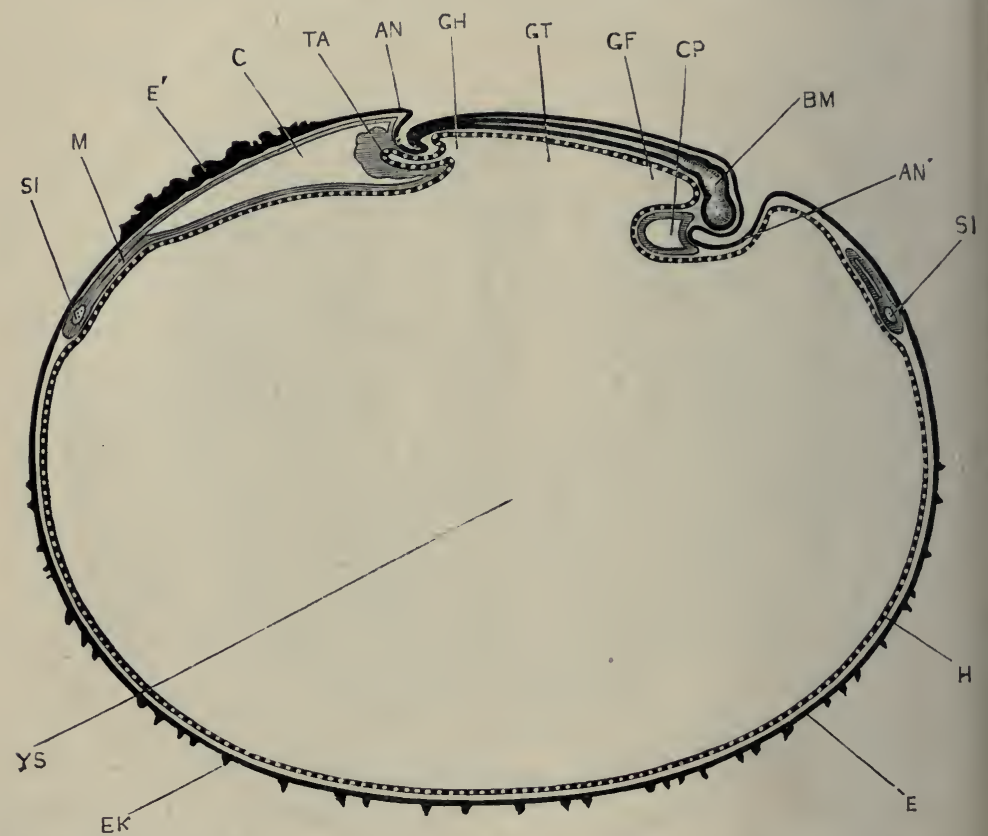

FIG. 146. - A median longitudinal, or sagittal, section through a Rabbit Embryo and blastodermic vesicle at the end of the ninth day. ( $C f$. Fig. 145.) (In part after Van Beneden and Julin.) $\times 10$.

AN, tail foll of amnion. AN', proamnion. BM, mirl-brain. C, extra-embryonie part of the colom or boly-cavity. $\mathbf{C P}$, periearlial cavity. $\mathbf{E}$, epiblast. $\mathbf{E}$ ', thiekened epilblast by which the blastodennie vesiele is attaehed to the uterus (cf. Fig. 169). EK, epiblastic villi. GF, fore-gut. $\mathbf{G H}$, hini-grit. $\mathbf{G T}$, mid-gut. $\mathbf{H}$, hyjoblast. $\mathbf{M}$, mesolulast. SI, sinus terminalis. TA, alluntois. YS, eavity of yolk-sac, or blastodermie vesicle.

(Fig. 146), lying with its dorsal surface upwards, towards the wall of the nterus, and its ventral surface downwards towards the yolk-sac. From this time, however, the dorsal surface of the embryo grows more rapidly than the ventral surface, and the whole embryo in consequence becomes strongly flexed. By the end of the tenth day (Fig. 147), while the midalle portion of the 
body, to which the yolk-stalk is attached, remains in the same position as before, the head and neck, which have greatly increased in size, are bent downwards at right angles to the trunk, and, pushing down the wall of the yolk-sac before them, appear to project into this latter: the head and neck are, however, really separated from the cavity of the yolk-sac, as shown in Fig. 147, by the wall of the sac itself. The hinder or tail end of the embryo, the basal part of which is alone shown in

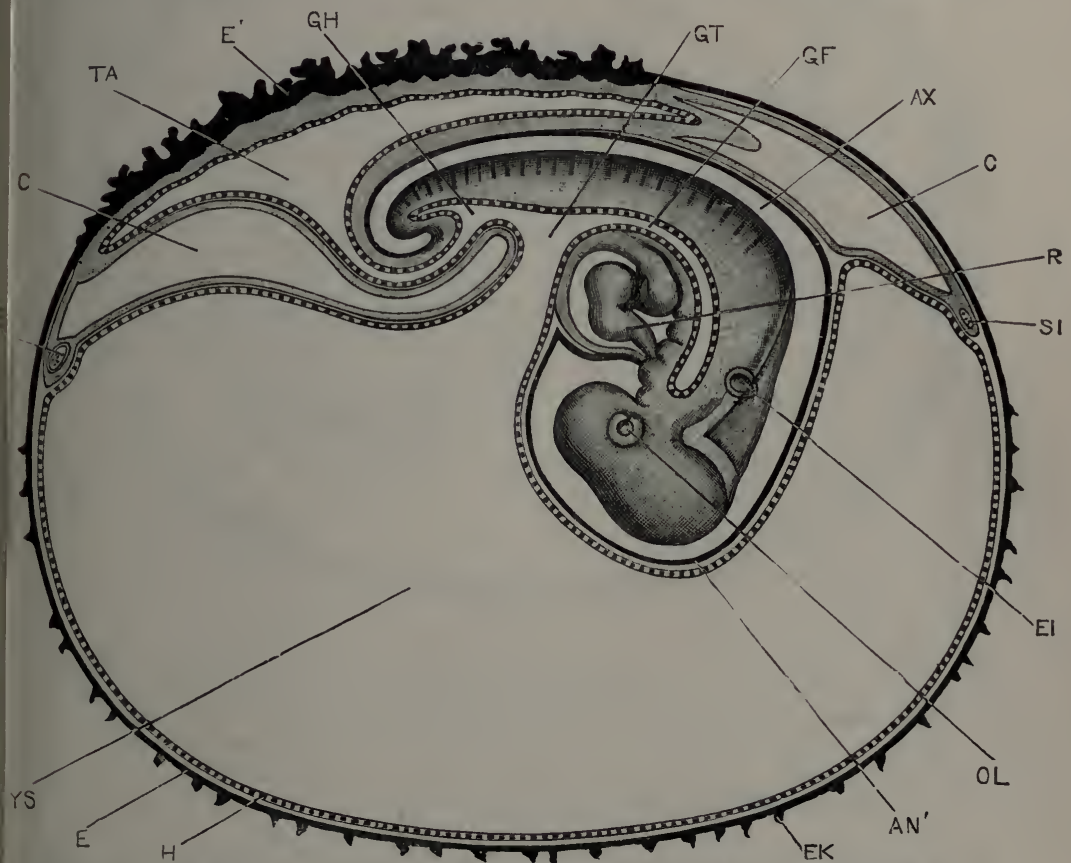

FIG. 147.-A Rabbit Embryo and blastodermic vesicle at the end of the tenth day. The embryo is represented in surface view from the right side, the course of the alimentary canal being indicated by the broad dotted line; the blastodermic vesicle is shown in median longitudinal, or sagittal section. The greater part of the tail, which in the natural condition is twisted spirally, has been removed. (In part after Van Beneden and Julin.) $\times 10$.

$\mathbf{A} \mathbf{N}^{\prime}$, proamnion. AX, amnionic cavity, between the inner or true amnion and the embryo. $\mathbf{C}$, extra-embryouric part of the cœlom or borly-cavity. $\mathbf{E}$, epiblast. $\mathbf{E}$ ', thickened epiblast, by which the blastodermic vesicle is attached to the uterus, and from which the fotal part of the placenta is formed. $\mathrm{EI}$, auditory vesicle. $\mathbf{E K}$, epiblastic villi. $\mathbf{G F}$, foregut. GH, hind-gut. GT, mid-gut. H, hypoblast. OL, leus of eve. $R$, heart. SI, sinus terminalis. TA, cavity of allantois. $\mathbf{Y}$, cavity of yolk-sac or blastodermic vesicle.

Fig. 147, has also grown considerably, and is wrapped spirally round the stalk of the allantois.

By the twelfth day the embryo has acquired the form shown 
in Fig. 148. The sereral divisions of the brain are clearly recognisable, as are also the nose, and the eyes and ears. On the

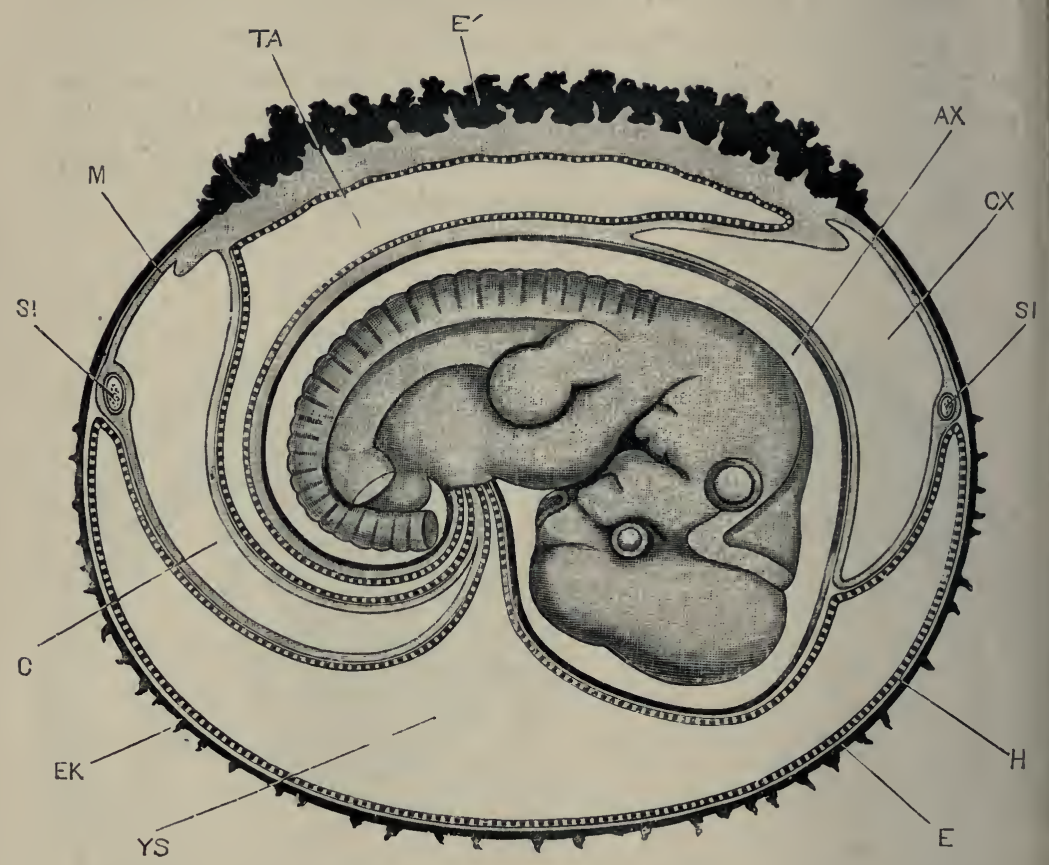

FIG. 148.-A Rabbit Embryo and foetal appendages at the end of the twelfth day. The embryo is represented in surface view from the right side; the yolk-sacand fotal membranes are shown in median longitudinal, or sagittal section. The hind-limb and part of the tail have been removed to allow the yolk-stalk and allantoic stalk to be fully seen. In part after Van Beneden and Julin. $\times 8$.

AX, amnionic cavity, between the inner or true amnion and the embryo, C, CX, extra-embryonic part of the colom or body-cavity. E, epiblast. E', ectoplacenta, or thickener part of the epiblast, from which the placenta is formerl. EK, epiblastic villi. H, hypoblast. $\mathbf{M}$, mesoblast. SI, sinus terminalis. TA, cavity of allantois. $\mathbf{Y}$, cavity of yolk-sac or blastodermic vesicle.

sides of the head and neck the visceral arches and clefts are well seen; and both fore and hind limbs have attained considerable size, and show indications of division into their several segments.

The twelfth-day rabbit embryo corresponds closely in form and in structure to a chick embryo of the middle of the sixth day, and is of very nearly the same actual size. The chief points of difference between the two are the much smaller size of the 
brain and of the sense organs, more especially the eye, in the rabbit.

The first trace of the neural groove appears in the chick embryo about the eighteenth hour of incubation, and in the rabbit embryo early on the eighth day. Starting from this stage, the rate of development is approximately the same in the two embryos; the twelfth day rabbit embryo corresponding to the chick embryo about the mirldle of the sixth clay.

By the twentieth day the rabbit embryo has attained the shape and size shown in Fig. 149 ; in grade of development, and also in actual dimensions, it corresponds very closely to a chick embryo of the twelfth day.

'The young rabbit is bor'n on the thirtieth day, i.e. about twenty-two days from the time of first appearance of the neural groove, the earliest formed organ in the body. The chick is hatched on the twenty-first day of incubation, or rather more than twenty days from the same starting-point. The young rabbit at birth is of considerably gieater bulk than the chick

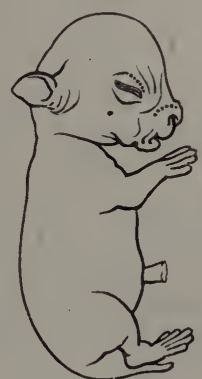

FIG. 149.-A Rabbit Embryo of the twentietl. day, seen from the right side. The rows of spots. round the nose and above the eye, and the single large spot below the eye, represent hair follicles, the last-mentioned one being of especial size. $\times 1$. on hatching, but is in a far more helpless condition; the eyelids are still nnited together, and the young animal is quite incapable of looking after itself, and would perish but for the supply of milk afforded it by the mother.

\section{The Yolk-sac.}

'The yolk-sac is the extra-embryonic portion of the blastodermic vesicle; i.e. the part which is left after the embryo is constricted off by the head, tail, and side folds.

The yolk-sac (Figs. 146, 147, 148, rs), though corresponding in its mode of formation, and in its relations to the embryo, with the yolk-sac of the chick embryo ( $c f$. Fig. 100), differs from this latter in one very important respect. The yolk-sac of the bird is filled with food matter for the nutrition of the embryo, and affords the supply of nourishment at the expense of which the 
whole development is effected. The yolk-sac of the Mammal, on the other hand, is a thin-walled vesicle, containing fluid, but no food matter.

Hence the causes that led to the formation of a yolk-sac in the bird, i.e. the necessity of constricting off the active from the inactive part of the egg in order to avoid undue distortion of the embryo, will not come into play in the case of the Mammal ; and the formation of a yolk-sac by the rabbit embryo must be explained as due to an inherited tendency, and compels us to infer that Mammals are descended from ancestors which produced large eggs, provided with much foodyolk. Further evidence in support of this view has already been given in the earlier portions of this chapter.

With regard to the structure of the yolk-sac of the rabbit embryo, it will be seen in Fig. 146 that the wall of the upper portion, rather less than half the entire surface, consists of all three embryonic layers-epiblast, mesoblast, and hypoblastexcepting only a small patch (Figs. $145,116, \mathrm{Ax}^{\prime}$ ), immediately in front of the head of the embryo, which will be referred to shortly. The wall of the lower half of the yolk-sac contains no mesoblast, but is formed of epiblast and hypoblast alone.

In the mesoblast of the upper half of the yolk-sac, bloodvessels are present, forming the vitelline circulation, or circulation of the vascular area (Fig. 145). The margin of this vascular area, or, what is the same thing, the margin of the mesoblast, is indicated by a circular vessel, the sinus terminalis (Figs. 145, $146,147,148$, SI), into which the vitelline artery opens, and from which the blood is distributed over the vascular area before it is returned to the heart by the vitelline veins.

By the downward projection of the head of the embryo on the tenth day (Fig. 147), the upper wall of the yolk-sac becomes driven ventralwards, and during the succeeding days, as the embryo gets bigger (Fig. 148), this doubling up of the yolk-sac becomes more and more marked. By the thirteenth day the two layers, vascular and non-vascular, are almost in contact with each other, and the cavity of the yolk-sac is practically obliterated.

The outer or non-vascular wall, which is in contact with the wall of the uterus (cf. Fig. 170, YL), now breaks down and becomes absorbed. Portions of it persist for a time; but by about the sixteenth day it has practically disappeared, and the 
vascular, or original upper wall of the yolk-sac, comes into contact with the wall of the uterus, the hypoblast of the yolk-sac lying in contact with the uterine epithelium.

About the eighth day, irregular epiblastic buds (Figs. 146, $117, \mathrm{EK}$ ) arise from the surface of the lower, or non-vascular, half of the yolk-sac. These acquire close attachment to the mucous membrane of the uterus, and aid in fixing the blastodermic vesicle in position, while it is possible that they have also some nutritive function. 'They begin to degenerate about the ninth day, and by the fourteenth or fifteenth day have disappeared.

\section{The Amnion.}

'The amnion of the rabbit, while agreeing in most respects with that of the chick, differs from this in the prominent share taken by the tail-fold, which, as was first pointed out by Van Beneden and Julin, practically forms the whole amnionic covering of the embryo.

On the ninth day, as already mentioned, there is, immediately in front of the head of the embryo, a patch of the blastoderm, roughly circular in outline (Figs. $115,146, \mathrm{Av}^{\prime}$ ), into which the mesoblast does not yet extend, and which therefore consists of epiblast and hypoblast alone. This patch is termed the proamnion, and corresponds to the similarly named structure in the chick.

'The rapid growth of the head of the embryo forwards, and then downwards, depresses the pro-amnion so as to form a deep pocket, projecting into the yolk-sac. This is a well-marked feature on the tenth and eleventh days (Fig. 147, $\mathrm{AN}^{\prime}$ ), but from the twelfth day onwards it becomes less obvious (Fig. 148), owing to the general depression of the upper wall of the yolksac which is then occurring.

The pro-amnion, as a special part of the wall of the yolksac, has only a temporary existence. The mesoblast gradually invades it from the sides, spreading inwards between the epiblast and hypoblast, and, on the three-layered condition being definitely attained, the pro-amnion as such ceases to exist.

The amnion itself is formed almost entirely by the tail-fold, aided to a slight extent by the side-folds. The tail-fold of the amnion (Fig. 146, Ax) is formed immediately behind the 
tail end of the embryo, partly by depression of the embryo into the yolk-sac, and partly by the actual uprising of a fold of the somatopleure, or body wall.

After it is once started, the amnion grows rapidly, and by the end of the tenth day has spread forwards so as to roof over the whole body of the embryo. In front of the embryo it meets and fuses with the somatopleure, at the anterior border of the pro-amnionic pit (Fig. 147).

Apart from the prominent share taken by the tail-fold, the formation and relations of the amnion are practically the same in the rabbit as in the chick. As the amnion is a fold of somatopleure (Figs. 146, 147), the space between its inner and outer layers is necessarily continuous, as in the chick, with the cœlom or body cavity of the embryo.

\section{The Allantois.}

The allantois arises, on the ninth day (Fig. 146, TA), as a hollow diverticulum from the ventral surface of the hinder end of the alimentary canal, appearing almost like a posterior prolongation of the embryo itself. It consists, at first, of a thick wall of mesoblast, in which the allantoic vessels develop very early, and a lining of hypoblastic epithelium ; and from its first appearance it is in very close relation with the amnion. or actually continuous with this (Fig. 146).

As the amnion extends forwards, the allantois grows with it, spreading rapidly between its two layers, and over the back of the embryo (Fig. 147, 148, TA). Owing to its early fusion with the outer layer of the amnion, the upper surface of the allantois lies practically in contact with the wall of the uterus.

The cavity of the allantois is at first small, but from the tenth to the twelfth days it enlarges very greatly (Figs. 11.7. $148, \mathrm{TA})$.

\section{The Placenta.}

The placenta is the organ through which the embryo receives, from the blood of the mother, the nutriment by which it is enabled to develop. It is a structure of great importance and great complexity; the mode of its formation will be dealt with fully at the end of this chapter, but a brief outline may be given here. in order to render its relations to the blood-vessels and other organs of the embryo more intelligible. 
Up to the seventh day the blastodermic vesicle lies quite freely in the uterus (Fig. 168), but towards the end of the seventh day it begins to acquire adhesions to the uterine wall. These are effected partly by the small epiblastic villi of the lower pole of the vesicle (Fig. 116, EK), but principally by the epiblast cells of the vascular area : these latter proliferating freely over a horse-shoe-shaped patch (Fig. 145, E' ), which surrounds the sides and hinder end of the embryonic region; and growing out into irregular tags and processes, which adhere firmly to the wall of the uterus. By the ninth day (Figs. 145, 146, and 169), this adhesion has become so firm, that, if the blastodermic vesicle is pulled away from the uterus, the thickened epithelium over this horse-shoe area is torn from the vesicle and remains attached to the uterine wall.

By the tenth day (Fig. 147), the allantois has come into extensive contact with the wall of the blastodermic vesicle beneath this area of attachment; and the blood-vessels of the allantois are thus brought very close to the uterine vessels of the mother. By a further series of changes, which will be fully described later on in this chapter, the surface of contact between the maternal and foetal blood-vessels is greatly increased, and the highly complicated structure of the placenta is gradually elaborated ( $c f$. Fig. 170).

\section{DEVELOPMENT OF THE NERVOUS SYSTEM.}

\section{General Account.}

In the rabbit, as in the chick and other Vertebrates, the nervous system is established very early. The neural groove (Fig. 144, NG) appears, about half way through the eighth day, as a shallow longitudinal depression, in front of the primitive streak, and bordered laterally by the neural folds.

The neural groove rapidly increases, both in length and in depth. By the end of the ninth day (Fig. 145) the lips of the groove have met and fused along the greater part of their length, though still remaining separate at both the anterior and posterior ends. The distinction between the wider anterior part, or brain, and the narrower posterior portion, or spinal cord, is very evident; and the vesicles of the fore-brain, mid-brain, BM, and hind-brain are already well established. 
The general history of the development of the nervous system, both central and peripheral, of the rabbit is closely similar to that of the chick, and it will be only necessary to describe in the present section the points of more special interest, and in particular those characteristic of Mammals as contrasted with Birds.

\section{The Brain.}

Cranial flexure commences towards the end of the ninth day (Fig. 146), before closure of the neural canal is completed; and proceeds rapidly. By the tenth day (Fig. 147) the brain and spinal cord are closed along their whole length; cranial flexure is strongly pronounced; and the head of the embryo, mainly owing to the rapid growth of the brain, has acquired a shape, and proportions, similar to those of a chick embryo towarls the close of the fourth day of incubation.

By the twelfth day (Fig. 161) the head has increased considerably in size, and, when the embryo is viewed from the side, appear's to be bent twice at right angles; the first bend being near the junction of the brain and spinal cord, opposite the reference line $\mathrm{HY}_{\mathrm{Y}}$ in Fig. 161; and the second bend being marked by the mid-brain, BM, which forms the most prominent part of the brain at this stage.

Sagittal sections of twelve-day embryos (Fig. 150) show that flexure has really taken place to a far greater extent than is apparent from the surface. Following the floor of the brain from behind forwards, there is first, at the junction of spinal cord and mid-brain, between the reference lines $\mathrm{TH}$ and $\mathrm{CH}$ in Fig. 150, a rather sharp bend, ventralwards, of nearly $90^{\circ}$; this is corrected a little further forwards by a second and more gradual bend dorsalwards, at the level of the reference line P'r. At the base of the mid-brain, BM, there is a very sharp and sudden bend of about $180^{\circ}$, by which the infundibulum, $\mathrm{IN}$, and the floor of the hind-brain are brought almost into contact with each other. In front of the infundibulum the floor of the brain again bends dorsalwards, and nearly at right angles.

These flexures are even more strongly marked in the later stages of development, the angles formed by them becoming sharper and more pronounced. This is well shown in Fig. 151 , which represents the condition of the brain on the eighteenth day, as seen in sagittal section. The extreme sharpness of the 


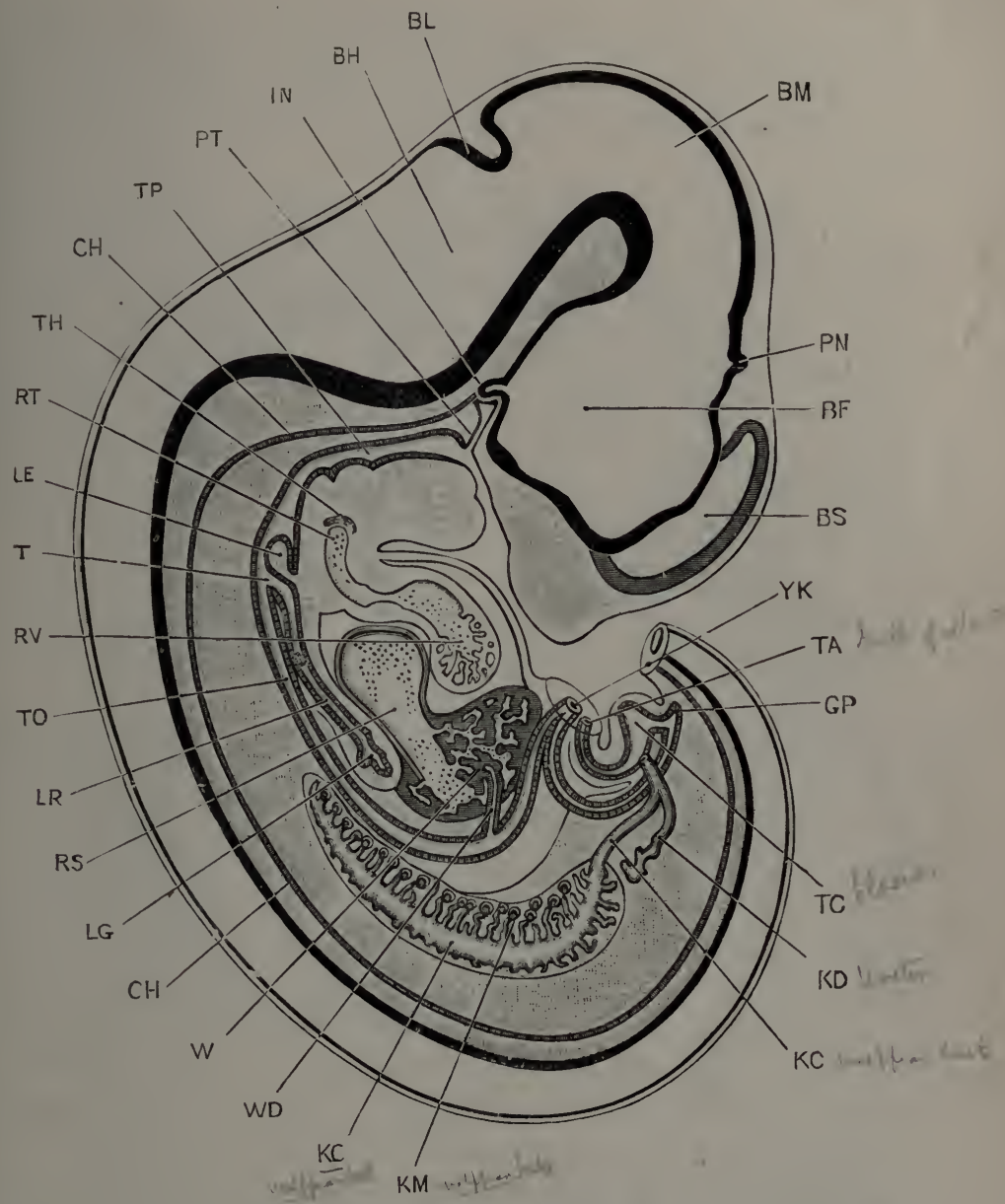

FIG. 150.-A median longitudinal, or sagittal section through a Rabbit Embryo at the end of the tweifth day. The section is a strictly median one except in two respects: the cerebral hemisphere of the left side has been introduced in order to render the figure more complete; and the Wolffian body and ureter of the right side. The terminal portion of the tail has been removed. (Compare Fig. 161 for a surface view of an embryo of the same age. $) \times 14$.

$\mathbf{B F}$, eavity of fore-brain or thalamencephalon. BH, envity of hind-brain, or fourth ventricle. BI, ecrehellum. BM, eavity of mill-brain. BS, eavity of cerebral hemisphere, or lateral ventriele. $\mathbf{C H}$, notochord. GP, post-anal gut. IN, finger-like urocess of infundibulum. KC, Wolffias duet. KD, ureter. KM, Wolffian body. LE, epiglottis. LG, lung. IR, trachen. PN, pineal body. PT, pituitary body. RS, sims venosus. RT, truneus arteriosts. RV, ventricle of heart. T, glottis. TA, stalk of allantois, cut sliort. TC, cloatei. TH, thyroid bods. TO, œsophagus. TP, pharynx. W, liver. WD, bile duct. $\mathbf{Y}$, yolk-stalk, cut short. 
bend at the junction of the spinal cord and brain, between the reference lines RP and oI in Fig. 151, is very characteristic of mammalian embryos at this stage; while the sharp bend at the base of the mid-brain is quite as conspicuous as in the earlier stage.

The general relations of the brain to the head are much the same in the rabbit embryo as in the chick. In the early stages (Figs. 147, 148, and 150) the brain forms practically the whole of the head, and determines its shape almost exclusively; but in the later stages (Figs. 149, 151), as the parts of the face assume definite shape, and grow forwards to form the snout, the brain becomes thrown more and more on to the dorsal surface, and ultimately to the posterior part of the head, and takes a much less prominent share in determining the general contour.

In dealing with the several parts of the brain it will be convenient to commence with the thalamencephalon, and the structures developed from, or in connection with it.

The thalamencephalon (Fig. 150, BF) is the anterior cerebral vesicle, or fore-brain, of the early stages ( $c f$. Figs. 145, 146). From it the optic vesicles arise as lateral outgrowths; these appear very early, and attain some size before the roof of the fore-brain is closed (Fig. 145); their further development, and their conversion into the essential parts of the eyes, will be described in the next section of this chapter, p. 387 .

The side walls of the thalamencephalon thicken very quickly, to form the optic thalami (Fig. 155, BU); and, owing to this thickening of its sides, the central cavity, or third ventricle, becomes reduced to a vertical cleft, very narrow from side to side. The anterior wall of the thalamencephalon remains thin, as the lamina terminalis (Fig. 151, BT), lying between the roots of the two cerebral hemispheres.

The roof of the thalamencephalon remains thin, consisting in the greater part of its extent of a single layer of epithelial cells, devoid of nervous elements of any kind. From this roof, rather behind the middle of its length, the pineal body arises about the twelfth day, as a hollow median papilla (Fig. 150, rN). This rapidly lengthens, forming a tubular and backwardly directed diverticulum of the brain. Its distal end dilates (Fig. 151, Px), to form a slightly expanded vesicle, from the sides of which 
irregular hollow outgrowths arise : at a later stage these outgrowths become solid, and separate completely from the stalk.

In front of the pineal body the roof of the third ventricle,

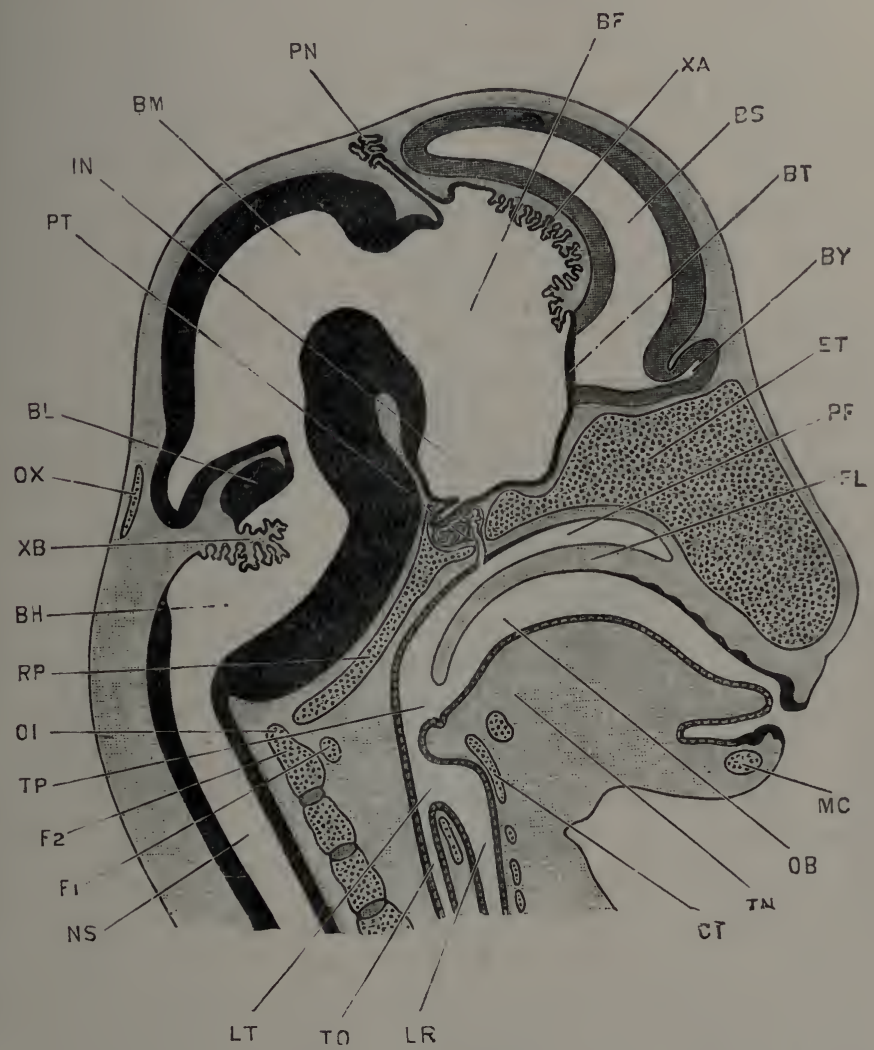

FIf. 1.jl.- $\Lambda$ median longitudinal, or sagrittal section through the head of a Rabbit Embryo of the eighteenth day. (Compare Fig. 149 for a surface view of an embryo at a slightly later stacre.) $\times 10$.

BF, cavity of thalameneephalon, or thiril ventricle. $\mathbf{B H}$, eavity of mednlla ohlongatil, or fourtl ventricle. BL, eerebellum. BM, eavity of mir-brain. BS, cavity of cerelmal hemisphere, or lateral ventricle. BT, lamina terminalis. BY, eavity of olfietury lobe. CT, thirroil cartilage. ET, mesetlmoill eartilage. F.1, first cervieal "1) atlas vertebra. F.2. veroull cervieal or nxis vertebra. IN, infunilibulum. IR, traceliea. LT, glottis. MC, Mcekel's centilage. NS, central canal of spinal cord. OB. lncenl eavity: OI, olmitoil process of axis vertebra. OX, supranceipital etrtilage. PF, justerior narial ehamber. PI, palate. PN, pineal body. PT, pitnitury bolly. RP, basilar plate. TN, tongue. TO, œsoplagus. TP, pharynx. $\mathbf{X A}$, churoid plexus of thirl ventricle. $\mathbf{X} \mathbf{B}$, clusoil plexus of fourth ventricle.

which is here excessively thin, becomes thrown into folds, which hang down into the cavity of the ventricle (Fig. 151, xA). Between these folds blood-vessels pass in, from the vascular meso- 
blast outside the brain, and give rise to the choroid plexus of the third ventricle.

The floor of the thalamencephalon also remains thin, though not so thin as the roof. The anterior part of the floor is crossed by a shallow transverse groove, which is prolonged outwards into the optic stalks (Figs. 150, 151, and 155). The posterior part of the floor gives rise to the infundibulum. This is a median, thin-walled depression, from the hinder end of which a hollow finger-like diverticulum arises on the tenth day ( $c f$. Fig. $150, \mathrm{IN})$; this diverticulum lies, from the first, in very close relation with the anterior, end of the notochord, and with the pocket-like outgrowth from the stomatodrum (Fig. 150, PT), which gives rise to the pituitary body. This anterior end of the notochord is ultimately absorbed and obliterated; but the infundibular diverticulum and the pituitary body remain in intimate relation throughout life; the diverticulum forming what is spoken of, in the adult rabbit, as the posterior lobe of the pituitary body.

The further development of the pituitary body itself, which may conveniently be dealt with here, is as follows. The stomatodreal diverticulum (Fig. 150, PT) dilates at its blind end, and gives off from this terminal dilatation hollow outgrowths; these branch freely (Fig. 151, PT), but in the later stages of development become solid. The central dilated cavity of the pituitary body persists; it retains its communication with the buccal cavity, through the tubular stalk, for some time. The formation of the palate (Fig. 151, PL) leaves the pituitary stalk in communication with the narial passage, but cuts it off from direct communication with the buccal cavity. In the later stages, the pituitary stalk loses its connection with the narial passage, and becomes obliterated.

The cerebral hemispheres arise, as in the chick, in the first instance as a median anterior prolongation of the thalamencephalon, which may be termed the vesicle of the hemispheres. 'This soon becomes divided by an inwardly projecting fold of its anterior wall into right and left lobes, which by further growth become the cerebral hemispheres; the median anterior wall of the vesicle, between the bases of the hemispheres, persisting as the lamina terminalis.

From their mode of formation the hemispheres are necessarily 
hollow ; and their cavities, the lateral ventricles, retain throughout life their communications with the third ventricle through the foramina of Monro, a pair of apertures which are at first wide, but which gradually become reduced, by thickening of their lips, to narrow slits.

The cerebral hemispheres first become prominent about the twelfth day (Fig. 150, Bs); from this date they grow actively,

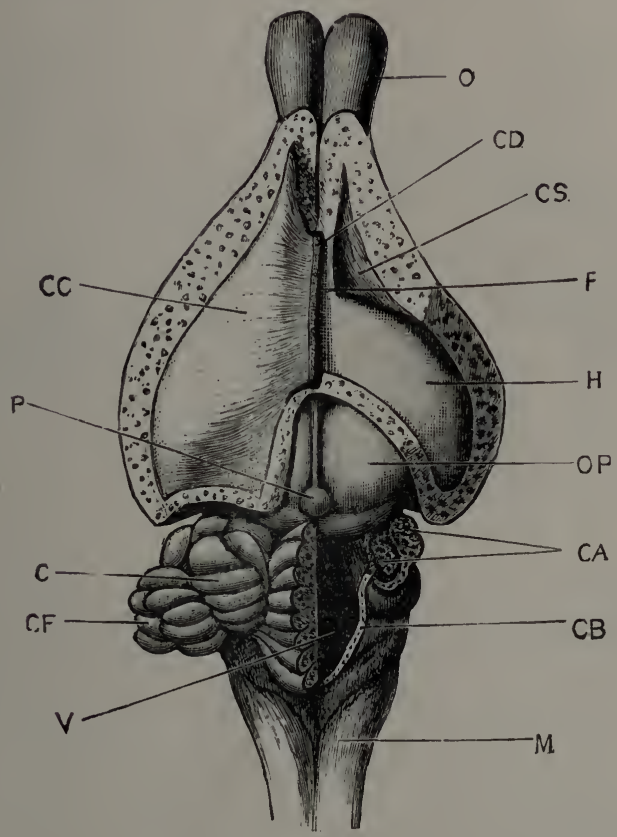

FIG. 152.-The brain dissected from abore. Enlarged. (From Marshall and Hurst.)

C, lateral lobe of cerebellum. CA, pillars of cerebellum. CB, cut elge of velum medullæ posterius. $\mathbf{C C}$, corpus callosum : the riglit half is removed. $\mathbf{C D}$, cut edge of eorpus callosum. CF, floccular lobe of cerebellum. CS, corpus striatum. $\mathbf{E}$, anterior limit of boly of fornix. H, hipjweampius major. M, medulla oblongata. Ó, olfactory lobc. $\mathrm{OP}$, anterior optic lobe. $\mathbf{P}$, jineal bolly. $\mathbf{V}$, fourth ventricle.

extending forwards, and still more rapidly backwards, so as to overlie and cover the roof of the thalamencephalon, and at a later stage the mid-brain as well (cf. Figs. 151 and 152).

The wall of each hemisphere is at first thin on all sides, and the cavity, or lateral ventricle, is consequently large (Fig. 151). The inner wall remains thin, but the outer wall (Fig. 152) thickens considerably in the later stages of development; while 
the hinder ends of the hemispheres thicken still more, to form the corpora striata (Fig. 155, BI), a pair of prominent swellings, lying in front and to the outer sides of the optic thalami, BU, and separated from these by well-marked grooves.

The hippocampus major (Fig. 152, H) is a prominent curved ridge, projecting into the lateral ventricle, and extending round into its descending cornu; it is really an inwardly projecting

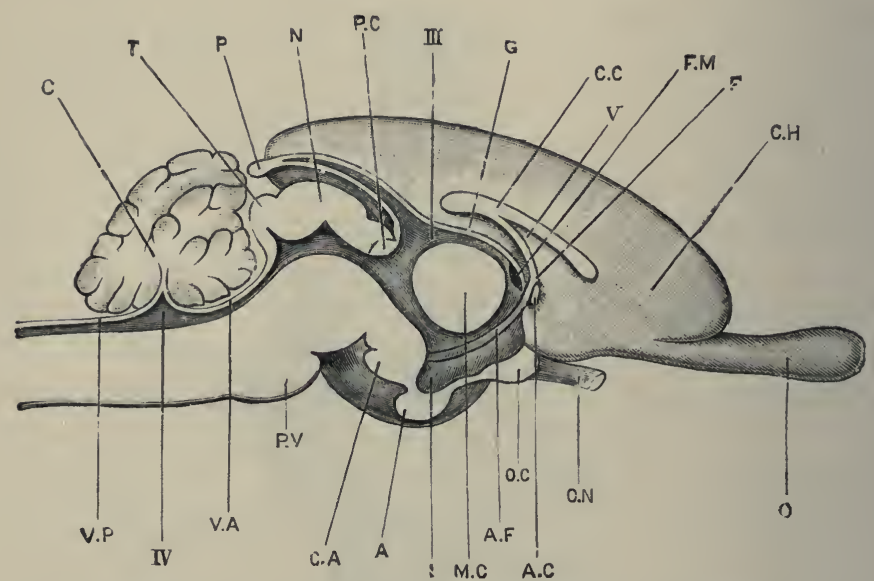

FIG. 153.-A longitudinal and vertical section of the brain of an adult Rabbit, taken in the median plane. (From Marshall and Hurst.)

A, pituitary body. AC, anterior eommissure. AF, anterior pillar of the fornix, seen in the wall of the third rentriele. $\mathbf{C}$, cerebellum. $\mathbf{C A}$, corpus albicans. $\mathbf{C C}$, corpus callosum. $\mathbf{C H}$, inner surface of left cerebral hemisphere. F, borly of the fornix. F'M, foramen of Monro. G, velum interpositum. I, infundibulium. MC, midale commissure. $\mathbf{N}$, anterior lohe of corpora quadrigemina, or 'nates.' $\mathbf{O}$, olfactory lobe. OC, optic chiasma. ON, left optie nerve. $\mathbf{P}$, pineal body. $\mathbf{P C}$, posterior commissure. PV, pons Varolii. T, posterior lobe of corpora quadrigemina, or 'testis.' VA, velum merhnlla anterius, or valve of Vienssens. VP, velum medullæ posterius.

III, third ventriele. IV, fourth ventricle. V, fifth ventriele.

fold of the wall of the hemisphere, formed by a deep groove on the surface of its inner wall.

The choroid plexus of the lateral ventricle (Fig. 155, x) is a somewhat similar, but much thinner fold of the inner wall of each hemisphere, between the two layers of which blood-vessels, $\mathrm{XD}$, pass in freely. It lies immediately below the hippocampal fold.

The commissural bands between the two hemispheres are very characteristic structures in Mammals, in which they attain a much higher development than in other Vertebrates. The most important of these are the corpus callosum, the fornix, and 
the anterior, middle, and posterior commissures of the third ventricle. Their development is complicated, and difficult to follow.

In front of the lamina terminalis, the two hemispheres extend forwards side by side, very close to each other (Fig. 152), but separated by a median cleft in which lies the connective tissue lamina from which the fal $x$ cerebri is formed. At the hinder end of this cleft, just in front of the lamina terminalis, the walls of the two hemispheres come in contact and fuse; and from this fused patch, which is somewhat triangular in shape as seen in sagittal section, the commissural bands are formed. The corpus callosum (Fig. 153, CC), the most important of them, is formed from the dorsal part of the fused patch; it develops from before backwards, the anterior end being formed first. The fornix, in which the fibres are mainly longitudinal in direction, is formed from the ventral part of the patch; and the anterior commissure from its hinder end, just in front of the lamina terminalis. It is not quite clear whether the fifth ventricle (Fig. 15:3, v) is formed by the breaking down of the central part of the fused patch, or is merely a persistent part of the original cleft between the two hemispheres.

The surfaces of the hemispheres are at first smooth, and even in the adult rabbit are only slightly convoluted. The convolutions arise as foldings or groores of the surface, extending to a greater or less depth, and are classed as primary or secondary according to whether they are folds involving the whole thickness of the wall of the hemisphere, or mere groores in its substance.

The olfactory lobes appear, about the fourteenth day, as a pair of hollow outgrowths from the ventral surface of the anterior ends of the cerebral hemispheres; by the eighteenth day (lig. $151,13 \mathrm{x})$ they have become prominent structures.

The mid-brain. In the early stages, up to about the twelfth day (Fig. 150), the mid-brain is very imperfectly marked oft from the fore-brain ; later on (Fig. 151), the boundary between the two divisions becomes well defined.

As compared with the chick, the micl-brain of the rabbit is of rather smaller size, and less prominent: it is further distinguished by its tendency to grow backwards over the hindbrain, a tendency already present on the twelfth day (Fig. 1.50), 
but much more pronounced in the later stages, the posterior lobes of the mid-brain on the eighteenth day (Fig. 151) completely overlapping the cerebellum, BL.

The roof of the mid-brain gives rise to the corpora quadrigemina. A transverse furrow first appears, dividing it into a larger anterior, and a smaller posterior portion; the anterior portion soon becomes divided, by a median longitudinal groove, into the anterior lobes of the corpora quadrigemina, or nates; the posterior portion, overhanging the cerebellum, is not divided until a much later stage.

'The floor of the mid-brain forms the very sharp bend at the base of the brain which has already been noticed (Figs. 150 and 151); as in the chick, it becomes greatly thickened on the formation of the longitudinal pillars of nerve fibres known as the crura cerebri (Fig. 154, CC), which connect the optic thalami and corpora striata with the hind brain.

The cerebellum is developed from the roof of the anterior part of the hind-brain, in much the same way as in the chick. On the tenth day (Fig. 147) a slight thickening is formed across the roof of the anterior end of the hind-brain; by the twelfth day this has become more conspicuous (Fig. 150, BL), but is still only a slightly thickened transverse band. By the eighteenth day (Fig. 151, BL) it has increased considerably in thickness; and, shortly after this stage, it becomes folded transversely on itself, as in the chick ( $c f$. Fig. 116, BL). Secondary foldings appear on its surface, and the complicated structure of the adult cerebellum is gradually acquired.

Of the several parts of the adult cerebellum ( $c f$. Fig. 152), the median lobe, or vermis, is the first to be formed; the lateral lobes and floccular lobes appearing at a much later date.

Immediately in front of the cerebellum, between it and the mid-brain, the roof of the brain becomes extremely thin, forming the velum medullæ anterius (Fig. 153, vA), which is ultimately reduced to a single layer of epithelial cells devoid of nervous elements.

The medulla oblongata is formed from the floor of the hind-brain, and from the part posterior to the cerebellum. 'The floor of the medulla oblongata remains thin in the actual mid- 
ventral plane (Fig. 158); the lateral parts of the floor, and the sicles as well, thicken very considerably. The roof of the fourth ventricle, as in the chick, widens out considerably (Fig. 158), and at an early stage becomes exceedingly thin. Immediately behind the cerebellum, the roof of the medulla remains comparatively smooth, as the velum medullæ posterius (Fig. 153, VI) ; but a short way further back it becomes thrown into a complicated series of folds (Fig. 151, XB), which hang down into the fourth ventricle, and between the layers of which the bloodvessels penetrate in large numbers to form the choroid plexus of the ventricle.

The walls of the medulla oblongata consist mainly of longitudinal fibres. 'The pons Varolii (Fig. 153 and 154, Pv), the great transverse band of nerve fibres which connects the two halves of the cerebellum together, develops very late; its position is indicated, about the eighteenth day, by the sharp rectangular bend in the floor of the medulla, opposite the cerebellum (Fig. 151).

Before leaving the brain, it should be noted that, in spite of the complicated foldings which various parts of it undergo, and the extreme thinness to which its walls are reduced in places, notably in the roof of the third, and in that of the fourth ventricle. the cavity of the brain remains a closed one, and its walls are not actually perforated at any place.

The membranes of the brain, i.e. the pia mater and dura mater, are connective-tissue structures, of mesoblastic origin.

\section{The Spinal Cord.}

The development of the spinal cord of the rabbit need not be described in detail, as in all essential respects it agrees with that of the chick.

\section{The Histological Development of the Brain and the Spinal Cord.}

This will be more fully dealt with in the chapter on Human Embryology.

In the spinal cord, the changes undergone are essentially similar to those in the chick. The original walls of the neural canal give rise mainly to the grey nervous matter, the innermost 
layer of cells forming the epithelial lining of the central canal. The white matter develops later; in the spinal cord it appears on the eleventh day, the ventral and lateral bands of white matter being formed practically simultaneously. The central canal of the spinal cord remains a narrow vertical cleft until about the sixteenth day, when its dorsal part becomes obliterated, as in the chick, preparatory to the formation of the dorsal fissure.

In the medulla oblongata, the arrangement of white and grey matter is essentially the same as in the spinal cord, the white matter forming, about the eleventh day, on the outer surface of the grey matter; in the later stages the relations between white and grey matter become much more complicated.

In the fore-brain the conditions are somewhat different. The walls of the hemispheres, which at first are very thin, become early differentiated into an outer layer of rounded elements, which later on give rise to grey matter, and an inner epithelial layer, which becomes the epithelial lining of the ventricle. About the sixteenth or seventeenth day, bands of white fibres grow upwards from the crura cerebri, through the optic thalami and corpora striata, and make their way between the two layers of the wall of the hemisphere; while a little later a very thin superficial layer of white matter forms on the surface of the brain, outside the grey matter. In this way the characteristic distribution of white and grey matter in the hemispheres of the adult is brought about.

\section{The Peripheral Nervous System.}

The general history of the peripheral nervous system is the same in the rabbit as in the chick; but the earliest stages of development have not yet been worked out in such detail. By the ninth day both spinal and cranial nerves are established, and by the eleventh day (Fig. 165) all the principal branches of distribution are present.

\section{The Cranial Nerves.}

I. The olfactory or first cranial nerve. The time of first appearance of the olfactory nerve in the rabbit has not been definitely determined. The nerve is, however, clearly recognisable, as a short stem, connecting the cerebral hemisphere with the olfactory pit, before the olfactory lobe is formed. 
II. The optic or second cranial nerve will be considered in the section dealing with the development of the eye, p. 387.

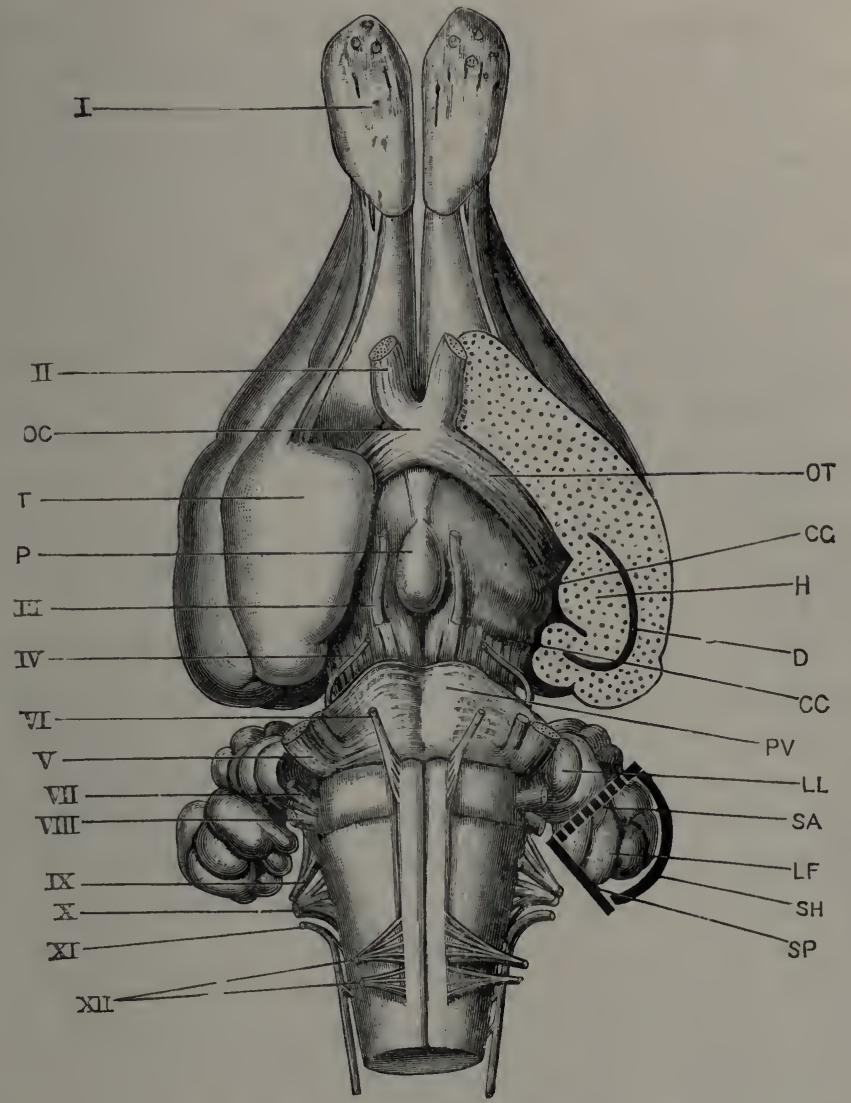

FIG. 154.-The brain of an adult Rabbit from the ventral surface. The greater part of the left temporal lobe has been sliced off horizontally. The planes of the three semicircular canals of the left side are indicated by the thick lines surrounding the floccular lobe of the cerebellum. (From Marshall and Hurst.) $\times 2$.

CC, crus cerebri. CG, corpus geniculatum. D, lescending cornu of left lateral ventricle. H, hippocampus major. LF, flocenlar lobe of cerebellum. LI, lateral lobe of cerebellum. OC, optic chiasma. OT, optic tract. $\mathbf{P}$, pituitary body. $\mathbf{P V}$, pons Varolii. SA, anterior vertical semicireular canal. SH, horizontal semicircular canal. SP, posterior vertical semicircular canal. T, temporal lobe of cerebral hemisplicre.

$\mathrm{I}$, olfactory lobe, with roots of olfactory nerves. II, optic nerve. III, third nerve or motor oculi. IV, fourth nerve. V, trigenimal nerve. VI, sixth nerve or abilucens. VII, facial nerve. VIII, aurlitory nerve. IX, ylosso-pharyngeal nerve. $\mathbf{X}$, pneumogastric nerve. XI, spinal accessory nerve. XII, hypoglossal nerve.

III, IV, and VI. The third, fourth, and sixth cranial nerves. There are no exact observations recorded on the development of 
these three nerves in the rabbit. From the time when they can be clearly recognised, about the eleventh day, their course and relations are the same as in the adult.

$\mathrm{V}$. The trigeminal or fifth cranial nerve. This nerve can be recognised on the ninth day, at a stage immediately after closure of the brain has been effected. The nerve appears at this stage as a pyriform ganglionic mass, the narrow end of which is in close contact with the dorsal surface of the brain, while the rest of the nerve lies close alongside the brain, extending about half way down its side. By the tenth day the roof of the hind-brain has widened very greatly, and the root of the fifth nerve is now attached to the junction of the thin roof and thickened side of the brain. Before the end of the tenth day, the nerve branches distally; and by the twelfth day the ophthalmic, maxillary, and mandibular branches are of considerable length, and have courses and relations very similar to those of the same nerves in a five-day chick embryo ( $c f$. Fig. 115).

VII and VIII. The facial or seventh, and auditory or eighth cranial nerves are, as in the chick, very intimately connected, or actually continuous with each other, from their first appearance. They can be recognised before the end of the ninth day; and by the tenth day the facial nerve has acquired its definite relation to the hyoid arch, as in a five-day chick embryo (Fig. 115). The auditory nerve appears to be continuous with the epithelium of the auditory vesicle from its earliest appearance.

IX. The glosso-pharyngeal, or ninth cranial nerve, as in the chick, arises by multiple roots from the side of the medulla oblongata, immediately behind the auditory vesicle. Its main stem lies in the first branchial arch.

$\mathrm{X}$. The pneumogastric, or vagus, or tenth cranial nerve has, in the rabbit embryo of the twelfth day, almost exactly the same course and relations as in a chick embryo of the fifth day (Fig. 115). It arises by a considerable number of roots from the side of the medulla oblongata, behind the roots of the glossopharyngeal nerve; the most anterior of the roots of the pneumogastric nerve is, as in the chick, directly continuous with the posterior root of the glosso-pharyngeal nerve; while the hindmost root of the pneumogastric is of great length, and runs backwards along the side of the medulla oblongata to the gan- 
glion of the first spinal nerve, with which it is closely connected (cf. Fig. 115). The roots of origin of the pneumogastric nerve converge to form a single large trunk, which lies immediately hehind the trunk of the glosso-pharyngeal nerve; it expands to form a large oval ganglion, beyond which it divides into a small branch to the second branchial arch, and a much larger visceral branch which runs backwards to the heart, lungs, and stomach.

XI. The spinal accessory or eleventh cranial nerve. The development of this nerve has not yet been determined in the rabbit.

XII. The hypoglossal or twelfth cranial nerve. Exactly opposite the roots of the pneumogastric nerve in rabbit embryos of the twelfth day, but arising from the medulla oblongata at a more ventral level, and nearer the median plane, is a second set of nerve roots. These are quite as numerous as the more dorsally placed pneumogastric roots, but are more slender; they converge, and unite to form the hypoglossal nerve. Anatomically, the roots of the pneumogastric and hypoglossal nerves, at this stage, have relations closely comparable to those between the dorsal and ventral roots of a spinal nerve; but it is not yet clear whether this comparison has any morphological value. The roots of the hypoglossal nerve are probably correctly regarded as belonging to the same category as the ventral spinal roots, but their relations to the roots of the pneumogastric must be considered at present as much more doubtful.

\section{The Spinal Nerves.}

The earliest stages in the development of the spinal nerves have not yet been described in the rabbit. The dorsal roots and the ganglia are clearly established by the end of the ninth lay; the ventral roots develop later, apparently during the tenth day.

The ganglia of the dorsal roots are at first of very considerable width; almost as wide, in fact, as the mesoblastic somites; so that on the twelfth day there are hardly any intervals between the spinal nerves, the successive ganglia being practically in contact with one another along the greater part of the length of the spinal cord.

Beyond the ganglia the nerves narrow rapidly, and have the normal proportions. The main divisions of the spinal nerves are 
early established, and are all present by the eleventh day (Fig. 165). The nerve trunk, beyond the ganglion, divides at once into a smaller dorsa! branch, $\mathrm{NN}^{\prime}$; and a larger ventral branch, $\mathrm{NN}$; each branch containing nerve fibres from both the dorsal and ventral roots of the nerve. The dorsal branch, $\mathrm{Nx}^{\prime}$, runs outwards and upwards, to the muscles and skin of the back; while the ventral or larger branch, NN, runs downwards in the body wall, and in the somites opposite the limbs sends branches into these latter.

\section{The Sympathetic Nervous System.}

This has received much attention, but several points concerning its early origin still remain in doubt. Before the end of the eleventh day it is already well established (Fig. 165, NY), consisting of a main ganglionated cord running along each side of the body, close to the dorsal surface of the aorta, and receiving branches from the ventral branches of the several spinal nerves as it passes these. Dr. Paterson, from observations chiefly on rat embryos, but partly on rabbits, concludes that the longitudinal cord is the first part of the sympathetic system to be developed, that it arises in the mesoblast entirely independently of the spinal nerves, and is at first devoid of ganglia; he believes that the connection of the longitudinal cord with the spinal nerves is a secondary one, and is effected by outgrowths from the ventral branches of the spinal nerves after the longitudinal cords are established. These observations are, however, so entirely at variance with what is known as to the development of the sympathetic nervous system in other Vertebrates, in which the sympathetic develops merely as a specialised portion of the spinal nervous system, that it seems preferable to suspend judgment on the matter, pending renewed investigations.

The connections of the spinal nerves with the longitudinal sympathetic cords are limited to the thoracic and lumbar regions, but the cords themselves extend forwards along the neck to the head, where they acquire connections with the hinder cranial nerves.

\section{The Supra-renal Bodies.}

The supra-renal or adrenal bodies, which in the adult rabbit form a pair of small, round, yellow bodies, a-little way in front of the kidneys, are developed from two distinct sources. 
The outer or cortical portion of the supra-renal body is developed from a mass of mesoblast cells which appears about the twelfth day, lying in front of the kidney, and between the aorta and the root of the mesentery.

The medullary portion of the supra-renal body is formed from a column of cells, which grows out from the longitudinal sympathetic cord about the fifteenth day, and makes its way into the mesoblastic mass which gives rise to the cortical layer. This mode of development agrees with what is known as to the formation of the supra-renal bodies in other Vertebrates, but leaves the real morphological meaning of these curious structures still undecided.

\section{THE DEVELOPIIENT OF THE SENSE ORGANS.}

\section{The Nose.}

There is very little of special interest about the olfactory organ of the rabbit, which resembles in most features of its development that of the chick. A special diverticulum arises at an early stage from each olfactory sac, which acquires a separate opening into the mouth, through the naso-palatine canal, and becomes the organ of Jacobson.

\section{The Eye.}

The general history of development of the eye of the rabbit is very similar to that of the chick; the formation of the optic vesicles as outgrowths of the fore-brain, the doubling up of the vesicles to form the optic cups, the pitting-in of the surface epiblast to form the lens, and the subsequent fate of the several parts being essentially the same in the two cases. One of the most marked points of difference is the much smaller size of the eye in the rabbit (cf. Figs. 115 and 148).

The optic vesicles arise as lateral outgrowtlis of the forebrain, at a very early stage. Before the end of the ninth day, i.e. before the fore-brain is closed dorsally, the optic vesicles (Fig. 145) are already conspicuous structures. The vesicles soon become constricted at their origins from the fore-brain, the constricted portions giving rise to the optic stalks. As the constrictions proceed from above downwards, the optic stalks remain 
connected with the ventral surface or floor of the fore-brain (Fig. 155).

The optic cup. On the tenth day, the doubling up of the optic vesicle to form the optic cup commences, and by the fourteenth day (Fig. 155) it is completed. The process of doubling up takes place in very much the same way as in the

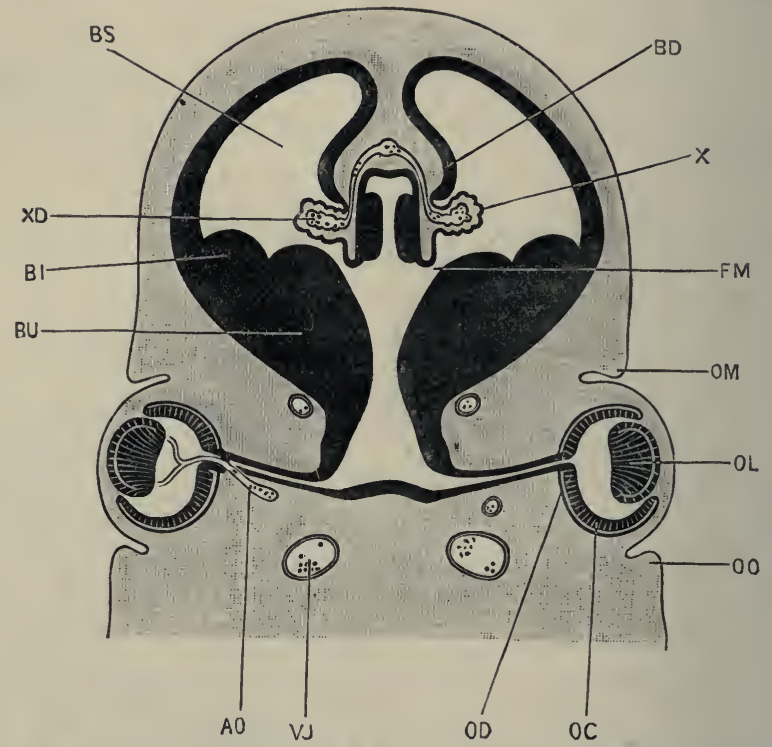

FIG. 155.-A transverse section across the head of a Rabbit Embryo of the fourteenth day, the section passing through the eyes, the fore-brain and the cerebral hemispheres. $\times 14$.

AO, central artery of retina, arising from interual carotid artery. BD, fold of the inner wall of the corebral hemisphere, which forms the hippocampus major. BI. corpus striatum. BS, lateral veutricle, or cavity of the cerebral hemisphere. BU, optic thalamus. $\mathbf{F} \mathbf{M}$, foramen of Mouro, leading from the third ventricle to the lateral ventricle. $\mathbf{O C}$, inner or retinal wall of optic cup. OD, outer or pigment wall of optic cup. OL, lens. OM, upper eyelid. OO, lower eyelid. VJ, jugular vein. $\mathbf{X}$, folr of the inner wall of the cerebral hemisphere, which forms the choroid plexus of the lateral ventricle. XD, blood-vessels of the choroid plexus.

chick; and, although occurring simultaneously with the formation of the lens, it is to be ascribed rather to a complicated mode of growth on the part of the walls of the optic vesicle itself, than to mechanical inpushing by the developing lens.

A choroidal fissure is formed along the ventral surface of the optic cup, as in the chick; the only important point of difference being that, in the rabbit embryo, the process of folding or 
doubling up is not confined to the optic cup itself, but extends a certain distance along the optic stalk towards the brain. The consequence is that the part of the optic stalk near to the eye (Fig. 155) is not a simple tube with thick walls, but a tube deeply grooved along its under surface by folding of its walls.

This groove, being continuous with the choroidal fissure, leads into the cavity of the optic cup, i.e. of the globe of the eye; and it is by running along this groove that the central artery of the retina, a branch of the internal carotid artery (Fig. 155, A0), gains admittance to the interior of the eye. This artery supplies the retina throughout life, and during the development of the eye supplies the vitreous body and the capsule of the lens as well.

Of the two walls of the optic cup, the distal or inner wall (Fig. 155, oc) is, from the first, much thicker than the proximal or outer wall, oD ; the difference being a very pronounced one by the fourteenth day. From the inner layer, oc, the entire thickness of the retina proper is developed, the rods and cones being processes from its outer surface, which do not appear until shortly before birth.

'The outer and thinner' wall of the optic cup, oD, becomes converted, as in Vertebrates generally, into the pigment layer of the retina; a stratum of hexagonal cells, closely fitted together, with which the retinal rods ultimately acquire very close relations. In the cells of this layer, granules of pigment are deposited at an early stage; and up to a late period of development the black colour of the eye is due to this layer, the choroid coat of the eye not developing, or acquiring pigment, until very near the time of birth ( $c f$. Fig. 156).

Near the free edge of the optic cup, the two layers are of approximately equal thickness (Fig. 156), and grow forwards in front of the lens to form the pigmented epithelium of the posterior surface of the iris.

The optic nerve. It is at present uncertain whether the fibres of the optic nerve of the rabbit are developed in situ, from the walls of the tubular optic stalk, or whether, as seems far more probable, they arise in the retina and grow inwards alung the optic stalk to the brain. 'The nerve fibres from the 
two eyes cross one another in the floor of the third ventricle, to form the optic chiasma (Fig. 154, OT), and then continue their growth upwards and backwards, round the walls of the fore-brain, to the mid-brain.

The lens. During the tenth day the deeper part of the surface epiblast, opposite the optic resicle, becomes thickened, forming the first rudiment of the lens. On the eleventh day this thickened patch becomes invaginated, forming the vesicle of the lens; the mouth of the pit narrows, and by the end of the twelfth day or early on the thirteenth day it closes, completing the vesicle, which soon separates from the surface epiblast.

From the first, the inner or deeper wall of the lens vesicle is much thicker than the outer wall. The inner wall continues to increase in thickness, through elongation of the cells composing it, until by the end of the fourteenth day (Fig. 155, OL), the cavity of the vesicle is almost completely obliterated.

The lens continues to grow rapidly, and throughout the later stages of development is of large proportionate size. Its structure and relations on the twenty-first day are shown in Fig. 156 , oL, where its inner surface is seen to be very strongly convex, and the outer surface less markedly so. The axial cells of the lens remain straight or nearly so, while the more marginal ones are curved in the direction indicated by the lines crossing the lens in the figure. The first formed part of the lens acts as a nucleus, round which successive layers of cells are added on in the later stages of development. These arise at the equator of the lens, and, increasing rapidly in length, spread on to the faces of the lens, over the ends of the first formed cells.

The lens, during the time of its formation, is invested by a sheath of mesoblast. According to Kölliker, this is present from the first as a thin layer, between the epiblastic thickening, which gives rise to the lens, and the optic vesicle; and is carried in by the lens as this becomes invaginated. Most other investigators maintain that it arises from mesoblast which gains admittance into the globe of the eye through the choroidal fissure. This mesoblastic investment of the lens is very vascular, the blood being brought to it by a branch of the central artery of the retina (Fig. 155, A0). This divides into very numerous branches on the inner or deeper surface of the lens, which 
extend round its margin and cover the outer surface as well. There are no veins which correspond exactly to this artery, the

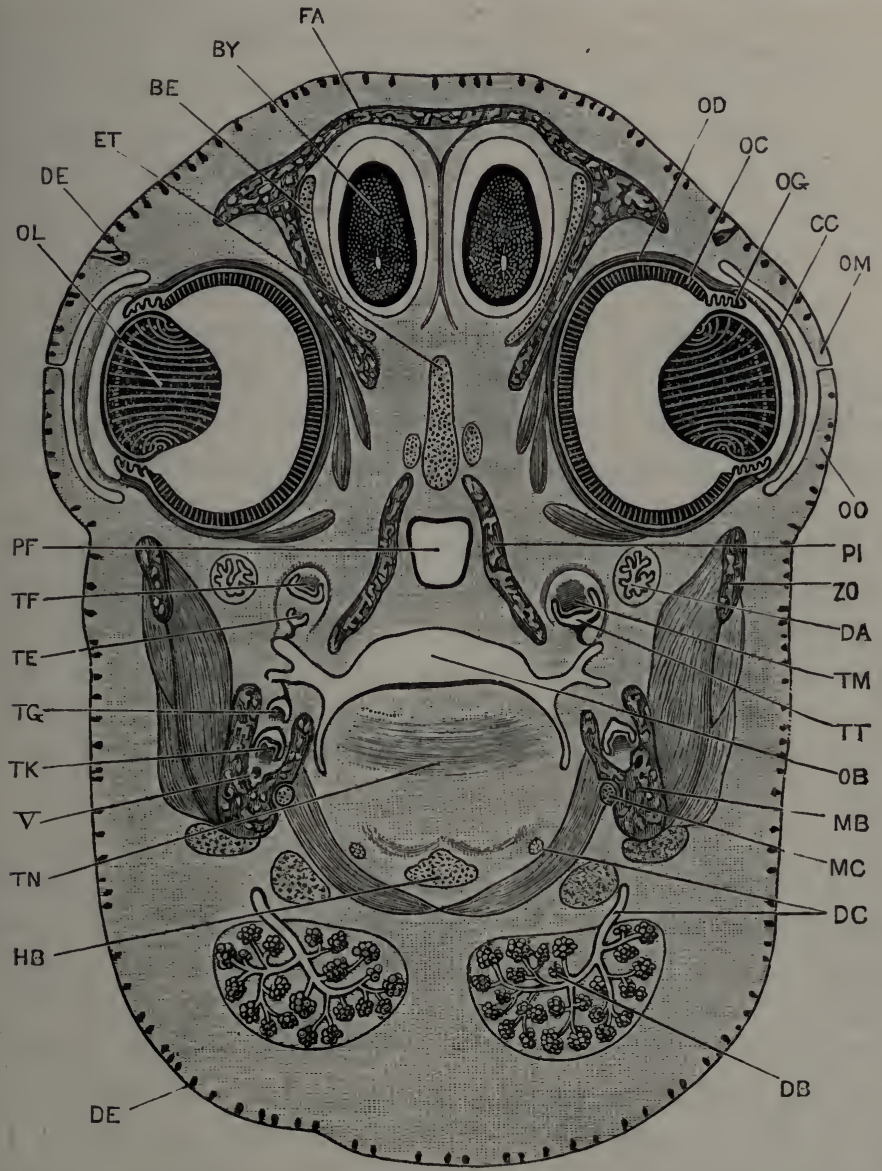

FIG. 156.-A transverse section across the head of a Rabbit Embryo of the twenty-first day, the section passing through the centres of the eyes (of. Figs. 149 and 151). $\times 8$.

BE, orbito-sphenoidal cartilage. BY, olfactory lobe. CC, cornea. DA, suborbital gland. DB, submaxillary gland. DC, dnet of submaxillary gland. DE, hair follicles. $\mathbf{E T}$, pre-sphenoidal cartilage. $\mathbf{F} \mathbf{A}$, frontal bone. HB, basihyal cartilage. IMB, mandible. MC, Meckel's cartilage. OB, buccal cavity. OC, inner or retinal layer of optic cup. OD, outer or pioment layer of optic cup. OG, iris. OL, lens. OM. upper eyelia. OO, lower evelid. PE', posterior narial passage. PI, palatine bone. TE, deciduons grinding tooth of upper jaw. TF, permanent grinding tooth of upper jaw. TG, deciruous grinding tooth of lower jaw. TK, permanent grinding tooth of lower jaw. TM, tooth papilla. TN, tongue. TT, enamel organ. ZO, zygoma. V, mandibular branch of trigeminal nerve.

blood being returned by veins in connection with the iris. This vascular investment to the lens is merely a provisional structure, 
serving for its nutrition during growth ; it disappears completely when the lens has reached its full size.

The vitreous body is derived from the mesoblast which grows into the cavity of the optic cup, through the choroidal fissure. It is extremely vascular in the early stages of its formation, receiving its blood from the central artery of the retina. There is no structure in the rabbit corresponding to the pecten of the bird.

The cornea (Fig. 156, cc) is formed, as in the chick, from mesoblast, which spreads in between the surface epiblast and the lens; and the anterior chamber of the eye is a space which appears at a rather late stage, between the cornea and the lens.

The choroid and sclerotic are formed, as in the chick, from the mesoblast surrounding the optic cup. They are developed very late; and on the twenty-first day (Fig. 156), when the cornea is well developed, the choroid and sclerotic are merely represented by a thin layer of connective tissue, devoid of pigment. The sclerotic of the rabbit is not cartilaginous at any stage.

The eyelids are folds of skin, above and below the eyeball. They appear early, and by the fourteenth day have attained some size (Fig. 155, om, oo). By the nineteenth or twentieth day they have grown completely over the eye, and meet each other along their free edges ( $c f$. Fig. 149); a little later (Fig. 156 , ом, oo) the edges of the two eyelids fuse together, the epidermal layers becoming continuous with each other; this fusion persists throughout the remaining period of development, and is the cause of the blindness characteristic of the young at birth.

The third eyelid, or nictitating membrane, is a similar fold of skin, arising at the inner angle of the eye, and lying between the other two eyelids and the eyeball.

The lacrymal glands arise as solid ingrowths of epiblast into the underlying connective tissue, which subsequently become hollowed out to form the cavities of the glands and ducts. 


\section{The Ear.}

The ear of the rabbit, like that of the chick, is derived from a pitting-in of the epiblast at the side of the hind-brain. By closure of its mouth, the pit becomes a vesicle or sac, imbedded in the mesoblast of the side of the head; and from the walls of this sac, which are of epiblastic origin, the epithelial lining of the vestibule and of its various prolongations is derived; the semicircular canals, cochlea, and other parts being formed by outgrowths or constrictions of the originally simple sac.

The mesoblast immediately surrounding the sac gives rise to the connective tissue wall of the auditory labyrinth, while the mesoblast a little distance off gives origin to the cartilaginous auditory capsule ( $c f$. Fig. 159). Between the labyrinth and the cartilaginous capsule a series of lymphatic spaces appear, filled with fluid, in which the labyrinth hangs suspended. Finally, important series of accessory organs, characteristic of air-breathing Vertebrates-the tympanic membrane, Eustachian tube, auditory meatus, auditory ossicles, and external ear-are formed, and acquire definite relations with the essential organ of hearing, i.e. the auditory vesicle itself.

The auditory vesicles arise in the rabbit, towards the end of the ninth day, as a pair of shallow depressions of the epiblast at the sides of the hind-brain. During the tenth day each pit deepens rapidly, and by the end of the day the mouth of the pit narrows and closes, converting the pit into the closed auditory sac or vesicle (Fig. 147, EI), which lies imbedded in the side wall of the head, opposite the first branchial arch.

The auditory vesicle is at first spherical, but soon becomes triangular in outline as seen in transverse sections. The dorsal angle of the triangle, which marks the place where the vesicle separates from the external epiblast, grows upwards as a long tubular process, the recessus vestibuli (Fig. 158, ER), which follows the curvature of the brain wall, and ends blindly at its dorsal extremity.

From the outer side of the vestibule, a wide lateral diverticulum arises, from which the semicircular canals are developed at a slightly later stage (Fig. 158, ED, EH). The ventral angle of the vestibule is prolonged downwards and inwards as a curved finger-like process, the cochlear canal (Fig. 158, EL). 
The auditory nerve (Fig. 158, vili) develops very early, as already noticed, and by the tenth day, if not indeed from its

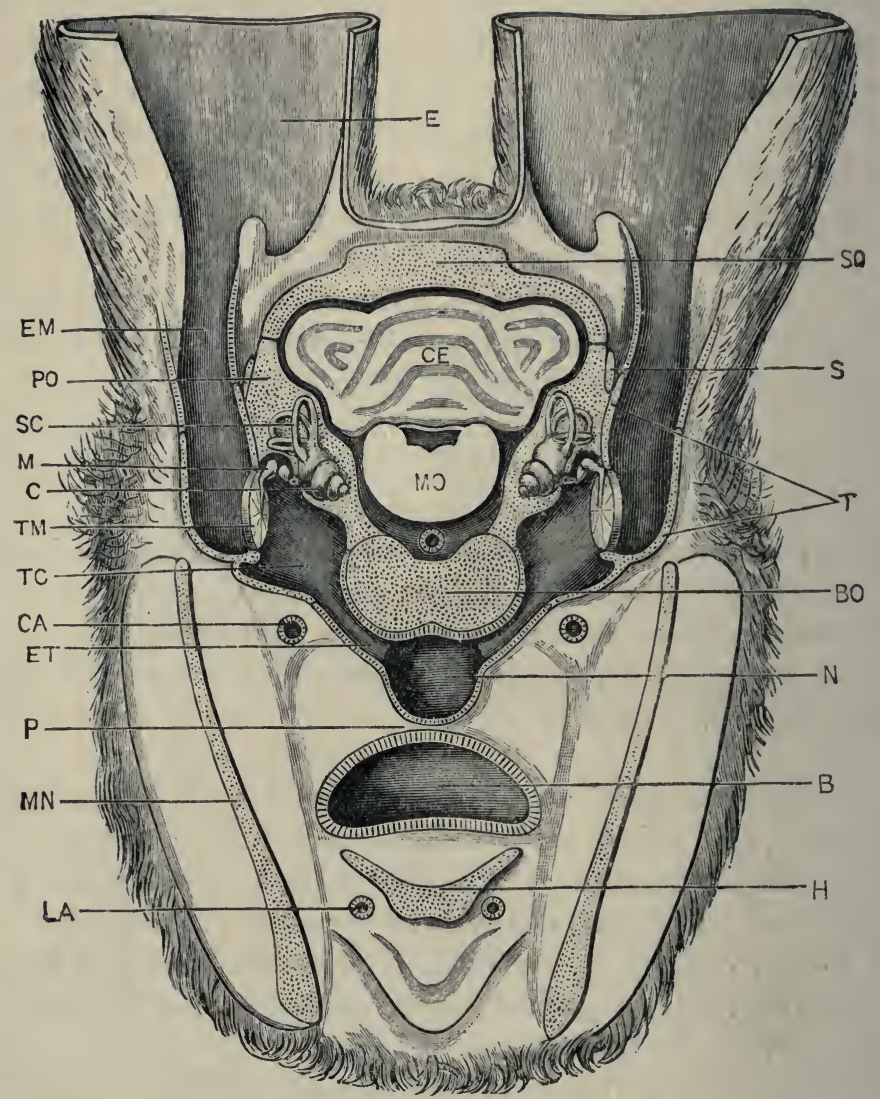

FIG. 157.-A diagrammatic section across the head of an adult Rabbit, to show the relations of the internal ear, tympanic cavity and membrane, and the auditory ossicles. The section is drawn as seen from the front, and is taken along a line joining the reference letters so and $\mathrm{M} N$ in Fig. 162 (p. 405). The external ears are cut short, close to their bases, and the floccular lobes of the cerebellum, which lie between the three semicircular canals of each side, are omitted entirely. (From Marshall and Hurst.)

B, buceal cavity. BO, basi-occipital. C, cochlea. CA, right external carotid artery. $\mathbf{C E}$, cerebellum. $\mathbf{E}$, external ear or piuna. EM, external auditory meatus. $\mathrm{ET}$, Eustachian tube. $\dot{H}$, body of the liyoid. I A, right lingual artery. M. malleus ; to its inner side are seen the incus and stapes. MN, mandible. MO, medulla oblongata. $\mathbf{N}$, posterior nasal chamber. $\mathbf{P}$, soft palate. $\mathbf{P O}$, periotic. S, post-tympanic process of squamosal. SC. anterior vertical semicircular canal. SO, supra-occipital. T, tympanic bone. TC, tympanic eavity. TM, tympanic membranc.

earliest appearance, is intimately connected with the inner wall of the vestibular sac. 
The condition on the fifteenth day is shown in Fig. 159, the section, on the left side, being taken at a level slightly anterior to that of the right side. The recessus vestibuli, ER, is still large, and is dilated at its upper end in a club-shaped manner. The three semicircular canals are. well established: they are formed, as in the chick and frog, from flattened saccular out-

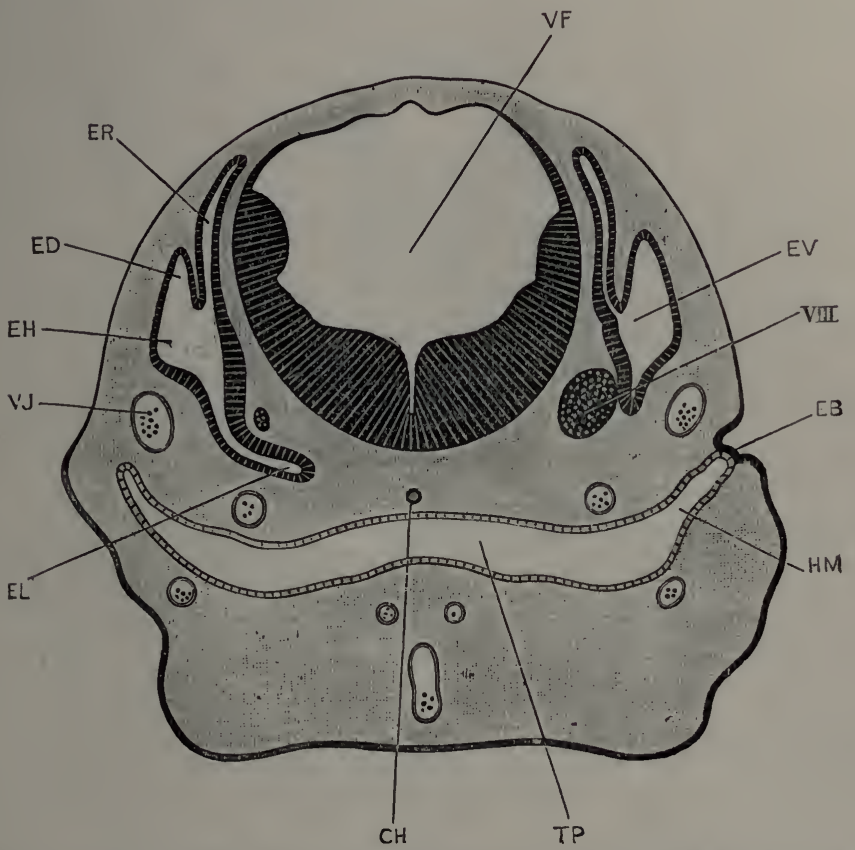

FIG. 158.-A transverse section across the head of a Rabbit Embryo at the end of the eleventh day, the section passing through the medulla oblongata, the ears, and the pharynx. The plane of section of the right half of the figure is slightly anterior to that of the left half. (Compare Fig. 147.) $\times 30$.

CH, notochorl. EB, membrane closing hyomandibular cleft. ED, common stem of the two vertical semicircular canals. EH, rudiment of the exterual or horizontal semicircular canal. EL, coclilear canal. ER, recessus vestibuli. EV, auditory vesicle. HM, hyomanilibular pouch. TP, pharynx. VF, fourth ventriclc. VJ, jugular vein. VIII, anditory nerve.

growths of the auditory vesicle, the two walls of each outgrowth coming in contact and fusing, so as to form a curved tube opening into the vestibule at both ends. The section (Fig. 159) passes through the stem common to the two vertical semicircular canals, ED, and also through the horizontal canal, kI. Each semicircular canal has already acquired an ampulla at one end. 
The body of the vestibule is partially divided by a constriction into a larger division, the utriculus, with which the semicircular canals are connected; and a smaller division, the sacculus, which opens through a narrow neck, the canalis reuniens, into the cochlear canal, EL. This latter is a tube of fairly

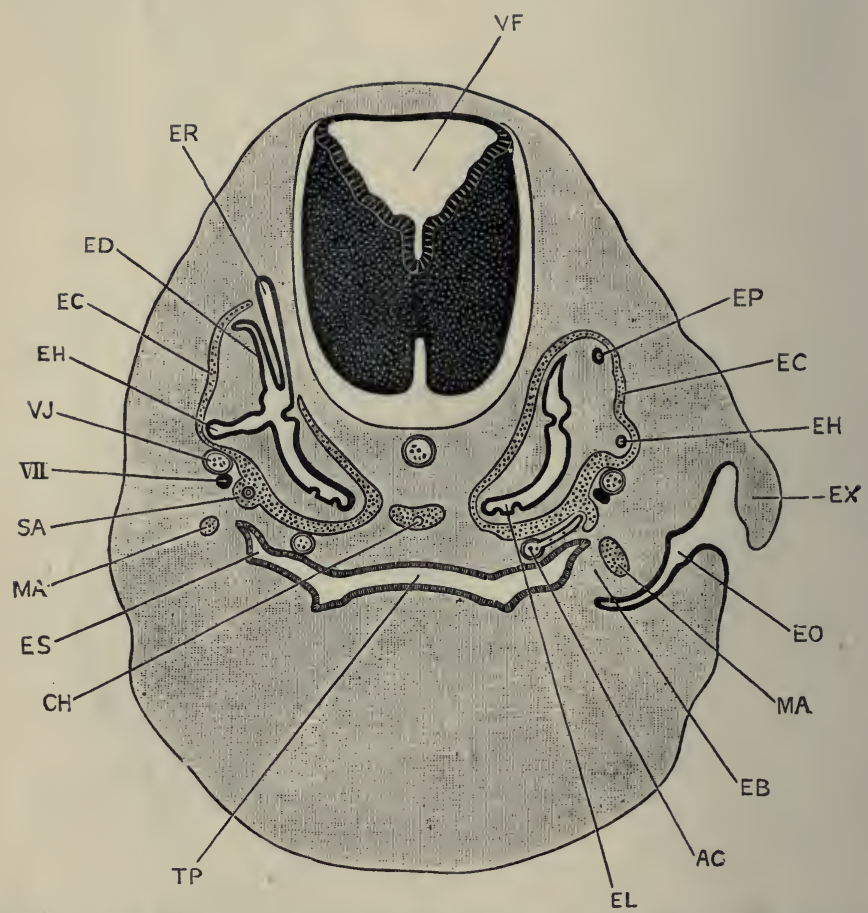

FIG. 159.-A transverse section across the head of a Rabbit Embryo of the fifteenth day, passing through the medulla oblongata, the ears, and the pharynx. The plane of section of the left side of the figure is slightly anterior to that of the right side. $\times 16$.

AC, carotill artery, giving off a small branch which runs through the areh of the stapes. CH, notochorl. EB, tympanic membrane. EC, cartilaginous auditory capsule. ED, common stem of the two vertical semicircular canals. EH, exterual or horizontal semicircular canal. EL, eochlear canal. EO, external auditory meatus. EP, posterior vertical semicircular canal. ER, recessus vestibuli. ES, Eustachian passage. EX, external ear. IMA, malleus. SA, stapes. TP, pharynx. VF, fourth ventricle. VJ, jugular vein. VII, facial or seventh cranial nerve.

uniform diameter, curved as shown in the figure, and with its wall markedly thicker along the inner than the outer side of the curve. A cartilaginous periotic capsule, $\mathrm{EC}$, is present, surrounding the ear, but at some little distance from it; the recessus vestibuli alone projecting beyond the capsule. 
$\Lambda t$ a stage a little later than that shown in Fig. 159, the cochlear canal, which up to this point has been only slightly curved, begins to form the spiral turns, so characteristic of the adult (Fig. 157, c), the twisting being brought about by growth in a spiral manner of the blind end of the canal.

The cochlear canal becomes the scala media of the cochlea in the adult. Immediately outside it the mesoblast becomes excavated to form a couple of tubular passages, the scala vestibuli and scala tympani, which lie respectively above and below the scala media or cochlear canal. The scala vestibuli and scala tympani commence at the basal end of the cochlea, and gradually extend along it towards its apex, following the turns of the spiral ; and ultimately, on reaching the apex, they open into each other, though not until a very late stage of development. From the epithelium of the floor of the cochlear canal, or basilar membrane, the organ of Corti is developed; while the roof of the cochlear canal, separating it from the scala vestibuli, is spoken of in the adult as the membrane of Reissner.

At the base of the cochlea, the scala vestibuli opens into the peri-lymphatic space surrounding the central part of the vestibule, while the scala tympani is closed at its base by the membrane of the fenestra rotunda.

Similar peri-lymphatic passages are formed, by excavation of the mesoblast, around the semicircular canals.

As in the frog, there is at first a single patch of the epithelium of the auditory vesicle with which the auditory nerve is continuous. 'This single, large patch becomes ultimately broken up into several smaller ones, which by growth of the intervening strips of epithelium are separated further and further from one another until they reach their adult positions.

The accessory auditory apparatus of the rabbit is, in a general way, similar to that of the frog or the chick, but is more complicated.

The Eustachian tube (Fig. 157, ET) is formed from the hyomandibular gill-pouch. This reaches very close to the surface in the early stages of development, but does not open to the exterior at any period. On the eleventh day (Fig. 158, HM) the hyomandibular pouch reaches almost to the surface, the hypoblast of the pouch meeting the epiblast at the bottom of 
the external groove, so that the cleft is at this stage closed only by a very thin branchial membrane, $\mathrm{EB}$, formed of epiblastic and hypoblastic layers without any intervening mesoblast. As shown in the figure, the hyomandibular pouch lies at this stage some distance ventral to the ear, and the two structures are completely independent of each other.

By the fifteenth day (Fig. 159) the conditions have changed materially. A thick layer of mesoblast has grown in between the epiblast and hypoblast of the branchial membrane, so that the hyomandibular pouch is now separated from the surface of the head by a thick plate, $\mathrm{EB}$, which becomes later on the tympanic membrane. Further, by growth upwards of its lips, and through the general thickening of the side walls of the head, the shallow hyomandibular groove of the earlier stage is converted into a deep pit, Eo, the external auditory meatus, the margin of which is already commencing to grow out as the rudiment of the pinna or external ear ( $c f$. Figs. 149 and 157).

In the later stages ( $c f$. Fig. 157), the external meatus, EM, becomes much longer, and the pinna attains enormous dimensions; the tympanic membrane, TM, becomes relatively much thinner than at the fifteenth day; while the Eustachian passage becomes more distinctly tubular, and, owing to the formation of the palate, $P$, now opens into the posterior narial chamber instead of directly into the buccal cavity.

With regard to the auditory ossicles of the rabbit, it is difficult to speak with certainty. The stapes (Fig. 159, SA) forms, about the fifteenth day, as a ring of cartilage, which from its first appearance is in close connection with the outer wall of the periotic capsule, and apparently continuous with this, at the place where the fenestra ovalis is formed a little later. The ring-like form of the stapes is apparently due, as shown in Fig. 159, to its being formed around a small branch of the carotid artery, AC.

Concerning the origin of the other two auditory ossicles of the mammal, the malleus and incus (Figs. 157, M, and 159, MA), there has been much discussion. While it appears very probable that they are formed in connection with the cartilaginous bars of one or more of the visceral arches, investigators differ widely as to whether both are developed from the mandibular bar, 
which is perhaps the most generally accepted view, or both from the hyoidean bar, or one from each of these bars. There are, at present, no recorded observations which determine the matter satisfactorily in the case of the rabbit.

The malleus can be recognised on the fifteenth day (Fig. 159, IA) ; it is, from its first appearance, imbedded in the substance of the tympanic membrane, $\mathrm{EB}$, and is for some time continuous witl the posterior end of the mandibular bar, or Meckel's cartilage.

\section{THE DEVELOPMENT OF THE DIGESTIVE SYSTEM.}

\section{A. The Alimentary Canal.}

\section{General Account.}

The general history of the development of the alimentary canal of the rabbit is closely similar to that of the chick. The greater part of the length of the canal is formed from the mesenteron, which, as in the chick, is a tubular cavity included within the embryo by the process of constriction, through which the embryo becomes separated from the yolk-sac ( $c f$. Figs. 146 and 147). Owing to this mode of formation of the mesenteron, it necessarily communicates with the cavity of the yolk-sac in the early stages, and so long as the yolk-stalk remains tubular. The mesenteron may, therefore, as in the chick, be divided into three lengths: fore-gut, mid-gut, and hind-gut; the fore-gut (Figs. 146 and 147, GF) being the anterior portion, in which roof, sides, and floor are all alike present; the hind-gut, $\mathrm{GH}$, being the similar portion at the hinder end of the body; and the mid-gut, GT, being the median portion, which opens through the yolk-stalk into the cavity of the yolk-sac, and which consequently has no floor. Fore-gut and hind-gut increase in length, at the expense of the mid-gut, as the embryo becomes more and more sharply constricted from the yolk-sac; and ultimately, when the yolk-stalk becomes solid, about the thirteenth day, the mid-gut as a separate division of the alimentary canal ceases to exist ( $c f$. Figs. 146, 147, and 150).

The mouth and anal openings are formed, as in other Vertebrates, by stomatodxal and proctodæal invaginations of the epiblast at the anterior and posterior ends of the embryo 
respectively, which meet and open into the mesenteron, and so place it in communication with the exterior.

The alimentary canal is at first straight, or merely follows the curvature of the body, and is situated immediately ventral to the notochord. It remains in this condition, throughout life, in the pharyngeal and osophageal regions, and also at its extreme hinder end; but along the rest of its extent it shifts ventralwards, remaining connected with the dorsal wall of the body cavity by a mesentery (Fig. 165, $\mathrm{MH}$ ). In the region of the small intestine the alimentary canal increases in length far more rapidly than the body of the embryo, and becomes in consequence thrown into folds, in order that it may be accommodated within the body cavity.

\section{The Stomatodæum.}

The relations of the stomatodral pit are practically the same as in the chick. Perforation of the stomatodæal membrane is effected at an early stage, before the end of the tenth day. The pituitary body arises, still earlier, as a diverticulum from the posterior and dorsal angle of the stomatodæal pit; its further development has already been described in the section dealing with the formation of the brain (p. 376).

\section{The Buccal Cavity and Pharynx.}

The pharyngeal region of the mesenteron is, from the first, distinguished by its great width (Figs. 158 and 159, TP). Early on the tenth day, the branchial pouches arise as paired diverticula from the sides of the pharynx; and, opposite to the outer ends of the branchial pouches, branchial grooves are formed on the surface of the neck, marking out the boundaries of the several visceral arches. The walls of the branchial pouches and of the corresponding branchial grooves come into close contact, a thin branchial membrane (Fig. 158, EB), consisting of epiblast and hypoblast, without any intervening mesoblast, alone separating the two. This membrane, however, remains intact; and in the rabbit none of the gill-clefts are ever completely formed, or open to the exterior at any stage of development. There are also no traces of gills, either external or internal, at any period in the rabbit.

The visceral arches are well developed, and on the twelfth 
day (Fig. 161) the maxillary, $\mathrm{MX}$, mandibular, $\mathrm{MN}$, hyoid, $\mathrm{HY}$, and first branchial arches form conspicuous ridge-like projections
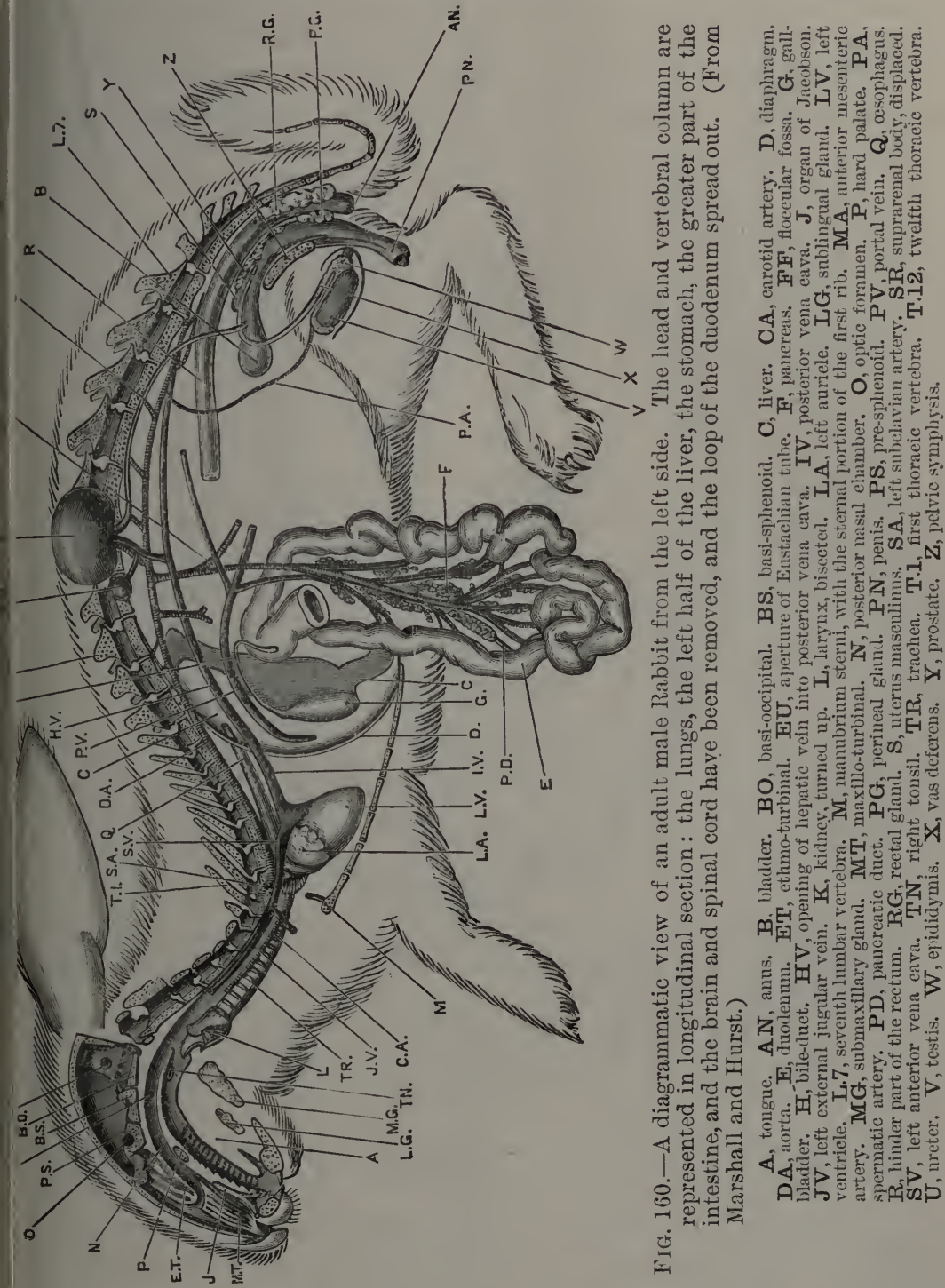

in the side walls of the pharyngeal region. Of the branchial grooves, or external depressions separating the successive arches, 
the hyomandibular groove, нм, is by far the most conspicuous. The further development of the hyomandibular groove, and the mode in which it gives rise to the external auditory meatus, have already been described in the section dealing with the ear (p. 397).

The tongue is developed as a swelling in the floor of the buccal cavity; it commences to form on the twelfth or thirteenth

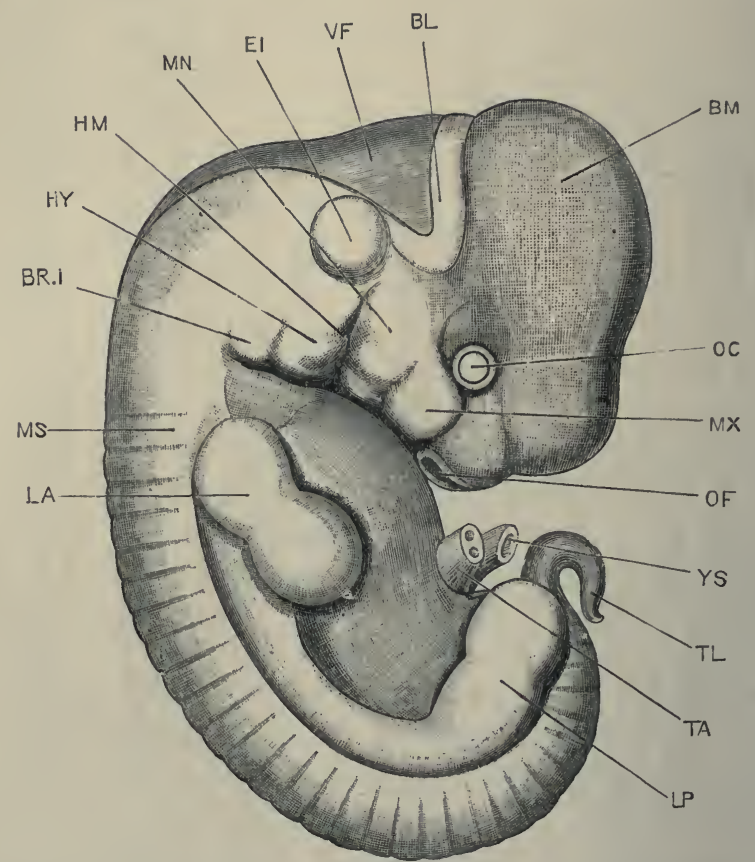

FIG. 161.-A Rabbit Embryo at the end of the twelfth day, seen from the right side. The yolk-stalk and allantoic stalk are cut short, close to the body of the embryo. $\times 9$.

BI, cerebellum. BM, mid-brain. BR.1, first branchial arch. EI, auditory vesicle. HM, hyomandibular groove. HY, hyoid arch. IA, fore limb. IP, hinit limb. MN, mandibular areh. MS, mesoblastic somite or protovertebra. IMX, maxillary arch. OC, eye. OF', olfactory pit. TA, allantoic stalk, cut short. 'TL, tail. V F , fourth ventricle of brain. YS, yolk-stalk, cut short.

day, and by the eighteenth day (Fig. 151, TN) has attained the form characteristic of the adult.

The boundary line between stomatodæum and mesenteron is impossible to fix absolutely, in the later stages of development; but its position may be approximately determined, if it be remembered that the stalk of the pituitary body (Fig. 151, PT) marks the posterior boundary of the stomatodæum in the mid- 
dorsal line; while, on the floor of the buccal cavity, the boundary line lies in front of the root of the tongue; the whole of the tongue being formed from the mesenteron, and being therefore covered with hypoblastic epithelium.

The palate is formed, about the fifteenth day, by a pair of horizontal ridges which grow inwards from the sides of the buccal cavity, and, meeting each other in the median plane, fuse to form a horizontal shelf (Fig. 151, PL), which separates the nasal chamber above from the buccal cavity below. The fusion of the two halves of the palate proceeds from before backwards; and the palate ends with a free posterior edge, behind which the nasal and buccal chambers are continuous with each other (Fig. 151, TP).

\section{The Esophagus.}

The hinder end of the pharynx narrows very rapidly, and passes abruptly into the straight tubular œsophagus (Figs. 150, and 151 , TO). It has not yet been determined whether the cesophagus of the rabbit, like that of the chick and tadpole, passes through a stage in which it is solid for a time.

\section{The Stomach and Intestine.}

The stomach becomes evident, as a distinct dilatation of the alimentary canal, about the thirteenth day; its long axis at first corresponds with that of the body, but later on it shifts its position, and becomes placed at first obliquely, and then almost directly across the body.

The intestine undergoes changes corresponding fairly closely with those already described for the chick. The lengthening of the intestine is effected almost entirely in two situations, giving rise to two ventrally directed loops. Of these, the proximal or duodenal loop is formed immediately behind the stomach, and in the rabbit attains a considerable length (Fig. $160, \mathrm{E})$. The distal or vitelline loop is formed by elongation of the >-shaped loop of the intestine already present on the twelfth day, and from the apex of which the yolk-stalk, YK, arises; the vitelline loop attains an enormous length in the rabbit.

A short length of the intestine, between the duodenal and ritelline loops, remains in the rabbit, as in the chick, stationary 
throughout the whole period of development; it is attached to the dorsal wall of the body cavity by a very short mesenterial fold, and is easily recognised in an adult rabbit.

A well-developed post-anal gut, or prolongation of the hinder end of the intestine into the tail, is present on the tenth and eleventh days. By the twelfth day (Fig. 150), the greater part of this has already disappeared; a small diverticulum of the cloacal cavity, GP, marks its basal portion, and detached fragments of it may persist for a time at intervals along the tail.

This post-anal gut is probably a secondary feature, and due, as in the frog, to the drawing out of the alimentary canal into the tail as this latter lengthens.

\section{The Proctodæum.}

The proctodæum in the rabbit is little more than the actual anal opening; it develops late, and is usually not formed until about the sixteenth day.

\section{B. Organs Developed in connection with the Alimentary Canal.}

\section{The Teeth.}

Teeth are cutaneous structures, developed from the mucous membrane covering the jaws. They appear in rabbit embryos during the third week, and are at first independent of the bones of the jaws; indeed, the upper teeth develop before the maxillary bones are formed (cf. Fig. 156). The jawbones, however, soon acquire close relations with the teeth, growing round them, and inclosing them in sockets.

In the rabbit, as in Mammals generally, there are two sets of teeth, known as milk or deciduous, and adult or permanent, respectively. The deciduous dentition of the rabbit is represented by the formula :-di. $\frac{2}{1}$; dc. $\frac{0}{0} ; \mathrm{dm} . \frac{3}{2}$; the corresponding formula for the permanent dentition being,

$$
\text { i. } \frac{2}{1} \text {, c. } \frac{0}{0}, \mathrm{pm} . \frac{3}{2}, \mathrm{~m} \cdot \frac{3}{3} \text {. }
$$

The milk, or deciduous, teeth in the rabbit are lost very early. The deciduous incisors, corresponding to the large chisel-shaped incisors of the permanent set, are very small, and are shed before the birth of the young rabbit. The second pair of deciduous 
incisors of the upper jaw are much larger, and persist as functional teeth for about three weeks after birth, lying wedged in between the large and small permanent incisors. For the first three weeks after birth there are therefore three upper incisors on each side in the rabbit; the first and third being the permanent incisors, and the middle one being the deciduous second incisor, which has not yet been shed. The deciduous molars (Fig. 156, TF, TG) are of considerable size, and persist

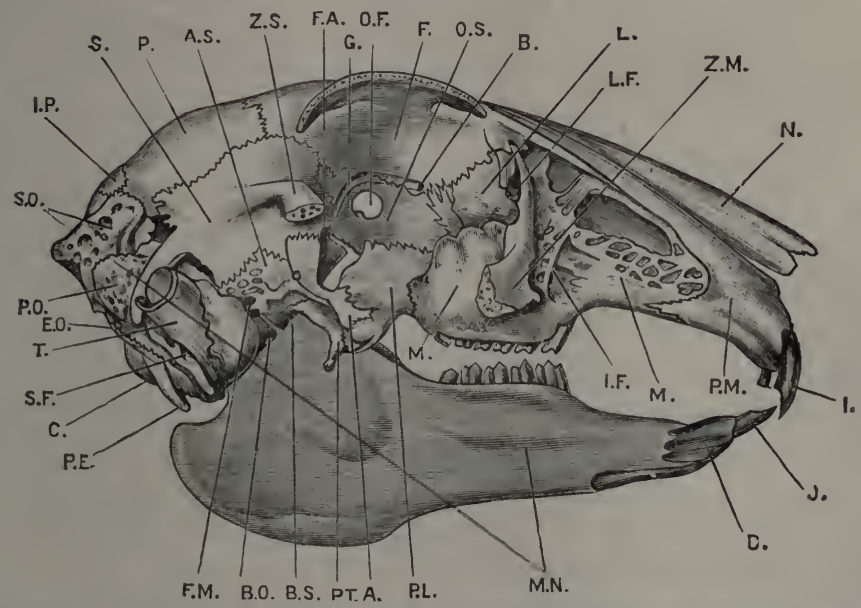

FIG. 162.-The skull of the Rabbit, from the right side. The middle portion of the zygomatic arch and the right half of the mandible have been removed. (From Marshall and Hurst.)

A, external pterygoicl process of ali-splienoil. AS, ali-splenoid. B, internal orbital foramen. BO, hasi-occipital. BS, basi-sphenoid. C, occipital condyle. D, mandibular sympliysis. $\mathbf{E O}$, ex-nceipital. $\mathrm{F}$, frontal. $\mathbf{F A}$, foramell lacernm anterius. $\mathbf{F} \mathbf{M}$, foramen lacerum medium. $\mathbf{G}$, orbital groove, for ophthalmic division of trigeminal nerve. I, anterior upper incisor. IF, infra-orbital foramen. IP, inter-parietal. J, lower incisor. I, lacrymal bone. LF', lacrymal foramen. $\mathbf{M}$, maxilla. MN, mandible. $\mathbf{N}$, nasal bone. OF, optic foramen. OS, orbito-sphenoil. $\boldsymbol{\perp}$, parietal. PE, parnccipital process of ex-occipital. PI, palatine bone. PMI, pre-maxilla. PO, periotic. PT, pterygoid. S, squamosal. SE', stylo-mastricl foramen. SO, supra-occipital. T, tympanic bone. ZM. zy.gomatic process of maxilla, cut short. ZS, zygomatic process of syunamosal, eut sliort.

until three or four weeks after birth, when they are pushed out by the permanent premolars developed beneath them.

A fully formed tooth consists chiefly of dentine, covered on its crown, or grinding surface, with a cap of a very hard and densely calcified substance, the enamel; and invested, especially round its deeper part or root, by a layer of bony substance, the cement. The dentine is hollowed out by the pulp cavity, in which are lodged the blood-vessels and nerves of the tooth. 
These gain admittance through a larger or smaller hole in the root, or fang, of the tooth; the hole remaining widely open throughout life in the rabbit, and other animals in which the teeth grow continuously throughout life, but becoming reduced to one or more very small apertures in the majority of Mammals, in which the teeth cease growing after they have reached their full size.

Teeth, as already noticed, are cutaneous structures; and of the substances of which the tooth consists, the enamel is formed from the epithelium, and the dentine and cement from the underlying connective tissue layer or dermis.

The first step in the formation of a tooth consists in an ingrowth from the deeper layer of the epithelium into the connective tissue of the gum (cf. Fig. 156). This ingrowth soon becomes hollow and flask-shaped, its deeper end dilating into a sac (Fig. 156, TT), while its superficial part forms a narrow solid neck or stalk, which remains in connection with the surface epithelium. Opposite the deeper end of the flask, the connective tissue of the gum becomes condensed to form the dental papilla (Fig. 156, TM). The deeper end of the epithelial flask, or enamel organ as it is called (Fig. 156, TT), now becomes closely applied to the dental papilla, which gradually acquires the definite shape of the crown of the tooth to which it is going to give rise.

The enamel organ (Fig. 156, TT), at this stage, is a flattened sac, consisting of outer and inner epithelial layers, and having its cavity occupied by a reticulum of stellate cells; the outer epithelial layer is still connected with the surface epithelium by a narrow stalk or string of cells, while the inner layer forms a cap, closely embracing the top and sides of the dental papilla.

This cap consists of a single layer of very regularly arranged, six-sided, columnar epithelial cells; and it is by calcification of the substance of these cells that the enamel layer of the tooth is produced. Calcification commences at the surface of the enamel organ next to the dental papilla, and gradually spreads outwards through the cells of the enamel organ.

The dentine is formed by calcification of the dental papilla, and is therefore of mesoblastic origin. Calcification appears first at the surface of the papilla next to the enamel organ, so that the crown of the tooth is the first part to be formed; and, 
when once completed, no further change in the shape of the crown can occur.

'The mode of formation of the dentine is as follows. The cells at the surface of the dental papilla form a single layer of finely granular nucleated cells, closely arranged side by side, and spoken of as odontoblasts. The most superficial parts of the odontoblasts become converted into, or else form by excretion, a gelatinous matrix in which calcification soon occurs, forming the dentine. The deeper parts of the odontoblasts, containing the nuclei, remain soft and unaltered; they give off fine processes torvards the surface of the tooth, which lie in channels in the dentine, these channels being the dentinal tubules of the adult. By a continuance of this process the dentine increases in thickness; the odontoblasts, which are the active agents in the process, forming a layer on the inner surface of the dentine, and sending out fine radial prolongations into the dentinal matrix.

The follicle, or tooth-sac, is formed by a condensation of the vascular mesoblast around the tooth. The cement is a thin layer of bone formed round the tooth by the wall of the follicle, which acts as the periosteal membrane.

The permanent teeth are formed in the same way as the deciduous teeth ; their enamel organs arising as outgrowths from the necks of those of the deciduous teeth (Fig. 156).

\section{The Thyroid Body.}

'The thyroid body of the rabbit arises early in the tenth day, as a median thickening of the epithelium of the floor of the pharynx, which grows downwards into the connective tissue immediately in front of the pericardial cavity. The stalk of connection with the pharyngeal floor narrows, and during the eleventh day disappears, leaving the thyroid as a solid epithelial body (Fig. 150, TH) embedded in the mesoblast of the floor of the pharynx, immediately in front of the truncus arteriosus, and between the roots of the carotid arches.

In the later stages the thyroid body widens transversely, giving off two lateral lobes which rapidly increase in size. A cavity appears in the median portion, and soon extends into the lateral lobes, from which outgrowths, some hollow and some solid, soon arise. As the heart shifts backwards into the thorax, 
the thyroid body also moves its position, coming into close relation with the upper rings of the trachea.

\section{The Thymus.}

The thymus of the rabbit is formed by bud-like outgrowths from the epithelium of one of the hinder branchial pouches. These buds first become conspicuous about the fourteenth day; they soon separate from the walls of the pharynx, and gradually shift backwards, increasing greatly in size as they do so, until they reach their final position at the anterior end of the thorax.

\section{The Lungs.}

The lungs arise in the rabbit, much as in the chick or frog, from the ventral wall of the mesenteron, at the place where it narrows, immediately behind the pharyngeal region, to form the œsophagus.

On the tenth day the cavity of the œsophagus, which is elsewhere circular in transverse section, becomes laterally compressed at its anterior end, immediately behind the pharyngeal region. By the outgrowth of two horizontal ridges from its side walls, which meet and unite in the median plane, a short length of the osophagus becomes divided into two tubes: of these, the dorsal tube is the œsophagus itself; while the ventral one, or laryngeal chamber, is a short tube, ending blindly behind, but opening in front into the osophagus through the orifice which afterwards becomes the glottis ( $c f$. Fig. 150).

From the laryngeal chamber the lungs arise, on the eleventh day, as a pair of lateral diverticula, which grow backwards along the dorsal part of the body cavity and the sides of the œsophagus (Fig. 150, LG).

The lungs, being thus formed as outgrowths from the alimentary canal, will, like the canal itself, have mesoblastic walls, lined by a hypoblastic epithelium.

On the twelfth day secondary ontgrowths arise from the main tube or bronchus of each lung, and these in the later stages branch freely to form the smaller bronchi, from the terminal branches of which the air cells are formed about the time of birth.

The branchings of the bronchi occur almost entirely towards the dorsal and outer surfaces of the lungs (Fig. 163), so that the original or main bronchial tubes, LB, lie close to the inner surfaces 
of the lungs. The smaller bronchi divide, for the most part, in a regular, dichotomous manner, as shown on the right-hand side of Fig. 163. The branching at first affects the hypoblastic lining alone, but about the thirteenth or fourteenth day (Fig. 163) the mesoblastic wall becomes divided by external

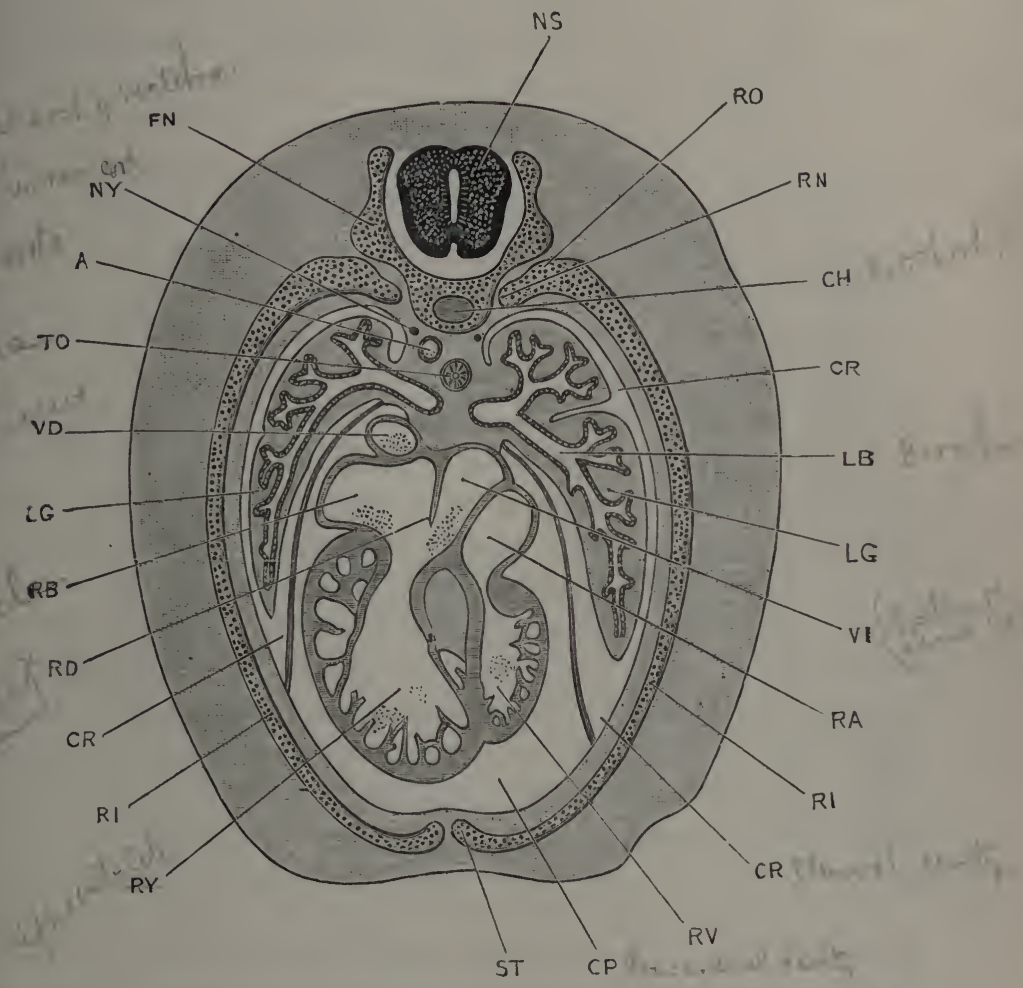

FIG. 163.-A transverse section across the thorax of a Rabbit Limbryo of the sixteenth day. $\times 15$.

A, llorsal aorta. CH, notochord. $\mathbf{C P}$, pericarlial eavity. $\mathbf{C R}$, pleural carity. FN, neural arch of vertebra. LB, bronchus. LG, lumg. NS, splinal cord. NY, simpathetic nerve corl. RA, right anriele. RB. left auricle. RD, inter-aurienlar septum. RI, rib. RN, capitulum of rib. RO, tubercle of ril. RV, riglit ventricle. RY. left ventricle. ST, ventral ent of rib, from which the sternmm is formed. TO, ocoplatisus. VD, left anterior vena cava. VI, posterior vena cava.

grooves or clefts, which mark out the boundaries of the main lobes of the lungs. The trachea, or median part of the air passage, is at first very short; but, as the neck elongates, and the lungs get carried back into the thorax, the trachea rapidly increases in length, and by the eighteenth day ( $c f$. Fig. 151) 
the proportions are not very unlike those of the adult. 'The glottis, L'T, at this stage is a longitudinal slit-like opening in the ventral wall of the osophagus; it is overhung by the epiglottis ( $c f$. Fig. 150, LE), a fold at the back of the pharynx, marked off from the tongue by a well-marked transverse groove. The glottis leads into a dilated laryngeal chamber (Fig. 1 $\tilde{1} 1$ ), which is continued down the neck as the trachea, LR. The thyroid, CT, cricoid, and tracheal cartilaginous rings are already present, and have the same relations as in the adult.

\section{The Liver.}

The liver of the rabbit, like so many other important organs, commences on the tenth day, arising as a diverticulum from the ventral surface of the mesenteron, about the junction of the stomach and duodenum. This diverticulum, which becomes the left bile duct of the adult, is directed ventralwards, and its blind end is in close relation with a thickened mass of condensed mesoblast which forms part of the ventral body-wall of the embryo, behind the heart, and in front of the yolk-stalk.

On the eleventh day, the right bile duct arises as an outgrowth from the left duct, close to its opening into the duodenum. From the lining epithelium of both right and left ducts, solid rods of hypoblast cells, the hepatic cylinders, grow out into the mass of condensed mesoblast around them. The hepatic cylinders branch and anastomose freely, forming a reticulum, the meshes of which are occupied by the connective tissue, in which blood-vessels early appear in large numbers.

By a series of further changes, which have not yet been accurately determined in the rabbit, the adult liver is formed. The cells of the reticulum become the hepatic cells, which are thus of hypoblastic origin; while some at least of the cylindrical rods become hollow, and form the bile passages, which communicate directly or indirectly with the bile ducts ( $c f$. Fig. $150, \mathrm{w})$.

The gall bladder arises on the eleventh day, as a diverticulum form the right bile duct.

The relations of the blood-vessels to the liver are much the same as in the chick, and will be described more fully in the section dealing with the development of the vascular system. It may be noticed here that in its early stages the liver has, as 
in the chick, especially close relations with the vitelline veins, through which the blood is returned to the embryo from the yolk-sac.

From the twelfth day onwards, the common part of the bile duct, where the right and left bile ducts join to enter the duodenum, lengthens rapidly, thus giving rise to the single bile duct of the adult rabbit (cf. Fig. 160, II).

\section{The Pancreas.}

The pancreas arises, on the twelfth day, as a swelling or bulging of the dorsal wall of the intestine, slightly further back than the bile duct, and opposite the yolk-stalk. On the eleventh day it becomes much more sharply defined, and constricted off from the gut as a somewhat pyriform, hollow sac, which opens into the dorsal wall of the intestine, and gives off small buds from its surface. In the succeeding days these buds enlarge, and give off other similar buds from their sides, and so form a compound gland of the racemose type. The pancreas, therefore, in its mode of development agrees closely with the salivary or other ordinary glands, and differs markedly from the liver.

As the alimentary canal is at first straight, and lies but a little distance ventral to the notochord (Fig. 150), the pancreas, in its early stages, is embedded in the dorsal wall of the body cavity, and wedged in between the intestine and the dorsal aorta. As the intestine lengthens, to form the duodenal loop, the pancreas is drawn down- with the mesentery, between the two limbs of the loop, and so attains its adult position (Fig. $160, \mathrm{~F})$.

\section{The Cloaca.}

On the twelfth day (Fig. 150), the rectum opens into a dilated cloacal chamber, TC, from which the stalk of the allantois, $\mathrm{TA}$, opens, and into which the Wolffian ducts, $\mathrm{KC}$, and ureters, $\mathrm{KD}$, discharge. This cloacal dilatation lies in a rounded cloacal papilla, which forms a well-marked projection on the ventral surface of the embryo, behind the allantoic and yolk stalks, and in front of the root of the tail (Fig. 1 50 ).

Just before entering the cloacal dilatation, the intestine makes a rather sharp bend ventralwards, the distinctness of which is slightly exaggerated in Fig. 150. Between the intestine and the 
cloacal dilatation, and separating the two structures from each other behind the point of opening of the Wolffian duct, is a septum of connective tissue, which is well seen in the figure, where it is crossed by the reference line, TC.

During the next three days this septum extends backwards, its growth being effected by the union, in the median plane, of two lateral ridges to form a median partition. This partition divides the cloacal chamber into two separate portions, a dorsal or rectal chamber, and a ventral or urino-genital chamber. The proctodæal opening is established by this time, and the partition, on reaching the surface, divides this opening into separate anal and mino-genital apertures, the partition itself forming the perinæum, or transverse septum between the two apertures.

The anal opening lies on the posterior surface of the cloacal papilla, almost in the angle between the papilla and the tail, so that the cloacal papilla is from this time concerned with the urino-genital organs alone. The cloacal, or genital papilla as it may now be termed, elongates considerably, and the urino-genital aperture is prolonged as a median groove along its dorsal or posterior surface. From this stage, development differs in the two sexes: in the male the two lips of the groove unite to form the penial urethra, the papilla itself becoming the corpus spongiosum of the penis. In the female the groove remains open, its borders forming the lips of the vulva.

\section{DEVELOPMENT OF THE HEART AND BLOOD- VESSELS.}

The general relations of the heart and its various cavities, and of the great arterial and venous trunks, and the changes which they undergo during development, are much the same in the rabbit as in the chick, and it will not be necessary to describe them in detail in this chapter. The changes in the leart itself, and especially the mode of formation of the septa, by which the several cavities are shut off one another, will require closer consideration.

Besides the vessels of the embryo itself, there are two extraembryonic vascular systems:-(i) the vitelline, or yolk-sac circulation, which is comparatively unimportant in the rabbit; and (ii) the allantoic or placental circulation, which is of the 
utmost importance, as it affords the means through which the embryo receives its supply of mutriment from the mother, and is enabled to effect the necessary respiratory and excretory interchanges.

The vitelline circulation will be dealt with in the present section; the relations of the allantoic ressels in the placenta will be treated separately, in the concluding section of this chapter.

\section{The Heart.}

The heart of the rabbit, like that of the chick, is formed by the union of the two vitelline veins, which return to the embryo the blood from the vascular area.

The vitelline veins are formed in the mesoblast of the splanchmopleure, and appear at an early stage of development, when the folding-off of the embryo from the yolk-sac, by the side folds, has only just commenced. The right and left vitelline veins, and consequently the two halves of the heart as well, are therefore at first a considerable distance apart; and in rabbit embryos of the ninth day (Fig. 145, R) they appear as a pair of tubes, lying along the sides of the head, opposite the hind-brain.

As the side-folds deepen, constricting off the embryo from the yolk-sac, the two tubes get carried round to the ventral surface of the embryo, where they lie close together, side by side. About the middle of the tenth day they fuse together to form a single tubular heart, lying in the floor of the pharyngeal region of the mesenteron, and having relations very similar to those of the heart in a chick embryo of about the thirtieth hour.

In the latter part of the tenth day, the heart, while it remains attached to the floor of the pharynx at both its ends, becomes free in the middle portion of its length; and, growing rapidly, hangs down iuto the body cavity as a loop, which soon becomes twisted on itself like a letter $\mathrm{S}$, and partially divided by constrictions into chambers (Fig. 147, R). The hinder or proximal limb of the heart, which receives the great veins, is the sinus venosus; the first loop of the $S$ is the auricular portion; the second loop is the ventricular portion; and the distal or anterior limb is the truncus arteriosus, from which the aortic arches arise as right and left branches. 
The later changes undergone by the heart are of very considerable interest, and have been described with great care by Born. It will be convenient to deal with the several cavities in order, beginning at the hinder or venous end of the heart.

The sinus venosus, on the tenth day, is a vessel running transversely across the body, and slightly enlarged at its two ends to form the right and left cornua or horns. Each horn receives three veins:-(i) the Cuvierian vein, which is formed by the junction of the anterior and posterior cardinal veins, returning venous blood from the body of the embryo generally; (ii) the vitelline vein, returning blood from the yolk-sac ; (iii) the allantoic vein, returning blood from the allantois. The sinus venosus is at this stage nearly symmetrical, and opens into the auricular cavity by a wide median aperture.

By the eleventh or twelfth day, the right horn of the sinus venosus has become much larger than the left horn. The allantoic and vitelline veins, in place of opening separately into the sinus venosus, now unite before reaching the heart, and discharge into the sinus through a single vein, the posterior vena cava. The posterior vena cava and the right Cuvierian vein, or right anterior vena cava as it is now termed, open into the larger or right horn of the sinus venosus; while the smaller left horn receives only the left Cuvierian vein, or left anterior vena cava. The opening from the sinus venosus into the auricle has now become more slit-like, and leads distinctly into the right half of the auricular chamber; the slit-like opening is bounded by two valve-like folds of the endocardial lining of the heart, which may be termed the right and left venous valves respectively.

At a later stage the sinus venosus becomes absorbed into the right auricle, of which it now forms part ; the three venæ cavæ opening separately into the auricular cavity. Of the two venous valves, the left one disappears, while the right one becomes the Eustachian valve, by which the blood from the posterior vena cava, and for a time that from the right anterior vena cava as well, is directed into the left auricle.

The auricular portion of the heart. The originally single auricular chamber becomes divided into right and left auricles by a septum, which arises during the twelfth day from the dorsal 
wall of the auricular chamber, and grows down into its cavity (cf. Fig. 163, RD). For a time the lower and posterior edge of the auricular septum is free, but during the fourteenth day it meets and fuses with a cushion-like thickening of the margin of the auriculo-ventricular aperture.

Before this fusion is completed, however, a new aperture, the foramen ovale, is formed in the dorsal and anterior part of the auricular septum, through which free communication between the two auricles is maintained up to the time of birth of the young rabbit.

The pulmonary veins develop rather late, and are of small size until near the time of birth; the two veins, from the right and left lungs respectively, unite to form a single vessel, which opens into the dorsal wall of the left auricle, very close to the auricular septum.

The ventricular portion of the heart. The ventricular cavity is at first single, and receives the blood from the auricular cavity through a transverse slit in its dorsal wall.

The division of the ventricular cavity into right and left ventricles is effected by a septum, which grows upwards from the apex of the ventricle towards the auriculo-ventricular aperture. This ventricular septum (cf. Fig. 163) appears about the twelfth day, and its position is indicated from an early period by a groove on the surface of the heart. The septum remains incomplete for some time, the two ventricles communicating above its free edge. About the fifteenth day the septum meets, and unites with, the cushion-like thickenings of the margin of the auriculo-ventricular aperture, and so completes the separation between the two ventricles.

The thickening of the wall of the ventricle is effected in the first instance, just as in the frog, by the ingrowth of muscular trabeculæ into the cavity, which unite to form a reticulum (cf. Fig. 163), the proper wall of the ventricle remaining thin. In the later stages, however, the outer walls of the ventricles thicken considerably throughout their entire substance. Up to the time of birth there is practically no difference in thickness between the walls of the right and left ventricles, the resistance to be overcome being approximately the same in the two cases. 
The truncus arteriosus becomes divided, much as in the chick or in the frog, by an internal longitudinal septum; which, arising between the roots of the systemic and pulmonary arches, grows backwards in a somewhat spiral course, dividing the truncus arteriosus into right or pulmonary, and left or aortic tubes. The septum continues its growth backwards until it meets the upper free edge of the ventricular septum, with which it fuses.

After the truncus arteriosus is thus divided internally, an external groove appears on its surface, opposite the internal septum; and this groove deepens until it splits the truncus arteriosus into two completely separate and independent vessels, of which the right one, or pulmonary trunk, arises from the right ventricle, and the left one, or aortic trunk, from the left ventricle.

The semilunar valves are formed by projections of the thickened endocardium at the roots of the pulmonary and aortic trunks: the valves are at first thick and soft, but later on become membranous.

\section{The Arteries.}

In the rabbit, as in the chick, five pairs of aortic arches are developed, which appear in order from before backwards. By the middle of the tenth day the first two pairs are present, in the mandibular and hyoidean arches respectively. By the end of the tenth day a third pair of aortic arches is present, in the first branchial arches; and before the end of the eleventh day the remaining two pairs are established, in the second and third branchial arches respectively.

Of these five pairs of aortic arches, the first two pairs, in the mandibular and hyoidean arches respectively, lose their connections with the dorsal aortæ during the eleventh day, and become reduced to the arteries of the floor of the mouth and of the tongue.

The aortic arches of the third pair, in the first branchial arches, persist as the carotid arteries. They retain for a time their connections at their dorsal ends with the fourth pair of arches, but ultimately lose these, and from this time send blood to the head alone; each divides into external and internal carotid arteries, supplying the parts outside and inside the skull respectively. 
The aortic arches of the fourth pair, in the second branchial arches, are the systemic arches, which by their union form the dorsal aorta. At first the vessels of the two sides, right and left, are of equal size, but from a very early period the left one becomes the larger, and ultimately forms the arch of the aorta in the adult. The right systemic arch persists for some time, but ultimately disappears, with the exception of its proximal part, which is said to give origin to the right subclavian artery.

The aortic arches of the fifth pair, in the third branchial arches, are the pulmonary arches : from them the pulmonary arteries arise as posteriorly directed branches. The pulmonary arches retain their connections with the dorsal aortæ throughout the whole period of intra-uterine life, up to the time of birth; these connections having, as in the chick embryo, a most important influence on the course of the circulation. At the time of birth, the part of each pulmonary arch between the origin of the pulmonary artery and the aorta ( $c f$. Fig. 128), a part known as the ductus arteriosus or ductus Botalli, becomes obliterated; and from this time the blood driven into the pulmonary arches by the right ventricle can no longer pass directly to the aorta, but is all sent through the pulmonary arteries to the lungs.

Zimmermann has found traces, in rabbit embryos of the eleventh day, of a pair of aortic arches between the systemic and pulmonary arches. This observation, if confirmed by future investigation, will be of considerable interest, as showing that the pulmonary arches of the rabbit are the sixth and not the fifth pair, and that the pulmonary arteries therefore arise in the rabbit from the same pair of arches as in the frog; in other words, that the pulmonary arteries are strictly corresponding structures in these two types.

As regards the arteries of the trunk, the two dorsal aorta are at first distinct along their whole length, and the allantoic arteries appear as though they were direct posterior continuations of the aortx. Later on, the two aortæ unite to form the definite clorsal aorta, which is continued as a narrow median candal artery to the hinder end of the embryo; the allantoic arteries from this time appearing as branches of the aorta. 


\section{The Veins.}

The general relations of the veins in the rabbit, and the changes which they undergo during development, are very similar to those described in the next chapter as seen in the human embryo, and will not be dealt with further in this section ( $c f$. pp. 578 to 583 ).

\section{The Course of the Circulation.}

It will be convenient to give here a brief account of the course of the circulation during the latter half of intra-uterine life, when the placental circulation is in full swing; and also a summary of the changes which occur at the time of birth.

As regards the heart, the ventricular septum is complete, as is also the septum of the truncus arteriosus. The auricular septum is, however, incomplete, the foramen ovale allowing blood to pass across directly from the right to the left auricle.

The blood is brought to the right auricle by the three venæ cavæ. Of these, the right and left anterior venæ cavæ-the Cuvierian veins of the earlier stages-return to the heart venous blood from the head and from part of the trunk of the embryo. This is received into the right auricle and driven by it into the right ventricle.

The blood in the posterior vena cava is derived from many sources. The main factors are the allantoic veins, which return to the heart the blood from the placenta, blood which is arterial both as regards nutritive matter and as regards its contained gases.

The other factors of the posterior vena cava are, the vitelline veins from the yolk-sac, which are small and comparatively unimportant; the mesenteric veins, which return venous blood from the alimentary canal of the embryo, and which are of small size; and the posterior vena cava itself, which returns blood from the kidneys and the hinder part of the body. Of these factors, the allantoic veins are so much the largest that the blood returned by the posterior vena cava to the heart may be rightly spoken of as arterial. This arterial blood is discharged into the right auricle, but never really enters the cavity of the auricle, since it is directed at once, by the Eustachian valve, through the foramen ovale into the left auricle, and driven thence into the left ventricle. 
The right ventricle is thus filled with venous blood, and the left ventricle with arterial blood. On the ventricular systole, the arterial blood from the left ventricle is driven through the aortic trunk and the carotid arteries to the head; while the venous blood from the right ventricle is driven through the pulmonary trunk and pulmonary arches into the dorsal aorta, and then backwards along the body, the greater part passing along the large allantoic arteries to the placenta.

'The changes that occur' at birth are practically the same as those which are effected in the chick on liatching ( $c f$. p. 314).

(i) The vitelline and allantoic circulations are stopped. The result of this is that the blood in the posterior vena cava is from this time venous, since the arterial supply previously brought by the allantoic veins is now cut off.

(ii) The ductus venosus, or direct passage through the liver, is closed. The effect of this change is that all the blood brought to the liver must now pass through its capillaries in order to get to the heart, whereas formerly the ductus venosus afforded a short cut by which the liver capillaries could be avoided.

(iii) The ductus arteriosus, or ductus Botalli, closes on both sides of the body. This renders it impossible for blood from the right ventricle to get directly into the aorta. All the blood from the right ventricle lias now to pass along the pulmonary arteries to the lungs, and the pulmonary vessels consequently dilate very considerably, to accommodate this increased quantity of blood. A further effect is that the dorsal aorta now receives its blood supply from the left ventricle instead of, as formerly, from the right ventricle; i.e. it now contains arterial, instead of venous blood.

(iv) The foramen ovale closes. This is effected at a rather later stage than the other changes. When it is completed, the blood from all three venæ cavæ enters the right auricle, and is driven from this into the right ventricle; while the only blood entering the left auricle is now the blood returned from the lungs by the pulmonary veins, vessels which up to the time of birth are comparatively small and insignificant, but which dilate very greatly as soon as lung breathing is established.

The circulation, by these changes, becomes that of the adult. A complete double circulation is established; the right and left 
sides of the heart are perfectly distinct from each other; and to get from one side to the other the blood must pass through either the pulmonary or the systemic circulation.

\section{The Circulation in the Yolk-sac.}

The circulation in the yolk-sac is definitely established by the tenth day, and presents some points of interest.

In the rabbit, as in other Vertebrates, the vitelline vessels are developed in the inner, or splanchnopleuric layer of the mesoblast, beyond the embryonal area (Figs. 146 and 147). The mesoblast, as already noticed, only extends over the upper half of the blastodermic vesicle; the lower half, or hemisphere, having a wall composed of epiblast and hypoblast alone. The boundary between these two halves is a sharp one, and is indicated by an annular vessel, the sinus terminalis (Figs. 146 and 147, SI), which runs round the margin of the mesoblast, and marks the outer limit of the vascular area.

The course of the vitelline vessels in the rabbit differs in some important respects from that of the chick. In the chick the vitelline arteries and veins lie in two layers, the reins being dorsal or superficial to the arteries; and the sinus terminalis is a vein, which collects the blood from the marginal part of the vascular area and returns it, by branches which form main factors of the vitelline veins, to the heart ( $c f$. Fig. 99).

In the rabbit, on the other hand, all the vessels of the vascular area lie in one plane. The vitelline arteries run straight backwards from the embryo, and open at once into the sinus terminalis, which is therefore an artery, and not, as in the chick, a vein. From the vitelline arteries themselves, and from the sinus terminalis, smaller arteries arise, which branch freely and end in capillaries; the capillaries unite to form veins which open finally into the vitelline veins themselves, a pair of large vessels which run in the vascular area, concentrically with the sinus terminalis, but about midway between this and the embryo. Opposite the anterior or head end of the embryo, the vitelline veins turn sharply backwards, and, entering the embryo along the yolkstalk, run forwards to the heart.

There are at first two vitelline arteries, and two vitelline veins. Of the two arteries, the left one soon becomes much the larger, the right one appearing as a mere branch of it. Both 
vitelline veins may persist, but more usually the right one becomes much reduced in size, or else atrophies completely.

In the rabbit, the vitelline circulation is of much less importance than in the chick, inasmuch as the nutrition of the rabbit embryo is effected, not by the yolk-sac, but by the placenta.

\section{DEVELOPMENT OF THE EXCRETORY SYSTEM.}

The general history of the development of the excretory organs and their ducts in the rabbit is much like that of the click.

No trace of a head kidney, or pronephros, has yet been described, and it may be assumed that this structure is either altogether absent, or else very small and rudimentary. A segmental, or Wolffian duct is early formed along each side of the body; and in connection with each duct a Wolffian body is developed, which is large in the embryo, but which becomes replaced functionally by the metanephros or permanent kidney in the later stages and in the adult animal. The Müllerian duct develops rather later than the Wolffian duct and Wolffian body; it lies very close to the Wolffian duct, but is apparently independent of this.

\section{The Wolffian Duct.}

The mode of development of the Wolffian duct in the rabbit has been much debated; the point in dispute being whether it is formed from mesoblast, or directly from the external epiblast.

According to the observations of Hensen, supported by Flemming, the Wolffian duct arises, early in the ninth day, as a solid ridge-like thickening of the epiblast (Fig. 164, KC), at the level of the fourth and fifth mesoblastic somites, and close to their onter borders. It soon separates from the epiblast, and then lies as a solid rod of cells between the epiblast and mesoblast; this rod grows rapidly backwards, becomes tubular by the formation of an axial cavity or lumen, and on the eleventh day reaches the hinder end of the body, and opens into the dorsal surface of the allantois, just in front of the union of the rectum and the allantois to form the cloaca ( $c f$. Fig. $150, \mathrm{KC}, \mathrm{TC}$ ).

There is no doubt that the Wolffian duct, in the early stages of its development, lies very close indeed to the epiblast, espe- 
cially at its hinder end; but the more recent and very careful observations of Martin show conclusively that it is merely a case of very close apposition, and that the duct is really of mesoblastic origin along its entire length : its mode of formation being practically the same as that already described in the case of the chick, p. 315 .

\section{The Wolffian Body.}

The Wolffian body commences to form, in the latter part of the ninth or early part of the tenth day, as a series of solid strings

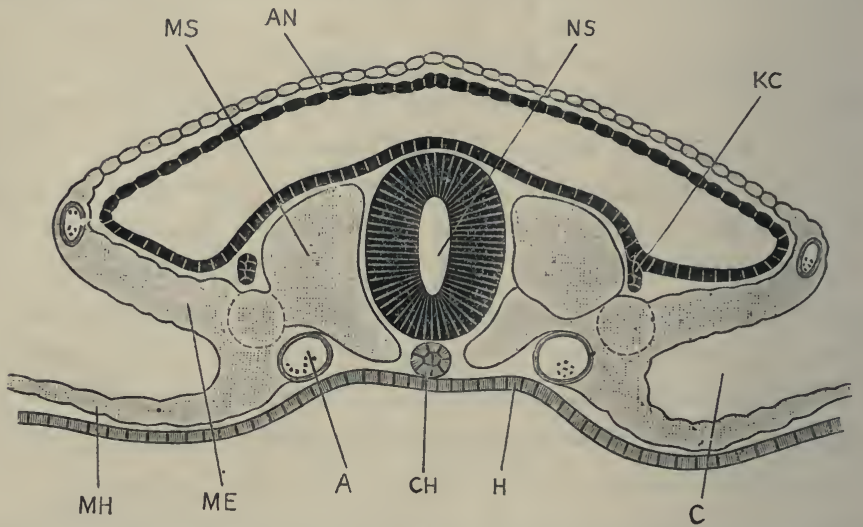

FIG. 164.-A transverse section across the body of a Rabbit Embryo of the early part of the tenth day, showing the supposed epiblastic origin of the Wolffian duct. (After Hensen.) $\times 75$.

A, dorsal aorta. AN, amnion. C, cœlom, or body cavity. CH, notochord. $\mathbf{H}$, hypoblast. KC, Wolffian duct. ME, somatopleuric layer of mesoblast. MH, splanchnopleuric layer of mesoblast. MS, mesoblastic somite or protovertebra. NS, central canal of spinal cord.

of cells, which lie to the inner side of the Wolffian duct along almost its entire length.

These strings of cells are stated to arise as ingrowths from the peritoneal epithelium; but the point is not definitely established, and from a very early stage the strings lie embedded in the mesoblast, and quite independent of the peritoneum. The strings soon become tubular, and are then spoken of as the Wolffian tubules. Each tubule opens at one end into the Wolffian duct; while its opposite, or blind end, becomes expanded into a vesicle, and then doubled up on itself to form a Malpighian body, into which a branch of the aorta quickly penetrates to form the glomerulus (Fig. 165, GM). The Wolffian tubules are not seg- 
mentally arranged; two or three corresponding to each somite in the region of the body in which they occur.

The relations of the Wolffian body to the blood-vessels are well seen in Fig. 165. The dorsal aorta, A, lies between the two Wolffian bodies, and gives off branches which supply the

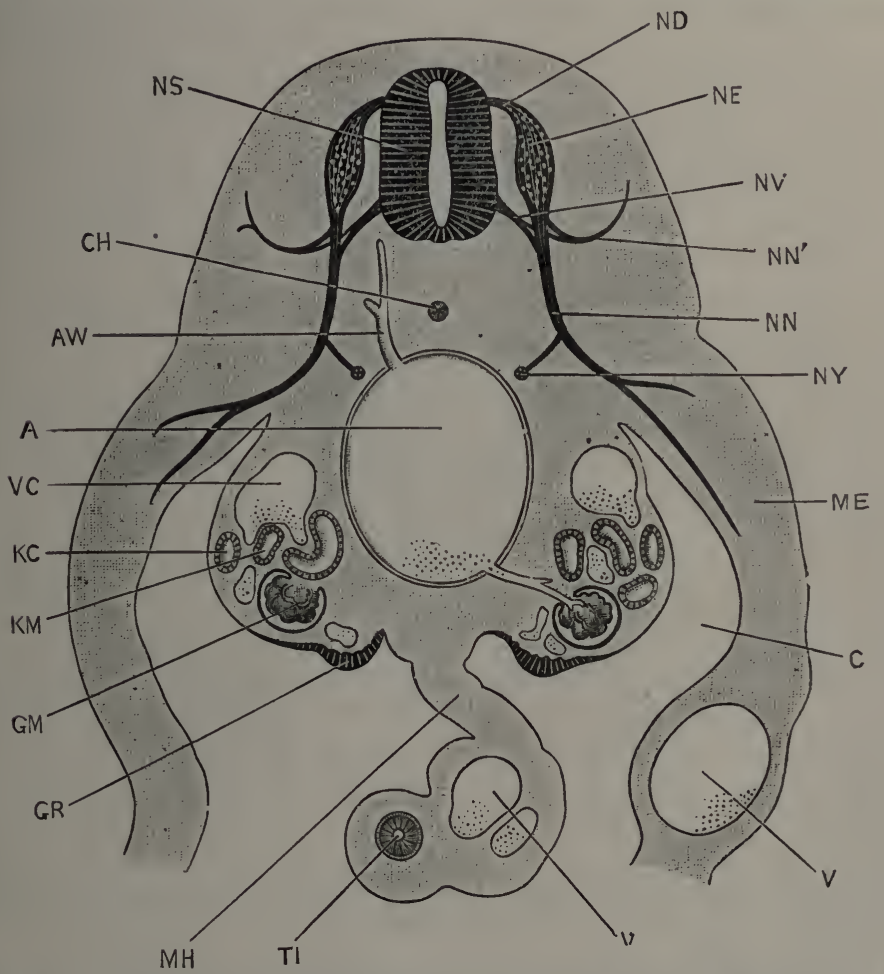

FIG. 165.-A transverse section across the body of a Rabbit Embryo at the end of the eleventh day. $\times 45$.

A, dorsal aorta. A W, lumbar arters. C, cœlom, or bolly cavity. CH, notochori. $\mathbf{G} \hat{\mathbf{M}}$, glomertulus of Malpighian body. GR, genital ridge. KC, Wolffian cluet. $\mathbf{K M}$, tubule of Wolffian body. ME, somatopleure. MH, mesentery. ND, dorsal root of spinal nerve. $\mathbf{N E}$, spinal ganglion. $\mathbf{N N}$, ventral division of spinal nerve. $\mathbf{N N}^{\prime}$, dorsal division of spinal nerve. NS, spiual cord. NV, ventral root of spinal nerve. NY, sympathetie ganglion. TI, iutestine. V, vein. Vं C, posterior cardinal vein.

glomeruli; while the posterior cardinal veins, vc, lie along their dorsal surfaces, and give off numerous branches, which lie in very close relation with the tubules, and from which the epithelial cells of the tubules withdraw the excretory products.

The Wolffian body increases rapidly in size, and soon becomes more compact and of more definite shape. Its position and rela- 
tions on the twelfth day are well seen in Fig. 150, where the transversely running Wolffian tubules, KM, opening into the longitudinal Wolffian duct, $\mathrm{KC}$, give the whole organ a somewhat comb-like appearance.

By the fourteenth day, the Wolffian body (Fig. 166, KM) has increased still further in size, especially at its hinder end; and

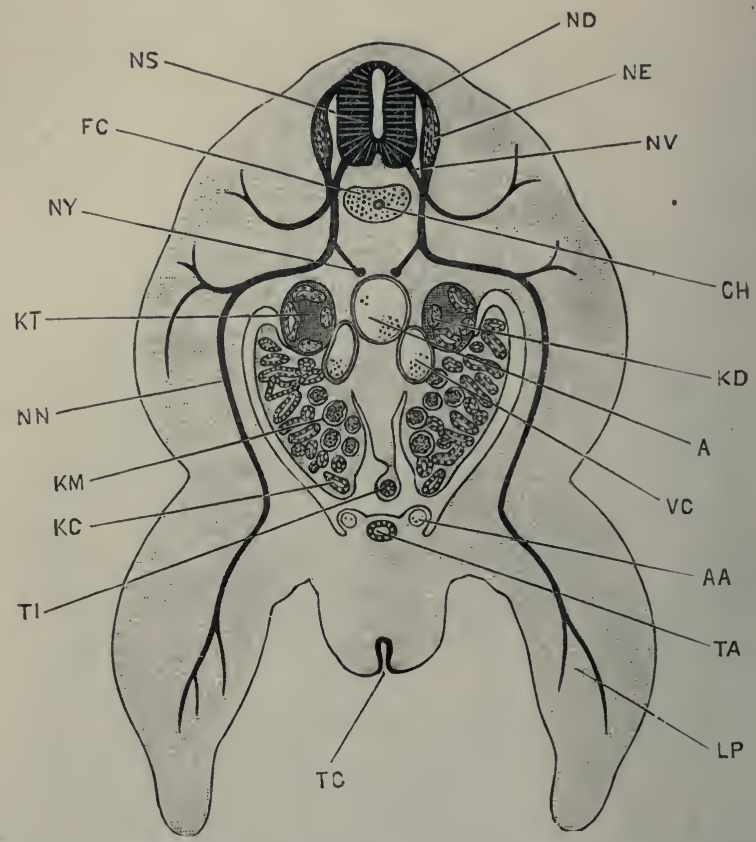

FIG. 166.-A transverse section across the hinder part of the body of a Rabbit

Embryo of the fourteenth day, the section passing through the hind limbs. $\times 12$.

$\mathbf{A}$, dorsal aorta. A A, allantoic artery. $\mathbf{C H}$, notochord. $\mathbf{F} \mathbf{C}$, centrum of vertebra. KC, Wolffian duct. KD, ureter, or metanepliric duct. KM, Wolffim bols. KT. kidney or metanephros. LP, hind limb. ND, dorsal root of spinal nerve. NE, spinal ganglion. NN, ventral division of spinal nerve. NS, spinal cord. NV, ventral ront of spinal nerve. NY, longitudinal sympathetic cord. TA. stalk of allantois. TC, cloacal aperture on cloacal papilla. TÍ, intestine. VC, posterior cardinal vein.

it remains of large size until within a short time of birth. It is the excretory organ of the embryo, and its great size and abundant vascular supply indicate that it is of considerable functional importance.

\section{The Kidney and Ureter.}

The adult kidney, or metanephros, develops in the rabbit in much the same manner as in the chick. 
On the eleventh day a diverticulum arises from each Wolffian duct, just before this reaches the cloaca, and grows forwards dorsal to the Wolffian duct. During the twelfth day this diverticulum (Fig. 150, KD) increases in length ; its blind anterior extremity dilates, and gives rise to branching tubular processes; while the mesoblast surrounding the processes becomes more compact than elsewhere, giving definite shape to the organ.

The structure formed in this way becomes the kidney, the original diverticulum from the Wolffian duct forming the ureter. 'The kidney extends forwards, dorsal to the Wolffian body, and overlapping this, so that both the Wolffian body and the kidney may be cut in the same transverse section (Fig. 166, KM and KT).

In the later stages, the lateral branches from the ureter subdivide, and elongate considerably to form the kichney tubules. There are for some time no Malpighian bodies in the kidney, but these are ultimately formed in connection with the blind ends of the tubules, in the same mamer as in the Wolffian body.

The kidneys are, from the first, compact bodies; they early acquire their characteristic shape (Fig. 160, k), and also their asymmetry in position, the right kidney moving some distance further forwards than the left.

The Wolffian duct and the ureter of each side open, on the twelfth day (Fig. $150, \mathrm{KC}, \mathrm{KD}$ ), by a common duct into the urino-genital sinus. In the later stages, by unequal growth in different directions, and by absorption of the common duct into the urino-genital sinus, the relations become altered; the Wolffian ducts still opening into the urino-genital sinus, but the ureters now opening directly into the bladder.

\section{The Miullerian Duct.}

The mode of the development of the Müllerian duct in the rabbit has not been very clearly ascertained. About the twelfth or thirteenth day it is present as a peritoneal funnel, lying to the inner side of the Wolffian body, close to its anterior end; from the funnel a duct arises which crosses over, dorsal to the Wolffian body, and then runs backwards a short distance, lying very close to the outer side of the Wolffian duct, and ending blindly behind. During the succeeding days the Miillerian 
duct grows slowly backwards, but does not reach the level of the urino-genital sinus until about the twentieth day.

\section{The Genital Ducts and Accessory Genital Organs.}

In the male, or buck rabbit, outgrowths from the tubules of the Wolffian body penetrate into the testis at a very early stage of development, forming the tubuliferous tissue already described, and giving rise ultimately to the vasa efferentia. The Wolffian body becomes greatly reduced in size, and is converted into the head of the epididymis ; the proximal part of the Wolffian duct, which is greatly convoluted, gives rise to the body and tail of the epididymis (Fig. 160,w); and the distal part of the duct forms the vas deferens, $x$.

The testes originally lie opposite the anterior ends of the Wolffian bodies, and attached to the dorsal wall of the abdomen; ultimately they shift their position from the dorsal to the ventral wall of the abdomen, and, passing through the inguinal rings, become lodged in a pair of pouch-like folds of the skin, the scrotal sacs (Fig. 160).

The Müllerian ducts, in the male rabbit, disappear completely. The uterus masculinus (Fig. 160, s) has been stated to be formed from their hinder or distal ends, but according to Kölliker it is derived from the Wolffian, and not from the Müllerian ducts ; these latter in the male never opening into, or even reaching, the urino-genital passage.

In the female, or doe rabbit, the Müllerian ducts become greatly enlarged, and form the oviducts. Their abdominal openings persist as the open fimbriated mouths of the Fallopian tubes ; the proximal portions of the ducts become the Fallopian tubes themselves; the middle portions become the uteri; and the terminal, or distal segments unite to form the vagina.

The Wolffian bodies, in the female, undergo degenerative changes ; they become greatly reduced in size, and are ultimately converted into the parovaria. The Wolffian ducts either disappear completely, or else small portions of them persist as rudimentary or vestigial structures. 


\section{DEVELOPMENT OF THE CGLOM.}

The colom, or body-cavity, of the rabbit appears, as in most Vertebrates, as a cleft in the mesoblast, formed by splitting, or rather by rearrangement, of its cells into two layers, somatic and splanchnic.

The colom appear's first on the eighth day, and by the ninth day (Fig. 146, c) has become a cavity of considerable size. It is not confined to the embryo, but stretches out beyond this, and in all directions, reaching almost to the margin of the mesoblast, indicated by the sinus terminalis, sr.

Immediately in front of the embryo, in the pro-amnion (Fig. 145, $\mathrm{Ax}^{\mathrm{l}}$ ), there is at first no mesoblast, and consequently no cœlom; but in the later stages, as the mesoblast invades the proamnion from its sides, the cœlomic cavity extends into this region also.

Within the embryo itself, the cœlom is confined to the body region, and does not extend forwards into the head. The abdominal portion of the colom presents no further changes of special interest, but in the thorax the development of the pericardial and pleural cavities, and also the formation of the diaphragm, require notice.

\section{The Pericardial Cavity.}

Early on the ninth day, the heart consists of two tubes, lying along the sides of the head, and widely separate from each other (Fig. 14.5, R). The parts of the cœlom into which these tubes project become later on the pericardial cavity, so that this cavity, like the heart itself, consists at first of two separate halves, right and left respectively.

As the side-folds deepen, the two halves of the heart are brought together beneath the pharynx; and, early on the tenth lay, the right and left halves of the pericardial cavity meet beneath the throat, and become continuous with each other.

The pericardial cavity (Fig. 147) is thus merely the anterior part of the general bolly-cavity or cœlom, and there is at first no boundary between the two, except the very imperfect partitions formed by. the right and left vitelline veins, where they diverge behind the heart.

Towards the close of the tenth day, and during the early 
part of the eleventh day, the pericardial cavity becomes shut off from the body cavity by a couple of septa. One of these, which is ventral in position, is formed by a thick transverse fold of the splanchnopleuric mesoblast, immediately behind the heart, and between this and the liver. The second, or dorsal septum is much thinner, and grows forwards from the walls of the Cuvierian veins to the anterior end of the body cavity. These two septa, between them, shut off the ventral and anterior portion of the cœlom as a pericardial cavity, distinct from the general body cavity (Figs. 150 and 163, CP).

\section{The Pleural Cavities.}

After the boxing-in of the pericardial cavity, by the dorsal and ventral septa, is completed, the general body cavity still extends forwards as a pair of pocket-like diverticula, dorsal to the pericardial cavity, and along the sides of the œsophagus. Into these pocket-like cavities the lungs hang freely, and the pockets themselves become the pleural cavities.

As the lungs enlarge, the pleural cavities, which at first lie entirely dorsal to the pericardial cavity, gradually extend downwards so as to embrace its sides (Fig. 163, CR), and ultimately reaching almost to the mid-ventral wall of the chest.

\section{The Diaphragm.}

The diaphragm is formed from a couple of septa, dorsal and ventral respectively, which arise independently, and are for some time quite distinct from each other.

Of these, the ventral septum is the thick transverse partition already described as forming the ventral part of the hinder wall of the pericardial cavity.

The dorsal septum of the diaphragm arises, on the thirteenth day, as a transverse fold of mesoblast, which grows downwards from the dorsal wall of the body cavity, just behind the $\mathrm{Cu}$ vierian veins. It has for a time a free ventral edge, crescentic in shape; but it ultimately meets, and fuses with, the ventral septum, or posterior wall of the pericardial cavity, thereby completing the diaphragm, and shutting off the pleural cavities from communication with the body cavity. 


\section{DEVELOPMENT OF THE MUSCULAR SYSTEM.}

'The majority of the body muscles are developed, as in the chick, from the muscle plates of the protovertebre, or mesoblastic somites.

The great dorsal muscles of the neck and trunk, and the muscles of the thoracic and abdominal walls, are derived directly from the muscle plates, but the origin of many of the other muscles is not determined with certainty. The muscles of the head arise independently of the muscle plates; and the muscles of the limbs also arise independently, and in situ. It is probable, however, that in both these cases the mode of development has undergone secondary modifications and abbreviations.

\section{DEVELOPMENT OF THE SKELETON.}

There are in the rabbit, as in the chick or frog, three stages in the development of the skeleton. The first, or earliest, stage is that in which the notochord is the only specially skeletal structure present; the second stage is that in which a cartilaginous skeleton is developed, not only in relation with the notochord, but in the head and limbs as well; while the third or final stage is characterised by the development of bone. which gradually becomes the dominant and essential constituent of the skeleton. Bones arise either as cartilage-bones, in direct connection with the cartilaginous skeleton ; or else independently of this, as membrane-bones.

It is important to remember that each of these stages is not a further development of the preceding stage, but an independently arising one, which displaces its predecessor. Thus the cartilaginous skeleton does not arise from the notochord, but outside this and independently of it, and gradually displaces and obliterates it; to be displaced in its turn by the bony skeleton. So too the lower jaw is not formed from Meckel's cartilage, but around it; and the formation of the bone leads ultimately to the obliteration of its cartilaginous predecessor.

The development of the skeleton of the rabbit has not yet been studied in detail, and there are many points on which our knowledge is still very incomplete. 


\section{The Vertebral Column.}

On the tenth day, the notochord, which up to this time has been the sole skeletal structure present, becomes surrounded by a membranous sheath; and, during the eleventh and twelfth days, a cartilaginous tube begins to form around this sheath.

By the fourteenth day, the cartilaginous tube is definitely established; and in it a distinction, as regards histological characters, is apparent, from the first, between the vertebral and the intervertebral regions. The tube thickens on its inner surface, and so begins to encroach upon the notochord. Opposite the centra of the vertebræ the notochord becomes constricted, and finally completely obliterated.

Between the successive vertebræ, in the intervertebral regions, the notochord remains of full width for a long time; and, according to Kölliker and others, it even persists throughout life, as part of the nucleus pulposus in the axes of the intervertebral ligaments.

From the vertebral centra the neural arches (Fig. 163, Fi) grow up at the sides of the spinal cord, during the fifteenth and sixteenth days; but the completion of the neural canal dorsally does not occur until a late stage.

The first two vertebræ undergo modifications similar to those already described in the bird; the centrum of the first vertebra or atlas (Fig. 167) separating from the rest of the vertebra, and fusing. with the centrum of the second, or axis, vertebra to form its odontoid process.

The transverse and other processes of the vertebra arise as outgrowths from the cartilaginous centra or from the neural arches.

\section{The Ribs and Sternum.}

The ribs (Fig. 163, RI) arise as bars of cartilage, in the connective tissue septa between the several muscle-segments or myotomes of the thorax. In the rabbit, the two or three most anterior ribs are at first continuous with the vertebræ, and appear as elongated transverse processes. At a later stage a joint is formed between the rib and the vertebra, and in this way the tubercular articulation is acquired (cf. Figs. 163 and 167). The head or capitulum of the rib develops as an outgrowth

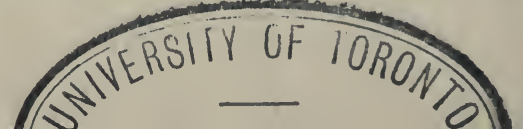



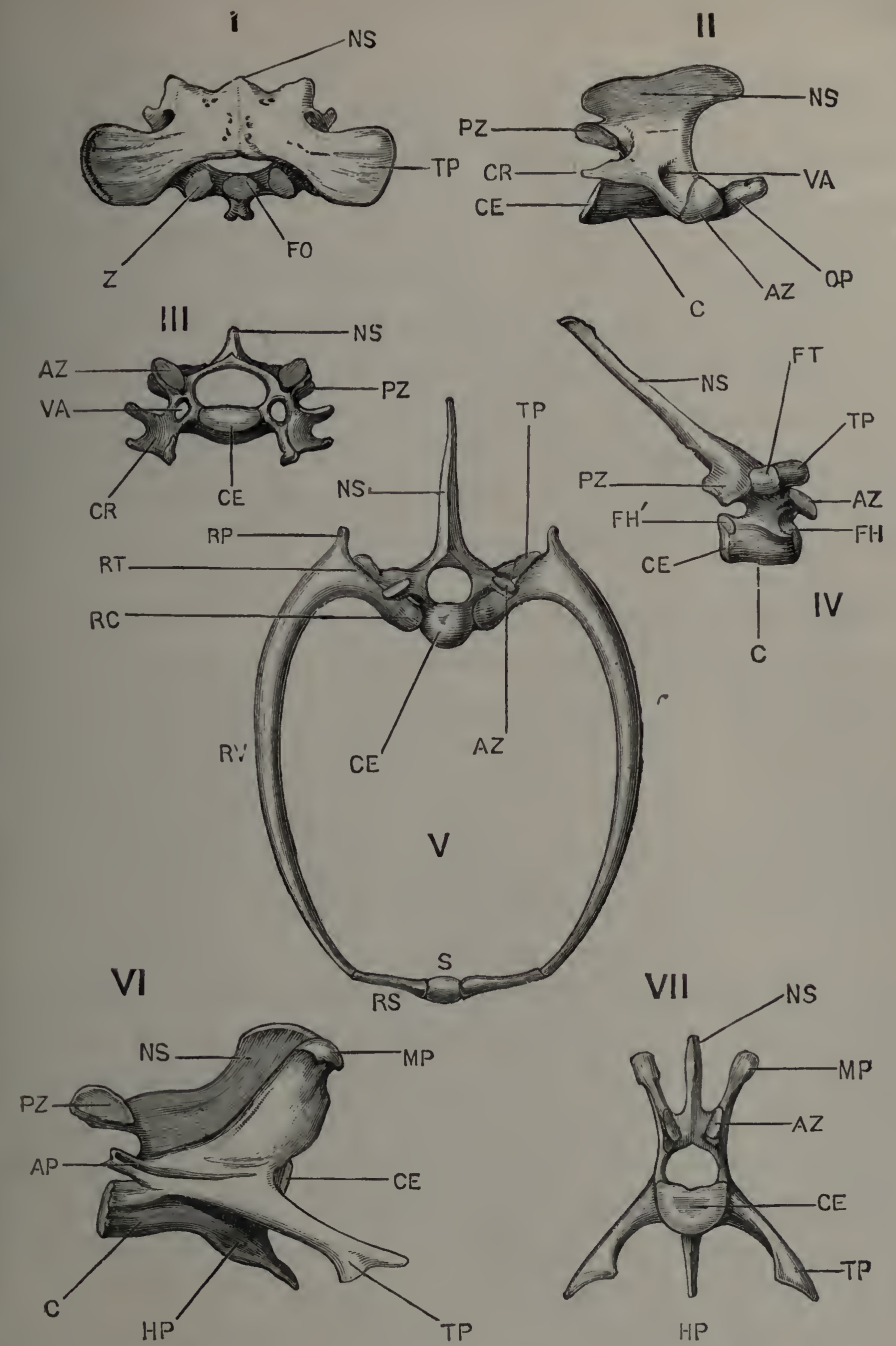

$r$

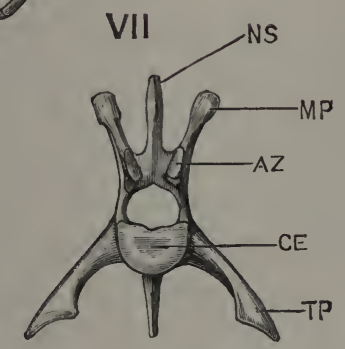

HP

FIG. 167.-Selected vertebræ from the Rabbit. (From Marshall and Hurst.)

I. First cervical vertebra, or atlas, from the dorsal surface. II. Second cervical vertebra, or axis, from the right side. III. Fifth cervical vertebra; anterior surface. IV. Fourth thoracic vertebra, from the right side. V. Fourth thoracic vertebra, and fourth pair of ribs; anterior surface. VI. Second lumbar vertebra, from the right side. VII. Sccond lumbar vertebra; anterior surface.

AP, anapophysis. AZ, anterior, or pre-zygapophysis. C, centrum. CE, epiphysis of centrum. $\mathbf{C R}$, eervical rib. $\mathbf{F H}$, facet for capitulum or hearl of the fourth rib. $\mathbf{F H}^{\prime}$, faeet for capitulum of the fifth rib. FO, facet for odontoil process. F'T, faect for tubercle of fourth rib. HP, hypapophysis. MP, metapophysis. NS, neural spine, or spinous process. $\mathrm{OP}$, oilontoid process. PZ, post-zygapophysis. RC, capitulum or hear of rib. RP, proeess of rib for attachment of ligaments. RS, sternal portion of rib. RT, tubercle of rib. RV, vertebral portion of rib. $\mathbf{S}$, steruum. TP, transverse process. V A, vertebrarterial canal. Z $\mathbf{Z}$, articular surface for axis vertebra.' 
from the proximal end of the rib, and does not articulate with the vertebra until a considerably later period. The posterior ribs, behind the first two or three, develop from the start independently of the vertebræ.

The sternum is formed in two halves, and from the ventral ends of the ribs. Wach rib is at first slightly dilated at its ventral end (Fig. 163, ST), and these enlarged ends of successive ribs, growing both anteriorly and posteriorly, meet and fuse, so as to form along either side a longitudinal cartilaginous bar, connecting the ventral ends of the ribs of its side of the body. The two bars, right and left, approach each other, meet in the median plane, and fuse to form the sternum.

3. The Skull ( $c f$. Figs. 160 and 162).

The cartilaginous skull of the rabbit is formed from the same essential elements - parachordals, trabeculæ, sense capsules, and visceral bars-as in the chick or frog; and the general relations of these parts to one another are very similar in the three animals. Cartilage appears in the head of the rabbit embryo about the fourteenth day, and by the sixteenth or seventeenth day the cartilaginous skull is practically completed.

The two parachordal cartilages fuse together very early to form the basilar plate (Fig. 151, RP), which underlies the medulla oblongata, and forms the floor of the hinder part of the skull. The edges of the basilar plate grow up at the sides of the brain, and fuse with the independently arising periotic capsules (Fig. 159, EC) ; and then, growing in towards each other, meet above the cerebellum to complete the occipital ring (Fig. 151, ox). In front of the supra-occipital cartilage, the roof of the skull remains membranous until the formation of the bones.

The trabeculæ are a pair of rods of cartilage, which are continuous at their hinder ends with the basilar plate: further forwards they lie at the sides of the pituitary body, and in front of this unite to form the ethmoidal plate (Fig. 151, ET). This latter is at first small, and never becomes so large as in the bird, but as the nose grows forwards, and the face assumes its definite form, the ethmoidal plate extends forwards with it, giving off from its upper surface a median vertical septum between the two olfactory organs. The cartilaginous olfactory capsules arise independently, but very early fuse with the ethmoidal septum. 
With regard to the cartilaginous bars developed in the visceral arches, the maxillary or palatopterygoid bar forms the basis of the upper jaw, but it is not clear whether it arises independently, or as an ontgrowth from the mandibular bar.

The mandibular bar is a rod of cartilage, which along the greater part of its length is known as Meckel's cartilage (Figs. 151 and $156, \mathrm{Mc}$ ) ; it forms the basis of the lower jaw, the bones of the mandible being formed around it, though not in direct connection with it, except at the chin.

The hyoid bar is at first cartilaginous along its whole length, but subsequently disappears in great part. Its lower or ventral end forms the basis of the anterior, or lesser cormu of the hyoid bone of the adult.

Of the first branchial bar, the only part that persists is the ventral end, which forms the basis of the posterior, or greater cormu of the hyoid bone. The body of the hyoid bone is formed from the median elements of the hyoid and first branchial arches.

The development of the auditory ossicles, and their relations to the mandibular and hyoid bars have already been considered in the section dealing with the development of the ear (p. 398).

Concerning the appendicular skeleton there is nothing special to note, except that Kölliker has shown that the clavicle in rabbit embryos of about the seventeenth day is cartilaginous; and that the clavicle, though presenting some peculiarities in the details of its mode of ossification, ought to be viewed as a cartilage bone, and not, as is commonly stated, as a membrane bone.

\section{DEVELOPMENT OF THE SKIN.}

\section{The Hairs.}

Hairs are epidermal structures, and are as characteristic of Iammals as are feathers of Birds. The first stage. in the formation of a hair consists in the growth of a small solid process from the deeper or mucous layer of the epidermis, into the underlying connective tissue (Fig. 156, DE). A small papilla of vascular connective tissue grows into the deeper end of the epidermal process, and serves for its nutrition. The hair itself 
is formed by cormification of the axial or central cells of the process, while the outer or peripheral cells form the hair-sheath, or follicle. The hair grows upwards from its base, and the free tip soon projects above the surface of the skin.

From the first, the hairs of the eyebrows, and of the upper lip and nose are of exceptionally large size (Fig. 149); and one particularly large hair, arising from the cheek immediately below the eye, forms a prominent feature in rabbit embryos from about the nineteenth day onwards, and is also very large in the adult rabbit.

\section{The Claws.}

The claws are formed by cornification of the epidermis at the ends of the fingers and toes. The layer of epidermis that becomes converted into the claw is not, in the first instance, the most superficial one, but is a special stratum, developed between the superficial and the deeper or Malpighian layers of the epidermis ; the Malpighian layer, with the underlying dermis, being modified to form the bed of the claw. The distal border of the claw soon projects freely at the end of the digit, and its further growth is effected by additions at its hinder or attached border, and to its under surface.

\section{The Mammary Glands.}

The mammary glands, like the other cutaneous glands, are formed by ingrowths of the epidermis into the underlying connective tissue. These ingrowths give off secondary branches, which are at first solid, but soon becorne hollow, and form the gland cavities; the ducts being derived from the original epidermal ingrowths.

\section{DEVELOPMENT OF THE PLACENTA.}

The placenta is the organ by which the nutrition of the embryo is effected during the period of its stay in the uterus; and it is through the placenta that the mammalian embryo is enabled to attain so large a size, and so high a grade of development at the time of birth, although formed from an ovum of extremely small size and almost devoid of food-yolk.

The placenta (Fig. 170) is formed partly from the mother, 
and partly from the embryo or foetus; the foetal element being supplied by the wall of the blastodermic vesicle, and by the allantois; and the maternal element by the part of the wall of the uterus to which the blastodermic vesicle becomes attached.

In the chick, the allantois (Fig. 101) attains a great size, and forms the respiratory organ of the embryo during the later stages of its development.

In the rabbit the allantois becomes still larger and more important, subserving nutrition as well as respiration. It becomes firmly attached to the wall of the uterus (Figs. 148 and 170 ), and then gives off, from its outer surface, vascular tufts or villi into the substance of the uterine wall. 'The vessels of these villi, which are derived from the allantoic arteries and veins, and are therefore continuous with the blood-vessels of the embryo, lie in close contact with the dilated maternal capillaries of the uterus. The intervening walls between the two sets of bloodvessels, fotal or allantoic, and maternal or uterine, become so greatly reduced in thickness that diffusion readily takes place between the two blood streams, through these very thin partitions. In this way the fotal blood derives nutrient matter from the maternal blood, and gives up to it the gaseous and other excretory matters that are formed in the embryo, as a necessary consequence of the chemical changes associated with its growth and development.

The actual details of development of the rabbit's placenta are extremely complicated, and the accounts given by different investigators are at variance with one another, even in points of primary importance. The most complete and consistent account is that given by Duval; it is supported in many important respects by Minot's investigations, and has afforded the basis on which the following description has been founded.

The mucous membrane of the uterus, in the unimpregnated condition, is thrown into six longitudinal folds, which project into the uterine cavity, and give it a stellate appearance in transverse section. Of these folds, two (Fig. 168, PK) lie on the side of the uterus next to the mesometrium, or mesenterial fold, MM, which attaches the uterus to the abdominal wall ; these are termed by Minot the placental folds or placental lobes. The second pair, or periplacental folds, P.I, lie at the sides of the 
uterus; and the third pair, or obplacental folds, lie opposite the placental folds, along the free or unattached border of the uterus.

It is from the mesometrial, or placental, folds alone that the maternal part of the placenta is derived: the periplacental and

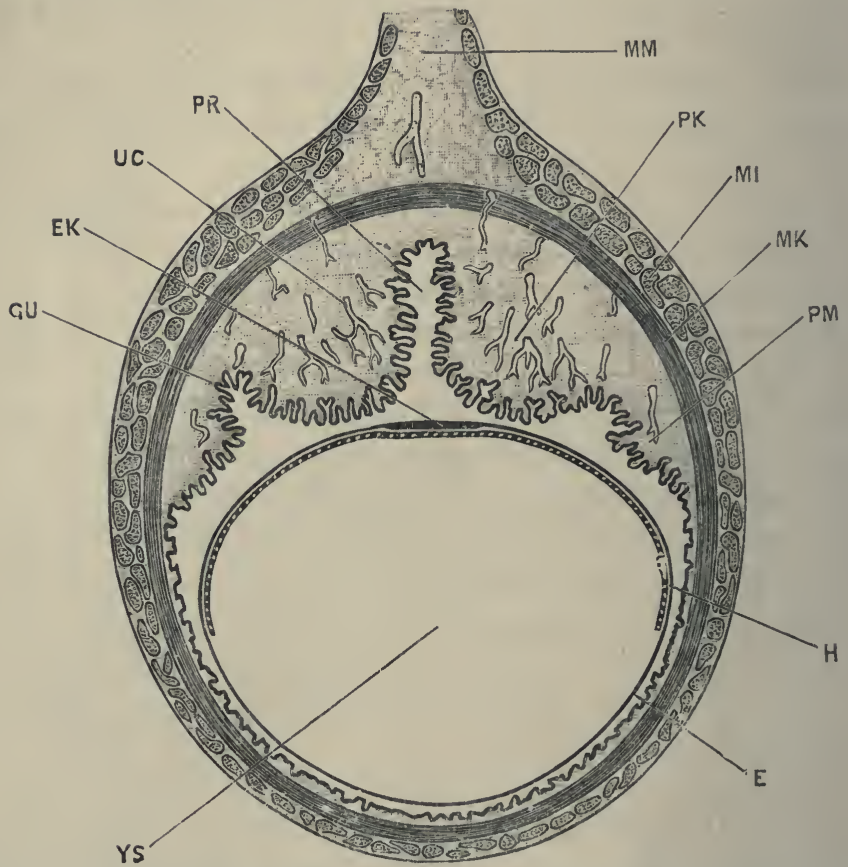

FIG. 168.-A transverse section across the uterus, with the contained blastodermic vesicle, of a Rabbit at the end of the seventh day. (In part after Duval.) $\times 12$.

E, epiblast of blastodermic vesicle. FK, thickened epiblast of embryonal area. GU, uterine glands. H, hypoblast of blastodermic vesicle. MI, outer or longiturinal muscles of the wall of the uterus. MK, inner or circular muscles of the wall of the uterus. MM, mesometrium, or mesenterial fold connecting the uterus with the dorsal wall of the abdomen. PK, placental fold of uterus. PM, periplacental fold of uterus. PR, meilian cleft between the two placental folds. UC, dilated capillaries of submucous layer of uterus. $\mathbf{Y} \mathbf{S}$, yolk-sac.

obplacental folds undergo considerable changes, but do not take any direct part in the formation of the placenta.

On the seventh day the blastodermic vesicles are spaced out along the uterus, and the swellings or loculi of the uterus, indicating their position, are well marked externally.

The blastodermic vesicle, the structure of which at this stage 
has already been described (p. 360), lies quite freely within the uterus, and the structure of the uterine walls is as follows (cf. Fig. 168).

The muscular walls of the uterus are well marked, consisting of outer longitudinal, MI, and inner circular layers, MK. Within the layer of circular muscles come the submucous and glandular layers. Of the six longitudinal folds of the nterus, the two placental folds, PK, form large and prominent ridges, separated by a deep median cleft, PR. The periplacental folds, PM, are similar, but much smaller; while the obplacental folds are no longer recognisable, having become flattened out and obliterated by the stretching, which this part of the wall of the uterus has undergone, to make room for the embryo.

The submucous layer, which is very thick in the placental folds, PK, but comparatively scanty elsewhere, consists of loose connective tissue, with very numerous, branched connectivetissue cells, and is very vascular. The blood-vessels, which are derived from the mesometrium, perforate the muscular walls of the uterus as small arteries and veins, and then dilate, within the submucous layer, into large but very thin-walled capillaries (Fig. 168, cc), which are especially numerous in the subglandular layer of connective tissue, immediately below the surface epithelium.

The epithelium lining the uterus is pitted to form the uterine glands, Gu, which are very deep and freely branched in the placental and periplacental folds ; while in the obplacental area, owing to the stretching which this part of the uterus has undergone, the mouths of the glands are greatly dilated, and the glands themselves widened out.

Early on the eighth day the attachment of the blastodermic vesicle to the wall of the uterus commences, and by the ninth day it is completed. The attachment is effected, as already noticed, by thickening and proliferation of the epiblast cells of the blastodermic vesicle over a horse-shoe shaped patch, the placental area, which surrounds the sides and hinder end of the embryo (Fig. 145, $\mathrm{E}^{\prime}$ ). The epiblast cells of this placental area become more numerous, by repeated divisions, and grow out into irregular processes which fuse firmly with the surface of the placental lobes of the uterus (Fig. 169, E). By this time, accord- 
ing to Duval, the uterine epithelium of the placental lobes has entirely disappeared, by absorption, though it remains unaltered in the deeper parts of the glands for some time longer: the embryonic epiblast of the placental area is, therefore, in direct contact with the connective tissue of the uterine wall.

This thickened epithelium of the placental area of the blastodermic vesicle is a structure of very great importance, and has been named by Duval the ectoplacenta. It serves in the first instance, as just noticed, to attach the embryo to the uterine wall, and in the later stages it plays a very prominent part in the formation of the placenta. It must be borne in mind throughout the following description that, if Duval's account is correct, the ectoplacenta is entirely of foetal origin, and is not derived, even in part, from the uterine epithelium. This is a point, however, on which difference of opinion obtains ; Strahl, for instance, maintaining that the ectoplacenta is formed by proliferation of the uterine epithelium, and not from the embryonic epiblast.

The embryo normally lies with its long axis coinciding with that of the blastodermic vesicle, and therefore with that of the uterus, so that a transverse section of the uterus cuts the embryo transversely (Fig. 169, $\mathrm{NG}$ ). The embryo is usually in the middle of the upper surface of the blastodermic vesicle, and lies opposite the deep cleft, PR, between the two placental lobes, The position of the embryo is, however, variable, especially in the earlier stages: it may lie obliquely across the vesicle; or may, more rarely, lie opposite one or other of the placental lobes, instead of opposite the cleft between them.

In the submucous layer of the placental lobes important changes occur during the ninth day. The capillaries dilate very considerably (Fig. 169, uc), becoming much larger than the arteries and veins in connection with them. They retain their simple epithelial walls, but thick adventitious perivascular walls are formed outside these by the surrounding connective-tissue cells. These perivascular cells are at first ordinary connectivetissue cells, which increase in number, draw in their processes, and become arranged in layers, two or three cells thick, around the capillaries. This perivascular thickening of the walls of the capillaries occurs throughout the greater part of the submucous layer, but does not affect the capillaries immediately beneath the 
surface of the uterus, nor those of the outermost layer, next to the circular muscles of the uterine wall.

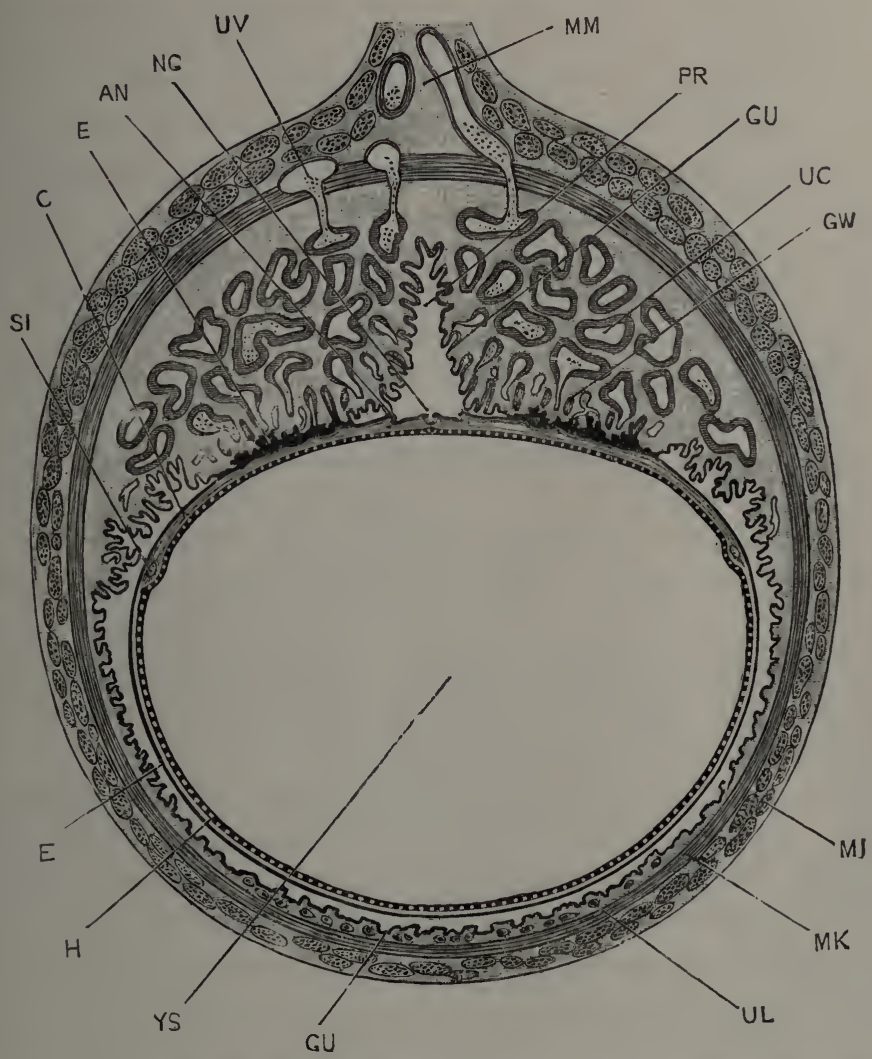

Fig. 169.-A transverse section across the uterus and the contained blastodermic vesicle of a Rabbit at the end of the ninth day. $C f$. Figs. 145 and 146 , in which the blastodermic vesicle and embryo of this age are shown in surface view and in sagittal section. (In part after Duval.) $\times 8$.

AN, side fold of the amnion. C, mesoblast of the upper wall of the blastodermic vesicle, beyond the embryonal area. $\mathbf{E}$, epiblast of the blastoilermic vesicle; the upper reference is $t$, the thickened epiblast of the placental area. GU, uterine glands. GW, morified uterine glands of placental region. H, hypoblast of blastodermic vesicle. MI, outer or longitudinal muscles of the wall of the uterus. MK, inner or circular muscles of

the wall of the uterus. MM, mesometrium. NG, neural groove of embryo, in transverse section. PR, merlian eleft between the placental lobes of the uterus. SI, sinus terminalis. UCC, eapillaries of placental region, with thickened perivascular walls. UL, giant cells. UV, blood-vessels of uterus. Y Y S, yolk-sac, or cavity of blastodermic vesicle.

During the tenth day, the ectoplacental epithelium increases greatly in thickness, and becomes excavated by irregular channels or lacunæ, which according to Duval open into the maternal 
or uterine capillaries. At the same time the inner or deeper surface of the ectoplacenta is becoming folded, and the mesoblast of the somatopleure grows in between these folds ( $c f$. Fig. $\left.147, \mathrm{E}^{\prime}\right)$. The uterine glands of the placental lobes have by this time almost disappeared ( $c f$. Fig. 169, Gw); and a little later they are completely absorbed. In the submucous layer the perivascular thickening of the walls of the capillaries has proceeded still further, while the capillaries themselves are even larger than before; and ultimately nearly the whole of the connective tissue of the submucous layer becomes converted into perivascular, or, as they are now termed, decidual cells.

In the periplacental lobes somewhat similar changes occur. The superficial epithelium of the uterus, and the epithelium lining the mouths of the glands degenerate and disappear; and perivascular thickenings of the capillary walls occur, although to a less marked extent than in the placental lobes.

In the obplacental region also, the uterine epithelium degenerates and becomes absorbed; but the epithelium of the glands themselves remains, and at a later stage, by spreading outwards from the mouths of the glands, reconstitutes an epithelial lining to this part of the uterus.

Towards the end of the tenth day, and during the eleventh day, the allantois is growing rapidly. As shown in Figs. 146 and 147 , the outer or mesoblastic wall of the allantois very early coalesces with the mesoblast of the outer layer of the amnion, opposite the placental area; and in this way the blood-vessels of the allantois are brought immediately beneath the ectoplacental epithelium, and consequently into close proximity with the dilated capillaries of the uterus: and the placenta is thus established.

The further development of the placenta consists mainly in a gradually increasing complication and elaboration, by which folds of the mesoblast, containing the allantoic vessels, are carried deeply into the ectoplacenta from its inner surface; while from the outer surface the maternal vessels extend in further, and in larger numbers, than before. This interdigitation of the fotal and maternal blood-vessels is accompanied by progressive thinning of the layer of ectoplacental epithelium 
intervening between them, until ultimately the two sets of blood-vessels are separated by exceedingly thin partitions.

The successive steps in this process are as follows; Duval's descriptions being mainly followed in the account here given.

From the tenth day onwards the growth of the vascular septa, or villi, from the allantois into the ectoplacenta proceeds very rapidly, so that the latter becomes cut up into a series of radially arranged columns, or lobules, within which lie the lacunæ opening into the maternal capillaries. At this stage the foctal blood is separated from the maternal blood by three structures:-(i) the endothelial wall of the foctal or allantoic capillaries; (ii) a layer, several cells in thickness, of the ectoplacental epithelium; (iii) the endothelial wall of the maternal capillaries. There is some doubt, however, with regard to the third layer; according to Duval, this has already disappeared, and the maternal ressels of the placenta are merely lacunar spaces hollowed out in the ectoplacental epithelium, and devoid of true walls.

During the twelfth to the fourteenth days, each of the ectoplacental columns or lobules becomes subdivided, by longitudinal folding of its walls, and ingrowth of septa, into a set of closely placed parallel tubules, the general direction of which is radial, i.e. vertical to the inner surface of the uterus.

These lobules, in the later stages, become larger and more minutely subdivided, and by the nineteenth or twentieth day the relations are as shown in Fig. 170. Each of the two placental lobes now consists of a number of lobules, $\mathrm{PH}$, which are somewhat fusiform in shape, radially arranged, and packed closely together side by side. Each lobule is further subdivided into a complicated system of branching tubular passages, which at each end of the lobule open into larger chambers, UP. 'Through these passages, which, according to Duval, are simply lacunæe excavated in the ectoplacental epithelium, the maternal blood circulates. Large afferent channels, derived from the uterine arteries, convey the maternal blood directly to the dilated chambers at the inner ends of the lobules, next to the surface of the uterus. From these chambers it flows back, through the complicated system of tubules of which the lobule consists, to the chambers at the outer ends of the lobules, from which it is 
carried away by efferent vessels which open into the uterine veins.

The foetal or allantoic vessels, AA, pass between the several lobules to their outer ends, and then return as thin-walled capillaries, which pass through the lobules, lying between the tubules into which these are divided; on reaching the inner surface of the uterus the capillaries open into the allantoic veins, VA, which return the blood from the placenta to the embryo.

In the later stages, from the twenty-fifth to the thirtieth day, the chief changes consist in the gradual thinning of the partitions separating the foetal and maternal streams in the lobules. The ectoplacental wall becomes gradually absorbed, more and more completely, until ultimately, according to Duval, a single layer of epithelial cells, the endothelial wall of the foetal capillary, is alone left between the two streams of blood.

The changes that occur in the deeper, or submucous part of the placenta require further notice. In the early stages (Fig. 169), the submucous layer is very thick, and the ectoplacenta very thin. By the nineteenth day (Fig. 170) the two have become of about equal thickness. From this time the submucous layer is the thinner of the two, and towards the close of gestation it becomes comparatively insignificant.

The chief changes that occur in the submucous layer during these later stages are:-a still further dilatation of the capillary vessels ; an increase in the decidual cells surrounding the capillaries; and the appearance in the subglandular region of a layer of special cells, spoken of as glycogenous cells. These latter are large, roundish, or ovoid vesicular bodies, each consisting of an outer capsule and a central multinucleate protoplasmic body, from which strands of protoplasm radiate outwards to the capsule. In the meshes between the strands lie faintly glistening glycogen masses. Each of these glycogenous cells is said to be formed by the fusion of a number, from three to six, of originally separate cells.

The perivascular and glycogenous cells are probably to be regarded as having some function in connection with the elaboration, or preparation, of the maternal blood, before it is sent to the placenta for the nourishment of the embryo. The blood 


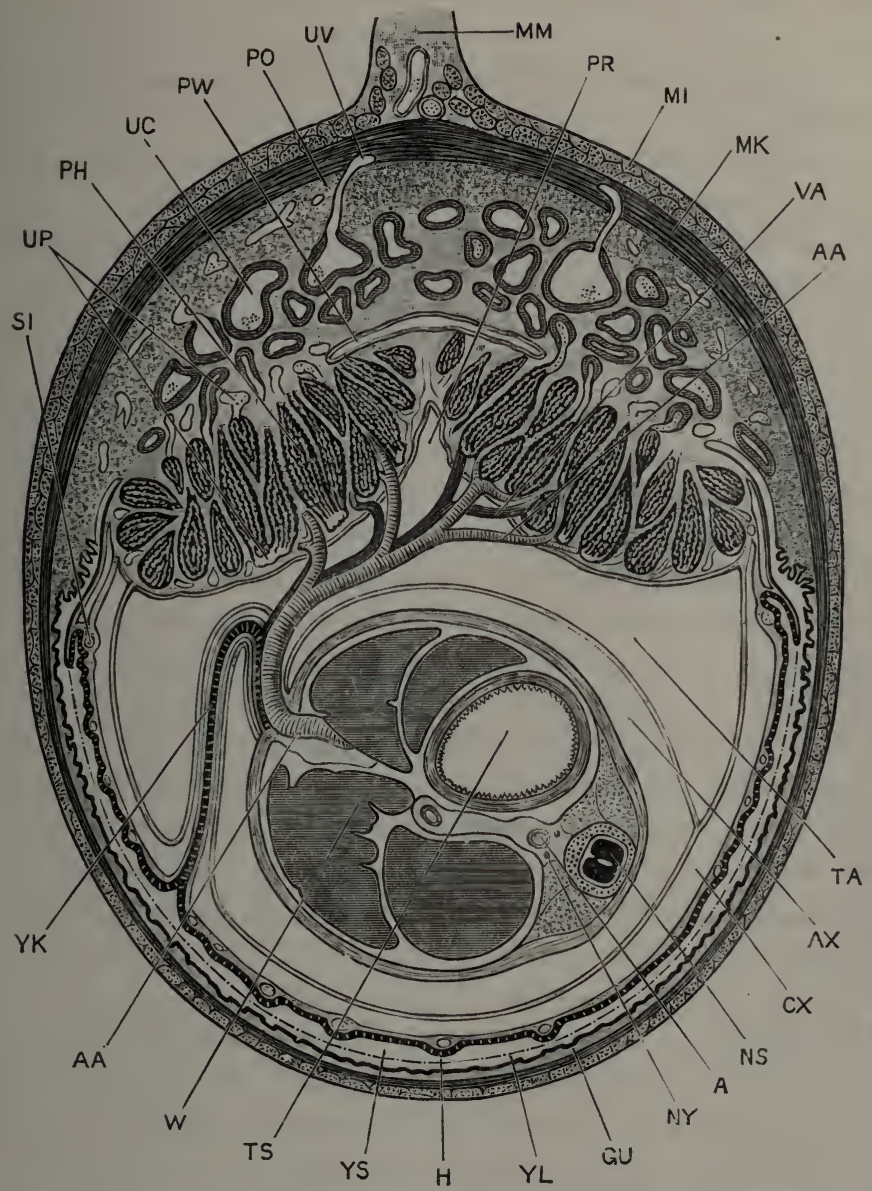

FIG. 170.-A transverse section across the uterus and the contained embryo of a Rabbit at the end of the nineteenth day. The embryo is cut transversely, about the middle of the body, the section passing through the yolk-stalk and allantoic stalk. $\times 3 \frac{1}{2}$.

$\mathbf{A}$, dorsal aorta of the embryo. AA, allantoic arterv. AX, amnionic cavits, between the inner or true amnion and the embryo. $\mathbf{C X}$, space between the inner and outer layers of the amnion (cf. Fig. 148). GU, uterine glands of obplacental region. $\mathrm{H}$, hypoblast of upper or vascular wall of yolk-sac. MI, onter or longitudinal muscles of the wall of the uterus. MK, inner or circnlar muscles of the wall of the uterus. MM, mesometrium. NS, spinal corl of the embryo. NY, sympathetic nerve cord. $\mathbf{P H}$, lobule of placenta. PO, region along which the separation of the placenta occurs at birth. PR, interplacental groove. PW, subplacental cavity. SI, sinus terminalis. TA, cavity of the allantois ( $c f$. Fig. 148). TS, stomach of embryo. UC, lilated uterine capillary, with thick perivascular wall. UP, utcrine or maternal sinuses of placenta. UV, blood-vessels of uterus. VA, allantoic vein. W, liver of embryo. $\mathbf{Y} \mathbf{K}$, yolk-stalk. $\mathbf{Y} \mathbf{L}$, dotted line representing the lower or non-rascular wall of the yolksac, now completely absorbed. Y Y S, cavity of yolk-sac, continums with the uterine eavity owing to absorption of the lower wall of the yolk-sae. 
in the maternal capillaries of the placenta is specially characterised by the relatively enormous number of leucocytes which it contains, at all stages from about the eleventh or twelfth day onwards.

Curious modifications occur in the lining epithelium of the maternal capillaries of the submucous layer during the formation of the placenta. The ordinary endothelial walls, which these vessels at first have, become replaced by a layer of irregular, thickened, and often columnar cells. According to Duval, this layer is formed by extension outwards of cells from the ectoplacenta along the interior of the maternal vessels; while Minot regards it as formed by degenerative changes in the proper endothelial wall of the capillaries.

In the obplacental region, and to a less extent in the periplacental region, certain peculiar cells, characterised chiefly by their enormous size, and hence spoken of as colossal or giant cells, appear in the submucous layer at an early stage (Fig. 169, UL). These are stated to be derived from the uterine epithelium of these regions; they form marked features from the ninth day onwards, but their function is entirely unknown. Cells of exceptional size are commonly associated with absorptive, rather than with formative changes, but the actual absorption occurring in this region of the uterus is comparatively slight in amount.

Parturition. The outer layer of the submucous connective tissue, next to the circular muscle layer of the uterus, is characterised by the small size of its blood-vessels (Fig. 170, Po); and it is along this line that separation takes place at the time of birth, the entire placenta, both fotal and maternal, coming away with the young animal.

The actual separation is effected by strong contractions of the muscles of the uterus. The hæmorrhage at parturition is but slight, partly because the blood-vessels along the plane of separation are small, and partly because of the rapidity with which complete contraction of the uterus is effected.

Long before the birth of the young animal, the mucous membrane of the obplacental region of the uterus has been completely re-established; this mucous membrane is attached to the muscular walls of the uterus by very loose connective 
tissue. Owing to the strong contraction of the muscles of the uterns at the time of birth of the young, the bare patch, from which the placenta has been separated, is at once greatly reduced in size, while the loosely attached mucous membrane of the obplacental region slips orer it, and closes the wound almost instantly. The complete regeneration of the uterine epithelium after parturition is effected with astonishing rapidity, and the doe is ready to receive the buck almost immediately after she has given birth to the young.

The placenta is commonly regarded as essentially an allantoic structure. But the facts, that the attachment to the uterus is first effected, not by the allantois, but by the epiblast of the blastodermic vesicle; and that the allantois merely utilises this attachment as a means of getting access to the uterus, suggest that the participation of the allantois in the formation of the placenta is probably a secondary and not a primitive character.

The further fact that changes occur in the mucous membrane of the obplacental and periplacental regions, similar to the earlier changes seen in the placental region, suggests that the area of attachment of the blastodermic vesicle to the uterus was originally a more extensive one. Minot has contended, from these and similar considerations, that the mammalian placenta was originally formed from the chorion, i.e. from the extra-embryonic part of the blastodermic vesicle, and not from the allantois; and the history of the formation of the placenta in the lower groups of Mammals strongly supports this view.

\section{List of the more important Publications dealing with the Development of the Rabbit.}

Barry, M.: 'Researches in Embryology.' Philosophical Transactions of the Royal Society. 1840.

Beneden, E, van: 'Recherches sur la Composition et la Signification de l'CEuf.' Bruxelles. 1870.

'Recherches sur l'Embryologie des Mammifères. La Formation des Feuillets chez le Lapin.' Archives de Biologie, i. 1880.

- Observations sur la Maturation, la Fécondation et la Segmentation de l'CEuf des Cheiroptères.' Archives de Biologie, i. 1880.

Beneden, E. van, and Julin, C.: 'Recherches sur la Formation des Annexes. F'œtales chez les Mammifères.' Archives de Biologie, v. $188 t$.

Bischoff, T. I. W.: 'Entwicklungsgeschichte des Kaninchen-Eies.' Braunschweig. 1842. 
Born, G.: 'Beitrïge zur Entwickelungsgeschichte des Säugethierherzens. Archiv für mikroskopische Anatomie, xxxiii. 1889.

'Coste, M.: 'Histoire générale et particulière du Développement des Corps organisés.' Paris. 1847-1859.

Duval, M.: 'Le Placenta des Rongeurs.' Journal de l'Anatomie et de la Physiologie, xxv. and xxvi. 1889-90.

Flemming, W.: 'Ueber die Bildung von Richtungsfiguren in Säugethiereiern beim Untergang Graaf'scher Follikel.' Archiv für Anatomie und Entwickelungsgeschichte. 1885 .

'Die ektoblastische Anlage des Urogenitalsystems beim Kaninchen.' Archiv für Anatomie und Entwickelungsgeschichte. 1886.

Foster, M., and Balfour, F. M.: 'The Elements of Embryology;' second edition by Sedgwick and Heape. 1883.

Fraser, A.: 'On the Development of the Ossicula Auditus in the Higher Mammalia.' Philosophical Transactions of the Royal Society. 1882.

Goette, A. : 'Beiträge zur vergleichenden Morphologie des Skeletssystems der Wirbelthiere.' Archiv für mikroskopische Anatomie, xiv. 1877.

Haddon, A. C. : 'Suggestions respecting the Epiblastic Origin of the Segmental Duct.' Scientific Proceedings of the Royal Dublin Society. 1887.

Hensen, V.: 'Beobachtungen über die Befruchtung und Entwicklung des Kaninchens und Meerschweinchens.' Archiv für Anatomie und Entwickelungsgeschichte. 1875 .

Hochstetter, F.: 'Ueber die ursprüngliche Hauptschlagader der hinteren Gliedmasse des Menschen und der Süugethiere.' Morphologisches Jahrbuch, xvi. 1890.

Hubrecht, A. A. W.: 'Studies in Mammalian Embryology: i. The Placentation of Erinaceus Europxus, with Remarks on the Phylogeny of the Placenta.' Quarterly Journal of Microscopical Science, xxx. 1889.

Huxley, T. H. : 'Evolution and the Arrangement of the Vertebrata.' Proceedings of the Zoological Society. 1880.

Kastschenko, N.: 'Das Schicksal der embryonalen Schlundspalten bei Säugethieren.' Archiv für mikroskopische Anatomie, xxx. 1887.

Kölliker, A.: 'Entwicklungsgeschichte des Menschen und der höheren Thiere.' Leipzig. 1879.

'Ueber die Chordahöhle und die Bildung der Chorda beim Kaninchen.' Sitzungsberichte der phys.-med. Gesellschaft in Würzburg. 1883. 1882.

'Die Entwicklung der Keimblätter des Kaninchens.' Leipzig.

Krause, W.: 'Die Anatomie des Kaninchens.' Leipzig. 1868.

Lockwood, C. B. : 'The Early Development of the Pericardium, Diaphragm, and Great Veins.' Philosophical Transactions of the Royal Society. 1888.

Löwe, L.: 'Beiträge zur Anatomie und zur Entwickelungsgeschichte des Nervensystems der Säugethiere und des Menschen.' Berlin. 1880.

Martin, E.: ' Ueber die Anlage der Urniere beim Kaninchen.' Archiv für Anatomie und Entwickelungsgeschichte. 1888.

Masius, J. : 'De la Genèse du Placenta chez le Lapin.' Archires de Biologie, ix. 1889.

Masquelin, H., and Swaen, A.: 'Premières phases du Développement du Placenta maternel chez le Lapin.' Archives de Biologie, i. 1880. 
Minot, C. S.: 'Uterus and Embryo. I. Rabbit. II. Man.' Journai of Morphology, ii. 1889.

'Die Placenta des Kaninchens.' Biologisches Centralblatt, x. 1890.

'Zur Morphologie der Blutkörperchen.' Anatomischer Anzeiger, v. 1890.

'A Theory of the Structure of the Placenta.' Anatomischer Anzeiger, vi. 1891 .

Owen, R. : 'Comparative Anatomy and Physiology of Vertebrates,' iii. 1868. Parker, W. K. : 'Mammalian Descent.' London, 1885.

Paterson, A. M.: 'Development of the Sympathetic Nervous System in Mammals.' Philosophical Transactions of the Royal Society. 1890.

Retterer, E.: 'Sur l'Origine et l'Evolution de la Région ano-génitale des Mammifères.' Journal de l'Anatomie et de la Physiologie, xxvi. 1890.

Robinson, A.: 'Observations upon the Development of the Segmentation Cavity, the Archenteron, the Germinal Layers, and the Amnion in Mammals.' Quarterly Journal of Microscopical Science, xxxiii. 1892.

Schäfer, E. A.: 'Quain's Anatomy.' 'Tenth edition, vol. i., part 1. 1890.

Strahl, H.: 'Zur Bildung der Cloake des Kaninchenembryo.' Archiv für Anatomie und Entwickelungsgeschichte. 1886.

'Untersuchungen über den Bau der Placenta.' Archiv für Anatomie und Entwickelungsgeschichte. 1889.

Tourneux, F.: 'Sur les Modifications que subit l'Euf de la Lapine pendant sa Migration dans l'Oviducte, et sur la durée de cette Migration.' Comptes Rendus de la Société de Biologie. Série ix., tome 1. Paris. 1889.

Vassaux, G.: 'Recherches sur les premières phases du Développement de l'Eil chez le Lapin.' Archives d'Ophthalmologie, viii. 1888.

Woodward, M. F.: 'On the Milk-dentition of Hyrax and of the Rabbit.' Proceedings of the Zoological Society. 1892.

Zimmermann, W.: 'Ueber einen zwischen Aorten- und Pulmonalbogen gelegenen Kiemenarterienbogen beim Kaninchen.' Anatomischer Anzeiger, iv. 1889. 


\section{Chapter VI. \\ THE DEVELOPMENT OF THE HUMAN EMBRYO.}

\section{PRELIMINARY ACCOUNT.}

The human embryo is developed from an egg, which, like that of other animals, is a single nucleated cell, derived from the peritoneal cells forming the outermost layer of the ovary. The human egg, or ovum, measures $0.2 \mathrm{~mm}$. in diameter, i.e. is rather less than double the diameter of the ovum of the rabbit.

The ovum, when ripe, is discharged from the ovary, and is taken up by the open mouth of the Fallopian tube or oviduct, down which it travels to the uterus, where it remains during the rest of the period of derelopment. Prior to the arrival of the ovum, the mucous membrane lining the uterus undergoes important changes, and gives rise to a special layer, the decidua, to which the ovum is attached and in which it becomes embedded. During gestation the cavity of the uterus gradually becomes filled up by the growth of the embryo and of its inclosing inembranes.

As the ovum is of very small size, the nutriment at the expense of which development takes place must be obtained from without. This is effected, as in the rabbit, by means of the placenta, an organ in which the blood-vessels of the embryo and throse of the wall of the uterus are brought into extensive contact, so that free interchange of contents can take place through their walls.

The process of fertilisation of the human ovum, and the early stages of development have not yet been seen; and of specimens showing the first formation of the embryo only a very limited number have been obtained, and very few of these in fit condition for microscopical investigation. Of later embryos, numerous specimens have been examined and described, and 
from a stage corresponding to about a forty-eight hour chick embryo, or to a nine-day rabbit embryo, the history of human development has been determined in considerable detail.

For this satisfactory condition of our knowledge we are very largely indebted to the labours of Professor His, whose detailed and careful descriptions, and splendid series of figures, form the basis on which the account in the present chapter has been in chief part founded.

The total period of human development is usually estimated at slightly under ten lunar months. The exact period cannot be ascertained, owing to the impossibility of determining the time at which fertilisation of the egg is effected, i.e. at which development commences.

The details of development of the human embryo are closely similar to those of the rabbit; the chief points of difference being:-(i) the far longer time occupied by the human embryo, more than nine times as long as the rabbit; (ii) the extreme slowness with which the early stages of development are effected in the human embryo; and (iii) the early stage at which the allantois is established in the human embryo, and the peculiar mode in which it is formed.

\section{THE HUMAN OVUM.}

\section{Formation of the Ovum.}

The earlier stages in the development of the ova are already completed in the female child before birth; and after birth the formation of ova only goes on for a very short time, and to a very limited extent. According to Bischoff, Waldeyer, Foulis, and others, the formation of new ova ceases about the age of two years; in other words, the ovaries of a female child already contain, at the end of the second year, all the ova that will ever be developed in them. As each ovum is morphologically a single cell, this means that an individual cell may live, and retain all its characteristic activities, for a period of forty-five years or more.

The early development of the human ova must, therefore, be studied, not in the woman or child, but in the embryo. The several stages of its formation are so closely similar to those 
already described in the rabbit that a detailed account will be unnecessary.

The germinal epithelium. In embryos of abont the fiftl week, the genital ridges appear, as a pair of longitudinal bands along the dorsal wall of the abdominal cavity, close to the inner borders of the Wolffian bodies. The ridges, which at first are merely caused by the epithelial cells becoming columnar in place of squamous in shape, rapidly increase in thickness, partly owing to actice division of the cells of the germinal epithelium covering the ridge, and partly owing to ingrowth of connective tissue along their axes.

At an early age, an intimate relation is established between each genital ridge and the corresponding Wolffian body, a number of rod-like outgrowths arising from the Malpighian bodies of the Wolffian body, and growing into the substance of the genital ridge. These rods subsequently become hollow, and form the so-called tubuliferous tissue of the ovary. This lies, at first, close beneath the germinal epithelium, but soon withdraws into the deeper part of the ovary; it has nothing to do with the formation of the ova, and merely requires mention on account of its great prominence during the early stages of development.

The germinal epithelium gives rise to the ova in much the same way as in the rabbit. In its earliest stages it is a single layer of columnar epithelium cells, with large nuclei, the cells measuring on an average about $0.014 \mathrm{~mm}$. in length by 0.007 $\mathrm{mm}$. in width.

By division of its cells, the germinal epithelium rapidly increases in thickness. The surface cells remain columnar, but the deeper cells, which are spherical or polygonal in shape, grow down into the connective-tissue stroma as irregular branching rods of cells, the egg columns (Fig. 171). By further growth inwards of the egg columns, accompanied by active growth outwards of the vascular connective tissue, the structure of the ovary rapidly becomes more complicated. In place of the original arrangement, of a layer of epithelial cells clothing a central connective-tissue core, there is now (Fig. 171) a superficial layer of columnar epithelium, $a$, beneath which is a reticular framework of connective tissue, the meshes of which are 
filled with irregular columns or rods of epithelial cells, arranged for the most part vertical to the surface.

The primitive ova. The columnar epithelial cells of the surface layer are at first all much the same size, but they do not long remain so. At an early period, about the sixth or seventh week, certain of the cells become conspicuous by their larger size and more spherical shape; these are the primitive ova (Fig. 171, c, c), each of which is capable of developing into a definitive or permanent ovum, and then, if fertilised, of giving

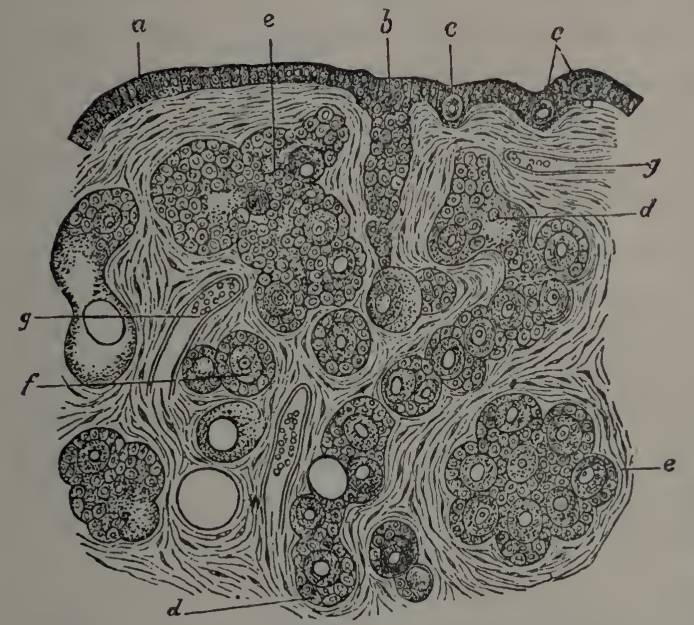

FIG. 171.-Part of a vertical section of the ovary of a new-born Infant.

(From Stricker's 'Histology.') $\times 150$.

$a$, superficial layer of columnar epithelium. $b$, plate of epithelial cells, formed by irregular growth of the ovary. $c c$, primitive ova. $d e$, nests of various shapes, containing ova and commencing follicles. $f$, isolated follicle with its contained ovum. $g$, bloodvessel.

rise to an embryo. Each of these enlarged epithelial cells is in fact a potential human being.

On the formation of the egg columns, by proliferation of the deeper surface of the germinal epithelium, the primitive ova are carried down into them in large numbers. As the egg columns penetrate deeper and deeper into the substance of the ovary, they become broken up, by further growth of the connective tissue, into groups or nests of cells (Fig. 171, $d, e$ ), each nest containing one or more primitive ova as well as a number of indifferent epithelial cells. In these nests a tendency sonn 
manifests itself for the smaller or indifferent cells to arrange themselves round the primitive ova, so as to inclose these in follicles (Fig. 171, $d, e, f$ ). At first there may be in a single nest several of these follicles, each containing a single ovum, but the continued growth of the connective tissue stroma gradually breaks up the nests, and tends to isolate the several follicles from one another, forming around each of them a separate connective tissue investment.

At the time of birth of the infant, the structure of the ovary is as shown in Fig. 171. The germinal epithelium, $a$, or superficial layer of columnar epithelial cells, is separated from the deeper layers of the ovary, at almost all parts, by a thin layer of connective tissue, the tunica albuginea. A little deeper down are seen large nests of epithelial cells, formed by proliferation from the deeper surface of the germinal epithelium, but cut off and isolated by growth of the connective tissue stroma. In these nests certain of the cells, the primitive ova, are distinguished by their larger size, and round these the smaller cells tend to arrange themselves so as to form capsules or follicles. In the deeper parts of the ovary the vascular connective tissue has, by its further growth, broken up the nests, and separated the follicles more or less completely from one another.

In passing from the exterior towards the deeper parts of the ovary, successive stages in the development of the ova are met with. In the superficial layer of columnar epithelial cells the earliest stages are seen; certain of these cells, the primitive ova, being of rather larger size than their neighbours.

Beneath this surface layer are large nests, composed of epithelial cells, which, except in the larger size of the primitive ova, differ but little from one another, and present no regularity of arrangement. In the more deeply placed nests, the cells immediately adjacent to the ova have arranged themselves round these latter so as to form follicles; but there are, in such nests, many cells of indifferent character, whose ultimate fate is still uncertain. Deeper still, the number of these indifferent cells is greatly diminished; and the follicles are larger, more clearly defined, and separated from one another by connective tissue trabeculæ.

In such a section, therefore, as in Fig. 171, the most deeply situated ova are the oldest and most mature, and have, in 
attaining their present position, passed in succession through the several stages which are met with in passing from the surface to the deeper parts of the ovary.

The primitive ova are spherical cells, from 0.05 to $0.07 \mathrm{~mm}$. in diameter, with granular and rather ill-clefined nuclei, and devoid of nuclear membranes. Each primitive ovum is inclosed in a follicle, consisting of a single layer of small cubical or flattened epithelial cells.

The permanent ova. About the time the egg follicles or capsules commence to form around the primitive ova, these latter undergo changes by which they become converted into the permanent ova. Primitive ova occur in both sexes, and the early stages in the development of the genital organs are the same in both; but the change to permanent ova occurs in the female only, and marks the establishment of sexuality.

The change, as in other animals, chiefly concerns the nucleus. In the primitive ovum this is uniformly granular, with a rather ill-defined outline; in the permanent ovum it becomes converted into a spherical vesicular body, of much larger size than before, with a sharply defined double-contoured wall, fluid contents, and a nuclear reticulum with one or more nucleolar enlargements at the nodes.

Besides the changes in the nucleus, the whole egg increases in size; its protoplasm, previously clear, becomes granular; and around the egg, between it and the follicle, a thin elastic investing membrane, the zona radiata, is formed.

The Graafian follicle. Each ovum is surrounded at first by a single layer of cells, derived, like the ovum itself, from the germinal epithelium. These cells are at first flattened, but very shortly become cubical or columnar in shape. Since they lie between the ovum and the blood-vessels of the ovary, the nutrient matter must pass through the follicular cells in order to reach the ovum; and it is probable that these cells do not merely transmit the food, but play some part in elaborating it.

A second layer of cells soon appears in each follicle, formed, as in the rabbit, between the original layer and the ovum, and probably by division of this originally single layer of cells into two. Shortly afterwards, by further division, the follicle 
becomes several cells thick. By splitting apart of these cells, accompanied by rapid growth of the outer layer, a cavity is formed in the thickness of the wall of the follicle; and this cavity, which is filled with fluid, rapilly increases in size, dividing the follicle into an outer wall, the tunica granulosa, and an inner one, or discus proligerus, which immediately invests the ovum (cf. p. 349).

The fully formed Graafian follicle ( $c f$. Fig. 133, GK, p. 347) is ovoid or ellipsoidal in shape: its walls consist of :-(i) an outer investment of vascular connective tissue, derived from the stroma of the ovary, and divisible into a rather ill-defined outer layer, the tunica fibrosa folliculi; and an inner well-marked layer of fine connective tissue, abundantly supplied with capillary blood-vessels, the tunica propria folliculi. (ii) Within this latter is the tunica granulosa (Fig. 133, GB), a thick layer of granular, spherical or polygonal cells. At one part, the tunica granulosa is much thickened, forming a roundish mass projecting into the cavity of the follicle; and embedded in the middle of this roundish mass, or discus proligerus, is the ovum, ow. The cells immediately surrounding the ovum are distinctly columnar in shape, while the remaining cells of the follicle are spherical or polygonal. The cavity of the follicle is filled by the watery liquor folliculi.

In the early stages of their formation (Fig. 133) the more mature Graafian follicles lie in the deepest parts of the ovary ; but, as they increase in size, their growth takes place in all directions ; and ultimately the outer walls of the follicles approach very close to the surface of the ovary, or actually push the superficial layer of epithelium and connective tissue of the ovary before them, and so form rounded external projections on its surface.

At the most prominent part of the ripe Graafian follicle is a small spot, the hilum folliculi, distinguished from the rest of the follicle by being devoid of blood-vessels : at this place, shortly after the follicle has reached its full dimensions, i.e. a diameter of from 1.25 to $4 \mathrm{~mm}$., rupture of the follicular wall occurs, and the ovum, together with the liquor folliculi, is discharged on the surface of the ovary.

This rupture of the wall of the Graafian follicle is due in part to fatty degeneration of the cells composing the wall; and in 
part to increased pressure on the follicle, caused by a sudden accession of blood to the ovary.

The ripe ovarian ovum. The ripe human ovum is a spherical cell, about $0.2 \mathrm{~mm}$. in diameter. It consists of a granular mass of protoplasm; within which is a nucleus, or germinal vesicle, about $0.045 \mathrm{~mm}$. in diameter, containing a nuclear reticulum and a conspicuous nucleolus or germinal spot. The ovum is invested by a transparent elastic membrane, the zona pellucida, which is about $0.01 \mathrm{~mm}$. thick.

Each Graafian follicle, as a rule, contains only a single ovum; in exceptional cases two ova, and in a few instances three, have been seen in the same follicle.

\section{The Corpus Luteum.}

After the discharge of the ovum, important changes occur in the Graafian follicle, leading to the formation of the body known as the corpus luteum, which occupies and fills up the cavity of the follicle.

'The corpus luteum is formed by rapid growth of the wall of the empty follicle, which becomes thrown into radial folds, projecting into the cavity of the follicle, and blocking this up almost completely. The folding involves both the follicular epithelium and the connective-tissue wall of the follicle, but the latter takes the most active share in the process. The characteristic vellow colour of the folded wall, which has given rise to the name corpus luteum, is due to large numbers of yellowish cells, derived apparently from the connective tissue stroma of the ovary. Between the two layers of each of the folds, bloodvessels pass in freely; and the central cavity of the follicle, which, by ingrowth of the radial folds, is reduced to an irregularly stellate space, becomes occupied by a cicatricial fibrons tissue, which is red in the early stages, but in the later ones becomes grey.

The subsequent changes in the corpus luteum differ considerably according to whether the ovum, which has been discharged from the follicle, (i) is fertilised and develops into an embryo; or (ii) is not fertilised, but dies without undergoing any further development.

In the latter case, i.e. if the ovum is not fertilised, the corpus 
luteum spurium, as it is then called, increases slightly in size for a few days; but ten or twelve days after the discharge of the ovum, commences to shrink, and disappears completely in a few iveeks' time.

If, however, the ovum that has escaped from the follicle is fertilised, and gives rise to an embryo, the corpus luteum, now spoken of as corpus luteum verum, or corpus luteum of pregnancy, does not reach its full development until two or three months after the discharge of the ovum. It persists throughout the greater part, or the whole, of pregnancy, contracting towards the close of the period to a small white stellate cicatrix, the corpus albicans, which may persist for some months after delivery.

The fully developed corpus luteum verum, or corpus luteum of pregnancy, is a firm body, larger than the original follicle, and attaining one-fourth, or even one-third, the size of the entire ovary (Fig. 255).

The presence of a corpus luteum verum in one of the ovaries is a matter of considerable medico-legal importance, inasmuch as it has been appealed to as positive evidence of pregnancy having occurred; but the best authorities now agree that there is no infallible sign by which the corpus luteum of pregnancy can be distinguished from that of the non-fertilised ovum. The differences between the two are chiefly those of size, and length of duration, and cannot always be relied on in determining disputed cases. The terms 'true' and 'false,' as applied to the two kinds of corpora lutea, appear, indeed, to be erroneous; as the two structures are essentially similar, and in many cases indistinguishable from each other.

\section{Ovulation.}

From the time of puberty, and throughout the whole of the child-bearing period of life, i.e. from about the fifteenth to about the forty-fifth year, the gradual maturation of the Graafian follicles, ending in rupture of the follicles and discharge of the ora, is continually going on ; and in the healthy condition this discharge of ova occurs, not in an indefinite manner, but at regular, and usually monthly intervals, one or more ova being set free at each period.

This periodical maturation and discharge of ova is spoken of as ovulation. It goes on independently of sexual intercourse, 
or of any kind of influence from the male; but it is possible that, as held by many authorities, the discharge of ova, though in no way dependent on sexual intercourse, may yet be hastened by this.

\section{Menstruation.}

Menstruation is the periodical discharge from the uterus of a certain amount of blood, mixed with mucus from the uterine glands, and with epithelial and connective-tissue cells, derived from disintegration of the mucous membrane of the uterus itself.

There is a close connection between menstruation and ovulation. Both processes commence at puberty, and last throughout the child-bearing period. They both recur periodically; and, further than this, the intervals are the same, and the two processes occur, as a rule, simultaneously. The true nature and extent of the connection between the two will be discussed after the nature of the menstrual process has been considered more fully.

During the period of pregnancy, that is, during the whole time that an ovum or embryo is developing within the uterus, menstruation ceases, recommencing six or seven weeks after the birth of the child. The normal occurrence of the menstrual periods may also be affected by a variety of accidental or pathological conditions, for the consideration of which reference must be made to works dealing with obstetrics.

Menstruation, i.e. the actual discharge from the uterus of blood and other matters, is not an isolated process, but is the terminal act of a series of changes, which occur at regular intervals in the walls of the uterus, and of which the sequence is as follows.

In the quiescent condition the uterus is lined by a smooth mucous membrane, of a soft, spongy consistence, and pale red colour. It consists of a single layer of ciliated epithelial cells, resting on a very delicate basement membrane, beneath which is the connective-tissue layer of the mucous membrane. This latter is about $1.5 \mathrm{~mm}$. in thickness, and consists of connective tissue, with very numerous connective-tissue cells, and traversed by irregularly arranged muscle fibres. It is attached by its outer surface to the muscular wall of the uterus. 
The epithelium lining the uterus is pitted to form the uterine glands. These (Fig. 175) are tubular glands, embedded in large numbers in the connective-tissue layer of the mucous membrane, vertically to the inner surface of the uterus ; they are straight, or slightly convoluted; their blind or outer ends are usually slightly dilated; and they secrete a transparent, glutinous, alkaline fluid.

Changes in the mucous membrane accompanying menstruation. These changes commence with congestion and tumefaction of the mucous membrane lining the entire uterus. This swells up considerably, becoming softer and more vascular than before, and forming ridge-like folds which project into the cavity of the uterus. The connective-tissue cells increase considerably in number, and the uterine glands become longer, wider, and more convoluted. The whole layer of mucous membrane increases in thickness from $1.5 \mathrm{~mm}$. to from 3 to $5 \mathrm{~mm}$.; while the glands increase in diameter from 0.08 to $0.12 \mathrm{~mm}$. This swollen and hypertrophied mucous membrane forms what is called the menstrual decidua.

At the menstrual period, the superficial layer of the mucous membrane, about a fourth of the entire thickness, breaks down and is thrown off, usually in detached fragments, but sometimes, in cases of dysmenorrhoea membranacea, as a single piece, forming a complete cast of the interior of the uterus. Fatty degeneration has been noticed in these cast-off cells, but only in the later stages, after the menstrual discharge has actually commenced.

This disintegration, and casting off, involves the loss of the epithelial lining of the uterine cavity, of the mouths of the uterine glands, and also of about one-fourth of the entire thickness of the swollen mucous membrane. It of necessity causes rupture of the blood-vessels of the detached portions, and so occasions more or less free hæmorrhage; and the blood so discharged, together with the broken-down mucous membrane of the uterus, and with a certain amount of mucus from the uterine glands, forms the menstrual or catamenial flow.

The menstrual flow lasts, as a rule, from three to five days, but may be protracted for a week or more. It is accompanied by nervous and other disturbances, which are fully described in works on obstetrics. 
At the commencement of a period, the menstrual discharge is viscous, consisting largely of mucus from the uterine glands, slightly tinged with blood; in the middle of the period the flow becomes almost pure blood; while towards the end it becomes paler, the mucus again preponderating. Owing to mixture with the uterine mucus, the blood of the menstrual flow does not coagulate. The total amount of the menstrual discharge is usually from four to six ounces; but this may be widely cleparted from in individual cases, either in the way of diminution or of excess.

On the cessation of the menstrual flow, the uterine epithelium is very quickly regenerated, spreading over the surface from the necks of the uterine glands. It is completely reformed within three or four days of the end of the menstrual period. After this re-establishment of the uterine mucous membrane, the nterus remains in a quiescent condition for from ten days to a fortnight; at the end of this time it begins to swell again, and the menstrual process is repeated. This repetition occurs, as already noticed, at intervals, usually of four weeks, throughout the whole child-bearing period; the only normal disturbing element being gestation, during which menstruation is in abeyance, recommencing a short time after the birth of the child.

\section{Explanation of the Menstrual Process.}

The complete menstrual cycle, occupying in typical cases twenty-eight days, may be divided into four stages, which follow one another in regular sequence.

(i) The first or constructive stage is characterised by swelling of the mucous membrane, enlargement of the uterine glands, and increase in the connective-tissue cells of the mucous membrane; it results in the formation of a menstrual decidua, lining the entire uterus.

(ii) The second or destructive stage includes what is ordinarily known as the menstrual or catamenial period. It is marked by abundant discharge of mucus from the enlarged glands, and by the disintegration and discharge from the uterus of the inner layer of the mucous membrane. It involves loss of the epithelial lining of the uterus and of the necks of the glands, and is accompanied by hæmorrhage.

(iii) The stage of repair comes next, during which the uterus is recovering from the destructive changes. The uterine 
epithelium is restored, by growth from the lips of the deeper parts of the uterine glands; and the swelling of the mucous membrane subsides.

(iv) The fourth stage is the period of quiescence, during which the uterus, having regained its normal structure, remains without further change until the commencement of the next sncceeding constructive stage.

The actual and relative durations of the several stages enumerated above are not determined with certainty, and are subject to individual variations. It will, perhaps, be right to assign about a week to the constructive stage ; rather less than a week (five days on an average) to the destructive stage ; three or four days to the stage of repair ; and twelve or fourteen days to the quiescent period; the four stages together occupying the twenty-eight days which make up the normal menstrual cycle.

Of the above four stages, the first and second require further attention; the fourth stage is the normal condition; and the third stage is merely the return of the uterus to the normal condition after a period of disturbance.

Concerning the first or constructive period, there is hardly any room for doubt that it is to be regarded as a preparation on the part of the uterus for the reception of an ovum.

The several stages of the process correspond closely, in essential respects, with those that occur in the placental lobes of the rabbit's uterus from about the fourth to the eighth day. In the rabbit, as in the human uterus, there occur swelling of the mucous and submucous tissues, increased vascularity, a large increase in the number of the connective-tissue cells, and a great enlargement of the uterine glands, which become larger, wider, and more freely branched. These changes, in the rabbit's uterus, are clearly related to the nutrition of the embryo, for it is to this hypertrophied and modified area of the uterine mucous membrane that the embryo becomes attached on the eighth day; and it is from this area that the maternal part of the placenta is formed.

The most important difference between the rabbit's and the human uterus, as regards these stages, is that in the rabbit the ovum, or rather the blastodermic vesicle, is present within the uterus during the whole of the series of changes, although it lies quite freely and does not acquire attachment until the eighth day; while in the human uterus, on the other hand, the men- 
strual constructive process goes on without the stimulus afforded by the presence of an ovum.

As regards the actual changes in the uterus itself, the resemblance between the two cases is so great that it seems necessary to suppose that their significance is the same ; and it must, therefore, be concluded that the human uterus periodically prepares itself, by the formation of a decidual lining, for the reception of an ovum; the process occurring at monthly intervals throughout the child-bearing period, and quite irrespectively of the presence or arrival of a fertilised ovum.

The second or destructive stage, constituting the act of menstruation in the ordinary sense of the term, is much more difficult to explain. At first sight it appears to consist simply in a rapid, and somewhat violent, undoing of the work accomplished in the preceding stage.

If, however, it is compared with the changes that take place in the rabbit's uterus during gestation, it is found that the human uterus at the end of the constructive period of menstruation has reached a stage corresponding to that of a rabbit's uterus at the end of the seventh or beginning of the eighth day of pregnancy, when the blastodermic vesicle is still lying freely within the uterus, but is just about to acquire its attachment.

In the rabbit this attachment is effected, early on the eighth day, by fusion of the wall of the blastodermic vesicle with the epithelium of the modified and hypertrophied placental lobes of the uterus (Fig. 169). This fusion is immediately followed, or rather is accompanied, by degenerative changes in the uterine mucous membrane opposite the area of attachment, which rapidly lead to absorption of the uterine epithelium, and of the mouths and necks of the uterine glands.

Similar changes occur during the formation of the human placenta, and will be described in the concluding section of this chapter; and inasmuch as the portion of the wall of the nterus which is concerned in the changes is the same in menstruation and in pregnancy, the menstrual discharge may be viewed, not merely as a destructive process, but as corresponding in a modified form to the rapid absorption of the same parts which occurs normally during pregnancy.

The constructive stage of menstruation, and, as just seen, the destructive stage as well, may be regarded as phases in the 
preparation of the uterus for the formation of a placenta; stages which can be carried up to a certain point without needing the stimulus of the presence of an ovum or embryo, but which, having reached a point at which further development is impossible without an embryo, stop abruptly. The constructive stage has been shown to be an active preparation of the uterus for the reception of a fertilised ovum; the succeeding or destructive stage is not to be regarded as a simple undoing of this preparation, but as a further continuance, in a modified form, of the act of preparation, which leaves the uterus in a condition in which, for further elaboration to occur, the presence of an embryo is indispensable.

\section{The Connection between Ovulation and Menstruation.}

Ovulation and menstruation, or the discharge of ova from the ovary, and of the disintegrated decidua from the uterus, are processes which occur periodically, and as a rule simultaneously; and it becomes a matter of interest to inquire into the nature of the connection between them.

The ovaries swell up, and become tender, at monthly intervals. The enlargement commences, as a rule, a few days before the menstrual period, attains its maximum about the time of the period, and gradually subsides after the period is over.

As the ovary is known to become congested just before the rupture of a Graafian follicle and the discharge of an ovum, it appears a fair inference that this discharge occurs about the same time as the menstrual flow, i.e. that ovulation and menstruation are practically simultaneous. However, although this may be, and probably is, the rule, yet it is far from being an invariable one. Thus Kölliker, on examining the ovaries of seven women who had died directly after menstruation, found that in two of the cases there was no fresh corpus luteum in either ovary; that is, that no ovum had been discharged at the time of menstruation; and Coste has cited similar instances.

Ovulation and menstruation may be assumed to occur as a rule about the same time, but it is by no means clear what is the precise nature of the connection between the two processes. Authorities differ as to the stage in the menstrual period at which ovulation occurs, the majority holding that it takes place 
two or three days before the commencement of the period, while others maintain that it happens at the middle, or even towards the end, of the period. It is very possible that there is no constancy in this particular respect.

A still more difficult point remains to be considered. The menstrual decidua is to be viewed as a preparation on the part of the uterus for the reception of an ovum; but it has still to be determined whether the decidua which is broken up and discharged at a given menstrual period is the one prepared for the ovum which is set free from the ovary at the same period, or for an ovum liberated at some previous or subsequent period. The question is one of great importance, as the means of determining the age of human embryos are very materially affected by the answer given to it.

The menstrual cycle has been seen to consist essentially in a periodically recurring preparation of the uterus for the reception of an ovum. It is important to determine, if possible, at what particular phase of the cycle, the uterus is in the condition most favourable for the reception of an ovum. Very different views have been expressed on this point, and two of these call for special notice.

(i) That the end of the constructive period is the natural and most favourable moment for the ovum to enter the uterus.

(ii) That the period of quiescence is the most favourable time.

In support of the former view, it is urged that the formation of the decidua is unintelligible except on the supposition that it is a preparation for the reception of the ovum; and that the analogy of the rabbit's uterus, in which the sequence of changes is strikingly similar, is in favour of the end of the constructive period, or perhaps the commencement of the destructive period, being the one specially concerned with the fixation of the ovum to the wall of the uterus.

It must be noticed, however, that if the normal time of attachment to the uterus is, in the human ovum, the end of the constructive period, i.e. the commencement of the menstrual period, then it is clear that the ovum which is to be attached cannot be the one discharged from the ovary at the same period. For the discharge of the ovum is practically coincident with the onset of the menstrual period; and the ovum, after leaving the 
ovary, has still, in order to reach the uterus, to travel along the entire length of the Fallopian tube, a passage which is known to take three days in the rabbit, and eight to ten days in the dog, and which in all probability takes at least a week in the human species. It follows that the decidua which is discharged at a given menstrual period cannot have been prepared for the ovum discharged at the same period, but must be the preparation for the ovum which was discharged at the preceding menstrual period.

The second view, that the period of quiescence in the menstrual cycle is the most favourable time for the entrance of the ovum into the uterus, leads to the same conclusion, inasmuch as the only ovum which could reach the uterus during the quiescent stage is the one discharged at the previous menstrual period.

In favour of this second view, that the quiescent period in the menstrual cycle is the most favourable time for the ovum to enter the uterus, the following considerations may be urged.

(a) A much greater range of time is given, within which the uterus is ready for the reception of an ovum. The quiescent period is the longest of the four stages which compose the menstrual cycle, lasting from twelve to fourteen days ; while, on the view that the completion of the constructive process marks the time at which the uterus is best fitted to receive an ovum, the range of time is limited to two or three days at most; and the longer period is more in accordance with what is known of the range of time within which conception may occur.

(b) The stages in the formation of the menstrual decidua have been compared, above, with the changes which occur in the uterus of a rabbit, from the fourth to the seventh or eighth day of pregnancy; and the close similarity between the two cases has been insisted on. It should now be noticed that these changes in the rabbit occur after the entrance of the ovum into the uterus; i.e. that in the rabbit the ovum enters the uterus while this latter is in the quiescent stage.

Neither of the above arguments is at all conclusive, and the question is still an open one. It must be repeated, however, that if either of these views is correct, the same conclusion follows with regard to the relation between ovulation and menstruation, viz. that the decidua of a particular menstrual period 
is related, not to the ovum discharged at that period, but to the ovum discharged at the preceding period.

It follows that there is no necessary connection between ovulation and the occurrence of the menstrual flow; a point which helps to explain the cases quoted by Kölliker, Coste, and others, in which there was no discharge of ova at the time of menstruation.

The fact that the two processes, ovulation and menstruation, occur normally at or about the same time, may perhaps be explained by the consideration that at the time of ovulation there is very considerable congestion of the ovaries and Fallopian tubes; and this, owing to the free communication between the ovarian and uterine arteries, must almost necessarily cause congestion of the uterus; and this determination of blood to the large and thin-walled vessels of the decidua is probably an important factor in causing the menstrual hæmorrhage.

\section{The Duration of Pregnancy.}

Much has been written on this point, and many elaborate tables have been compiled from which it appears:

(i) That there is no absolutely fixed period of gestation.

(ii) That there is no means of determining with certainty the commencement of gestation, as the precise time of fertilisation of the ovum cannot be ascertained.

It is customary to calculate the duration of pregnancy from the last occurring menstrual period; and this, if the argument given above is correct, will correspond with the discharge, from the ovary, of the ovum from which the child is developed. The most reliable estimates indicate a normal duration of pregnancy, dating from the last occurring menstrual period, of 270 to 280 days. This is, however, estimated by some authorities from the first day of the period; by others, and more usually, from the last day.

It is possible that the actual limits, in normal pregnancy, are not so wide as indicated above. Apart from the difficulty of determining the date of fertilisation, the chief causes of uncertainty arise from our ignorance of the length of time during which the ova and spermatozoa retain their vital activity, after leaving the ovary and testis respectively. 
Concerning the spermatozoa, we have very little precise knowledge. It is known that spermatozoa, introduced into the vagina, may retain their vitality, and presumably their fertilising power as well, for a week; and the fact that successful impregnation may occur at any time in the menstrual cycle, strengthened by the analogy of other animals, suggests that human spermatozoa may retain their power for considerably longer periods. It is stated that ripe spermatozoa may remain for months in the testis before being discharged, without losing their fertilising power.

The time taken by the spermatozoa to travel along the vagina, uterus, and Fallopian tube to the ovary, is not known, but is probably very short; in the rabbit it does not occupy more than a quarter of an hour to two hours.

If the ovum is not fertilised it soon dies. How long an ovum may retain its vitality, and capacity for fertilisation, is not known; indeed, no unfertilised human ovum has yet been seen, outside the ovary. Some experiments of Bischoff, on lower Mammals, point to the conclusion that, in these, the ovum, if not fertilised, dies in the lower part of the Fallopian tube, before reaching the uterus. Assuming that the human ovum also dies shortly before reaching the uterus; and assuming further, as is done by most authorities, that the human ovum takes at least eight days to travel down the Fallopian tube, it may be stated that the human ovum probably retains its vitality, and power of being fertilised, for some time, perhaps a week, after discharge from the ovary; but ultimately loses it, probably before reaching the uterus. This is, however, at present little more than speculation.

If the above considerations prove well founded, and if, as suggested above, the length of time during which an ovum remains alive and fertilisable, after leaving the ovary, is less than the interval between two successive periods of ovulation, it will follow that there must be certain times during which there are ova ready to be fertilised, and certain times during which there are none; i.e. that fertilisation can only be effected at certain recurring periods, and cannot occur in the intervals between these periods.

Concerning the respective lengths of these periods we have 
no certain knowledge, but it is commonly held that the intervals rluring which there are no ova capable of being fertilised are at least as long as the periods in which there are such ova. In other words, assuming that the ova discharged at a given menstrual period retain their vitality for from ten to fourteen days - a pure assumption-there would be an interval of about two weeks before the next menstrual period, i.e. before the next discharge of ova, and during this interval there would be no fertilisable ova in the oviduct, and fertilisation could not take place. Iny spermatozoa received during this interval would have to wait until its close, at the next period of ovulation, before they had a chance of meeting with ova capable of being fertilised.

There seems to be a general consensus of opinion that the first day or days after the cessation of the menstrual period are the most favourable time for fertilisation to take place. This is in complete accordance with what has been said above, both with regard to the ovum and the decidua, for the ovum will be lying within the Fallopian tube in a healthy fertilisable condition and easily accessible to the spermatozoa; while if the ovum takes another week or so to travel down the tube to the uterus it will enter this latter while it is in the quiescent state, which, it has been shown above, there is reason for regarding as the most favourable one for the reception of the ovum.

\section{Estimation of the Age of Human Embryos.}

It follows from what has been said above, that there is no means of determining with certainty the age of a human embryo prematurely discharged from the uterus; for development dates, not from the discharge of the ovum from the ovary, but from the moment of fertilisation; and this latter cannot be determined.

Ovulation is a process easily overlooked, but the fact that it occurs simultaneously with the menstrual periods renders its date readily determinable, but within certain limits only. The connection between the two processes is a loose one, and it is probable that ovulation may occur either from two to three days before a menstrual period, or during the period; giving a possible error of about a week in estimating the age of an embryo from the date of menstruation. 
Professor His, in the first part of his monograph on the development of the human embryo, laid down the following rule:-

"The age of an embryo is the time that has elapsed since the first day of the first omitted period.'

Thus, supposing the commencement of a menstrual period to be due on January 5 , and that when this time comes, the period is omitted; but that at some subsequent time, say February 9 , an embryo is aborted; then, according to Professor His' rule, the age of the embryo would be the interval between January 5 and February 9, i.e. thirty-five days.

In arriving at this result, Professor His argues in the following manner: The ovum leaves the ovary either at, or shortly before, the menstrual period; if it is fertilised, presumably by spermatozoa previously introduced, menstruation does not occur'; but the changes in the uterine mucous membrane, instead of, as usual, becoming retrogressive, either remain stationary or else continue to be progressive; and so prepare the uterus for the reception of the ovum. Hence the first omitted menstrual period corresponds in point of time with the fertilisation of the ovum; and hence the age of the embryo may be taken as the time that has elapsed since the first omitted period.

This method of calculation is, however, open to very grave objections, the more important of which are as follows :-

(i) There are strong reasons, which have been fully considered in the previous portion of this chapter, for regarding the decidua which is broken up and discharged at a menstrual period to be related, not to the ovum discharged from the ovary at the same period, but to the ovum discharged at the preceding period.

(ii) Professor His' rule assumes that the ovum is invariably fertilised on the first day of the first omitted period. There is no direct evidence in support of this; and the loose nature of the connection between ovulation and menstruation renders it highly improbable.

(iii) The rule assumes that the act of fertilisation of an ovum, which in all probability will not reach the uterus for at least a week, is able to arrest the degenerative changes already commenced in the decidua, to suddenly stop the menstrual flow that is on the verge of taking place, or has actually commenced, and 
to convert the retrogressive changes of the uterus into progressive ones.

(iv) The rule is not in accord with the well-established fact that, in order to insure pregnancy, the most favourable time for intercourse is shortly, or immediately, after the conclusion of a menstrual period. This is intelligible enough if the ovum to be fertilised is the one discharged at that period; but is hard to understand if, as the rule requires, these spermatozoa have to wait for a period of three weeks or more, until the next discharge of ova.

These objections are serious ones, and Professor His, in the second part of his work, recognises that the rule as originally formulated cannot apply to all cases. He quotes instances in which the dates were accurately recorded, and in which the fertilised ovum must have belonged to the last occurring period, and not to the first omitted one; he is of opinion, however, that the rule as stated above will still apply to the majority of cases.

This more recent view may be expressed graphically, thus. If $I$. is the first day of the last actually occurring menstrual period, and II. is the first day of the first omitted period; then the possible days of fertilisation are as follows:-

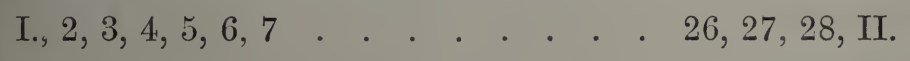

That is, an ovum discharged during an actually occurring period remains capable of fertilisation for a certain number of days, expressed in the formula as a week, commencing with I., and ending at 7. During this time it may be fertilised, either by spermatozoa received after the period is over, or received before the period and retained in the oviduct during it. In the case of these embryos the age should be calculated from I., the first day of the last actually occurring period.

On the other hand, Professor His, and others, maintain that there are possibilities of fertilisation at the other end of the series ; and that an ovum, discharged from the ovary a day or two before the next period, II., is due, may, if fertilised, stop that period from occurring; and in such cases, if they really happen, the age of the embryo should be calculated from the first omitted period, and not from the last occurring one. It is not yet certain which of these two possibilities is the normal mode of 
occurrence, but such evidence as we have is in favour of the former.

It is customary, however, to adopt His' original rule, and to estimate the age of human embryos from the first day of the first omitted period, and this method will be followed in this chapter. It must be repeated, however, that this is done merely from convenience, and from the absence of any other precisely formulated system. Viewed on its own merits, His' rule will certainly not apply generally.

\section{THE GENERAL HISTORY OF DEVELOPMENT OF THE HUMAN EMBRYO.}

In this section the earliest stages in the formation of the human embryo will be described, so far as they are at present known; and an account will be given of the external characters of the embryo at the several stages up to the time of birth.

These descriptions are in the great majority of cases taken from Professor His' monograph, and, as explained in the preceding section, the ages given are those assigned by him to the several stages. In the following sections the development of the nervous, digestive, and other systems will be considered in detail; and in the concluding section the placenta, the foctal membranes, and the relations of the embryo to the uterus will be described.

The actual length of an embryo is not always easy to determine, owing to the varying amount of flexure of the head and body at different stages. By the length of an embryo, in the following descriptions, is always meant the longest straight line that can be drawn through it in the sagittal plane. In the earliest stages of development this coincides fairly well with the longitudinal axis of the embryo (Figs. 176 to 179); from the beginning of the fourth week to the end of the fifth week (Figs. 200,203 , and 205) it is a line drawn from the prominent hump at the junction of the head and body, to the pelvic region; and from the end of the fifth week onwards, as the head is gradually lifted up by straightening of the neck (Figs. 211, 212), the line once more approximates to the longitudinal axis of the fœetus.

With regard to the general course of development, the first 
fortnight is occupied in preliminary processes, no trace of the embryo appearing until the twelfth or thirteenth day. From the end of the second to the end of the fourth week, the embryo is acquiring definite form, and the various organs and systems are being established. From the fourth to the sixth or seventh week there is a gradual change from the embryonic to the foetal form; the head becoming uplifted, the nose, ears, and lips established, the limbs divided by joints, and the fingers and toes formed. By the end of the second month, the general form is as shown in Fig. 212, and from this time onwards the further changes consist chiefly in increase of size, and in proportionately greater development of the limbs.

The changes that occur in the shape and size of the embryo up to the end of the second month are well shown in the series of outlines given in Figs. 176 to 178, 189 to 195, 199 to 203, 205, 211, and 212. These figures, which are borrowed from Professor His, are in each case five times the linear dimensions of the embryos themselves.

\section{The First Week.}

The fertilisation of the human ovum has not been studied. A single observation, by Nagel, of a ripe ovarian ovum, removed by operation and examined in a fresh condition, showed that two polar bodies were present, lying on the surface of the ovum within the zona pellucida.

There is no reason for supposing that fertilisation is effected in other than the normal manner; and it is probable that it takes place at or about the time the ovum leaves the ovary and enters the oviduct.

The segmentation of the human ovum has not been seen. It is highly probable, from analogy of other Mammals, that it occurs during the passage of the ovum along the Fallopian tube towards the uterus.

The ovum of the dog, which is slightly smaller than the human ovum, travels quickly along the first part of the oviduct but stays some days in the distal or lower part, where it undergoes segmentation, entering the uterus eight or ten days after leaving the ovary. Bischoff and others believe, though there is no direct evidence on the point, that the human ovum agrees in this respect fairly closely with that of the dog; undergoing 
segmentation in the lower part of the oviduct, and not entering the uterus until from eight to ten days, or perhaps longer, after the time of discharge from the ovary.

\section{The Second Week.}

Of ova or embryos which are believed to belong to the end of the second week a few examples have been described. These are of great interest, although there is room for doubt in some of these cases whether the specimens can be regarded as perfectly normal.

Reichert's ovum. The best known instance is an ovum described by Reichert, and believed to be of about the twelfth or thirteenth day. This ovum, which is represented four times the

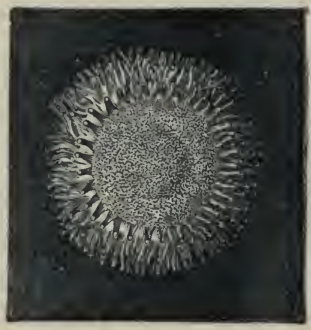

Fig. 172.

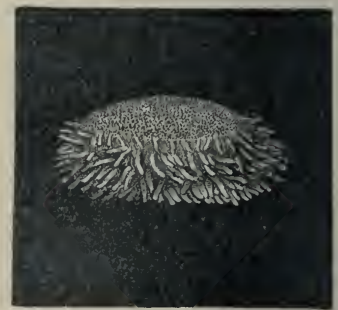

FIG. 173.

FIGS. 172 and 173.-Front and side views of Reichert's Ovum. (From Kölliker, after Reichert.) $\times 4$.

natural size in Figs. 172 and 173, was found, in situ, in the uterus of a woman who had committed suicide, and gave every indication of being perfectly normal.

The ovum was a vesicular body, lenticular in shape, and measuring $5.5 \mathrm{~mm}$. across its greater diameter, and $3.3 \mathrm{~mm}$. from side to side. Of the two surfaces, the one turned towards the wall of the uterus, the upper one in Fig. 173, was more convex than the opposite surface, which faced towards the cavity of the uterus. The margin of the vesicle was thickly fringed with villi, the largest of which were $0.2 \mathrm{~mm}$. long, and slightly branched; the middle portions of both surfaces were smooth, and devoid of villi; and in the centre of the more convex or uterine surface was a small circular spot (Fig. 172), 1.6 mm. in diameter, and of a darker colour than the rest of the vesicle.

The relations of the ovum to the uterus were as follows. The 
entire uterus was lined by a decidua, described as not differing in any special manner from an ordinary menstrual decidua, and forming the usual ridge-like projections into the cavity of the uterus. To one of these ridges, on the dorsal surface of the fundus of the uterus, the ovum was attached, the decidua spreading over it as a thin layer so as to completely encapsule it (cf. Fig. 175). The marginal villi were lescribed and figured by Reichert as penetrating a little distance into the enlarged uterine glands.

In the ovum itself there was no indication of primitive or neural grooves, nor of any other part of the embryo. The wall of the vesicle was described by Reichert as consisting of a single layer of flattened epithelial cells, prolonged

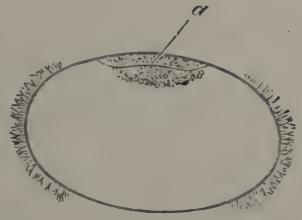

FIG. 174. - Diagrammatic section of Reichert's Ovum. (From His.) $\times 5$. $a$, the embryonal area. outwards to form the hollow villi. In the circular patch on the uterine surface, spoken of as the germinal or embryonal area, a second or inner layer of finely granular nucleated cells was present. The cavity of the vesicle was occupied by a gelatinous fluid, traversed by a network of fibres, and containing within it a rounded body attached to the germinal area.

Lining the whole vesicle was a second, fairly coherent membrane, with which the fibres were continuous. By Reichert this second membrane, the network of fibres, and the central rounded body were all alike considered to be artificial products, due to coagulation of the fluid contents of the vesicle by the alcohol in which the specimen was preserved.

Ova of similar appearance, and of apparently about the same age, have been described by Wharton Jones, Breuss, Kollmann, and others; and in none of these cases was any trace of an embryo present.

The position held by the ovum in relation to the uterus, in the case recorded by Kollmann, is shown in Fig. 175. The whole uterus was lined by a decidual membrane, DV; this was greatly thickened about the middle of the ventral wall, forming: the decidua serotina, Dw, to which the ovum, CV, was attached; the decidua extending over the ovum so as to completely encapsule it. The ovum itself was in the form of a hollow, thinwalled vesicle, with short branched villi projecting from its 
surface, apparently on all sides. The villi were stated not to penetrate into the uterine glands. The minute structure of the ovum, or blastodermic vesicle as it may more properly be called, was not ascertained.

The chief additional points learnt from these further

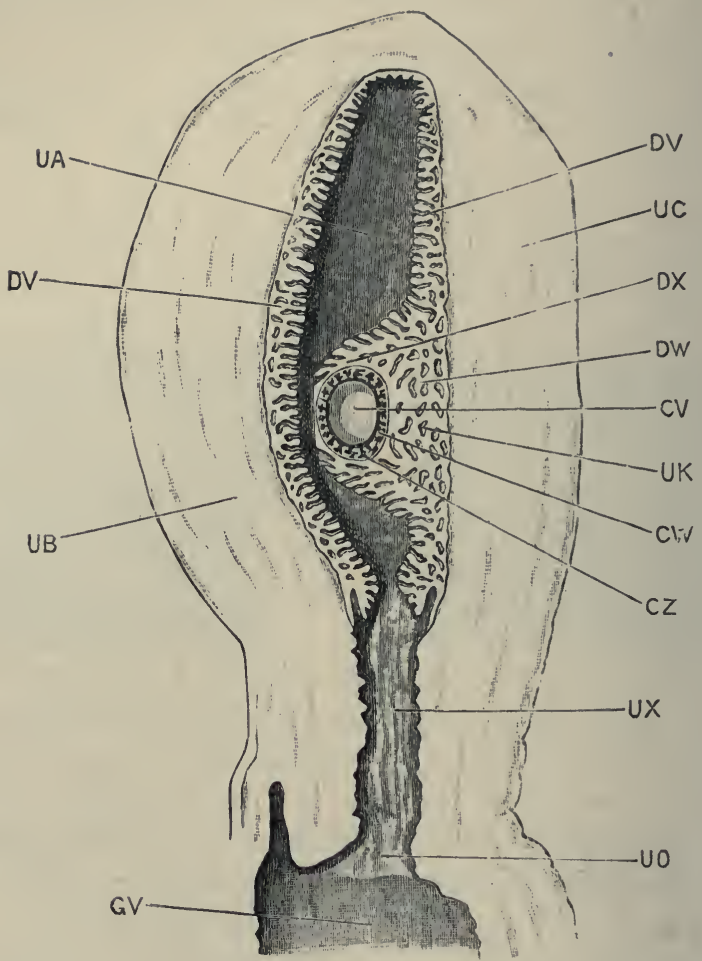

FIG. 175.-A longitudinal section of the Uterus with an Ovum in situ, estimated as about the thirteenth day. (After Kollmann.) $\times 1$.

CV, eavity of ovum or blastodermic vesiclc. CW, wall of blastodermic vesicle. CZ, villi projecting from wall of blastodermic vesicle. $\mathbf{D V}$, decidua vera. $\mathrm{DW}$, decidua serotina. DX, decidua reflexa. $\mathbf{G V}$, vagina. $\mathbf{U A}$, cavity of uterus. $\mathbf{U B}$, dorsal wall of uterus. UC, ventral wall of uterus. UO, os uteri. UKK, uterine glands. UX, cervix uteri.

specimens are: (i) that the rounded body described by Reichert as lying within the vesicle is made up of nucleated cells, and is apparently solid, and attached to the embryonal or germinal area; (ii) that there is strong reason for thinking that the wall of the vesicle really consists, not of a single layer of cells, but of two layers, of which the inner one, regarded by Reichert as a 
coagulation product, is of the nature of connective tissue, and therefore of mesoblastic origin. This latter point is, however, a doubtful one.

In the present state of our knowledge it is hardly possible to make any satisfactory comparison between these early human ova, and the stages already described as occurring in the rabbit. The difficulty is much increased by the absence of detailed histological description, and by the doubt as to whether the ova are in all respects normal. It must also be borne in mind that we are absolutely ignorant of the mode in which segmentation of the human ovum, and the immediately succeeding stages are effected; and that great uncertainty still exists with regard to the details of these processes in the rabbit.

As, however, the several human ova of the stage in question agree in a number of important points, and as, in the case of Reichert's ovum, there is every reason for regarding the specimen as normal, it is advisable to make such comparison as is possible between these ova and the several stages of development of such a Mammal as the rabbit.

In the first place, the complete absence of any trace of an embryo indicates that the stage is a very early one. In ova which there are strong reasons for regarding as but one or two days older, an embryo is present; so that the stage represented by Reichert's ovum may be described as one shortly before the first appearance of the embryo, and as corresponding in this respect with the blastodermic vesicle of a rabbit at about the fifth or sixth day.

In its vesicular character, the thinness of its walls, and the presence of a central embryonal area of different constitution to the rest of the wall, there are additional points of resemblance between Reichert's ovum and the blastodermic vesicle of a rabbit of the sixth day, or of a dog in the early part of the third week. There is also a close correspondence in actual size between these stages in the three instances.

Reichert was of opinion that this comparison was a true one; and the view is supported by $\mathrm{His}$, who gives in illustration of it the diagrammatic section (Fig. 174). His considers that the outer wall of the vesicle consists of epiblast only, and that the hypoblast forms the inner circular patch of cells in the embryonal area; he also regards the central rounded mass of 
cells as hypoblastic, and as destined to become hollowed out at a later stage to form the yolk-sac.

Concerning this comparison, it must be borne in mind that we have as yet no satisfactory knowledge of the histological structure of these early human ova, and that the stage is one about which much doubt exists even in the case of the rabbit. It must further be noticed that there are some points of importance which tell directly against the interpretation suggested by Reichert and His.

In the first place, there is nothing in the blastodermic vesicle of a rabbit on the sixth day that can be compared with the central mass of cells in Reichert's ovum.

Secondly, if His is right in interpreting this central mass of cells as the yolk-sac, then the yolk-sac of the human embryo is developed in a manner entirely different from that of the rabbit. In the rabbit (Fig. 146), the yolk-sac is part of the blastodermic vesicle itself, while in the human embryo it appears to be, from the first, independent of this. In other words, if the central mass of cells in Reichert's ovum is the yolk-sac, and the later stages strongly support this interpretation, then the wall of the vesicle of Reichert's ovum cannot correspond to the wall of the rabbit's blastodermic vesicle ( $c f$. Figs. 146 and 188).

Thirdly, there is strong reason for thinking, as already noticed, that the wall of Reichert's ovum is double; an inner mesoblastic lining being already present, as well as the outer epithelial layer. To this inner layer, if it really exists, there is nothing corresponding in the rabbit's blastodermic vesicle until a later stage.

On the whole, then, the evidence, while not excluding a general correspondence in grade of development between Reichert's ovum and a rabbit's blastodermic vesicle of about the sixth day, appears to be against a close or exact agreement between the two. There are features in Reichert's ovum which do not fit in with the processes of development as known in the rabbit, or in other Mammals; peculiarities which will probably not be understood until opportunity has occurred for study of the segmentation of the human ovum, and of the stages immediately following it. Light will perhaps be thrown on the question by investigations on Mammals more nearly allied to man than are rabbits or dogs. 


\section{Embryos of the Thirteenth and Fourteenth Days.}

His' embryo, E. One of the youngest human ova containing a distinct embryo was obtained by Professor His in 1869, and was carefully described by him under the distinguishing letter E. This embryo, which is at present deposited in the Anatomical Museum at Basle, is estimated to be about thirteen days old : it is represented from the right side in Fig. 176; and in diagrammatic sagittal section in Fig. 188.

The entire vesicle (Fig. 188) is a thin-walled sac, measuring $8.5 \mathrm{~mm}$. by $5.5 \mathrm{~mm}$. and covered all over with branched villi. The contained embryo (Fig. 176) is $2.1 \mathrm{~mm}$. long, and is attached at its hinder end, by a short thick stalk, to the inner surface of the vesicle. A slight constriction separates the

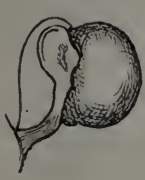

FIG. 176.

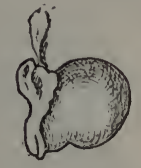

FIG. 177.

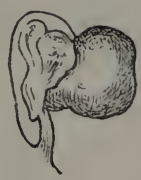

FIG. 178.

FIGS. 176, 177, 178.-Outline figures, from the right side, of three Human Embryos, estimated to be of the thirteenth or fourteenth days. (From His.) $\times 5$.

FIG. 176.--Embryo lettered by Professor His, E ( $c f$. Fig. 188).

Fig. 177.-Embryo described by Allen Thomson.

FiG. 178.-Embryo lettered by Professor His, SR (cf. Fig. 179).

embryo ventrally from the yolk-sac, which measures $2 \cdot 3$ by $1.6 \mathrm{~mm}$. Covering the embryo, but at a short distance from it, is a membranous fold, which is clearly the inner or true amnion. The embryo itself presents along its dorsal surface a shallow neural groove, bounded by prominent neural folds; and the only other organs visible on the surface are a pair of longitudinal folds, formed by the two halves of the heart, and lying between the anterior end of the embryo and the yolk-sac. From the heart, vessels can be traced, running over the surface of the yolk-sac.

His' embryo, SR. This is a well-preserved embryo of the thirteenth day, slightly older than the embryo E, but very similar to it in all important respects.

The entire vesicle measures 8 to $9 \mathrm{~mm}$. in diameter, and 
is covered over its whole surface by branched villi, as in the case of the embryo E ( $c f$. Fig. 188). The embryo (Figs. 178 and 179) is $2.2 \mathrm{~mm}$. long: it is attached to the inner surface of the vesicle by a short thick stalk, $\mathrm{TZ}$, and is separated from the yolksac, ys, by a slight constriction.

In the embryo itself, the head end, HD, is more markedly raised above the yolk-sac than in the embryo $\mathrm{E}$; and the neural groove, NG, is widely open along its whole length. The dorsal

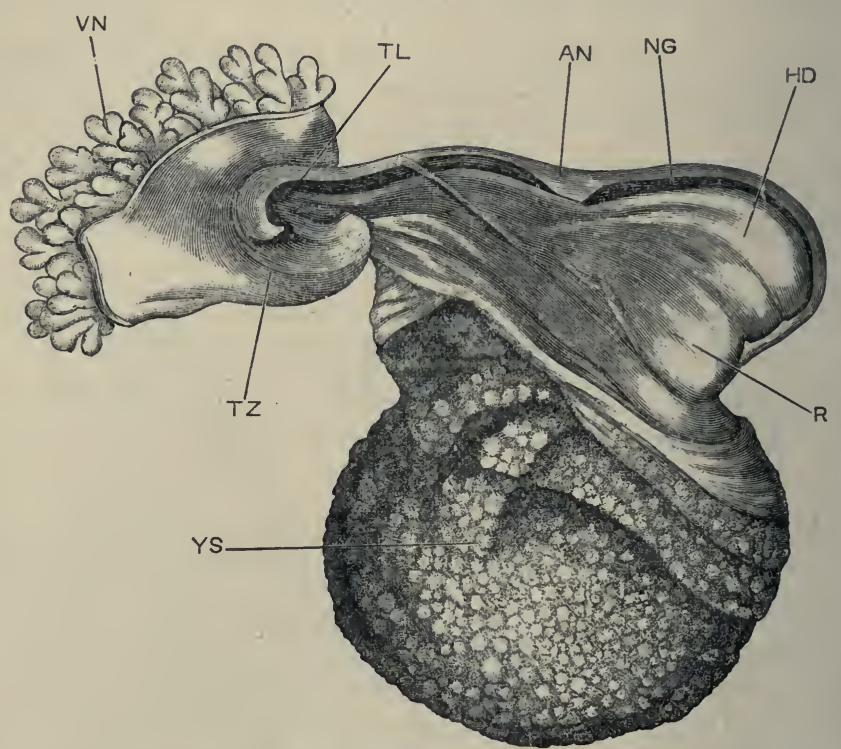

Fig. 179.- - Human Embryo lettered by Professor His, SR, and estimated as of the thirteenth day. The wall of the blastodermic vesicle has been removed, except the part to which the allantoic stalk is attached. (After His.) $\times 2 \tilde{5}$.

AN, inner or true amnion. HD, head end of the embryo. $\mathbf{R}$, heart. $\mathbf{N G}$, neural groove. TL, tail. TZ, allantoic stalk, connecting the embryo with the wall of the blastodermic vesicle. VN, villi. Y YS, yolk-sac.

surface of the embryo is somewhat sinuous in outline, presenting alternate convexities and concavities: the most anterior and largest swelling is formed by the head; then comes a concavity opposite the middle of the length of the yolk-sac; and then another marked convexity further back. The hinder end of the embryo projects freely as a short blunt tail, TL, beyond the stalk of attachment to the wall of the vesicle, Tz, which now arises from the ventral surface of the hinder end of the embryo. The two halves of the heart, $\mathrm{R}$, form prominent swellings between 
the head and the yolk-sac. 'There is as yet no trace of either visceral arches or clefts; and the dorsal surface of the embryo is enveloped in the thin membranous amnion, AN, which now lies rather closer to it than in the case of the embryo E.

Human embryos of about the same age as the embryos $\mathrm{E}$ and SR have been described by Allen Thomson (Fig. 177), Keibel, V. Spee (Figs. 180 to 184), Kollmann (Fig. 185), and others. These all agree in essential respects, and leave no doubt that the stage must be regarded as a perfectly normal one.

V. Spee's embryo, which was studied by means of sections, is of considerable importance, as it has shown the internal struc-

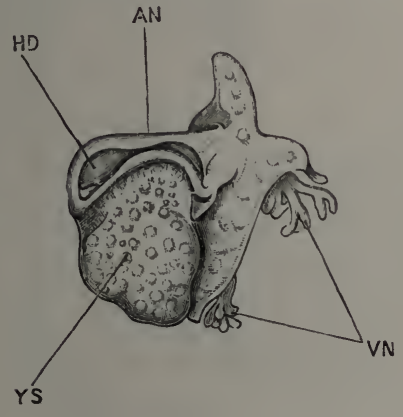

FIG. 180.

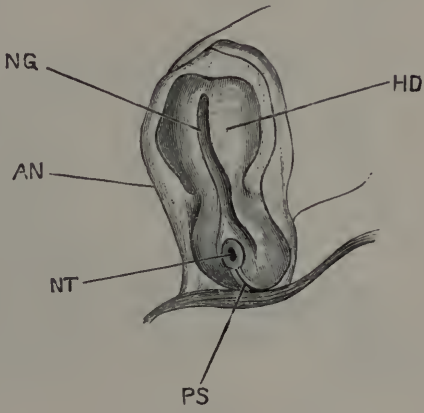

FrG. 181.

FIG. 180. - A Human Embryo of about the thirteenth day, from the left side: the wall of the blastodermic vesicle has been in chief part removed. (After V. Spee.) $\times 8$.

FiG. 181. The same embryo from the dorsal surface. (After V. Spee.) $\times 14$.

AN, inner or true amnion. HD, head ent of embryo. NG, neural groove. NT, neurenteric canal. PS, primitive streak. VN, villi of chorion. YS, yolk-sac.

ture, and the relations of the germinal layer's, at the stage in question.

This embryo is represented from the left side in Fig. 180, and from the dorsal surface in Fig. 181. It is, if anything, slightly younger than the embryo $\mathrm{E}$, and the constriction separating the embryo from the yolk-sac has hardly commenced to form. The head of the embryo (Fig. 181, HD) is wide and flat, and the neural groove is shallow. At the hinder end, the two neural folds diverge from each other, and embrace between them the anterior end of a well-marked primitive streak (Fig. 181, PS); and just in front of the primitive streak is a small but well- 
defined neurenteric passage, $\mathrm{NT}$, leading from the surface into the cavity of the yolk-sac.

The sections (Figs. 182-84) will render the relations of the parts clearer. Fig. 182, which passes across the anterior part of the head, shows the widely open neural groove, NG; the fore-gut,

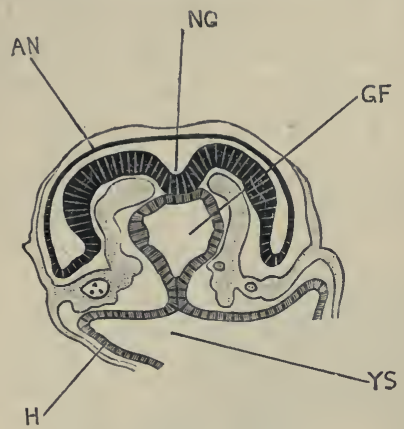

FIG. 182.

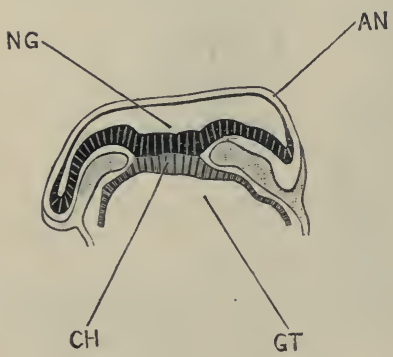

FIG. 183.

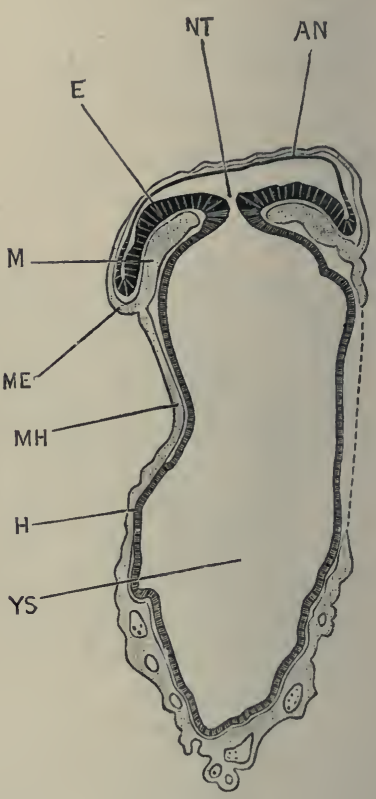

FIG. 184.

FIGS. 182-184.- Sections across the Human Embryo of the thirteenth day, represented in Figs. 180 and 181. (After v. Spee.) $\times 45$.

FIG. 182.- Transverse section across the head end of the embryo.

FIG. 183. - Transverse section across the middle of the body.

EIG. 184.-Transverse section across the hinder end of the embryo and the yolk-sac, the section passing through the neurenteric canal.

$\mathbf{A N}$, inner or true amnion. $\mathbf{C H}$, commencing notochord. $\mathbf{E}$, epiblast of the embryo. GF, fore-gut. $\mathbf{G T}$, mid-gut. $\mathbf{H}$, hypoblast. $\mathbf{M}$, mesoblast. $\mathbf{M} \mathbf{E}$, somatopleuric laver of mesoblast. MH, splanchnopleuric layer of mesoblast. NG, neural groove. NT, neurenteric canal. Y Y, cavity of yolk-sac.

GF, lined by hypoblast, and just shut off from the yolk-sac, Ys ; and the mesoblast, with its contained blood-vessels. Fig. 183 passes through the body region, and shows the commencing notochord, $\mathrm{CH}$, as a thickened plate of hypoblast cells in the mid-dorsal wall of the gut.

Fig. 184 passes through the neurenteric canal, NT, which 
places the mid-gut and yolk-sac in direct communication with the exterior. It will be noticed that the wall of the yolk-sac consists of an inner lining of hypoblast, $\mathrm{H}$, and an outer wall of mesoblast, $\mathrm{NH}$, in which are very numerous blood-vessels. On the right side the mesoblast was torn in the section, and is indicated by a dotted line in the figure.

The amnion, Ax, in all three figures is seen to consist of both epiblastic and mesoblastic layers.

Kollmann's embryo (Fig. 185) is rather older than the others described in this section, and may be estimated as of the fourteenth day. It affords an important transitional stage

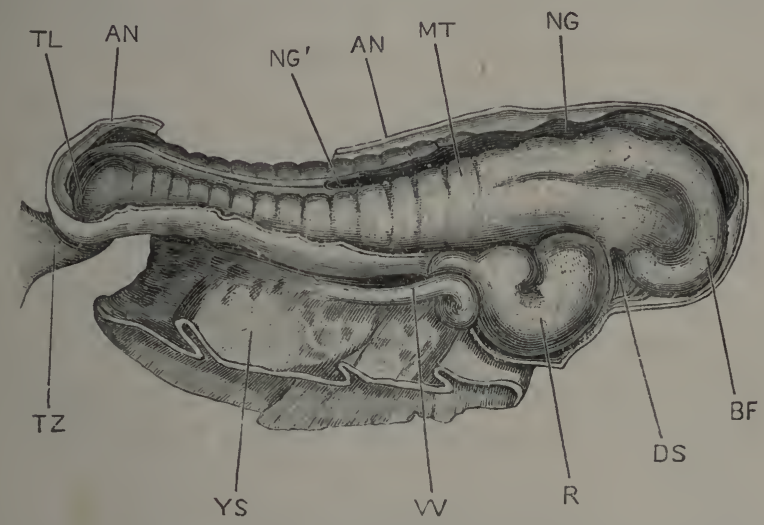

FIG. 185.-Human Embryo of about the fourteenth day, from the right side. The yolk-sac and the wall of the blastodermic vesicle have been removed. (After Kollmann.) $\times 27$.

AN, outer or true amnion. BF, fore-brain. DS, stomatodæum. MT, mesoblastic somite or protovertebra. $\mathbf{N G}$, neural groove. $\mathbf{N G}^{\prime}$, point behind which the neural groove is closed to form the neural tube. $R$, heart. $T I$, tail. TZ, allantoic stalk. W, vitelline vein. YS, yolk-stalk, cut short.

between the embryos $\mathrm{E}$ and $\mathrm{SR}$ on the one hand, and on the other the embryos of the third week, which will be described in the next section.

Kollmann's embryo (Fig. 185) measures $2.5 \mathrm{~mm}$. in length. As compared with the earlier embryos, the head is larger and more prominent; and the embryo is much more distinctly constricted off from the yolk-sac. The neural folds have met and fused, to complete the neural canal, in the hinder part of the body, but the neural groove is still widely open in the head and anterior part of the body. The brain vesicles are becoming evident, and the flexure of the anterior end of the head is already 
commencing. A distinct stomatodocal depression, DS, is present on the under surface of the head. The two halves of the heart, $\mathrm{n}$, have united; and the heart, now a single tube, is already twisted in a characteristic $S$ shape. Fourteen or fifteen pairs of mesoblastic somites, or protovertebræ, MT, are clearly visible from the surface.

There are as yet no traces of visceral arches or clefts, nor of eyes, ears, or limbs.

On comparing the embryos E and SR (Fig. 179), with the corresponding stages in the development of the rabbit, i.e. with rabbit embryos towards the end of the eighth day, before closure of the neural canal has occurred at any point, there are seen to be several points of difference.

The rabbit embryo at this stage is still on the surface of the vesicle, while the human embryo is already covered by a wellformed amnion. In connection with this separation from the surface there is a further point of difference; the human embryo is connected at its hinder end by a short thick stalk (Fig. $179, \mathrm{Tz}$ ) with the wall of the vesicle; while in the rabbit the tail fold is only just commencing, and the hinder end of the embryo is still directly continuous with the wall of the blastodermic vesicle.

This stalk of connection (Fig. 179, $\mathrm{Tz}$ ), between the embryo and the wall of the vesicle, arises from the under surface of the hinder end of the embryo, and its relations are practically identical with those of the allantois of a tenth-day rabbit embryo. As this stalk contains a tubular diverticulum of the hind-gut, and transmits the allantoic arteries and veins ( $c f$. Fig. 198), it clearly corresponds, at any rate in great part, to the rabbit's allantois, and may consequently be spoken of as the allantoic stalk.

As regards amnion and allantois, the main difference between the human embryo and the rabbit may be briefly expressed by saying, that both amnion and allantois develop in the human embryo at an earlier stage, relatively to the embryo itself, than is the case in the rabbit. The probable explanation of this precocious development of amnion and allantois, and comparatively late appearance of the embryo itself, in the human species, as compared with the rabbit, will be considered further on. 
Another difference of even greater importance between the two embryos is found in the relations of the yolk-sac in the two cases respectively, to which attention has already been directed. In the rabbit, the yolk-sac (Fig. 146, ys) is part of the blastodermic vesicle itself; while in the human embryo ( $c f$. Fig. 188) it lies freely within this. This difference is explained by Keibel as due to a relatively early extension of the splitting of the mesoblast, in the human embryo, right round the lower half of the blastodermic vesicle. In the rabbit (Fig. 147) the mesoblast, and consequently the cavity, c, between its layers, only extends halfway round the blastodermic vesicle, stopping at the sinus terminalis, ST. If, in the rabbit, the mesoblast, and the split between its somatic and splanchnic layers, were to extend round to the lower pole of the blastodermic vesicle, then the yolk-sac would be completely split away from the wall of the vesicle, and a condition similar to that of the human embryo would be attained.

No stages intermediate between Reichert's ovum and His' embryo E, or V. Spee's embryo, have yet been described. The gap, though probably only a slight one in actual time, is of great importance; for, while Reichert's ovum has no trace of an embryo, His' embryo E possesses neural groove and folds, heart and yolk-sac, and has both amnion and allantois well developed.

His has attempted to bridge over the interval, and has given a series of diagrams, reproduced in Figs. 186-188, showing hypothetical intermediate stages.

The figures represent diagrammatic longitudinal sections through embryos at successive stages of development, and should be compared with Fig. 174, which represents a similar section through Reichert's ovum.

In Fig. 186, which is a hypothetical stage, the commencement of the formation of the embryo is indicated. The embryonal or germinal area has become somewhat depressed, but at its anterior end, to the right in the figure, is lifted up by the commencing head fold.

In Fig. 187, also a hypothetical stage, the general depression of the embryonal area has increased, the embryo being pushed down into the blastodermic vesicle. The head fold has deepened, 
and the head end of the embryo is now more prominent, and is raised distinctly above the yolk-sac. At the hinder end of the embryo, to the left in the figure, the embryonal area still preserves its primitive connection with the chorion or wall of the vesicle. The head end of the embryo is covered over by the commencing head fold of the amnion.

Fig. 188 is a somewhat diagrammatic section, at a stage

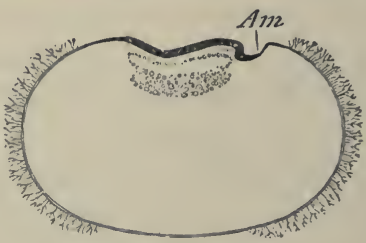

FIG. 186.

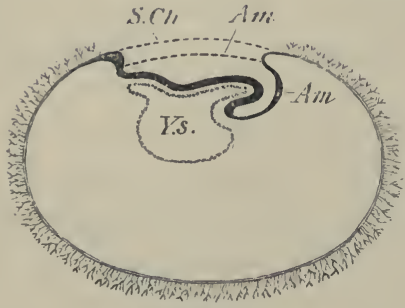

Fig. 187.

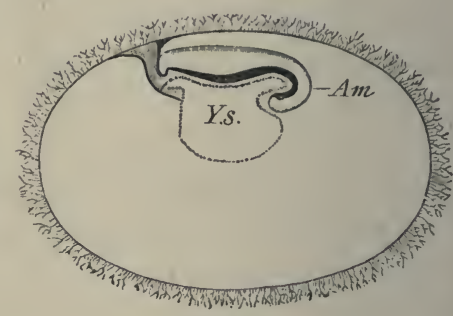

Fig. 188.

FIGS. 186-188.-Diagrammatic longitudinal sections through Human Embryos, representing hypothetical stages intermediate between Reichert's ovum and His' Embryos, E or SR. (From His.) $\times 5$.

Frg. 186.--Shows the commencement of the head fold of the embryo, and of the amnion.

FIG. 187.- A rather later stage, in which the embryo is depressed into the blastodermic vesicle, but still remains in connection with the wall of the vesicle through the allantoic stalk. The dotted lines indicate, hypothetically, the further growth of the amnion.

FIG. 188.-A later stage, equivalent to that of His' embryos E or SR (Fig. 179). The amnion is complete, and the villi extend the whole way round the vesicle.

$A m$, inner layer of the amnion, or true amnion. S.Ch, dotted line representing the future extension of the outer layer of the amnion. $Y s$, yolk-sac.

corresponding to that of the embryos $\mathrm{E}$ or SR (Fig. 179). The changes necessary to derive it from the stage shown in Fig. 187 are very slight. The hinder end of the embryonal area now forms the thick allantoic stalk, connecting the embryo with the chorion; and a tubular diverticulum of the ventral wall of the hind gut, the allantois proper, now extends some way along the stalk. The amnion extends over the whole back of the embryo ; 
a change due possibly, as Professor His suggests, to backward growth of the head fold of the amnion of the earlier stages, as indicated by the dotted lines in Fig. 187 ; but more probably caused, at any rate in part, by approximation and meeting of the side folds of the amnion along the mid-dorsal line.

His' diagrams (Figs. 186 to 188) undoubtedly give an intelligible and consistent theory concerning the development of the human embryo from the stage indicated by Reichert's ovum to that of the embryos $\mathrm{E}$ and SR, and it is greatly to be hoped that opportunity may occur for testing their correctness by actual observation.

If the transition occurs in the way suggested by Professor His, then both the amnion and the allantois of the human embryo present features differing widely from those of the rabbit. The amnion has no tail fold, which is almost the only part developed in the rabbit; while the allantois is, from the first, continuous with the chorion.

Even in the rabbit, however, an approach is made towards the mode of development of the allantois supposed to occur in the human embryo; the mesoblast of the allantois in the rabbit being, from the first, continuous with the mesoblast of the tail fold of the amnion (Fig. 146), and very early fusing with the chorion as well (Fig. 147).

The precocious development of the allantois, which is one of the most striking points about the human embryo, may be connected with the precocious appearance of the vascular layer of mesoblast lining the blastodermic vesicle; and both features, in so far as they are exceptional, may be regarded as examples of the tendency to shortening or abbreviation of the processes of development, which is so constantly encountered by the student of embryology.

The establishment of a vascular connection between the embryo and the chorion, and so indirectly with the mother, is the characteristic feature of mammalian development; and it is not surprising to find, in the most highly developed of all Mammals, this feature thrown back to an earlier stage than that at which it originally appeared; and hurried on prematurely, even at the expense, as it would seem, of the embryo itself, whose development is unusually retarded. 
The germinal layers of the human embryo. But little can be said on this point at present. Concerning the mode of establishment and of differentiation of the germinal layers, we know nothing. In Reichert's ovum the outer wall of the vesicle consists of a single layer of epithelial cells, which from Reichert's figures appear to be flattened, or pavement cells, and which must almost certainly be epiblastic. The central mass of cells, forming the yolk-sac, is certainly hypoblastic; and so also, in all probability, is the thick disc of granular cells forming the deeper layer of the embryonal area.

Whether mesoblast is, or is not, present in Reichert's ovum is a disputed point; but at a stage not much later, in the embryos E and SR, layers of vascular mesoblast are present, not only in the embryo itself, but covering the outer surface of the yolk-sac, and lining the wall of the blastodermic vesicle as well (cf. Figs. 182, 183, and 184). The mode of origin, and the time of appearance, of the mesoblast are unknown; but from the stage represented by the embryos E and SR, the history of the layer is practically the same as in the rabbit or chick.

\section{The Third Week.}

During the third week, the embryo assumes more definite form. The neural canal is closed along its whole length; the brain vesicles, optic vesicles, and auditory sacs are formed; the visceral arches and clefts develop; and the head and neck acquire their characteristic embryonic shape. The embryo increases considerably in size; the constriction between embryo and yolk-sac becomes much more marked; and towards the end of the week the first rudiments of the limbs appear.

During this time, development proceeds rather slowly, the changes passed through in the course of the week corresponding roughly to those effected during the second and third days in a chick embryo.

Only a limited number of embryos of the third week have been described, not much more than a dozen in all, and only a few of these were in satisfactory condition for detailed examination. Outlines of some of the more important specimens are given in Figs. 189 to 195, and more detailed drawings on a larger scale in Figs. 196, 197, and 198. 
Coste's embryo(Fig. 196). An embryo described and figured by Coste, about the precise age of which there is some doubt, appears to belong to the commencement of the third week. The whole vesicle measures $16.2 \mathrm{~mm}$. along its greater diameter, and is covered externally with short, slightly branched villi. The embryo is attached to the inner surface of the vesicle by a short

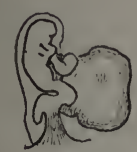

F'IG. 189.

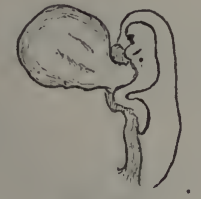

FIG. 190.

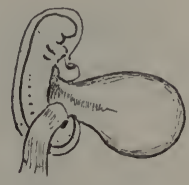

Fig. 191.

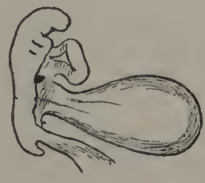

FIG. 192.

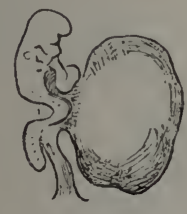

F'IG. 193.

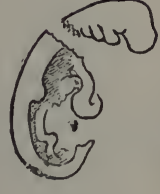

FIG. 194.

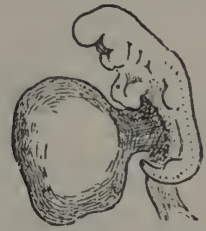

FIG. 195.

FIGS. 189 to 195.-Outline figures of seven Human Embryos of the third week. (From His.) $\times 5$.

Fig. 189.- Embryo lettered by Professor His, $\mathrm{Lg}$, and estimated as fifteen days old. ( $C f$. Fig. 197, p. 489.)

FIG. 190.--Embryo lettered by Professor His, Sch, and estimated as fifteen days old.

FIG. 191.-Embryo lettered by Professor His, M, and estimated as eighteen days old.

FIG. 192.-Embryo figured by Professur Allen Thomson, and probably about eighteen days old.

Frg. 193.-Embryo lettered by Professor His, BB, and estimated as about eighteen days old.

FIG. 194.-Embryo lettered by Professor His, Kln, and estimated as about twenty days old.

FIG. 195.-Embryo lettered by Professor His, Lr, and estimated as twenty or twenty-one days old. (Cf. Fig. 198, p. 491.)

thick allantoic stalk, A.s. The head end of the embryo is well developed, and raised freely above the yolk-sac; but the body is still so closely connected with the yolk-sac that a distinct yolkstalk can hardly be said to be present. The body of the embryo is concave upwards, and the tail has a well-marked upward direction. In the neck, three visceral arches are visible as thickenings, but the grooves between them are only faintly indicated. 
Below the neck, in the angle between the embryo and the yolk-sac, is the heart, $\mathrm{H}$, a large tube sharply twisted on itself. Blood-vessels are present in the wall of the yolk-sac, YS, and also in the allantoic stalk, from which latter they pass into the wall of the blastodermic vesicle, the inner layer of which is vas-

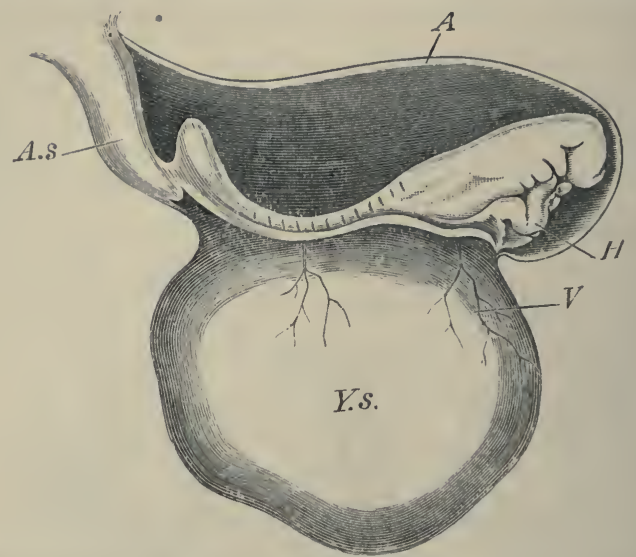

Fig. 196.-Human Embryo at the commencement of the third week. (From $\mathrm{H}$ is, after Coste.) $\times 15$.

$A$, inner or true amnion. $A s$, allantoic stalk. $H$, heart. $V$, blood-vessel of yolk-sac. $Y$ s, jolk-sac.

cular throughout its whole extent, although the blood-vessels do not penetrate into the villi.

The middle portion of the embryo is clearly divided into protovertebræ, but there are no traces of limbs.

Embryo lettered by Professor His, Lg (Figs. 189 and 197), and estimated as fifteen days old. The entire blastodermic vesicle in this case measures 17 by $11 \mathrm{~mm}$., and is covered with villi, except at two patches at opposite poles of the vesicle. The embryo (Fig. 197) is rather closely invested by the amnion, AN, and is connected with the wall of the vesicle by a short thick allantoic stalk, TZ. The yolk-sac, ys, is nearly spherical, and about $2 \mathrm{~mm}$. in diameter, and is still widely continuous with the ventral surface of the embryo.

The most marked feature in the general form of the embryo is the very sharp bend in the middle of the back, opposite the yolk-sac. A similar and equally sharp bend has been noticed in other embryos of about the same age (cf. Figs. 190, 193), but 
it is not yet certain whether it is to be regarded as a normal feature. His has suggested that it may possibly be caused by the embryo tending to increase in length more rapidly than the closely fitting amnion will allow it to; the embryo consequently becoming bent at the place where its ventral wall is weakest, i.e. opposite the yolk-sac.

In the head, cranial flexure is well marked, the anterior part of the head being bent down at right angles to the hinder part,

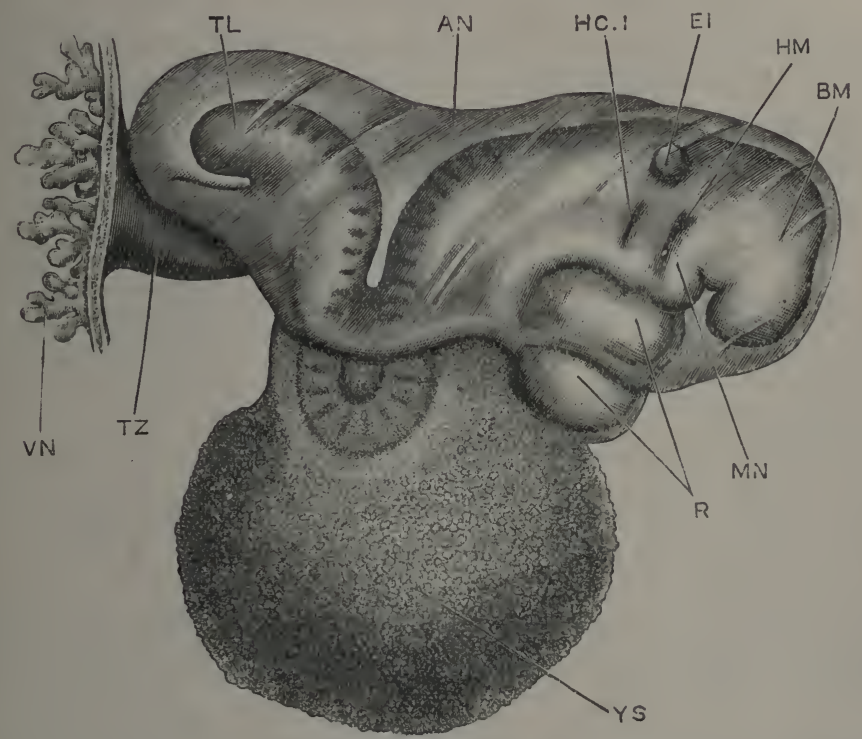

FIG. 197.- Human Embryo lettered by Professor His, Lg, and estimated as fifteen days old. The wall of the blastodermic vesicle has been removed, except the portion with which the allantoic stalk is continuous. (From His.) $\times 30$.

AN, inner or true amnion. BM, mid-brain. EI, auditory pit. HC.I, first branchial cleft. HM, hyomandibular cleft. MN, mandibular arch. R, heart. TI, tail. TZ, allantoic stalk. VN, villi of chorion. YS, yolk-sac.

and the fore-brain being in consequence carried round to the under surface of the head. The prominent angle of the brain is formed by the mid-brain, BM, behind which comes the nearly straight hind-brain.

At the sides of the fore-brain are lateral swellings, caused by the outgrowing optic vesicles, but there is as yet no trace of the lens. The auditory pits, Er, are a pair of shallow depressions, with widely open mouths, at the sides of the hind-brain. 
At each side of the neck there are a couple of slit-like depressions, HM, and HC.1, transverse to the axis of the embryo. These are the external grooves, which lie opposite the hyomandibular and first branchial gill-pouches, or diverticula from the pharynx. The epiblastic grooves and the corresponding hypoblastic pharyngeal pouches lie in close contact with one another, but do not communicate, so that there are at this stage no complete gill-clefts, or actual perforations in the wall of the neck.

The hyoid arch is the ridge or strip of the neck lying between the first branchial and hyomandibular grooves, HC.1 and Hм. The mandibular arch, $\mathrm{MN}$, forms a much more prominent ridge, in front of the hyomandibular groove; and, wedged in between the dorsal end of the mandibular arch and the under surface of the head, is the comparatively small maxillary arch.

The stomatodæum, or mouth depression, is a shallow pit on the under surface of the head, bounded in front by the head itself, at the sides by the maxillary arches, and behind by the mandibular arches. It does not yet open into the fore-gut (cf. Fig. 232).

The heart, $\mathrm{R}$, is large, and lies immediately below the branchial region of the neck, between this and the yolk-sac; it is a single tube, twisted so as to form a strongly curved loop, with its convexity towards the right side of the embryo.

In the body region, the outlines of the somites or protovertebræ can be seen through the skin; about thirty-five pairs being already present. The tail, TL, forms a prominent rounded stump; and from the under surface of its base the short thick allantoic stalk, TZ, arises, which attaches the embryo to the chorion. There is as yet no trace of the limbs.

An embryo lettered by Professor His, Lr (Figs. 195 and 198), and estimated as twenty or twenty-one days old, may be taken as typical of the condition attained by the end of the third week.

Apart from the great increase in size, best seen by comparing Figs. 189 and 195, p. 487, the chief points in which the embryo Lr differs from the embryo Lg, are:-The narrowing of the yolkstalk, by which the embryo is separated more markedly from the yolk-sac; and the almost complete disappearance of the sharp bend in the back, opposite the yolk-sac, which is so 
curiously characteristic of the embryo $\mathrm{Lg}$, and of others of the same age.

The greatest length of the embryo Lr, measured in a straight line, from the swelling caused by the mid-brain to the rounded hinder end of the body, is $4.2 \mathrm{~mm}$.

In the head, the outlines of the several brain vesicles can be

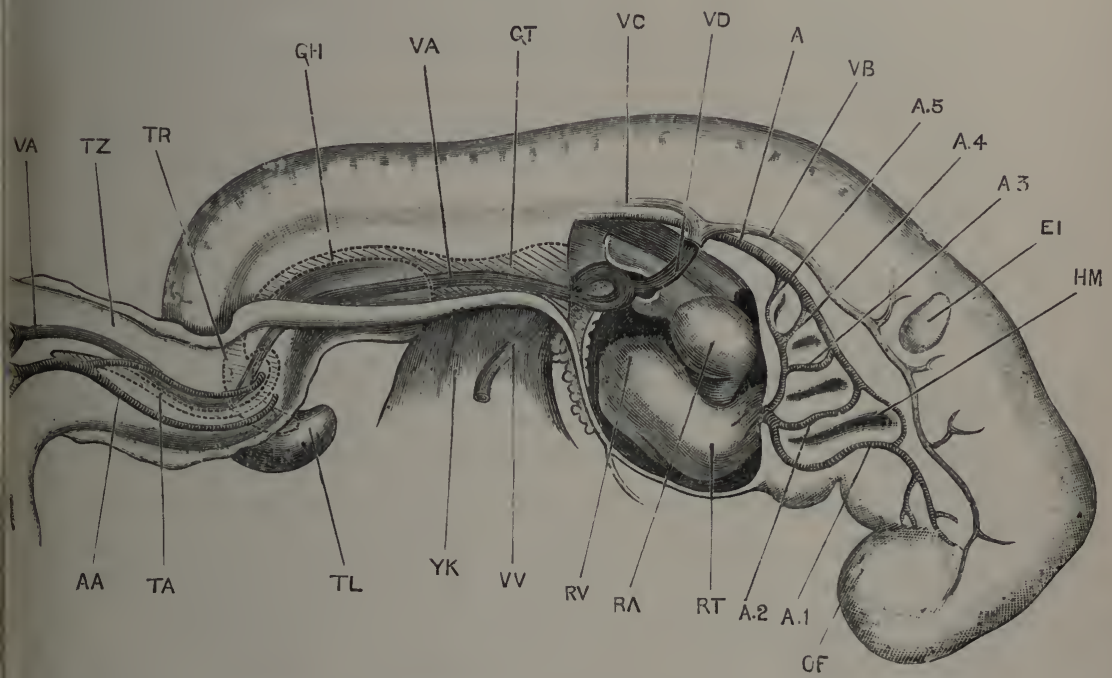

Fig. 198.-Human Embryo lettered by Professor His, Lr, and estimated as twenty or twenty-one days old. The right wall of the pericardial cavity has been removed, to expose the heart; and the arteries, the veins, and the hinder part of the alimentary canal are represented as though the embryo were transparent. (From His.) $\times 23$.

\begin{abstract}
A, dorsal aorta. A.1, first aortic arch, in the mandibular arch. A.2, second aortic arch, in the hyoid arch. A.3, third aortic arch, in the first branchial arch. $\mathbf{A . 4}$, fourth aortic arch, in the second branchial arch. A.5, fiftl aortic arch, in the third branchial arch. AA, allantoic artery. EI, anditory vesicle. GH, hind-gut. GT, mid-gut, opening into the yolk-stalk. HM, hyomandibular cleft or groove. OF, olfactory pit. RA' right auricle. RT, truncus arteriosus. $R V$, ventricle. TA, diverticulum of hind-gut into allantoic stalk. TL, tail. TR, cloaca. TZ, allantoic stalk. VA, allantoic vein. VB, anterior cardinal vein. VC, posterior cardinal vein. VD, Cuvierian vein. VV, vitelline vein. YK, yolk-stalk.
\end{abstract}

recognised fairly accurately through the skin; and the proportions have not altered very greatly from those of the earlier embryo, Lg. The axes of the fore-brain and of the hind-brain are still at right angles to each other, the mid-brain forming a prominent rounded swelling at their junction. The fore-brain is wider from side to side than before, owing to the optic vesicles, which project outwards from its sides. 
'The olfactory pits, of, are a pair of shallow depressions, on the under surface of the extreme anterior end of the head, above the mouth. The lens of the eye has not yet commenced to form ; and the auditory vesicles, EI, are now a pair of closed sacs, embedded in the mesoblast at the sides of the hind-brain.

The gill-cleft region is a triangular patch on each side of the neck, with the apex directed backwards, and is bounded both dorsally and ventrally by shallow grooves. The hyomandibular cleft, HM, and the first, second, and third branchial clefts are all represented by pharyngeal pouches, and by external grooves corresponding to them; but none of the clefts are actually perforated.

The stomatodæal pit is much deeper, and more clearly defined than before, owing to uprising of its lips: it now opens into the fore-gut.

The heart, RA, RV, RT, is larger than before, and its several divisions are more clearly marked off from one another by constrictions. The whole heart has shifted backwards to a certain extent, the greater part of it now lying behind the gillcleft region.

The somites, or protovertebræ, are more distinct than before. A pair of longitudinal ridges which run along the sides of the body, ventral to the somites, are spoken of as the Wolffian ridges. Each of these ridges is more prominent at two places, opposite the posterior end of the heart, and opposite the allantoic stalk respectively. These more prominent parts of the continuous Wolffian ridges are the rudiments of the arms and legs.

\section{The Fourth Week.}

The fourth week is marked by a great increase in the size of the embryo, growth being relatively more active at this than at any other period.

In the early part of the fourth week (Figs. 199-201), the flexure of both head and body is very strongly marked, the embryo being rolled up on itself so that the head and tail touch or even overlap, and the outline of the entire embryo being approximately circular. The several parts of the head are more conspicuous than before; the visceral clefts and arches are more clearly defined; the nose and ear are more prominent; the heart is very large; the Wolffian ridges are still present as con- 
tinuous structures, but the enlargements of the ridges, which become later the arms and legs, are rapidly increasing in size.

Towards the end of the fourth week (Figs. 202, 203, 204),

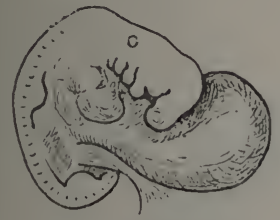

FIG. 199.

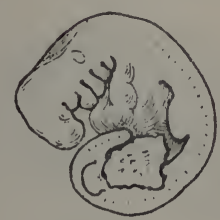

FIG. 200.

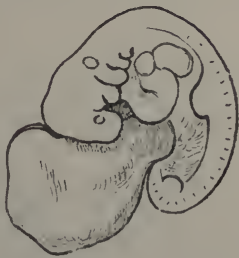

FIG. 201.

FIG. 199-201.-Outline figures of three Human Embryos, estimated as about twenty-three days old. (From His.) $\times 5$.

FIG. 199.-Embryo figured and described by Coste. For a figure of the uterus and blastodermic vesicle from which this embryo was obtained, see Fig. 255, p. 608 .

FIG. 200.- Embryo lettered by Professor His $\alpha$.

FIG. 201.-Embryo figured and described by Allen Thomson. By a mistake of the engraver's this figure has been reversed; and it is really the right side of the embryo, and not the left, that is shown.

the embryo acquires a very characteristic form, corresponding closely in shape, in size, and in internal structure with a chick

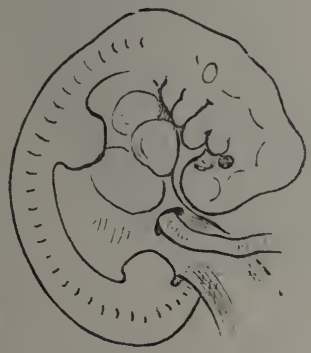

FIG. 202

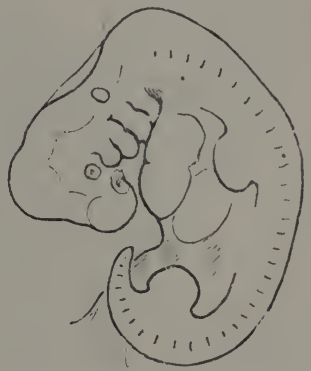

FIG. 203.

Figs. 202 and 203.-Outline figures of two Human Embryos, estimated as twenty-seven days old. (From His.) $\times 5$.

FIG. 202.-Embryo lettered by Professor His, B.

FIG. 203.-Embryo lettered by Professor His, A.

embryo at the end of the fourth day, or a rabbit embryo of the eleventh day.

The embryo (Fig. 204), which measures $7.5 \mathrm{~mm}$. along its longest diameter, is still strongly flexed. The back is rather straighter than before, but, owing to the very sharp bend in 
the cervical region, at the junction of the head and trunk, the under surface of the head is still almost in contact with the tail. The several parts of the brain are readily distinguished

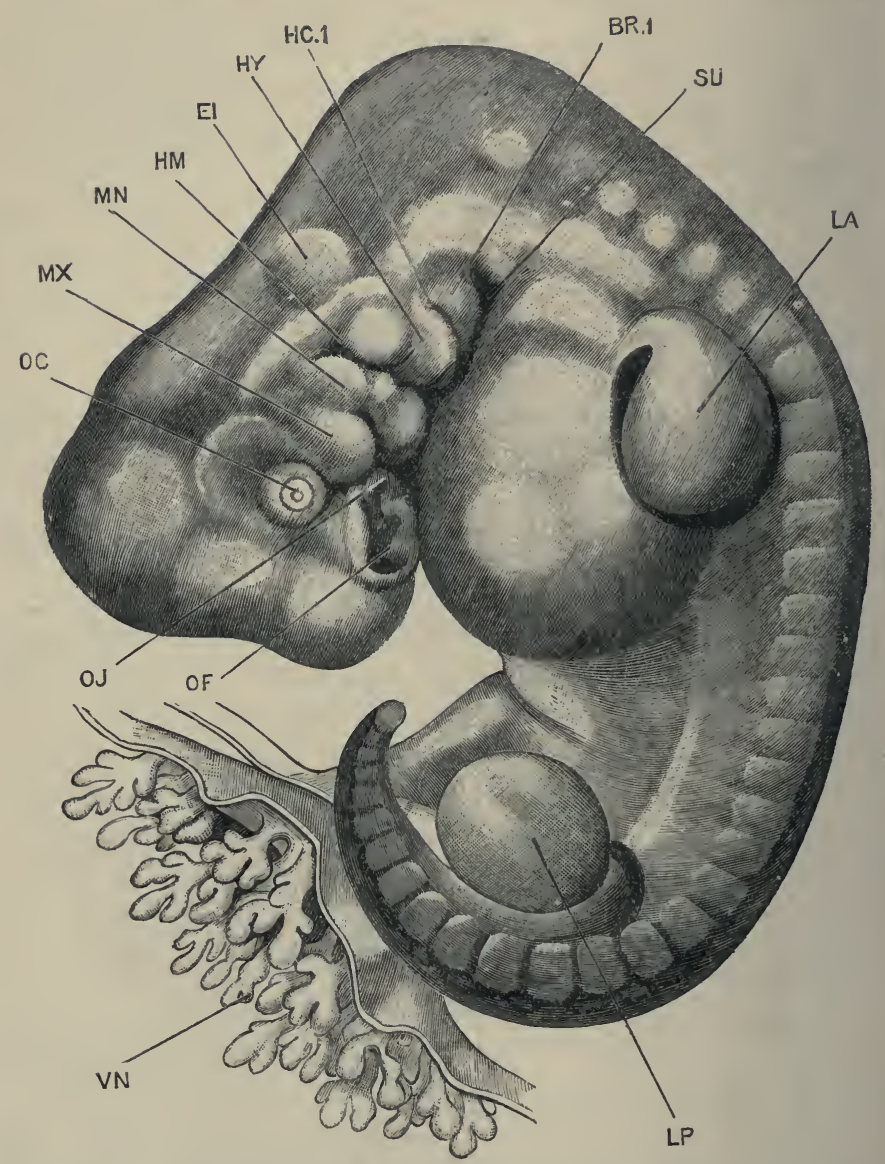

Fig. 204.-Human Embryo lettered by Professor His, A, and estimated as twenty-seven days old. (From His.) $\times 13$.

BR.1, first branchial arch. EI, auditory vesicle. HC.1, first branchial cleft. HM, hyomandibular cleft. HY, hyoid arch. LA, fore-limb or arm. LP, hind-limb or leg. IMN, mandibular arch. MX, maxillary arch. OC, lens. OF, olfactory pit. OJ, Jacobson's organ. SU, sinus præcervicalis. VN, villi of chorion.

through the skin, the mid-brain being especially prominent. The nerve ganglia, both cranial and spinal, are well developed, and form swellings that are clearly visible from the surface.

The olfactory pits, of, on the under surface of the fore part 
of the head, are larger and deeper than before, and are bordered by prominent lips, with somewhat irregular outlines. At the inner and ventral corner of each olfactory pit there is a small but deep notch, oJ, with a sharply defined border: from this notch the organ of Jacobson is developed.

The eyes are very much smaller than in a chick embryo at c corresponding stage of development. The lens is present as a small circular pit, with an open mouth, oc.

The auditory vesicles, EI, appear from the surface as a pair of rounded swellings, just above the dorsal ends of the hyoid arches, HY.

The visceral arches have undergone considerable modification. The maxillary arch, $\mathrm{Mx}$, lies immediately behind the eye; it is larger than before, but is still much smaller than the arches next behind it. The mandibular arch, MN, is the largest of the series, and is partially divided by a constriction, about the middle of its length, into dorsal and ventral portions. The hyoid arch, HY, is nearly as large as the mandibular arch, and is also constricted across its middle. The first branchial arch, BR.1, lies behind the hyoid arch, and is of much smaller size than this. A still smaller second branchial arch is present, but is not visible from the surface, being overlapped and concealed by the first (cf. Fig. 239, p. 552).

With regard to the visceral clefts, it is probable that none are open at this, or indeed at any period in development; but the point has not been determined with absolute certainty. Behind the first branchial arch there is, on each side of the neck, a deep pit, the sinus præcervicalis, su. This is a blind pocket, (cf. Fig. 239), formed by a process of telescoping, through which the hinder pairs of branchial arches are carried forwards, so as to lie between the anterior arches, instead of behind these. The sinus præcervicalis does not open into the pharynx or into any other cavity, and at a later stage it is obliterated by fusion of its anterior and posterior walls with each other ( $c f$. Fig. 240, su).

The mouth ( $c f$. Fig. 206) is much wider from side to side than in the earlier stages; it is bounded in front by the fronto-nasal process, at the sides by the maxillary arches, and behind by the mandibular arches.

In the body of the embryo, thirty-five pairs of somites, or protovertebræ, are clearly visible; of these, eight are cervical, 
twelve thoracic, five lumbar, five sacral, and five caudal. The tail projects freely as a short conical process.

The fore and hind limbs, LA, LP, are flattened buds, with rounded margins; they are about as long as they are wide, and show as yet no trace of a division into segments, or into fingers and toes. 'The outer surface of each limb is its extensor surface; and the inner, facing the body of the embryo, is the flexor surface. The root of attachment of the fore-limb, or arm, extends from the fifth cervical to the second thoracic somite; and the attachment of the hind-limb, from the fourth or fifth lumbar somite to the third or fourth sacral. The Wolffian ridge connecting the arm and leg of each side is still present, but is inconspicuous.

The heart is of great size, and its several divisions can be easily recognised through the thin wall of the pericardial cavity. The liver, which is also large, forms a prominent swelling' between the heart and the fore-limbs.

The yolk-sac is about the size of the head and neck of the embryo; and the yolk-stalk is now long and slender. The inner, or true, amnion is a thin transparent membrane which invests the embryo rather closely; and the allantoic stalk, which lies to the right of the tail, and to the left of the yolkstalk, is about $2 \mathrm{~mm}$. long, and rather more than $1 \mathrm{~mm}$. in diameter.

\section{The Fifth Week.}

The fifth week is marked by great increase in size of the whole embryo, and especially of the head; by further straightening of the back, and uplifting of the head; by the more definite formation of the face; and by rapid growth of the limbs.

The cervical flexure, at the junction of the head and body, is still very conspicuous, and throughout the greater part of the fifth week the greatest length of the embryo is, as in the fourth week, a line drawn from this cervical prominence to the sacral curvature. At the close of the fifth week, the head becomes lifted up more markedly, and the length of the embryo, about $15 \mathrm{~mm}$., is now represented by a line drawn from the top of the midbrain to the sacral curve (cf. Fig. 211).

Throughout the fifth week, the head of the embryo grows rapidly, and by the end of the week it forms, with the neck, about 
half of the entire embryo. The shape of the head is still determined almost entirely by the brain, of which the several divisions are clearly recognisable from the surface. All parts of the brain increase considerably in size, and more especially the cerebral hemispheres.

During the fifth week the face is gradually acquiring definite form, and the features are becoming established.

'The olfactory pits deepen considerably; and their inner and outer borders become raised into prominent lips. The inner borders are formed by the lateral margins of the fronto-nasal process, which grow out as two rounded wings, the processus globu-

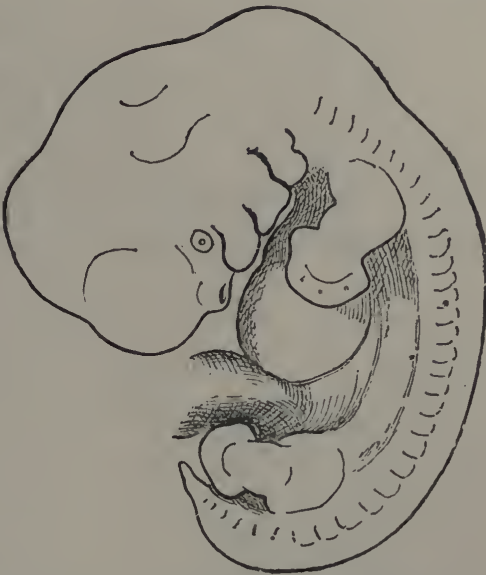

Fig. 205.-Human Embryo lettered by Professor His, $\mathrm{Rg}$, and estimated as thirty-two or thirty-three days old. (From His.) $\times 5$. lares (Figs. 206, 207, Fo). The outer borders are formed by the lateral frontal processes, which separate the olfactory pits from the eyes.

The lower margin of each olfactory pit is incomplete, and between the processus globularis and the lateral frontal process there is a deep nasal groove (Fig. 206), leading from the olfactory pit to the stomatodæum. Towards the end of the fifth week, the maxillary arches (Fig. 207, nx) become more prominent, and growing inwards meet the processus globulares, Fo, and fuse with these; thus bridging over the nasal grooves, and converting them into short tubes, the posterior narial passages, which lead from the olfactory pits to the mouth. At the same time the apertures of the olfactory pits become narrowed, to form the external nostrils.

The bridge of the nose is formed from the median part the fronto-nasal process (Figs. 206, 207, FP). At the commencement of the fifth week this is a triangular area, slightly depressed below the level of the surrounding parts; but towards the close of the week, a blunt process appears in the centre of 
the area, formed by a sagittal fold of its surface, and gradually grows forwards to form the bridge of the nose. For some time the nose is very short and inconspicuous, and the nostrils very far apart; but towards the end of the second month ( $c f$. Figs. 213 and 214), the nose grows forwards more prominently, and the nostrils are brought closer together. The alæ nasi, forming the outer borders of the nostrils, are developed from the lateral nasal processes.

The mouth changes its shape very markedly during the fifth week. At the beginning of the week (Fig. 206, ns) it is a

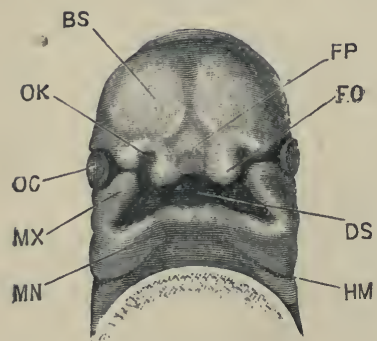

FIG. 206.

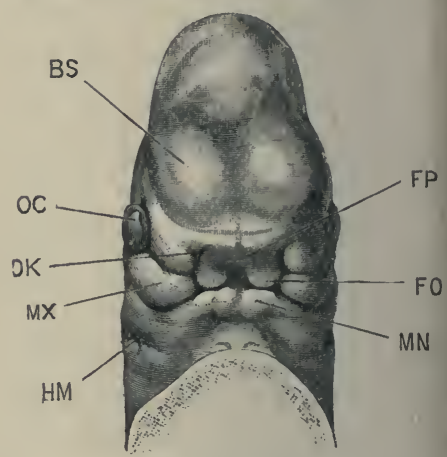

FIG. 207.

FIG. 206.- The under surface of the head of a Human Embryo lettered by Professor His, Hn, and estimated as about twenty-nine days old. (From His.) $\times 7 \frac{1}{2}$.

FIG. 207.-The under surface of the head of a Human Embryo lettered by Professor His, C.II, and estimated as about thirty-four days old. (From His.) $\times 5$.

BS, cerebral hemisphere. DS, stomatodæum. FO, processus globularis, or lateral portion of fronto-nasal process. F'P, median portion of fronto-nasal process. HM hyomandibular cleft. MN, mandibular arch. MX, maxillary arch. OC, eje. OK, olfactory pit.

wide opening, extending transversely across the under surface of the head; but before the end of the week (Fig. 207) it has become greatly reduced in size, by convergence of the maxillary arches and the processus globulares, and is now a narrow transverse slit. Between the maxillary arch and the lateral nasal process of each side is a depression, the lacrymal groove, which at first (Fig. 206) leads into the stomatodæum, but which on the completion of the narial passage opens into this latter (Fig. 207).

In the region of the visceral arches and clefts, important changes occur. The tendency of the anterior arches to grow backwards over the hinder arches, or, as it may be better 
expressed, the telescoping of the hinder arches within the anterior ones ( $c f$. Fig. 239), has been already referred to. At the end of the fourth week (Fig. 201), the second branchial arch is overlapped by the first, BR.1, and is completely concealed by this in surface views. Early in the fifth week, the second branchial arch is in its turn overlapped by the hyoid arch; and from about the thirtieth day onwards (Fig. 205) the only arches visible on the surface of the neck are the maxillary, mandibular, and hyoidean. Behind the hyoidean arch is the deep fissure caused by the sinus præcervicalis ( $c f$. Fig. 240, su), which must not be mistaken for a visceral cleft.

During the fifth week the borders of the hyomandibular cleft become more prominent, and gradually give rise to the folds from which the external ear is developed, in the following manner.

At the end of the fourth week (Fig. 204), the hyomandibular cleft, HM, is a deep groove between the mandibular and hyoid arches, and rmming about halfway across the head. The mandibular arch is divided by a slight constriction, about the middle of its length, into dorsal and ventral portions: of these, the ventral portion bears at its upper and posterior border a small rounded process, well shown in the figure, and named the tuberculum tragicum; while the dorsal portion of the arch, to which the reference line, $\mathrm{NN}$, runs, is the tuberculum anterius helicis. Opposite the dorsal end of the hyomandibular cleft is a longitudinal ridge, the tuberculum intermedium helicis.

The hyoid arch is divided, by two transverse constrictions, into dorsal, middle, and ventral lobes : of these, the dorsal lobe is named the tuberculum anthelicis; the middle lobe, to which the reference line, $\mathrm{HY}$, in Fig. 20\% runs, is the tuberculum antitragicum; and the ventral lobe, which is the smallest of the three, is the tuberculum lobulare.

In the course of the fifth week, these swellings assume more definite form, and gradually give rise to the several parts of the external ear or pinna. The tuberculum anterius helicis (Fig. 208,2 ), and tuberculum intermedium (3) unite together, and with a vertical ridge, the cauda (3c), which arises along the posterior border of the hyoid arch, to form the horse-shoe shaped marginal rim, or helix, of the ear. The ventral ends of the hyoid and mandibular arches fuse, and so give more definite shape to the hyomandibular cleft, which latter becomes the external auditory 
meatus. The tuberculum lobulare (6) fuses with the lower end of the cauda helicis (Fig. 209), and at a later stage grows ventralwards to give rise to the lobule of the ear. The tubercula anthelicis, tragicum, and antitragicum, give rise to the antihelix, tragus, and antitragus respectively of the adult ear.

The body of the embryo presents no external cliaracters of special interest during the fifth week. Owing to the increasing thickness of the muscular and connective tissue walls, the outlines of the internal organs are less distinctly seen from the surface than in the earlier stages.

The limbs undergo important changes during the week, and

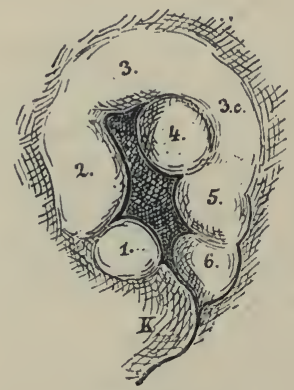

FIG. 208.

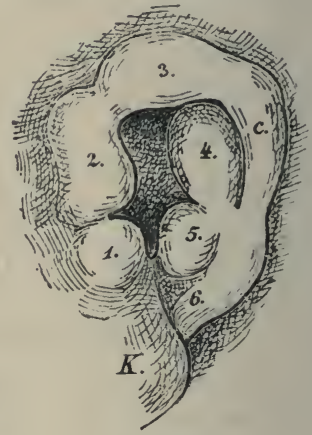

FIG, 209.

FIG. 208.-The left ear of a Human Embryo lettered by Professor His, Br.2, and estimated as thirty-five days old. (From His.) $\times 20$.

Fig. 209.- The left ear of a Human Embryo, lettered by Professor His, Dr, and estimated as thirty-eight days old. (From His.) $\times 20$.

1, tuberculum tragicum. 2, tuberculum anterius helicis. 3 , tuberculum intermedium helicis. $3 c$ and $c$, cauda helicis. 4 , tuberculum anthelicis. 5 , tuberculum antitragicum. 6, tuberculum lobulare.

afford ready means of determining the age of the embryo. In the early part of the fifth week they become divided, first into two, then into three segments. By the middle of the week this division is well marked, the terminal segments, i.e. the hands and feet, forming broad flattened terminal plates, with free rounded margins. A day or two later (Fig. 205), a distinction appears in the hand, between a more swollen basal part, and a thin flattened marginal part; and towards the close of the week the first traces of fingers appear, as small lobes at the boundary between the basal and marginal portions, which soon extend to the free edge, but do not project beyond this until the sixth week. 
The hind-limb is slightly behind the fore-limb in its development, and at the end of the fifth week the toes are only just commencing to appear.

The fore- and hind-limbs of each side are still connected by a low and inconspicuous Wolffian ridge. During the fifth week the tail (Fig. 205) is more conspicuous than at any other stage

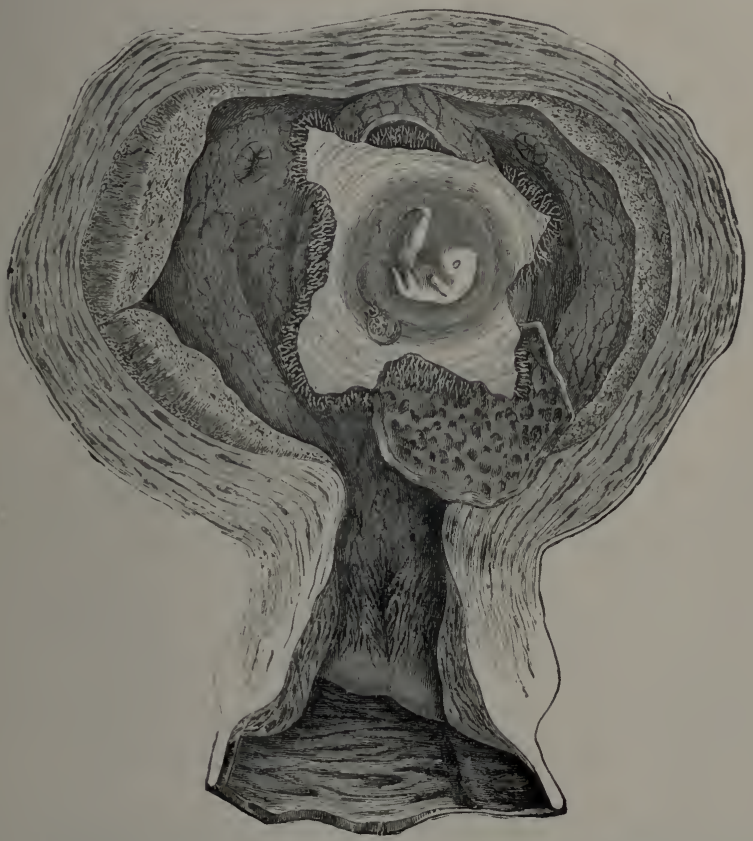

FIG. 210.-A Pregnant Uterus of about the fortieth day. The uterus has been opened from the ventral surface, and the decidua reflexa and chorion cut through by a crucial incision, and the flaps turned aside to expose the embryo. The embryo is still inclosed in the amnion, and the small yolksac, with its long stalk, are seen lying between the amnion and the chorion. At the upper part of the figure the apertures of the Fallopian tubes are seen. (From Kölliker, after Coste.) $\times \frac{3}{5}$.

in development; it is a thin pointed projection, usually bent either laterally, or backwards, by the pressure of adjacent parts.

\section{The Sixth Week.}

During the sixth week the embryo increases in size, though not so rapidly as in the earlier stages. At the commencement of the week it is about $15 \mathrm{~mm}$. long, and at the close about 19 or $20 \mathrm{~mm}$, but the actual measurements depend rather on the 
degree to which the head is lifted up, by straightening of the cervical flexure, than on any real increase in the dimensions.

The position of the embryo within the uterus, about the fortieth day, is shown in Fig. 210. The embryo is connected with the placenta by a thick allantoic stalk. The yolk-stalk is long and thin : its proximal part is bound up with the allantoic stalk in a sheath formed round both by the inner or true amnion; while its distal portion, ending in the small yolk-sac, lies between the amnion and the chorion. The amnion is a transparent sac some distance from the embryo.

The embryo itself is rapidly assuming more definite shape, and by the end of the week is distinctly human in appearance. Owing to the thickening of the muscles and of the subcutaneous connective tissue, and the formation of skeletal elements, the shape of the embryo as a whole, and especially of the head, is much less dependent on the internal organs than in the earlier stages.

The head is still of great size. The face has made considerable progress, and the features are now well established. The nose is larger than before, but is still very broad and flat. The eyelids are commencing to form, as folds of skin, above and below the eyes. The lips appear as folds at the margins of the jaws, but only reach a small development during the sixth week: the red ridge of each lip arises independently, and not until a much later period; about the middle of the third month.

Up to the end of the fifth week there is a distinct notch in the median plane where the two mandibular arches meet: during the sixth week this notch is gradually filled up, and the chin formed as a median projection.

The external ear makes considerable progress during the week (Figs. 209, 211), and by its close the relations and proportions of the several parts are readily comparable with those of the adult.

Apart from the external auditory meatus and the external ear, the visceral clefts and arches are no longer recognisable. The sinus præcervicalis has closed, and the neck is becoming established as a constricted region between the head and trunk.

The limbs have increased considerably in size, the upper arm and thigh in particular being much longer than before. 
The fingers project beyond the margin of the hand by the middle of the sixth week; and the toes become clearly established, although they do not reach the margin of the foot until the early part of the seventh week. The elbow and knee project outwards at first, but towards the end of the sixth week the limbs

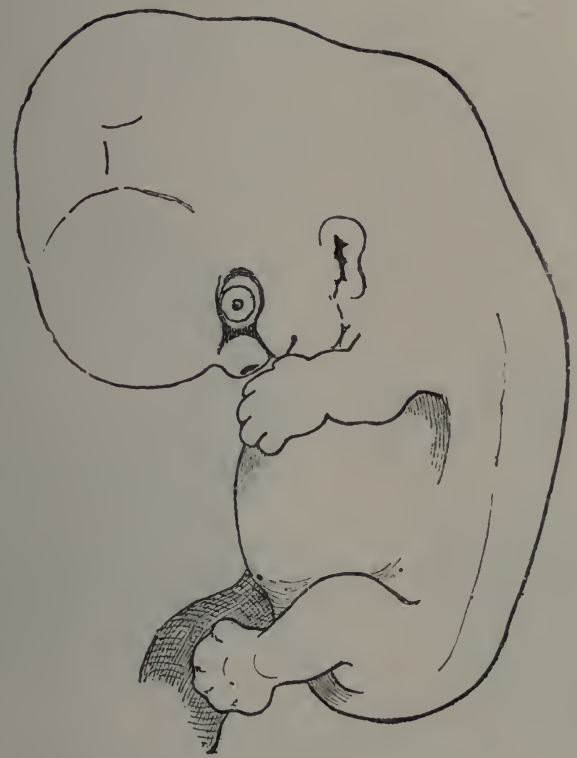

FiG. 211. -Human Embryo about the middle of the sixth week.

(From His.) $\times 5$.

become rotated so as to lie alongside the body, the elbow being directed backwards, and the knee forwards.

The tail is less conspicuous than before, and owing to the growth of adjacent parts is gradually becoming incorporated in the body.

\section{The Second Month.}

At the end of the second month the embryo measures from 25 to $30 \mathrm{~mm}$. in length, and weighs from 12 to 20 grammes.

The cervical flexure has almost disappeared: the head is well lifted up, and is still of very large size, forming nearly half the entire embryo. The eyelids, nose, lips, and external ear have all made considerable advance; the nose is still broad and flat, and the nostrils wide apart, though much closer than 
before. The median part of the upper lip is formed by the two processus globulares, which meet and fuse shortly before the end of the second month. The cheeks are now well formed.

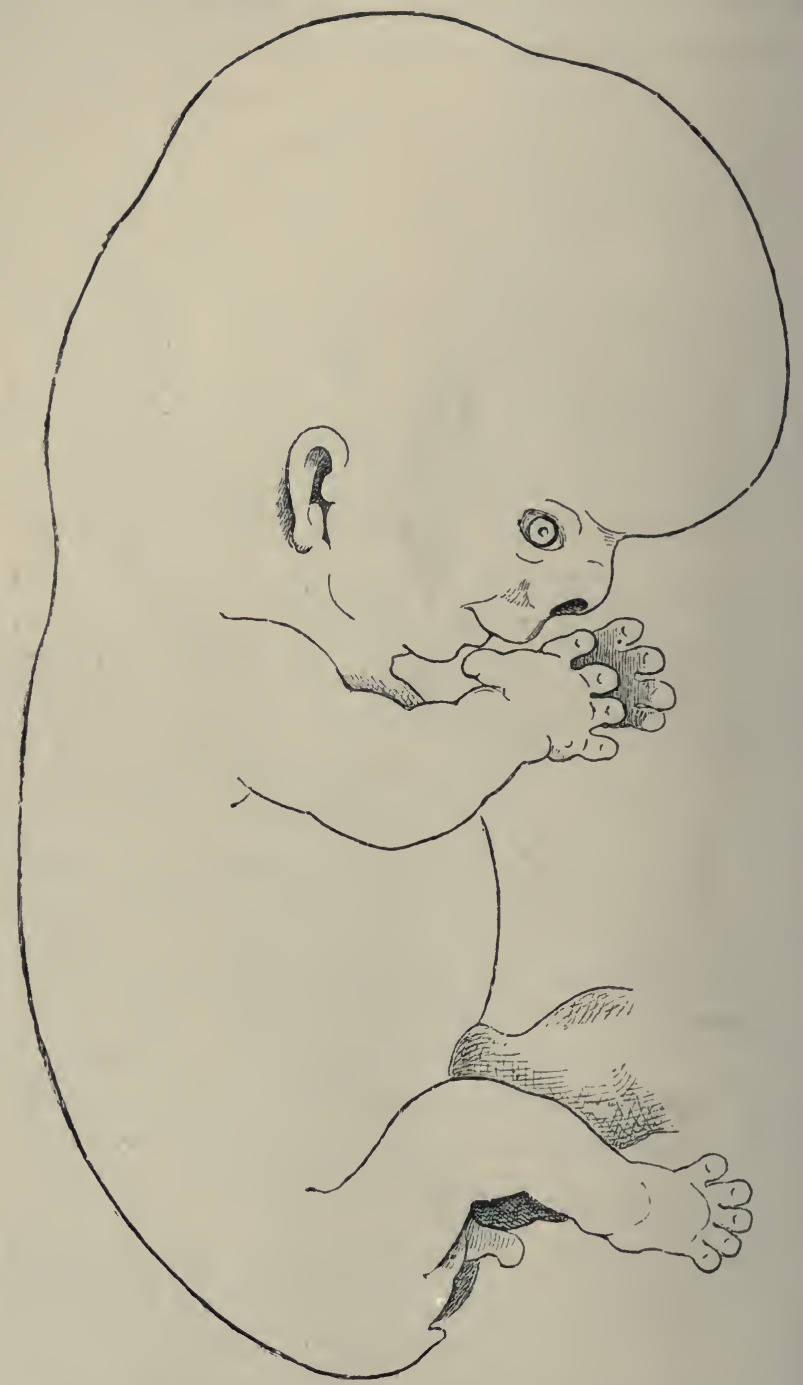

Fig. 212.-Human Embryo at the end of the second month. (From His.) $\times 5$.

The limbs project some distance beyond the body; and the 
fore-limb, which is still the larger of the two, has the characteristic shape of the human arm. The thumb is clearly marked off from the fingers, and the deltoid swelling at the shoulder is already prominent. The leg is smaller than the arm, and is so directed that the soles of the feet are apposed.

The neck is well marked, though short. The ventral wall of the body is completely formed. The umbilical cord, which attaches the embryo to the placenta, is about 8 or $10 \mathrm{~mm}$. long: it is as a rule straight, but may be slightly twisted on itself. It is formed by the allantoic stalk and yolk-stalk, bound together

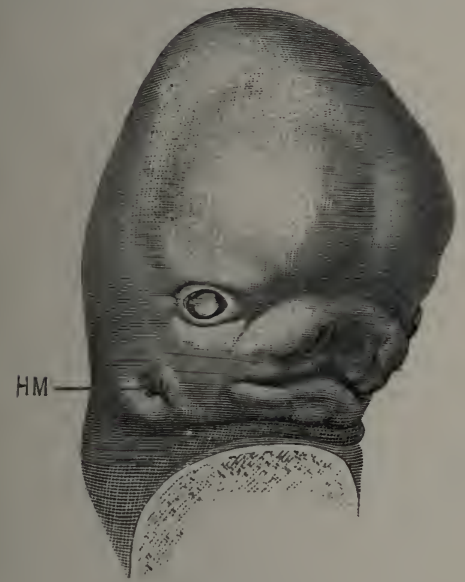

FiG. 213.

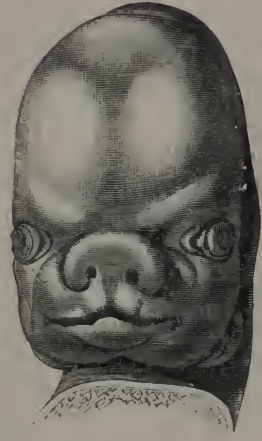

FIG. 214.

FIG. 213.-Head of a Human Embryo at the end of the seventh week. HM, external auditory meatus. (From His.) $\times 5$.

FiG. 214.-Head of a Human Embryo at the end of the second month. (From His.) $\times 3$.

by the amnion, and it still contains at its base a loop of the intestine.

From the end of the second month, when the definite human form is established, up to the time of birth, it is customary to use the term fotus in place of embryo.

\section{The Third Month.}

At the end of the third month the fotus measures about $7 \mathrm{~cm}$. in length, or 9 to $10 \mathrm{~cm}$. if the legs be included, and weighs from 100 to 125 grammes.

The head is still very large relatively to the rest of the body, 
but not nearly so much so as in the earlier stages: the lips and eyelids are closed, and the helix of the ear is folded down so as to almost close the meatus. The neck is longer than before. The limbs, though small, have acquired their definite shape and proportions; and nails are present, as thin plates, on both fingers and toes. The integument is slightly firmer than before, but is still very thin, transparent, and rose-coloured.

Up to this stage a loop of the intestine has been situated in the allantoic stalk, and therefore outside the embryo; but by the end of the third month this loop is withdrawn, and the whole alimentary canal, which has increased greatly in length, is from this time situated within the abdominal cavity.

During the third month, the external genital organs become established. The history of their development will be given in the section dealing with the organs of reproduction.

\section{The Fourth Month.}

At the end of the fourth month the foetus measures 12 to 13 $\mathrm{cm}$. in length, from the vertex of the head to the coccyx; or from 16 to $20 \mathrm{~cm}$. if the legs be included. The weight is usually from 230 to 260 grammes.

The skin is of a rosy colour, and is much firmer than before. Short whitish hairs appear on the head, and a slight down on other parts of the body. The eyelids, nostrils, and lips are all closed. The chin, which has hitherto been inconspicuous, begins to become prominent. The legs and arms are of about equal length: and the external sexual characters are usually wellmarked.

The anus is open, and the duodenum contains meconium of a light greyish-white colour. The umbilicus, or point of origin of the umbilical cord, is low down, close to the pubes. In the skull, the bones are still far from meeting one another, so that the sutures and fontanelles are very wide. The muscles are more fully developed than before, and may give rise to distinct movements of the fotus. In abortions at this period the fœtus rnay live for some hours.

\section{The Fifth Month.}

By the end of the fifth month the foctus measures about $20 \mathrm{~cm}$. in length; or, if the legs be included, 25 to $27 \mathrm{~cm}$. The average weight is about half a kilogramme. 
The skin is more consistent than before, and presents on its surface at certain places small patches of sebaceous matter. Hairs are more extensively developed than before, but are still devoid of any distinct colour. The legs are now longer than the arms, and the nails are well formed. The umbilicus is further forward than in the preceding month, and is now some distance in front of the pubes.

The head is still very large in proportion to the other parts. The heart, liver, and kidneys are also disproportionately large. The small intestine contains meconium, which, owing to the secretion of bile, is now of a pale greenish-yellow colour. The gall-bladder is of some size. Ossification has commenced in the pubes, and in the os calcis.

\section{The Sixth Month.}

The total length of the foetus at the end of the sixth month, measured from the vertex to the heels, is from 30 to $32 \mathrm{~cm}$. The weight is very variable; its average amount is about a kilogramme.

The skin is of a dirty reddish colour, and much wrinkled; it is covered, at any rate in the axillix and groins, with a sebaceous deposit. The hairs are more strongly developed, and of a darker colour than before. Both eyelashes and eyebrows have commenced to appear.

The umbilicus is still further forward than before, and the meconium in the intestine is darker and more viscous. The testes of the male have not yet descended into the scrotum, but are situated within the abdominal cavity, lying on the psoas muscles, immediately behind the kidneys.

The sternum is well developed, and has commenced to ossify. The nails reach to the ends of the fingers, and extend about a quarter of the way round them.

\section{The Seventh Month.}

The total length of the foetus at the end of the seventh month, measured from the vertex to the heels, is about 35 or $36 \mathrm{~cm}$., and the weight averages about $1 \frac{1}{2}$ kilogramme.

The skin is still of a dirty reddish colour, but not so dark as before. There is an increased deposit of fat in the cellular tissue, causing the body to appear more plump and 
round. The hair's are plentiful, and about 5 or $6 \mathrm{~mm}$. in length.

The several bones forming the roof of the skull become strongly convex, the central portion of each, from which ossification starts, forming a very evident prominence. The eyelids, which have been closed since reaching their full size in the fourth month, now open.

The whole of the large intestine is filled with a dark olivegreen viscous meconium. The liver is still very large relatively to the whole body, and is of a deep brownish red colour.

The testes have, as a rule, descended as far as the inguinal rings, and may even have entered the inguinal canals.

The end of the seventh month is of interest, as being perhaps the earliest period at which the foetus can be born with any reasonable chance of surviving.

\section{The Eighth Month.}

During the eighth month the increase in bulk is more marked than that in length. At the end of the month the total length of the foetus, from the head to the coccyx, is about $28 \mathrm{~cm}$.; and from the head to the heels about $40 \mathrm{~cm}$. The weight varies from 2 to $2 \frac{1}{2}$ kilogrammes.

The skin is of a brighter flesh colour than before, and is covered all over with the sebaceous deposit known as 'vernix caseosa.' This substance, which usually makes its appearance about the middle of gestation, was formerly considered to be a deposit formed from the liquor amnii, but appears rather to consist of matter formed by the cutaneous glands of the foetus, mixed with dead epithelial cells. It varies much in quantity in different cases, and is always more abundant in certain situations, notably the head, axillæ, and groins.

The chin is now far more prominent than before, the lower jaw equalling the upper in length. One of the testes, usually the left one, has passed through the inguinal canal into the scrotum, while the other is, as a rule, still in the canal. There is no ossification in the lower epiphysis of the femur.

\section{The Ninth Month.}

At the full time the foetus measures about $35 \mathrm{~cm}$. from the head to the coccyx, and $50 \mathrm{~cm}$. from the head to the heels. The weight is, on the average, from 3 to $3 \frac{1}{2}$ kilogrammes. 
The skin is paler than before. The subcutaneous connective tissue is filled with fat, giving roundness and firmness to the body and limbs. The hair is thick, long, and fairly abundant on the head, while the down has begun to disappear from the body.

The umbilicus is almost exactly in the middle of the body, or slightly behind this point. Both testes are, as a rule, in the scrotum, which has now a corrugated surface.

Ossification has commenced in the centre of the cartilage at the lower end of the femur. This is the first epiphysial ossification to appear in the body, and is often the only one present at full time. Ossification has sometimes commenced in the proximal epiphyses of the tibia and humerus; but while the presence of these centres is a sure sign of full time having been reached, their absence does not, without further evidence, indicate premature delivery.

\section{DEVELOPMENT OF THE NERVOUS SYSTEM.}

The general history of development of the human nervous system is the same as in other Vertebrates. Certain points, especially in connection with the brain, will require detailed notice; and, with regard to the histological development of the nervous elements, recent researches by His, and others, have shown that human embryos are well suited for the most minute investigations.

\section{The Brain.}

a. General account. It will be convenient to give first a general account of the development of the brain, and of its condition at successive stages, and then a more detailed description of parts, such as the cerebral hemispheres, which are of special interest.

The second week. In the youngest human embryos, such as His' embryos E and SR (Figs. 176, 178, and 179), estimated as about thirteen days old, the neural groove is widely open along its whole length, but by comparison with later embryos it is possible to determine, even at this stage, the several regions of the brain.

Thus, in Fig. 179, the dorsal concavity, opposite the refer- 
ence line AN, marks the junction of the brain and spinal cord; the highest point of the cephalic convexity, close to the reference line HD, is the region of the mid-brain; and the part in front of this is the fore-brain, which is already flexed ventralwards.

The third week. By the fifteenth day (Figs. 197, p. 489, and 232, p. 545) the neural canal is closed along its whole length, except at the extreme hinder end ; the several divisions of the brain-fore-brain, mid-brain, and hind-brain-are well established; and cranial flexure is strongly marked, a sharp bend of about 90 degrees taking place opposite the mid-brain, by which the fore-brain is brought down to the under surface of the head.

The fore-brain is of considerable length ; its most anterior part is the vesicle of the hemispheres, a short, rounded, and comparatively inconspicuous dilatation, which as yet shows no trace of division into right and left hemispheres. The thalamencephalon, or fore-brain proper (Fig. 232, BF), is long, and compressed laterally; from its sides arise the optic vesicles, Bо, which project outwards and slightly backwards, and are already constricted at their bases to form the optic stalks. The floor of the thalamencephalon is produced downwards behind the optic stalks into a shallow pit, the infundibulum.

The mid-brain, BM, is small and rounded; it is separated by a constriction from the fore-brain in front, and by a much sharper one, the isthmus, from the hind-brain.

The hind-brain is the widest as well as the longest part of the brain; it is widest in front, and gradually tapers posteriorly as it passes into the spinal cord. The roof of the hind-brain is very thin, except at its anterior end, where a slightly thickened transverse band, BL, marks the commencement of the cerebellum.

During the third week the brain rapidly increases in size, and by the end of the week has attained the proportions shown in Fig. 215. The several divisions of the brain are more distinctly marked off from one another, and the vesicle of the hemispheres, BS, and the cerebellum, BL, are more conspicuous than before.

The cervical flexure, by which the entire head is bent ventral- 
wards on the body, is commencing to appear at the junction of brain and spinal cord ; it is shown in Fig. 215, at a level between the reference lines HC. 3 and CIr.

The fourth week. By the end of the fourth week the shape of the brain is as shown in Fig. 216. The flexure at the level of the mid-brain, or mesencephalic flexure as it may be termed, has increased greatly in extent, and now amounts to about $180^{\circ}$,

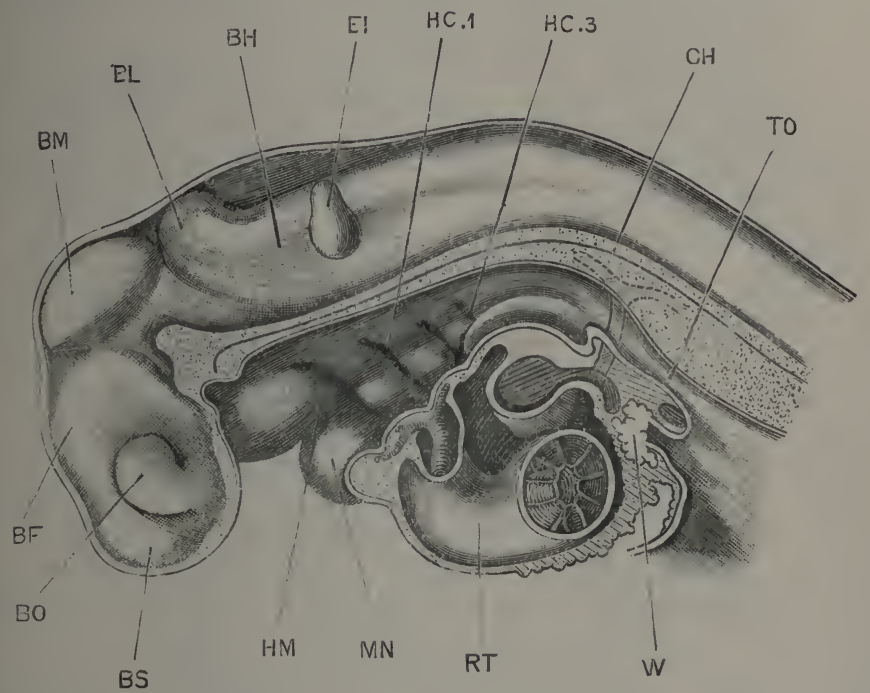

FIG. 215.-The head and fore part of the body of a Human Embryo lettered by Professor His, Lr, and estimated as twenty or twenty-one days old. (Cf. Fig. 198.) The brain is exposed from the left side; the rest of the embryo is represented in sagittal section. (From His.) $\times 28$.

BF, thalamencephalon. BH, hind-brain, or medulla oblongata. BL, cerebellum. $\mathbf{B M}$, mid-brain. BO, optic vesicle. BS, vesicle of the cerebral hemispheres. $\mathbf{C H}$, notochorr. EI, autlitory vesicle. HC.1, first branchial pouch. HC.3, third branchial pouch. HM, lyomandibular pouch. MN, mandibular arch. RT, truncus arteriosus. TO, cesolliagus. W, liver.

the infundibulum and the hind-brain almost touching each other. The cervical flexure, marking the junction of the brain and spinal cord, at the level of the reference line A.5, is also much more pronounced than before, and forms an angle of about $90^{\circ}$. A third, or metencephalic flexure, with the concavity directed dorsalwards, is commencing to form opposite the cerebellum, at the level of the reference line PT; at a slightly later stage this flexure becomes very strongly marked.

As regards the individual parts of the brain, the vesicle of 
the hemispheres has greatly increased, and is now divided by a median fold into right and left hemispheres, BS, which are

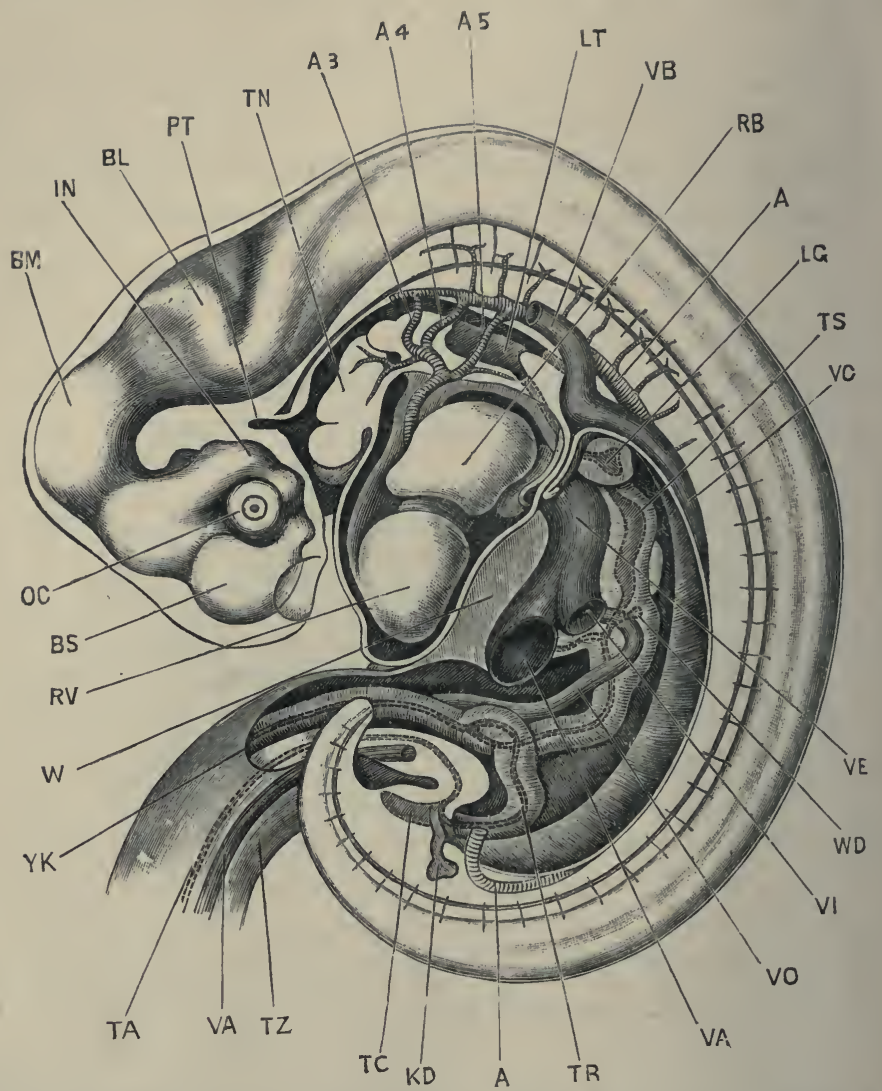

FIG. 216.-Human Embryo lettered by Professor His, Pr, and estimated as twenty-eight days old. The brain is exposed from the left side; and the body of the embryo has been dissected to show the heart and aortic arches, and the alimentary canal. (From His.) $\times 9$.

A, dorsal aorta. A.3, third aortic arch, or carotid arch. $\mathbf{A . 4}$, fourth aortic arch, or systemic arch. A.5, fifth aortic arch, or pulmonary arch. BI, cerebellum. BM mid-brain. BS, cerebral hemisphere. IN, infundibulum. $\mathbf{K} \mathbf{D}$, ureter. $\mathbf{L} \mathbf{G}$, lung. LT, laryngeal chamber. OC optic cup. PT, pituitary diverticulum from stomatodæum. RB, left auricle. RV, ventricle. TA, eavity of allantoic stalk. TC, cloaca. TN, tongue. TR, intestine. TS, stomach. TZ, umbilical stalk. VA, allantoic vein. $\mathbf{V} \mathbf{B}$, anterior cardinal vein. $\mathbf{V C}$, posterior cardinal vein. $\mathbf{V E}$, meatus venosus. VI, posterior vena cava. VO, vitelline vein. W, liver. WD, bile duct. YK, yolk-stalk.

already commencing to grow backwards over the thalamencephalon. This latter is very deep dorso-ventrally, but compressed laterally ; the infundibulum, IN, is of considerable size; 


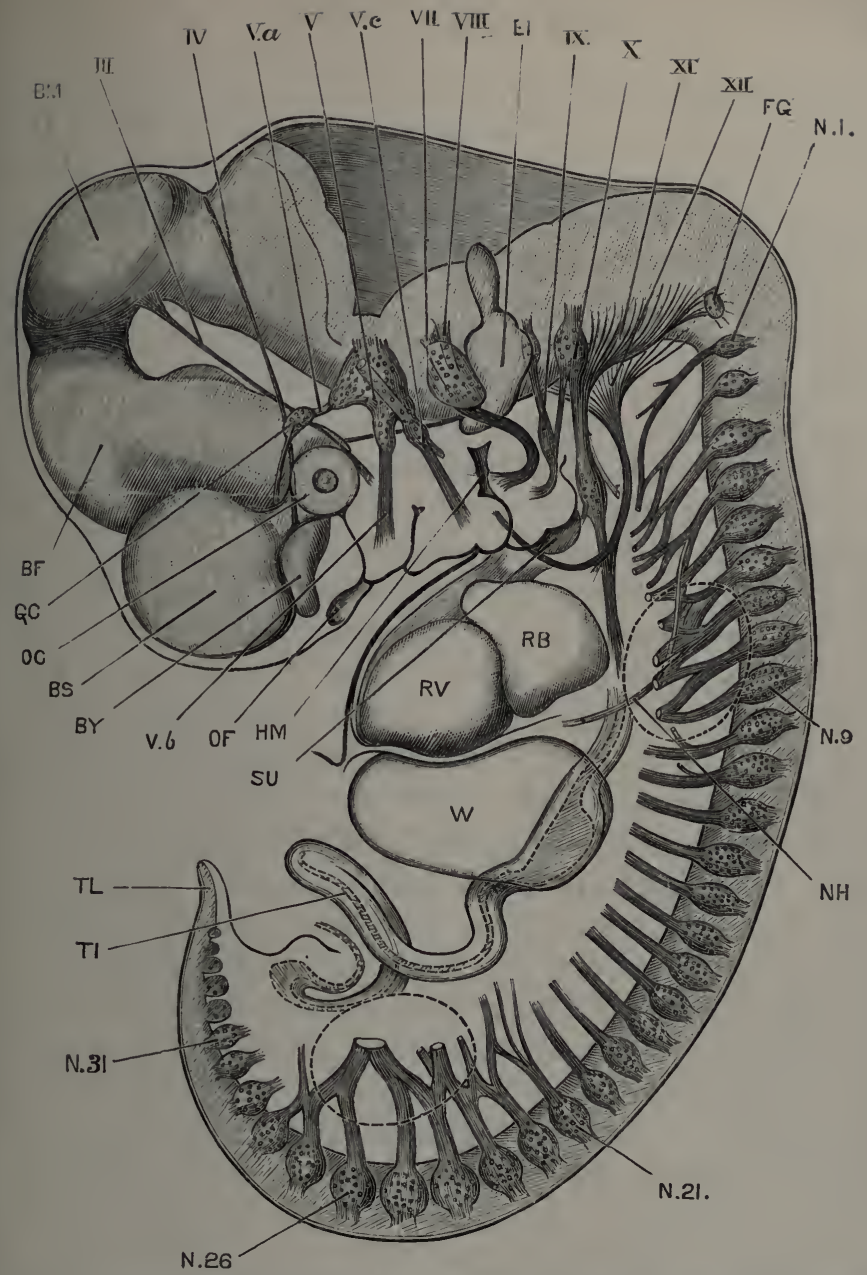

FIG. 227.-Diagrammatic figure of a Human Embryo, lettered by Professor His, Ko, and estimated as thirty-one days old. The brain and spinal cord, and the cranial and spinal nerves are shown, and certain of the other organs are represented in outline. The bases of the fore and hind limbs are indicated by the dotted outlines. In all cases in which the full length of the nerve is not shown, the end is represented as though cut across. (After His.) $\times 10$.

BF, thalamencephalon. BM, mid-brain. BS, cerebral hemisphere. BY, olfaetory lobe. EI, auditory vesicle. FG, Froriep's ganglion. GC, ciliary ganglion. HM, hyomandibular cleft, or external auditory meatus. N.1, ganglion of first cervical nerve. N.9, ganglion of first thoracic nerve. N.21, ganglion of first lumbar nerve. N.26, ganglion of first sacral nerve. N.31, ganglion of first coccygeal nerve. NH, phrenic werve. OC, optic cup. OF, olfactory pit. RB, left auricle. RV, ventricle. SU, sinus præcervicalis. TI, vitelline loop of intestine. TL, tail. W, liver. III, third cranial werve. IV, fourth cranial nerve. V, Gasserian ganglion. V $a$, ophthalmic branch of fifth, or trigeminal nerve. $\mathbf{V} b$, maxillary branch of fifth, or trigeminal nerve. Vc, manlibular branch of fifth, or trigeminal nerve. VII, ganglion of the seventh, or facial nerve. VIII, ganglion of the eiglith, or auditory nerve. IX, niuth, or glossnpharyngcal nerve. $\mathbf{X}$, ganglion of the root of the tentl, or pneumogastric nerve. $\mathbf{X I}$, roots of the eleventh, or spinal accessory nerve. XII, roots of the twelfth, or hypoglossal nerve. 
The nerves of the first set, i.e. the motor nerves, have localised centres of origin in the brain : the nerves of the second set, or sensory nerves, are not definitely localised in the brain, except by the points at which the fibres enter the brain. The two groups of nerves arise independently, as in the case of the spinal nerves. They may retain their independence, forming purely motor, or purely sensory nerves; or they may become more or less closely associated with one another to form nerves of mixed, motor and sensory, function.

The course of the cranial nerves in the early stages of their development is curiously straight (Fig. 227); their main direction, like that of the spinal nerves, being at right angles to the axis of the head, or brain, at their points of origin. This initial course is liable to disturbance through shifting relations of the parts with which the nerves are in connection, or through growth of the skeletal or other neighbouring parts. Thus the facial nerve is at first straight, but, owing to the telescoping of the hinder visceral arches within the anterior ones, its course becomes much modified (Fig. 227, viI).

In many instances some further explanation is required: thus the glossopharyngeal nerve extends forwards in front of its proper territory, in order to reach the circumvallate papillæ of the tongue; while the facial nerve extends forwards to the forehead. An interesting case is the extension of the pneumogastric nerve to the heart, lungs, and stomach. The posterior limit of the head may be taken as indicated by the hinder border of the second branchial arch; or, in the adult, by the boundary line between the thyroid and cricoid cartilages, if Callender and His are right in regarding the thyroid cartilage as developed from the cartilage of the second branchial arch. In any case, the heart, lungs, and stomach are, in the adult, far behind the head region. It must be remembered, however, that the heart originally lies between the ventral ends of the visceral arches, and that the lungs arise from the floor of the pharynx, so that both heart and lungs really lie within the proper area of distribution of the pneumogastric nerve. The stomach, however, does not do so, and in order to reach it the pneumogastric nerve must pass beyond the limits of its own territory.

In describing the cranial nerves individually it will be con-

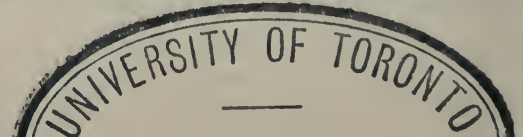


venient to arrange them in two groups, in accordance with the distinction laid down above, and to describe the nerves of each group in order, from behind forwards.

Group A. Nerves arising from groups of neuroblasts in the substance of the brain, in the same way as the motor or ventral roots of the spinal nerves.

To this group belong the third, fourth, and sixth nerves; the motor root of the trigeminal nerve; the facial nerve; the motor roots of the glossopharyngeal and pneumogastric nerves; and the spinal accessory and hypoglossal nerves.

Along the spinal cord, the motor roots all leave the cord at the same horizontal level, the sole exception being at the anterior end of the cervical region, where the hinder roots of the spinal accessory nerve arise at a level dorsal to that of the motor spinal roots. In the brain there are two series of motor roots, a ventral series and a lateral series; the ventral series including the hypoglossal, the sixth, and perhaps the fourth and third nerves as well; and the lateral series including the anterior roots of the spinal accessory, and the motor roots of the pneumogastric, glossopharyngeal, facial, and trigeminal nerves.

The hypoglossal, or twelfth cranial nerve (Fig. 227, XII), arises by a long series of roots, each formed by a bundle of axis cylinders which arise as outgrowths from a group of neuroblasts in the ventro-lateral wall of the medulla oblongata (Fig. 228, xir). The roots commence just in front of the motor root of the first spinal nerve, and in line with this, and extend forwards to the level of the glossopharyngeal nerve and the posterior border of the auditory vesicle.

The mode of origin, and the position and relations of these roots, strongly suggest a comparison with the ventral or motor spinal roots.

In sheep embryos Froriep describes a dorsal ganglionic root of the hypoglossal nerve, in addition to the ventral roots, so the comparison with a spinal nerve or nerves seems quite legitimate. In human embryos at the end of the fourth week, and beginning: of the fifth week, His has described a small ganglion, which he names Froriep's ganglion (Fig. 227, FG), lying immediately in front of the first cervical ganglion, x.1, and in line with this. Froriep's ganglion is small and gives off no nerves at all, and at a slightly later stage it disappears altogether; it appears, how- 
ever, to correspond to the ganglion described by Froriep in sheep embryos as forming a true dorsal root to the hypoglossal nerve.

It is probable, therefore, that the hypoglossal nerve is to be regarded as formed by the rentral roots of one or more nerves equivalent to spinal nerves; and of which the dorsal root, or

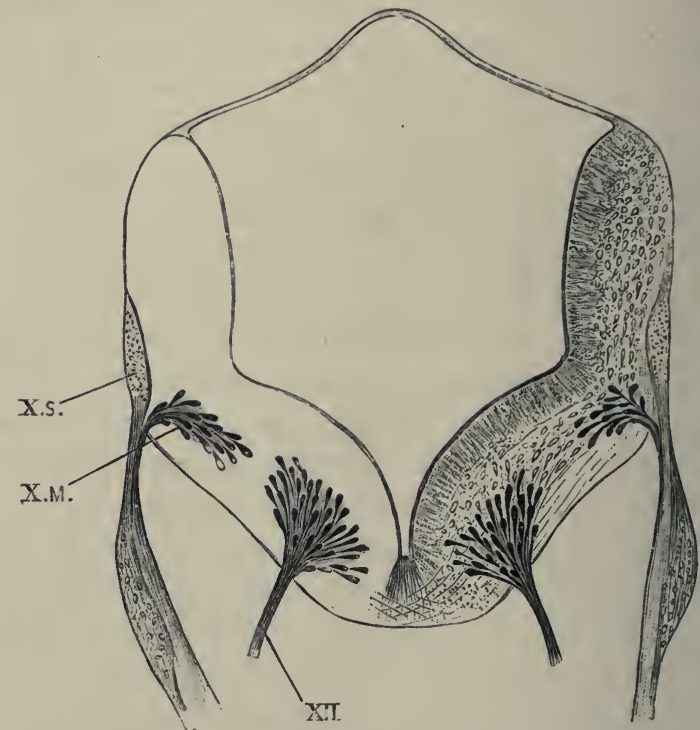

HIG. 228.- Transverse section across the medulla oblongata of a Human Embryo, lettered by Professor His, Ko, and estimated as thirty-one days old. The embryo is the same one as that represented in Fig. 227, and the section passes through one of the roots of the hypoglossal nerve, and through both the motor and sensory roots of the pneumogastric nerve. (From His.) $\times 40$.

$\mathbf{X} . \mathbf{M}$, motor root of pneumogastric nerve. $\mathbf{X} . \mathbf{s}$, sensory root of pneumogastric nerve. XII, root of hypoglossal nerve.

roots, are represented in man by the rudimentary Froriep's ganglion alone.

The spinal accessory, or eleventh cranial nerve (Fig. 227, xI), arises by a number of roots, formed by outgrowths from groups of neuroblasts in the side of the medulla oblongata; the roots lying at a level dorsal to that of the hypoglossal roots, and at the junction of the ventro-lateral and dorso-lateral regions of the medulla (cf. Fig. 228).

The roots of the spinal accessory nerve are very numerous. At the beginning of the fifth week (Fig. 227) the most posterior 
root lies in close relation with Froriep's ganglion, FG, and a very little distance in front of the first cervical nerve; while the most anterior root lies just behind the pneumogastric nerve. The cervical roots of the spinal accessory nerve do not appear until a later stage, a probable indication that the spinal accessory is to be regarded as a cranial rather than as a spinal nerve.

The motor roots of the pneumogastric, or tenth cranial nerve (Figs. 227 and 228, xм). These lie immediately in front of the anterior roots of the spinal accessory nerve, and in line with them. They arise from groups of neuroblasts in the walls of the medulla oblongata (Fig. 228), in a manner precisely similar to that in which the ventral spinal roots are formed. The nerve fibres converge to form small bundles which leave the medulla immediately ventral to the much larger and more conspicuous sensory root (Fig. 228, x.s), by which they are covered and more or less completely concealed.

The motor roots of the glossopharyngeal, or ninth cranial nerve, are exactly similar to those of the pnemmogastric; they lie immediately in front of these, and in line with them, and in transverse sections present an appearance practically identical with that shown for the pneumogastric nerve in Fig. 228, x.x.

The facial, or seventh cranial nerve (Fig. 227, vII), arises from a group of neuroblasts in the side wall of the medulla oblongata, opposite the auditory vesicle. The bundle of axis cylinders, formed as outgrowths of the neuroblasts, does not at once pass out from the medulla, but runs forwards a short distance in its substance, and emerges immediately below the auditory nerve and in very close relation with this. The root of the facial nerve lies in line with the motor roots of the glossopharyngeal and pneumogastric nerves, i.e. it belongs to the lateral series of motor roots.

The chorda tympani is present early in the fifth week as an anterior branch of the facial nerve, which runs in the tympanic membrane, but does not yet reach the trigeminal nerve.

The sixth cranial nerve belong's to the ventral series of motor roots. It arises from several groups of neuroblasts which lie in the ventro-lateral area of the medulla oblongata, in line with the hypoglossal roots, and vertically below the root of the auditory nerve, i.e. a short distance anterior to the root of the facial nerve. The sixth nerve, after emerging from the brain, 
runs almost directly forwards, lying to the inner side of the Gasserian ganglion, and reaches the external rectus muscle early in the fifth week. The nerve is drawn, though not named, in Fig. 227, as a thin band emerging from the ventral surface of the brain immediately below the ganglion of the facial and auditory nerves, and running horizontally forwards towards the hinder border of the eye.

The motor root of the trigeminal, or fifth cranial nerve, lies at a level slightly ventral to the motor roots of the facial, glossopharyngeal, and pneumogastric nerves, but clearly belongs to the lateral rather than to the ventral series of roots. It lies to the inner side of the Gasserian ganglion, and in close relation with this, and is, in its early stages, slightly anterior to this in position.

The fourth cranial nerve (Fig. 227, IV), though leaving the brain on the mid-dorsal surface, is stated by His to arise from a group of neuroblasts on the ventral surface of the isthmus, or constricted neck between the hind-and mid-brains. These roots lie close to the mid-ventral plane, and clearly belong to the ventral series. From this origin the fibres of the fourth nerve run up, in the sides of the brain, to its dorsal surface, cross those of the opposite side in the mid-dorsal plane, and finally emerge from the brain as the definite nerves.

Though very slender, the fourth nerves are of considerable length by the early part of the fifth week (Fig. 227), already reaching to the level of the eye.

The fourth nerve has long been a source of trouble to morphologists. Professor His' observations on its development in human embryos will, if confirmed and extended to other Vertebrates, throw a very welcome light on the problem, showing that, in spite of the peculiar position at which it leaves the brain, the fourth nerve really belongs to the category of ventral or motor roots.

The third cranial nerve (Fig. 227, III) arises from a group of neuroblasts in the floor of the mid-brain, rather further apart than the other ventral roots, but belonging to the same series.

Group B. The nerves included in this series arise from groups of neuroblasts, not in the brain, but in the ganglia, i.e. they are developed in the same manner as the dorsal or sensory roots of the spinal nerves. 
To this group belong the sensory roots of the pneumogastric and glossopharyngeal nerves; the auditory nerve; the sensory root of the trigeminal nerve; and probably the olfactory nerve as well.

In the head there are four primary ganglion masses, those of the fifth, eighth, ninth, and tenth cranial nerves. Whether these are connected in the early stages, to form a continuous neural ridge along each side, has not yet been ascertained; neither has the precise mode in which the permanent connection of these ganglia with the brain is acquired been determined.

The four ganglionic masses are clearly visible at the end of the third week. During the fourth week they become gradually divided up, each giving rise to two or more ganglia, which, by further elongation of the connecting nerve strands, move apart to a greater or less distance from one another.

The sensory root of the pneumogastric, or tenth cranial nerve, is from the first in close relation with the motor root, being attached to the brain immediately dorsal to this latter (Figs. 227 and $228, \mathrm{x} . \mathrm{s}$ ). The ganglion is at first single, but by the end of the fourth week it becomes divided into a proximal and smaller part, the ganglion of the root; and a distal, larger, and fusiform part, the ganglion of the trunk (Fig. 227). In the later stages these two ganglia move some distance apart, owing to lengthening: of the nerve trunk between them. The ganglion of the root is connected with the distal, or petrous, ganglion of the glossopharyngeal nerve by an oblique commissural band, well seen in Fig. 227 : whether this is a persistent remnant of an originally continuous neural ridge has not been determined.

By the end of the fourth week, the superior and inferior laryngeal nerves are present, and also a large branch extending down the osophagus towards the stomach.

The sensory root of the glossopharyngeal, or ninth cranial nerve, is very similar to that of the pneumogastric, but of smaller size. The ganglion early divides into a proximal 'jugular' portion, and a distal 'petrous' portion (Fig. 227). The nerve itself is straight in the early stages, but becomes curved forwards at its ventral end (Fig. 227) as the first branchial arch, with which it is specially associated, is carried forwards along the inner side of the hyoid arch ( $c f$. Fig. 240).

The auditory nerve. In the case of the auditory ganglion of 
the human embryo, nerve fibres have been traced growing out from the nerve cells of the ganglion into the brain, the attachment taking place immediately dorsal to the point of emergence of the facial nerve. Beyond its root of attachment, the ganglion of the auditory nerve divides into two main portions, the cochlear and vestibular ganglia: these diverge from each other, and between them the root of the facial nerve is wedged. The auditory ganglia very early acquire connection with the wall of the auditory vesicle, and the several ganglia of the adult ear are formed by further division of the two ganglia of the embryo.

It is stated that the geniculate ganglion of the facial nerve is derived from the same ganglionic mass from which the auditory ganglion is formed.

The sensory root of the trigeminal, or fifth cranial nerve. The ganglion of the trigeminal nerve is, from the first, of great size (Fig. 227). The three principal branches of the nerveophthalmic, maxillary, and mandibular (Fig. 227, v, a b c)-are already present, and of large size, before the end of the fourth week; as these nerves lengthen, the originally single ganglion gradually breaks up, small portions becoming detached, and moving out along the growing nerve stems. In this way, early in the fifth week, the ciliary, sphenopalatine, and otic ganglia are established; the submaxillary ganglion is not separated until a rather later stage. The main ganglion persists as the Gasserian ganglion of the adult, and the motor root of the trigeminal nerve lies along its inner side, and in close contact with it (Fig. 227, v).

The optic nerve. The optic vesicle and optic stalk are parts of the brain, and cannot be compared with nerves, either sensory or motor. There is, however, strong reason to think that the actual optic nerve fibres do not arise in the optic stalk, but are formed independently, as outgrowths from the retinal cells which grow inwards to the brain, following the line of the optic stalk, but being fundamentally independent of this. Even then, however, inasmuch as the retina is developmentally part of the brain, the optic nerve would rather resemble the intra-cerebral fibres of the brain than the ordinary sensory nerves; and for the present the relations of the optic nerves to the other nerves must be left undecided. 
The olfactory nerve. According to the observations of Professor His, the mode of development of the olfactory nerve in the human embryo is as follows. The olfactory lobe is formed as an outgrowth of the cerebral hemisphere towards the end of the fourth week, and very early becomes divided by a transverse constriction into anterior or distal, and posterior or proximal portions.

At this stage, in embryos of from twenty-seven to twentyeight days, although the olfactory pit is well developed (Figs: 201 and 227), there is, according to His, no trace of either the olfactory ganglion or olfactory nerve. A day or two later, the olfactory epithelium begins to undergo changes similar to those which occur in the wall of the brain, or spinal cord, preparatory to the appearance of nerves. Neuroblasts are formed near its inner or deeper surface : these soon become pyriform, and give off processes which grow into the mesoblast, and towards the brain. There is thus, early in the fifth week, a mass of neuroblasts forming a ganglion in direct connection with the olfactory epithelium: from the ganglion, nerve fibres grow out towards the brain, but do not yet reach this. By the end of the fifth week the nerve fibres reach the olfactory lobe, meeting it at the constriction separating the proximal and distal portions, and thus placing the olfactory epithelium in connection with the brain.

During the second month the distal portion, or bulb of the olfactory lobe, whicl at first lies entirely in front of the nerve, becomes bent down so as to lie in contact with this; and by the end of the second month the olfactory nerve arises by a number of fibres from the olfactory bulb, instead of by a single stem from the olfactory lobe behind the bulb, as in the earlier stages.

The roots of the adult olfactory nerve are formed by bundles of ascending or centripetal nerve fibres, which grow from the ganglion into the brain; they are already present at, or shortly after, the end of the second month.

At first sight this account appears to differ widely from the descriptions given above of the development of the other sensory nerves; but the differences are not really so great as they seen. In the case of all sensory nerves the connection with the brain, or spinal cord, is acquired by growth of nerve processes centri- 
petally, from the ganglia into the brain or cord: the ganglia themselves, though developed in close relation with the brain and cord, are not really parts of these, but are independent structures. 'The formation of neuroblasts in the olfactory epithelium presents no difficulty, when it is remembered that the wall of the brain or spinal cord is itself merely a specialised portion of the surface epithelium; while, finally, it has been shown in earlier parts of this book that in other Vertebrates, such as the frog and chick for example, the surface epithelium may, in the hinder cranial nerves, take a direct share in the formation of the nerve ganglia.

It is probable indeed that the mode of development of the olfactory nerve described above, as observed in human embryos, lepresents a more primitive type of nerve development, from which that of the other sensory nerves has been derived.

\section{The Sympathetic Nervous System.}

The mode of development of the sympathetic nervous system has not been accurately determined in human embryos. From the close resemblance in the mode of development of other parts of the nervous system, it is probable that it takes place in essentially the same manner as described above in the case of the chick and rabbit.

\section{THE DEVELOPMENT OF THE SENSE ORGANS.}

\section{The Nose.}

All the essential points in the development of the olfactory organ have been already described. The formation of the olfactory pit, the organ of Jacobson, the external nostrils, the bridge and alæ of the nose, and the posterior narial passages, are dealt with on pp. 494 to 498; and the development of the olfactory lobe and olfactory nerve on pp. 517 and 537 .

During the third month the olfactory pits, which are at first simple, become greatly complicated by folding of their walls; and in this way the nasal labyrinth, supported by the turbinal scrolls, is established. The accessory cavities communicating with the nose, i.e. the antrum, and the frontal, sphenoidal, and ethmoidal sinuses, are not established until a later stage. 


\section{The Eye.}

The mode of development of the human eye is so closely similar to that of the rabbit that it will be needless to describe it in detail.

The optic vesicles appear as lateral outgrowths of the forebrain as early as the fifteenth day (Fig. 232, во). They soon become constricted at their bases, and then doubled up to form the optic cups, in the same manner as in other Vertebrates. Owing to the mode in which this doubling up is effected, a choroidal fissure is left, leading into the cavity of the cup; and, as in the rabbit, the choroidal fissure extends a little way along: the optic stalk, towards the brain. Throughout all the earlier stages of development (Fig. 204), the eye is very small, as in Mammals generally, standing in this respect in marked contrast to the eye of the chick embryo at a corresponding stage of development ( $c f$. Fig. 115).

The inner wall of the optic cup (cf. Fig. 155) is from the first the thicker of the two; and by the end of the fourth week is at least four times as thick as the outer wall.

The lens develops late. In embryos three weeks old it is still an open pit: at four weeks the mouth of the pit has closed (Fig. 204), and from this time the cavity of the lens vesicle becomes rapidly filled up by elongation of the cells forming its inner or deeper wall. During the whole period of its development, the lens is enveloped in a vascular capsule which serves for its nourishment. New cells are constantly added on round the equator of the lens, and growth continues until the time of birth, when the vascular capsule atrophies and disappears.

The vitreous body is formed of mesoblast, which makes its way into the cavity of the optic cup through the choroidal fissure: it is very vascular during the earlier stages of its formation.

The cornea is formed from a layer of mesoblast which grows across the front of the eye, between the outer conjunctival epithelium and the lens, towards the end of the second month. In the deeper part of this layer a cavity appears, which becomes the anterior chamber of the eye. The thick layer of mesoblast in front of this chamber becomes the cornea, while the much thinner layer between the chamber and the lens forms the 
anterior wall of the lens capsule. The cornea becomes transparent during the fourth month, at which time it is strongly convex, more so than in the adult. The cornea is very thick from the first, and much thicker than the sclerotic; and at the time of birth it is said to be absolutely thicker than in the adult.

The choroid is a very vascular layer, in which pigment begins to appear towards the end of the second month. The occasional occurrence in the adult human eye of a non-pigmented streak along the under surface of the eyeball, or even of complete fissure of the iris, or coloboma iridis, is commonly attributed to incomplete closure of the choroidal fissure. Inasmuch, however, as the choroidal fissure concerns primarily the optic cup alone, and not the choroid coat, it is probable that coloboma iridis should not be regarded as a simple case of arrested development, but as involving some further pathological process as well. The choroidal fissure normally closes about the seventh week.

The retina is formed, as in other Vertebrates, from the inner and thicker layer of the optic cup. After its first establishment, it grows for a time more rapidly than the outer coats of the eyeball, and consequently becomes thrown into folds which during the second month project freely into the cavity of the eye. In the later stages it becomes flattened out again. The rods and cones are formed as outgrowths from the outer surface of the thickened inner layer of the optic cup: they do not appear until very late; shortly before the time of birth.

The mode of origin of the fibres of the optic nerve of the human eye has not been determined with exactness. It appear's certain, however, that the nerve fibres are not formed out of the walls of the optic stalk, and it is probable that they arise as outgrowths from neuroblasts in the retina itself, which grow inwards towards the brain along the path afforded by the optic stalk; and on reaching the base of the thalamencephalon pass across to the opposite side of the brain, thus forming the optic chiasma, and continue their course up the sides of the brain as the optic tracts, until they finally reach the corpora quadrigemina.

The eyelids appear, towards the close of the second month, as folds of skin above and below the eyeball (Fig. 213); they 
unite with each other, thus closing the eye, about the third or fourth month, and separate again shortly before birth.

The lacrymal duct is formed along the line of the lacrymal groove, as a linear depression running from the eye to the nose, along the line of meeting of the external nasal process and the maxillary arch (Fig. 207). The duct itself arises as a solid rod of epithelial cells split off from the floor of the groove: it becomes sinuous at an early stage, and from its sides the lacrymal glands arise as solid branched outgrowths, with dilated, hollow, bulb-like ends. At a later stage the solid cords become hollow along their axes, and converted into the lacrymal ducts. At the inner canthus of each eye the duct bifurcates, while still a solid rod, to form the rudiments of the upper and lower lacrymal canals.

The third eyelid, or plica semilunaris, which is rudimentary in man, arises as a small fold of the conjunctiva at the inner canthus of the eye, within the upper and lower eyelids.

\section{The Ear.}

The ears appear as a pair of open pits at the sides of the hind-brain on the fifteenth day (Fig. 197, EI). Almost directly afterwards the mouths of the pits close, and the vesicles, thus formed, separate from the skin. The original mouth of each pit lengthens out into an elongated neck, the recessus labyrinthi (Fig. 229, ER), while the vesicle itself forms a flattened sac, $\mathrm{EV}$, somewhat oval in outline, and lying embedded in the connective tissue at the side of the hind-brain.

At the commencement of the fifth week the auditory vesicle becomes more irregular in shape. Its ventral and anterior end (Fig. 227, EI) grows forwards as a short blunt process, which forms the rudiment of the cochlea; while, near its dorsal end, three flattened projections appear on its outer surface, which are the first stages in the formation of the three semicircular canals.

By the end of the fifth week (Fig. 230), the auditory vesicle has increased considerably in size, and its main divisions are well established. The body of the vesicle is divided by a fold into two main portions : a dorsal division, or utriculus, UT ; and a ventral division, or sacculus, S. From the utriculus the three semicircular canals arise, the two vertical canals, EA, EP, being 
already separated from the vesicle, except at their ends, while the horizontal canal, $\mathrm{EH}$, is still a wide, flattened, pouch-like outgrowth from the vesicle. From the sacculus, the cochlea, EL, arises as a short, blunt, anteriorly directed outgrowth. The recessus labyrinthi, ER, is much larger than before, and is divided at its lower part by a partition into two tubes, of which one opens into the sacculus and the other into the utriculus; these tubes affording the sole communication between the two chambers, sacculus and utriculus, of the auditory vesicle.
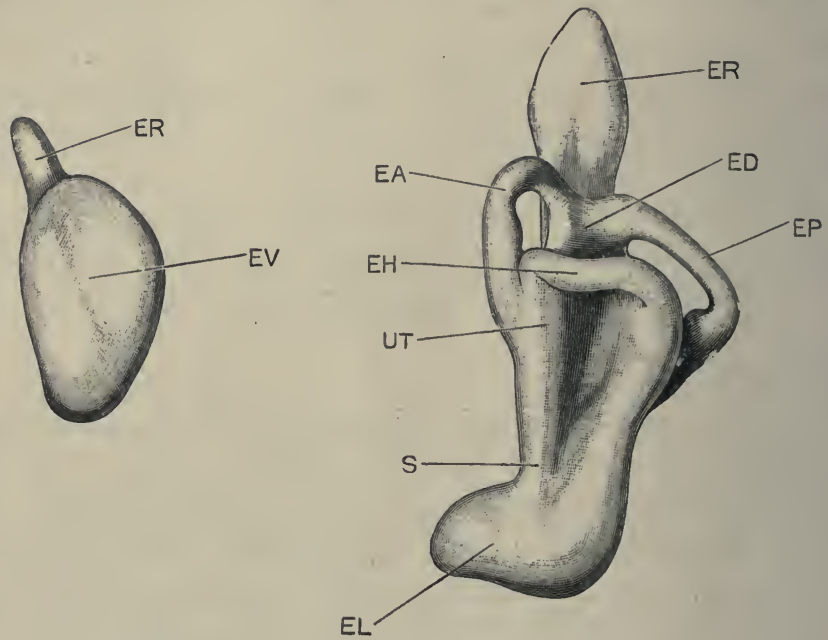

FIG. 229.

FIG. 230.

FIG. 229.-The left auditory vesicle of a Human Embryo four weeks old, seen from the outer surface ( $c f$. Fig. 227). From W. His, jun. $\times 35$.

FIG. 230.- The left auditory vesicle of a Human Embryo five weeks old, seen from the outer surface. From W. His, jun. $\times 35$.

EA, anterior vertical semicircular canal. ED, common stem of the two vertical semicircular canals. EH, horizontal or external semicircular canal. EL, cochlen. EP, posterior vertical semicircular canal. ER, recessus labyrinthi. EV, auditory vesicle. S, sacculus. UT, utriculus.

By the eighth week, the shape and proportions of the internal ear are as shown in Fig. 231. The sacculus, s, and utriculus, -UT, are now comparatively small parts of the internal ear. The semicircular canals have greatly increased in length; and the cochlea, EL, has grown enormously, and is rolled up spirally on itself.

The auditory nerve, as already noticed ( $\mathrm{p} .536)$, very early becomes continuous with the auditory epithelium: it soon 
divides into two main portions, the restibular and cochlear ganglia, and from these, by further division, the several nerve endings of the adult ear are derived.

The epithelial cells of the auditory vesicle, which, it will be remembered, are derived directly from the surface epidermis of the head, become variously modified in different parts of the resicle. Over the greater part of its surface they remain flat pavement cells, while opposite the nerve endings they become altered into the hair-cells, rods of Corti, sense cells of the ampullæe, and other specialised structures.

The mesoblast of the side of the head, in which the auditory:

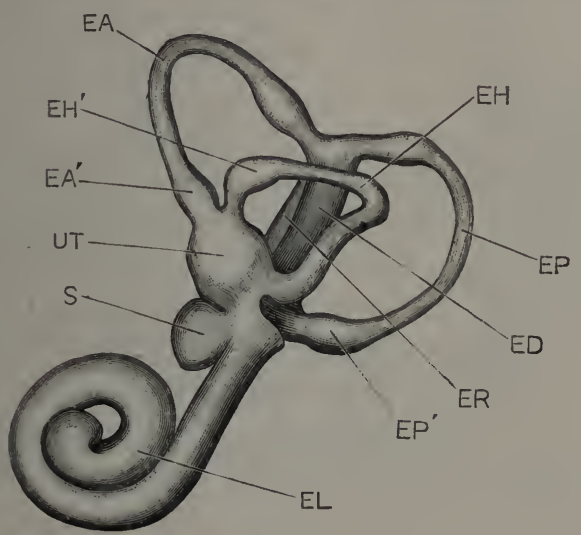

FIG. 231. - The left auditory vesicle, or internal ear, of a Human Embryo of

the eighth week, seen from the left side. From W. His, jun. $\times 17$.

EA, anterior vertical semicircular canal. EA', ampulla of anterior vertical semicircular eanal. ED, common stem of the two vertical semicircular canals. EH, horizontal semicircular canal. EH', ampulla of horizontal semicircular canal. EI, cochlea. EP, posterior vertical semicircular canal. EP', ampulla of posterior vertical semicircular cinal. ER, recessus labyrinthi. S, sacculus. UT, utriculus.

vesicle is embedded, undergoes important changes. The layer in immediate contact with the epithelial vesicle becomes closely connected with this, and forms the connective tissue wall of the labyrinth; at a little distance from the labyrinth the mesoblast becomes converted into cartilage, which forms the periotic capsule. Between the cartilaginous capsule and the labyrinth itself, the intervening mesoblast breaks down to form the perilymphatic spaces surrounding the vestibule and the semicircular canals, and the two lymphatic canals-scala tympani and scala vestibuliwhich lie above and below the scala media, or cochlear outgrowth from the labyrinth. 
In the later stages the cartilaginous periotic capsule becomes replaced by bone. 'This is in chief part spongy bone, but is lined on the surface towards the labyrinth by layers of compact bone, formed by the periosteal membrane. The modiolus and septa of the cochlea, as well as the osseous spiral lamina, are formed wholly in connective tissue, without any preformation in cartilage.

\section{The Accessory Auditory Organs.}

The Eustachian tube and tympanic cavity are formed from the hyomandibular pouch, or diverticulum from the pharynx. The pouch does not quite reach the surface at any stage of development: the membrane closing it at its outer end becomes the tympanic membrane; and the groove or depression on the surface of the head, opposite the hyomandibular pouch, becomes, as already noticed, the external auditory meatus; the external ear, or pinna, being formed from a series of processes developed round its margin (vide pp. 499, 500).

Up to the time of birth, the Eustachian tube, and the tympanic cavity itself in great part, are practically obliterated; their walls being brought into contact with each other by the development of very abundant, gelatinous, connective tissue in their substance. This is absorbed about the time of birth, and the tympanic cavity and Eustachian tube opened out.

\section{DEVELOPMENT OF THE DTGESTIVE SYSTEM.}

\section{General Account.}

The alimentary canal of the human embryo, like that of the chick, is at first merely the part of the cavity of the yolk-sac which is included within the embryo, as this is constricted off by the head-, side-, and tail-folds ( $c f$. Fig. 188).

As the constriction deepens, to form the yolk-stalk, the part of the cavity within the embryo, or mesenteron, gradually becomes more and more sharply separated from the cavity of the yolk-sac proper ; the two cavities still, however, communicating freely through the yolk-stalk.

The mesenteron soon acquires a definite tubular form, and on the fifteenth day has the shape and relations shown in Fig. 232. 
It consists of three parts, fore-gut, mid-gut, and hind-gut, which are approximately equal in length.

The fore-gut is widened transversely at its anterior end to form the pharynx, TP, which is separated in front by a thin, obliquely placed septum, DU, from the bottom of the stomatodral, or mouth invagination, DS. Behind the pharynx, the fore-gut narrows to form a short tubular portion, the œsophagus, which lies immediately above the heart. Behind the œsophagus is a fusiform dilatation, the stomach, Ts, beyond which the fore-gut

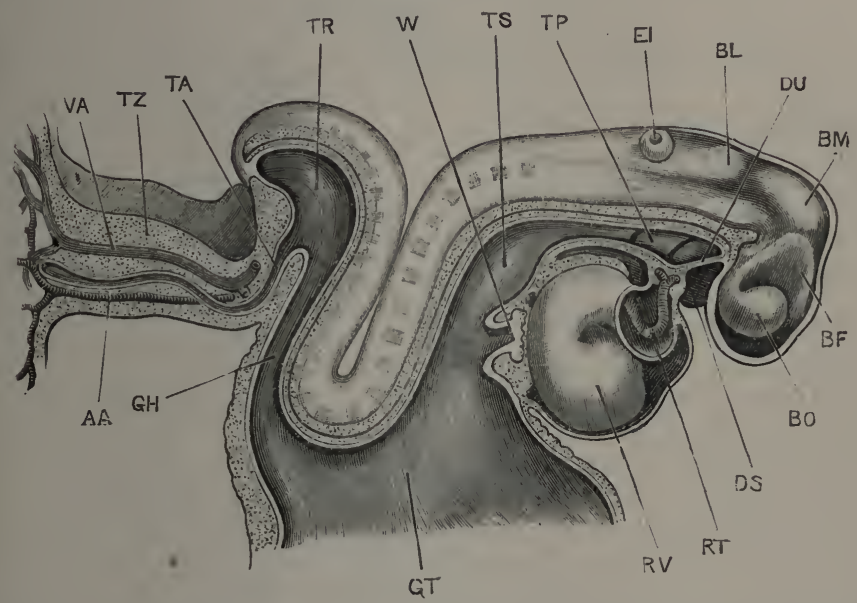

Fig. 232.-Human Embryo, lettered by Professor His, Lg, and estimated as fifteen days old ( $f f$. Fig. 197). The brain and heart are exposed from the right side; the alimentary canal and the yolk-stalk are represented in median sagittal sectiou. (From His.) $\times 30$.

A A, allantoic artcry. BF, thalamencephalon. BL, cerebellum. BM, mid-brain. BO, optic vesicle. DS, stomatodæum. DU, septum between stomatodæum and pharynx. EI, auditory pit. GH, hind-gut. GT, mill-gut and yolk-stalk. RT, truncus arteriosus. RV, ventricular portion of heart. TA, allantoic diverticulum. TP, pharyngeal region of fore-gut. TR, cloacal dilatation of hind-gut. TS, stomach. TZ, allantoic stalk. VA, allantoic vein. W, liver.

passes into the mid-gut, GT, which latter opens through the wide yolk-stalk into the yolk-sac. The hind-gut, GH, is at first narrow and tubular; but at its hinder end it dilates to form the large cloacal chamber, TR, from the ventral surface of which the allantois, TA, arises as a narrow tubular diverticulum. There is as yet no trace of a proctodæal, or anal invagination.

In embryos about a day older than the one represented in Fig. 232, i.e. of about the sixteenth day, the stomatodæal septum is perforated, and the mouth opening established 
(cf. Fig. 215). The proctodæal opening is not formed until a much later stage, about the end of the fifth week, and is a perforation of the integument rather than a distinct pit.

During the fourth week the alimentary canal rapidly assumes more definite form. 'The pharynx (Figs. 216 and 233) remains of great width from side to side, and in connection with it the gillpouches, lungs, and other important structures are formed. 'The œsophagus rapidly increases in length as the neck elongates; and

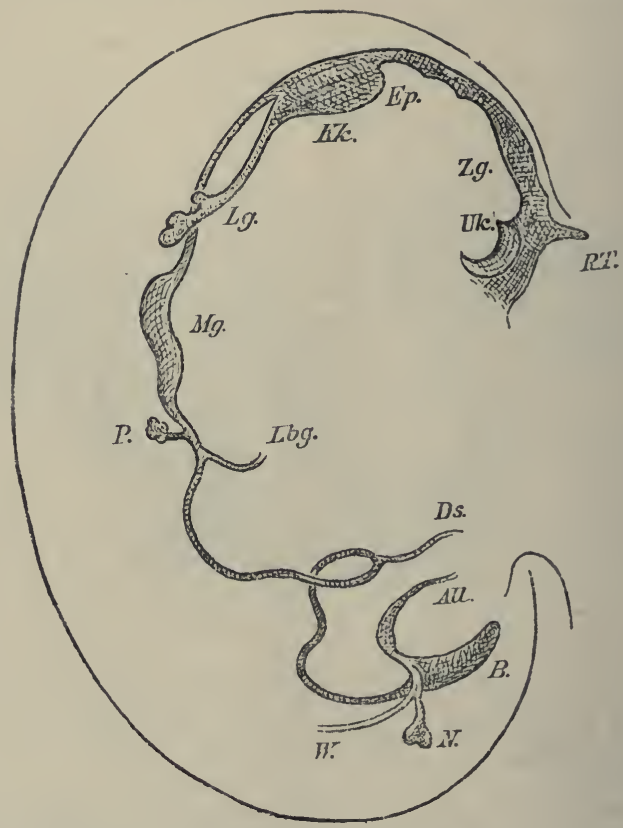

FIG. 233.-Outline figure of the alimentary canal of a Human Embryo, lettered by Professor His, Pr, and estimated as twenty-eight days old ( $c f$. Fig. 216). The figure is drawn from the right side, and the cavity of the alimentary canal is alone represented, not the thickness of its walls. The curved line bounding the figure on the left is the notochord. (From His.) $\times 15$.

$A l l$, allantois. $B$, cloaca. $D s$, yolk-stalk. $E p$, epiglottis. $K k$, laryngeal chamber. $L . b g$, bile-duct. $L g$, lung. $M g$, stomach. $N$, ureter. $P$, pancreas. $R T$, pituitary bocly. $U k$, mandibular arch. $W$, Wolffian duct. $Z g$, tongue.

the stomach becomes a more conspicuous dilatation. The intestine is long, narrow, and tubular; it forms a prominent, ventrally directed vitelline loop, from the apex of which the narrow yolkstalk arises, connecting the intestine with the yolk-sac.

The alimentary canal is at first (Fig. 232) closely attached 
to the dorsal wall of the body along its whole length, lying immediately ventral to the notochord; and is hence equal in length to the part of the body in which it lies. During the fourth week, the intestine grows much more rapidly than the body of the embryo, and becomes thrown into loops which project ventralwards (Fig. 233). A small duodenal loop is formed immediately beyond the stomach, and opposite the bile-duct,

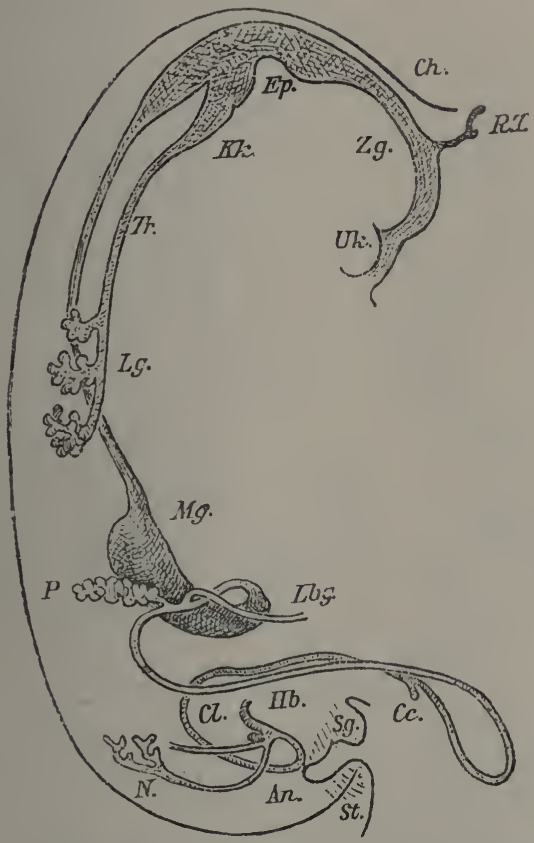

FIG. 234.-Outline figure of the alimentary canal of a Human Embryo, lettered by Professor His, Sch, and estimated as thirty-five days old. The figure is drawn from the right side, and the cavity of the alimentary canal is alone represented, not the thickness of its walls. The curved line bounding the figure on the left is the notochord. (From His.) $\times 10$. $A n$, position at which the auus will be formed. $C c$, creum. $C h$, notochord. $C l$, rectum. $E p$, epiglottis. $H b$, basal portion of allantois, which becomes the bladder. $K k$, larynx. $L b g$, bile-duct. $L g$, lung. $M g$, stomach. $N$, rudiment of permanent kidney or metanephros. $I^{2}$, pancreas. $R T$, pituitary body. Sg, clitoro-penis. Sl, tail. $T r$, trachea. Uk, mandible, or lower jaw. $Z y$, tongue.

$L h y$; and a much larger vitelline loop is formed lower down, from the apex of which the yolk-stalk, $D s$, arises. As the intestine lengthens, its attachment to the dorsal wall of the body becomes drawn out into a thin vertical sheet of mesoblast, the mesentery, between the layers of which the blood-vessels of the alimentary canal run. 
During the fifth week (Figs. 234, 235, and 236), the cosophagus lengthens very greatly; the stomach in consequence shifts backwards, and at the same time acquires its characteristic shape (Fig. 236, Mg), and becomes placed across the body instead of along it. The vitelline loop of the intestine (Fig. 234) passes out some distance beyond the body; it lengthens

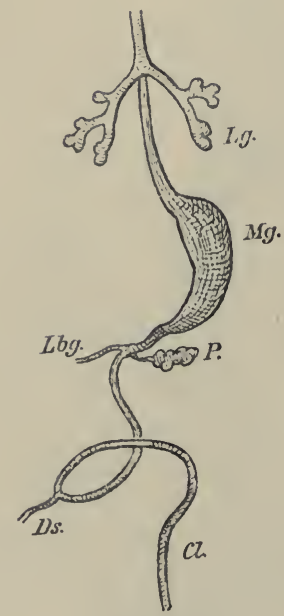

FIG. 235.

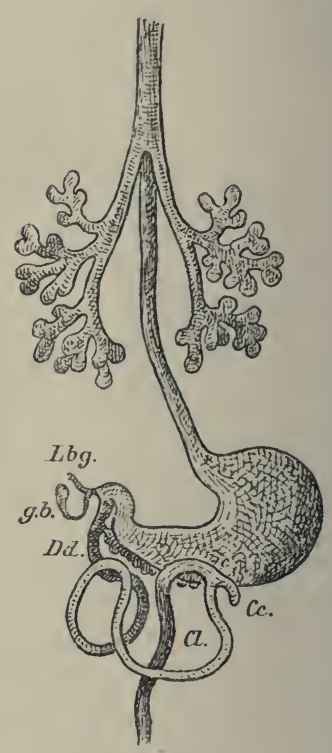

FIG. 236.

FIG. 235.-Outline figure of the alimentary canal of a Human Embryo, estimated as thirty-two days old. The figure is drawn from the ventral surface, and the cavity of the alimentary canal is alone represented, not the thickness of its walls. (From His.) $\times 12$.

FIG. 236.-Outline figure of the alimentary canal of a Human Embryo, estimated as thirty-five days old. The figure is drawn from the ventral surface, and the cavity of the alimentary canal is alone represented, not the thickness of its walls. (From His.) $\times 10$.

$C c$, cæcum. $C l$, rectum. $D d$, duodenum. $D s$, yolk-stalk. $g b$, gall bladder. $L b !$, bile-duct. $L g$, lung. $\mathbf{M g}$, stomach. $P$, pancreas.

considerably, and becomes at the same time twisted on itself. Before the end of the week, the tubular yolk-stalk (Fig. 235, Ds) separates from the intestine, although detached portions of the tube may persist along the yolk-stalk for some time longer. The cæcum, $C c$, arises during the fifth week as a diverticulum from the distal limb of the vitelline loop, not far from the point of attachment of the yolk-stalk. 
During the fifth week the cloaca, which up to this time has been a single dilated chamber (Fig. 233), becomes divided, by the growth backwards of a septum from the angle between the allantoic stalk and the intestine, into two separate tubes; of these, the dorsal one (Fig. 234, Cl) is continuous with the intestine and forms the rectum; while the ventral one, $H b$, receives the allantoic stalk, and the Wolffian ducts and ureters, and forms the urino-genital passage.

The septum which thus divides the cloaca into rectal and urino-genital chambers is formed by the union in the median plane of two lateral folds or ridges, which arise from its sides; it reaches the surface of the body just below the root of the tail, about the end of the fifth week (Fig. 234). The proctodral opening is formed about the same time, but it is not certain whether this takes place before or after the completion of the septum : in the former case there would be for a short time a single cloacal aperture; in the latter case the rectal and urinogenital apertures would be distinct from the first.

The later stages in the development of the part of the alimentary canal from the œsophagus to the rectum present few features of special interest. The epithelium lining the cosophagus is ciliated during the fifth and sixth months, and perhaps for a longer period.

The mucous membrane of the stomach is smooth up to the end of the second month; during the third month it becomes much folded, especially at the pyloric end, and in the course of the fourth month the glands commence to develop. In the intestine the villi appear towards the end of the second month, and the glands of Lieberkiuhn about the beginning of the fourth month. The large intestine is at first closely similar to the small intestine, and contains numerous villi, which about the fourth or fifth month become united by folds of the mucous membrane to form a honeycomb pattern. Peyer's patches appear about the sixth month.

\section{The Pharynx.}

The pharynx requires special notice on account of the importance of the structures developed in connection with it.

From the first the pharynx is distinguished from the rest of the length of the alimentary canal by its great width. 
At its first formation (Fig. 237) the pharynx is of approximately uniform width along its whole length; but at an early stage the anterior part widens very greatly and the whole pharynx becomes funnel-shaped, with the apex directed backwards (Figs. 238 and 239).

The condition of the pharynx on the fifteenth day is shown in horizontal section in Fig. 237, which should be compared

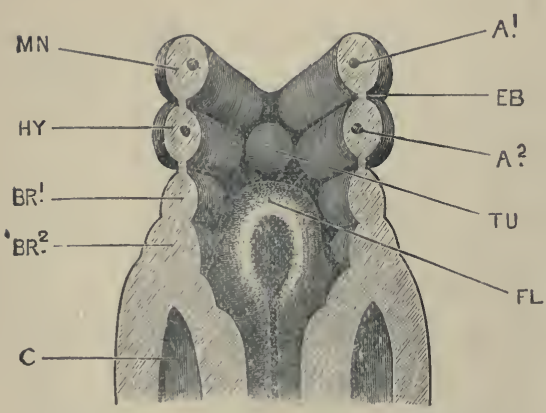

FIG. 237.-The floor of the pharynx of a Human Embryo fifteen days old, seen 1. from above. (Cf. Figs. 197 and 232.) $\approx$ (From His.) $\times 50$.

A1, first aortic arch, in the mandibular arch. A.2, second aortic arch, in the hyoid arch. BR1, first branchial arch. BR2, second branchial arch. C, body cavity, or eœlom. EB, membrane closing the hyomandibular cleft, which becomes afterwards the tympanic membrane. FI, furcula. HY, livoid arch. $\mathrm{MN}$, mandibular arch. TU', tuberculum impar. with Figs. 197 and 232, which represent the same embryo in surface view, and in sagittal section. The visceral arches are seen to form prominent ridges projecting into the pharynx, and separated from one another by grooves, the visceral pouches. Of the visceral arches, the mandibular, MN, and hyoidean, HY, are well developed; and behind these the first and second branchial arches, $\mathrm{BR}^{1}$ and $\mathrm{BR}^{2}$, are recognisable, though less clearly defined.

The hyomandibular and first branchial pouches are well formed; and corresponding to them on the outer surface of the pharynx are well-marked external visceral grooves, clearly seen in surface views of the embryo (Fig. 197, HM, $\mathrm{HC}^{\mathrm{l}}$ ).' The corresponding visceral pouches and grooves, on the inner and outer surfaces of the pharynx respectively, do not quite meet, but are separated by thin membranous partitions, of which the most anterior one, EB, between the mandibular and hyoidean arches, becomes ultimately the tympanic membrane.

Further back, there are less strongly marked second branchial, and third branchial pouches or grooves on the inner surface of the pharynx, with slight indications of corresponding visceral grooves on the outer surface.

Towards the end of the third week, and in the early part of 
the fourth week, the hinder visceral arches, and the pouches separating them from one another, become much more clearly defined: the pharynx also changes its shape, becoming much wider in front, and narrowing posteriorly towards the œsophagus (Fig. 238).

The mandibular, hyoid, and first and second branchial arches are well defined (Fig. 238, $\mathrm{Mr}, \mathrm{HY}, \mathrm{BR}^{1}$, and $\mathrm{BR}^{2}$ ), the hyoid arch being especially large. Both the internal visceral pouches, and the external visceral grooves between the successive arches are well marked. There is some doubt as to whether any of the gill-clefts are actually open in the human embryo; such evidence

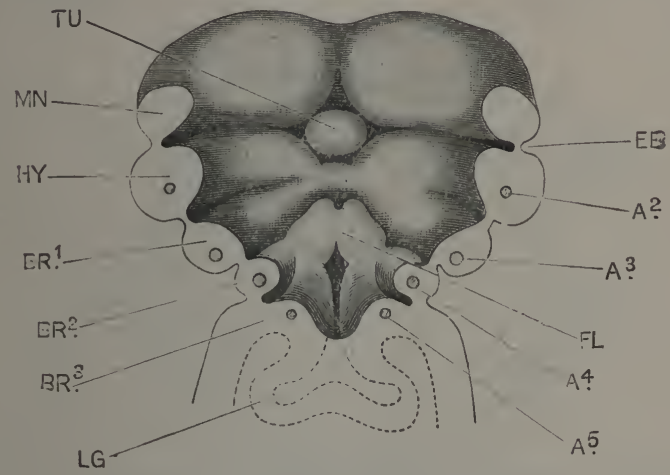

FiG. 238.-The floor of the pharynx of a Human Embryo, twenty-three days old, seen from above. Cf. Fig. 243, which represents the same embryo. (From His.) $\times 30$.

A2, second anric arch, in the hyoidean areh. A.3, third aortic arch, in the first branchial arch. A4, fourth aortic arch, in the second branchial arch. A5, fifth aortic areh, in the third branchial areh. BR1, first branchial arch. BR2, second branchial areh. BR3, third branchial arch. FB, membrane elosing the hyomandibular cleft, which afterwarls becomes the tympanic membrane. FL, fureula. HY, hyoid areh. LG, lung. MN, mantibular arch. TU, tuberculum impar.

as has been obtained points to the conclusion that none of the clefts are really completed either at this or any other stage in development; the visceral pouches and the corresponding visceral grooves being always separated by thin partitions, as at EB in Fig. 238.

The second branchial arch, $\mathrm{BR}^{2}$, is bounded posteriorly by the conspicuous and deep third branchial pouch; irnmediately behind this is a ridge, $\mathrm{BR}^{3}$, projecting into the cavity of the pharynx, and bounding laterally the entrance to the cosophagus. Although there is no external ridge on the surface of the embryo corresponding to this internal ridge, yet its relations to other 
organs, and more especially the fact that in it, as in the anterior arches, an aortic arch, or branch of the truncus arteriosus, $A^{5}$, is present, show that the ridge in question, $\mathrm{BR}^{3}$, is really a third branchial arch.

In Fig. 238 it is seen that the second branchial arches, $\mathrm{BR}^{2}$, not only lie nearer the middle line than the first branchial arches, $\mathrm{BR}^{1}$, but are also in part overlapped by these. During the latter part of the fourth week, this overlapping becomes much more marked, the posterior visceral arches shifting forwards, and being telescoped within the arches in front of them.

In Fig. 239 the condition at the end of the fourth week is shown, at which time the first branchial arches have com-

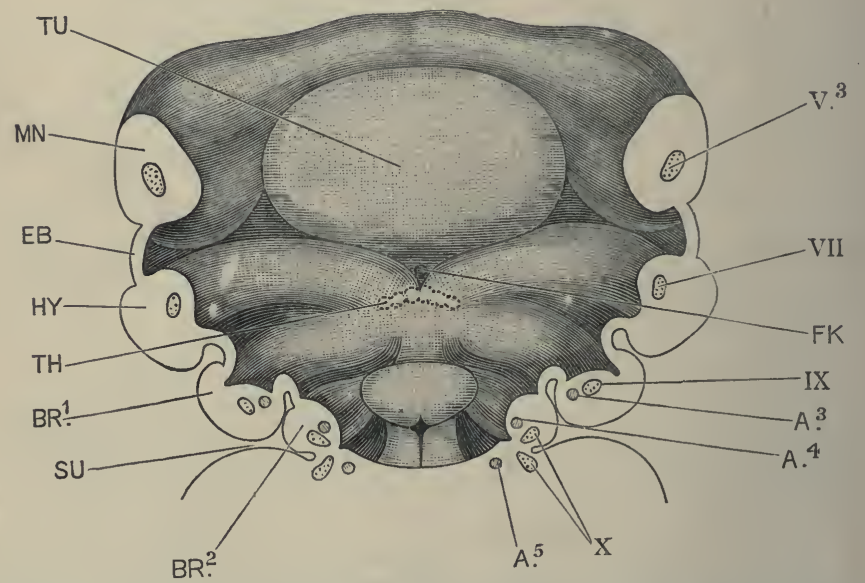

FIG. 239.-The floor of the pharynx of a Human Embryo twenty-eight days old, seen from above. $C f$. Fig. 216, which represents the same embryo. (From His.) $\times 30$.

A.3, third aortic arch, in the first branchial arch. A.4, fourth aortic arch, in the second branchial arch. A.5, fifth aortic arch, in the third branchial arch. BR.1, first branchial arch. BR.2, second branchial arch. EB, membrane closing the lyomandibular cleft, which afterwaris becomes the tympanic membrane. FK, foramen cxeum. HY hyoid arch. IMN, mandibular arch. SU, sinus præcervicalis. TH, median thyroid rudiment. TU, tuberculum impar. V.3. mandibular branch of trigeminal nerve. VII, hyoidean branch of facial nerve. IX, glosso-pharyngeal nerve. X, branchial branches of pnenmogastric nerve.

pletely overlapped the second branchial arches, $\mathrm{BR}^{2}$, so as to conceal them in surface views of the embryo.

During the fifth week the first branchial arches are in their turn overlapped and concealed by the hyoid arches (Fig. 240), so that in surface views of embryos of this age none of the arches behind the hyoid can be seen (cf. Fig. 205). 
By this telescoping of the visceral arches a deep cleft is formed at each side of the neck, extending round to its ventral surface, and dividing the pharyngeal region from the trunk. This cleft, which presents a certain resemblance to the opercular cavity of a tadpole, is the sinus præcervicalis (Fig. 240, Su); it ultimately becomes obliterated by fusion of its anterior and posterior walls.

\section{The Upper Lip and the Palate.}

The fronto-nasal process consists, as already described, of a median area (Fig. 240, FP), and two lateral lobes, the processus

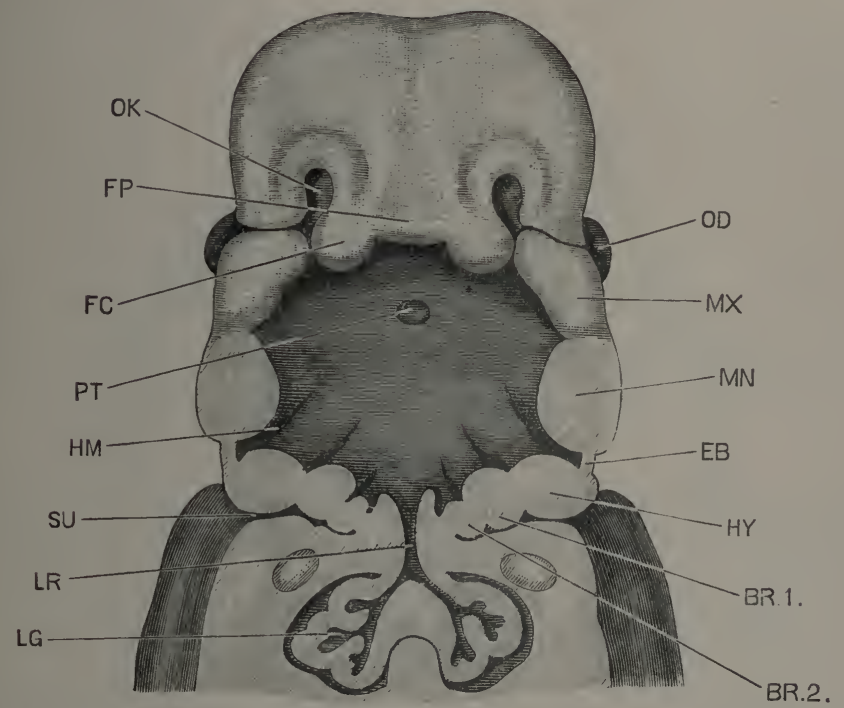

FIG. 240.-The head and neck of a Human Embryo thirty-two days old, seen from the ventral surface. The floor of the mouth and pharynx has been removed. Cf. Fig. 205, which is an outline figure of the same embryo. (From His.) $\times 12$.

BR.1, first branclial arch. BR.2, second branchial areh. EB, membrane closing the hyomandibular cleft. which afterwards becomes the tympanic membrane. FC, processus globularis. FP, merlian part of fronto-nasal process. HM, lyyomandibular pouch. HY, hyoid arch. LG, lung. IR, larynx. MN, mandibular arel.. MX. maxillary arch. OD, eye. OK, mouth of olfactory pit, or exterual nostril. PT, pituitary body. SU, sinus præcervicalis.

globulares, FC. The processus globulares form the inner lips of the nasal grooves, which connect the olfactory pits with the mouth, and of which the outer lips are formed by the inner edges of the maxillary arches, $\mathrm{Mx}$. By fusion of their inner and outer lips, the nasal grooves become converted into the posterior 
nasal passages, a pair of short tubes leading from the olfactary pits to the fore part of the roof of the mouth, into which they open in much the same position as the posterior nares in an adult frog.

At a later stage, after the outgrowth of the median part or bridge of the nose, the two processus globulares meet each other in the median plane, and fuse to form the median part of the upper lip (cf. Figs. 207 and 241).

There are, thus, in the upper lip three sutural lines : a median one, where the inner borders of the two processus globulares meet and fuse with each other; and a pair of lateral ones, where

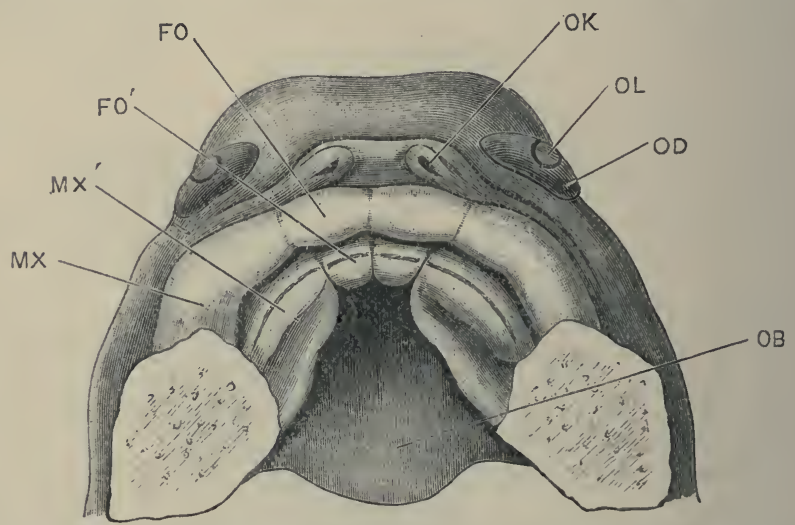

FIG. 241.--The roof of the mouth of a Human Embryo about two and a half months old, showing the mode of formation of the palate. (From His.) $\times 10$.

FO, processus globularis. $\mathbf{F O}^{\prime}$, palatal process of processus globularis. MX, maxillary arch $\mathbf{M X}^{\prime}$, palatal process of maxillary arch. $\mathrm{OB}$, mouth cavity. OD, eye. OK, aperture of olfactory pit, or nostril. OL, lens.

the outer borders of the processus globulares meet and fuse with the inner ends of the maxillary arches.

The median cleft is the one which persists throughout life in the hare or rabbit, but it is doubtful whether it ever remains open in man; what is called hare--lip in man being due to imperfect closure of one or other of the lateral clefts.

The palate is formed, as regards its most anterior portion, by a pair of horizontal shelf-like outgrowths from the processus globulares (Fig. 241, $\mathrm{FO}^{\prime}$ ), which meet and fuse in the median plane. The rest of the palate, comprising the greater part of its length, is formed by two similar outgrowths, $\mathrm{Mx}^{\prime}$, from 
the inner surfaces of the maxillary arches. The palatal processes grow rapidly, and by the beginning of the third month the anterior ends of the maxillary processes, $\mathbf{M x}^{\prime}$, have met and fused with each other in the median plane, immediately behind the premaxillary processes, or outgrowths from the processus globulares, $\mathrm{FO}^{\prime}$. A small aperture is left in the median plane between the four palatal processes, and persists as the foramen incisivum. The completion of the palate is effected by the extension backwards of the fusion of the inner edges of the maxillary processes, towards their hinder ends. Occasionally the union fails to take place properly, and the malformation known as cleft palate results.

By the formation of the palate, the anterior part of the mouth cavity becomes divided into dorsal or nasal, and ventral or buccal portions, and the communication between the posterior nostrils and the buccal cavity is shifted backwards to the level of the hinder edge of the palate.

The septum narium is formed in the first instance by upgrowths from the inner edges of the palatal processes, which fuse together in the median plane, and grow dorsalwards as a partition, dividing the nasal chamber into right and left halves.

\section{The Tongue.}

The tongue arises from the floor of the fore-gut, so that its epithelial covering is entirely of hypoblastic origin. It is formed from two l'udiments, which are at first completely separate from each other; an anterior median swelling, the tuberculum impar, from which the body and tip of the tongue are developed; and a posterior $\mathrm{V}$-shaped ridge, which gives rise to the root of the tongue.

On the fifteenth day (Fig. 237) the ventral ends of the mandibular arches, Mx, almost meet each other in the median plane; the ventral ends of the hyoid arches, HY, are some little distance from each other; and the ventral ends of the first and second branchial arches, BR.1, BR.2, are still further apart. There is thus left in the floor of the pharynx, between the ventral ends of the visceral arches, a triangular, mesobranchial area, the apex of which is directed forwards. From the dorsal surface of this area the tongue is developed; while the heart (Fig. 232) lies immediately beneath it. 
At the anterior end of the mesobranchial area, between the ventral ends of the mandibular and hyoid arches, is a small rounded elevation, the tuberculum impar (Fig. 237, TU). Behind this, and between the ventral ends of the first and second branchial arches, there is a much larger elevation, with prominent rounded margins and a median longitudinal furrow. This is the furcula (Fig. 237, FL); and from it the epiglottis will be developed at a later stage, while the median groove will become the glottis.

The furcula lies at first immediately behind the tuberculum impar; but in the early part of the fourth week (Fig. 238) the two become separated by a transverse ridge, formed from the ventral ends of the hyoid and first branchial arches, which unite together and extend across the floor of the mouth. This ridge

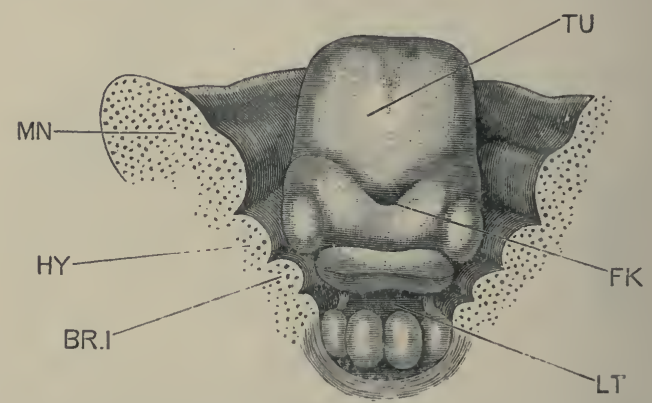

Fig. 242.-The tongue and floor of the mouth of a Human Embryo at the end of the second month. (From His.)

BR.1, first branchial arch. FK, foramen cæcum. HY, hyoid arch. LT, glottis. MIN, mandibular arch. TU, body of the tongue, formed from the tuberculum impar.

soon grows forwards at the sides of the tuberculum impar, embracing it like a $\mathbf{V}$. At the angle of the $\mathrm{V}$, between the ridge and the tuberculum, a small backwardly directed pit is formed, the mouth of which becomes the foramen cæcum (Fig. 239 , FK), while the pit itself becomes the median portion of the thyroid body, TH.

The median part of the transverse ridge soon becomes marked off by lateral grooves, and fusing with the tuberculum impar gives rise to the root of the tongue (Fig. 242). The $\mathrm{V}$-shaped groove, marking the boundary between the two originally separate elements of which the tongue consists, is very conspicuous throughout development, and is often well 
marked in the adult: it is always indicated, in the median plane, by the foramen creum (Fig. 242, FK). The line of circumvallate papill:, which appears during the third month, lies immediately in front of this groove, and therefore in the part of the tongue formed from the tuberculum impar: immediately in front of the foramen crecum, and sometimes surrounding it, is a single, very deeply depressed circumvallate papilla.

The double origin of the tongue is indicated by its nerve supply; the body and tip of the tongue, developed from the tuberculum impar, are supplied by the gustatory branch of the trigeminal nerve; while the root and sides of the tongue, developed from the transverse ridge, are supplied by the glossopharyngeal. It must be noted, however, that in order to reach the circumvallate papillæ the branches of the glosso-pharyngeal nerve have to overstep the boundary between the two parts of the tongue, and invade the part formed from the tuberculum impar.

\section{J. The Thyroid Body.}

The thyroid body is formed from three independently arising rudiments, which remain distinct until a rather late stage in development : (i) a middle thyroid rudiment (Fig. 239, тH), which is a deep pit commencing at the foramen crcum, at the junction of the body and root of the tongue, and extending downwards and backwards in the floor of the mouth; and (ii) a pair of lateral thyroid rudiments, which are outgrowths of epithelium from the floor of the mouth at the sides of the larynx, in close relation with the third branchial pouches.

The middle thyroid rudiment, which appears about the middle of the fourth week, consists at first of a short tubular duct, which divides at its blind end into right and left lobes (Fig. 239, TH). During the fifth week the median duct, or thyro-glossal duct, elongates rapidly, growing downwards and backwards until its bifurcated distal end lies opposite the larynx, or upper end of the trachea. During this rapid growth the duct usually loses its lumen, and becomes a solid rod of epithelial cells extending, in the median plane, from the foramen crecum to the trachea.

Towards the end of the fifth week, this epithelial cord usually becomes broken up in the middle part of its course into a number of detached fragments; and a little later it becomes 
still furtler interrupted by the formation of the cartilaginous body of the hyoid, which lies exactly in its path.

The paired lateral thyroid rudiments early separate from the epithelium, and form a pair of lobed masses lying at the sides of the larynx, and of considerably larger size than the bifurcated median rudiment. At a later stage they shift still further back, so as to lie alongside the trachea, and then fuse with the median rudiment to form the definite thyroid body. The median rudiment gives rise to the isthmus of the adult thyroid, and probably to parts of the lateral lobes as well; the greater part of the lateral lobes, however, are formed from the much larger lateral rudiments.

At an early stage the lobes are excavated by a number of detached cavities, which become the vesicles of the adult thyroid From the history of their development it follows that the epithelial walls of these vesicles are of hypoblastic origin.

The duct or stalk of the middle thyroid rudiment usually disappears in great part; detached portions of it not uncommonly persist as accessory suprahyoid or epihyoid bodies, or as cysts.

Occasionally the upper part of the stalk persists as a tube, the lingual duct, extending from the foramen crecum, on the dorsum of the tongue, backwards and downwards towards the body of the hyoid, or actually reaching this in some cases.

The lower or posterior part of the stalk may also occasionally persist, forming the so-called pyramid of the thyroid, a somewhat pyriform body, enlarged and saccular at its lower or posterior end, and tapering upwards to a fibrous cord which is attached to the dorsal surface of the hyoid bone. The pyramid is apparently formed by persistence and enlargement of one of the two branches into which the stalk bifurcates at its lower end. When present, it is usually single, but cases have occurred in which two pyramids were found, due apparently to persistence of both branches of the bifurcation.

\section{The Thymus.}

The thymus is a paired organ of epithelial origin, developed in connection with the second and third branchial clefts, and perhaps the first branchial cleft as well.

It appears about the middle of the fifth week; but as to the precise mode of its formation there is still some doubt. Born 
maintains that the thymus of man, like that of other Vertebrates, is developed from the hypoblastic lining of the pharynx; His' observations, on the other hand, support an epiblastic origin; the thymus, according to him, being formed from the epiblastic walls of the sinus præcervicalis, the deep fissure at the side of the neck caused by the overlapping of the hinder visceral arches by the more anterior ones ( $c f$. Fig. 240, su).

The thymus gradually shifts backwards towards the root of the neck, extending along the pneumogastric nerve and carotid artery almost as far as the heart. It attains a great size in later foetal life, and continues to increase after birth up to about the end of the second year, when it measures two inches or more in length.

\section{The Salivary Glands.}

The salivary glands commence to form early in the second month, and by the end of the month have attained a considerable size. The ducts arise as grooves of the buccal epithelium, which by fusion of their lips become tubes; the glands themselves are, at first, solid outgrowths of epithelial cells, which later become hollowed out by extension of the cavities of the ducts into their substance. The submaxillary glands appear first, then the parotid, and lastly the sublingual glands.

\section{The Teeth.}

The teeth are developed in man in very much the same way as in the rabbit. In embryos about seven weeks old the epithelium becomes thickened along the border of each jaw, and the deeper or Malpighian layer of the epithelium grows down into the substance of the jaw as a continuous keel-like ridge, the common enamel germ. This soon becomes enlarged at intervals to form the enamel organs of the milk or deciduous teeth, while between the enamel organs the ridge becomes less conspicuous, and ultimately disappears.

Each enamel organ is flask-shaped, consisting of a terminal enlarged portion, buried deeply in the jaw, and a narrow neck or stalk which connects the enlarged part with the surface epithelium of the jaw. Opposite each enamel organ the connective tissue of the jaw becomes more compactly arranged to form the dental papilla ( $c f$. Fig. 156, TM). The dental papilla soon becomes moulded into the shape of the future tooth, and 
the enamel organ becomes closely fitted, like a cap, over the surface of the papilla, which acquires the form of the crown of the future tooth.

From the dental papilla the main substance of the tooth, or dentine, is formed in the following manner. On the surface of the papilla next the enamel organ a layer of special cells, the odontoblasts, appear. These form, by excretion on their outer surfaces, a dense matrix in which fine filamentous processes of the odontoblasts are embedded; by calcification of the matrix the dentinal substance is formed, the dentinal tubules being the narrow channels in the matrix occupied by the processes of the odontoblasts. The first formed part of the dentine is the outermost layer of the crown of the tooth, and this layer thickens by further formation of dentine on its inner surface, the odontoblasts gradually withdrawing further and further from the surface, as the dentine increases in thickness.

The enamel is formed from the layer of epithelial cells of the enamel organ which lies in immediate contact with the dental papilla. This layer consists of closely set, columnar or prismatic cells, and it is by direct calcification of these cells that the enamel is produced. The rest of the enamel organ is merely nutritive in function, and does not give rise directly to any part of the tooth.

The crown is thius the first part of the tooth to be formed. After it is completed, the tooth increases in length by the further formation of dentine round the lower part of the papilla. The aperture at the base of the tooth is at first a widely open one; but, as the tooth approaches its full size, the aperture becomes gradually narrowed to form the root or fang of the tooth. In the case of the grinding teeth, the aperture becomes divided by bridges of dentine into two or three separate openings, and by elongation of the margins of these openings the double or triple fangs of the adult tooth are produced. At the apex of each Iang a minute hole remains, through which the blood-vessels and nerves gain admittance to the pulp of the tooth, which latter is the part of the dental papilla that remains encapsuled in the middle of the tooth after its completion.

The cement, or outermost layer of the fully formed tooth, is a bony deposit developed from the connective-tissue sheath which surrounds it. 
On the first appearance of the bony jaws, the teeth lie in continuous grooves extending all round their free borders. Partitions are soon formed, dividing these grooves into separate compartments or alveoli, which grow round the teeth so as to closely embrace them.

The order of appearance of the milk teeth takes place in regular sequence; but the actual dates at which the several teeth emerge, or are 'cut,' vary within certain limits. The cutting of the milk teeth usually commences about seven months after birth, and is completed by the end of the second year. The central lower incisors appear first, about the seventh month ; the upper incisors two or three months later; a few months later still the lower lateral incisors, and the first premolars; four or five months later the canines; and about the end of the second year, the second premolars.

The permanent teeth are developed in the same manner as the milk teeth. From the stalk or neck of the enamel organ of each milk tooth, a small outgrowth arises at a very early stage, about the sixteenth week, which becomes the enamel organ of the corresponding permanent tooth. A dental papilla is formed opposite each enamel organ, and the permanent teeth are formed in the jaw, a little way behind and below the corresponding milk teeth, and in precisely similar fashion.

The three hinder grinding, or molar teeth, which have no milk predecessors, are formed by extension backwards of the original common enamel germ, from which the milk teeth are developed. The enamel organ for the first permanent molar appears about the fifteenth week of embryonic life; that for the second permanent molar about seven months after birth; and that for the third permanent molar, or ' wisdom tooth,' not until the third year.

The eruption, or cutting, of the permanent teeth of the lower jaw takes place at the following dates, the teeth of the upper jaw usually appearing a little later :-

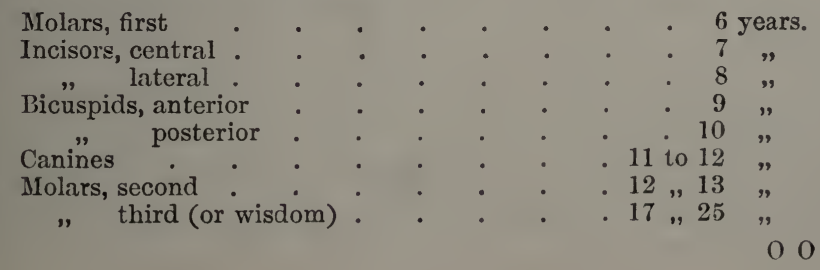




\section{The Lungs.}

On the fifteenth day (Fig. 237), a swelling is present on the floor of the pharynx, opposite the first, second, and third branchial arches; and along the middle of this swelling, or furcula, FL, there runs a longitudinal groove.

By the sixteenth day this groove is much more pronounced, and its posterior end leads into a short blind pocket.

By the end of the third week the pocket has become much deeper, extending backwards, ventral to the œsaphagus and independently of this; and its hinder end is split into right and left lobes ( $c f$. Fig. 238, LG). These lobes are the rudiments of the lungs; the tube leading to them is the trachea; and the slit-like opening, or groove, on the floor of the pharynx is the future glottis.

During the fourth week the lungs grow rapidly, extending back alongside the œsophagus, and dorsal to the heart (Figs. 216 and 233, $L g$ ); their distal ends are enlarged and are commencing to divide into lobes. The right lung has three terminal buds or lobes, and the left lung two; these buds forming the rudiments of the five lobes of the adult lungs.

During the fifth week the lungs continue to increase rapidly ; the main lobes elongate greatly, and give rise to secondary and tertiary buds, which end in slightly expanded ampullæ (Figs. 235 and $236, L g)$.

The further development of the lungs consists in a continuation of the process of budding, by which new tubules and ampullæ arise from the older ones, either by dichotomous division, or, as in the later stages, by lateral branching. The air cells appear, as closely set pouchings of the walls of the ampullæ, which are not recognisable until the time of birth.

The trachea is at first short, but rapidly elongates during the fifth and following weeks. The larynx first becomes evident, as a dilatation of the anterior part of the trachea, towards the end of the fifth week (Fig. 234). The vocal cords, and the ventricles of the larynx, are not formed until about the fourth month.

The anterior, median part of the furcula becomes the epiglottis (Fig. 233, $E p$ ) ; while the lateral ridges give rise to the ary-epiglottic folds and the arytenoid cartilages. The thyroid cartilage is said by Callender, and by His, to be formed from the 
cartilages of the second branchial arches, though Kölliker believes it to arise independently.

The lungs, as they grow backwards, project into the dorsal part of the body cavity, pushing before them the peritoneal lining of the cavity, which forms their pleural covering. At a later stage, the portions of the body cavity into which the lungs hang are shut off, by the diaphragm and pericardium, from the rest of the cavity, and become the definite pleural sacs.

\section{The Liver.}

The liver is present on the fifteenth day (Fig. 232, w) as a short, hollow diverticulum, with a compact mass of cells at its blind end, arising from the ventral wall of the fore-gut and the anterior wall of the yolk-stalk, immediately behind the heart.

By the end of the third week (Fig. 215, w) the liver is of larger size, and the bile-duct, or wide tubular passage connecting the liver with the gut, is longer than before, but otherwise the relations are much the same as in the earlier stage.

During the fourth week the liver enlarges very rapidly (Figs. 216,243, w). It consists of a close network of anastomosing epithelial cylinders, the development of which has not been followed accurately: the meshes of the network are chiefly occupied by blood-vessels, which are present in large numbers, and of great size. The development and relations of these blood-vessels of the liver will be described in the next section of this chapter.

The rapid growth of the liver continues during the succeeding weeks. In the second half of gestation it is rather less marked in proportion to the other viscera, but even at the end of pregnancy the weight of the liver is to that of the whole embryo as 1 to 18 , while in the adult it is only 1 to 36 . After birth the liver diminishes rapidly, both in size and weight, owing to the cutting off of the blood supply previously brought to it by the allantoic veins.

The bile-duct rapidly lengthens during the fourth week; and the gall-bladder appears, as a diverticulum of the bile-duct, before the end of the fifth week (Fig. 236, g.b).

The large size of the liver during almost the whole period of gestation, and its abundant vascular supply, indicate that it must be of great physiological importance. It probably serves to modify in some way the nutrient material brought from the 
placenta by the allantoic veins; and it almost certainly acts as an important excretory organ during embryonic and foetal life.

The brown, or greenish-brown, mass known as meconium, which occurs in the small intestine from the third to the fifth month, and lower down, in the large intestine and rectum, during the later months of pregnancy, contains bile in considerable quantity, as well as mucus, and epithelial and other débris.

\section{The Pancreas.}

The pancreas arises, towards the close of the fourth week, as a dorsally directed diverticulum from the duodenum, almost opposite the opening of the bile-duct (Figs. 233 and 234, P), and lying in the thickness of the mesentery which attaches the duodenum to the dorsal body-wall. The pancreas grows rapidly, giving oft lobed offshoots, from which the acini and their ducts are formed. The original diverticulum from the duodenum persists as the pancreatic duct; it at first opens a little distance from the bile-duct, but ultimately the two ducts lie close alongside each other, and open into the duodenum by a single orifice.

\section{The Mesentery.}

The mesentery is the thin vertical sheet of mesoblast which slings the stomach and intestine to the body-wall. The relations of the mesentery are at first extremely simple, but as the intestine lengthens, and especially as it becomes thrown into convolutions, they become greatly complicated. The attachment of the dorsal border of the mesentery to the body-wall remains comparatively unmodified throughout life, though at certain places oblique or transverse lines of attachment are acquired in addition to, or in place of, the original simple longitudinal attachment.

The part of the mesentery which attaches the stomach to the body-wall, commonly spoken of as the mesogaster, undergoes special modification. The stomach originally lies lengthways along the body (Fig. 233, Mg, and 243), the mesogaster being attached along the border which will afterwards become the greater curvature of the stomach, and the pancreas (Fig. 233, P) lying in the thickness of the mesogaster near its posterior limit. When the stomach shifts its position, and becomes placed transversely across the body (Fig. 236), its original left side becomes ventral, and its right side dorsal; while the mesogaster remains attached along what is now the posterior border of the stomach. 
This part of the mesogaster, along the posterior border, or greater curvature of the stomach, becomes produced into a double fold or sac, the great omentum, which hangs down, like a curtain, over the coiled mass of the intestine, close to the ventral wall of the abdomen.

Shortly after birth, the two layers of the omental sac coalesce, so that the omentum becomes a single membranous layer, in which fat early tends to accumulate.

The clorsal part of the mesogaster, which is attached to the dorsal body-wall, and in the thickness of which the pancreas is contained, comes into close contact with the layer of mesentery suspending the transverse colon, and ultimately fuses completely with this; a change which causes the pancreas to appear to lie altogether dorsal to the mesentery, instead of in its substance.

\section{THE DEVELOPMENT OF THE CIRCULATORY SYSTEM.}

The general history of the development of the blood-vessels in man, their relations at the different periods of embryonic and of foetal life, and the changes by which at the time of birth the adult circulation is established, are closely similar to those already described in the rabbit. Certain differences have been noticed in the mode of formation of the valves of the heart, and in the development of the great veins, more especially of those in relation with the liver; these are, however, of comparatively small importance, and are possibly, in some cases, due to the difficulty of obtaining human embryos in satisfactory histological condition, and of the particular age desired.

In the following account, which is based mainly on the descriptions of Professor His, the development of the heart will be dealt with first, then that of the arteries and the veins, and finally a brief description will be given of the course of the circulation in the embryo and foetus, and of the changes which occur at birth.

\section{Development of the Heart.}

General Account. The early stages in the development of the heart in the human embryo are known very imperfectly, and only as regards the external form of the organ. 
In the youngest human embryos, as in the corresponding stages of the rabbit, the heart consists of two symmetrical and perfectly distinct halves. On the thirteenth day (Fig. 179, R) the heart is present as a pair of straight tubes lying along the sides of the anterior end of the embryo, between the neural folds and the yolk-sac, and in connection at their hinder ends with the vessels which return the blood from the yolk-sac.

At a slightly later stage (Fig. 185, R), the two halves of the heart have united to form a single tube, which is already twisted on itself.

By the fifteenth day (Figs. 197 and 232), the heart has advanced considerably in development, and forms a prominent swelling on the under surface of the embryo, between the head and the yolk-sac. It is a single tube, of considerable size; attached, both in front and behind, to the floor of the fore-gut, but free along the middle portion of its length, which is twisted into a prominent S-shaped loop. The dorsal and posterior end of the loop is the auricular portion of the heart ; and is separated by a slight constriction, the canalis auricularis, from the succeeding or ventricular portion (Fig. 232, Rv). This forms the widest and most prominent part of the loop; it runs almost transversely across the body from the left to the right side, and then turns forwards rather sharply, and passes into the truncus arteriosus, RT, or terminal limb of the loop. The anterior end of the truncus arteriosus (Fig. 232) is attached to the floor of the fore-gut, very far forwards, opposite the mandibular arches.

The wall of the heart (Fig. 232) is double along its entire length, consisting of an outer mesoblastic tube, in which musclecells are already present on the fifteenth day, and an inner endothelial tube, the origin of which has not been determined. The endothelial tube is very much smaller than the muscular tube, and the space between the two is occupied by a gelatinous substance traversed by fine radial fibres, apparently of the nature of connective tissue (cf. Fig. 215).

During the third week the heart continues to increase rapidly in size, and its several divisions become more clearly marked off from one another by constrictions. By the end of the third week it has reached the condition shown in Figs. 198 and 215. The auricular portion (Fig. 198, RA) is much larger than before; it is very wide from side to side, and is produced into conspicuous 
ear-like appendages. A marked constriction, the canalis auricularis, separates it from the ventricular portion. This latter, $\mathrm{RV}$, is shaped something like the adult stomach, and lies almost

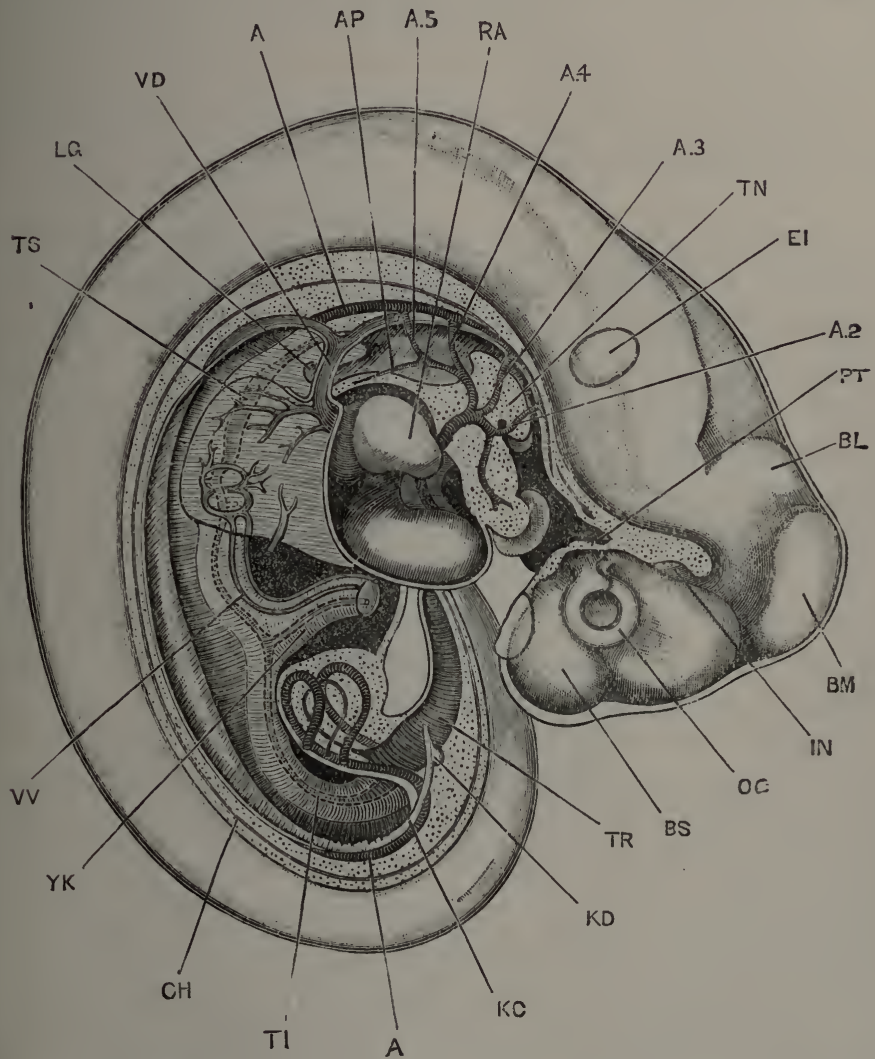

FIG. 243.-Human Embryo, lettered by Professor His, Bl, and estimated as twenty-three days old. The brain and spinal cord are exposed from the right side ; and the body is dissected to show the heart, the blood-vessels, and the alimentary canal. (From His.) $\times 20$.

A, dorsal aorta. A.2, second aortic arch, in the hyoidean arch. A.3, third aortic, or carotid arch, in the first branchial arch. A.4, fourth aortic, or systemic arch, in the second branchial arch. A.5, fifth aortic, or puluonary arch, in the third branchial arels. AP, pulmonary artery. BL, cerebellum. BM, mill-hraiu. BS, cerebral hemisphere. CH, notochoril. EI, ear. IN, infundibulum. KC, Wolffian duct. KD, ureter. LG, hung. OC, optic cup. PT, pituitary boty. RA, right auricle. TI, intestine TN, tongue. TR, rectum. TS, stomach. VD, Cuvierian vein. VV, vitelline veiu YK, yolk-stalk.

directly across the body; its right-hand or distal end bends sharply forwards, and passes into the truncus arteriosus, RT, which is attached to the floor of the fore-gut rather further back 
than before, opposite the hyoidean and first branchial arches (Fig. 215). The structure of the heart is the same as in the earlier stages, except that the muscular elements have increased considerably. The wide space between the muscular and the endothelial walls is well shown in Fig. 215, as is also the fibrous network connecting the two walls.

In describing the further development of the heart it will be convenient to take the several divisions one by one.

The sinus venosus. The blood is returned to the heart by three main veins on each side:-the Cuvierian vein (Fig. 243, vD), from the body of the embryo; the vitelline vein, from the yolk-sac; and the allantoic vein, from the placenta. 'These three pairs of veins form by their union a single large vessel, the sinus venosus, which runs transversely across the body, immediately in front of the liver, and opens through a median ape $\vec{i}$ ture into the auricular portion of the heart.

The sinus venosus is at first situated behind the diaphragm, but during the fourth week it gradually extends over, and in front of this, and so comes to lie in the pericardial cavity, immediately behind the auricle (Fig. 243).

Towards the end of the fourth week the sinus venosus becomes placed somewhat obliquely, in place of transversely, across the body; at the same time its right side becomes larger than the left, and the opening into the auricular cavity, which was at first median, shifts so as to lead distinctly into the right side of the auricle (Fig. 244, Rs). During the fifth week, the opening from the sinus venosus into the auricle widens out very considerably, so that the sinus becomes part of the auricle itself, and ceases to exist as a separate cavity. The left horn of the sinus venosus, which now only receives the left Cuvierian vein, retains its independence more completely, and persists as the coronary sinus.

The auricles. The auricular chamber is at first single, but towards the end of the fourth week it becomes imperfectly divided into the right and left auricles (Fig. 244). The division is indicated externally by a slight constriction, and more markedly by the outgrowth of the auricular appendices, which very early show characteristic crenations along their margins. 
Seen from within, the auricular portion of the heart has, at the end of the fourth week, the appearance shown in Fig. 244. Opposite the external constriction, a fold, sK, the septum superius, projects into the cavity from its anterior end and ventral wall, and reduces the communication ketween the two auricles to a rather

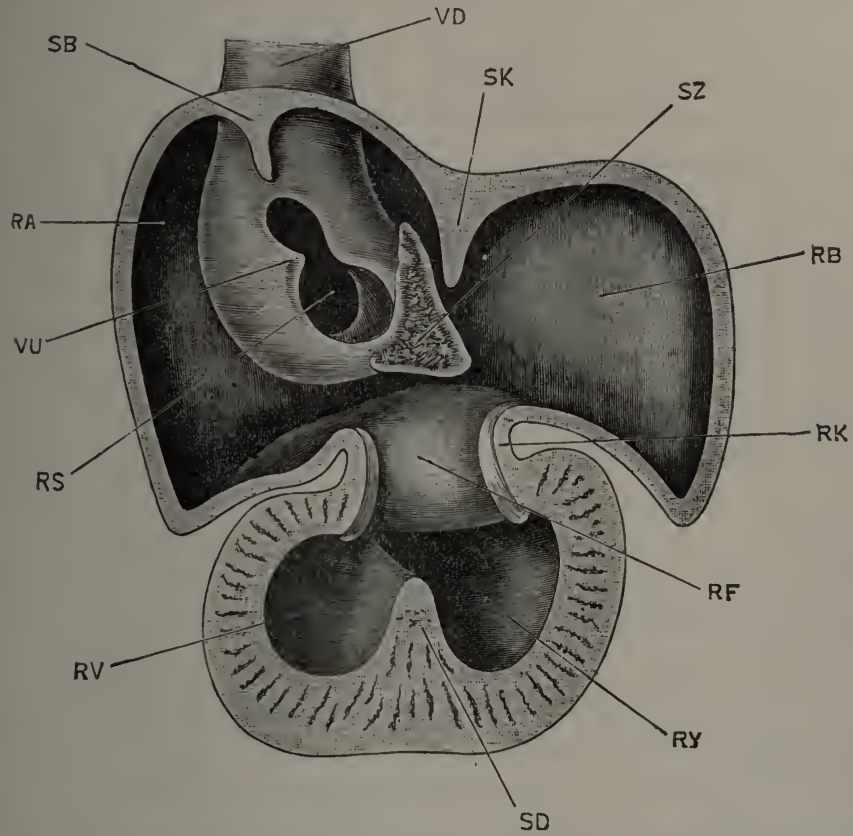

Fig. 244.-The dorsal half of the heart of a Human Embryo, twenty-eight days old, seen from within. The heart has been bisected lengthways, and the ventral half removed. (From His.) $\times 32$.

RA, riglt auricle. $\mathrm{RB}$, left auricle. $\mathrm{RF}$, auriculo-ventricular a perture. $\mathbf{R K}$, canalis auricularis. $\mathbf{R S}$, opening of sinus venosus into right auricle. $\mathbf{R V}$, right ventricle. RY, left ventricle. SB, septum spurium. SD, septum inferius. SK, septum superius. SZ, spina vestibuli. VD, right vena cava anterior. VU, Eustachian valre.

small circular aperture, nearer the dorsal than the ventral surface.

The conspicuous projection into the dorsal part of the right auricle, shown in the figure, is caused by the sinus venosus. 'The aperture from the sinus venosus into the auricle is an obliquely placed slit, RS, of which the outer lip is thickened, and forms the Eustachian valve, vu; while the opposite, or inner lip, is a thin fold, which at the lower end of the slit passes into a triangular thickening of connective tissue, the spina vestibuli, 
sz, projecting into, and partially blocking up, the aperture between the right and left auricles. This spina vestibuli, according to Professor His, plays an important part in the formation of both the interauricular and interventricular septa.

An additional fold, the septum spurium, SB, projects into the cavity of the right auricle, opposite the upper end of the slit-like opening of the sinus venosus; it is a transient structure, and ultimately disappears completely.

Of the two auricles, the right one, RA, is at first (Fig. 244) much the larger. The walls of the auricles, like the rest of the heart, consist of two layers, muscular and endothelial; these are at first some distance apart, but about the twenty-third day they come in contact, and unite firmly to form the definite auricular wall. The connective-tissue elements of the wall are derived apparently from the gelatinous matter which originally separates the muscular and endothelial walls from each other.

The interauricular septum is formed, according to His, by coalescence of the septum superius (Fig. 244, SK) with the spina vestibuli, Sz; the latter growing downwards towards the ventricle as a thickened plug, which before the end of the fifth week divides the originally single auriculo-ventricular aperture into separate right and left openings.

It is not quite clear whether the foramen ovale in the human embryo is merely due to the interauricular septum remaining incomplete dorsally; or whether it is a new aperture formed in the dorsal part of the septum, as described by Born in the case of the rabbit.

The canalis auricularis, at the beginning of the fourth week (Fig. 243), is a short, straight, and rather narrow tube, connecting the auricular and ventricular portions of the heart. In the latter part of the fourth week this purtion of the heart shortens somewhat, the auricular and ventricular portions approach each other, and the canalis auricularis becomes telescoped within them (Fig. 244, RK), projecting partly into the auricular and partly into the ventricular cavity, and being no longer visible from the surface except as a sharply marked annular constriction.

The lumen of the canalis auricularis becomes at the same time reduced to a nurrow transverse slit, its dorsal and ventral walls thickening to form a pair of endothelial cushions, which 
fuse with the lower border of the spina vestibuli to complete the interauricular septum, and from which also the auriculoventricular valves are derived.

The ventricles. The ventricular cavity becomes partially divided towards the close of the fourth week by a fold, the septum inferius (Fig. 244, SD), which arises from its dorsal and posterior wall, and the position of which is indicated externally by a slight groove on the surface of the heart. The completion of the interventricular septum is a somewhat complicated process, and will be described after the truncus arteriosus has been dealt with.

The ventricular wall consists at first of an outer muscular tube, and an inner and much smaller endothelial tube, the two tubes being separated by a considerable quantity of gelatinous comnective tissue (cf. Fig. 215). The thickening of the ventricular wall is effected, in the first instance, by the outgrowth of bands from the muscular tube into the gelatinous tissue: these bands interlace and unite with one another to form a spongework of muscular trabeculæ. The gelatinous tissue now becomes greatly reduced in amount, so that the endothelial and muscular walls are brought much closer together, and the endothelium becomes moulded to the surface of the muscular wall, covering the trabeculæ, and lining the depressions of the spongework. The wall of the ventricle is now in much the same condition as it remains in throughout life in the frog. In the later stages of development, the outer, compact muscular wall thickens very considerably, and the spongework becomes less conspicuous, forming ultimately the columnæ carneæ.

The walls of the two ventricles are of equal thickness throughout almost the whole of fotal life, as the resistance to be overcome by the two is approximately equal until the time of birth.

The truncus arteriosus. In the truncus arteriosus the most important change is the formation of the aortic septum, by which the single tube becomes divided into two, lying side by side, which become the systemic and pulmonary trunks respectively; or, in the adult, the ascending aorta and the pulmonary artery.

This division of the truncus arteriosus is effected by two 
longitudinal ridge-like thickenings of the endothelial lining, which, arising from opposite sides, encroach on the lumen, reducing it to a slit, dumb-bell shaped in section; by further growth, the two ridges meet each other and fuse, so as to divide the lumen into two completely separate passages.

The endothelial ridges, and consequently the septum itself, appear first at the distal end of the truncus arteriosus, between the origins of the systemic and pulmonary aortic arches, and gradually extend backwards towards the ventricle. The septum first appears towards the end of the fourth week; and is completed before the end of the fifth week; it has a slightly spiral course, so that the two tubes, into which it divides the truncus arteriosus, are respectively dorsal and ventral at the proximal end, next to the ventricle, and right and left at the distal end of the truncus.

Of the two tubes, the one (Fig. 245, Rx) which lies dorsally at its proximal end, and on the right side distally, is the systemic trunk ; the other, $\mathrm{RW}$, which is ventral proximally, and on the left side distally, is the pulmonary trunk; and the same relations are retained throughout life by the ascending aorta and the root of the pulmonary artery, into which the trunks develop respectively.

The separation of the systemic and pulmonary trunks at first concerns their internal cavities alone; but it is soon followed by the appearance of external grooves, which deepen until they completely separate the two trunks from each other.

The interventricular septum. The truncus arteriosus originally arises from the right-hand corner of the ventricular cavity, and the two trunks into which it becomes split retain for a time the same relations. In other words, at a time when the interventricular septum is already partially formed (Fig. 244, $\mathrm{SD})$, both the systemic and pulmonary trunks arise from the right ventricle, and the left ventricle has for a time no outlet, except through the right ventricle.

The completion of the interventricular septum has to be effected in such a way that while the pulmonary trunk is left in connection with the right ventricle, the systemic trunk shall be cut off from the right ventricle and placed in communication with the left ventricle.

The formation of the interventricular septum is consequently somewhat complicated. The greater part of the septum is 
formed from the septum inferius (Fig. 244, SD), but it is completed above, partly by the lower edge of the interauricular septum, and partly by a prolongation of the aortic septum, which divides the truncus arteriosus into systemic and pulmonary trunks.

The aortic septum grows back beyond the truncus arteriosus, so as to project a certain distance into the ventricular cavity; it then fuses with the free lower edge of the interauricular septum, in such a way as to cut off the systemic trunk from the right ventricle, and to place it in communication with the left ventricle; while finally the septum inferius extends so as to meet and fuse with the interauricular septum, and so completes the separation of the ventricles from each other.

The valves of the heart. The outer flaps of the auriculoventricular valves, both mitral and tricuspid, are formed from the lower lips of the canalis auricularis, which hang down into the ventricular cavity (Fig. 244); the inner flaps of the valves are derived from the lower edge of the interauricular septum. The valves are at first very thick and soft, and only later become thin and membranous.

The semilunar valves are formed, about the end of the fifth week, as cushion-like thickenings of the endothelium, which soon become hollowed out into pockets.

\section{The Arteries.}

The general plan of arrangement of the arteries in the human embryo is the same as in other Vertebrates; and has already been described, in previous chapters, in the case of the rabbit, the chick, and the frog.

From the anterior end of the truncus arteriosus a series of pairs of aortic arches arise, which run round the sides of the pharynx, lying in the visceral arches (Fig. 243). On reaching the dorsal surface of the pharynx, the aortic arches of each side open into a longitudinal vessel, the aorta. The two aortæ run backwards along the body, ventral to the notochord; they are at first separate along their whole length, but early fuse together in the hinder part of their course to form the definite dorsal aorta. From the dorsal aorta, vitelline arteries are given off to the yolksac; and at the posterior end of the embryo the aorta divides 
into the two large allantoic arteries, which carry blood from the embryo to the placenta.

The aortic arches of man, as of other Vertebrates, are developed in order from before backwards.

At the fifteenth day (Figs. 197 and 232) there are two pairs of aortic arches present, lying in the mandibular and hyoidean, arches, and corresponding, therefore, to the most anterior pairs in rabbit, chick, or frog embryos. By the sixteenth day three additional pairs have appeared, in the first, second, and third branchial arches; and up to the end of the third week all five pairs are still present (Fig. 198, A.1-A.5).

The point of attachment of the truncus arteriosus to the floor of the mouth shifts backwards during development, as already noticed, and at the end of the third week is opposite the hyoidean and first branchial arches. The truncus arteriosus, at this stage, immediately on entering the floor of the mouth, divides into two branches on each side (Fig. 198). Of these, the anterior branch runs forwards, and divides into the mandibular, A.1, and hyoidean, A.2, aortic arches ; while the posterior branch runs backwards, and divides into the three hinder aortic arches, A.3, A.4, A.5.

The aortic arches diminish in size from before backwards (Fig. 198); and, owing to the funnel-like shape of the pharynx (cf. Fig. 238), the hinder arches lie much nearer the median plane than do those further forward.

All five pairs of arches are complete, opening at their dorsal ends into the aortæ (Fig. 198). In front of the first, or mandibular arch, each aorta is continued forwards as the internal carotid artery, which runs along the side of the brain, and gives off branches supplying this.

During the fourth week important changes occur in the aortic arches, closely comparable with those already described in other Vertebrates, and leading to the establishment of the adult scheme of circulation.

Early in the fourth week (Fig. 243) the middle portion of the first, or mandibular, aortic arch of each side becomes obliterated, and disappears; and very shortly afterwards the corresponding portion of the second, or hyoidean, aortic arch disappears in the same fashion.

By the end of the fourth week the condition of the aortic 
arches is as shown in Fig. 216. The mandibular and hyoidean aortic arches have lost their connection with the aortæ. Their rentral or proximal ends persist as the external carotid arteries and their various branches; the mandibular arch, according to His, giving rise to the external and internal maxillary arteries, and the temporal artery; while from the second, or hyoidean arch, the lingual and ascending pharyngeal arteries arise, and perhaps also the occipital and posterior auricular arteries.

The third aortic arch, A.3, in the first branchial arch, remains complete. As seen from the side (Fig. 216), it is somewhat S-shaped, its curvature being such that the direction of flow of the blood in it is naturally forwards, along the internal carotid artery, towards the head.

The fourth and fifth aortic arches, A.4 and A.5, are both complete, opening at their dorsal ends into the aortæ. From the fifth arches, near their ventral ends, the pulmonary arteries arise, early in the fourth week, as small branches which run backwards to the lungs (Fig. 243, AP).

During the fifth week further changes of importance occur. The division of the truncus arteriosus, by formation of the aortic septum, is completed, and the systemic and pulmonary trunks are now entirely independent of each other; the systemic trunk (Figs. 245, 246, Rx) remaining in connection with the fourth and third aortic arches, and with the persisting remnants of the second and first arches as well ; while the pulmonary trunk, Rw, communicates with the fifth pair of aortic arches alone.

The portion of the aorta between the dorsal ends of the third and fourth, or, as we may now call them, the carotid and systemic arches, disappears (Fig. 245).

The third, or carotid arch, becomes more directly continuous with the anterior prolongation of the aorta, the two vessels together forming the internal carotid artery, AI; while the common carotid artery (Figs. 24.5, 246) is formed by lengthening of the arch at its origin from the systemic trunk.

Towards the end of the fifth week the heart travels rapidly backwards, as the neck elongates; this causes great lengthening of the common carotid artery (Fig. 246, AE), and straightening of the course of the internal carotid artery. It further leads, among other changes, to the pulling out of the laryngeal branch of the pneumogastric nerve, to form its recurrent loop. 
In the early part of the fifth week, the left fourth, or systemic arch, becomes distinctly larger than the corresponding arch of the right side; and this difference soon becomes more pronounced. By the end of the fifth week the fourth right arch is not only markedly smaller than the left arch, but has lost its connection with the aorta, and now forms only the vertebral and subclavian arteries of the right side.

The fifth aortic arch of the right side disappears, beyond the origin of the right pulmonary artery. The fifth left arch,

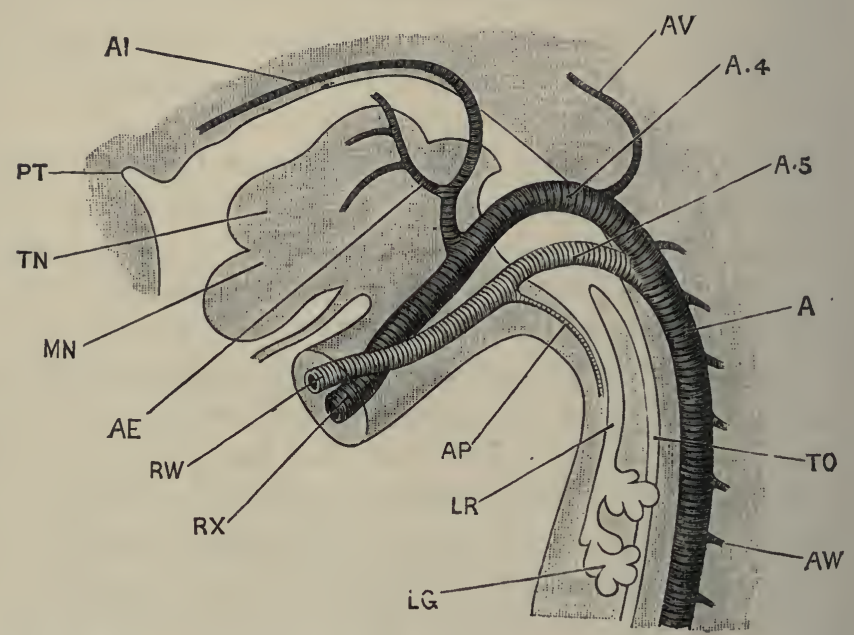

FIG. 245.-The aortic arches of a Human Embryo thirty-two days old, from the left side. (From His.) $\times 18$.

A, dorsal aorta. A.4, fourth, or systemic aortic arch. A.5, fiftl, or pulmonary aortic arch. $\mathrm{AE}$, exterual carotil artery. AI, internal carotid arterv. $\mathrm{AP}$, pulmonary artery. AV, vertebral artery. A W, intervertebral or segmental arteries. $L G$, lung. LR, trachea. MN, mandible, or lower jaw. PT, pituitary diverticulum from moutl. RW, pulmonary trunk. RX, systemic trunk. TN, tongue. TO, œsoplagus.

however, remains of large size up to the close of fœtal life; the portion of the arch between the root of the left pulmonary artery and the dorsal aorta being known as the ductus arteriosus (Figs. 245 and 246, A.5).

The dorsal aorta and its branches. The point at which the two aortæ unite, to form the single dorsal aorta, is about the junction of the cervical and dorsal regions, in embryos at the end of the fourth week, but the exact position varies considerably in different specimens. As the union proceeds-backwards, the dorsal aorta increases considerably in size, and its diameter in 
the lumbar region is more than double that in the anterior thoracic region. At the hinder end of the lumbar region the aorta divides into the right and left allantoic arteries, which run along the allantoic stalk to the placenta, and which, at any rate in the early stages, appear as direct continuations of the aorta rather than as branches of it.

The proximal ends, or roots, of the allantoic arteries persist throughout life as the common iliac arteries, from which the external iliac arteries arise as branches, on the formation of the

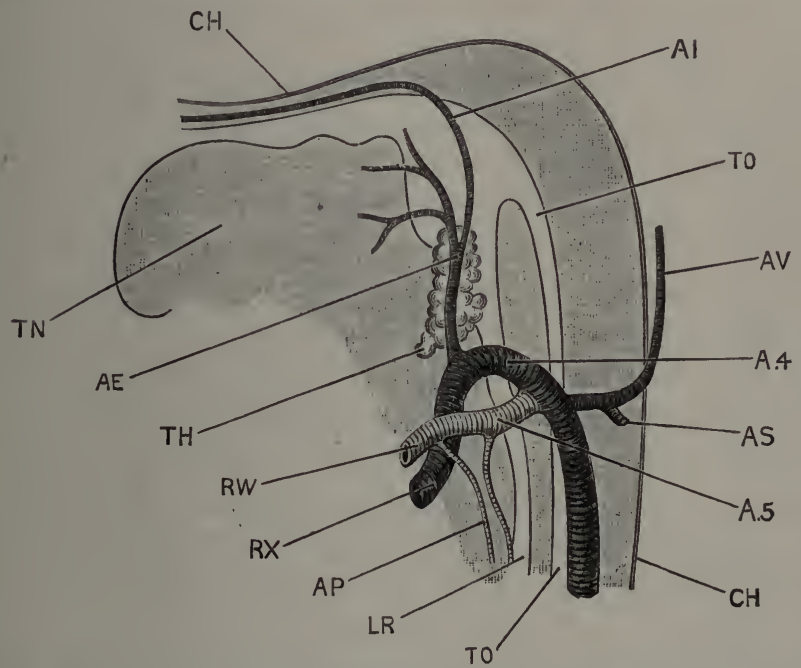

FIG. 246.-The aortic arches of a Human Embryo thirty-five days old, from the left side. (From His.) $\times 30$.

A.4, fourth, or systemic aortic arch. A.5, fifth, or pulmonary aortic arch. A. common carotid artery, at its point of division into internal and external carotid arteries. AI, internal carotid artery. AP, pulmonary artery. AS, subclavian artery. AV, vertebral artery. CH, notochord. IR, trachea. RW, pulmonary trunk. RX, systemic trunk. TH, thyroid body. TN, tongue. TO, œsophagus.

hind limbs. The hypogastric arteries are the abdominal, or intra-fœtal, portions of the allantoic arteries, beyond the origin of the internal iliac arteries; their cavities become obliterated after birth, but their walls persist as solid cords, crossing the sides of the bladder obliquely, and running forwards and upwards to the umbilicus.

The vertebral arteries appear, about the twenty-fourth day, as a pair of longitudinal trunks running along the sides of the brain, and extending from the level of the ears to the commence- 
ment of the cervical region. They have at first no communication with the other vessels, but towards the end. of the fourth week their anterior ends unite to form the median basilar artery, which becomes connected with the internal carotid arteries to form the circle of Willis. About the same time, a series of paired segmental or intervertebral arteries arise, as branches from the dorsal wall of the aorta, along the cervical and thoracic regions (Fig. 245, Aw), and supply the spinal cord. One, or more, of the anterior pairs of these intervertebral arteries become continuous with the hinder ends of the vertebral arteries (Fig. $24.5, \mathrm{Av}$ ), which thus acquire their connection with the aortæ. In the later stages, some of the intervertebral arteries further back become connected in similar fashion with one another, and with the vertebral artery; and by the acquisition of these posterior connections, with simultaneous loss of the older and more anterior ones, the point of origin of the vertebral artery from the aorta is gradually shifted backwards to the root of the neck.

The subclavian arteries arise as branches of the vertebral arteries (Fig. 246, AS) ; but, as the fore limbs increase in size, the relative proportions of the two vessels soon become reversed, and the vertebral arteries appear as branches of the subclavians.

From the sides of the dorsal aorta a series of pairs of arteries arise, which supply the Wolffian bodies. The cœliac axis is from the first a median artery ; it arises from the ventral wall of the aorta, in the anterior thoracic region, and gradually shifts backwards, until its adult point of origin, opposite the last thoracic vertebra, is attained.

In the development of the aorta, and in that of all the other arteries as well, the wall of the vessel consists at first of a single layer of endothelial cells. Outside this, the layer of circular muscle-fibres is formed from the surrounding mesoblast, early in the third week. At a later stage a layer of connective tissue is formed between the muscular and the endothelial layers, but it is not clear from what source this connective tissue is derived. His suggests that it is formed directly from the blood in the blood-vessel itself.

\section{The Veins.}

The general arrangement, and mode of development, of the veins in man is the same as in the rabbit. The most important 
differences consist in the disappearance of the left anterior vena cava, and in certain modifications in connection with the veins of the liver.

In the latter part of the third week (Fig. 198), the blood is returned to the heart by three pairs of veins, of approximately equal size :- the Cuvierian, vitelline, and allantoic veins.

Of these, the Cuvierian veins, vD, return blood from the embryo itself, and are formed on each side, as in the rabbit and the chick, by the union of an anterior cardinal or jugular vein, vB, from the head, with a posterior cardinal vein, vc, from the trunk.

The vitelline veins, vv, return blood from the yolk-sac, and enter the embryo by the yolk-stalk.

The allantoic veins, va, return blood from the placenta; they enter the embryo along the allantoic stalk, and run forwards in the side walls of the body to the heart.

The veins are at first of equal size on the two sides of the body, and by the union of the six veins the transversely placed sinus venosus is formed. In following their further development it will be convenient to take the several veins separately.

The vitelline veins are comparatively small, as in Mammals generally, owing to the small size of the yolk-sac. They lie-in the splanchnopleuric mesoblast, and, after entering the embryo at the umbilicus, run forwards along the sides of the alimentary canal to the sinus venosus (Fig. 243, vv). The vitelline veins are closely associated with the liver, and they become surrounded by this as it is developed; furthermore, the principal changes which they undergo are in connection with the vascular supply of the liver.

Early in the fourth week, about the twenty-third day (Fig. 243), the vitelline veins become interrupted as they pass through the liver, breaking up into a set of afferent hepatic vessels supplying the liver, and a set of efferent hepatic vessels conveying the blood from the liver to the heart. The afferent and efferent hepatic vessels are connected by capillaries only, so that all the blood entering the liver by the vitelline veins must traverse the substance of the liver in order to reach the heart.

About the same time, the right and left vitelline veins become connected together, immediately before they enter the 
liver, by three transverse commissural vessels. Two of these commissural vessels pass ventral to the duodenum, while the third, or middle one, is dorsal to it ; and the three together form two vascular rings, or sinus annulares, encircling the duodenum (Fig. 243). From the anterior ring, afferent vessels arise which carry blood into the liver.

At a slightly later stage, during the latter part of the fourth

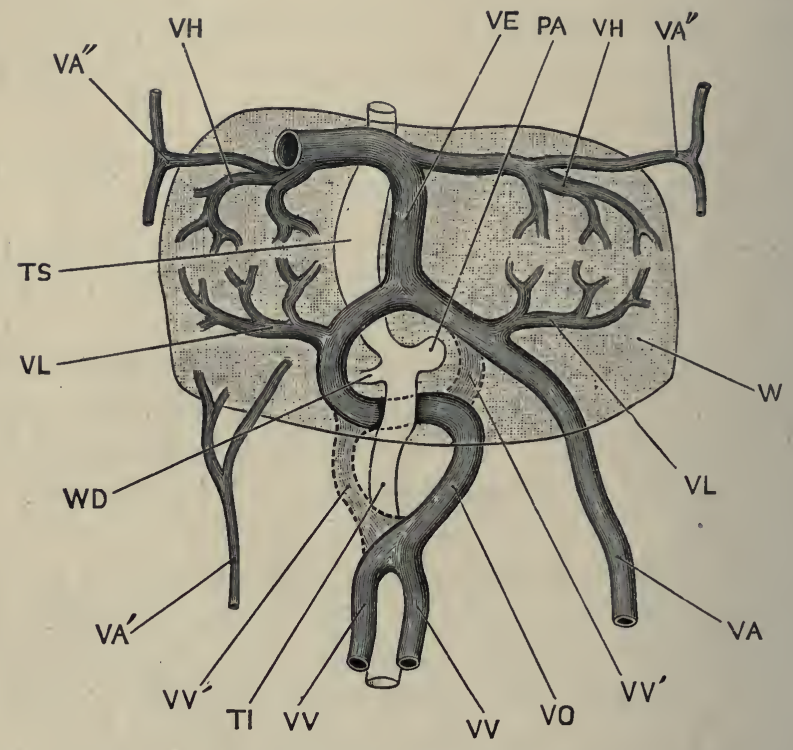

FIG. 247.-The liver and the veins in connection with it of a Human Embryo twenty-four or twenty-five days old, seen from the ventral surface. (From His.) PA, pancreas. TI, intestine. TS, stomach. VA, left allantoic vein. VA',
right allantoic vein. $\mathbf{V} \mathbf{A}^{\prime \prime}$, anterior detached portions of the allantoic veins. VE, ductus venosus, or vena Arantii. VH, efferent hepatic vessel. VI, afferent hepatic vessel. VO, hepatic portal vein. $\mathbf{V V}$, vitelline vein. VV', portions of the sinus annulares which disappear. $\mathbf{W}$, liver. $\mathbf{W} \mathbf{D}$, bile duct.

week, the right and left vitelline veins unite to form a single vein, which is joined, before it reaches the liver, by veins returning blood from the intestine, and which may from this time be spoken of as the hepatic portal vein.

Of the two sinus annulares, the left half of the anterior one, and the right half of the posterior one, disappear; the persistent portions. form a single vessel (Fig. 247, vo), which becomes the anterior part of the hepatic portal vein, and which, from 
the mode of its development, runs round the duodenum with the spiral course characteristic of the vein in the adult.

The allantoic veins are at first paired, but they soon fuse together at their hinder ends, within the allantoic stalk, to form a single vessel ; further forwards, within the embryo itself, they remain separate, running in the side walls of the body, close to the base of the amnion folds (Fig. 198).

During the fourth week, both allantoic veins lose their connection with the sinus venosus. The right allantoic vein (Fig. 247, vA'), which is now much the smaller of the two, breaks up into two sets of vessels : an anterior set, $\mathrm{vA}^{\prime \prime}$, which run in the body wall, and join the efferent hepatic vessels as these leave the liver; and a posterior set, $\mathrm{VA}^{\prime}$, which disappear at a slightly later stage.

'The left allantoic vein, va, which is much larger than the right, also divides into two sets of vessels : an anterior set, $\mathrm{vA}^{\prime \prime}$, which resemble those of the right side; and a large posterior vessel, va, which joins the anterior sinus annularis, or hepatic portal vein, just as this enters the liver substance.

The ductus venosus. At about the twenty-third day, both the vitelline and the allantoic vessels have lost their direct connections with the sinus venosus, and in order to reach the heart the blood in these vessels must traverse the liver capillaries. A direct communicating passage is now established between the portal vein, just before it enters the liver, and the right hepatic vein just before this reaches the sinus venosus. This communication (Fig. 247, vE) is the ductus venosus, sometimes called the vena ascendens or vena Arantii; it enlarges very rapidly, and affords a wide and direct path by which the blood from the placenta can reach the heart without passing through the liver capillaries.

In rabbit and chick embryos the ductus venosus is the persistent anterior part of the fused vitelline veins ; in man, according to Professor His, whose descriptions have been followed above, it is, as just described, an entirely new vessel.

The posterior vena cava is a very insignificant vein in the earlier stages. It is formed by the junction of the iliac veins, 
and does not appear until the hind-limbs have begun to become prominent. It joins the ductus venosus as this emerges from the liver.

The Cuvierian veins. Each Cuvierian vein (Fig. 198, v\&) is formed by the junction of an anterior and a posterior cardinal vein. The anterior cardinal vein persists as the external jugular vein, and is joined later on by the internal jugular and subclavian veins.

The posterior cardinal veins disappear, in the middle part of their course, on the replacement of the Wolffian bodies, with which they are specially related, by the permanent kidneys. The hinder ends of the veins become the internal iliac veins, and acquire connections with the allantoic veins. The anterior portion of the right posterior cardinal vein gives rise to the azygos vein.

The Cuvierian veins themselves run at first transversely; but, as the heart shifts backwards, their direction becomes at first oblique, and finally longitudinal.

The right Cuvierian vein persists as the anterior vena cava. The left Cuvierian vein undergoes important changes: up to the end of the second month it is as large as the right vein; but during the third month a communicating vessel is formed between the left and right Cuvierian veins, just behind the junction of the jugular and subclavian veins. Through this communicating branch, which is very large and has a somewhat oblique course, the blood from the left jugular and subclavian veins is carried across to the right Cuvierian vein, instead of returning to the heart as before by the left Cuvierian vein. The, left Cuvierian vein, having no longer any function to perform, shrinks up and becomes obliterated more or less completely. Portions may persist, either as fibrous cords, or as venous channels of greater or less size; and the posterior end, where it opens into the sinus venosus, is said to give rise to the coronary sinus.

The pulmonary veins appear late, about the end of the fifth week : they open into the left auricle, close to the interauricular septum. At first there is only a single opening into the auricle, but at a later stage, about the fourth month, there are two 
openings, and in slightly older fotuses all four openings are present; the change being apparently due to the opening out of the originally single orifice, and the absorption of the vein, as far as its tirst branches, into the wall of the auricle; much in the same way as the sinus venosus is opened out, and made part of the wall of the right auricle.

\section{The Course of the Circulation during the first Four Months of Gestation.}

In the early stages, up to the end of the first month, the blood brought back to the heart-whether from the body of the embryo itself, from the placenta, or from the yolk-sac-is poured into the sinus venosus, and thence, through a median slit-like aperture, into the single auricular cavity. Complete mixture of the streams from the several sources must necessarily occur, in both the sinus venosus and the auricle, and the blood driven out through the truncus arteriosus by the ventricle will be of a mixed character.

After the sinus venosus is taken into the heart, in the early part of the second month, there are for a time three separate openings into the right auricle: those of the right and left Cuvierian veins, and of the posterior vena cava respectively. The auricular septum is now partially formed, but there is still free communication between the two auricles through the foramen ovale. Of the three veins, the opening of the posterior vena cava lies nearest to the foramen ovale; and the Eustachian valve, a fold of the wall of the auricle along the right-hand side of the opening, tends to direct the blood from the posterior vena cava through the foramen ovale into the left auricle. The foramen ovale is at this stage a mere aperture in the auricular septum, not guarded by valves, so that a certain amount of direct mixture of the blood returned to the auricle by the different veins must of necessity take place.

During the third month, the transverse communication from the left to the right Cuvierian vein is being established; and by the end of the fourth month the left Cuvierian vein has practically disappeared, the whole of the blood from both sides of the head, and from both fore limbs, being returned by the right Cuvierian vein, or anterior vena cava as it may now be called. Neglecting the coronary sinus, which is comparatively 
insignificant, there are at this stage only two vessels returning blood to the right auricle: the anterior vena cava, which returns venous blood from both sides of the head, and from both fore limbs; and the posterior vena cava, which brings back blood, mainly arterial in character, from the placenta, and also from the hinder part of the body of the embryo, and from the yolk-sac.

During the fourth month the foramen ovale, which has hitherto been a free opening, becomes partially blocked up by a fold, which acts as a valve, allowing blood to pass from the right to the left auricle, but obstructing its return in the opposite direction.

The Eustachian valve becomes larger at the same time; and partly owing to its increased size, and partly to slight changes in the position and direction of the opening of the posterior vena cava, the whole of the blood returned by this latter vessel is now discharged through the foramen ovale into the left auricle.

\section{The Course of the Circulation during the Latter Half of Ges- tation.}

During the latter four months or so of gestation the course of the circulation is as follows :-

The right auricle receives blood from three sources-

(i) From the anterior vena cava.

(ii) From the coronary sinus.

(iii) From the posterior vena cava.

The anterior vena cava returns venous blood from both sides of the head, and from both fore-limbs.

The coronary sinus, which is the persistent terminal portion of the original left anterior vena cava, returns venous blood from the walls of the heart itself.

The posterior vena cava, which is much the largest of the three, returns blood: (a) from the linder part of the body, and especially the kidneys and the hind limbs; and (b) from the placenta, the intestine and the liver. The latter of these two streams requires further consideration.

Of the two allantoic veins, by which the blood was returned from the placenta in the earlier stages, the right one has disappeared. The left allantoic vein, which is very large, enters the body at the umbilicus, and runs forwards to the hinder border of the liver; here it is joined by the hepatic portal vein, 
which returns blood from the intestine, and is formed in part from the vitelline veins of the earlier stages.

On reaching the liver, the blood has two alternative routes open to it, by either of which it can reach the posterior vena cava. Part of the blood is conveyed by the afferent hepatic vessels into the substance of the liver, from which it is returned by the efferent hepatic vessels, or hepatic veins, to the posterior vena cava; the greater part, however, continues straight onwards through the wide ductus venosus, and so reaches the posterior vena cava without having traversed the liver.

The blood brought back to the heart by the posterior vena cava is thus derived very largely from the allantoic vein, and in part from the renal veins; it is therefore purer as regards gaseous constituents, and freedom from nitrogenous excretory matters, and is richer in nutrient matters, than the blood returned by the anterior vena cava; and the blood in the anterior and in the posterior venæ cavæ may consequently be contrasted as venous and arterial respectively.

The venous blood brought to the right auricle by the anterior vena cava passes, on the auricular contraction, into the right ventricle. From the ventricle it is driven along the pulmonary trunk (Fig. 246, RW); a small portion passes along the pulmonary arteries, AP, to the lungs, but as the lungs are in an unexpanded condition there is considerable resistance to the entrance of blood into the pulmonary vessels, and only an insignificant portion of the stream takes this path. Nearly the whole of the venous blood in the pulmonary trunk passes along the ductus arteriosus (Fig. 246, A.5) to the dorsal aorta, down which it courses to the bifurcation of the aorta into the two common iliac arteries; then down these latter, and partly along the external iliac arteries to the hind limbs, but mainly along the allantoic arteries to the placenta, where it gains nutrient matter and oxygen, and from which it is returned to the foetus by the allantoic vein.

The arterial blood brought to the right auricle by the posterior vena cava does not really enter the cavity of the right auricle, but is directed at once, by the Eustachian valve, through the foramen ovale into the left auricle, which also receives the very small quantity of blood returned from the lungs by the pulmonary veins. From the left auricle the blood passes into 
the left ventricle, and is thence driven along the systemic trunk (Fig. 246, Rx), and through the carotid and subclavian arteries to the head and fore-limbs.

It is probable that very little, if any, blood from the left. ventricle gets into the dorsal aorta, for this is already filled, through the ductus arteriosus, from the right ventricle; and as the two ventricles have at this stage walls of about equal thickness, and presumably of equal strength, there will be as strong a tendency for the blood of the right ventricle to pass forwards along the arch of the aorta, as for the blood from the left ventricle to pass backwards along the dorsal aorta.

Theoretically, the aorta might be ligatured just in front of the point at which the ductus arteriosus joins it, without in any way disturbing the foetal circulation; and instances of malformation have occurred, in which the aorta was completely obliterated at this place, and yet development in other respects. proceeded normally. Such a malformation, though causing no disturbance of the circulation so long as the fotus is receiving nourishment through the placenta, is fatal at the time of birth, as the arterial supply of the whole body behind the arms is then cut off.

\section{The Changes in the Circulation at the Time of Birth.}

At birth, the placental circulation is arrested, and the allantoic and vitelline vessels are interrupted; and, as the lungs become inflated, the pulmonary circulation is fully established.

In connection with this shifting of the seat of respiration, from the placenta to the lungs, important changes are effected in the circulation, the principal of which are:

(i) Shrinking and obliteration of the ductus arteriosus, and of the hypogastric, or allantoic, arteries.

(ii) Obliteration of the ductus renosus, and of the part of the allantoic vein within the body of the child.

(iii) Closure of the foramen ovale.

By these changes it is brought about that the blood in the posterior vena cava, which is now entirely venous, is no longer able to get into the left auricle, owing to closure of the foramen ovale, but passes, with that of the anterior vena cava, from the right auricle to the right ventricle. From the right ventricle, owing to the obliteration of the ductus arteriosus, it can no 
longer reach the aorta, but passes entirely along the pulmonary arteries to the lungs. From the lungs it is returned by the pulmonary veins, which are now greatly enlarged, to the left auricle, and so to the left ventricle, which drives it not only to the head and upper limbs, but also along the dorsal aorta to the hinder part of the body.

By obliteration of the ductus venosus, all the blood in the hepatic portal vein is compelled to pass through the capillaries of the liver in order to reach the posterior vena cava. In other words, by these three changes-obliteration of the ductus arteriosus, obliteration of the ductus venosus, and closure of the foramen ovale-the foetal circulation has been converted into that of the adult.

These changes do not occur immediately on birth, nor are they effected simultaneously.

Obliteration of the allantoic or hypogastric arteries occurs first; it is effected partly by contraction of the entire vessels, but chiefly by thickening of their inner coats, and is usually completed by the third or fourth day after birth.

The allantoic veins and the ductus venosus remain open rather longer, but are generally obliterated by the sixth or seventh day.

The ductus arteriosus, according to Allen Thomson, ' is rarely found open after the eighth or tenth day, and by three weeks it has, in almost all instances, become completely impervious.'

Closure of the foramen ovale is the last of the changes to be completed. The closure is at first effected merely by the valve, which projects into the left auricle, being kept closely applied to the margin of the aperture by pressure of the increased quantity of blood now returning by the pulmonary veins. At a later stage the edge of the valve gradually coalesces with the margin of the opening, but the union-often remains incomplete for some months; and it not unfrequently happens that an oblique valvular aperture, large enough to admit a probe, persists for the first year of infancy, and may even be permanent throughout life, in which case a direct passage of venous blood into the left auricle is liable to occur, especially on overexertion. 


\section{DEVELOPMENT OF THE URINARY ORGANS.}

The general history of development of the urinary organs in man is the same as in the rabbit. Paired Wolffian ducts and Wolffian bodies appear first; these form the excretory organs of the early stages, and attain a considerable size during the second month, after which time they gradually shrink, ultimately losing their excretory function, and becoming modified to form accessory parts of the reproductive system.

The permanent or adult kidneys arise, as in the rabbit, as outgrowths from the hinder ends of the Wolffian ducts: from the third month onwards they replace the Wolffian bodies as the functional excretory organs.

A pair of Müllerian ducts is formed, independently of the Wolffian ducts, and in the female becomes modified to form the oviducts, uterus, and vagina. The head-kidney, if present at all, is in a very rudimentary and evanescent condition.

\section{The Wolffian Duct and Wolffian Body.}

According to Kollmann, the Wolffian ducts appear, about the fourteenth day, as a pair of longitudinal grooves of the external epiblast, just below the level of the myotomes (Fig. 248, Kc). By the middle of the third week the ducts are tubular, and lie embedded in the mesoblast of the intermediate cell mass. It is not yet certain, however, whether the tubular duct is formed by closure of the lips of the groove, or by splitting off of a rod of cells from the thickened floor of the groove, which subsequently acquires a lumen, and becomes tubular: while the observations recorded in the case of rabbit embryos render it possible that the origin of the Wolffian duct from the epiblast may prove to be apparent rather than real ( $c f$. p. 421).

The Wolffian ducts at first end blindly behind, but about the end of the third week or beginning of the fourth week they grow back to the cloaca, and open into its sides (Fig. 243, KC).

The Wolffian bodies appear about the eighteenth day as a pair of longitudinal ridge-like thickenings of the dorsal wall of the body cavity, one on each side of the mesentery. These soon becorne more prominent, and by the beginning of the fourth week extend from about the sixth cervical to the last lumbar somite. 
Each Wolffian body consists at first of rods of cells, which appear to arise independently of the Wolffian duct. The rods soon become S-shaped: early in the fourth week they acquire axial cavities, and so become tubes; and by the end of the week the tubes, or Wolffian tubules as they may now be termed, grow towards the Wolffian duct and open into it. The opposite, or

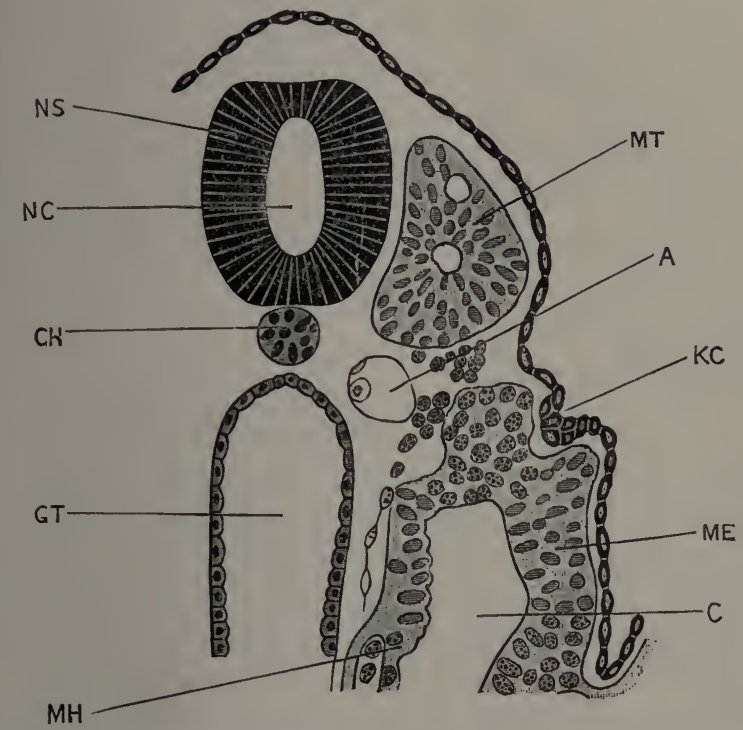

FiG. 248.-Transverse section across the body of a Human Embryo, estimated as fourteen days old. For a figure of the whole embryo, see Fig. 185, p. 481. The embryo had thirteen pairs of mesoblastic somites, and the section figured passes through the tenth pair. (From Kollmann.) $\times 240$.

A, aorta. C, cœlom. CH, notochord. GT, mid-gut. KC, Wolffian duct. $\mathbf{M E}$, somatopleuric layer of mesoblast. MH, splanchnopleuric layer of mesoblast. IMT, myotome or mesoblastic somite. NC, central canal of spinal cord. NS, spinal cord.

closed ends of the tubules become dilated, and then invaginated to form Malpighian bodies, the glomeruli being derived from branches of the aorta which penetrate into the Wolffian body along its whole length; while the veins open into the largeposterior cardinal veins, which are intimately associated with the Wolffian bodies from their first appearance.

The Malpighian bodies are more abundant along the inner side of each Wolffian body, while the duct lies along its outerborder, except at the hinder end, where it crosses to the inner side.

During the second month the Wolffian bodies grow rapidly: 
the Malpighian bodies increase greatly, both in number and in size; and new Wolffian'tubules are formed, apparently by budding from the old ones. In each tubule the part next the Malpighian body, which is probably the secreting portion, has thicker walls, formed of larger epithelial cells, than the more distal part which opens into the Wolffian duct.

The Wolffian body reaches its greatest development about the eighth week, from which time it slowly diminishes in size. Degeneration commences, and proceeds more actively at the anterior end of the Wolffian body, which from the first has lagged behind the rest of the organ in development. Ultimately the whole structure becomes affected; by the fifth month the Malpighian bodies have almost entirely disappeared, and in the end the Wolffian body becomes reduced to an accessory part of the reproductive apparatus.

\section{The Kidney and Ureter.}

The ureter arises on each side as a diverticulum from the hinder end of the Wolffian duct, in the early part of the fourth week (Fig. 243, KD). This soon acquires an independent opening into the cloaca, a little way behind that of the Wolffian duct (Figs. 216, KD, and 233, N). At its opposite or blind end the ureter grows forwards, between the hinder end of the Wolffian body and the vertebræ. It dilates to form a somewhat elongated sac, which is the pelvis of the future kidney; and from this sac branching tubular diverticula grow out (Fig. 231, s), and become the urinary tubules. These rapidly increase in number and in length; Malpighian bodies are formed in connection with their distal ends, and the kidney structure is definitely acquired by the end of the second month, at which time the degeneration of the Wolffian body commences.

The bladder is formed by dilatation of the basal or proximal part of the allantois. Beyond the bladder the allantoic stalk loses its cavity and becomes a solid rod, the urachus, leading from the bladder to the umbilicus. The lumen usually disappears early in the fifth week, but it may persist for a much longer time, or even be present in the adult.

\section{The Muillerian Duct.}

About the end of the fourth week, a longitudinal ridge-like thickening of the peritoneum appears along the outer side of each 
of the Wolffian bodies. The ridge lies close to the Wolffian duct, and extends along its whole length, but is quite independent of this.

Early in the fifth week, the Müllerian duct is formed in this ridge; it is a narrow straight tube, lying along the outer side of the Wolffian duct, but distinct from this. Its anterior end opens into the body cavity by an elongated slit-like mouth, situated in a patch of thickened peritoneal epithelium, a little way in front of the anterior end of the Wolffian body. Posteriorly, the Müllerian duct ends blindly.

By the eighth week the Müllerian duct has undergone some changes. It commences in front with a wide funnel-like mouth, the margins of which are already slightly fimbriated. Behind this mouth, the duct runs straight backwards for some distance, along the outer side of the Wolffian body, then turns sharply inwards, crosses ventral to the Wolffian duct, and continues backwards in close contact with the Müllerian duct of the opposite side ; it still ends blindly behind.

In the male, the Müllerian ducts begin to atrophy shortly after reaching this stage. In the female, they undergo further development, and give rise to the oviducts, uterus, and vagina, as will be described in the section dealing with the accessory organs of reproduction.

\section{The Head-kidney.}

Janosik has described, in an embryo eighteen to nineteen days old, what he thinks may prove to be a rudimentary pronephros, in the form of a couple of peritoneal funnels just in front of the anterior end of the Wolffian duct; the anterior funnel having close to it a structure not unlike an external glomerulus. The early development and subsequent fate of these structures have not yet been determined.

\section{THE DEVELOPMENT OF THE REPRODUCTIVE ORGANS.}

\section{The Essential Reproductive Organs.}

These have already been described, in the introductory portion of this chapter (pp. 4.49 to 457 ); but a few further details may conveniently be added here.

In embryos thirty-two days old ( $c f$. Fig. 205), the genital 
ridges are present as a pair of bands of epithelium, many cells thick, and lying along the inner sides of the Wolffian bodies. Primitive ova are already present, and, according to Nagel, are found not only in the genital ridges themselves, but also beyond their limits, and especially in the thickened epithelium in the neighbourhood of the Müllerian ducts. This may perhaps be taken as an indication that the genital epithelium was originally less sharply circumscribed than at present.

Nagel has shown that distinct differences may be detected in the genital ridges of the two sexes from as early a period as thirty-two days; and he is inclined to doubt whether there is absolute identity at any time, even in the earliest stages.

In the male, the genital ridge, at thirty-three days, shows a fairly definite arrangement of the cells in strings; these form a network of tortuous anastomosing cords, arranged somewhat regularly, and bound together by connective tissue. Embedded in the cellular cords are the primitive sperm cells. These are comparatively few in number; their formation ceases at an early stage, in embryos of about six or seven weeks, on the completion of the tunica albuginea; but in the later stages, although no new primitive sperm cells are formed from the germinal epithelium, those which are already present increase freely by division. The cellular cords themselves become converted into the seminal canals, which are thus derived directly from the germinal epithelium.

In the female, the primitive ova, in embryos of thirty-three days, are much more numerous than the primitive sperm cells of the male. They are found in various phases of development, and the formation of new primitive ova continues until about the close of gestation. It is very doubtful whether any new primitive ova are formed after birth, and by some authorities their formation is believed to stop about the seventh month. The tendency for the smaller cells to become grouped around the primitive ova, and so form follicles, is evident even in the fifth week, and affords a good clue by which a young ovary may be distinguished from a young testis, and the sex of the embryo thus determined.

\section{The Accessory Reproductive Organs.}

As in the rabbit, the chick, and indeed the great majority of Vertebrates, the genital ducts of the human embryo are formed 
from tubes which originally belong to the excretory system; the oviducts being formed from the Müllerian ducts, and the vasa deferentia of the male from the Wolffian ducts; while other portions of the embryonic excretory apparatus persist in a modified $\mathrm{or}$ vestigial form, as accessory organs in relation with the reproductive system.

\section{a. In the Male.}

The Miillerian ducts begin to atrophy about the middle of the third month, and ultimately disappear completely along the greater part of their length. The anterior end of the Müllerian duct may persist, and in connection with it the hydatids of Morgagni are believed to be formed; this name being given to one or more small pedunculated bodies, lying between the testis and the head of the epididymis. One of these bodies is of larger size, and more constant occurrence, than the others.

It is stated that the posterior ends of the Muillerian ducts unite together, and give rise to the uterus masculinus, a small pocket-like diverticulum from the dorsal wall of the prostatic portion of the urethra, a quarter to half an inch in depth, and bearing on its margins the slit-like openings of the vasa deferentia. The statement, however, needs confirmation.

The Wolffian body and Wolffian duct. The greater part of the Wolffian body disappears, but the anterior end becomes intimately connected with the testis, and persists throughout life. From the Wolffian tubules of this anterior end tubular outgrowths arise, which during the fourth month grow into the substance of the testis, and give rise to the vasa efferentia; these soon become connected with the seminal tubes, which latter, according to Nagel, are formed directly from the germinal epithelium. The anterior Wolffian tubules become the coni vasculosi; and the Wolffian duct is converted, in front, into the extremely tortuous epididymis, and further back into the vas deferens.

The structures known as the vasa aberrantia, a series of tortuous tubular diverticula from the lower end of the epididymis; and the parepididymis, or organ of Giraldès, are probably persistent portions of some of the hinder Wolffian tubules.

\section{b. In the Female.}

The Muillerian ducts, at the beginning of the third month, are still quite distinct from each other. Their anterior ends, 
with the abdominal openings, are widely separate; their posterior portions lie side by side, between and slightly dorsal to the Wolffian ducts, and bound up with these by connective tissue, to form what is spoken of as the genital cord. The Müllerian ducts still end blindly behind.

Towards the end of the third month, the two Müllerian ducts fuse together, opposite the middle third of the genital cord ; and from this point the fusion extends rather rapidly forwards, and much more slowly backwards. The fused portion, or uterovaginal canal, enlarges steadily, especially in its transverse diameter. By the beginning of the fourth month, a distinction appears between the uterine and vaginal portions of the canal; the proximal portion, or uterus, being lined by a columnar epithelium, and the distal portion, or vagina, by a squamous epithelium.

During the fourth month, the boundary line between the uterus and vagina becomes a much sharper one. The uterus becomes considerably dilated : the vagina, on the other hand, is flattened dorso-ventrally; and, by proliferation of its epithelial cells, its lumen becomes completely blocked up for a time, reappearing in the course of the fifth month.

The two Müllerian ducts thus give rise to the whole length of the female passages; the anterior or proximal ends of the ducts remaining distinct from each other, and forming the oviducts or Fallopian tubes; while the posterior or distal portions fuse together, and give rise to the uterus and vagina.

The fusion of the two halves of the uterus is not completed until the latter part of the fourth month; and the occasional retention of a more or less complete uterine septum, even in the adult, is due to imperfect fusion of the two originally distinct ducts.

The cervix uteri is established during the fifth month, at the time when the lumen of the vagina is reappearing. The folds of the wall of the cervix, spoken of as the arbor vitæ, appear during the fourth month; while the differentiation of the muscular walls, and of the enormously developed muscularis mucosæ commences in the sixth month. The uterine epithelium is devoid of cilia during the whole of foetal life ; and up to the time of birth there are no glands in the body of the uterus. Glands are, however, present in the cervix, and apparently 
secrete the plug of mucus which commonly occupies the os uteri at the time of birth.

The Wolffian body. In the fernale, outgrowths from the anterior. Wolffian tubules into the ovary occur, similar to those which in the male give rise to the vasa efferentia; but they do not give rise to any adult structure.

A number of the Wolffian tubules of the anterior end of the Wolffian body persist throughout life, forming the structure known as the parovarium (Fig. 249, a), sometimes called the

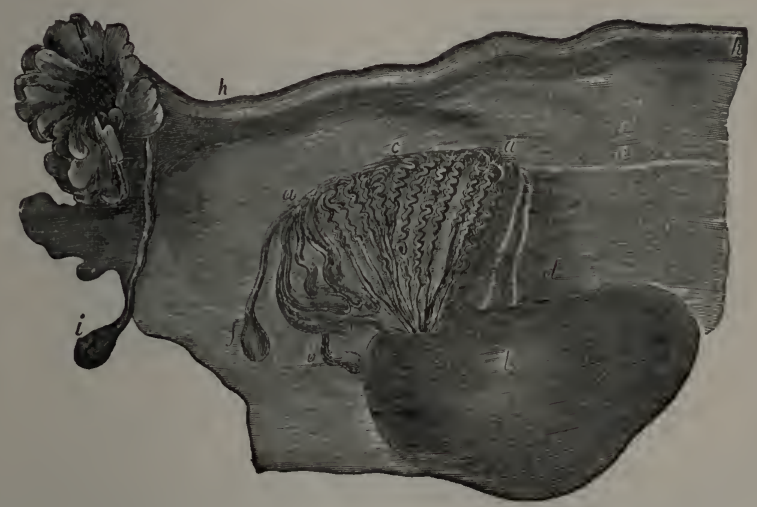

FIG. 249.- The adult Ovary, Parovarium, and Fallopian tube. From Quain 'Anatomy.' (After Kobelt.)

a. $a$, parovarium, epoophoron, or organ of Rosenmiller ; formed from the anterior end of the Wolffian body. $b$, remains of some of the anterior Wolffian tubules, sometimes forming hydatids. $c$, the longitudinal duct of the parovarinm, formed from the anterior end of the Wolffian duct. d, rudimentary Wolffian tubules. e, atrophied remains of the Wolffian duct, or duct of Gaertner. $f$, the terminal bulb or hydatid. $h$, Fallopian tube. $i$, hydatid attacher to the end of the Fallopian tube. $l$, ovary.

epoophoron or organ of Rosenmüller; a series of transverse tubes which run, with a somewhat tortuous course, in the fold of peritoneum between the ovary and the Fallopian tube, and are connected with the anterior end of the ovary.

A small portion of the hinder part of the Wolffian body may persist as a rudimentary structure, the paroophoron, lying in the peritoneum opposite the hinder end of the ovary.

The Wolffian duct persists, in front, as the longitudinal duct of the parovarium (Fig. 249, c), into which the transverse tubules, $a$, open, and which corresponds to the epididymis of the male. The hinder part of the Wolffian duct usually disappears, but it may persist along a greater or less portion of its length as 
the duct of Gaertner, running alongside the Fallopian tube (Fig. $249, e)$, and sometimes extending along the walls of the uterus, or even as far as the vagina.

\section{The External Genital Organs.}

These are practically identical in the two sexes, in the early stages of their development; the distinction between male and female, as regards the external genital organs, not being evident until about the ninth or tenth week.

At the end of the fifth week (Fig. 234), the septum dividing the rectum from the urino-genital passage has almost reached the surface, but the two passages apparently open by a single cloacal aperture. Immediately in front of this aperture is a

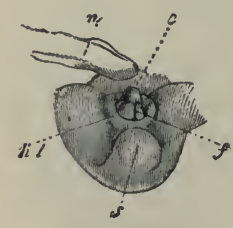

FIG. 250.

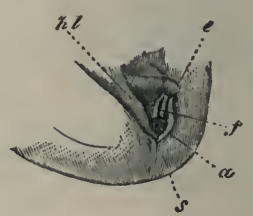

FIG. 251.

FIG. 250.--The external genitalia of a Human Embryo of about the ninth week (probably rather younger). (From Kölliker, after Ecker.) $\times 2$.

$c$, genital tubercle, or elitoro-penis. $f$, groove, continuous with urino-genital passage. $h l$, labio-scrotal folds. $n$, umbilical cord. $s$, coceygeal region.

FIG. 251.-The external genitalia of a Human Embryo of about the tenth week. (From Kölliker, after Ecker.) $\times 2$.

a, anus. $e$, genital tubercle, or clitoro-penis. $f$, genital groove, continuous with urino-genital aperture. $h l$, labio-scrotal folds. $s$, coccygeal region.

small conical projection, $s g$, the genital tubercle or clitoro-penis. The posterior surface of this tubercle is marked by a longitudinal groove, which leads, through the cloacal aperture, into the urino-genital passage; the lips of the groove are slightly swollen, and are continuous with the lips of the cloacal opening, which form the inner sexual folds. The tip of the genital tubercle is expanded into a small knob, the glans.

A little later, towards the end of the second month, the septum between the urino-genital passage and the rectum reaches the surface, dividing the cloacal aperture into two separate openings, an anterior or urino-genital (Fig. 251, $f$ ), and a posterior or anal (Fig. 251, a).

Up to this time the course of development is practically the 
same in all embryos, but from about the tenth week differences become apparent between the two sexes.

In the male, the genital tubercle elongates, and forms the penis. The lips of the groove, along the posterior surface of the tubercle, meet and fuse to form the canal of the penis, or penial urethra; and, by a similar fusion of the lips of the urino-genital opening, the penial urethra and urino-genital passage become directly continuous with each other. The glans penis is at first solid, but towards the end of the third month the groove extends forwards along it, and, gradually closing from behind forwards, carries the opening of the urethral canal to the apex of the glans. The prepuce appears, towards the end of the third

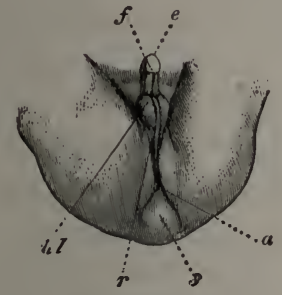

FIG. 252.

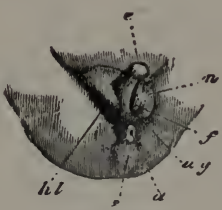

FIT. 253.

FIG. 252.--The external genitalia of a male Human Embryo towards the end of the third month. (From Kölliker, after Eicker.)

$a$, anns. e, penis. $f$, raphé formerl by union of lips of genital groove. $h l$, scrotum. $r$, aphe formed by union of the two lialves of the scrotnm. $s$, cuccyx.

FIG. 253.-The external genitalia of a female Human Embryo towards the end of the third month. (From Kölliker, after Ecker.)

$a$, anns. e, clitoris. $f$, genital gronve. $u g$, nrino-genital aperture. $h l$, labia majora. $n$, labia minora. or iuner genital folds. $s$, coceygeal region.

month, as a fold of skin round the base of the glans, and is at first interrupted ventrally by the urethral groove.

The scrotum is formed from a pair of folds of skin, the labioscrotal or outer genital folds, which arise at the sides of the urino-genital opening, and ultimately unite with each other in the median plane behind the penis (Fig. 252, $h l$ ).

In the female the genital tubercle remains small and becomes the clitoris (Fig. 253,e); and the genital groove remains open. The inner genital folds (Fig. 2ऽ3, $n$ ), at the sides of the urinogenital opening, become the labia minora or nymphæ; while the outer genital, or labio-scrotal folds, $h l$, become the labia majora, and, in front, the mons Veneris. 
The urino-genital canal shortens considerably in the female, so as to bring the aperture of the urethra close to the surface.

The above changes are usually completed in both sexes by the end of the third month; but they may be delayed until a much later date.

\section{THE FETAL MEMBRANES AND THE PLACEN'TA.}

1. The amnion is the thin transparent membrane which invests the embryo like a sac, covering its dorsal surface and sides (cf. Fig. 197, Av) - p. 489 .

The mode of formation of the amnion in the human embryo has not yet been determined. In Reichert's ovum, estimated to be twelve or thirteen days old, there was no trace of an $($ f 472 ) amnion present (Figs. 172 and 173); while in the embryos ( $k$ 477 $\mathrm{E}$ and SR (Figs. 176, 178, 179), which are believed to be of the thirteenth day, the amnion is already fully formed. Figs. 186 h. 484 to 188 show the mode in which the development of the amnion is believed to occur, by growth backwards of a fold of the wall of the blastodermic vesicle over the embryo; but the figures are purely hypothetical, and the intermediate stages which they represent have not been seen.

Of the two layers of which the amnion consists, the outer one ( $c f$. Fig. 188) is simply a part of the wall of the blastodermic vesicle, and it is usual to limit the term amnion to the inner layer, which more immediately invests the embryo. The space between this inner layer, or amnion, and the embryo is spoken of as the amnionic cavity, and is filled with fluid.

The rate of growth of the amnion, as compared with that of the embryo itself, varies considerably at different periods of development. On its first formation, about the thirteenth day, the amnion invests the embryo fairly closely (Fig. 179). During the third week the amnion grows rather more rapidly, so that the space between it and the embryo enlarges somewhat (Fig. 196). During the fourth week, as at the corresponding stages in the rabbit or chick, the embryo grows considerably, and at the end of the week the amnion invests it very closely.

During the second month the amnion enlarges much more rapidly, and the amnionic cavity becomes a space of considerable size, filled by the liquor amnii (Fig. 254). ' Owing to this 
increase in its dimensions, the amnion forms a sheath around the umbilical cord, and also comes into close contact with the wall of the blastodermic vesicle over the whole extent of its inner surface.

The liquor amnii, which occupies the amnionic cavity, between the amnion and the embryo, varies much in quantity at different periods of gestation. It is apparently most abundant about the fifth or sixth month. Its actual quantity is difficult to fix, as it varies greatly in different cases; when in excess, i.e. more than about $1 \frac{1}{2}$ litre, it constitutes the affection known as hydrops amnii.

The liquor amnii contains urea, especially during the later months of gestation; this appears to be a true excretory product, separated by the kidneys of the fotus, and discharged through the urino-genital aperture into the amnionic cavity.

Structurally, the human amnion consists, like that of the rabbit or chick, of a single layer of epiblast cells, supported on a thin layer of mesoblast ( $c f$. Figs. 182 to 184). The mesoblast consists of a homogeneous matrix, with embedded cells; while the epiblastic epithelium is, according to Minot, noteworthy on account of the distinctness with which the intercellular bridges of protoplasm, connecting the several cells with one another, can be made out; the boundaries between adjacent cells being formed, not by divisional planes, but by lines of vacuoles, between which the protoplasmic bodies of the cells are directly continuous with one another.

\section{The Umbilical Cord.}

The umbilical cord, which connects the embryo with the placenta (Fig. 254 ), is formed in the first instance by the allantoic stalk (Fig. 179, 197, Tz). This stalk, in the human embryo, is directly continuous, from the first, with both the embryo and the wall of the blastodermic vesicle (Figs. 186 to 188), and has the appearance of a direct prolongation backwards of the hinder end of the embryo. On the formation of the tail, the allantoic stalk is gradually driven down to the ventral surface of the embryo ( $c f$. Figs. 179, 197, 198, TL and TZ), and acquires the position and relations characteristic of it in rabbit or chick embryos at corresponding periods.

The chief purpose of the allantoic stalk is to afford a path, 
along which the allantoic vessels can pass from the embryo to the placenta (Fig. 198, AA, va) ; and the explanation of the pre-

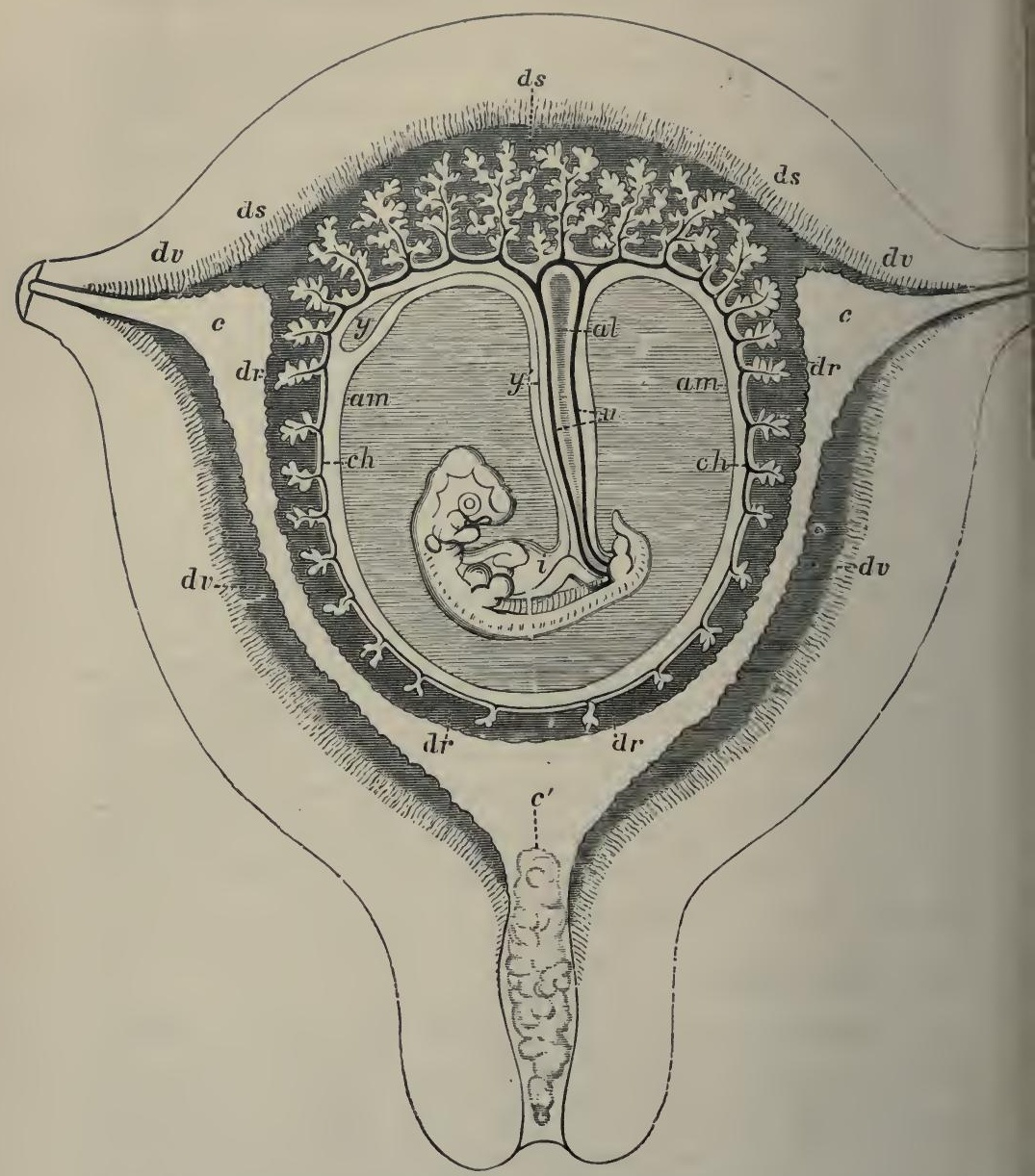

FIG. 254.-A diagrammatic section of the pregnant Human Uterus at the seventh or eighth week. (From Quain's 'Anatomy,' after Allen Thomson.)

al, allantoic stalk. am, true amnion; the part sharled horizontally, between the amnion and the embryo, is the amnionic cavity. $c$, cavity of the uterus. $c^{\prime}$, plug of mucus in the cervix uteri. ch, chorion. $d r$, decidua reflexa. ds, decidua serotina. dv, decidua vera. $i$, intestine of embryo, $u$, allantoic arteries. $y$, yolk-sac. $y^{\prime}$, yolk-stalk.

cocious appearance of the allantoic stalk, and probably also of the peculiarities of its development, in human embryos, is to be found in the importance of establishing vascular relations 
between the embryo and the mother at as early a period as possible.

In the later stages of development, the yolk-stalk, or pedicle of the yolk-sac (Fig. 254, $y^{\prime}$ ), becomes closely applied to the allantoic stalk, and bound up with it in a sheath formed by the spreading amnion, am; and it is to the compound structure formed of these elements that the name umbilical cord is given.

The umbilical cord increases considerably in length during development. About the middle of gestation it is usually from 13 to $21 \mathrm{~cm}$. long, and from 9 to $11 \mathrm{~mm}$. thick. At the time of birth, its average length is from 48 to $60 \mathrm{~cm}$., and its thickness 11 to $13 \mathrm{~mm}$.; but it is liable to very great individual variations. It may be as short as $12 \mathrm{~cm}$.; or, on the other hand, may attain a length of $167 \mathrm{~cm}$.

The umbilical cord is almost invariably twisted spirally on itself, and the cause of this twisting, which commences about the middle of the second month, has been the subject of much discussion. If examined more closely, it is found that all the constituents of the cord are not twisted to the same extent; the spirals described by the allantoic arteries being always more numerous, and closer together, than those of the whole cord, or than those of the veins round which the arteries appear to twist. The twisting appears to be due to the allantoic arteries increasing in length more rapidly than the other constituents of the cord, and so being compelled to adopt a tortuous instead of a straight course. The allantoic arteries may describe as many as thirty or forty complete turns in passing from the fotus to the placenta.

As the spiral growth involves the whole umbilical cord, and this cord is fixed at its placental end, it is clear that, as the cord twists, the embryo must rotate in the liquor amnii. 'The cord may become twisted round the neck of the fotus, and may even be tied in knots: these knots being produced by the cord, at an early stage of development, becoming thrown into a loop, and the embryo then floating through the loop.

Structure of the umbilical cord. The fully developed umbilical cord consists of the following structures (Fig. 254) :-

1. The sheath formed around it by the amnion. This invests the cord very closely, except at its insertion into the placenta. 
2. The right and left allantoic arteries, $u$. These are usually quite distinct from each other along the greater part of the length of the cord, but just before reaching the placenta are almost invariably united by an anastomotic branch.

3. The allantoic vein. This has thinner walls than the arteries, and has also, according to Kölliker, rudimentary valves. There are at first two allantoic veins, but the right one is, almost from the first, smaller than the left, and disappears completely about the fourth week.

4. 'The epithelial lining' of the allantoic cavity. During the first and early part of the second month, the allantoic stalk is hollow, its cavity extending from the cloaca of the fotus along the whole length of the cord, as far as the wall of the uterus. Later on, in the third or fourth month, the cavity becomes constricted, or altogether obliterated. Isolated portions of it may, however, persist, especially at the proximal or fœtal end of the cord, up to the time of birth.

5. The yolk-stalk and its vessels, the vitelline arteries and veins. These usually disappear during development, and are seldom to be distinguished in the cord at full time. The yolkstalk at first lies in a groove in the allantoic stalk; but it soon becomes completely surrounded by this latter, and then ceases to be distinguishable.

6. The Whartonian jelly: this forms the matrix of the cord, in which are embedded the various structures described above. It consists of a complex network of branching connective-tissue cells, embedded in a clear gelatinous matrix. Immediately beneath the surface epithelium, and around the blood-vessels and the allantoic cavity, the connective-tissue meshwork is rather denser than elsewhere; and, in the matrix, fibres are developed, especially during the later months of gestation.

7. Up to the close of the third month, the end of the umbilical cord next the embryo contains, as already noticed, a locp of the intestine (Fig. 254, i), but after this date the alimentary canal is, as a rule, completely withdrawn into the body of the fotus.

\section{The Chorion.}

The term chorion has been used in very different senses by writers on embryology. It is convenient to employ it for that part of the blastoderm, or blastodermic vesicle, which is not direct $]_{j}$ 
concerned in the formation of the embryo. It is usual to exclude the amnion from the definition; but in the case of the human embryo the outer layer of the amnion, or 'false amnion' as it is commonly called in other Vertebrates, is so directly continuous with the wall of the vesicle that it is better to include it under the same name.

'Thus in Reichert's ovum (Fig. 174) the chorion is the whole wall of the vesicle, except the embryonic area, $a$. In His' embryo E (Fig. 188), the chorion forms the entire wall of the vesicle, the embryo being now depressed within its cavity.

It is the chorion which comes in contact with the walls of the uterus (Figs. 254, 255), and it is from the chorion that the foetal part of the placenta is developed.

The human chorion is remarkable for its very early and complete separation from the yolk-sac (Figs. 186 to 188); and also for the very early period at which villi are developed from its outer surface.

Structurally, the chorion consists of an outer layer of epiblast, which from the first is two cells thick; and an inner and thicker layer of mesoblast, which very early becomes vascular, the bloodvessels being derived from the allantoic arteries and veins, which reach the chorion along the allantoic stalk, and which are, of course, directly continuous with the blood-vessels of the embryo.

In Reichert's ovum (Figs. 172, 173, 174), the villi are confined to a broad marginal zone round the equator, the centres of the two flattened surfaces forming bare patches. At a very slightly later period, in His' embryo $\mathrm{E}$, and in others of about the thirteenth day (Figs. 175, 188), the villi cover the entire surface of the chorion.

The chorionic villi consist at first entirely of epiblast. They arise as solid buds of epiblast, which become hollow as they increase in size; and at a later stage the mesoblast grows into them along their axes, carrying the blood-vessels with it. During the fourth week the villi grow actively; they branch freely, and in a very irregular manner. They penetrate the decidua, or modified mucous membrane of the uterus, to a slight depth; but do not, as was formerly believed to be the case, grow into the uterine glands. They become attached to the decidua at their tips, but remain free along the rest of their length. As in their 
first appearance, so also during the later stages of their growth, the epithelial layer, or epiblast, is always in advance of the mesoblastic connective-tissue core; the villi presenting lateral processes, or knobs, caused by local thickenings of the epithelium, into which at a later stage the vascular connective tissue penetrates.

The villi are at first of uniform size over the whole surface of the chorion (Fig. 188); but towards the end of the second month, or early in the third, they begin to develop unequally. Opposite the decidua serotina, or part of the uterine wall to which the ovum is directly attached (Fig. ${ }^{6.60} 254, d s$ ), the villi increase greatly in size and in complexity, forming ultimately the fotal part of the placenta. Over the rest of the surface of the chorion, opposite the decidua reflexa, $d r$, the villi, on the contrary, begin to shrink; the blood-vessels which supply them undergoing at the same time a gradual diminution in size.

In this way a distinction is established between the chorion frondosum, opposite the decidua serotina, which is very vascular, and beset with closely placed and richly branched villi; and the chorion læve, opposite the decidua reflexa, which is a thin transparent membrane, with no blood-vessels, and connected with the decidua reflexa merely by a few scattered, slightly branched, and inconspicuous villi. By the end of the fourth month, the villi of the chorion læve have almost completely disappeared, except from a narrow fringe round the margin of the placenta, where they persist until the close of gestation.

Up to the end of the third month, the villi can be fairly readily withdrawn from the crypts of the decidua in which they are lodged, and the foetal and maternal structures thus separated from each other ; but, after the placenta is definitely established, the connection between the fotal and maternal elements becomes so intimate that complete separation is no longer practicable.

The epithelium of the chorion frondosum undergoes important changes during the later months of gestation. Of the two layers of cells of which it consists from the first, the inner or deeper layer becomes thickened in irregular patches, very variable in number and in size; the individual cells are also very irregular, and show signs of degenerative changes. The outer, or surface layer of epithelium undergoes more extensive changes. The cell boundaries become lost, and the cell bodies 
run together to form a dense stratum, in which the nuclei remain visible for a time; ultimately the nuclei disappear, and the whole layer becomes modified into a hyaline, very refractive substance, permeated by numerous channels, so as to present a reticular appearance, and absorbing staining reagents very readily. This substance, formed by degeneration of the surface epithelial cells of the chorion, has been described, before its epithelial origin was known, as canalised fibrin.

Over the villi, the deeper or cellular layer of the epithelium disappears in great part, persisting only in isolated patches. The surface cells become converted in great part into a fibrin layer, similar to that of the chorion frondosum itself.

In the chorion lreve the epithelium retains its cellular character, and no fibrin layer is formed.

\section{The Decidua.}

The decidua is the mucous membrane of the pregnant uterus. The early stages in its formation are, so far as they are known, identical with those by which the catamenial or menstrual decidua is formed. The mucous membrane becomes thicker and more pulpy than in its quiescent condition; the blood-vessels enlarge; the glands elongate, and their deeper ends become tortuous and dilated; the deeper part of the mucosa becomes crowded with modified, and apparently proliferating connective-tissue cells; and the surface epithelium, lining the uterus, together with the immediately underlying connective tissue, show a tendency to disintegrate.

Up to this point, the formation of the catamenial decidua and of the decidua of pregnancy appear to be identical; the sole difference between the two is that, in the former, the processes having reached a certain point, stop and then become retrogressive, the decidua being broken up and discharged, together with a certain amount of blood, as the menstrual fluid; while, on the other hand, in the case of the decidua of pregnancy, development, after reaching the point mentioned, does not stop, but proceeds to further stages of elaboration.

The difference between the two courses seems to depend solely on the presence of a fertilised ovum within the uterus in the latter case, and on the absence of such an ovum in the former; so that the catamenial decidua may be viewed as a 
preparation on the part of the uterus for an ovum which never reaches it; the decidua, after waiting a certain time, becoming broken up and discharged. If, however, impregnation is effected, and a fertilised ovum reaches the uterus, a new stimulus is set up, and the developmental processes, instead of stopping, go on to further stages, and so give rise to the decidua of pregnancy.

Prior to the arrival of the ovum in the uterus, the decidua forms a complete lining to the uterus It does not cover the orifices of the Fallopian tubes (Fig. 254 ), which remain open throughout the greater part or the whole of pregnancy; neither does it extend into the cervix uteri, but stops abruptly at the os internum. With these exceptions, the decidua forms a layer, of approximately uniform thickness and structure, covering all parts of the uterine wall.

It seems to be, to a great extent, a matter of chance with what part of the uterus the ovum will come in contact, on entering its cavity; and it is therefore important that all parts of the surface should be equally prepared to receive it. In the great majority of cases, the attachment of the ovum is in the neighbourhood of the fundus, usually rather to one side of the median line, and more frequently on the dorsal than the ventral surface. It may, however, be situated in almost any part of the uterus; and its position may become a point of much practical importance. Ercolani has suggested that the ovum, on entering the uterus, is prevented from at once sinking to the cervix, by the fluid secreted by the utricular glands of the uterus, and that it floats on the surface of this fluid, until it comes in contact with, and adheres to, the wall of the uterus; the actual place of contact would in this case vary considerably, according to the amount of fluid in the uterus at the time.

The youngest ovum yet found in situ within the human uterus, that described by Reichert, was not simply attached to the decidua, but completely embedded in this ( $c f$. Fig. 175); a relation which is retained throughout the whole period of gestation (Fig. 254).

There has been some discussion as to the mode in which this encapsuling of the ovum is brought about; there are no direct observations on the point in the case of human embryos, but the fact that the openinge of the uterine glands occur on both surfaces of the encapsuling layer of the decidua, together 
with the known facts in regard to other Mammals, render it practically certain that the view first advanced by Sharpey is correct, and that, immediately after the ovum has attached itself to the uterine wall, the decidua grows up as a fold around and over it, so as to encapsule it; the object being, partly to maintain the ovum in contact with the uterine wall; and partly, perhaps mainly, to provide an increased extent of vascular surface from which the embryonic villi can draw nutriment.

The fold of the decidua which incloses, or encapsules, the ovum is spoken of as the decidua reflexa; it is at first very thin (Fig. 175, Dx, and Fig. 254, dr), but it has the same structure as the other parts of the decidua. In its early stages it is exceedingly vascular, the vessels converging from its margin to a small patch of a cicatricial appearance on its most prominent part, which probably indicates the point of meeting, and fusion of the folds by which it is formed.

The part of the decidua to which the ovum is directly attached, and from which the decidua reflexa is developed, is called the decidua serotina (Fig. 175, DW, and Fig. 254, ds); while the term decidua vera is given to the whole of the rest of the decidua, which lines the cavity of the uterus, but has no direct relation with the embryo (Fig. 175, Dv, and Fig. 254, dv).

The decidua vera plays no part in the nourishment of the embryo, and during the latter half of pregnancy becomes greatly reduced in thickness, and undergoes degenerative changes; that it should be formed at all is due, as already noticed, to the fact that, as it is quite uncertain with which particular part of the uterine wall the ovum will come in contact, all parts must be ready in the first instance to receive it.

The fact that the decidua vera, though lining the greater part of the uterus, takes no share in the nutrition of the embryo, and after attaining a certain stage, first stops, and then undergoes retrograde development, renders the comparison between the menstrual decidua and the decidua of pregnancy a still closer one; it further helps to render intelligible the not very uncommon cases in which menstruation takes place at least once after conception has occurred; and also those much rarer cases in which it has been stated to occur regularly throughout the greater part or even the whole of pregnancy.

The decidua reflexa and decidua serotina are at first of very 
small extent (Fig. 175). However, as the chorionic vesicle with its contained embryo increases in size, the decidua reflexa necessarily grows with it. This growth is at first more rapid than

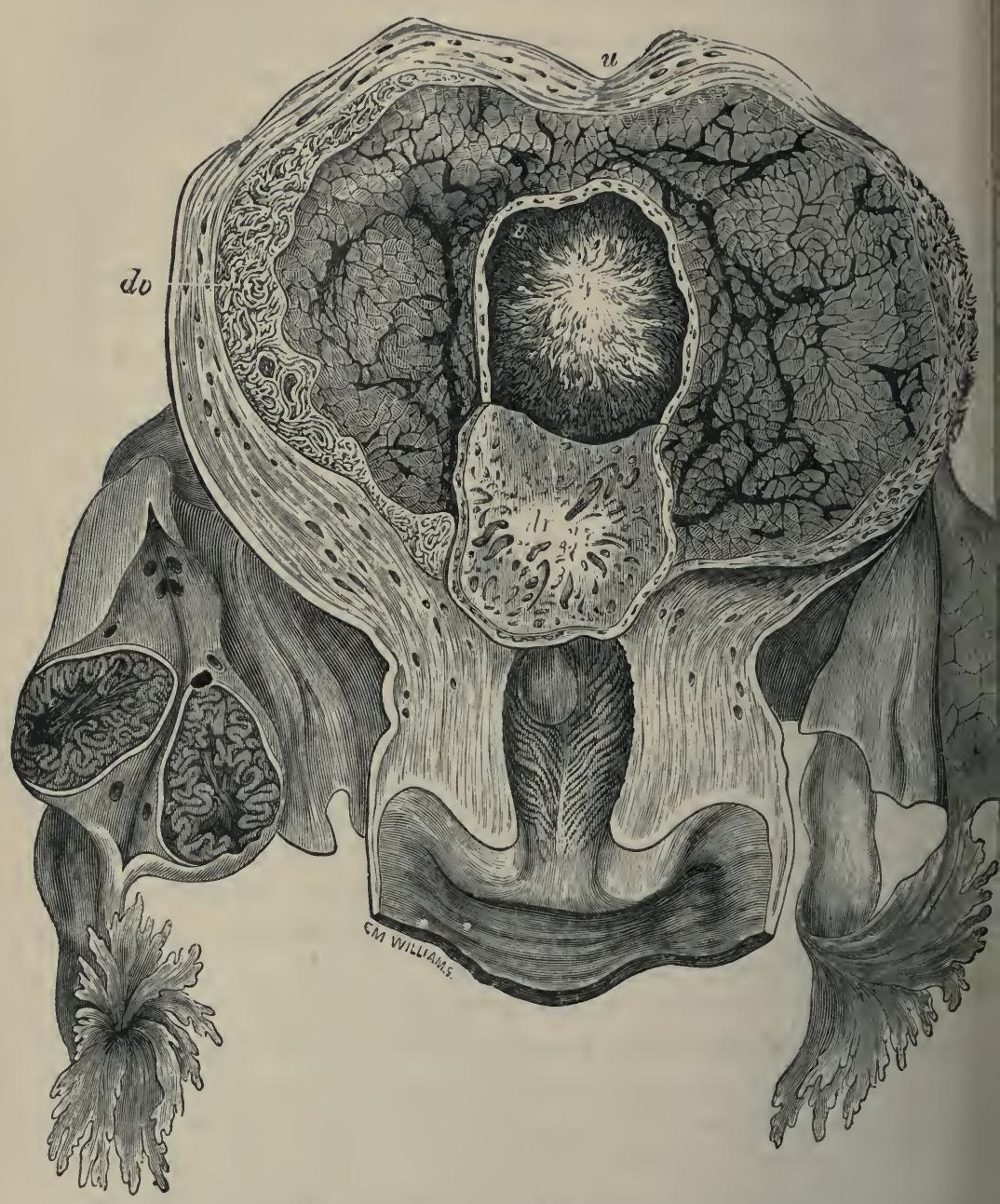

FIG. 255.-A pregnant Human Uterus of about the twenty-fifth day. The uterus has been cut open longitudinally from the ventral surface; the decidua reflexa has been cut open, and the flap turned down to expose the chorionic vesicle; and the right ovary has been bisected to show the large corpus luteum. The embryo that was taken from this chorionic vesicle is shown in Fig. 199. (From Quain's 'Anatomy,' after Coste.) $a r$, decidua reflexa. $d v$, decidua vera. $o$, cavity of the decidua reflexa, in which the chorionic vesicle is lying. $u$, uterus. 
that of the uterus as a whole, and in consequence the decidua reflexa ultimately comes in contact with the decidua vera, and so completely obliterates the cavity of the uterus ( $c f$. Fig. 254). This usually occurs about the sixth month: the two layers, decidua reflexa and decidua vera, are generally described as not only coming in contact, but as fusing more or less completely together, so as to form a single membrane; but according to Minot's observations, the decidua reflexa, which early undergoes degenerative changes, is entirely absorbed by the sixth month, so that the chorion comes into contact with the decidua vera.

So long as the uterine cavity remains an actual one, i.e. up to the time when the chorion meets with the decidua vera, there remains an open passage from the vagina, through the uterus and along the Fallopian tube, to the ovary; and it is, at least theoretically, possible for spermatozoa to reach the ovary, and for what is termed super-foetation to occur.

The decidua serotina is simply the part of the decidua with which the impregnated ovum comes in contact, on entering the uterus, and to which it adheres; and it is at first, therefore, identical in structure with the decidua vera. It very early, however, acquires special characters, owing to the chorionic villi of the ovum becoming intimately connected with it. For some time longer, the decidua serotina and decidua reflexa still remain closely similar to each other; but towards the end of the second month (Fig. 254), the chorionic villi opposite the decidua reflexa begin to diminish in size and importance, and to show signs of degenerative changes; while those in connection with the decidua serotina become much larger and more complicated. The relations between the fœtal villi and the maternal tissues become still more intricate, and gradually the complex and elaborate structure of the fully formed placenta is acquired.

With regard to the detailed changes that take place in the different parts of the decidua during pregnancy, our knowledge is still imperfect in many important respects.

In the region lined by the decidua vera, the mucous membrane becomes greatly thickened; and the uterine glands become dilated and elongated, and acquire exceedingly tortuous courses. By the end of the fifth month the mucous membrane 
is nearly half an inch thick. The surface layer, about one-fourth of the entire thickness, is spoken of as the stratum compactum : in it the gland tubes remain comparatively straight and narrow, while in the interglandular tissue numbers of large, epitheliumlike, decidual cells appear, apparently formed by modification of connective-tissue corpuscles. The deeper three-fourths of the thickness of the mucous membrane, or stratum spongiosum, has a different structure, the gland tubes being greatly dilated and very irregular in shape, and their lining epithelium consisting of flattened or cubical, in place of columnar cells.

After the fifth month, by which time the chorion has met with the decidua vera so as to obliterate the cavity of the uterus, the decidua vera gradually becomes thinner and less vascular, and undergoes degenerative changes, leading ultimately to the almost complete disappearance of the glands, with the exception of their deepest or outermost ends.

The decidua reflexa goes through changes of a very similar kind: the glands first become dilated and lengthened, and then, as the decidua reflexa becomes more and more distended through the enlargement of the chorionic vesicle, the glands gradually atrophy, and the entire layer degenerates, and ultimately completely disappears.

In the decidua serotina, from which the maternal portion of the placenta is formed, the changes have been followed in more detail, but are still only imperfectly known. There is the same division into two layers as in the decidua vera: (i) an inner or superficial layer, from which the surface epithelium and all traces of the uterine glands disappear entirely, and in which decidual cells are developed in large numbers; and (ii) an outer or deeper layer, in which the gland cavities remain as irregular clefts, from which the epithelium has disappeared; except in the very outermost layer, in immediate contact with the muscular wall of the uterus, where the outer or blind ends of the glands persist, greatly compressed, but retaining their epithelium. It is from these outer ends of the glands that the epithelial lining of the uterus is regenerated, after the separation of the placenta.

The further changes that occur in the decidua serotina, and more especially the relations of the blood-vessels, are described in the next section. 


\section{The Placenta.}

The fully formed placenta, at the close of gestation, is a discoidal or cake-shaped body, of spongy consistency, measuring from 16 to $21 \mathrm{~cm}$. in diameter and 3 to $4 \mathrm{~cm}$. in thickness. It is attached to, or rather forms part of, the inner wall of the uterus ( $c f$. Fig. 254), and to its inner or free surface, usually a little distance from its centre, the umbilical cord is attached, the opposite end of the cord being connected with the fotus.

The placenta consists of outer or maternal, and inner or foetal layers, derived respectively from the decidua serotina, and from the chorion. The distinction between the fotal and the maternal elements is easily made in the early stages of development; but in the fully formed placenta, owing to the intricacy of the relations between the chorionic villi and the maternal blood-vessels, and the profound histological modifications which almost all parts undergo, it becomes a matter of the greatest difficulty to determine the real nature of the several structures met with; and there are important points, more especially in regard to the relations of the maternal blood-vessels, on which our knowledge still remains imperfect and unsatisfactory.

The placenta consists of three chief layers or strata : (i) an inner layer formed by the chorion; (ii) an outer layer formeả by the decidua serotina, or modified mucous membrane of the uterus; (iii) a middle or intermediate layer, which is much thicker than the other two, forming four-fifths or more of the entire thickness of the placenta, and which consists of the intricately branched fotal villi, together with the maternal sinuses with which these are in relation.

Of these three layers, the inner one is distinctly fœtal, and the outer one maternal in origin; the middle layer is fotal as regards the villi themselves, but the precise relations of the maternal vessels are still undecided. This middle, or villous zone is the characteristic, and the functionally active part of the placenta. At the margin of the placenta it thins out and disappears, and the inner and outer layers, chorionic and decidual, come into contact with each other in the region of the decidua reflexa.

The inner or chorionic layer of the placenta has already been described (pp. 601 to 605). The amnion is closely united with its inner or free surface over its whole extent. The outer surface, 
towards the villous zone, is characterised by the presence of patches of the substance spoken of as canalised fibrin, and shown by Minot to be produced by a peculiar mode of degeneration of the surface epithelial cells of the chorion. Round the margin of the placenta, the chorionic and decidual layers are so intimately fused that it is impossible to make out a boundary line between them : the decidual cells invade the chorion, and the two layers undergo degenerative changes in common.

The outer or decidual layer of the placenta, formed by the decidua serotina (Fig. 254, ds), is about $1.5 \mathrm{~mm}$. thick, and shows the same distinction into inner or compact, and outer or spongy layers already noticed in the decidua vera. Both layers contain very numerous decidual cells, formed, as elsewhere, by modification of connective-tissue cells. The decidual cells of the compact layer are smaller and more crowded than those of the spongy layer. Their actual size varies very greatly, and the largest ones may contain as many as ten nuclei.

The uterine glands have disappeared from the compact layer of the decidua, but their outer or blind ends persist in the spongy layer as irregular slit-like cavities, filled for the most part with fine granular matter, and retaining in- places their lining of glandular epithelium. The outer surface of the spongy layer is closely united with the muscular wall of the uterus ; and groups of decidual cells may penetrate between the muscle fibres at places.

The middle or villous zone of the placenta is much the thickest of the three, and is also the most important, and the most complicated. It may be described, roughly, as a huge sinus filled with maternal blood, and divided up into a labyrinth of intercommunicating loculi by a framework of fibrous bands and partitions, which run across between the decidual and chorionic walls; the loculi being occupied by forests of arborescent villi, which arise from the chorionic wall and are richly supplied with capillaries derived from the allantoic vessels of the fœetus.

The villi branch extraordinarily freely: their stems arise. from the chorion, and the majority of the branches end freely, but many are attached either to the uterine decidua, or to the partitions separating the loculi.

The fotal blood-vessels. The blood is carried from the foetus to the placenta by the two allantoic arteries, which run in the 
substance of the umbilical cord. On reaching the placenta the arteries branch freely, and very irregularly; the branches spread over the surface of the placenta, between the amnion and the chorion, and dip rather suddenly into the substance of the placenta. Within this they branch freely, and approach the uterine surface of the placenta by a series of terrace-like steps, spreading out horizontally, and then dipping suddenly towards the surface, two or three times in succession; ultimately they enter the villi, and follow their branches to their finest ramifications.

The capillaries of the villi, into which the arteries finally pass, are variable in diameter, and exhibit irregular dilatations and constrictions ; their average size is, however, very large, as they frequently admit from four to six red-blood corpuscles abreast.

The capillaries unite at their further ends to form veins, which follow generally the same paths as the arteries, and finally leave the placenta as the allantoic vein, which, running along the umbilical cord, returns the blood from the placenta to the fœtus.

The fotal vessels thus form a closed set of blood-vessels, which have proper walls of their own, of the usual structure, along their entire course, and which show no special peculiarities except the large size of the capillaries in the villi.

The maternal blood-vessels are derived directly from the uterine arteries and veins. The arteries, which are of comparatively small size, run with a very tortuous course, from which their name ' curling arteries' is derived, through both the spongy and compact layers of the decidua, and open suddenly into the great sinuses or loculi of the placenta. From these sinuses the blood is returned by veins, which run very obliquely through the decidua, and eventually join the veins of the muscular wall of the uterus.

The fœtal villi are thus bathed by a slowly moving stream of maternal blood; and the necessary nutritive and respiratory interchanges must be effected in this middle or villous zone of the placenta. Nothing is known as to the exact mode in which these are carried on; whether by simple diffusion, or whether aided or modified by active participation of epithelial or other cellular elements. The one thing that is quite clear is, that in the 
placenta the foctal and maternal blood streams are kept apart, and that no actual mixing of blood from the two sources can occur.

The real nature of the sinuses, or loculi, in which the maternal blood lies has been much discussed, and is not yet determined with certainty.

It was formerly held that they are produced by enormous dilatation of the capillaries, which normally connect the uterine arteries and veins together; and Waldeyer has shown that these sinuses have a distinct epithelial lining, continuous with that of the uterine vessels.

Kölliker and Langhans point out that, on the fœetal side of the placenta, the walls of the sinuses are formed by the chorion, and show no trace of decidual structure; they, therefore, suggest that the sinuses are not really maternal capillaries, but are spaces between the maternal and fotal portions of the placenta, i.e. between the decidua and the chorion, into which the blood has penetrated by extravasation, or by rupture of the uterine vessels, consequent on the degeneration which the uterine mucous membrane is known to undergo.

On the other hand, a comparison of what is known concerning the human uterus, with the facts ascertained in regard to the formation of the placenta in the rabbit and in other Mammals, leads to the belief that the sinuses may prove to be spaces formed by absorption, not within the maternal tissues, but in the chorionic epithelium itself; in which case the whole thickness of the villous zone of the placenta would be of foetal origin.

\section{Separation of the Placenta at Birth.}

In parturition, the contraction of the muscular walls of the uterus, and the consequent pressure on the uterine contents, more especially upon the amnionic fluid, causes the investing membranes of the embryo, i.e. the combined decidua vera and decidua reflexa, the chorion, and the amnion, to bulge through the os uteri. On rupture of the membranes, the amnionic fluid first escapes, and subsequently the fœetus is expelled.

The further contraction of the uterus detaches the placenta from the uterine wall, the plane of separation passing through the outer or spongy layer of the decidua, in which the deeper parts of the uterine glands still persist; and the placenta, with 
the decidua, the chorion, and the amnion forming a membranous fringe around it, is in its turn expelled as the after-birth. The continuation of the uterine contraction, after the expulsion of the placenta, checks, and in normal cases reduces to a minimum, the hæmorrhage that necessarily results from the tearing across of the maternal vessels along the plane of separation of the placenta.

The deepest part of the spongy layer of the decidua, in which are the blind ends of the uterine glands, remains in the uterus as a thin lining to the muscular walls; and, from the epithelium of these persistent parts of the glands, the entire uterine epithelium is speedily regenerated.

\section{List of the more important Publications dealing with, or bearing on the Development of Man.}

Ackeren, F. v.: 'Beiträge zur Entwicklungsgeschichte der weiblichen Sexualorgane des Menschen.' Zeitschrift für wissenschaftliche Zoologie, xlviii. 1889.

Allen, F. J. : ' On the Cause of the Twisting of the Umbilical Cord, illustrated by Mechanical Models.' Journal of Anatomy and Physiology, xxvi. 1892.

Born, G. : 'Ueber die Derivate der embryonalen Schlundbogen und Schlundspalten bei Säugethieren.' Archiv für mikroskopische Anatomie, xxii. 1883.

Bowen, J. T.: 'The Epitrichial Layer of the Human Epidermis.' Anatomischer Anzeiger, iv. 1889.

Broca, A : 'Contribution à l'Etude du Développement de la Face.' Annales de Gynécologie, xxviii. 1887.

Chiarugi, G. : 'Anatomie d'un Embryon Humain de la Longueur de mm. $2 \cdot 6$ en ligne droite.' Archives Italiennes de Biologie, xii.

Coste, M.: 'Histoire Générale et Particulière du Développement des Corps Organisés.' 1847-1859.

Cunningharn, D. J.: 'The Complete Fissures of the Human Cerebrum, and their Significance in connection with the Growth of the Hemisphere and the Appearance of the Occipital Lobe.' Journal of Anatomy and Physiology, xxiv. 1890.

Darwin, C. : 'The Descent of Man.' 2nd edition. 1874.

Dursy, E. : 'Zur Entwickelungsgeschichte des Kopfes des Menschen und der höheren Wirbelthiere.' 1869.

Ecker, A. : 'Icones Physiologicæ.' 1851-59.

Ercolani, G. B.: 'Nuove Ricerche di Anatomia Normale e Patolegica sulla Placenta dei Mammiferi e della donna.' Mem. d. Accademia d. Scienz. d. Bologna. 1883.

Erdl, M. P.: 'Die Entwickelung der Leibesform des Menschen.' 1846. 
Farre, A.: Article 'Uterus and its Appendages.' Todd's Cyclopæclia of Anatomy and Physiology, v. 1858.

Fol, H.: 'Description d'un Embryon Humain de cinq millimètres et six dixièmes.' Recueil Zool. Suisse. 1884.

Froriep, A.: ' Ueber ein Ganglion des Hypoglossus und Wirbelanlagen in der Occipitalregion.' Archiv für Anatomie und Physiologie: Anatomische Abtheilung. 1882.

Gottschalk: 'Ein Uterus gravidus aus der fünften Woche der Lebenden entnommen.' Archiv für Gynäkologie, xxix. 1887.

'Beitrag zur Entwickelungsgeschichte der menschlichen Placenta.' Archiv für Gynäkologie, xxxvii. 1890.

Heinz: 'Bau und Entwicklung der menschlichen Placenta.' Archiv für Gynäkologie, xxxiii. 1888.

Hensen, V.: 'Die Physiologie der Zeugung.' Hermann's Handbuch der Physiologie, vi. 1881.

Beitrag zur Morphologie der Körperform und des Gehirns des menschlichen Embryos.' Archiv für Anatomie und Physiologie. 1877.

Hertwig, O.: 'Lehrbuch der Entwicklungsgeschichte des Menschen und der Wirbelthiere' Dritte Auflage. 1890.

His, W.: 'Anatomie menschlicher Embryonen.' 1880-85.

'Mittheilungen zur Embryologie der Säugethiere und des Menschen.' Archiv für Anatomie und Physiologie : Anatomische Abtheilung. 1881.

'Ueber das Auftreten der weissen Substanz und der Wurzelfasern am Rückenmark menschlicher Embryonen.' Archiv für Anatomie und Physiologie: Anatomische Abtheilung. 1883.

' Zur Geschichte des menschlichen Rückenmarkes und der Nervenwurzeln.' Abhandlungen der mathematisch-physischen Classe der königl. sächsischen Gesellschaft der Wissenschaften.' Bd. xiii. No. 6. 1886.

'Ueber den Sinus præcervicalis und über die Thymusanlage.' Archiv für Anatomie und Entwickelungsgeschichte. 1886.

' Zur Bildungsgeschichte der Lungen beim menschlichen Embryo.' Archiv für Anatomie und Entwickelungsgeschichte. 1887.

'Die Entwickelung der ersten Nervenbahnen beim menschlichen Embryo.' Archiv für Anatomie und Entwickelungsgeschichte. 1887.

' Zur Geschichte des Gehirns sowie der centralen und peripherischen Nervenbahnen beim menschlichen Embryo.' Abhandlungen der matbematisch-physischen Classe der königl. sächsischen Gesellschaft der Wissenschaften. Bd. xiv., No. 7. 1888.

'Ueber die embryonale Entwickelung der Nervenbahnen.' Anatomischer Anzeiger, iii. 1888.

'Schlundspalten und Thymusanlage.' Archiv für Anatomie und Entwickelungsgeschichte. 1889.

'Die Neuroblasten und deren Entstehung im embryonalen Mark.' Archiv für Anatomie und Entwickelungsgeschichte. 1889.

'Zur Anatomie des Ohrläppchens.' Archiv für Anatomie und Entwickelungsgeschichte. 1889.

'Die Formentwickelung des menschlichen Vorderhirns vom Encle des ersten bis zum Beginn des dritten Monats.' Abhandlungen der mathematisch-physischen Classe der königl. sächsischen Gesellschaft der Wissenschaften. Bd. xv. No. 8. 1889. 
' Histogenese und Zusammenhang der Nervenelemente.' Archiv für Anatomie und Entwickelungsgeschichte.' Supplement-Band. 1890.

'Der Tractus thyreoglossus und seine Beziehungen zum Zungenbein.' Archiv für Anatomie und Entwickelungsgeschichte. 1891.

' Die Entwicklung der menschlichen und thierischen Physiognomien.' Archiv für Anatomie und Entwickelungsgeschichte. 1892.

His, W., junior: ' Zur Entwickelungsgeschichte des Acustico-Facialgebietes beim Menschen.' Archiv für Anatomie und Entwickelungsgeschichte, Supplement-Band. 1889.

Janosik, J. : 'Zwei junge menschliche Embryonen.' Archiv für mikroskopische Anatomie, xxx. 1887.

Kanthack, A. A.: 'The Thyreo-Glossal Duct.' Journal of Anatomy and Physiology, xxv. 1891.

Kastschenko, N. : 'Das menschliche Chorionepithel und dessen Rolle bei den Placenta.' Archiv für Anatomie und Entwickelungsgeschichte. 1885.

Keibel, F.: 'Zur Entwicklungsgeschichte der menschlichen Placenta.' Anatomischer Anzeiger, iv. 1889.

'Ein sehr junges menschliches Ei.' Archiv für Anatomie und Entwickelungsgeschichte. 1890.

' Ein menschlicher Embryo mit scheinbar bläschenförmiger Allantois.' Archiv für Anatomie und Entwickelungsgeschichte. 1891.

' Ueber den Schwanz des menschlichen Embryo.' Archiv für Anatomie und Entwickelungsgeschichte. 1891.

Klaatsch, H.: 'Zur Morphologie der Mesenterialbildungen am Darmkanal der Wirbelthiere, ii. Theil, Säugethiere.' Morphologisches Jahrbuch, xviii. 1892.

Kölliker, A: 'Entwicklungsgeschichte des Menschen und der höheren Thiere.' Zweite Auflage. 1879.

'Zur Entwickelung des Auges und Geruchsorganes menschlicher Embryonen.' Festschrift der Schweizer Universität Zürich gewidmet. 1883.

Kollmann, J. : ‘ Die menschlichen Eier von 6 Mm. Grösse.’ Archiv für Anatomie und Physiologie: Anatomische Abtheilung. 1879.

'Die Körperform menschlicher normaler und pathologischer Embryonen.' Archiv für Anatomie und Entwickelungsgeschichte, Supplement-Band. 1889.

'Die Entwickelung der Chorda dorsalis bei dem Menschen.' Anatomischer Anzeiger, v. 1890.

'Die Rumpfsegmente menschlicher Embryonen von 13 bis 35 Urwirbeln.' Archiv für Anatomie und Entwickelungsgeschichte. 1891.

Krause, W. : 'Ueber die Allantois des Menschen.' Archiv für Anatomie und Physiologie, 1875 and 1876 ; and Zoologischer Anzeiger, iv. 1881.

Kundrat and Engelmann: 'Untersuchungen über die Uterusschleimlaut.' Stricker's Med. Jahrbuch. 1873.

Kuppfer, C.: 'Decidua und Ei des Menschen am Ende des ersten Monats.' Münchener medizinische Wochenschrift, xxxv. 1888.

Langhans, Th.: 'Untersuchungen über die menschliche Placenta.' Archiv für Anatomie und Physoiologie, 1877.

'Ueber die Zellschicht des menschlichen Chorion.' Henle's Festgabe. 1882.

Lannelongue et Ménard : 'Affections Congénitales.' 1891. 
Lenhossék, M. v.: 'Die Entwickelung der Ganglionanlagen bei dem menschlichen Embryo.' Archiv für Anatomie und Entwickelungsgeschichte. 1891.

Leopold, G.: 'Studien über die Uterusschleimhaut während Menstruation, Schwangerschaft und Wochenbett.' Archiv für Gynäkologic, xi. xii. 1877.

'Ueber den Bau der Placenta.' Archiv für Gynäkologie, xxxv. 1889. Lockwood, C. B.: 'The Development and Transition of the Testis, Normal and Abnormal.' Journal of Anatomy and Physiology, xxi. and xxii. 1887 and 1888.

Mall, F. : 'A Human Embryo twenty-six days old.' Journal of Morphology, v. 1891.

Marshall, C. F.: 'The Thyro-glossal Duct, or Canal of His.' Journal of Anatomy and Physiology, xxvi. 1891.

Meuron, P. de : 'Recherches sur le Développement du Thymus et de la Glande 'Thyroïde.' 1886.

Meyer, H.: 'Die Entwicklung der Urniere beim Menschen.' Archiv für mikroskopische Anatomie, xxxvi. 1890.

Minot, C. S.: 'The Early Stages of Human Development.' The New York Medical Journal. 1885.

'Uterus and Embryo. II. Man.' Journal of Morphology, ii. 1889.

'On the Fate of the Human Decidua Reflexa.' Anatomischer Anzeiger, v. 1890.

Articles 'Chorion,' 'Placenta,' etc., in Buck's Reference Handbook of the Medical Sciences.

'Human Embryology.' 1892.

Nagel, W.: 'Das menschliche Ei.' Archiv für mikroskopische Anatomie, xxxi. 1888.

'Ueber den Wolff'schen Körper des menschlichen Embryo.' Zeitschrift für Geburtshülfe und Gynäkologie. 1889.

'Ueber die Entwicklung des Urogenitalsystems des Menschen.' Archiv für mikroskopische Anatomie, xxxiv. 1889.

' Ueber das Vorkommen von Primordialeiern ausserhalb der Keimdrüsenanlage beim Menschen.' Anatomischer Anzeiger, iv. 1889.

'Ueber die Entwickelung des Uterus und der Vagina beim Menschen.' Archiv für mikroskopische Anatomie, xxxvii. 1891.

'Ueber die Entwickelung der Urethra und des Dammes beim Menschen.' Archiv für mikroskopische Anatomie, xl. 1892.

Phisalix, C. : 'Etude d'un Embryon Humain de 10 millimetres.' Archives de Zoologie expérimentale et générale. 1888.

Reichert, C.B.: 'Beschreibung einer frühzeitigen menschlichen Frucht im bläschenförmigen Bildungszustande.' 1873.

Schäfer, E. A. : Quain's 'Elements of Anatomy.' 10th edition, vol. i. part 1: Embryology. 1890.

Schlesinger, W.: 'Ein menschliches Ei aus sehr früher Zeit der Schwangerschaft.' Internationale klinische Rundschau, ii. 1888.

Schwalbe, G. : 'Das Darwin'sche Spitzohr beim menschliches Embryo.' Anatomischer Anzeiger, iv. 1889.

Selenka, E.: 'Zur Entstehung der Placenta des Menschen.' Biologisches Centralblatt, x. 1891.

Spee, F. 'Beobachtungen an einer menschlichen Keimscheibe mit offener 
Medullarrinne und Canalis neurentericus.' 'Archiv für Anatomie und Entwickelungsgeschichte. 1889.

Tait, L. : 'Menstruation and the Ovaries.' Lancet. 1888.

Toldt, C.: 'Ueber die Altersbestimmung menschlicher Embryonen.' Prager med. Wochenschrift. 1879.

'Bau und Wachsthumsveränderungen der Gekröse des menschlichen Darmkanales.' Wiener Denkschriften. 1879.

'Die Darmgekröse und Netze im gesetzmässsigen und im gesetzwidrigen Zustand.' Wiener Denkschriften. 1889.

Tourneux, F.: 'Sur le Développement et l'Evolution du Tubercule Génital chez le Fotus Humain dans les deux sexes, avec quelques remarques concernant le Développement des Glandes Prostatiques.' Journal de l'Anatomie et de la Physiologie, xxv. 1889.

Tourneux and Legay: ' Mémoire sur le Développement de l'Utérus et du Vagin.' Journal de l'Anatomie et de la Physiologie, xx. 1884.

Vierordt, H.: 'Das Massenwachsthum der Körperorgane des Menschen.' Archiv für Anatomie und Entwickelungsgeschichte, Supplement-Band. 1890.

Viti, A. : ' $L$ ' amnios umano nella sua Genesi e Struttura ed in Rapporto all' Origine del Liquido amniotico.' 1886.

Vrolik, W.: 'Die Frucht des Menschen und der Säugethiere.' 1854.

Wagner, R.: 'Icones Physiologicæ; Tabulæ Physiologiam et Geneseos Historiam illustrantes.' 1839.

Waldeyer, W. : Article ' Ovary and Parovarium,' in Stricker's Manual of Human and Comparative Histology, New Sydenham Society's translation, vol. ii. 1872.

'Ueber den Placentakreislauf des Menschen.' Sitzb. d. kgl. preuss. Akad. d. Wiss. zu Berlin. 1887.

'Bemerkungen über den Bau der Menschen- und Affen-Placenta.' Archiv für mikroskopische Anatomie, xxxv. 1890.

Zimmermann : 'Rekonstruktion eines menschlichen Embryos von $7 \mathrm{Mm}$. Länge aus der 4. Woche.' Verhandlungen der anatomischen Gesellschaft. 1889. 



\section{N D E X}

ACCESSORY auditory apparatus : Frog, 143-144 ; Chick, 281 ; Rabbit, 397-399 ; Man, 544

Accessory genital organs : Chick, 319-320 ; Rabbit, 426; Man, 592-596

Accessory thyroid body: Frog, 182; Chich, 286

Acetabulum : Frog, 213

Adolescent period: Amphioxus, 87-89

Adrenal body : Chick, 320

After-birth : Man, 615

Age of embryo, estimation of : Chick, 223 ; Rabbit, 352 ; Man, 467-470

Air cell : Rabbit, 408

Air sac: Chick, 292

Alæ nasi : Mran, 498

Alecithal eggs: 7,17

Alimentary canal: Amphioxus, 69-71 ; Frog, 105-109, 145-157; Chick, 281296 ; Rabbit, 399-404; Man, 544-565

Allantoic stalk: Man, 482, 484, 496, 599, 602

Allantois : Chick, 227, 246-247, 294-296; Rabbit, 370, 435; Man, 484, 485, 545

Amnion: Chick, 245-247 ; Rabbit, 369-370 ; Man, 477, 479, 496, 598-599

AмpнioxUs, 37-89 : structure of adult, 37-44; morphological importance, $44-46$; general account of development, $46-48$; early embryonic development, 48-61; condition at hatching, 61 ; later embryonic development, $61-72$; condition at close of embryonic period, 72-73; larval period, 73-87; adolescent period, $87-89$; bibliography, 89

Anatomy: Amphioxus, 37-44

Anterior cerebral vesicle: Chick, 252; Rabbit, 374

Anterior commissure of brain: Rabbit, 379 ; Man, 518

Anterior gut diverticula: Amphioxus, 70

Anterior nares : Frog, 136; Chich, 275, 289; Man, 498

Antihelix: Man, 500

Antitragus : Man, 500

Antrum : Man, 538

Anus: Amphioxus, 71; Frog, 149; Chick, 291-292; Rabbit, 404; Man, 546, 549

Anus of Rusconi: Frog, 107, 110

Aorta, cardiac: Amphioxus, 41, 71

dorsal: Amphioxus, 42; Frog, 169, 179-182; Chick, 307 ; Rabbit, 417 ; Man, 573, 576-578

Aortic arch: Frog, 178-179; Chick, 303-307; Rabbit, 416-417 ; Man, 573576

Aortic trunk: Rabbit, 416

Appendicular skeleton: Frog, 212-215 ; Chick, 332-335 ; Rabbit, 433

Apteria: Chick, 337

Aqueous humour : Chick, 280 
Arch of aorta: Chick, 306; Rabbit, 417; Man, 576

Archenteron: 22 ; Amphioxus, 54, 60

Area opaca : Chick, 224, 235

Area pellucida: Chick, 224, 235-236

Area vasculosa: Chick, 224, 242

Arm : Man, 492, 496, 500

Arteries: Frog, 178-183 ; Click, 303-307 ; Rabbit, 416-417; Man, 573-578

Artery, allantoic : Chick, 307; Rabbit, 417 ; Man, 577, 602, 612 anterior cerebral : Frog, 180

anterior commissural : Frog, 180

anterior palatine: Frog, 180

ascending pharyngeal: Man, 575

basilar: Frog, 180 ; MIan, 578

carotid: Frog, 180; Chick, 305 ; Rabbit, 416; Man, 575

caudal : Rabbit, 417

central, of retina : Rabbit, 389

cœliac axis : Man, 578

cutaneous : Frog, 183

ductus arteriosus : Chick, 307, 314 ; Rabbit, 417; Man, 576, 585, 586

external carotid: Chick, 305 ; Rabbit, 416 ; Man, 575

external maxillary : Man, 575

hypogastric : Man, 577

iliac: Man, 577

internal carotid : Chick, 305 ; Rabbit, 416; Man, 574

internal maxillary : Man, 575

intervertebral: Man, 578

lingual: Frog, 180; Chick, 305; Man, 575

mandibular: Chick, 305

occipital : Man, 575

pharyngeal : Frog, 180

posterior auricular : Man, 575

posterior commissural: Frog, 180

pulmonary: Frog, 182; Chick, 306; Rabbit, 417; Man, 575

subclavian: Chick, 306; Rabbit, 417 ; Man, 576, 578

temporal : Man, 575

thyroid: Frog, 180

umbilical: Chick, 307

vertebral: Man, $576,577,578$

Asymmetry : Amphioxus, 47, 68, 72, 87

Atlas vertebra: Chick, 327 ; Rabbit, 430

Atrial cavity : Ampliioxus, 41, 82-84

Atrial pore: Amplioxus, 41,83

Attachment of blastodermic vesicle to uterus : Rabbit, 371, 437

Attachment of ovum to uterus: Man, 606-607

Auditory capsule : Frog, 143, 204; Chick, 328, 331; Rabbit, 396 ; Man, 543-544

Auditory organ: Frog, 139-144; Chick, 280-281; Rabbit, 393-399; Man, $541-544$

Auditory ossicle : Frog, 144, 209; Chick, 281, 330 ; Rabbit, 398-399

Auditory pit: Frog, 139, 140; Chick, 280 ; Rabbit, 393; Man, 489, 541

Auditory vesicle: Frog, 139, 140; Chick, 280; Rabbit, 393; Man, 492, 495, 541

Auditory vestibule : Frog, 139; Rabbit, 396

Auricle, of heart: Frog, 168; Chick, 300-303 ; Rabbit, 414-415; Man, 566, $567,568-571$

Auricular septum: Frog, 168; Chick, 301; Rabbit, 415 ; Man, 568-571

Axis cylinder, of nerve : Man, 524, 526-527, 528

Axis vertebra: Chick, 327 ; Rabbit, 430

Barb : Chick, 336

Barbule: Chick, 336

Basibranchial : Chick, 331 
Basihyal: Irog, 206 ; Chick, 330

Basilar membrane: Rablit, 397

Basilar plate: Chich, 329; Rahlirt, 432

Beak: Frog, 151; Chick, 267, 289

Bibliography : Introductory chapter, 35-36; Amphioxus, 89 ; Frog, 215-218; Chick, 337-340; Rabbit, 445-447; Man, 615-619

Bile duct: Frog, 156; Chick, 293; liabbit, 410 ; Mlan, 563

Bladder: Frog, 156 ; Man, 590

Blastocol: Amphioxus, 51, 52 ; Frog, 103 ; Chick, 234

Blastoderm : Chick, 224, 234, 235-237

Blastodermic vesicle: 1ial,bit, 354-356; Man, 474

Blastomere, 19 ; Amphioxus, 49-52 ; Frog, 102 ; Ilablit, 352_354

Blastophore, 12

Blastopore: Amphioxus, 54-56, 59; Frog, 107, 110-111, 114-115, 149; Chick, 239,242

Blastula : Amplicoxus, 52; Frog, 103-104

Blood corpuscles: Frog, 165

Bloorl-vessels : Amplivoxus, 41-42, 71-72 ; Frog, 165-185; Chick, 296-315 ; liablit, 412-421; Man, 565-587

Blood-vessels of placenta: Man, 612-614

Body cavity : Amphioxus, 41, 60, 68, 84-86 ; Chick, 243-244, 321-322 ; Rabbit, $427-428$

Bones of skull : Frog, 210; Chich, 331-332

Brachial plexus : Man, 527

Brain: Frog, 117-126 ; Chick, 252-261 ; Rabbit, 372-381; Man, 509-521

Brain vesicles : Frog, 118 ; Chich, 252 ; Rabbit, 371 ; Man, 481, 491

Branchial aortic arch: Chick, 305 ; Ralbbit, 416-417 ; Man, 574-576

Branchial arch: Frog, 159; Chick, 284, 330; Rabbit, 401; Man, 495, 499, $550-552$

Branchial bar : Frog, 206-207, 208-209; Chick, 330, 331 ; Rabbit, 433

Branchial blood-vessels : Frog, 169-179

of first branchial arch, 171-174

of second branchial arch, 174

of third branchial arch, 174

of fourth branchial arch, 174

of hyoid arch, 175-176

of mandibular arch, 176

changes at time of metamorphosis, 176-179

Branchial cleft : Frog, 92, 157-160; Chick, 285 ; Mun, 495

Branchial groove : Rablit, 400 ; Man, 490

Branchial membrane : 1iabbit, 400

Branchial pouch: Frog, 157-160; Chick, 283 ; Rablit, 400; Man, 550-551

Bridge of nose : Man, 497

Bronchi : Rablit, 408

Buccal cavity : Amphioxus, 40, 81 ; Frog, 150 ; Iiablit, 400-403

Buccal hood: Ampllioxus, 40, 81

Buccal orifice : Amphioxus, 39

Buccal tentacles: Amphioxus, 81

Bulbus olfactorius : Man, 517

Bursa Fabricii : Chick, 291

Crecum : Man, 548

Calcarine sulcus: Man, 519

Canalis auricularis : Man, 566, 567, 570

Canalis reuniens: Rablit, 396

Canalised fibrin : Man, 605, 612

Capitulum of rib : liabbit, 430

Capsule of lens: Chick, 277; IIan, 539-540

Cardiac aorta: Almplio.xus, 41, 71

Carotid arch: Frog, 178

Carotid gland: Frog, 181-182 
Carpus : Chick, 333

Cartilage-bone: Frog, 209 ; Chick, 324, 331

Catamenial flow : Man, 458

Cavity of amnion: Chick, 246; Man, 598

Cauda: Man, 499

Cement : Rabbit, 407 ; Man, 560

Cenogenetic characters, 28

Central canal of spinal cord: Chick, 250; Rabbit, 382

Central nervous system: Frog, 113-126; Chick, 247-261; Rabbit, 363, 371 382; Man, 509-528

Centrifugal cranial nerves : Man, 528-530

Centripetal cranial nerves: Man, 528-530

Centrolecithal egg, 7, 22

Ceratobranchial: Chick, 330

Ceratohyal: Frog, 206 ; Chick, 330

Cerebellum: Frog, 122; Chick, 254-256 ; Rabbit, 380 ; Man, 510, 521

Cerebral hemisphere : Frog, 125-126; Chick, 260-261 ; Rabbit, 376-379; Man, $512,513,514,516-520$

Cervical flexure: Man, 496, 510, 511

Cervical plexus : Man, 527

Cervical rib: Chick, 327

Cervix uteri : Man, 594

Chalaza, 231

Changes in circulation at birth : Rabbit, 419-420; Man, 586-587

Changes in circulation on hatching: Chick, 314-315

Cheek: Man, 504

Chiasma, optic: Frog, 139; Chick, 259; Rabbit, 390; Man, 514, 520, 540

CHICK, 219-340: general account, 219-228; the egg, 228-232; the early stages of development, 232-247; the nervous system, $247-274$; the sense organs, 274-281; the alimentary canal, 281-296; the heart and blood-vessels, 296-315; the urinary organs, 315-320 ; the body cavity and the muscular system, $321-323$; the skeleton, 324-335; the feathers, 335-337;

Chin : Man, 502 bibliography, 337-340

Chorion : Man, 602-605, 611

Chorion frondosum : MIan, 604

Chorion læve: Man, 604

Chorionic villi : Man, 603, 609

Choroid : Rabbit, 392 ; Man, 540

Choroidal fissure: Chick, 278; Rabbit, 388; Män, 539

Choroid plexus of fourth ventricle: Frog, 122; Chick, 254;Rabbit, 381

Choroid plexus of lateral ventricle: Rabbit, $378 ;$ Man, 516

Choroid plexus of third ventricle : Frog, 124;Chick, 258; Rabbit, 375-376 ; Man, 520

Chromatin loops : 14,15

Ciliary ganglion: Chick, 268; Man, 536

Circulation in yolk-sac: Rabbit, 420-421

Circulatory system : Amphioxus, 41-42, 71-72 ; Frog, 165-185; Chick, 296315; Rabbit, 412-421; Man, 565-587

Circumvallate papilla : Man, 557

Clavicle: Frog, 213; Chick, $332 ;$ Rabbit, 433

Claw : Rabbit, 434

Cleft palate : Man, 555

Clitoris : Man, 597

Clitoro-penis : Man, 596

Cloaca : Rabbit, 411-412; Man, 545, 549, 596

Cloacal papilla : Rabbit, 411

Cloacal septum : Rabbit, 412

Club-shaped gland: Amphioxus, 70-71, 72, 79-80

Cochlea: Frog, 141 ; Rabbit, 393, 397; Man, 541-544 
INDEX. Ccelom: Amphioxus, 41, 60, 68, 70, 84-86; Chich, 243-244, 321-322 ; Rabbit,

Colomic canal: Amphioxus, 41

Coloboma iridis: Man, 540

Colossal cells : Rabbit, 444

Columella: Frog, 209; Chick, 330

Columnæ carnex: ('hick, 303

Commissures of brain : Rabbit, 378-379; Man, 517-518

Commissures of spinal cord: Man, 525

Comparison of early stages in Amphioxus and in Frng, 112

Comparison of early stages in Rabbit and in Man, 475-476

Comparison of Mammalian development with that of other Vertebrates, 341344

Complete segmentation, $19,22,353$

Concrescence of lip of blastopore : Frog, 111

Condensation of ancestral history, 30

Coni vasculosi : Man, 593

Conjunctiva: Chich, 280

Convolutions of hemispheres: Rabbit, 379; Man, 518-520

Coracoid : Frog, 213; Chick, 332

Cornea: Chick, 280 ; Rabbit, 392 ; Man, 539-540

Coronary sinus : $\mathbf{M}$ Tan, 568, 582

Corpora adiposa : Frog, 198

Corpora quadrigemina: 1Rabbit, 380 ; Man, 514

Corpora striata : Chick, 260; Rabbit, 378; Man, 516-517

Corpus albicans : $\mathbf{M}$ an, 518

Corpus callosum : Rabbit, 379 ; Man, 518

Corpus luteum : Rabbit, 349 ; Man, 455-456

Corpus luteum spurium: Man, 456

Corpus luteum verum : Man, 456

Corpus mammillare: Man, 520

Corpus sponoiosum: Rabbit, 412

Corti, organ of : Rabbit, 397; Man, 543

Coste's embryo : Man, 487-488

Course of circulation in embryo : Chick, 311-314; Rabbit, 418-421; Man, $583-586$

Cranial flexure: Frog, 118, 120; Chick, 260; Rabbit, 372; Man, 489

Cranial nerves: Froy, 128-134; Chick, 262-271 ; Rabbit, 382-385 ; Man, 528_ 538

Cranium : Frog, 20:3-205 ; Chick, 328-330

Critical stage: Amphioxus, 47,75

Crura cerebri : Frog, 123; Chick, 256; Rabbit, 380 ; Man, 521

Cutaneous sense organs: Frog, 144-145

Cutis layer : Amphioxus, 85

Decidua of menstruation : Man. 458, 463-465

Decirlua of pregnancy : Man, 605-610

Decidua reflexa: Man, 607-609, 610

Decidua serotina: Man, 473, 607-609, 610, 611, 612

Decirlua vera : Man, 607,609-610

Decidual cells : Rabbit, 440; Man, 612

Deciduous dentition: Rablit, 404; Man, 561

Degeneration : Amphioxus, 45

Dental papilla : Frog, 211 ; Rabbit, 406; Man, 559-561

Dentine : Rabbit, 406-407; Man, 560

Descent of testis : Rabbit, 426 ; Man, 507, 508, 509

Deutoplasm : 4

Development : general account of, 1-3, 24-34; Amphioxus, 37-89; Frog, 90_

Diaphragm : Rabbit, 428

218; Chick, 219-340; Habbit, 341-447; Man, 448-619

S $\mathbf{s}$ 
Digestive system : Amphioxus, 69-71; Frog, 105-109, 145-157; Chick, 281296 ; Rabbit, 399-412; Man, 54t-565

Discoidal segmentation : 22

Discus proligerus: Rubbit, 349 ; Man, 454

Distortion of ancestral characters : 30

Dorsal fissure of spinal cord: Chick, 250; Man, 527

Dorsal roots of spinal nerves: Amphioxus, 43 ; Frog, 127-128; Chick, 271273 ; Rabbit, 385-386; Man, 525-528

Down feathers: Chick, 335

Duct of Gaertner: Man, 596

Ductus arteriosus: Chick, 307, 314; Rabbit, 417, 419; Man, 576, 585, 586

Ductus Botalli : Chick, 307; Rabbit, 417, 419

Ductus endolymphaticus : Frog, 142

Ductus venosus : Chick, 310, 314 ; Rabbit, 419; Man, 581, 585, 586, 587

Duodenal loop of intestine: Chick, 290; Rabbit, 403; Man, 547

Dura mater: Rabbit, 381

Duration of development : Amphioxus, 48; Frog, 91-94 ; Chick, 221 ; Rabbit, $350 ;$ Man, 449, 465-467

Duration of pregnancy: Man, 465-467

Ear: Frog, 139-144; Chick, 280-281 ; Rabbit, 393-399 ; Man, 541-544

Early stages of development: Amphioxus, 48-61 : Frog, 100-112 ; Chich, 232

Ectoplacenta: Rabbit, 438 247 ; Rabbit, 351-362; Man, 470-486

Ectoplacental lobule: Rabbit, 441

Egg: structure, 3-7; maturation, 7-11; fertilisation, 11-13; unicellular character, 32

Egg: Amphioxus, 43-44, 49; Frog, 94-100 ; Hen, 220-222, 228-232 ; Rabbit, $344-351$; Man, 448-455

Egg column : Man, 450-451

Egg shell: Hen, 220, 229

Eighth month: Man, 508

Fmbryo, formation of : Chick, 222-228 ; Rabbit, 362-367 ; Man, 472-486

Embryonal area : Rabbit, 357-361; Man, 473

Embryonic period: Amphioxus, 48-73

Enamel: Frog, 211; Rabbit, 406 ; Man, 560

Enamel organ: Rabbit, 406; Man, 559-561

Endostyle: Amplioxus, 40, 79

Enterocœlic cavity: Amphioxus, 60, 243

Epiblast: 22, 23-24; Amphioxus, 53, 56 ; Frog, 104-105 ; Chick, 234, 236 ; Rabbit, 358, 361 ; MFan, 475, 486

Epiblastic buds of yolk-sac: Rabbit, 369

Epibranchial groove: Amphioxus, 40

Epicoracoid: Frog, 213

Epidermic layer of epiblast: Frog, 105, 113

Epididymis : Chick, 320 ; Rabbit, 426 ; Man, 593

Epigenesis : 220

Epiglottis : Man, 556

Epiphysial ossification: Man, 509

Epipleural cavity : Amphioxus, 41

Epoophoron: Mfan, 595

Equal segmentation: 19, 22

Estimation of age of embryo: Chick, 223 ; Rabbit, 352 ; Man, 467-470

Ethmoidal plate: Chick, 329, 331 ; Rabbit, 432

Ethmoidal sinus : Man, 538

Eustachian tube: Frog, 143 ; Chick, 285 ; Rabbit, 397 ; Man, 544

Eustachian valve : Chich, 303 ; Rabbit, 418; Man, 569, 583, 584, 585

Excretory organ: Frog, 185-197 ; Chick, 315--320 ; Rabbit, 421-426 ; Mran, 588591

External auditory meatus : Chick, 285 ; Rabbit, 398, 402 ; Man, 499-500, 644

External ear: Rabbit, 398; Man, 499-500, 502, 544 
External genital organs : Man, 506, 596-598

External gills : Frog, 160-161

Eye: Amphioxus, 43, 63; Frrog, 136-139; Chick, 275-280 ; Rabbit, 387-392 ;

Eyebrow : Man; 507

$$
\text { Man, 495, 539-541 }
$$

Eyelash: IIan, 507

Eyelid:Chick, 280; Rabbit, 392; Man, 540-541

Face: Chick, 287-289; Man, 497-498, 502, 503

Fallopian tube: Rabbit, 426 ; Man, 594

False amnion: Chick, 246

Falx cerebri : Rabbit, 379 ; Man, 516

Fang of tooth: Man, 560

Fat body : Frog, 198

Featner : Chick, 335-337

Feather papilla: Chick, 335-336

Feat ures : Man, 497-498, 502, 503

Fenestra ovalis: Frog, 204, 209; Chick, 330; Rabbit, 398

Fenestra rotunda: Rabbit, 3.97

Fertilisation: 11-13,15-17, 32; 1mphioxus, 49; Frog, 91, 99-100; Hen, 222,

Fifth month : Man, 506-507

$231-232$; Rabbit, 351 ; Man, 448, 466-467, 471

Fifth ventricle: Rabbit, 379 ; Man, 517

Fifth week: Man, 496-501

Fin: Amphioxus, 37, 85

Finger: Man, 500, 503

First week : Man, $471-472$

Fissura arcuata : Man, 519

Fissure of spinal cord: Chick, 250: Man, 527

Flexure of embryo: Chick, 226: Rabbit, 364-365; Man, 478, 481, 488-491

Floccular lobe of cerebellum: Rabbit, 380

Foctal membranes: Man, 598-605

Fœtal vessels of placenta: Rabbit, 442 ; Man, 612-613

Fotal villi : Man, 609, 611, 612, 613

Follicle cells : Chick, 229; Rabbit, 346; Man, 452-455

Food yolk: 4-7, 19, 29; Frog, 100-101; Chick, 221-222

Foot: Chick, 335 ; Man, 500

Foramen cæcum : Man, 556

Foramen incisivum: Man, 555

Foramen of Monro: Chick, 261 ; Rabbit, 377 ; Man, 516

Foramen ovale: Chick, 303, 315; Rabbit, 415, 419; Man, 570, 584-587

Forc-brain : Frog, 118, 119; Chick, 252; Rabbit, 374; IIan, 510

Fore-gut: Chick, 281; Rabbit, 399; Man, 545

Fore-limb: Frog, 215; Chick,332-334; Man, 500

Formative cell: Chick, 235

Fornix : Rabbit, 379; Man, 518

Fourth month: Man, 506

Fourth ventricle: I'rog, $122 ;$ Chick, 254; Rabbit, 381

Fourth week: Man, 492-496

Froc, 90-218: general account of development, 90-94; the egg, 94-100; the early stages of development, 100-112; the nervous system, 112-134; the sense organs, 134-145; the alimentary canal, 145-157; the gills and gill clefts, 157-164; the heart and blood-vessels, 165-185; the urinary and reproductive organs, 185-198; the skeleton and the teeth, 198-215;

Frontal lobe of brain: Man, 517 bibliography, 215-218

Frontal sinus: Man, 538

Fronto-nasal process: Chick, 275, 287; Man, 497, 553

Froriep's ganglion: Man, 531

Furcula: C'lick, 332; Man, 556, 562 
Gaertner, duct of : Man, 596

Gall bladder : Frog, 156; Chick, 293; Rabbit, 410; Man, 563

Ganglion, auditory: Man, 536

ciliary: Chick, 268; Man, 536

cochlear: Man, 536, 543

Froriep's: Man, 531

Gasserian: Frog, 133; Chick, 269 ; Man, 536

geniculate: Man, $\mathbf{5 3 6}$

of glossopharyngeal nerve : Man, 535

of pneumogastric nerre : Man, 535

olfactory : Ifan, 537

otic: Man, 536

sphenopalatine : Man, 536

spinal : Chick, 272; Man, 525-526

submaxillary : Man, 536

vestibular: Man, 536,543

Gasserian ganglion: Froq, 133; Chick, 269; Man, 536

Gastrocœl: Amphioxus, 54

Gastrula: Ampliuxus, 53-57

General account of development of embryo: Amphioxus, 46-48; Frog, 90-

Genital cord: Man, 594

94; Chick, 219-228 ; Rabbit, 362-371; Man, 470-509

Genital ducts : Frog, 193-197; Chick, 319-320 ; Rabbit, 426 ; Man, 592-595

Genital organs, external: Man, 596-598

Genital papilla: Rabbit, 412 ; Man, 596

Genital ridge: Frog, 94, 197; Hen, 229; Rabbit, 345; Man, 450, 592

Germinal area : Man, 473

Germinal cell : Man, 523

Germinal disc : 19 ; Chich, 222, 232-235

Germinal epithelium : Hen, 229 ; Rabbit, 345 ; Man, 450-451

Germinal layers: 22-24; Amphioxus, 53; Frog, 104-110; Chick, 235-242 ; Rabbit, 356-361 ; Man, 486

Gèrminal vesicle:Amphioxus, 49; Frog, 95-96; Hen, 230; Man, 453

Gestation: Rabbit, 34t; Man, 465-467

Giant cell : Rabbit, 444

Gill : Frog, 92, 160-163

Gill arch: Amphioxus, 40 : Frog, 159

Gill cleft: Amphioxns, 40, 71, 75-78, 87-88; Frog, 92, 157-160 ; Chick, 283, 285 ; Man, 490, 492, 495, 551

Gill pouch: Chick, 283; Man, 490, 550-551

Giraldès, organ of : Man, 593

Gizzard: Chick, 290

Gland, carotid: Frog, 181-182

club-shaped: Amphioxus, 70-71, 72, 79-80

lacrymal: Chick, 280; Rabbit, 392; Man, 541

mammary : Rabbit, 434

of Lieberkühn : Man, 549

of stomach : Man, 549

salivary: Man, 559

uterine : Rabbit, 437; Man, 458, 459, 610, 612

Glans penis : Mau, 596, 597

Glenoid cavity : Frog, 212

Glomerulus: Frog, 192; Chick, 318; Rabbit, 422 ; Nan, 589

Glomerulus of head kidney : F'rog, 182, 190-191

Glottis : Frog, 154; Rabbit, 408; Man, 556, 562

Glycogenous cells : Rabbit, 442

Gonoblast: 12 ; Frog, 95, 197

Gonotome: Amplioxus, 89

Graafian follicle : Rabbit, 348-349; Man, 453-455

Grey matter of brain : Rabbit, 382

Gut diverticula : Amphimxus, 70 
IIair : Rabbit, 433-434; Man, 506, 509

Hand : Man, 500

Hare-lip: Man, 554

Hatching: Cliick, 228

Head cavity: Amphioxus, 70

Head fold: Chick, 226 ; Rabbit, 363 ; Man, 478

Head kidney: F'rog, 185-191 ; Chick, 318-319; Rabbit, 421 ; Man, 591

Heart : Frog, 167-169 ; Chich, 298-303; Rabbit,413-416 ; Man, 477, 478, 482,

Helix : Man, 499 $490,492,496,565-573$

Hemisphere, cerebral : Frog, 125-126 ; Chick, 260-261 ; Rabbit, 376-379 ; Ian, 512, 513, 514, 516-520

Hepatic cell: Frog, 156 ; Rabbit, 410

Hilum folliculi : Man, 454

Hind-brain : Frog, 118, 119 ; Chick, 252-256 ; Rabbit, 380-381 ; Man, 510

Hind-gut: Chick, 281; Rabbit, 399; Man, 545

Hindlimb: Frog, 215; Chick, 335; Man, 501, 503

Hippocampal sulcus: Man, 519

Hippocampus major : Rabbit, 378

His' embryo E : Man, 477

His' embryo Lg: Man, 488-490

His' embryo Lr: Man, 490-492

His' embryo SR : Man, 477-479

Histological development of brain and spinal cord : Rabbit, 381-382; Man, 521-527

Holoblastic segmentation : $19,22,353$

Horny jaw : Frog, 151-152

Horny teeth : Frog, 150-151

HUMAN EMBRYo: first week, 471-472 second week, 472-486

third week, 486-492

fourth week, 492-496

fifth week, 496-501

sixth week, 501-503

second month, 503-505

third month, $505-506$

fourth month, 506

fifth month, 506-507

sixth month, 507

seventh month, 507-508

eighth month, 508

Hydatid of Morgagni : Man, 593

ninth month, 508-509

Hyoid arch: Frog, 159; Chich, 284, 330; Man, 490, 495, 550-552

Hyoid bar: Frog, 206, 208; Chick, 330, 331; Rabbit, 433

Hyoidean aortic arch : Chick, 305; Rabbit, 416; Man, 574-575

Hyomandibular gill-cleft: Frog, 159 ; Chick, 285

Hyomandibular gill-pouch: Frog, 159-160; Rabbit, 397-398 ; Man, 544

Hypoblast : 22, 23, 24; Amphioxus, 53, 56; Frog, 109; Chick, 237; Rabbit, 358,$361 ;$ MIan, 475,486

Hypothetical stages between Reichert's ovum and His' embryo E: Man, $483-485$

Ilium : Frog, 214; Chick, 334

Impregnation: 12 ; Chich, 232 ; Rabbit, 350-351

Incubation: Chick, 223

Incus : Rabbit, 398-399

Infundibulum: Frog, 124; Chick, 258; Rabbit, 376; Man 510, 520

Inner amnion: Chick, 245; Man, 477

Inner nasal process : Chick, 288

Internal gills : Frog, 161-163 
Interorbital plate: ${ }^{-}$Chick, 330

Intestine: Amphioxus, 40; Frog, 147-148; Chick, 290-291; Rabbit,403-404; Man, 546-549

Iris : Chick, 279 ; Rabbit, 389

Ischium: Frog, 214; Chick, 334

Isthmus: Man, 510

Jacobson, organ of : Frog, 136; Rabbit, 387 ; Man, 495

Kidney: Frog, 191-193; Chick, 319 ; Rabbit, 424-425; Mfan, 590

Kollmann's embryo : Man, 481-482

Labia majora : Man, 597

Labia minora : Man, 597

Labial cartilage : Frog, 203, 205, 207

Labial cavity : Frog, 150

Labio-scrotal fold : Man, 597

Lacrymal duct: Chick, 280 ; Rahbit, 392 ; Man, 541

Lacrymal gland: C'hick, 280; Rabbit, 392; Man, 541

Lacrymal groove: Chick, 288; Man, 498

Lamina terminalis : Chick, 256; Rablit, 374, 376 ; Man, 517

Larval period: Amphioxus, 73-87

Laryngeal chamber : Rabbit, 408; Man, 562

Lateral frontal process: Man, 497

Lateral plate: Amphioxus, 84; Chick, 244

Lateral ventricle: Frog, 126; chick, 260; Rahbit, 377 ; Man, 516

Leg: Frog, 215; Chick, 335; Man, 492, 496, 501

Length of embryo: Man, 470-471

Lens, of eye : Frog, 137; Chick, 277 ; Rabbit, 390-392 ; Man, 495, 539

Limbs : Frog, 92-93, 212-215 ; Chick, 332-335 ; Man, 492, 496, 500, 502, 505

Lingual duct: Man, 558

Lip : Frog, 150-151; Man, 502, 553-554

Liquor amnii : Man, 598

Liquor folliculi : Man, 454

Liver: Amptioxus, 40 ; Frog, 154-156 ; Chick, 292-294 ; Rabbit, 410-411 ;

Lobule of ear : Man, 500 Man, 496, 563-564

Lower layer cells : Frog, 104; Chich, 234, 236

Iumbo-sacral plexus: Man, 527

Lung : Frog, 154; Chick, 292 ; Rabbit, 408-410; Man, 562-563

Lymphatics : Frog, 185

Malleus: Rabbit, 398-399

Malpighian body: Frog, 192; Chick, 318; Rabbit, 422; Man, 589

Mammalian developmert, general characters of : 341-344

Mammalian placenta, a chorionic structure : 445

Mammary gestation : 344

Mammary gland : Ralbit, 434

MAN, 448-619: preliminary account, 448-449; the human ovum, 449-470; general history of the development of the human embryo, 470-509; nervous system. 509-538; sense organs, 538-544; digestive system, 544-565; circulatory system, 565-587; urinary organs, 588-591; reproductive organs, 591-598 ; foetal membranes and placenta, 598-615; bibliography, 615619

Mandibular aortic arch: Chick, 305; Rabbit, 416; Man, 574, 575

Mandibular arch: Chick, 283, 287, 330; Rabbit, 401; Man, 490,"495, 499, $550-551$

Mandibular bar: Frog, 205; Chick, 330, 331; Rabbit, 433

Mantle layer of spinal cord: Man, 524, 528

Manubrium sterni : Chick, 332 
Manus: Frog, 215; Click, 333-334

Maternal vessels of placenta: Rabbit, 441; Man, 613_614

Maturation of egg : 7-11; Frog, 96-99; Hen, 230-231 ; Rabbit, 349-350; Man, 471

Maxillary arch: Chick, 288; Rabbit, 401; Man, 490, 495, 553-555

Measurement of embryo: Mfan, 470-471

Meatus venosus : Chick, 293, 309

Meckel's cartilage: Frog, 205, 206, 208; Chick, 330; Rabbit, 399, 433

Meconium: Man, 506, 507, 508, 564

Medulla oblongata: Frog, 122; Chick, 253; Rabbit, 380-381; Man, 521

Membrana limitans interna: Man, 523

Membrane bones: Frog, 210; Chick, 324, 332

Membrane of Reissner : Rabbit, 397

Menstrual cycle : Man, 459-460

Menstrual decidua: Man, 458, 463-465

Menstrual discharge: Man, 458-459

Menstruation : Man, 457-465

Menstruation, connection with ovulation : Man, 462-465

Meroblastic segmentation: 22 ; Chick, 232

Mesencephalic flexure: Man, 511, 513

Mesenteron: Amphioxus, 69 ; Frog, 105-109, 145-148; Chick, 281-282 ; Rabbit, 399 ; Man, 544-549

Mesentery : Frog, 148; Chick, 291; Rabbit, 400 ; Man, 547, 564-565

Mesoblast: 22, 23, 24; Amphioxus, 60-61; Frog, 109-110; Chick, 237, 239242, 297-298; Rabbit, 361-362; Man, 476, 481, 483, 486

Mesoblastic somite: Amphioxis, 60-61, 65-69, 84-87; Chick, 242-244, 322 323,324 ; Man, 482, 490, 495

Mesobranchial area: Man, 555, 556

Mesogaster : Man, 564

Metacarpal: Chick, 333-334

Metamorphosis : 30; H'rog, 93-94

Mletanephros: Chick, 319; Rabbit, 424-425

Metapleural fold: Ampliioxus, 37, 82-84

Metatarsal: Chick, 335

Metazoa, characters of : $1,2,3,27$

Metencephalic flexure: IIan, 511, 513

Mid-brain: Frog, 118, 119; Chick, 252, 256; Rabbit, 379-380; Man, 510, 520-521

Middle cerebral vesicle: Chick, 252; Rabbit, 379

Mid-gut : Chick, 281; Rabbit, 399 ; Man, 545

Milk dentition : Rabbit, 404 ; Man, 561

Mitral valve : Man, 573

Modiolus of cochlea : Man, 544

Mons Veneris : Man, 597

Iorphological importance of Amphioxus, 44-46

Motor cranial nerves: Man, 528-534

Motor roots of spinal nerves: Frog, 128; Chick, 273 ; Rabbit, 385; Man, 524

Mouth: Amphioxus, 40, 71, 80-81; Frog, 148; Chick, 287; Man, 495, 545

Müllerian duct: Frog, 195-197; Chick, 318, 319, 320; Rabbit, 425, 426; Man, 590-591, 593-594

Muscle plate: Chick, 322-323; Rabbit, 429

Muscular system: Chick, 322 2323 ; Rabbit, 429

Musculi papillares : Chick, 303

Myelospongium : Man, 523, 528

Myocœl : Amphioxus, 85-86, 89

Myotome: Amphioxus, 38, 67 ; Froy, 199; Chick, 323

Nail : Man, 506, 507

Nasal groove: Chick, 288; Man, 497, 553

Neck: Man, 502

Nephrostome of head kidney : Frog, 187-190 
Nephrostome of Wolffian body: Frog, 192; Chick, 317-318

Nerve, chorda tympani : Man, 533

cranial: Frong, 128-134; Chick, 262-271 ; Rabbit, 382-385 ; Man, 528538

dorsal: Amphioxus, 43

early development of : Frog, 126-128; Chick, 261-262; Man, 521528

lateral line: Frog, 130

mandibular: Frog, 132, 133; Chick, 269 ; Rabbit, 384 : Man, 536

maxillary: Frog, 133; Chick, 269; Rabbit, 384; Man, 536

ophthalmic: Frog, 132, 133; Chick, 269 ; Rabbit, 381; Man, 536

phrenic: Man, 527

recurrent laryngeal : Man, 575

spinal: Frog, 128; Chick, 271-274; Rabbit, 385-386 ; Man, 524-528

sympathetic: Frog, 134; ('hick, 274; Rabbit, 386; MFan, 538

ventral : Amphioxus, 43

1st cranial (olfactory): Frog, 133-134; Chick, 260, 266-267; Rabbit, 382 ; Man, 537-538

2nd cranial (optic): Frog, 133, 138-139;Chick, 267-268, 279; Rabbit, 383,$389 ; \operatorname{Man}, 536,540$

3rd cranial (oculomotor): Frog, 133; Chick, 268; Rabbit, 383; Man, 534

4th cranial (pathetic): Fron, 133; Chich, 268; Rabbit, 383; Man, 534

5th cranial (trigeminal): Frog, 132-133; C'hick, 269; Rabbit, 384; Mran, 534, 536, 557

6th cranial (ahducent): Frog, 132 ; Chick, 269; Rabbit, 383 ; Man, 533

7th cranial (facial); Frog, 132; Chick, 264-266, 269; Rabbit, 384; Man, 533

8th cranial (auditory): Frog, 131, 140; Chick, 270; Rabbit, 384; Man, $535-536,542$

9th cranial (glossopharyngeal): Frog, 131; Chick, 270; Rabbit, 384; Man, 533, 535, 557

10th cranial (pneumogastric or vagus): Frog, 129-131; Chick, 270$271 ;$ Rabbit, 384 ; Man, 533, 535

11 th cranial (spinal accessory): Chick, 271 ; Rabbit, 385 ; Man, $532-$ 533

12th cranial (hypoglossal): Chick, 271; Rabbit, 385; Man, 531-532

Nerve cell : Ifan, 526

Nerve fibre: Man, 526-527

Nervous layer of epiblast: Frog, 105, 113

Nervous system: Amphioxus, 42-43, 57-60, 62-63; Frog, 112-134; Chick, $247-274$; Rabbit, 371-387; Man, 509-53s

Neural canal: Amphioxus, 58,62-63; Man, 481, 510

Neural crest: Frog, 127; Chick, 263; Man, 526

Neural fold: Amphioxus, 58-59; Frog, 113-114; Chick, 248-249 ; Rabbit, 363,371 ; Man, 477

Neural groove: Frog, 113-114; Chick, 247 ; Rabbit, 363, 371 ; Man, 477, 480, 481,509

Neural plate: Amphioxus, 58; Frog, 113, 127 ; Chick, 247

Neural ridge: Frog, 127; Chick, 261, 263; Man, 526

Neural tube : Amphioxus, 59-60, 63; Frog, 114-116, ; Chick, 248; Man, 510

Neurenteric canal: Amphioxus, 59, 63, 69; Frog, 115, 149, 156; Chick, 250)$252 ;$ Man, 480

Neuroblast: Frog, 139 ; C'hich, 249 ; Man, 523-524, 526, 528, 537

Neuropore: Amiphioxus, 60, 63, 69

Nictitating membrane : Chick, 280; Rabbit, 392

Ninth month: Man, 508-509

Nose : Frog, 135-136 ; Chick.274-275 ; Rabbit, 387 ; Man, 497-498, 502, 5s s

Nostril : Frog, 136 ; Chick, 275, 289; Man, 498

Notochord: Amphioxus, 37, 61, 63-65; Fron, 109, 198-201 ; Chick, 242, 324 327; Rabbit, 430; Man, 480 
Nuclear skein : 8, 15; Frog, 97

Nutrition of embryo: Amphioxus, 57, 62; Frog, 92; Rabbit, 370, 435; Man,

Nymphæ: Man, 597 448

Obplacental folds of uterus: Rabbit, 436

Occipital lobe of brain: Man, 517

Oclontoblast: Rabbit, 407; Man, 560

Esophagus: Frog, 153-154; Chick, 289-290 ; Rabbit, 403 ; Man, 545, 546, 548

Olfactory capsule: Frog, 204 ; Chick, 331; Rabbit, 432

Olfactory lobe: Frog, 126 ; Chick, 260, 267 ; Rabbit, 379 ; Man, 513, 517, 537

Ulfactory organ: Amplioxus, 43; Frog, 135-136; Chick, 266-267, 274-275;

Omentum : Man, 565 II an, 492, 494-495, 497, 538

Omission of ancestral stages : 28,30

Omosternum : Frog, 213

Opercular cavity : Frog; 161

Opercular fold: Frog, 92, 16]

Opercular spout : lirog, 161

Optic bulb: Frog, 137

Optic chiasma : Frog, 139 ; Chick, 259 ; Rabbit, 390 ; Man, 514, 520, 540

()ptic cup: Frog, 138; Chick, 277-279; Rabbit, 388-389; Man, 539

Optic lobe: Frog, 123; Chick, 256

Uptic stalk: Frog, 125, 137-139; Chick, 267, 279; Rabbit, 387; Man, 510, 536

Optic thalami : Frog, 123 ; Chick, 256; Rabbit, 374; Man, 520

Optic vesicle: Frog, 125, 136-137; Chich, 252, 260, 267, 275-276; Rabbit,

Ora serrata: Chich, 279 374,$387 ; \operatorname{Man}, 510,539$

Organ of Corti : Rabbit, 397 ; Man, 543

Organ of Giraldès : Man, 593

Organ of Jacobson: Frog, 136; Rabbit, 387; Man, 495

()rgan of Rosenmüller: Man, 595

Outer amnion : Chick, 246

Outer nasal process : Chich, 288

Ovary: Amphioxus, 43; Frog, 197 ; Hen, 228; Rabbit, 345-349; Man, 450_ 453

Oviduct : Frog, 195-197 ; Hen, 228-229 ; Chick, 319, 320 ; Rabbit, 426 ; Man, 594

Oviposition: Amplioxus, 44, 46; Frog, 90-91, 99

Ovulation: Rabbit, 350-351; Man, 456-457

Ovulation, connection with menstruation: MIan, 462-465

Ovum: structure, 3-7; maturation, 7-11 ; fertilisation, 11-13; unicellular character, 32

Ovum : Amphioxus, 43-44,49; Frog, 94-100; Hen, 220-222, 228-232 ; Rabbit, $344-351$; Man, 448-455

Palate : Chick, 275 ; Rabbit, 403; Man, 554-555

Palatopterygoid bar: Frog, 205, 206, 207-208; Rabbit, 433

Palingenetic characters: 28

Pancreas: F'rog, 156; Chick, 294; Rabbit, 411; Man, 564

Pancreatic duct: Chick, 294; Rabbit, 411; Man, 564

P'arachordal cartilage : Frog, 203, 204, 207; Chick, 328, 331 ; Rabbit, 432

Parepididymis : Man, 593

Parietal lobe of brain: Man, 517

Parieto-occipital sulcus: Man, 519

Paroophoron: Man, 595

l'arovarium: Chick, 320; Rabbit, 426; Man, 595

Parthenogenesis : $15,16,17,32$

Partial segmentation : 22 
Parturition: Rabbit, 444-445; Man, 614-615

Pecten : Chick, 279

Pectoral girdle: Frog, 212-213; Chick, 332

Pelagic life of Amphioxus, 47

Pelvic girdle : Frog, 213-215; Chick, 334

Pelvic symphysis : Frog, 213

Penis : Man, 597

Pericardial cavity : Frog, 167, 169; Chick, 321-322 ; Rabbit, 427-428

Perilymph : Frog, 142 ; Man, 543

Periotic capsule: Irrog, 143, 204; Chick, 328, 331 ; Rabbit, 396, 432 ; Man, $543-544$

Peripheral nervous system : Frog, 126-134; Chick, 261-274; Rabbit, 382-386 ; Man, 521-538

Periplacental folds of uterus : Rabbit, 435

Peritoneum: Chick, 321

Perivascular cell : Rabbit, 438

Perivitelline fluid: Frog, 98, 100

Permanent dentition : Rabbit, 404: Man, 561

Permanent ova: Frog, 95 ; Chick, 230; Rabbit, 346-347 ; Man, 453

Pes : Chick, 335

Peyer's patch : Man, 549

Pharynx : Amphioxus, 40, 75-80 ; Frog, 152 ; Chick, 283-286 ; Rabbit, 400_

Phrenic nerve : Man, 527 $403 ;$ Man, 545, 546, 549-553

Pia mater : Rabbit, 381

Pineal body: Frog, 123; Chick, 256-257; Rabbit, 374; Man, 520

Pinna: Rabbit, 398; Man, 499-500, 544

Pituitary body: Frog, 124-125; Chick, 259, 289; Rabbit, 376, 400

Placenta : Rabbit, 343, 370-371, 434-445 ; Man, 611-615

Placental area of blastodermic vesicle : Rabbit, 437

Placental folds of uterus: Rabbit, 435

Pleural cavity: Chick, 322 ; Rabbit, 428 ; Man, 563

Plica semilunaris ; Man, 541

Polar body : 8-11, 15-17; Amphioxus, 49 ; Frog, 97-99 ; Hen, 231 ; Rabbit, 349,350 ; Man, 471

Polar mesoblast cells : Amphioxus, 55, 60, 65

Pons Varolii : Rabbit, 381 ; Man, 514, 521

Position of embryo: Chick, 225, 228; Rabbit, 438

Postanal gut: Frog, 156-157 ; Rabbit, 404

Postbranchial body : Frog, 164

Posterior nares : Frog, 136, 148 ; Chick, 275, 289 ; Man, 497, 553-554

Præoral pit: Amphioxus, 70, 81-82

Precoracoid: Frog, 213

Preformation : 219

Pregnancy, duration of : Man, 465-467

Prepubic process : Chick, 334

Prepuce : $M a n, 597$

Primary gill-slits : Amphioxus, 75̃-78

Primary reproductive cells : 12

Primary sulci : Man, 518-519

Primitive groove: F'rog,111; Chick, 238; Rabbit, 361

Primitive ova : 12 ; Frog, 95 ; Chich, 229-230; liabbit, 345-346 ; Man, 451453,592

Primitive sperm cells : IIan, 592

Primitive streak: Frog, 111, 149; Chick, 237-239, 240, 252; Rabbit, 361; $\operatorname{Man}, 479$

Pro-amnion : Chick, 241, 245 ; Rabbit, 369

Processus globularis : Man, 497, 504, 553-554

Proctodæum: Frog, 145, 149-150 ; Chick, 283, 291-292; Rabbit, 404; Man, 546,549

Pronephros: Frog, 185-191; Chick, 318-319; Rabbit, 421 
Pronucleus, female : 8, 13, 14; Frog, $100 ;{ }^{\mathbb{3}}$ Rabbit, 351

Pronucleus, male : 13,$14 ;$ Frog, 100

Protovertebra: Amphioxus, 84-85; Chick, 244,322, 324 ; Man, 482, 490, 495

Protovertebral segmentation: Chick, 326

Protozoa: characters of, 1-2 ; reproduction of, 34

Pterylia: Chick, 337

Pubes: Frog, 214 ; Chick, 334

Pulmo-cutaneous arch : $7 \% \circ \%, 179$

Pulmonary arch : Rabbit, 417

Pulmonary trunk: Chick, 306 ; Rabbit, 416 ; Man, 572, 573, 575

Pulp cavity of tooth: Frog, 211

Pupil: Chick, 279

Pygostyle: C'hick, 328

Pylangium : Frog, 168, 169

Pyramid of thyroid: Man, 558

Quadrate cartilage : Frog, 205-206, 207-208; Chick, 330

Quill : Chick, 336

RABBIT, 341-447: preliminary account, 341-344; the egg, 344-351; the early stages of development, 351-362; general history of the embryo, $362-371$; nervous system, 371-387 ; sense organs, 387-399; digestive system, 399-412 ; heart and blood-vessels, 412-421 ; excretory system, 421-426 ; cœlom, 427-428 ; muscular system, 429 ; skeleton, 429433 ; skin, 433-434 ; placenta, 434-445 ; bibliography, 445-447

Rate of development : Amphioxus, 48; Frog, 91 ; Rabbit, 342, 367

Rauber's layer : Rabbit, 358, 361

Recapitulation theory: 24-34, 45, 90

Recessus labyrinthi : Man, 541-542

Recessus vestibuli ; Frog, 142 ; Rabbit, 393, 395 ; Man, 541, 542

Rectal diverticula; Chick, 291

Rectification of cranial flexure: Frog, 120

Rectum : Chick, 291; Man, 549

Regeneration of uterine epithelium : Rabbit, 445; Man, 615

Reichert's ovum : Man, 472-476

Rejuvenescence : 17

Reproductive organs: Amplioxus, 88-89 ; Frog, 94-96, 197-198; Chick, 228_ $230,319-320$; Rabbit, $344-346,426$; Man, 449-455, 591598

Respiration of embryo: Chick, 228, 294; Rabbit, 435 ; Man, 613

Retina: Frog, 138; Chick, 278; Rabbit, 389; Man, 540

Rib : Chick, 327-328; Rabbit, 430-432; Man, 549

Ripening of egg : 7-11; Frog,96-99 ; Hen, 230-231 ; Rabbit, 349-350; Man, 471

Roots of cranial nerves : Man, 531, 534-535

Roots of spinal nerves: Man, 524-526

Rosenmüller, organ of : Man, 595

Sacculus: Frog, 141 ; Rabbit, 396 ; Man, 541-5 $\$ 2$

Saccus endolymphaticus: Frog, 142

Sacral vertebra : Chick, 328

Salivary gland: MIan, 559

Scala media: Rabbit, 397 ; Man, 543

Scala tympani : Rabbit, 397 ; Man, 543

Scala vestibuli : Rabbit, 397 ; Man, 543

Scapula: Fron, 213; Chick, 332

Schizocol : Chick, 243

Sclerotic: Rabbit, 392

Scrotal sac: Rabbit, 426

Scrotum : Man, 597

Second month: Man, 503-505 
Second week : Man, 472-486

Secondary gill-slits: Amphioxus, 76-78

Secondary sulci : Man, $519-520$

Segmental duct : Frog, 186-190, 193-194

Segmentation of egg: 14-15, 17-22, 27; Amphioxus, 49-52; Fvog, 100-104; Chick, 232-235; Rabbit, 352-354; Man, 471-472

Segmentation cavity : Amphioxus, 51-52; Frog, 103, 108-10.) ; Chich, 234

Segmentation nucleus: 13, 14; Frog, 100, 102

Semicircular canal : Frog, 141 ; Rabbit, 393, 395 ; Man, 541-542

Semilunar valve: Chick, 302; Rabbit, 416; Man, 573

Sense capsule : F'rog, 204-205 ; Chick, 328-330

Sense organs: Frog, 134-145; Chich, 274-281; Rabbit, 387-399; Man, 538-544

Sensory cranial nerves: Man, 528-530, 534-538

Sensory roots of spinal nerves: Anphioxus, 43 ; Frng, 127-128; Chick, 271273 ; Rabbit, 385-386; Man, 525-528

Separation of placenta: Rabbit, 444 ; Man, 614

Septum inferius: Man, 571, 573

Septum narium: Man, 555

Septum of truncus arteriosus : Frog, 168; Chick, 301, 302, 305; Rabbit, 416 ; Man, 571-572

Septum spurium : Man, 570

Septum superius : Man, 569

Seventh month: Man, 507-508

Sex, origin of: $34-35$

Sexual differentiation: Frog, 95; Rabbit, 346; Man, 453

Shell membrane: Hen, 220, 229

Sinus præcervicalis : MIan, 495, 502, 553, 559

Sinus terminalis : Chick, 310 ; Rabbit, 368, 420; Man, 483

Sinus venosus: Frog, 168, 183; Chick, 309; Rabbit, 413, 414; Man, 568, 579

Sinuses of placenta: Man, 612,614

Sixth month: Man, 507

Sixth week : MIan, 501-503

Size of eggs : 342

Skeleton: Frog, 198-215 ; Chick, 324-335; Rabbit, 429-433

Skin: Rabbit, $433-434$

Skull: Frog, 201-210; Chick, 328-332 ; Rabbit, 432-433

Somatic layer of mesoblast: Chick, 243 ; Rabbit, 427

Somatopleure : Chick, 243

Somite: Amphioxus, 60-61, 65-69, 84-87; Chick, 244

Spawn: Frog, 91, 99

Spawning period: Amphioxus, 46 ; Frog, 90

Spee's embryo: $\operatorname{Man}, 479-481$

Spermatozoon: 12, 13; Amphioxus, 44; Frog, 99-100 ; Chick, 232 ; Man, 466

Sphenoidal sinus: Man, 538

Spina vestibuli : Man, 569-571

Spinal cord : Frog, 116-117; Chich, 249-252 ; Rabbit, 381 ; Man, 521-528

Spinal nerves: Frog, 128; Chick, 271-274; Rabbit, 385-386; Man, 521-528

Splanchnic layer of mesoblast: Chick, 243 ; Rabbit, 427

Splanchnocœl : Amphioxus, 85-86

Splanchnopleure: Chick, 243

Spleen : Frog, i 85

Splitting of mesoblast: Chick, 243-244, 246; Rabbit, 427 ; Man, 483

Spongioblast: Man, 523-524

Stapes: Frog, 204, 209; Chick, 330 ; Rabbit, 398

Sternum: Frog, 213; Chick, 332; Rabbit, 432

Stomach: Chick, 290; Rabbit, 403 ; Man, 545, 546, 548

Stomatodæum: Frog, 145, 148-149 ; Chick, 259, 281, 286-289; Rabbit, 100 Man, $482,490,492,545$

Stratum compactum of decidua : Man, 610

Stratum spongiosum of decidua: Man, 610 
Subgerminal cavity : Chick, 235-236

Sucker : Frog, 92

Sulci : Man, 518-520

Superficial segmentation : 22

Super-foetation : Man, 609

Supra-plexus : Frog, 124

Suprarenal body: Chick, 320 ; Rabbit, 386-387

Suprascapula: Frog, 213

Sylvian aqueduct: Frog, 123; Chick, 256; Man, 520

Sylvian fissure : Man, 514, 516, 519

Sympathetic nerve system: Frog, 134; Chick, 274; Rabbit, 386-387; IIan, 538

Synangium: Frog, 169

Systemic arch: Frog, 178; Rabbit, 417

Systemic trunk: Chick, 306; Rabbit, 416; Man, 572, 573, 575

Tadpole : Frog, 92-94

Tail : Man, 492, 496, 503

Tarsus: ('hick, 335

Teeth: Tadpole, 150-151; Frog, 210-211; Rabbit, 404-407 ; Man, 559-561

'T'elolecithal egg : 7, 19; Frog, 101

Temporo-sphenoidal lobe of brain : Man, 517

Testis : Amphioxus, 44; Rabbit, 426; Man,

Thalamencephalon: Frog, 123-124; Chick, 256-260; Rabbit, 374-376; Man, 510,520

Third month: Man, 505-506

Third ventricle: Frog, 123-124; Chick, 256; Rabbit, 374

Third week: Man, 486-492

Thymus : Frog, 164; Chick, 286; Rabbit, 408; Man, 558-559

Thyroglossal duct: Man, 557

Thyrohyal: Prog, 209

Thyroid body: Frog, 152-153; Chick, 285-286 ; Rabbit, 407-408; Man, 557558

Thyroid cartilage: Man, 562

Toe : Man, 501, 503

Tongue : Frog, 152 ; Chick, 286 ; Rabbit, 402-403 ; Man, 555-557

Tongue-bar: Amphioxus, 78, 88

Tonsil : Frog, 163

Tooth sac: Rabbit, 407

Trabecula cranii : Frog, 203-205 ; Chick, 329, 331; Rabbit, 432

'Trachea: Chich, 292; Rabbit, 409 ; Man, 562

'Tractus olfactorius : Man, 517

Tragus : MITan, 500

'Tricuspid valve : Man, 573

Trigonum olfact orium: Man, 517

True amnion: Chick, 245 ; Man, 477

Truncus arteriosus: Frog, 168-169 ; Chick, 300-303, 305-306 ; Rabbit, 416 ; Man, $566,567,571-573,574$

Tuberculum anterius helicis : Mran, 499

Tuberculum anthelicis : MIan, 499

Tuberculum antitragicum : Man, 499

Tuberculum impar: Man, 555-557

Tuberculum intermedium helicis : Man, 499

Tuberculum lobulare: Man, 499

Tuberculum of rib: Rabbit, 430

Tuberculum tragicum: IIan, 499

Tubuliferous tissue: Frog, 197; Rabbit, 345, 426; Man, 450

Tunica albuginea: Rabbit, $346^{\circ} ;$ Man, 452

Tunica fibrosa folliculi : Man, 454

Tunica granulosa: Rabbit, 349 ; Man, 45 !

Tunica propria folliculi : Man, 454 
Twisting of umbilical cord: Man, 601

'Tympanic cartilage : Frog, 143-144

Tympanic cavity: Frog, 143 ; Chick, 285; Rabbit, 398; Man, 544

'Tympanic membrane: Frog, 143; Chick, 285 ; Rabbit, 398 ; Man, 544, 550

Tympano-Eustachian passage: Frog, 143; Chick, 285; Rabbit, 397; Man, 544

Umbilical cord : Man, 505, 599-602

Umbilicus : Man, 506, 507, 509

Unequal segmentation: 19,22

Urachus : Man, 590

Ureter: Frog, 193-195; Chick, 319; Rabbit, 425 ; Man, 590

Urethral canal: Man, 597

Urinary organs: Frog, 185-197; Chick, 315-320 ; Rabbit, 421-426 ; Man, 588-591

Urinary tubules : Man, 590

Urinogenital passage: Rabbit, 412, 425 ; Man, 549, 596

Urohyal : Frog, 206

Urosacral vertebra: Chick, 328

Urostyle : Frog, 199-201

Uterine gestation: 344

Uterine glands : Rabbit, 437; Man, 458-459, 609-610, 612, 614-615

Uterus : Hen, 231 ; Rabbit, 426 ; Man, 594

Uterus masculinus: Rabbit, 426 ; Man, 593

Utriculus : Frog, 141; Rabbit, 396; Man, 541-542

Vagina : Rabbit, 426 ; Man, 594

Valves of heart: Frog, 167-169; Chick, 301-303; Rabbit, 414-416; Man, 573

Vasa aberrantia : Man, 593

Vasa efferentia: Frog, 197; Chick, 320; Rabbit, 426; Man, 593

Vas deferens: Chich, 320; Rabbit, $426 ;$ Man, 593

Veins: Frog, 183-185; Chick, 308-311; Rabbit, 418; Man, 578-583

Vein, afferent hepatic : Chick, 309; Man, 579

allantoic: Chick, 310, 314 ; Rabbit, 414 ; Man, 568, 581, 602, 613

anterior abdominal : Froy, 184

anterior cardinal : Frog, 183; Chick, 308; Man, 582

anterior vena cava : Chick, 308-309; Rabbit, 414

coronary sinus : Man, 582

Cuvierian: Frog, 184; Chick, 308; Rabbit, 414 ; Man, 568, 582

ductus venosus: Chick, 310, 314; Man, 581, 585, 586, 587

efferent hepatic: Chick, 310; Man, 579

external jugular: $\mathbf{M a n}, 582$

facial : Frog, 183

hepatic: Frog, 183

hepatic portal: Man, 580,581

iliac : Man, 581, 582

inferior jugular: Frog, 184

internal jugular: Man, 582

jugular: Frog, 183 ; Chick, 309 ; Man, 582

meatus venosus: Chick, 293,309, 311

mesenteric: C'hick, 310

pectoral : Chick, 309

posterior cardinal : Frog, 183; Chick, 308; Rabbit, 423; Man, 582

posterior vena cava: H'rog, 184; Chick, 309-311; Rabbit, 414; Man, 581

pulmonary: Frog, 185; Man, 582

renal portal : Frog, 184

sinus annularis: Man, 580,581

subclavian : Man, 582

vena Arantii : Man, 581

vena ascendens : MIan, 581 
Vein, vertebral : Chick, 309

vitelline: Frog, 183; Chich, 300, 309, 310, 314 ; Rabbit, 413, 414, 420 ;

Velar plates : Frog, 163 IIon, 568, 579-581, 602

Velar tentacles: Amphioxus, 80,81

Velum: Amphioxus, 40, 80

Velum medullæ anterius : Rabbit, 380

Velum medullæ posterius : Rabbit, 381

Venous valves of heart: Rabbit, 414

Ventral commissure of spinal cord: Man, 525

Ventral fissure of spinal cord: Chick, 250; Man, 527

Ventral roots of spinal nerves: Frog, 128; Chick, 273; Rabbit, 385; Man, 524

Ventricles of brain: Frog, 116, 122-123; see also Third, Fourth, Fifth, and Lateral Ventricles

Ventricle of heart: Frog, 168-169; Chick, 300-303; Rabbit, 415; Man, 566, 567,571

Ventricular septum : chick, 301, 302; Rabbit, 415; Man, 572-573

Vermis : Rabbit, 380

Vernix caseosa : Man, $\mathbf{5 0 8}$

Vertebral column: Frog, 198-201; Chick, 324-328; Rabbit, 430

Vertebral plate : Chick, 244

Vertebral segmentation : Chick, 327

Vesicle of brain : Frog, 118; Chick, 252; Rabbit, 371; Man, 481, 491

Vesicle of hemispheres : Frog, 126;Chick, 260; Rabbit,376; Man, 510, 516

Vesicula seminalis : Frog, 194-195

Villi of intestine : Man, 549

Villi of placenta : Rabbit, 441

Villous zone of placenta: Man, 612

Visceral arch: Frog, 159; Chick, 283-284; Rabbit, 400-401; Man, 487, 495, $498-499,550-553$

Visceral cleft: Frog, 92, 157-160; Chick, 283, 285 ; Man, 490, 492, 495, 551

Visceral groove: Chick, 283; 1Rabbit, 400; Man, 550,551

Visceral pouch: Frog, 157-160; Chick, 283; Rabbit, 400; Man, 550, 551

Visceral skeleton: Frog, 205-207; Chick, 330-331

Vitality of ovum: Man, 466

Vitelline circulation : Rabbit, 420-421

Vitelline loop of intestine : Chick, 290; Rabbit, 403; Man, 547, 548

Vitelline membrane: Amphioxus, 49; Frog, 96; Hen, 229; Rabbit, 349

Vitreous body : Rabbit, 392; Man, 539

Vocal cord: Man, 562

Vulva: Rabbit, 412

Whartonian jelly : Maan, 602

White matter of brain : Rabbit, 382

White matter of spinal cord: Chick, 250 ; Rabbit, 382

White of egg : Hen, 220, 229, 231

White yolk: Hen, 230

Wing: Chick, 332-334

Wolffian body: Frog, 191-193; Chick, 316-318, 320; Rabbit, 422-424 ; Man, $588-590,593,595$

Wolffian duct: Frog, 193-195; Chick, 315-316, 320; Rabbit, 421-422 ; Man. $588,593,595-596$

Wolffian ridge : $\mathbb{M}$ Ian, $492,496,501$

Wolffian tubules: Frog, 191-193; Chick, 316-318; Rabbit, 422; Man, 589, 593

Xiphisternum : Frog, 213

Yellow yolk: Hen, 230

Yolk: 4-7, 19, 29; Frog, 96 ; Chick, 220-222, 229 
Yolk-cells : Frog, 104, 105, 146

Yolk-plug: Frog, 107, 111, 147

Yolk-sac: Chick, 222, 226; Rabbit, 367-369; Man, 476, 483, 496, 544

Yolk-spheres : Hen, 230

Yolk-stalk: Chick, 226 ; Rabbit, 399, 403; Man, 544, 548, 601, 602

Zona pellucida : Man, 455

Zona radiata: Rabbit, 348 ; Man, 453

PRINTED BY 


\section{SIIITH, ELDER, \& CO.'S PUBLICATIONS.}

The FUNCTIONS of the BRAIN. By DAVID FERRIER, M.D., LL.D., F.R.S., Professor of Forensie Medicine, King's College; Physician to King's College Hospital; Physician to the National Hospital for the Paralysed and Epileptic. Secon.l Edition, Rewritten, with many New Illustrations, 8vo. $18 s$.

'No scientific medieal man's library ean be / own work, as well as those of other observers said to be complete without Dr. Ferrier's book,

which contains the records of the author's

The LOCALISATION of CEREBRAL DISEASE. By DAVID FERRIER, M.D., F.R.S., Assistant-Physician to King's College Huspital: Professor of Forensic Medicine, King's College. With numerous Illustrations. 8 vo. $7 s .6 d$.

\section{A COURSE of PRACTICAL HISTOLOGY. By EdWARD ALBERT SCHÄFER,} Assistant-Professor of Physiology, University College. With numerous Illustrations. Crown 8vo. 10s. $6 d$.

'We are very much pleased with $\mathrm{Mr}$. | treatise will find that he has not only gained Schäfer's book. It is clearly written, well and originally illustrated, and possesses the inerit of being both concise and complete. In conclusion we may say that anv student who has gone fairly over the ground covered by this

a mastery over the modern methods of microseopical investigation, but a sound knowledge of the several structures of which the body is composed.'-LANCET.

ATLAS of HISTOLOGY. By E. KLEIN, M.D., F.R.S., Lecturer on Histology at St. Bartholomew's Ho-pital Medical School, and NoBLE SmirH, F.R.C.S. Edin. L.R.C.P. Lond., \&c.; Surgeon to the All Saints' Children's. Hospital, and Orthopædic Surgeon to the British Home for Incurables. A complete Representation of the Nicroscopic Structure: of Simple and Compound Tissues of Man aud the bigher Animals, in carefully executed coloured engravings, with Explanatory Text of the Figures, and a Concise Account of the hitherto ascertainerl facts in Histology. Rnyal 4to. with 48 Coloured Plates, bound in half-leather, price $£ 4.4 s$.; or in 13 parts, price $6 s$. each.

MANUAL OP PATHOLOGICAL HISTOLOGY. By CORNIL and RANVIER. Translated by authority from the New and Re-written French Edition, with the original Illustrations. Vol. I. Histolngy of the Tissues. Demy 8vo. 21s. Vol. II. Special Pathological Histology. Lesions of the Orgaus. Part I. Demy 8vo. 12s. Part II. Demy 8vo. 16s.

'An admirable exposition of all that is known respecting the morbid histology of the tissues and organs of the human body. . . . We should be glad to see it in the hands of all medical students. and of all those who wish to keep themselves informed of the pre-

sent state of pathology:-LoNDON MEDICAI. RECORD.

'We may safely recommend the work as the foremost text-book of its class, and we are certain that it will now be widely studied by many to whom the original was a closed book.

LANCET.

A TEXT-BOOK of PRACTICAL HISTOLOGY. With 30 Outline Plates, 1 Coloured Plate and 27 Wood Eugravings. By WILLIA.M STIRLING, M.D., Sc.D., F.R.S.E. Regius Professor of the Institutes of Medicine in the University of Aberdeen. Demy 4 to. price $14 s$.

ELEMENTS of HUMAN PHYSIOLOGY. By Dr. L. HERMANN, Professor of Physiology in the University of Zuirich. Second Edition. Entirely recast from the Sixth German Edition, with very copious Additions and many additional Woodcuts, by ArThur Gamake, M.D., F.R.S., Brackenbury Professor of Physiology in Owens College, Manchester, and Examiner in Physiology in the University of Edinburgh. Demy 8vo.16s.

ELLIS'S DEMONSTRATIONS of ANATOMY : being a Guide to the Knowledge of the Human Body by Dissection. Eleventh Edition. Edited and revised by G. D. ThANE, Professor of Anatomy, University College, London. With 252 Ilustrations. Small 8 vo. $12 s .6 d$.

ILLUSTRATIONS of DISSECTIONS. In a Series of Original Coloured Plates, the size of Life, repre-enting the Dissection of the Human Bods. By G. V. Ellis and G. H. FonD. Imperial folin. 2 vols. balf-bound in morocco, $26.6 s$. May also be bad in Parts separately. Parts 1 to 28, 3s.6d. each: Part 29, 5s.

'With these plates, and such as these, by / secting room. With such plates as these, the his side, the learner will be well guided in his dissection: and, under their guidance, he may safely continue his study when out of the dissurgeon will be fully reminded of all that is needful in anatomy when engaged in planning an operation.-MEDICAL TIMES.

London: SMITH, ELDER, \& CO., 15 Waterloo Place. 
The DESCRIPTIVE ATLAS of ANATOMY. A Representation of the Anatomv of the Human Tsodv. In 92 Roval 4to. Plates. containing 550 lllustrations. Introducing Heitzmann's Figurea. considerably modified, and with many Original Drawings frnm Nature. By NoвI, SiIrH, F.R.C.S. Edin., L.R.C.P. Innd.: Surgeon th the All Saints' Children's Hospital and Orthopædic Surgeon to the British Home for Incurables. Bound in half-lenther, price $25 s$.

'Certainlv one of the most remarkahle pnb. lications of the dav. The great alvantage which it, presents is that all the attachments of bones, the artories, veins, \&c., are copiouslv lettered and described in sit $\iota$; and the arteries and veins are coloured. The hook is one of great utility and merit, and reflects credit on the artist, and alan on those who have produced it.'-Britrish Medical JodRnal.

A DIRECTOR F for the DISSECTION of the HUMAN BODY. By Jofr CLELAND, M.D., "F.R.S., Professnr of Anatomy in the University of Glasgow. Third Edition, thoroughly revised bv JOHN YULE MACKAY, M.D., Senior Demonstrator of Anatomy in the University of Glasgow. Fep. $8 \nabla 0.3 s, 6 d$.

A HANDBOOK of OPHTHALMIC SCIENCE and PRACTICE. Illustrated by numerous Woodeuts and Chromo-lithogranhs of Microscopic Drawings of Disenses of the Fundus and other Parts of the Fve. By HRNRY JuLer, F.R.C.S., Junior Ophthalmic Surgann. St. Mary's Hospital : Senior Surgenn, Royal Westminster Ophthalmic Hospital ; late Clinical Assistant, Moorfields ; Demonstrator of Anatomy, St. Mary's Hospital, London. 8 ro. $18 s$.

- Altogether the book is one of which we are able to sneak in terms of unqualified approval, and will d ubtles a at once take its place as a favourite, trustworthy, and explicit guide

in the study and treatment of diseases of the eye and disorders of the vision, and, as such. be extensively used by practitioners and students.'-British MedicaL JourxaL.

\section{A TREATISE on the THEORY and PRACTICE of MEDICINE. By} JoHN SYer BuIsTowE, M.D. Lond., Fellow and formerly Censor of the Royal College of Physicians; Senior Physician to, and Joint-Lecturer on Mericine at, St. Thomas's Hospital; President of the Society of Medical Officers of Health ; Examiner in Medicine to the Royal College of Surgenns; formerly Examiner in Medicine to the University of London, and Lecturer on General Pathology and on Physiology at St. Thomas's Hospital. Seventh Edition. 870. price 21s.

'The busy practitioner will be able by its perusal to keep ahreast with the great progress which scientific medicine bas made within the past few years, and for which he has neither the time nor frequently the opportunity to

consult larger treatises, monographs, and journals. The style of the work is plain and lucid; though condensed it is never bald. As an accurate and praiseworthy guide, it is of the highest order of merit.'

BOSTON MEDICAL AND SURGICAL JouRNat.

CLINICAL MANUAL for the STUDY of MEDICAL CASES. Edited br James Finlayson, M.D., Pnysician and Lecturer on Clinical Medicine in the Glasgow: Western Infirmary. With Special Chapters by Prof. GAIRDNER on the Physiognomy of Disease; 1)r. AleXaNder RoBerTson on Insanity: Dr. Joseph COATs on the Throat; Professor STephesson on Disorders of the Female Organs; Dr. SAmson Gemmell on Physical Diagnosis. Third and Cheaper Edition, Revised, with Illustrations. Crown 8vo. 8s. $6 d$.

An INDEX of SYMPTOMS as an AID to DIAGNOSIS. By RALPII Winivingon LFFTwich, M.D., late Assistant-Physician to the East London Children's Hospital. Fep. 8vo. $5 s$.

* * Each Symptom is followed by an Alphabetical List of Diseases in which it occurs.

A IMANUAL of GENERAL PATHOLOGY. By Joseph F. PAyne, M.D., F.R.C.P., late Fellow of Magdalen College. Oxford; Physician and Joint-Lecturer on Patholozical Anatomy at St. Thomas's Hospital : Examiner in Pathology in the University of Oxford; late Examiner in Merlicine to the Royal College of Surgeons of Fingland, \&c. With Illustrations. Crown 8vo. 12s.6d.

A PRACTICAL TREATISE On URINARY and RENAL DISEASES, incluaing URINARY DEPOSITS. Illustrated by numerous Cases and Engravings. Fourtli Edition. Reviserl and Enlarged. By Sir WM. RoBERTs, M.D.. F.R.S., Assisted by Rober'T MACG Uilze, M.D. Lond.. Member of the Royal College of Physicians, London; late Pathologist to the Alanchester Royal Infirmary. Small 8v0.12s. $6 \mathrm{~d}$.

London: SMITH, ELDER, \& CO., 15 Waterloo Place. 


\section{SHITH, ELDER, \& CO.'S PUBLICATIONS.}

A TREATISE on the SCIENCE and PRACTICE of MIDWIFERY. By W. S. PL.IYFAlk, M.U., F.K.C.Y., Physician-Accoucheur to H.l. and R.H. tl.e Duchess of Edwburgh ; Protessor of Obstetric Medicine in King's College; Physiciun for tlie Diseases of Women und Children to King's College Hospital; Cousulting Physician to the General Lying-in Hospital, and to the Evelina Hospital for Children; late Presideut of the Obstetrical society of Londou ; Examiuer in Midwi, ery to the University of Loadon, and to the Royal College of Physicians. 2 vols. demy 8 vo. Seventh Edition. With

187 Illustrations and 6 Plates. 28s.

'These volumes take position in the Lighest $\mid$ it to practitioners, teachers, and students. rank of obstetric worke. - LANCE's.

"We need scarcely say that we recommend $f$ in second to no similar treatise in our

A SYSTEM of OBSTETRIC MEDICINE and SURGERY, THEORETICAL and Clinical. For the student and rractitioner. By IluBer'T BakNes, M.D., Obstetric I'hysician to the St. George's Hospital; Consulting Puysician to the Chelsea Hospital for Women, dic.; and FAxcuUR'T Barnes, M.L., Physician to the Royal Maternity Charity and to the British Lying-in Hospital ; Obstetric Physician to the Great Northeru Hospital ; Pnysician to the Chelsea Huspital for Wumen. Complete in 2 vols. with numerous Illustrations. 8vo. Vol, I., 18s.; Vol. II., 20s.

'The book needs but to be read to be appreciated. The illustrations are numerous and well executed, contributing materially to the usefulness of the work. The book is well "got up," handy in size, and will un.

questionably prove a valuable companion, whether to the student acquiring knowledge of obstetrics, or to the busy practitioner anxious to keep pace with the tinies.'

British Mikdical Journal.

A MANUAL of MIDWIFERY for MIDWIVES. By FANCOURT BARNEs, M.D. Aver., M.R.C.P. Lund.; Yhysleian to the Royal Maternity Charity; Phy:ician to the British Lying-in Hospital; Oustetric Physician to the Great Nurthern Hospital; Physician to the Chelsea Hospital for Women. Fitth Edition. Crown 8vo. With uumerous Illustrations. $6 s$.

A SYNOPTICAL GUIDE to the STUDY of OBSTETRICS : being an Aid to the student in the Class-room, in Private study, and in 1 reparng for Examications. By Robert BarNes, M.D., Ubstetric Physician to St. Geurge's Huspital; Consulting Physician to the Cnelsea Hospital for Women, dcc. Crown 8vo. $3 s$.

CLINICAL and PATHOLOGICAL OBSERVATIONS on TUMOURS of the UVAK 1 , HALLUPIAN ' UBE, and BRUAU LIGAMLN'. By ALBAN H. G. DORA., F.R.C.S., Assistant-surgeon to the Samaritan Free Hospital, formerly Anatomical aud Pathological Assistant to the Museum of the liojal College of surgeons of En land. With 32 Illustratiuns. 8 vo. 1 Us. 6 .

A TREATISE on SURGERY: its Principles and Practice. By T. Holmes, M.A. Cantab., H.K.C.S., Consulting Surgeon to St. George's Hospital; Memb. Associé de la Soc. de Chir. de Paris. Filta Edition. Edited by T. PICKERlNe P1CK, Surgeon to, and Lecturer on Sursery at, st. George's Horpital; Senior Surgeon, Victuria Hospital for Childreu; Member of the Court of Examiners, Royal College of Surgeons of England. With 428 Illustrations. Royal \&vo. 31 s.

'Mr. Pick has reviserl the whole book most thoroughly, has carefully brought it up to date, and uas incorporated a grtat deal of the mouern teaching in patbology und practical surge ry. ... We are sure that this edicon will largely add to the great reputation which this texc book has for a loug time had. - LANCLT.

'This edition may fairly claim to have been brought thoruugbly up to the levtl of the most recent knowledge, and no doubt will retain the place which this favourite handbook has gained as one of the staudard treatises on surgery.'-BRitish Medical JuURNaL.

A DICTIONARY of PRACTICAL SURGERY. By VARIOUS BitTisi

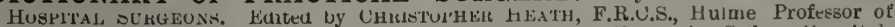
Clinical surgery in University College, London; Surgeun to University College Hospital; Member of the Cuuncil aud Court of Examiners of the Royal College of Surgeons ut Euglund. In 2 vols. comprising 1,864 pages. Turd biation. Koyal 8 vo. prlce $32 s .$, bouni in cloth ; or, in half-morocco, marbied edges, $42 s$.

'Altogether the work is a! credit to the euergy wî author $₫$, editor, aud publisher, and will rank highly wherever Biitish Suigery is consulted, and whererer the linglish lamguave is understood. All its cuntributur's are to be congratulated upoul its appearauce.'

BHTTISII MEDical JoURNaL.

'The Dietionary will stand undoubtedly as one of the ruost trustworthy works of reference publishel in modern times upon practical surgery, and will form a valuable and almost essential occupant or the practitioners' librars.' LONDUX IIEDICAL RECORD.

London: SMITH, ELDER, \& CO., 15 Waterloo Place. 


\section{SAIITH, ELDER, \& CO.'S PUBLICATITONS.}

An INDEX of SURGERY; being a Concise Classification of the Main Facts and Theories of Surgery for the Use of Senior Students and others. By C. IB. KEETLEY, F.R.C.S., Surgeon to the West London Hospital, and to the Surgical-Aid Society. Fourth Edition. Crown 8vo. 10s. $6 d$.

- Will prove truly valuable, and will, we trust, for many years be kept up to the imperious demands of surgical progress. The system of arrangement is just what the system in such a publication should ever be, purely alphabetical, aud the text is written in as elegant and intelligible English as can be expected in condensations and abridgments.'

BRitish MindCar, JoURNaL.

'Mr. Kectley's work fills a gap that has long

existed in the educational literature of surgery. Previous at'empts have been made to produce a book of the same character, but, compared with the "Index," they have all been crude and ineffectual. We heartily congratulate $\mathrm{Mr}$. Keetley on his performance, and as heartily thank him for conferring a real boon on medical students by thia much-needed and excellently executed aid to the study of surgery.'

MEdical News.

The SURGERY of DEFORMITIES. A Manual for Students and Practitioners. By Noble SMiTh, F.R.C.s. Edin., L.R.C.P. Lond., Surgeon to the All Saints' Children's Hospital; and Orthopædic Surgeon to the British Home for Incurables. With 118 Illustrations. Crown $8 \mathrm{vo} .10 s .6 d$.

'The woodcuts show very practically the points which they are intended to illustrate, and materially help the reader. We can recommend this as one of the most practical, useful, and able handbooks of Orthopædic Surgery. It is one which will be alike popular and useful with practitioners and students, and establishes for its author a high position in the department of science and practice

which his handbook illustrates.'-BritisH MEDICAL JourNal.

"This is a sound practical guide to the treat. ment of bodily deformities, based evidently upon personal observation and experience.... We can cordially recommend the work as a gnide to busy practitioners who will find in it just what they want clearly set forth and illustrated.'-LONnov MEDICAL RECORD.

ANTISEPTIC SURGERY : its Principles, Practice, History, and Results. By W. Watson Cheyne, M.B., F.R.C.S.. Assistant-Surgeon to King's College Hospital, and Demonstrator of Surgical Pathology in King's College. With 145 Illnstrations. 8vo. 21 s.

'In the volume before us $\mathrm{Mr}$. Cheyne has made a very valuable addition to surgical literature. The intimate professional relations of Mr. Cheyne with Professor Lister give a special importance and value to this work; for while

Mr. Lister's resulta and views have hitherto been published only fragmentarily in journals and transactions of learned societies, Mr. Cheyne's book affords a trustworthy and complete statement of them.'-LANCET.

DISEASES of the BONES: their PATHology, Diagnosis, and TreatMENT. By Thomas JuNks, F.R.C.S. Eng., B.S. Lond., Surgeon to the Manchester Royal Infirmary; Lecturer on Prantical Surgery in the Owens College, Victoria University; Consulting Surgeon to the Children's Hospital, Pendlebury, Manchester. With 7 Chromolithographic Plates, 9 Etchings, and 77 Woodouts. 8 vo. 12s. $6 d$.

\section{A MANUAL of DENTAL SURGERY and PATHOLOGY. By ALFRED}

COLEMAN, L.R.C.P., F.R.C.S. Exam., L.D.S., \&c. ; Senior Vental Surgeon and Lecturer on Dental Surgery to St. Bartholomew's and the Dental Hospital of London; Member of Board of Examiners in Dental Surgery, Rnyal College of Surgeons ; late President Odontological Society of Great Britain. With 388 Illustrations. Crown 8vo. 12s. $6 d$.

' It is always satisfactory to come across a jects on which he has especially worked, nor manual written by an author thoroughly minimise in importance others to which he lias acquainted with his subject, and with a just given less attention. The manual is well sense of proportion, not disposed to magnify balanced, clear, simple, and exact, and is an exdetails because they happen to include subcellent student's book.'

LONDON MERICAL RECORD.

MANUAL of the ANTISEPTIC TREATMENT of WOUNDS. For Students and Practitioners. By W. WATson CHEYNE, M.B., F.R.C.S., Assistant-Surgeon to King's College Hospital; Surgeon to the Paddington Green Children's Hospital, \&c. With Illustrations. Crown 8 vo. $4 s, 6 d$.

A SYSTEM of SURGERY: Pathological, Diagnostic, Therapeutic, and Operative. By SAMUEL D. Gross, M.D., LL.D., D.C.L. Oxon. Sixth Edition, greatly Enlarged and thoroughly Revised. With upwards of J,600 Illustrations. 2 vols, 8 ro. £3. 10s.

London: SMITH, ELDER, \& CO., 15 Waterloo Place. 


\section{SMII'H, ELDER, \& CO.'S PUBLICATIONS.}

\section{GENERAL and DIFFERENTIAL DIAGNOSIS of OVARIAN TUMOURS,}

with special Reference to the Operation of Uvariotomy, and Occasional Pathological and 'I herapeutical Considerations. By WAshington L. A'tleE, M.D. With 39 Illustrations. 8vo. 20s.

The QUESTION of REST for WOMEN DURING MENSTRUATION. By Mary PUTwaM JAcoBI, M.D., Professor of Materia Medica in the Women's Medical College, New York. With Illustrations. Demy 8vo. 12s.

A GUIDE to THERAPEUTICS. By Robert Farquharson, M.P., M.D. Edin., F.R.C.P. Lond., late Lecturer on Materia Medica at St. Mary's Hospital Medical School, \&c. Fourth Edition. Thoroughly revised. Crown 8ro. $7 s .6 d$.

THERAPEUTICS : its Principles and Practice. By H. C. Wood, M.D., LL.D., Professor of Materia Medica and Therapeutics and Clinical Professor of Diseases of the Nervous System in the University of Pennsylvania. Being the Seventh Edition of 'A TREATISE on THERAPEUTICS,' Rearranged, Rewritten, and Enlarged. 8vo. 16s.

OCULAR THERAPEUTICS. By L. DE Wecker, Professor of Clinical Ophthalmology, Puris. Trauslated and Edited by LiTTox FonBEs, M.A., M.D., F.R.G.S., late Clinical Assistant, Koyal London Ophthalmic Hospital. With Illustrations. Demy 8vo. 16s.

A PRACTICAL TREATISE on the DISEASES of the HEART and GREAT VESsELS, including the Principles of their Physical Diagnosis. By WALTER HAYLE WALShe, M.D. Fourth Edition, thoroughly Revised and greatly Enlarged. Demy 8 vo. $16 s$.

A PRACTICAL TREATISE on DISEASES of the LUNGS, including the Prunciples of Physical Diagnosis and Notes on Climate. By WAlter HAYLE WALshe, M.D. Fourth Edition, Kevised and much Enlarged. Demy 8vo. 16 s.

AUSCULTATION and PERCUSSION, together with the other Methods of Physical Examination of the Chest. By SAMUEL GEE, M.D. With Illustrations. Third Ldition. Fep. 8vo. $6 s$.

SKIN DISEASES, including their Definitions, Symptoms, Diagnosis, Prognosis, Morbid Anatomy, and Treatment. A Manual for Students and Practitioners. By Malcolm Morilis, Surgeon to the Skin Department, St. Mary's Hospital, and Lecturer on Dermatology in the Medical School. With Illustratious. Crown 8vo. 7s. 6d.

\section{HANDBOOK of MODERN CHEMISTRY, INORGANIC and ORGANIC.}

By the late MEYmotr TiDY, M.B., F.C.S., Professor of Chemistry and of Medical Jurisprudence and Public Health at the London Hospital; one of the Official Analysts to the Home Office; Medical Officer of Health for Islington; late Deputy Medical Officer of Health and Public Analyst for the City of London; Master of Surgery, \&c. \&c. Second Edition. Revised and Enlarged. Demy 8 vo. 18 .

The ELEMENTS of PHYSIOLOGICAL and PATHOLOGICAL CHEMISTliY. A Handbook for Medical Students and Practitioners. By T. Crasstolx Charles, M.D., F.C.S., \&c., Dlaster of Surgery; Lecturer on Practical Physiology, St. Thomas's Hospital, London; ; late Medical Registrar, St. Tliomas's Hospital, \&c. \&c. With 38 Woodcut Illustrations and 1 Chromo-lithograph. Demy 8vo.12s.6d.

London: SMITH, ELDER, \& CO., 15 Waterloo Place. 
SCHOOL HYGIENE AND DISEASES INCIDENTAL TO SCHOOL

LIFE. By Robert Farquharson, M.P., M.D. Edin., F.R.C.P. Lond., late Lecturer on Materia Medica at St. Mary's Hospital Medical School, \&c. Crown 8 vo. 7 s, $6 d$.

NOTES FROM SICK ROOMS. By Mrs. Leslie Stephen. Crown 8 vo. limp cloth, $2 s$.

FIRST AID TO THE INJURED. Five Ambulance Lectures by Dr. Friedricil Esmarch. Translated from the German by H.R.H. Princess Christian. Second Edition. With Illustrations. Post 8 vo. 2s.

OUTLINES OF NAVAL HYGIENE. By John D. Macdonald, M.D., F.R.S., Inspector-General R.N., Professor of Naval Hygiene, Army Medical School, Netley. Crown 8vo. With Illustrations. 7s. $6 d$.

THE YOUNG DOCTOR'S FUTURE: or, What shall be my Practice? By E. Diver, M.D. Being some Account of Appointments in the Mercantile Marine, Emigration Agencies, the Army and the Navy. With Hints as to PoorLaw Appointments, Clubs, \&c., and the Method of Cieneral Practice. Second Edition. Crown 8vo. 4 s.

CREMation of The DEAD. By William Eassie, C.E. Crown 8vo. With Illustrations. 8s. $6 d$.

\section{THE CURATIVE EFFECTS OF BATHS AND WATERS : being} a Handbook to the Spas of Europe. By Dr. J. Braun. With a Sketch on the Balneotherapeutic and Climatic Treatment of Pulmonary Consumption, by Dr. L. Rohden. An Abridged Translation from the Third German Edition, with Notes. By Hermann Weber, M.D., F.R.C.P. London, Physician to the German Hospital. Demy 8 vo. 18 s.

THE DOMESTIC MANAGEMENT OF CHILDREN. By P. M. Braidwood, M.D., Surgeon to the Wirral Hospital for Sick Children. 2s. $6 d$.

HOUSEHOLD MEDICINE : Containing a Familiar Description of Diseases, their Nature, Causes, and Symptoms, the most approved Methods of Treatment, the Properties and Uses of Remedies, \&c., and Rules for the Management of the Sick Room. Expressiy adapted for Family Use. By JoHn Gardner, M.D. Twelfth Edition, With numerous Illustrations. Demy 8vo. 12s. $6 d$.

To this Edition is added a plan for reading it systematically, to enable the non-professional reader to acquire a sound knowledge of the medical sciences, such as every well-educated person should possess; with an account of Homœeopathy, Hydropathy, and all the Systems of Medicine extant, Baths, Spas, Mineral Waters, \&c. \&c.

LEGAL MEDICINE. Part I., with Illustrations, royal 8vo. 25 s. Part II., royal 8vo. 2rs. By the late Charles Meymoti Tidy, M.B., F.C.S., Master of Surgery, Professor of Chemistry and of Forensic MIedicine at the London Hospital, Official Analyst to the Home Office, Medical Officer of Health fo Islington, late Deputy Medical Officer of Health and Public Analyst for the City of London, \&c.

A JUNIOR COURSE OF PRACTICAL ZOOLOGY. By A. Milnes Marshall, M.D., D.Sc., M.A., F.R.S., Professor in the Victoria University ; Beyer Professor of Zoology in Owens College; late Fellow of St. John's College, Cambridge. Assisted by C. Herbert HURST, Ph.D., Lecturer in the Victoria University, Demonstrator and Assistant Lecturer in Zoology, Owens College, Manchester. Third Edition, revised. With additional Illustrations. Crown 8vo. ros. $6 d$.

London: SMITH, ELDER, \& CO., 15 Waterloo Place. 
A work absolutely indispensable to every well-furnished library.'-THE TrMEs.

Price 15s. net per volume, in cloth; or in half-moroceo, marbled edges, 20s. net.

\title{
DICTIONARY Of NATIONAL BIOGRAPHY.
}

\section{Edited by LESLIE STEPHEN and SIDNEY LEE.}

Volume I. was published on January I, I885, and a volume has been issued every three months since that date.

\author{
A further Volume will be published on January I, April I, July I, and \\ October I of each year until the completion of the work.
}

\section{EXTRACTS FROM A FEW PRESS NOTICES OF THE WORK.}

TRUTH.- 'I am glad you share my admiration for Mr. Stephen's magnum opus THE MAGNUM OPUS OF OUR GENERATION"The Dictionary of National Biography." A dictionary of the kind had been attempted so often before by the strongest men-publishers and editors - of the day that I hardly expected it to succeed. No one expected such a success as it has so far achieved.'

The Athenæum. - 'The latest volumes of Mr. Stephen's Dictionary are FULL OF IMPORTANT AND INTERESTING ARTICLES. Great names strike the eye constantly as we turn the pages. ... Altogether the volumes are good reading. What is more important, the atticles, whether they are on small or great personages, are nearly all up to the high standard which has been set in the earlier portions of the work, and occasionally above it.'

Saturday Review.- 'From the name we have cited it will be seen that great pain have been taken with that portion of the Dictionary which relates to modern times, and this has been rightly done; for often nothing is more difficult than to tind a concise record of the life of a man who belonged to our own times or to those just preceding them. Consistently enough, the Editor has been careful to keep the work reasonably up to date.'

The Manchester Examiner And TIMES.- 'This magnificent work of reference has now left the tentative stage of its existence far behind, and Mr. Leslie Stephen and his contributors are well under weigh. ... We extend a hearty welcome to the latest instalment of a most magnificent work, in which both the editing and the writing appear still to improve.'
The Quarterly Review.-'A "DicTIONARY OF NATIONAL BIOGRAPHY,' or WHICH THE COUNTRY MAY BE JUSTI.Y PROUD, which, though it may need correct. ing and supplementing, will probably never be superseded, and which, in unity of conception and aim, in the number of the names inserted, in fulness and accuracy of details, in the care and precision with which the authorities are cited, and in the bibliographical information given, will not only be immeasurably superior to any work of the kind which has been produced in Great Britain, but will as far surpass the German and Belgian biographical dictionaries now in progress, as these two important undertakings are in advance of the two great French collections, which until lately reigned supreme in the department of Biography.'

The Spectator. - 'As each volume of the Dictionary appears, its merits become more conspicuous. . . The book ought to commend itself to as wide a circle of buyers as the "Encyclopædia Britannica."

The Lancet. - Its contents show no falling off in accuracy and completeness, so far as by a critical examination we have been able to discover, from those of the previous volumes, of which we have on all occasions spoken with praise. When completed, THE DICTIONARY WILL BE WELL NIGH INVALUABLF.

Tine Pall Mall Gazette.- 'As to the general execution, we can only repeat the high praise which it has been our pleasing duty to bestow on former volumes. To find a name omitted that should have been inserted is well-nigh impossible.'

London: SMITH, ELDER, \& CO., I5 Waterloo Place. 
THIRD EDITION. Imperial 8vo. price $\ell_{\text {I. II s. } 6 d .}$

\section{ANATOMY FOR ARTISTS.}

BY JOHN MARSHALL, F.R.S., F.R.C.S.

Professor of Anatomy Royal Academy of Arts; late Lecturer on Anatomy at the Government School of Design, South Kensington; Professor of Surgery in University College, London; Senior Surzeon to the University College Hospital, \&c., \&c.

lilustrated with 220 Original Drawings on Wood, by J. S. Cuthbert. Engraved by J. \& G. Nicholls.

BY THE SAME AUTHOR.

Folio, price $8 s$. in wrapper; or in portfolio, price $9 s$.

A RULE OF PROPORTION FOR THE HUMAN FIGURE.

Illustrated by JOHN S. CUTHBERT.

FOURTH EDITION. 4to. with folio Atlas, 2 Is.

\section{A DESCRIPTION OF THE HUIMAN BODY :} ITS STRUCTURE AND FUNCTIONS.

Ili.ustrated by Physiological Diagrams.

Designed for the use of Teachers in Schools, and of Young Men destined for the Medical Profession, and for popular instruction generally.

The Work contains 260 quarto pages of Text bound in cloth, and 240 Coloured Illustrations, arranged in II folio Plates, measuring 15 inches by $7 \frac{1}{2}$, in a limp cover.

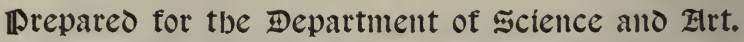 A SERIES OF LIFE-SIZED ANATOMICAL DIAGRAMS}

Specially adapted for Schools of Art and Art Students.

Seven Dia'grams, Life-size on paper, $7 \mathrm{ft}$. by $3 \mathrm{ft}$. gin. Each sheet sold separately, price I2.s. $6 d$., coloured in fac-simile of the Original Drawings: or $€ \mathrm{I}$. 1s. each, selected Proofs mounted on canvas, with rollers, and varnished. Explanatory Key, price Is.

To Teachers and Students of Artistic Anatomy this Series of Diagrams will be invaluable. Suspended on the walls of the lecture room or studio, they will not fail to secure, through the medium of the eye, that familiar acquaintance with the principal points in the osseous and muscular systems so indispensable to the Art Student.

\section{PHYSIOLOGICAL DIAGRAMS.}

An entirely New Edition, extended and Revised by the Author.

Eleven Diagrams, Life-size, each on paper $7 \mathrm{ft}$. by $3 \mathrm{ft}$. $9 \mathrm{in}$. . coloured in fac-simile of the Originals. I2s. $6 d$. each sheet; or selected Proofs, more highly coloured, mounted on canvas, with rollers, and varnished, price $£$ I. Is. each. Explanatory Key, price Is.

In the new Edition each subject has been re-drawn on the zinc, and, under the supervision of the Author, important additions have been made to the series, so as to render it as complete as possible and consistent with present state of Science.

** A detailed list of the Diagrams will be sent post free on application.

London: SMITH, ELDER, \& CO, I5 Waterloo Place. 





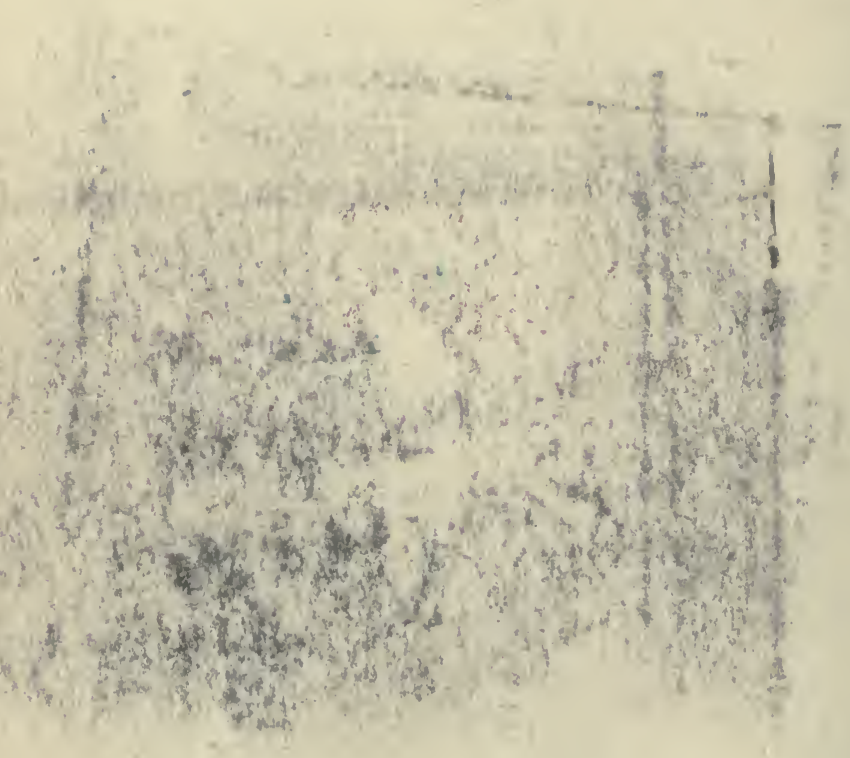


QL Marshall, Arthur Milnes

959 Vertebrate embryology

M37

Biological

in Medical

\section{PLEASE DO NOT REMOVE \\ CARDS OR SLIPS FROM THIS POCKET}

UNIVERSITY OF TORONTO LIBRARY 
